\title{
An Algorithm to Estimate the Lateral Position of Wheel-Rail Contact and Corresponding Rail Profile Radius
}

\author{
By \\ JARED G. VANDERWEES \\ A Thesis submitted to the Faculty of Graduate Studies of \\ The University of Manitoba \\ in partial fulfillment of the requirements for the degree of \\ MASTER OF SCIENCE
}

Department of Civil Engineering

University of Manitoba

Winnipeg, Manitoba

Copyright $@ 2018$ by Jared Vanderwees 


\section{ABSTRACT}

This research develops, verifies, and validates an algorithm to estimate the lateral position of wheel-rail contact and corresponding rail profile radius. The lateral contact position and the contact radius are two rail profile performance measures developed by this research that enable more refined characterization of the rail profile and its influence on rail wear and vehicle dynamics. This work directly builds on the past work by Hornaday $(2006,2010)$ and Abadpour \& Alfa (2007), both of which focused on the concept of the top-of-rail measurement. More broadly, the algorithm contributes to rail maintenance research and practice by developing new measures of performance based solely on commonly available rail profile data.

This research develops an algorithm using MATLAB ${ }^{\circledR}$ code based on the needs identified by the literature and review of current practice in the rail maintenance industry. The algorithm is an automated process that estimates the lateral position of wheel-rail contact and corresponding rail profile radii along a rail segment. The algorithm

- uses measured rail profile data as an input;

- applies rigid contact theory to model contact between a linear wheel profile and the rail profile

- calculates the lateral contact position and contact radius in the MATLAB environment;

- provides the results in graphic or numeric form on a profile-by-profile basis as well as summary statistics for each rail segment; and

- $\quad$ produces expected results when subjected to verification and validation tests.

The validation process analyzes the reasonableness of algorithm output against a series 
of expected results using rail profile data from selected tangent segments of a closed loop, captive fleet, North American rail transit property. The algorithm output does not significantly deviate from any of the expected results, and as a result, the algorithm is considered validated. 


\section{ACKNOWLEDGEMENTS}

Thank-you Dr. Regehr. Your encouragement, guidance, respectfulness, and passion for transportation engineering makes you not only a great advisor and professor, but an outstanding role model as well. Back in 2015, as a recent B.Sc. graduate with no career direction, I was in a meeting that you sat in on when you asked, "Would you consider a M.Sc. degree?" Back then, I could not have imagined what a wonderful experience this would turn out to be. Thank-you for believing in me.

To Gord Bachinsky, Teever Handal, Shane Kweens, and the rest of the Advanced Rail Management (ARM) staff: I am extremely grateful for your time, support, and guidance throughout this project. Your knowledge and passion for the railroad is clearly evident.

To Auja, Giuseppe, Caleb, Maryam, Steve and the rest of my UMTIG family: wow, what a journey! From all the memorable trips to office talks, waffle Christmas parties, and vying for stars during the roundabout, I feel so incredibly lucky to have spent these last three years with all of you. And then there is Karen, our UMTIG mom. The talks, the chips, the "you deserve it" encouragement, and the work you do makes UMTIG such an enjoyable and welcoming place to be. Everyday should be Karen Appreciation Day!

To my Mom, Dad, my brothers Steve and Mike, and my sister-in-law Felicia: you have unconditionally supported me not only through this degree, but also through all of life's ups-and-downs. I cannot say thank-you enough!

I would like to acknowledge the financial assistance provided by the Natural Sciences and Engineering Research Council of Canada, the American Railway Engineering and Maintenance-of-Way Association, and Transportation Association of Canada. 


\section{TABLE OF CONTENTS}

ACKNOWLEDGEMENTS

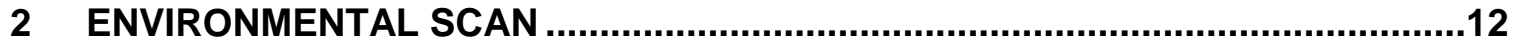

2.1 WHEEL-RAIL CONTACT AND WEAR ....................................................

2.2 OPTICAL RAIL PROFILE MONITORING .............................................. 16

2.2.1 Fundamental Concepts ............................................................. 16

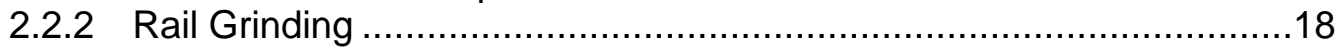

2.3 RAIL PROFILE PERFORMANCE MEASURES ..................................... 19

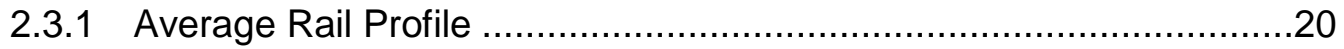

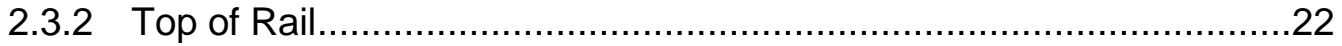

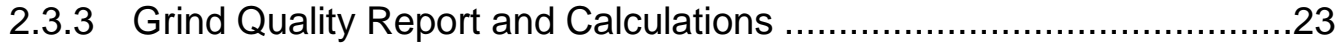

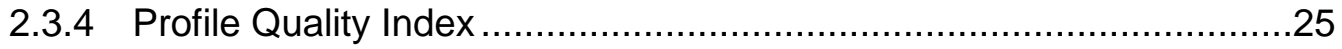

2.4 WHEEL-RAIL CONTACT MODELING APPROACHES ..............................27

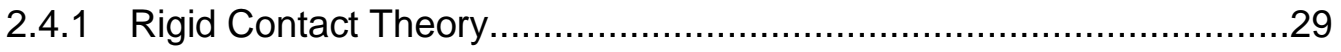

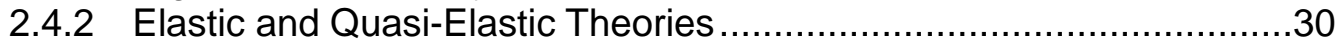

2.5 WHEEL-RAIL CONTACT RELATED SOFTWARE .................................

3 METHODOLOGY

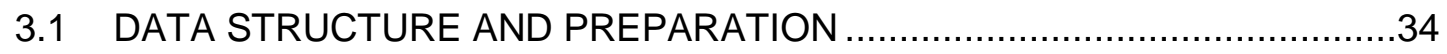

3.1.1 Track Segments, Rail Segments, and Rail Profiles ..........................34

3.1.2 Rail Profile Text Files .....................................................................

3.1.3 Exporting Rail Profile Data from Rangecam ................................41

3.1.4 Folder Structure and Naming Requirements .................................42

3.1.5 Sorting the Rail Profile Text Files ..................................................45

3.2 DESCRIPTION OF THE ALGORITHM ..............................................46

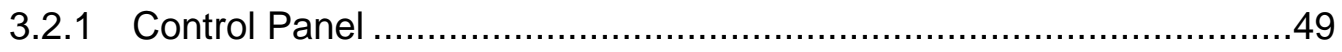

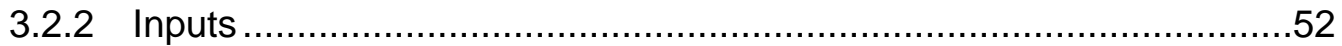

3.2.3 Analyzing Rail Profiles of a Rail Segment.....................................54

3.2.3.1 Text File to MATLAB ...........................................58

3.2.3.2 Plot Rail Profile......................................................63

3.2.3.3 Removing and Replacing Similar $\boldsymbol{x}, \boldsymbol{y}$-Coordinates............65

3.2.3.4 Correcting Order of $\boldsymbol{x}, \boldsymbol{y}$-Coordinates..........................68 
3.2.3.5 Rotate Rail Profile ..................................................69

3.2.3.6 Find Monotonically Increasing Series of $\boldsymbol{x}, \boldsymbol{y}$-Coordinates...74

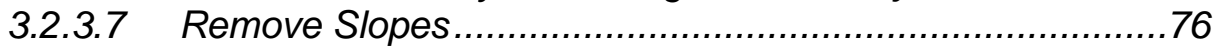

3.2.3.8 Transform Matrix ......................................................... 79

3.2.3.9 Smooth Rail Profile Data ..............................................82

3.2.3.10 Create Surface Slope Polynomial......................................85

3.2.3.11 Determining the Contact Point........................................86

3.2.3.12 Determining the Rail Profile Radius .................................. 91

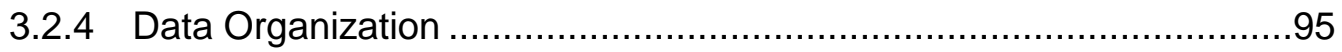

3.2.5 Output to Microsoft Excel .........................................................99

3.2.5.1 MATLAB Algorithm..................................................100

3.2.5.2 Track Segment Excel File............................................101

3.3 VERIFICATION OF THE PROCEDURE ................................................111

4 ALGORITHM VALIDATION THROUGH THE ANALYSIS OF RAIL PROFILE

DATA

4.1 SELECTION AND CLEANING OF VALIDATION DATA ….......................113

4.2 VALIDATION OF THE PROCEDURE ................................................119

4.2.1 Analysis of Track Segments .....................................................119

4.2.2 Algorithm Validation Criteria and Results ...................................124

4.2.2.1 Expected Result 1 ..................................................125

4.2.2.2 Expected Result 2 ................................................127

4.2.2.3 Expected Result 3 ................................................132

4.2.2.4 Expected Result 4 ..............................................137

4.2.2.5 Summary of Validation ...............................................141

5 CONCLUSION AND RECOMMENDATIONS ..................................................143

5.1 SUMMARY OF KEY FINDINGS AND LIMITATIONS .............................143

5.1.1 Key Contributions and Findings...................................................143

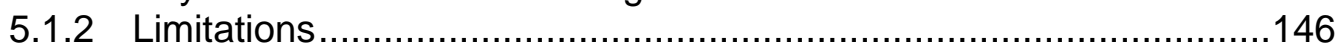

5.1.2.1 Inclusion of only Tangent Segments in the Validation.......146

5.1.2.2 The Simplification of the Wheel Profile to a Straight Line.. 148

5.1.2.3 Lack of Independent Ground Truth Data ........................148

5.1.2.4 Limited Rail Transit Property Maintenance Information ....149

5.1.2.5 The Applicability of the Algorithm Using Other Rail Profile

Data Sources and Future Implementation .....................................150

5.2 RECOMMENDATIONS FOR FUTURE RESEARCH AND WORK ................150

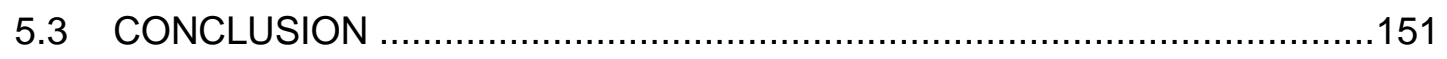

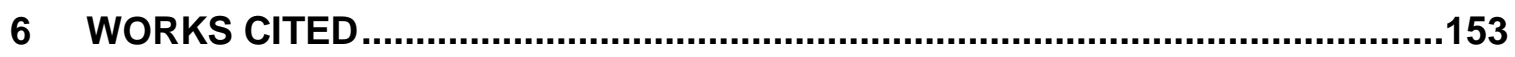

Appendix A: Procedure to Export Rail Profile Data from Rangecam

Appendix B: Procedure to Export the Track Segment Information from Rangecam

Appendix C: Rail Profile Text File Sorting Subroutine

Appendix D: Northbound Track Segment Analysis and Sensitivity Analysis Results

Appendix E: Southbound Track Segment Analysis and Sensitivity Analysis Results 


\section{LIST OF TABLES}

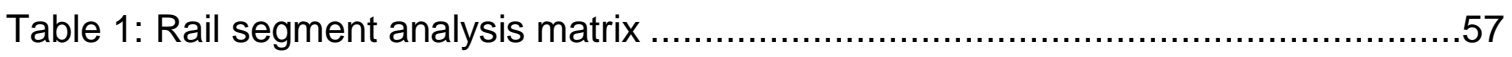

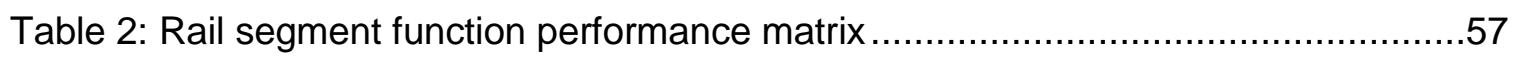

Table 3: Example of selection unique $x, y$-coordinates from the temporary matrix (a) and

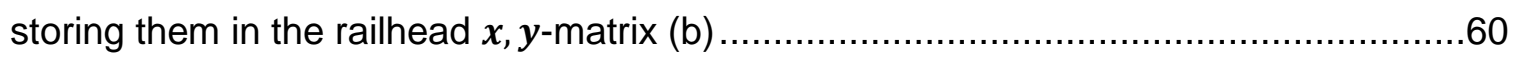

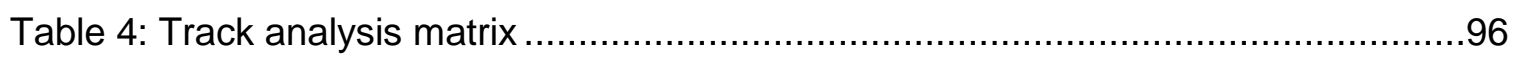

Table 5: Examples of the rail segment statistics tables containing statistics for (a) the rail

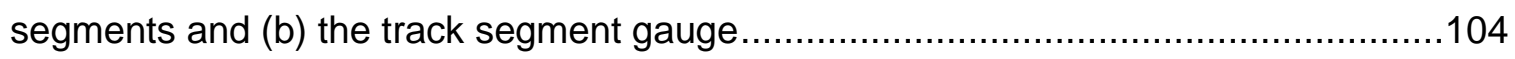

Table 6: Smoothing function performance table ...............................................107

Table 7: Least squares, fitted circle function performance table .............................107

Table 8: Rail segment statistic table from verification test using identical rail profiles ...111

Table 9: Rail segment statistics table from verification test using unworn 136 -lb rail

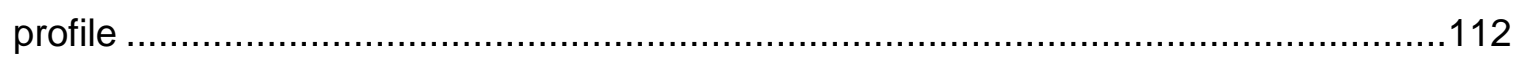

Table 10: Selected northbound track segments ..............................................115

Table 11: Selected southbound track segments .............................................116

Table 12: Six wheel slope conditions used in validation ......................................119

Table 13: NB-4 mean lateral contact positions................................................127

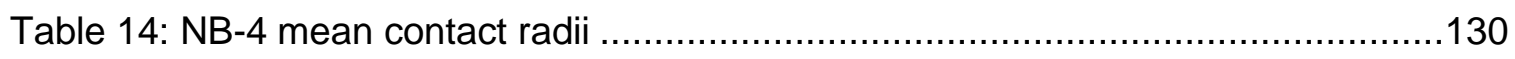

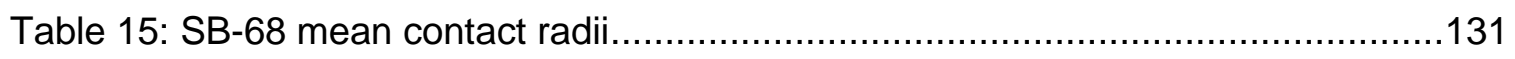

Table 16: NB-14 standard deviation of the lateral contact position...........................139

Table 17: NB-60 standard deviation of the lateral contact position............................141

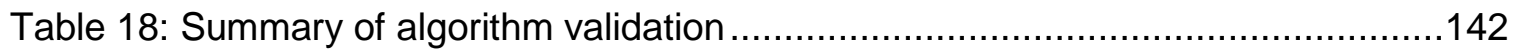




\section{LIST OF FIGURES}

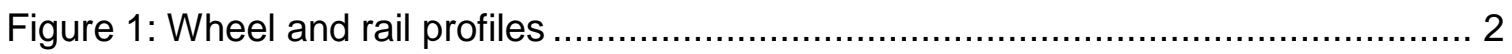

Figure 2: Grind template superimposed over a rail profile ..................................... 3

Figure 3: Wheel profile transformation to the algorithm's linear wheel profile ................ 7

Figure 4: Contact point and lateral contact position example ..................................10

Figure 5: Track level and wheel slope example ..............................................11

Figure 6: Wheel contact radius in (a) curve track and (b) tangent track ......................13

Figure 7: Optical rail sensor placement and alignment relative to the track.................17

Figure 8: Average rail profile (red line) of the rail segment profiles (purple lines) ..........21

Figure 9: Grind zone boundaries and crown radius...............................................24

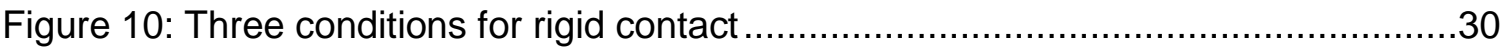

Figure 11: Segmentation: track segments, rail segments, and rail profiles....................35

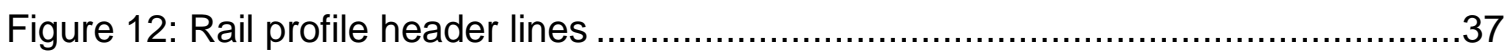

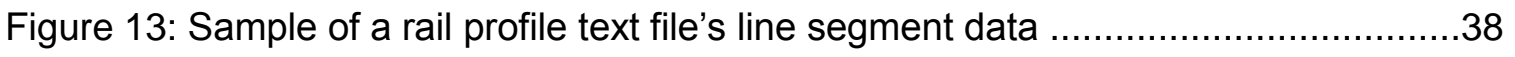

Figure 14: Example of line segments of a rail profile text file in Cartesian coordinates ..39

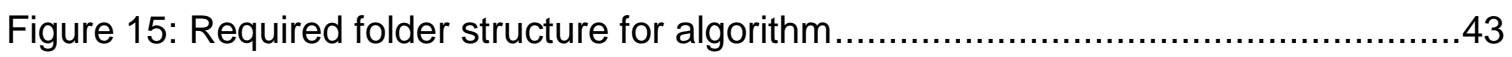

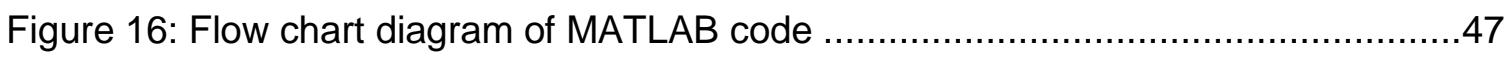

Figure 17: Simplified flow diagram of main functions ........................................ 49

Figure 18: Simplified flow diagram of analyze rail functions ..................................49

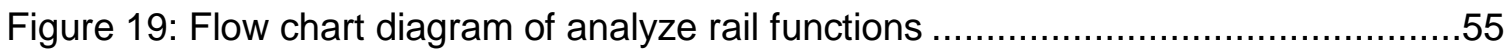

Figure 20: $\boldsymbol{x}, \boldsymbol{y}$-coordinates from a railhead $\boldsymbol{x}, \boldsymbol{y}$-matrix representing the railhead .........61

Figure 21: Example of an expected railhead profile shape...................................62

Figure 22: Example of a railhead profile removed from the analysis .........................63

Figure 23: The post track analysis left rail profiles plot (a) and right rail profiles plot (b).65 
Figure 24: Example of removing and replacing similar $x, y$-coordinates. .67

Figure 25: Rail profile in original orientation prior to rotation .................................. 71

Figure 26: Rail profile rotated by cant angle with a superimposed wheel profile ...........72

Figure 27: Rail profile rotated by cant angle and then rotated by the wheel slope.........72

Figure 28: Comparison of rail profile before and after rotation using a 1:5 wheel slope .73

Figure 29: Largest set of $\boldsymbol{x}, \boldsymbol{y}$-coordinates that are monotonically increasing $. . . \ldots \ldots \ldots \ldots . . . . . .75$

Figure 30: Removal of $\boldsymbol{x}, \boldsymbol{y}$-coordinates with average slopes larger than 20 degrees $\ldots . . .78$

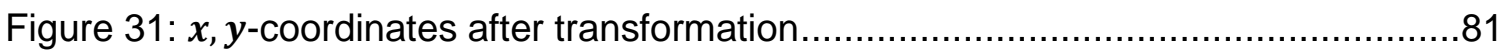

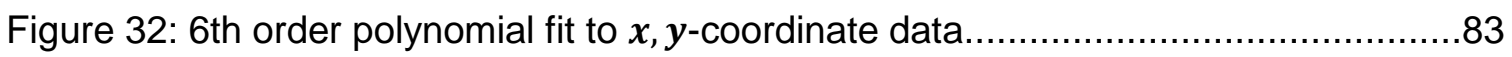

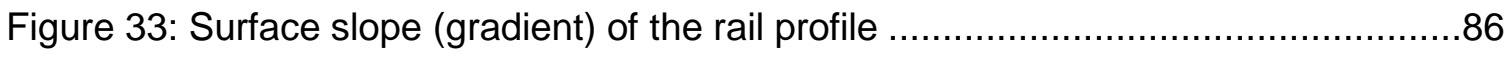

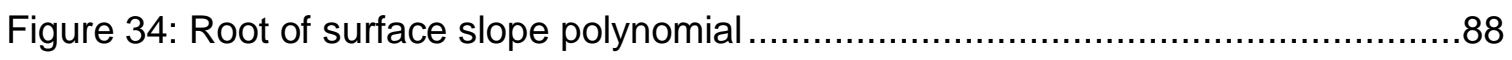

Figure 35: An example rail profile with two potential contact points ............................89

Figure 36: Interpenetration and potential contact points concept .............................90

Figure 37: The lateral contact point in the original orientation (1:5 wheel slope) ...........91

Figure 38: Fitting least squared, constant radius circle to radius $\boldsymbol{x}, \boldsymbol{y}$-matrix data..........94

Figure 39: Location of the track segment information tables...............................102

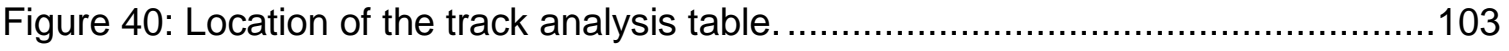

Figure 41: Location of the rail segment statistics tables ...................................... 104

Figure 42: A reformatted version of the rail segment graphs .................................105

Figure 43: Location of the rail segment graphs ...................................................106

Figure 44: Reformatted analysis performance graph: average distance between profile

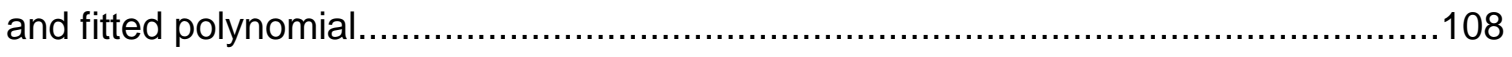

Figure 45: Reformatted analysis performance graph: average distance between profile

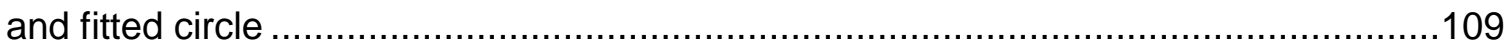

Figure 46: One-page summary of a track analysis ..........................................110 
Figure 47: 136-lb rail profile with fitted contact radius circle

Figure 48: An example of rail profile removed due to miss-measurement.

Figure 49: An example of a rail profile removed due to the inclusion of the rail web as part of the railhead. 118

Figure 50: The approximate locations of the wheel slopes used in the validation on the transit property specified wheel profile. .120

Figure 51: Comparison of transit property and AAR standard wheel profiles 121

Figure 52: Example of a track segment sensitivity analysis .123

Figure 53: Location of the lateral contact position with changes in wheel slope .126

Figure 54: NB-4 left (blue) and right (red) lateral contact point sensitivity analysis. .127

Figure 55: Contact radius relationship to the lateral contact position .128

Figure 56: NB-4 left (blue) and right (red) contact radius sensitivity analysis .130

Figure 57: SB-68 left (blue) and right (red) contact radius sensitivity analysis. .131

Figure 58: SB-68 rail profiles .132

Figure 59: Worn vs. unworn lateral contact position with identical wheel slopes .133

Figure 60: NB-19 left (blue) and right (red) lateral contact position sensitivity analysis 135 Figure 61: SB-13 left (blue) and right (red) lateral contact position sensitivity analysis 135 Figure 62: SB-45 left (blue) and right (red) lateral contact position sensitivity analysis 136 Figure 63: SB-45 rail profiles .137

Figure 64: Sensitivity of the lateral contact position on the top of the railhead 138

Figure 65: NB-14 left (blue) and right (red) lateral contact point sensitivity analysis .....139 Figure 66: NB-60 left (blue) and right (red) lateral contact position sensitivity analysis 140 Figure 67: NB-60 rail profiles .140

Figure 68: Wheelset shift and contact angles during curve segments..... 147 


\section{INTRODUCTION}

\subsection{PURPOSE}

The purpose of this research is to develop, verify, and validate an algorithm to estimate the lateral position of wheel-rail contact and corresponding rail profile radius. The algorithm uses $M A T L A B^{\circledR}$ code and rail profile data sampled from an in-service rail segment to estimate the lateral position of wheel-rail contact and corresponding radius of rail profiles along that rail segment. This research verifies the algorithm by comparing the algorithm output to known values of control rail profiles. It validates the algorithm by evaluating the reasonableness of the algorithm output for selected track segments using rail profile data from a rail transit property. To evaluate reasonableness, the research compares the algorithm output to a series of expected results. Ultimately, this research assists the current effort within the rail maintenance industry to develop tools for the monitoring and management of the wheel-rail interface.

\subsection{BACKGROUND AND NEED}

The dynamic forces generated by railway rolling stock act on the track through the contact patch between the wheel and the surface of the rail. Among other influences, wheel-rail contact depends on the geometries of the radial cross-section of the wheel (i.e., the wheel profile) and the transverse cross-section of the rail (i.e., the rail profile) (Iwnicki, Björklund, \& Enblom, 2009). Figure 1 shows an example of the wheel and rail profiles as a system. The nature of contact between the wheel and the rail is complex, but when optimized, can improve safety and operational performance while minimizing environmental impact, operational costs, and maintenance costs (Lunden \& Paulsson, 2009). 


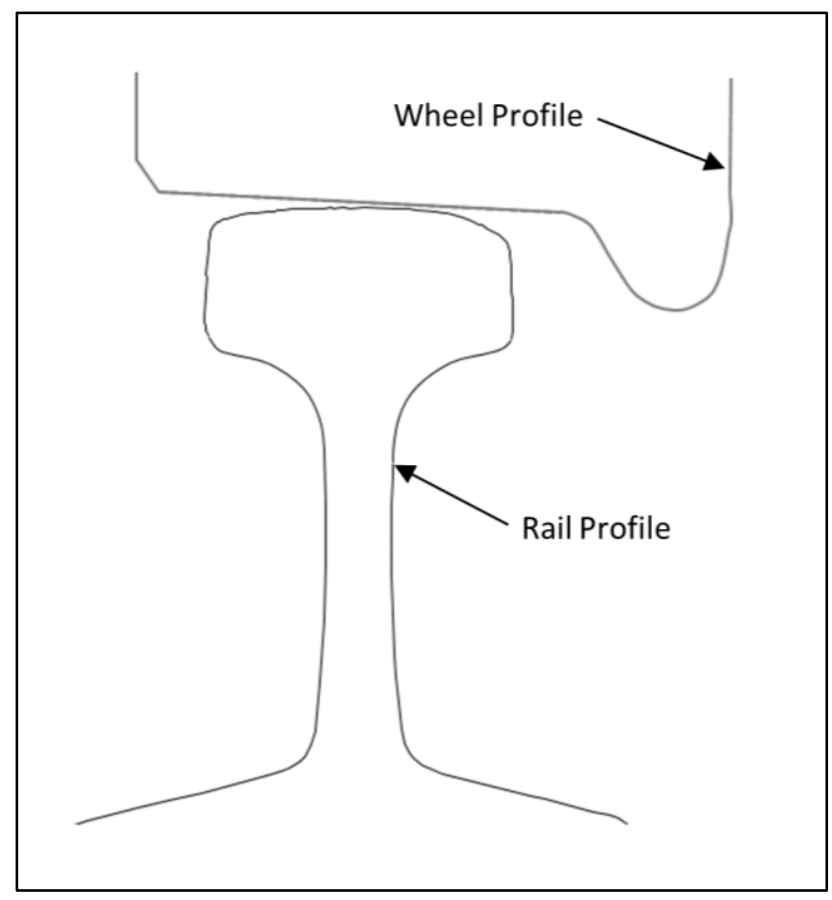

Figure 1: Wheel and rail profiles

Deterioration (i.e., wear) of both the rail and the vehicle's wheel change the shape and location of the contact patch, resulting in undesirable increases in contact forces and poor vehicle dynamics (Lewis \& Olofsson, 2009). The cause and effect of rail deterioration is a positive feedback loop that results in increased maintenance costs and safety implications (e.g., derailment). Proactive monitoring and management of the wheel-rail interface can lead to performance improvements and extended asset life (Grassie, 2009; Lunden \& Paulsson, 2009).

Many rail transit properties and heavy haul railroads measure and monitor the shape of the rail to support asset management decisions (G. Bachinsky, personal communication, 2017). One of the methods of measuring the rail profile uses a non-contact optical measurement system fitted to a hi-rail vehicle. Rail profile measurements provide fundamental inputs for the development of rail grinding programs, often through the application of processing software such as Holland ${ }^{\circledR}$ 's Rangecam ${ }^{\circledR}$. Rail maintenance 
personnel - either in-house personnel or consultants - use pre-grind measurements of representative rail profiles to develop rail grinding programs that restore the rail profile in a cost-effective manner with minimal loss of railhead material. This entails an appropriate selection of grind templates and the specification of grind frequency and intensity (i.e., depth). After performing maintenance, personnel compare post-grind measurements to pre-grind data and grind templates to measure improvement in rail shape and evaluate the effectiveness of grinding programs over time. Figure 2 shows a grind template superimposed over a rail profile.

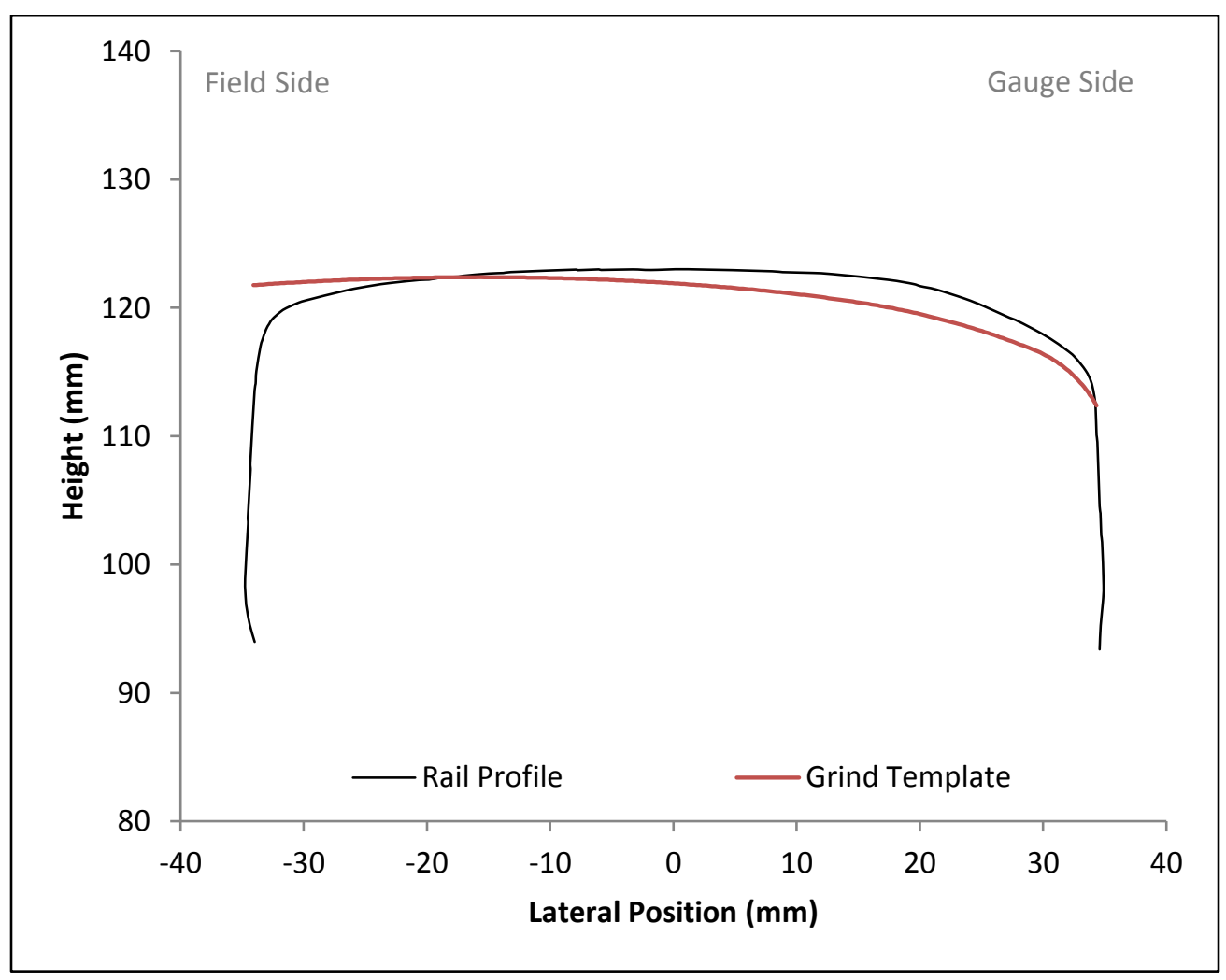

Figure 2: Grind template superimposed over a rail profile

Despite the availability of rail profile data, there is a need to better utilize these data to develop more comprehensive performance indicators that support more proactive rail maintenance programs. Rail researchers have focused on the development of 
representative mean profiles and the implementation of indicators such as the grind quality indices (GQI) (Regehr, Grande, Regehr, \& Bachinsky, 2017; Tunna, 2009); however, additional information is available from the rail profile data that can better characterize wheel-rail contact. More specifically related to the scope of this research, Hornaday $(2006,2010)$ investigated the benefits of measuring the variation of the top-ofrail (i.e., the uppermost point in the cross-section of the rail) of a rail segment and Abadpour \& Alfa (2007) developed a MATLAB code to measure the top-of-rail position. This research directly builds on these past research works.

This research proposes two new indicators of rail wear and performance: the lateral location of wheel-rail contact on a rail profile and the corresponding rail profile radius around the contact area. These indicators - which are extractable from rail profile data enable more refined characterization of the rail profile and its influence on rail wear and vehicle dynamics.

Incorporating the algorithm developed in this research into the rail maintenance industry's current practice will improve proactive maintenance approaches in both shortterm and long-term applications.

\subsection{OBJECTIVES AND SCOPE}

The objectives of this research are to

- develop an algorithm using MALTAB code to efficiently estimate the lateral position of wheel-rail contact and corresponding rail profile radius of rail profiles sampled from a rail segment;

- verify the algorithm by comparing algorithm output to known values of control rail profiles; and 
- validate the algorithm through the analysis of a selection of track segments from an in-service rail transit property.

The following points define the scope of the thesis:

- The validation process utilizes rail profile data from a rail transit property. While the algorithm can produce results with heavy haul railroad data, validation of heavy haul output is beyond the scope of this research.

- Collection of the rail profile data utilized in this research is to support current maintenance operations on a rail transit property and not for research purposes. Current data collection practice results in irregular maintenance records, irregular intervals between data collection runs, and temporal gaps between track maintenance efforts and data collection runs.

- Temporal comparison (i.e., performance indicator trending) of a track segment is not part of this research due to the current data collection practice.

- This research excludes track segments defined by curves, entry spirals, and exit spirals from the analysis. While the algorithm can run using rail profile data from curve and spiral segments, the limits of certain functions in the MATLAB code and the complexity of wheel-rail interaction in these types of segments results in this research only considering tangent segments.

- The algorithm executes using a specific rail profile data structure that influences certain steps within the MATLAB code. Other sources of rail profile data may not have the same data structure. While the methodological contributions of this 
research still apply, the algorithm will require certain adjustments based on the different data structures.

\subsection{APPROACH}

To understand the current state of knowledge regarding this topic, this research includes an environmental scan. The environmental scan provides background and context for the need this research addresses and contextualizes this research within the realm of wheel-rail interaction knowledge.

This research develops an algorithm based on the needs recognized by the environmental scan and current practice in the rail maintenance industry. The algorithm is an automated process using MATLAB code that estimates the lateral position of wheel-rail contact and corresponding rail profile radii along a rail segment. The following points provide an overview of the algorithm. The algorithm

- uses measured rail profile data as an input;

- applies rigid contact theory to model contact between a linear wheel profile and the rail profile

- calculates the lateral contact position and contact radius in the MATLAB environment;

- provides the results in graphic or numeric form on a profile-by-profile basis as well as summary statistics for each rail segment; and

- is verified and then validated using a selection of rail profile data from an in-service rail transit property.

This research takes a rail-centric approach. The algorithm

- uses field measured rail profile data while representing the wheel profile linearly 
(Figure 3 illustrates the transformation of a typical wheel profile to a linear wheel profile);

- measures the location of wheel-rail contact relative to the rail profile; and - measures radii of the rail profile.

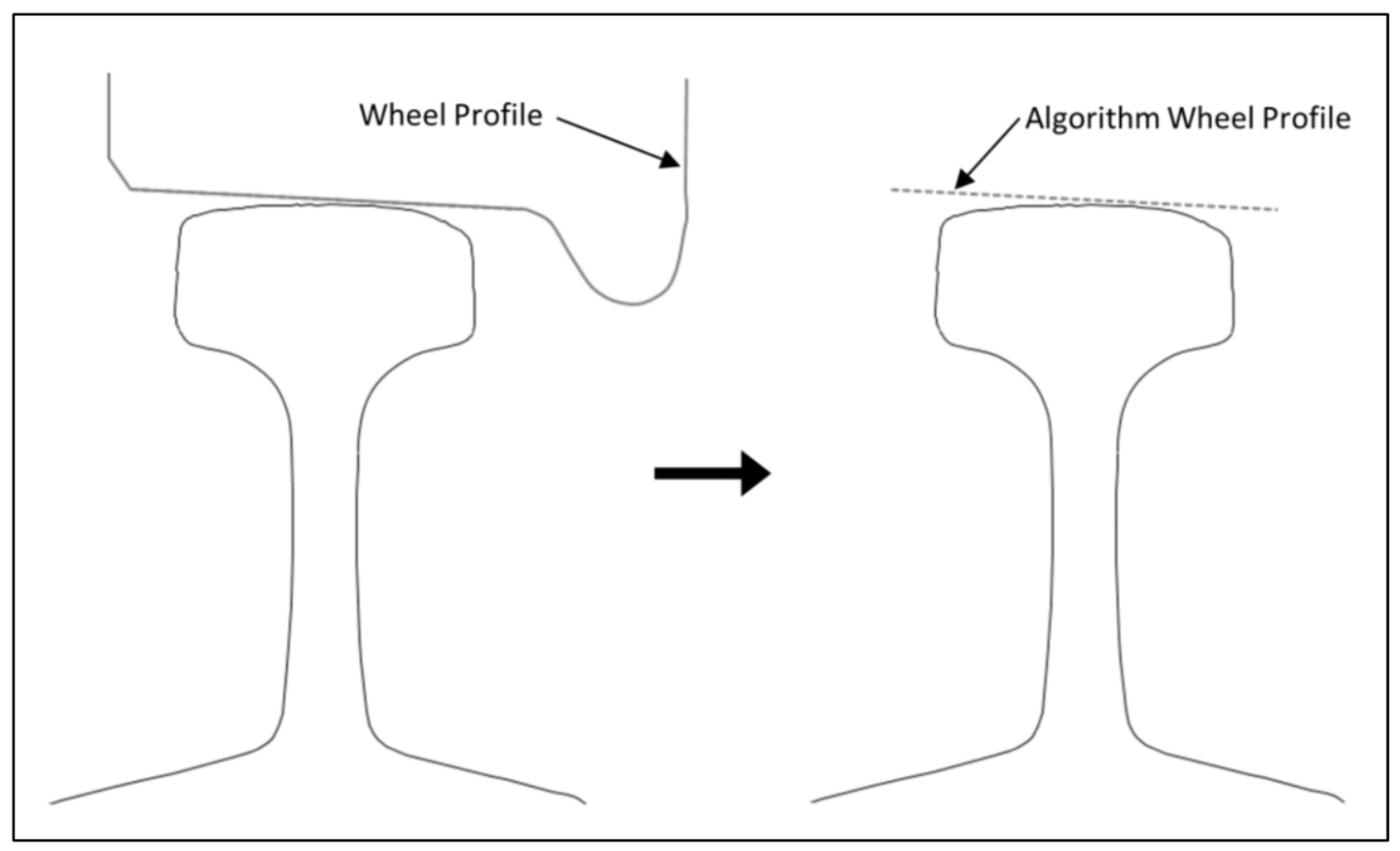

Figure 3: Wheel profile transformation to the algorithm's linear wheel profile

This research does not try to relate the lateral position of wheel-rail contact and corresponding rail profile radius to other variables, influences, and industry performance measures (e.g., GQI), but instead develops an algorithm and performance indicators that provide new information on wheel-rail contact and help support current rail maintenance efforts.

\subsection{THESIS ORGANIZATION}

The thesis consists of five chapters, including this introductory chapter. 
Chapter 2 presents findings from an environmental scan about fundamental principles, practices, and research efforts pertaining to this research. Specifically, the environmental scan focuses on five areas related to the rail maintenance industry: (1) wheel-rail contact and wear, (2) optical rail profile monitoring, (3) rail profile performance measures, (4) wheel-rail contact modeling approaches, and (5) wheel-rail contact related software.

Chapter 3 describes the methodology this research uses to develop and verify an algorithm to estimate the lateral position of wheel-rail contact and corresponding rail profile radius. Specifically, this chapter discusses: (1) data structure and preparation, (2) description of the algorithm, and (3) verification of the algorithm.

Chapter 4 describes the validation of the algorithm through the analysis of rail profile data from a North American rail transit property. Specifically, this chapter discusses: (1) the selection and cleaning of validation data and (2) the validation of the algorithm.

Chapter 5 concludes this research by summarizing the key findings and providing recommendations for future research.

\subsection{TERMINOLOGY}

ASCII text file: a plain text file that uses American Standard Code for Information Interchange (ASCII) characters (Industrial Metrics / Holland LP, 2012).

Cant Angle: the degree in which the rail profile is rotated to the gauge side of the rail measured from the vertical centreline of the rail profile web in reference to track level (Industrial Metrics / Holland LP, 2012). 
Contact Band $(\mathrm{mm})$ : an area of the railhead profile where contact of the train wheels typically occur.

Contact Radius $(\mathrm{mm})$ : the radius of the rail profile in the $\pm 9 \mathrm{~mm}$ window centred on the lateral contact point. The window around the lateral contact point simulates the width of the wheel-rail contact area.

Field Side: the side of the rail where the face points away from the track.

Gauge Side: the side of the rail where the face guides the wheel flanges and points towards the opposite rail.

Contact Point $(\mathrm{mm})$ : a point on the rail profile where the wheel profile makes contact when applying rigid contact theory. Figure 4 shows an example of the contact point.

Lateral Contact Position (mm): the $x$-value of the contact point of a rail profile, measured in millimetres from the vertical centerline of the web. Positive $x$-values are located on the gauge side of the centerline, negative $x$-values are located on the field side of the centerline. Figure 4 shows an example of the lateral contact position.

Lateral Variation $(\mathrm{mm})$ : the change in lateral position of a point (e.g., the lateral contact point) along a series of rail profiles representing a rail segment.

Rail: a steel bar that guides railway vehicles; a track uses two rails.

Railhead: the top section of the rail profile, affixed the rail web, where the wheel contacts the rail. 


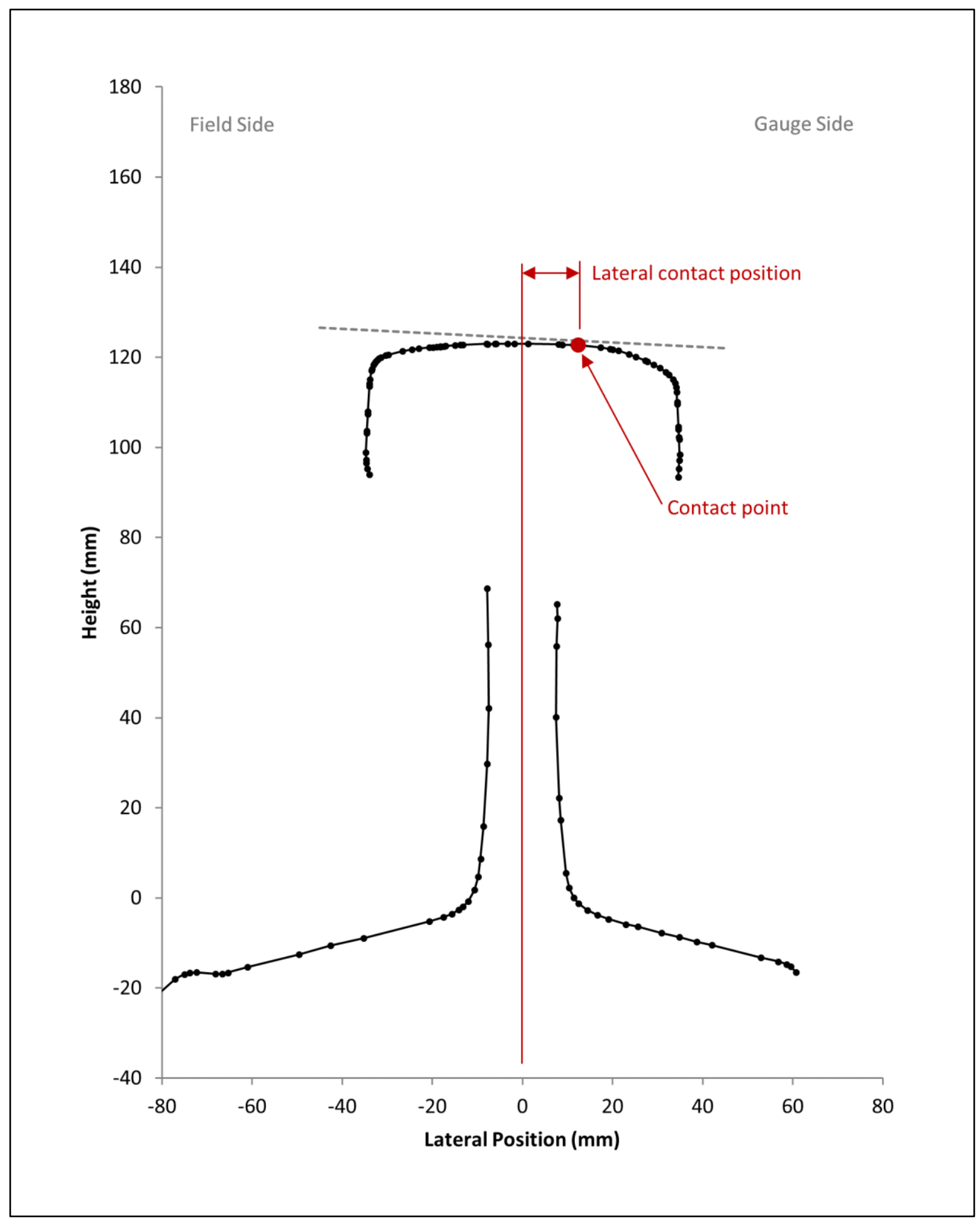

Figure 4: Contact point and lateral contact position example

Rail Profile: the cross-section of a rail. When converted into Cartesian coordinates, the centreline of the web is located at $x=0$. 
Rail Web: connects the railhead to the foot (i.e., bottom flange) of the rail profile.

Segment (mi., ft., or $\mathrm{km}$ ): a length of rail or track, typically segmented in curve and tangent sections.

Top of Rail: the uppermost point in the rail profile.

Track: for this research, the track refers to both the left and right rail of a segment.

Track Level: an imaginary line tangent to both the left railhead and right railhead (Industrial Metrics / Holland LP, 2012). Figure 5 shows an example of track level.

Wheel Profile: the radial cross section of the car or vehicle wheel (Iwnicki et al., 2009).

Wheel Slope: the slope that the wheel profile tread makes with track level. Figure 5 shows an example of wheel slope.

Wheelset: the wheel-axle assembly containing the left and right wheels fixed to the axle (Lewis \& Olofsson, 2009).

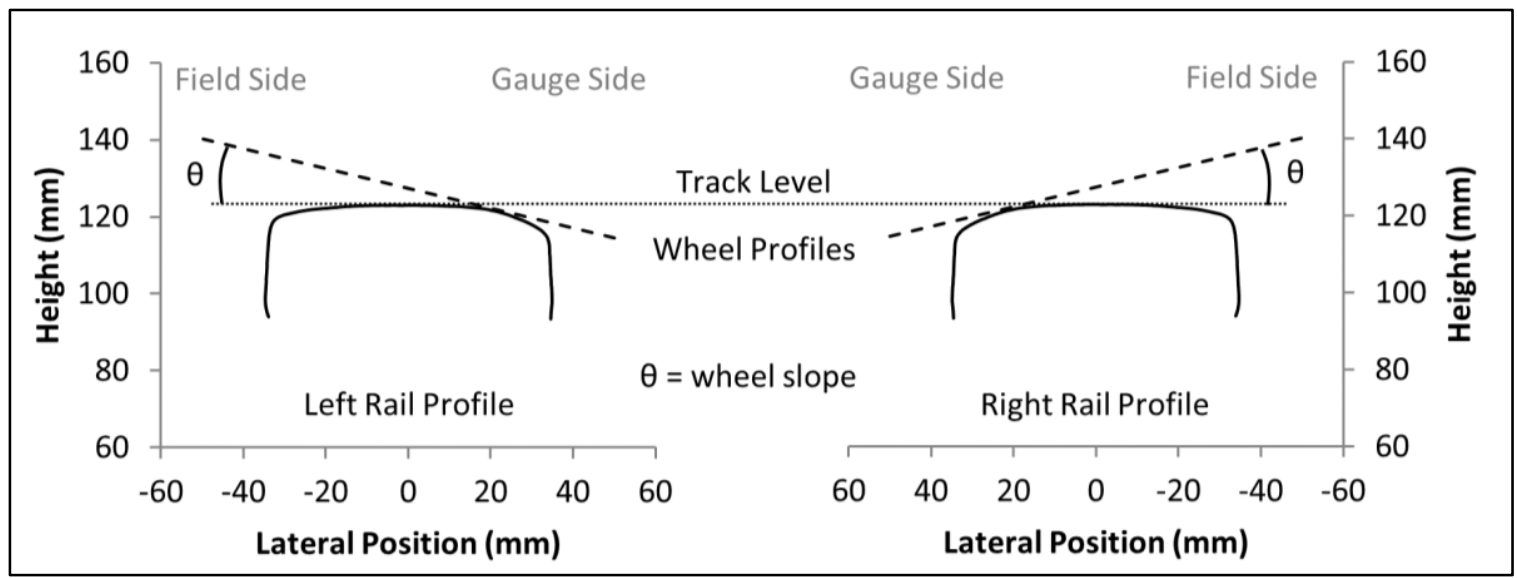

Figure 5: Track level and wheel slope example 


\section{ENVIRONMENTAL SCAN}

This chapter presents findings from an environmental scan about fundamental principles, practices, and research efforts pertaining to this research. Specifically, the environmental scan focuses on five areas related to the rail maintenance industry: (1) wheel-rail contact and wear, (2) optical rail profile monitoring, (3) rail profile performance measures, (4) wheel-rail contact modeling approaches, and (5) wheel-rail contact related software.

\subsection{WHEEL-RAIL CONTACT AND WEAR}

This section provides a high-level overview of contact mechanics and wear, two key aspects of the tribology of wheel-rail contact. Wheel-rail contact refers to the contact made between a flanged, conical rail vehicle wheel contacting the rail of a track, allowing a rail vehicle to safely transverse a track at speed with minimal rolling resistance despite high axle loads (Lunden \& Paulsson, 2009). Although this is a simple idea, wheel-rail contact is complex in nature and requires an interdisciplinary approach to understand fully. The type of contact between the wheel and the rail directly impacts wheel and rail wear, train energy consumption, and vehicle/track maintenance costs (Wu, 2006). Ultimately, optimizing wheel-rail contact will improve system performance.

A typical railway wheelset consists of two wheels fixed on an axle such that the matching radii on the two tapered wheels have identical rotating velocities (Tournay, 2001). The effective radius of a wheel changes based on the location of contact between the wheel and rail. The term contact radius refers to the effective radius of the wheel that is in contact with the rail. The design of the wheel and the rail allows a wheelset - as part of a bogie - to navigate both curve and tangent segments of track: 
- For steering around a curve, the wheelset shifts laterally on the track resulting in a difference in contact radii where the contact radius of the outside wheel is greater than the inside wheel, as seen in Figure 6 (a). The difference between contact radii results in larger rotational velocity in the outside wheel compared to the inside wheel (i.e., steering forces), allowing the wheelset to navigate the curve (Tournay, 2001).

- For tangent track, the contact radius for each wheel is theoretically identical, as seen in Figure 6 (b), resulting in no steering forces. If the wheelset experiences a disturbance, it can self-correct using the same principles for steering around a curve (Tournay, 2001).

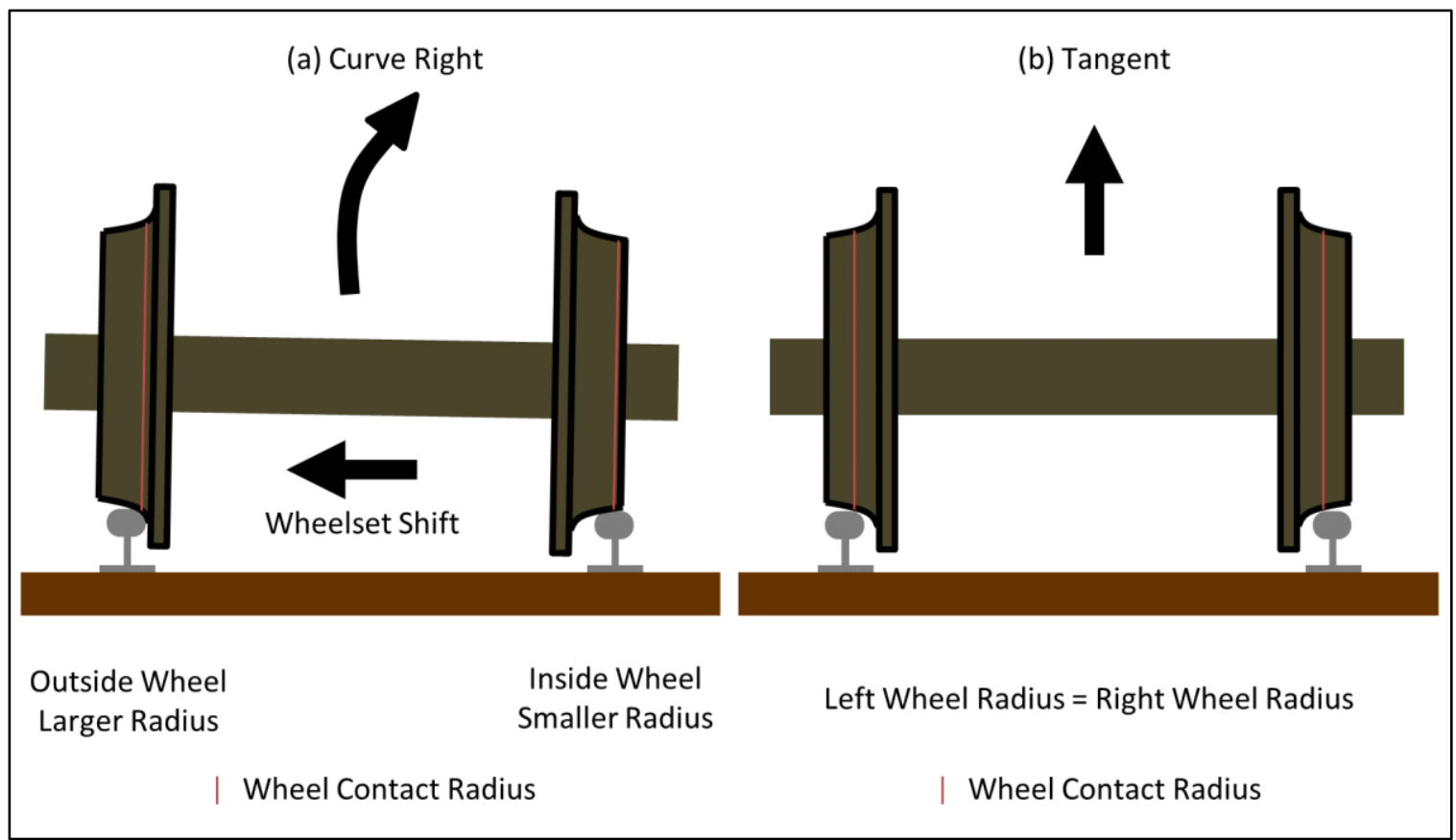

Figure 6: Wheel contact radius in (a) curve track and (b) tangent track

The preceding principles represent ideal situations. Due to a combination of track and wheel imperfections, superelevation of the track, and running speed of the train, the type of contact developed between the geometries of the wheel profile and rail profile are not 
always ideal. Bad contact development can lead to undesirable performance characteristics such as hunting (i.e., sustained kinetic oscillation of a wheelset) or flanging (i.e., contact between the rail and the flange of the wheel) (Tournay, 2001). Understanding the geometry of transverse wheel profiles and rail profiles and the resulting location of contact is an important part of understanding on-track vehicle dynamics.

The curvature of track, placement of the wheel in the bogie, and the wheel and rail geometry impact the location, size, and shape of the contact patch and forces between the wheel and the rail (Lewis \& Olofsson, 2009). Due to the imperfect nature of the wheel-rail system, the size and shape of the contact patch varies as a train travels along the track; typically, the size of the contact patch is approximately $1 \mathrm{~cm}^{2}$. Over time, the location, size, and shape of the contact patch will change due to wearing of both rail and wheel material.

Compatibility between the wheel profile and rail profile geometries is crucial for optimizing wheel-rail contact. The manufacturing and maintenance processes for wheels allows for a continuous array of potential wheel profiles while the manufacturing process for rails allow for only a discrete amount of rail profiles (Parsons Brinckerhoff, Incorporated, 2012). Furthermore, wearing processes transform both the wheel and rail profiles over time, resulting in a continuous array of potential in-service wheel-rail profile combinations.

An industry measure of the capability of wheel and rail profiles is conformality, which characterizes how well a wheel profile conforms to the rail profile. There are three distinct conformality categories: non-conformal, conformal, and too closely conformal 
(Magel, 2018b). Conformality affects both the number of contact points between the wheel and the rail as well as how the wheel transfers forces to the rail. Industry experts indicate that the design of the wheel profile and rail profile should ideally achieve conformal one-point or two point contact (Magel, 2018b). Non-conformal profiles or too closely conformal profiles lead to localized high stress transfer, accelerated wheel and rail wear, and poor steering performance (Magel, 2018b; Parsons Brinckerhoff, Incorporated, 2012).

Wear is defined as the "loss or displacement of material from a contacting surface" (Lewis \& Olofsson, 2009, pg. 40). Sliding wear and rolling wear are the two common wear types found in wheel-rail contact:

- Sliding is the lateral movement of the wheel relative to the rail due to a difference in velocity. Examples of sliding wearing mechanisms are oxidative wear, galling wear, abrasive wear, and thermal breakdown of surface material (Lewis \& Olofsson, 2009).

- Rolling wear typically refers to material fatigue due to cyclic loading. Material fatigue leads to cracking of the rail surface material that will propagate into the railhead if a railway does not provide proper maintenance to the rails. The rail industry uses the term rolling contact fatigue (RCF) to define these cracks (Lewis \& Olofsson, 2009).

Due to these wearing mechanisms, the geometry of the wheel profile and rail profile can deviate significantly from the specified unworn profiles, with the rate of change being a function of rail use, rail material, environmental conditions, and the condition of wheelrail contact. The rail industry categorizes wear in terms of mild, severe, or catastrophic 
wear (Lewis \& Olofsson, 2009). The definition of these classifications is not by numerical values but by observation of sudden increases in wearing rate of the materials from mild to severe to catastrophic.

\subsection{OPTICAL RAIL PROFILE MONITORING}

Rail data is a fundamental input in both wheel-rail contact algorithms and the creation of rail grinding programs. To gather data, the rail industry typically uses non-destructive testing to measure the shape of the rail profile and detect the existence of flaws and defects that may lead to failure of the rail (Cannon, Edel, Grassie, \& Sawley, 2003). Examples of typical non-destructive rail monitoring methods are visual inspection, magnetic particle inspection, optical rail profile measurement, eddy current, ultrasound, and radiography (Regehr, 2016). This research utilizes optical rail measurement data; therefore, this section focuses on optical rail measurement and its importance in rail grinding.

\subsubsection{Fundamental Concepts}

Compared to older methods of rail profile data collection - such as visual inspection by rail personnel - optical rail profile measurement allows for efficient and accurate measurement of discrete rail profiles at a quicker collection rate for longer track segments in less time (Kalay, 2001). Optical rail profile measurements achieve these

performance benefits with optical sensor rail profile measuring system attached to rail vehicle or a hi-rail vehicle.

A typical optical rail profile measurement system contains two optical sensors for each rail mounted to a high rail vehicle (Kalay, 2001). The location and orientation of the two optical sensors above and to either side of the rail profile, as Figure 7 illustrates, provide 
coverage of the majority of the rail profile; typically, the optical sensors do not cover the area where the railhead connects to the rail web and the bottom of the rail base. The set-up of optical rail measurement systems may differ depending on the area of the rail the railway desires to measure.

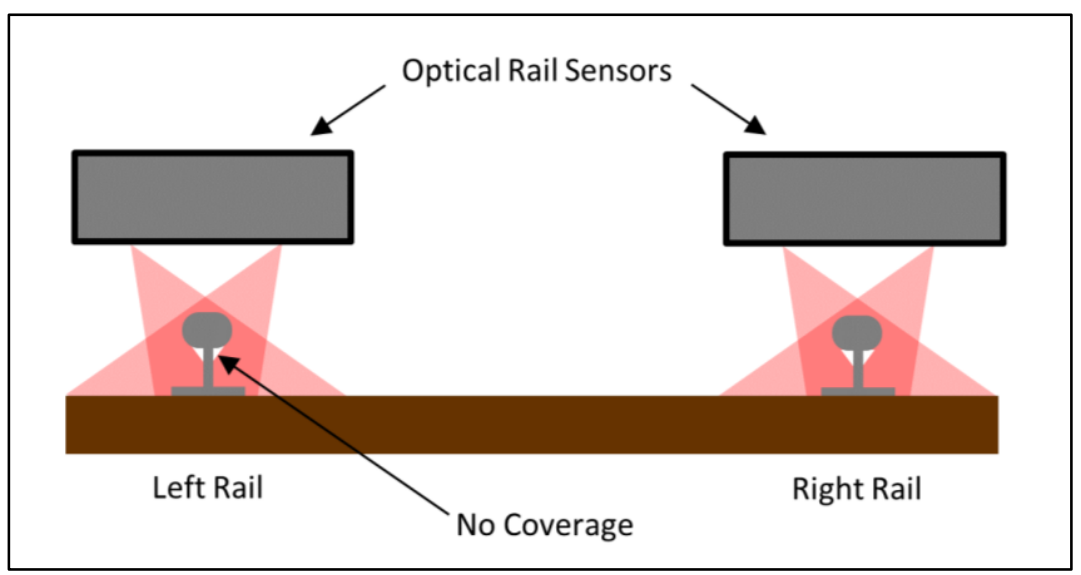

Figure 7: Optical rail sensor placement and alignment relative to the track

Using optical triangulation, an on-board computer converts and amalgamates the optical sensor information into a series of $x, y$-coordinates representing the rail profile (Chiacchiari \& Loprencipe, 2015). Additionally, devices on the hi-rail vehicle record the location of the profile in space (i.e., longitudinal position and cant angle). Calculation of prominent rail profile measurements occurs after rail profile measurement, during the processing in Rangecam.

The interval spacing of data collection points along the length of a rail segment is typically set at approximately $1.5 \mathrm{~m}$ (5 ft.) (G. Bachinsky, personal communication, 2018). In certain applications where necessary, the optical rail measurement system can achieve interval spacing of $0.15 \mathrm{~m}$ (6 in.). The combination of the hi-rail vehicle speed, the optical sensor equipment, and the onboard processing power determines the achievable minimum spacing. 
The accuracy of the rail profile measurement is dependent on many factors, such as

- image resolution;

- measurement method;

- the rail amalgamation process;

- $\quad$ system calibration;

- the speed of the hi-rail vehicle;

- the presence of vegetation or other objects that may obstruct the optical sensors;

- other light sources (e.g., sunlight); or

- items that reflect light (e.g., precipitation, rail material reflectiveness) (Regehr, 2016; G. Bachinsky, personal communication, 2016).

\subsubsection{Rail Grinding}

Rail profile data from an optical rail measurement system enables the development of an effective grinding program and for post-grind evaluation (Industrial Metrics, 2006).

In the rail maintenance industry, grinding refers to the removal of railhead material (sometimes termed artificial wear), reshaping the rail profile. The purpose of rail grinding is to improve wheel-rail interaction and to remove surface or near surface defects (e.g. corrugation, rolling contact fatigue) found in the rail before the defects worsen and potentially cause rail failure (Grassie, 2009; Roney, 2001). The process of rail grinding typically uses a series of circular grind stones at set angles mounted to a rail vehicle to remove the necessary rail material (Solomon, 2001). In terms of asset management, the goal of rail grinding is to extend the life of a rail.

When developing a grinding program, rail profile data - representing rail conditions just prior to maintenance - aids in the selection of a grind template (i.e, the desired shape of 
the rail after grinding) and determining the amount of rail material removal required (Industrial Metrics, 2006; G. Bachinsky, personal communication, 2017). Selection of the grind template requires a systematic review the wheel-rail interface, including current and future wheel profiles, track geometry, rail profiles, and track usage (Roney, 2001). Once selected, a grind analyst program superimposes the grind template onto the measured rail profiles to minimize material removal while still removing rail flaws/defects and achieving the desired shape (Industrial Metrics, 2006). Using a particular rail grinder, the amount of required rail material removal determines the number of passes a grinding machine will need to achieve the desired rail shape.

When evaluating the grinding activities, rail profile data collected after grinding allows for the comparison of the post-grind rail shape to the desired template (Industrial Metrics, 2006). Ideally, there will be minimal difference between the post-grind rail shape and the desired template. Companies can use this post-grind evaluation to evaluate their own grinding efforts or to demonstrate to clients that grinding performance meets specified targets.

\subsection{RAIL PROFILE PERFORMANCE MEASURES}

In recent years, there has been an increase in the use of performance measures in the transportation industry (Cambridge Systematics, 2006). However, performance measures are relatively unstructured and underutilized within the rail maintenance industry resulting in a lack of information despite adequate data collection (Parida, Kumar, Galar, \& Stenström, 2015; G. Bachinsky, personal communication, 2017). In the case of the wheel-rail interface, there are very few established performance measures. Establishing performance measures for wheel-rail contact will increase the value of the data collected and the wheel-rail contact tools developed, which can help improve asset 
management decisions (G. Bachinsky, personal communication, 2017).

The NCHRP classifies performance measures using three categories: inputs, outputs, and outcomes (Cambridge Systematics, 2006). Input performance measures track the amount of resources used to monitor and manage the wheel-rail interface (J. Regehr, internal report). Output performance measures quantify the scope and magnitude of maintenance efforts. Outcome performance measures quantify the impact of maintenance activity on the performance of railway operations. Further performance measures that relate inputs, output, and outcomes are also possible.

The performance measures described in this section relate to the rail component of the wheel-rail interface, which aligns with the scope of this research. Under the inputs, outputs and outcomes classification, all the performance measures in this literature review classify as outputs as well as serve as a foundation for some outcome-based measures.

\subsubsection{Average Rail Profile}

The average rail profile is a measure of the mean profile shape of $n$ measured rail profiles in a rail segment. The need for an average rail profile originates from industry rail grinding practices where a user or computer software compares a segment representative rail profile and a desired rail profile (Regehr et al., 2017). Current practice of selecting a representative rail profile for a track segment can lack both repeatability and objectivity. Automated calculation of an average rail profile improves on the repeatability and objectivity of user selection of a representative profile.

In response to this need, Regehr et al. (2017) develop a procedure in MATLAB that determines the average rail profile from a series of rail profiles representing a rail 
segment. The input data is optically measured rail profile data exported from Rangecam. The procedure normalizes all rail profiles based on the top of rail and then transforms the unevenly distributed Cartesian coordinates into polar coordinates on a set of predetermined superimposed radial lines. The average of the coordinates on each of the superimposed lines represents the average rail profile. Figure 8 shows the average rail profile of a rail segment. The steps within the procedure are dependant on the output structure of the rail profile data generated by Rangecam; therefore, the procedure is not equipped for widespread industry use.

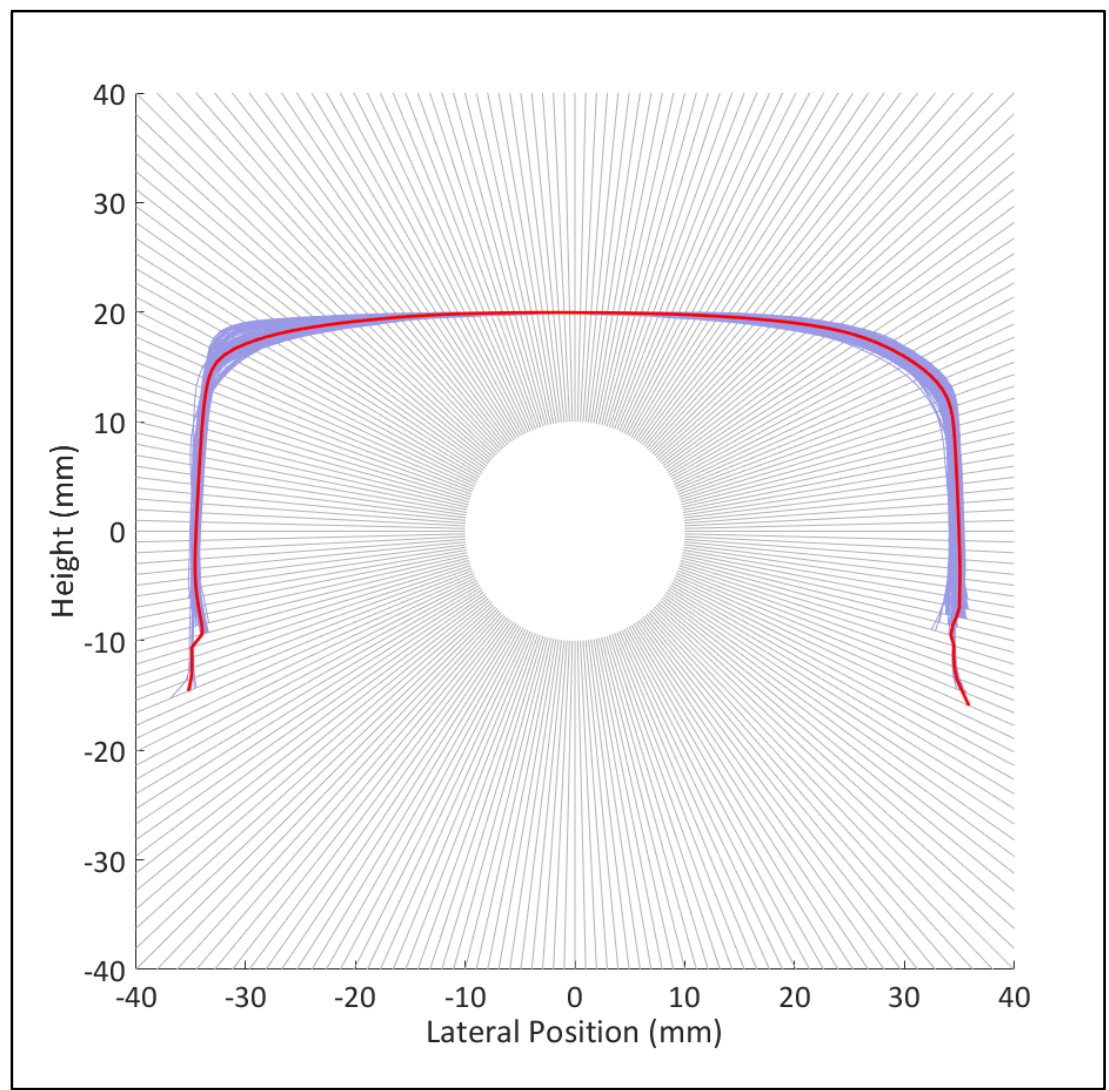

Figure 8: Average rail profile (red line) of the rail segment profiles (purple lines)

Regehr et al. (2017) recognize two future opportunities for the average rail profile, namely the integration of the average rail profile with other rail profile measures and the potential to re-segment rail by its profile shape rather than by track geometry (i.e., 
tangents, spirals, curves).

\subsubsection{Top of Rail}

There are two main performance measures related to the top of rail: the lateral position of the top of the rail profile - a rail profile performance measure - and the variation of the top of the rail profile along a rail segment - a rail segment performance measure.

Hornaday $(2006,2010)$ investigates the benefits that calculating the top of rail position of rail profiles along a rail segment could achieve in rail maintenance practices. To calculate the top of rail, Hornaday uses Excel to analyze optically measured rail profile $x, y$-coordinate data. Within Excel, he fits a second order polynomial to all rail profile points with a $y$-value within $0.5 \mathrm{~mm}$ of $y_{\max }$. The top of rail is the peak of the fitted polynomial, reported as the lateral distance from the centre of the rail (i.e., the top of rail $x$-value). Hornaday completes this process for each rail profile in the rail segment.

Hornaday (2006) makes two main conclusions based on the investigation and analysis using rail profile data from a heavy haul property. The first conclusion is that manually calculating the top of rail in Excel is a time consuming and labour-intensive task. The second conclusion is that the standard deviation of the top of rail along a rail segment is the most likely predictor of current or potential gauge face wear. Specifically, a larger deviation of the top of rail in tangent track increases the probability of a difference in wheel rolling radii leading to undesirable wheel truck steering and potential premature wearing of rail. However, later studies by Hornaday (2010) question that conclusion as a preliminary analysis of a transit property found no correlation between top of rail variations and gauge/vertical rail wear. 
Abadpour \& Alfa (2007) subsequently developed a polynomial MATLAB code to model the top of rail of a rail profile using rail profile $x, y$-coordinate data. The procedure selects all coordinates larger or equal to the threshold, $t$, below $y_{\max }$ of the rail profile data, fits an $m$ degree polynomial to the selected coordinates, and then calculates the peak of the polynomial using either a closed form solution when $m=2$ or a MATLAB optimizer function when $m>2$. The procedure was tested on single rail profiles and rail segments.

Abadpour \& Alfa (2007) discuss the choice of threshold values, $t$, and degree of the polynomial, $m$. They conclude that the selection of the threshold or degree of the polynomial is highly sensitive to the other; selecting an inappropriate value with respect to the other can lead to the inaccurate calculation of the top of rail location. Additionally, they mention that a second-degree polynomial can be a poor choice, as it may not accurately represent the local shape around the top of rail.

\subsubsection{Grind Quality Report and Calculations}

The grind quality report is a report Rangecam creates from a set of optically measured rail profiles; the report contains two rail profile performance measures: (1) the difference of the measured rail profiles from a selected desired rail profile averaged over each rail segment and (2) the average crown radius of the measured rail profiles averaged over each rail segment. Rangecam's Grind Analyst manual suggests utilizing the grind quality report

- before rail maintenance grinding programs for planning purposes and to measure the amount of material to be removed; and

- after grinding efforts to compare the post-grind rail to the grind template for evaluation of maintenance efforts (Industrial Metrics, 2006). 
The first grind quality calculation measures the difference between a measured rail profile and a desired rail profile in four zones: the extreme gauge zone, the gauge zone, the center zone, and the field zone (Industrial Metrics, 2006). Figure 9 shows the location of the four different zones and the crown radius. This grind quality calculation does not use the extreme field zone as it has no significant impact on wheel-rail interaction. Rangecam defines the zones in reference to the vertical centerline of an unworn rail profile.

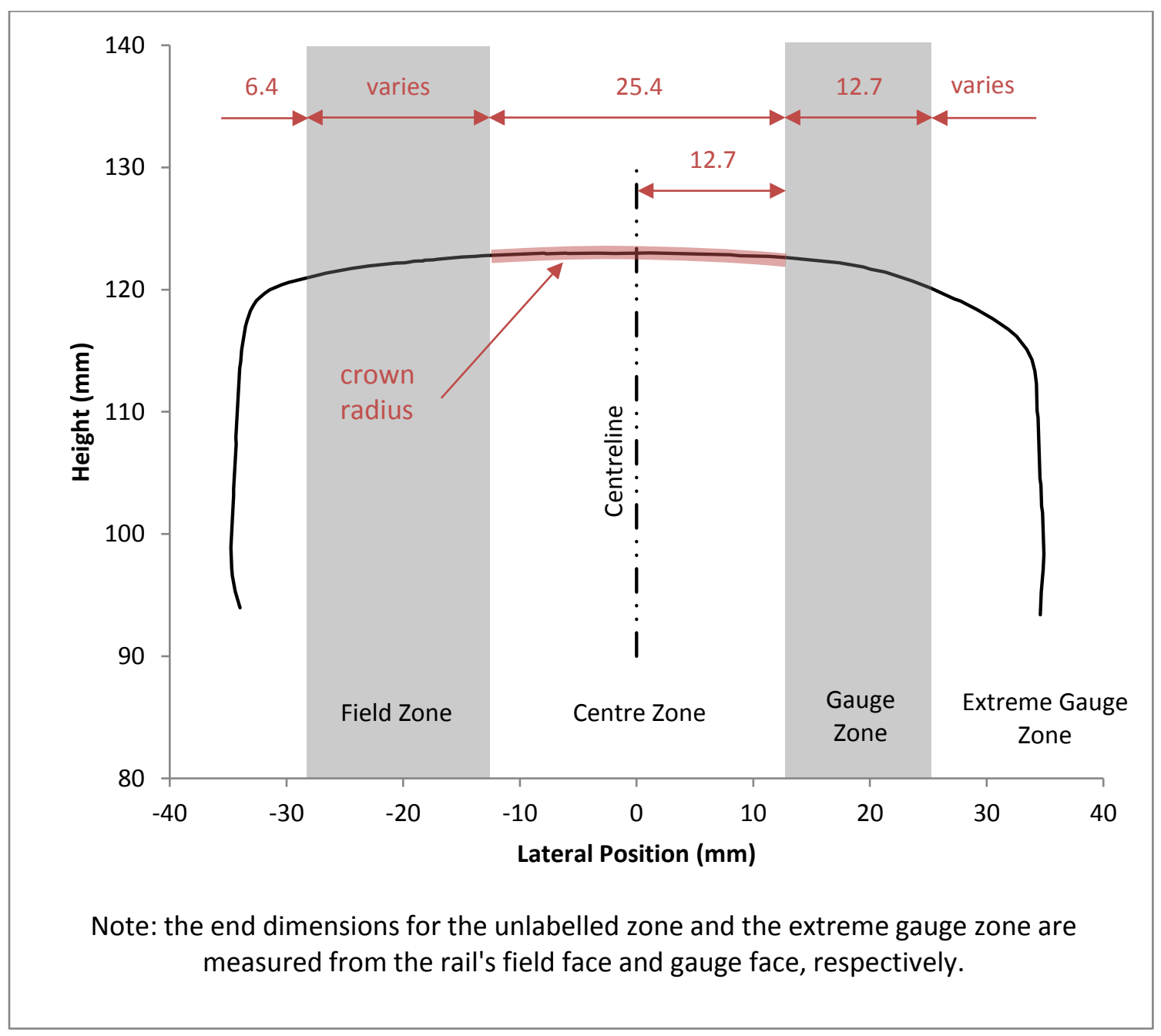

Figure 9: Grind zone boundaries and crown radius

The second grind quality calculation determines the average crown radius, which is the 
radius of the rail profile in the center zone. Figure 9 shows the location of the crown radius. Users can select which method Rangecam will use to calculate the crown radius (Industrial Metrics, 2006):

- The fit to all points method evenly resamples the uneven distribution of coordinates in the centre zone and fits a least squares circle to the resampled data.

- The fit to 3-points method fits a circle to the intersections of the rail profile with the two centre zone boundaries and the vertical centerline of the rail profile.

The grind quality report summarizes the results from the two grind quality calculations. The report divides the analyzed track section into geometric track segments (Industrial Metrics, 2006). Rangecam provides start and end mileage, geometry, and curve information (if applicable) for each track segment. The report then divides each track segment into the two rail segments. Rangecam provides the compass direction, grind template used, high or low rail (if applicable), and the five measurements (i.e., four grind zones and crown radius) for each rail segment. Each of the five measurements reports the average of that particular measurement across all rail profiles for that rail segment.

\subsubsection{Profile Quality Index}

The profile quality index (PQI) is a quantitative metric that describes the difference between an optically measured rail profile and the selected desired rail profile (Zarembski, Palese, \& Euston, 2005). The selected desired rail profile can be either

- an unworn rail profile for a temporal analysis of rail wear; or

- a grind template for pre-grind planning or post-grind evaluation (G. Bachinsky, 
personal communication, 2016).

This research will use the term PQI to describe what the rail maintenance traditionally calls the grind quality index (GQI). New industry nomenclature states that GQI is a composite performance measure made up a series of indices such as the PQI (i.e., the traditional GQI), surface quality index (SQI), and corrugation quality index (CQI) (G. Bachinsky, personal communication, 2018).

There are two methods for calculating a PQI. While both indices measure the difference between a measured and desired profile and provide a quantitative result, the indices use different numeric scales and are not directly relatable.

The first method measures the vertical difference between the average measurements given in the Rangecam Grind Quality report for a rail in a track segment and a superimposed rail template (G. Bachinsky, personal communication, 2016). The PQI calculation uses only three average measurement zones: the extreme gauge zone, gauge zone, and the center zone. The linear method omits the field zone, as it does not have a significant impact on wheel-rail interaction. The linear method also omits the crown radius; no sources state the reason for this omission.

The PQI equation is

$$
\begin{gathered}
P Q I=(\mid \text { Extreme Gauge Zone Difference }|+| \text { Gauge Zone Difference } \mid \\
+\mid \text { Center Zone Difference } \mid) * 1000
\end{gathered}
$$

The PQI has a lower limit of zero (indicates the rail profile matches the desired rail profile in each of the three zones) and an infinite upper limit (higher indices indicate a larger difference between the measured and desired rail profiles). Since the Rangecam Grind 
Quality report provides average measurements for a rail segment, the user applies the equation once per rail segment, resulting in a rail segment PQI.

The second method, developed by Zarembski, Palese, \& Euston (2005), compares an difference curve to an acceptance envelope. The difference curve is the difference between the measured rail profile and the selected desired rail profile. The acceptance envelope is the allowable difference curve tolerances. The PQI is "the percentage of area of the difference curve below the acceptance envelope" (Zarembski et al., 2005, pg. 47). The area method applies the PQI area method once for each rail of each track segment. The overall track segment PQI is the average PQI of the two rail segments.

From a rail profile perspective, the PQI is the "percentage of the railhead that matches the desired rail profile, within the allowable error of the acceptance envelope" (Zarembski et al., 2005, pg. 46-47). Since the area method PQI is a percentage, its range is from zero (the difference curve falls entirely outside the acceptance envelope) to 100 (the difference curve falls entirely within the acceptance envelope). The authors state that a railroad requires further grinding if the PQI is below 100 .

The acceptance envelope can vary across the railhead, as smaller railroad-defined tolerances are placed on the gauge side of the rail profile due to its importance in wheelrail contact relative to the field side (Zarembski et al., 2005). The area method is incompatible for a temporal analysis using an unworn rail profile since grinding tolerances define the acceptance envelope.

\subsection{WHEEL-RAIL CONTACT MODELING APPROACHES}

The ability to model wheel-rail contact is one of the key aspects of monitoring and managing the wheel-rail interface given that the industry collects wheel and rail data 
independent of each other. More specifically, many wheel-rail interface and vehicle dynamics software - covered later in this chapter - utilize wheel-rail contact models to predict both the forces and location of wheel-rail contact (Bosso, Spiryagin, Gugliotta, \& Somà, 2013). To model wheel-rail contact, three approaches exist which range in accuracy, complexity, and efficiency.

The solution to the problem of wheel-rail contact divides into four smaller problems (Bosso et al., 2013). The geometrical problem deals with the type of wheel-rail contact the location of contact on the profiles. The normal problem deals with the size and shape of the wheel-rail contact patch and the resulting pressures. The kinematical problem deals with the condition of creepage between the wheel and rail. The tangential problem deals with determining the tangential forces generated between the wheel and the rail.

While no problem is independent of another, solutions exist for each of the four problems, ranging in accuracy, complexity, computation time, and dependence on the other three problems (de Pater, 1988). Often simpler solutions are less accurate, require less computation time, and have less consideration for the other three problems.

The three approaches to calculating the state of wheel-rail contact (i.e., geometrical problem) and the resulting normal forces are

- $\quad$ rigid contact;

- elastic contact; and

- quasi-elastic contact theory.

This environmental scan focuses on rigid contact theory due to the applications of this theory within current wheel-rail interface research efforts. 


\subsubsection{Rigid Contact Theory}

Rigid contact theory ignores the elastic-plastic properties of both the wheel and rail material and considers them as rigid bodies (Bosso et al., 2013). Using rigid contact theory, the contact between the wheel and the rail occurs at a point and not over an area since there is no deformation of the wheel or rail due to any contact forces, as illustrated by the red dot in Figure 10b.

Rigid contact must satisfy three conditions (Bosso et al., 2013; T. Handal, personal communication, 2017):

- When both the wheel profile and rail profile are continuous and monotone, the profiles will be parallel at the point of contact (Figure 10a), such that

$$
\theta_{\text {wheel }}=\theta_{\text {rail }}
$$

where $\theta_{\text {wheel }}$ is the wheel profile surface angle and $\theta_{\text {rail }}$ is the rail profile surface angle.

- The point of contact between the rail must occupy the same space (Figure 10b), such that

$$
x_{\text {wheel }}, y_{\text {wheel }}=x_{\text {rail }}, y_{\text {rail }}
$$

where $x_{\text {wheel }}, y_{\text {wheel }}$ is the contact point on the wheel profile and $x_{\text {rail }}, y_{\text {rail }}$ is the contact point on the rail profile, both measured on the same coordinate system.

- The wheel and rail cannot interpenetrate (Figure 10c), such that

$$
y_{\text {rail }} \leq y_{\text {wheel }} \text { for a given a value } x
$$


where $y_{\text {rail }}$ and $y_{\text {wheel }}$ are $y$-values on the rail and wheel profiles, respectively, for a particular $x$-value.

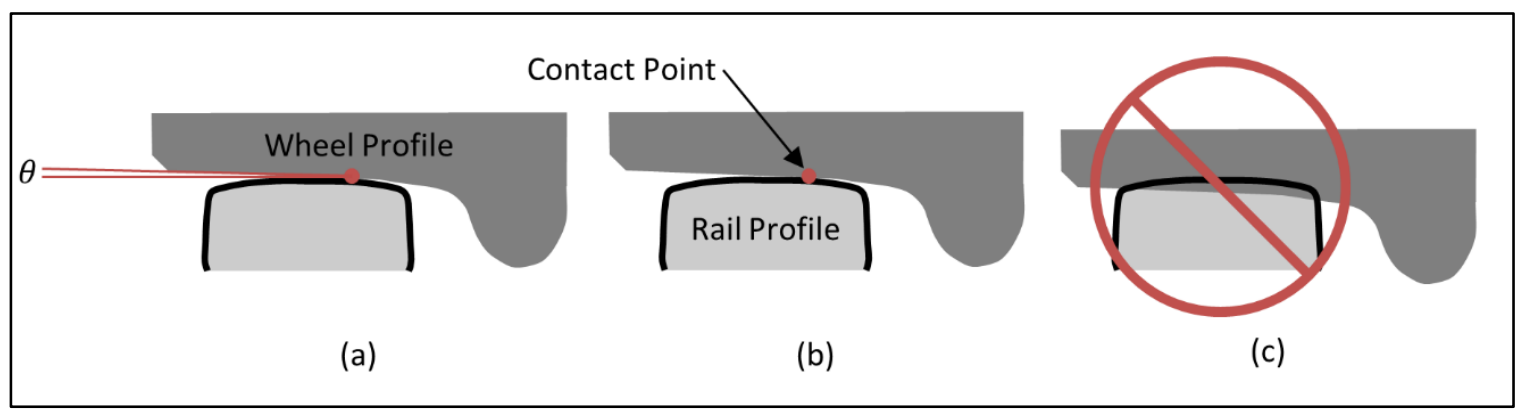

Figure 10: Three conditions for rigid contact

Rigid contact theory has three main limitations, it cannot

- calculate multiple points of contact between the wheel and the rail;

- account for the uplift action of the wheel due to track irregularities or other processes that may generate uplift (i.e., does not consider vertical movement); or

- $\quad$ solve for worn wheel and rail profiles analytically (Bosso et al., 2013).

Bosso et al. (2013) state that the benefit of using rigid contact theory is that computer programming can easily incorporate the three rigid contact conditions due to their analytical nature, resulting in a relatively high computational efficiency. Due to these benefits, multi-body contact software commonly use rigid contact theory.

\subsubsection{Elastic and Quasi-Elastic Theories}

Elastic contact theory considers both the wheel and rail as elastic bodies with a single elastic element modeling constraint in the normal direction (Bosso et al., 2013). When in contact, the elastic deformation of the wheel and rail results in a contact patch. Elastic contact theory allows for the determination of multiple contact points and the evaluation of worn profiles. The results using elastic contact theory are more accurate than rigid 
contact theory. Limitations of elastic contact theory include high computation requirements due to the numerical methods applied and the high requirements for data quality and quantity to properly apply this method (Bosso et al., 2013). Constant stiffness, Hertzian, multi-Herzian and non-Hertzian methods are well-known solutions to the elastic contact problem (Bosso et al., 2013), but are not discussed further in this research.

Models applying quasi-elastic contact theory use simple algebraic equations of worn wheel and rail profiles resulting in less computational time than elastic contact models (Bosso et al., 2013). Schupp, Weidemann, \& Mauer, (2004) develop the quasi-elastic contact model where the "results are obtained by an opportune regularization of the function defining the profile distance, no longer expressed as a point by point function, but as an average function" (Schupp et al., 2004, pg. 8). The authors state that the main limitation of quasi-elastic contact theory is that it cannot account for uplift action of the wheel.

\subsection{WHEEL-RAIL CONTACT RELATED SOFTWARE}

The exponential increase in accessible computing power over the past decades has lead to increases in rail data collection and the development of software capable of accurately and efficiently characterizing wheel-rail contact. This section gives a brief overview of the three commonly used algorithms for calculating contact conditions and creep forces as well as provides a list of currently available software related to wheel-rail contact. Finally, this section discusses proprietary wheel-rail software.

Most current software related to wheel-rail contact uses one of three algorithms to calculate contact conditions and creep forces: CONTACT ${ }^{\circledR}, \mathrm{USETAB}^{\circledR}$, and $\mathrm{FASTSIM}^{\circledR}$ 
(Vollebregt, 2008). More specifically, these algorithms calculate the

- size, shape, and location of the wheel-rail contact patch;

- stresses;

- displacements;

- areas of adhesion and slip; and

- areas of rolling and sliding (VORtech b.v., n.d.-a).

CONTACT utilizes Kalker's complete theory (non-Hertzian contact) which results in a relatively slow calculation time but high relative accuracy (Iwnicki et al., 2009; Vollebregt, 2008). CONTACT is an add-on to programs such as MATLAB, GENSYS ${ }^{\circledR}$, SIMPACK Rail ${ }^{\circledR}$, and Universal Mechanism ${ }^{\circledR}$ (VORtech b.v., n.d.-e). Pricing for CONTACT varies, but is free in certain restricted iterations (VORtech b.v., n.d.-d).

FASTSIM utilizes Kalker's simplified theory which solves problems quicker than CONTACT but only yields approximate results (Vollebregt, 2008; VORtech b.v., n.d.-c). Using FASTSIM, the calculation of the traction distribution between the wheel and rail is not recommended (VORtech b.v., n.d.-c). FASTSIM is an add-on to programs such as GENSYS, SIMPACK Rail, and Universal Mechanism.

USETAB works by interpolating pre-calculated tables from CONTACT to calculate wheel-rail creep forces (Vollebregt, 2008; VORtech b.v., n.d.-b). USETAB is similar to FASTSIM in that it allows for quick calculations and only yields approximate results; however, the USETAB results are more accurate than FASTSIM $(1.5 \%$ and $15 \%$ estimated error, respectively) (Shabana, Zaazaa, \& Sugiyama, 2007). Programs such as VAMPIRE ${ }^{\circledR}$ and NUCARS ${ }^{\oplus}$ use USETAB for simulations (VORtech b.v., n.d.-b).

Most multi-body dynamic behavior software utilizes CONTACT, FASTSIM, or USETAB 
to simulate rail vehicle dynamics on a track (Shabana et al., 2007). The following list provides examples for common industry software for multi-body dynamic behavior studies: NUCARS, GENSYS, VAMPIRE, SIMPACK, CONPOL ${ }^{\circledR}, \mathrm{CONTACT} \mathrm{PC92}^{\circledR}$, DYNARAIL ${ }^{\circledR}$, LaGer $^{\circledR}$, OCREC $^{\circledR}$, TDS CONTACT ${ }^{\circledR}$, VOCOLIN $^{\circledR}$ (Iwnicki et al., 2009). All software give near identical prediction for the location of the contact patches, but there are differences in the size and shape of contact (Iwnicki et al., 2009).

In addition to the foregoing programs identified in the literature, proprietary software also exists about which there is little publicly available documentation. An example of proprietary software is $\operatorname{VPUMMEL}^{\circledR}$ (Magel, 2018a). VPUMMEL is a vehicle-rail interaction tool developed by the National Research Council of Canada that evaluates wheel-rail interaction over a large number of wheel and rail profile cases using Hertzian contact theory (Magel, Kalousek, \& Caldwell, 2005). One of the outcomes of utilizing VPUMMEL are the selection of target wheel and rail profiles. Very little formal documentation exists for the VPUMMEL software.

To summarize, available wheel-rail software varies in terms of purpose, efficiency, and the contact theories they implement. These variations may limit their effectiveness for monitoring the performance of the wheel-rail interface for extensive rail networks. 


\section{METHODOLOGY}

This chapter describes the methodology to develop and verify an algorithm to estimate the lateral position of wheel-rail contact and corresponding rail profile radius. Specifically, this chapter: (1) discusses data structure and preparation, (2) describes the algorithm, and (3) verifies the algorithm.

\subsection{DATA STRUCTURE AND PREPARATION}

This section provides a detailed review of the rail profile, rail segment, and track segment data structures as well as the folder structures that store the rail profile information. This review provides background information about the methodology of this research, as proper data exporting methods, preparation, and organization are necessary prior to running the algorithm's MATLAB code to avoid execution errors or erroneous results.

The structure of the data this research uses falls into two primary categories: (1) the Rangecam output data structures and (2) the algorithm's folder structure requirements. The Rangecam output data structures influence certain steps within the algorithm; these data structures are essentially not modifiable due to the proprietary nature of Rangecam and the high level of effort that would be required to modify the output files. The algorithm's folder structure requirements ensure the algorithm executes correctly and ensure consistent organization of rail profile information.

\subsubsection{Track Segments, Rail Segments, and Rail Profiles}

Common practice in rail profile monitoring is to disaggregate the rail property into segments that share similar characteristics, such as geometry, wear patterns, and the 
type of wheel-rail contact developed. This research adopts the typical segmentation of track segments, rail segments, and rail profiles. The hierarchy and features of the track segments, rail segments, and rail profiles follow, supported by Figure 11:

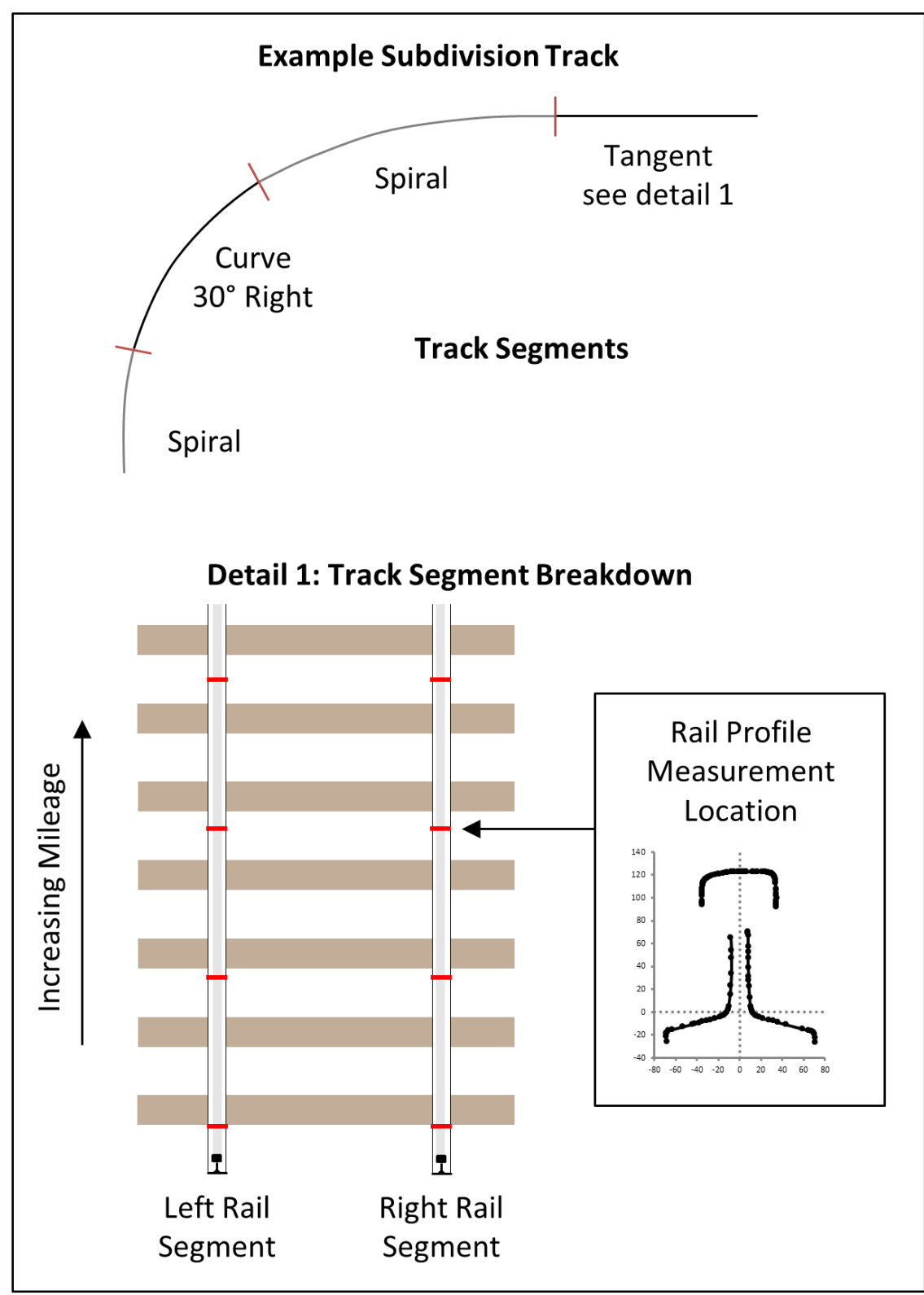

Figure 11: Segmentation: track segments, rail segments, and rail profiles

- A subdivision rail line divides into track segments.

- Segmentation typically occurs at points where track geometry changes 
(e.g., tangent to curve).

- A curve track segment may include the entry and exit spirals.

- The degree and direction of curve further differentiates track segments.

- The measurement process assigns a unique identification code to each curve track segment in the form:

\section{C\#(identification-number)}

e.g., C\#381

- A track segment includes a left and right rail segment.

- The measurement process designates the handedness (i.e., left or right) of a rail segment with respect to increasing topology mileage of the subdivision.

- The rail measurement process assigns a side-of-track compass point $(\mathrm{N}, \mathrm{S}, \mathrm{E}, \mathrm{W})$ to each rail segment. As an example, if the direction of increasing mileage of a subdivision is South, the measurement process assigns the compass point $W$ to the left rail segment and the compass point $E$ to the right rail segment.

- A series of measured rail profiles represent a rail segment.

- The number of rail profiles measured depends on the length of the segment and the acquisition rate of rail profile data by the hi-rail vehicle.

- Measurements of the left and right rail profile typically occur simultaneously at the same segment mile-point.

\subsubsection{Rail Profile Text Files}

During the rail profile data export process, Rangecam writes the data for each measured 
rail profile into a unique text file; this research refers to these files as rail profile text files. There are three main parts of a rail profile text file:

- the profile header lines;

- $\quad$ the profile detail lines; and

- the text file name.

The profile header lines are the first eight lines of a rail profile text file and contain the metadata on the rail profile. Rangecam delineates the metadata using spaces between values. Figure 12 shows the profile header lines in a rail profile text file.

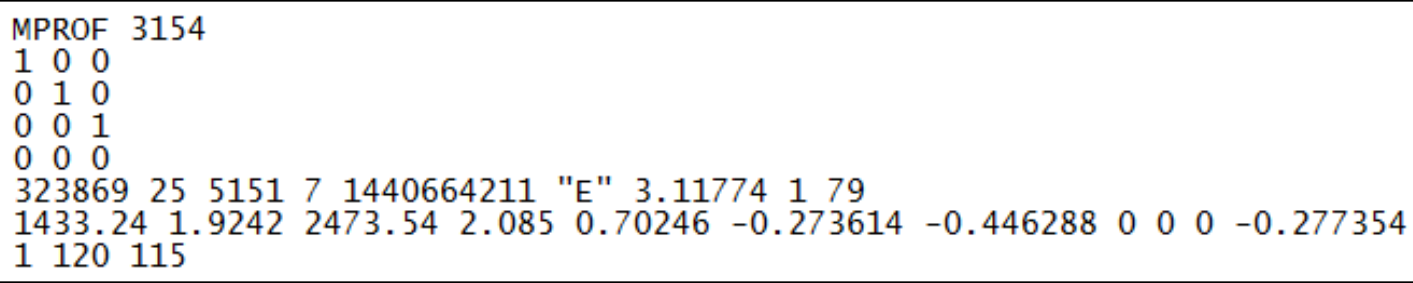

Figure 12: Rail profile header lines

The following points characterize the features of the profile header lines:

- The first line contains the profile object number.

- Lines two through five contain the transformation matrix that Rangecam applies to the rail profile to orient it correctly in the software. Examples of rail profile transformations include mirroring and rotating. A unique transformation matrix exists for each rail profile; however, a bulk export of rail profiles in Rangecam the export method this research uses - results in a non-transforming identity matrix for all rail profiles exported, regardless of the transformation needed to correctly orient the rail profile. 
- Lines six through eight contain the rail profiles' location in space and common rail profile wear measurements. More specifically, these lines include the

○ mile-point location;

o side-of-track designation by compass point (N,S,E,W);

- time of data acquisition;

o track gauge at mile-point location;

○ cant angle;

- location of the first line segment that represents the railhead;

$\circ$ number of line segments that represent the railhead;

○ gauge point wear; and

- vertical wear at rail centerline.

The profile detail lines are the remaining $p$ rows in the rail profile text file that describe the line segments which represent the measured rail profile; each row describes a single line segment. Rangecam defines each of the $p$ line segments by a set of start $x, y$ coordinates, end $x, y$-coordinates, and an arc curvature $\left(x_{1}, y_{1}, x_{2}, y_{2}\right.$, and $\alpha$, respectively), delineated with a space between values, as seen in Figure 4. Figure 14 provides a sample of the representation of the rail profile in Cartesian coordinates.

$\begin{array}{llllll}-34.290 & 104.902 & -34.643 & 108.164 & 0 \\ -34.643 & 108.164 & -34.720 & 110.618 & 0 \\ -34.720 & 110.618 & -34.702 & 111.217 & 0 \\ -34.702 & 111.217 & -34.636 & 111.766 & 0 \\ -34.636 & 111.766 & -34.497 & 116.464 & 0 \\ -34.497 & 116.464 & -34.530 & 117.015 & 0 \\ -34.530 & 117.015 & -34.426 & 120.513 & 0 \\ -34.426 & 120.513 & -34.460 & 121.064 & 0 \\ -34.460 & 121.064 & -34.292 & 126.712 & 0 \\ -34.292 & 126.712 & -34.029 & 128.855 & 0 \\ -34.029 & 128.855 & -33.915 & 129.302 & 0 \\ -33.915 & 129.302 & -33.587 & 130.243 & 0 \\ -33.587 & 130.243 & -33.162 & 131.080 & 0 \\ -33.162 & 131.080 & -32.639 & 131.865 & 0 \\ -32.639 & 131.865 & -31.608 & 132.885 & 0\end{array}$

Figure 13: Sample of a rail profile text file's line segment data 


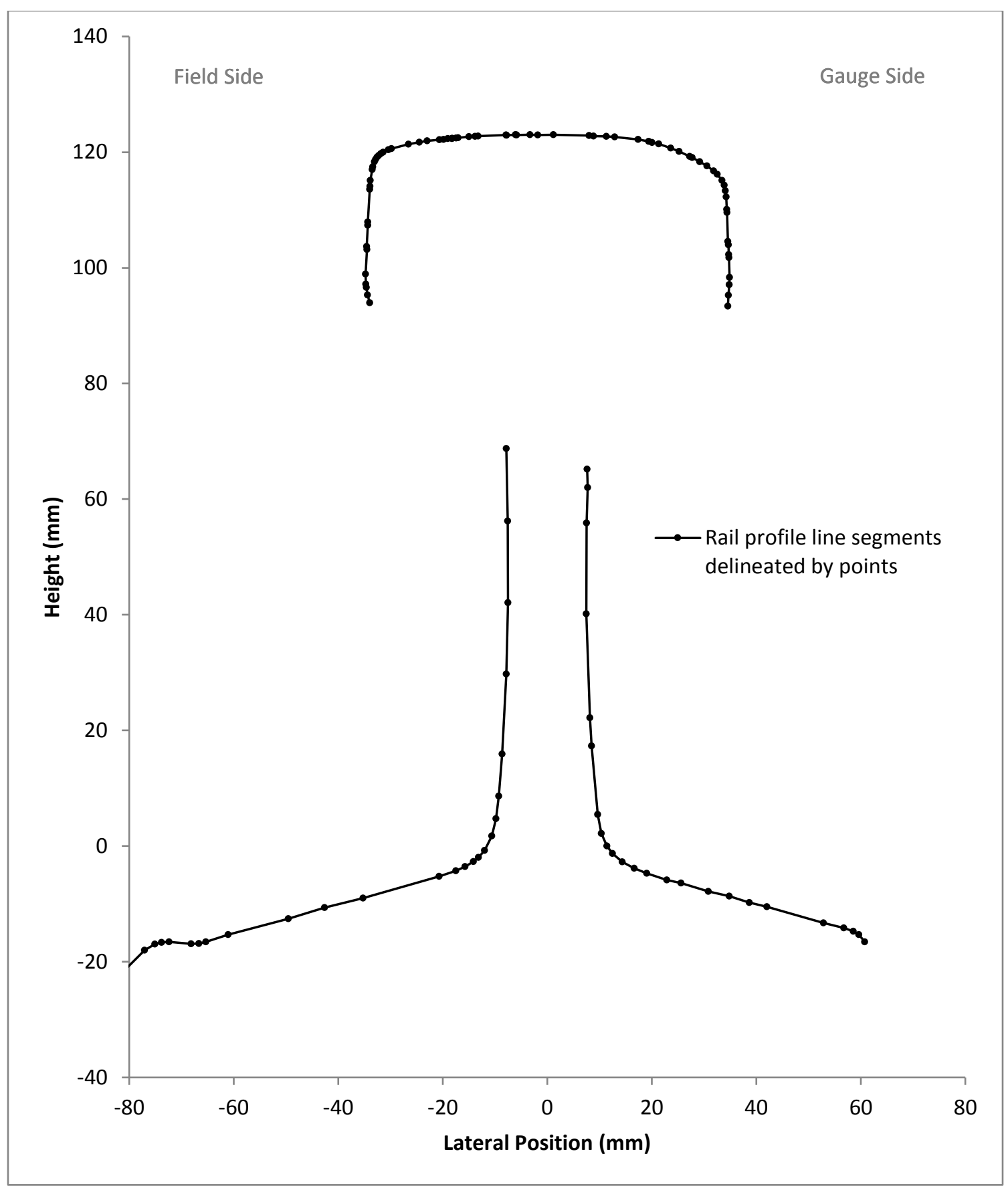

Figure 14: Example of line segments of a rail profile text file in Cartesian coordinates

The following points describe line segment features and how they represent a rail profile:

- The Rangecam export process models the rail profile using straight-line segments; thus, the arc curvature of a line segment, $a$, is always equal to 0 . 
- The number of line segments that represent a rail profile varies.

- The line segments are not a set length.

- The distribution of line segments varies among rail profiles.

- The line segments that represent the head of the rail profile are on consecutive rows in the rail profile text file.

- The optical rail measurement system that obtained the data in this research cannot measure the area where the railhead connects to the rail web due to the location of the optical sensors on the hi-rail vehicle.

- Adjacent line segments are continuous along either side of the web and around the top of the railhead. However, if the optical sensors cannot measure an area of the rail profile there will be a disruption in the line segment continuity. Section 2.2.1 (Fundamental Concepts) covers factors that affect rail profile measurement.

- Rangecam defines the origin of the coordinate system at the base of the rail profile.

- The measured rail profile is oriented in the Cartesian coordinate system by a least squares fit of the non-wearing parts of the measured profile to an unworn rail profile oriented vertically with the vertical web center line at $x=0$. This research refers to this orientation of the measured rail profile as the original orientation.

- Rangecam does not mirror the Cartesian coordinate system for a rail profile on 
the opposite side of the track; $x$-values of coordinates on the gauge side of the railhead will have $x>0$ for the left rail of a track and $x<0$ for the right rail of a track.

The text file name uniquely identifies the rail profile text file and contains information about the profile's location. When exporting rail profile text files, Rangecam automatically creates a file name for each profile in the form:

(subdivision name)_(track code)_(mileage integer)_(mileage decimal)_(side-of-track designation)_(run number)_(profile sequence number).

Detailed understanding of the composition of the rail profile text file name is not critical to this research, as the algorithm does not directly extract any information from the name of the rail profile text file. However, after the Rangecam export, the ordering by file name of the rail profiles in a folder presents complications when trying to sort larger numbers of rail profiles into the proper folder structure. Section 3.1.3 (Exporting Rail Profile Data from Rangecam) details the ordering of rail profiles after the Rangecam export. Section 3.1.5 (Sorting the Rail Profile Text Files) details how the order of rail profiles after the Rangecam export affects the sorting the rail profiles in the proper file structure.

\subsubsection{Exporting Rail Profile Data from Rangecam}

The input data for the algorithm are the rail profile text files that the user exports from Rangecam. Within the Rangecam software, the user executes the software's bulk export option. Appendix A details the procedure to export rail profile data from Rangecam.

Rangecam export process stores the entire run of rail profile text files in a single folder on the local computer drive. When sorted by name, the rail profile text files are in 
increasing mileage order and alternate left and right rail sides (i.e., if no rail profiles are missing, every other rail profile text file will be from the same rail side of the track).

\subsubsection{Folder Structure and Naming Requirements}

Prior to running the algorithm, the user must organize the rail profile text files into the proper folder structure; furthermore, the folders within the folder structure must be named correctly. This section covers the folder structure requirements and accompanying folder-naming convention, and provides recommendations for higherlevel folder organization.

The algorithm relies on the folder structure requirements and accompanying foldernaming convention during execution. If the folder structure does not meet the requirements, the algorithm will output erroneous analysis results or execution will fail. If the folders do not use the naming convention, the algorithm's naming of the output files will be less descriptive and a portion of the analysis metadata in the output file will be incorrect.

From a data-management standpoint, the folder structure requirements and accompanying folder-naming convention ensure a consistent organization of rail profile text files among different analyses and improve the user's ability to locate files and understand what the folders contain.

To maintain consistency, the required folder structure emulates the rail segmentation hierarchy discussed in Section 3.1.1 (Track Segments, Rail Segments, and Rail Profiles). Figure 12 shows the hierarchy of the required folder structure followed by details about the different folder requirements and the folder name convention: 


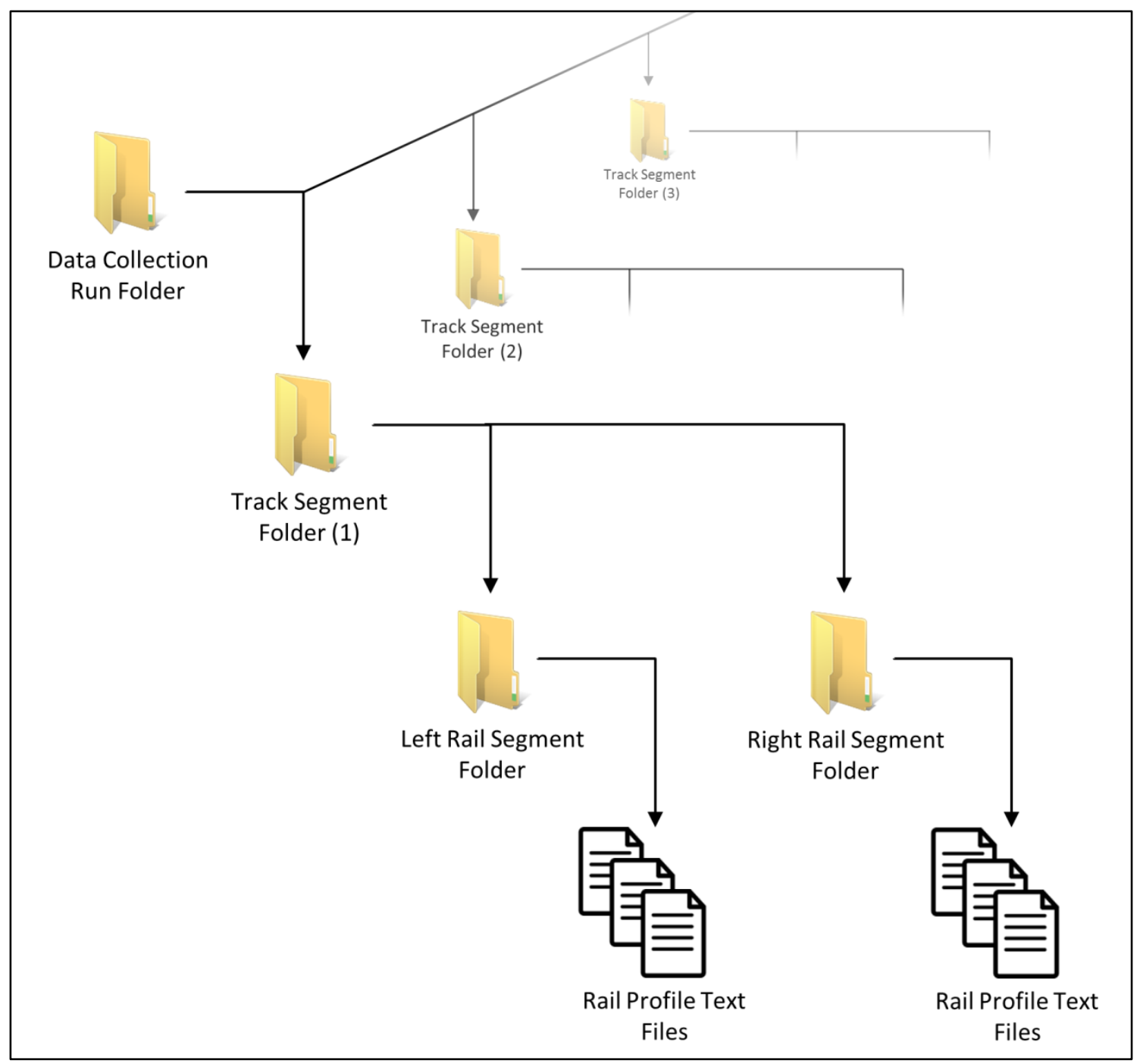

Figure 15: Required folder structure for algorithm

- The data collection run folder contains the track segments folders. The naming convention for the data collection folder is the date of the data collection run in the following year-month-day form:

\section{YYYYMMDD}

e.g., 20150827

- The track segment folder contains the left rail segment folder and the right rail segment folder. The naming convention of the track segment folders follow. 
For tangents:

(segment sequence number) Tangent (start mileage)-(end mileage)

e.g., 4 Tangent $0.375-1.123$

For curves:

(segment sequence number) (curve number) (degree and direction of curve) (start mileage)-(end mileage)

e.g., 18 C\#381 1.2L 3.804-3.984

The segment sequence number of a track segment starts at one for the segment with the lowest mileage and increases by one for every consecutive track segment. The sequence number properly orders the track segment folders in increasing mileage order within the data collection folder.

- The rail segment folder contains the rail profile text files that represent that particular rail segment. The name of the rail segment folder is the side-of-track designation the measurement process assigns to that rail segment. The names of the two rail segment folders in the track segment folder are Left Rail and Right Rail.

In addition to the required folder structure, this research also recommends that the user store multiple data collection run folders from a single subdivision in a single subdivision track folder. The rail subdivision folder does not directly affect the execution of the algorithm but improves overall data organization. 


\subsubsection{Sorting the Rail Profile Text Files}

This section covers two methods a user can implement to sort the rail profile text files into the correct folder structure as described in the previous section (Section 3.1.4, Folder Structure and Naming Requirements). The two sorting methods are

- manually sorting; and

- an automated sorting subroutine.

The need to sort the rail profiles is due to Rangecam's bulk export process, which does not have the capability to organize rail profiles in the file structure required for the algorithm.

The manual sorting method involves the user creating the folder structure and then manually sorting each rail profile text file into the correct rail segment folder. Manually moving a large number of files is an arduous process due to the inability to select a series of rail profiles in the Rangecam export folder that would go to the same rail segment folder. See Section 3.1.3. (Exporting Rail Profile Data from Rangecam) for

more detail about the ordering of rail profiles following the Rangecam export. The manual method is beneficial when the user only wants to analyze a small sample of rail profiles or if the user is making discrete changes to the outcome of the automated sorting subroutine.

This research develops an automated sorting subroutine using MALTAB code to improve the sorting of larger numbers of rail profile text files. Using the track segment report and the location of the folder containing Rangecam exported rail profile text files, the subroutine automatically creates the proper folder structure, applying the developed naming convention, and then sorts the rail profile text files into the appropriate rail 
segment folders. Appendix B details the procedure to export the track segment information from Rangecam. Appendix $\mathrm{C}$ details the rail profile text file sorting subroutine.

The user can initiate the algorithm's MATLAB code after sorting the rail profile text files.

\subsection{DESCRIPTION OF THE ALGORITHM}

This section describes the algorithm developed for this research. The algorithm calculates the lateral position of wheel/rail contact and the radius of the rail profile around the contact point.

The algorithm runs outside of the Rangecam software suite, but requires rail profile data outputs from Rangecam. Consequently, the structure of the Rangecam data (covered in Section 3.1, Data Structure and Preparation) influences certain steps within the algorithm; therefore, these steps will require adaptation for file types other than those exported from Rangecam. Nevertheless, the rail industry can use the main concepts this research develops to assist in rail maintenance practices.

The algorithm runs a series of functions to calculate the lateral contact position and corresponding contact radius for each rail profile of a track segment. Figure 16 shows a flow chart diagram of the algorithm. At a high-level, the algorithm

- $\quad$ prompts the user to select a track segment for analysis;

- analyzes each rail profile of the left rail segment, storing the lateral contact position, contact radius, and required metadata for each rail profile within that segment;

- $\quad$ performs the same analysis on the right rail segment; and 
- collates the data from both rail segments and exports the results to Excel.

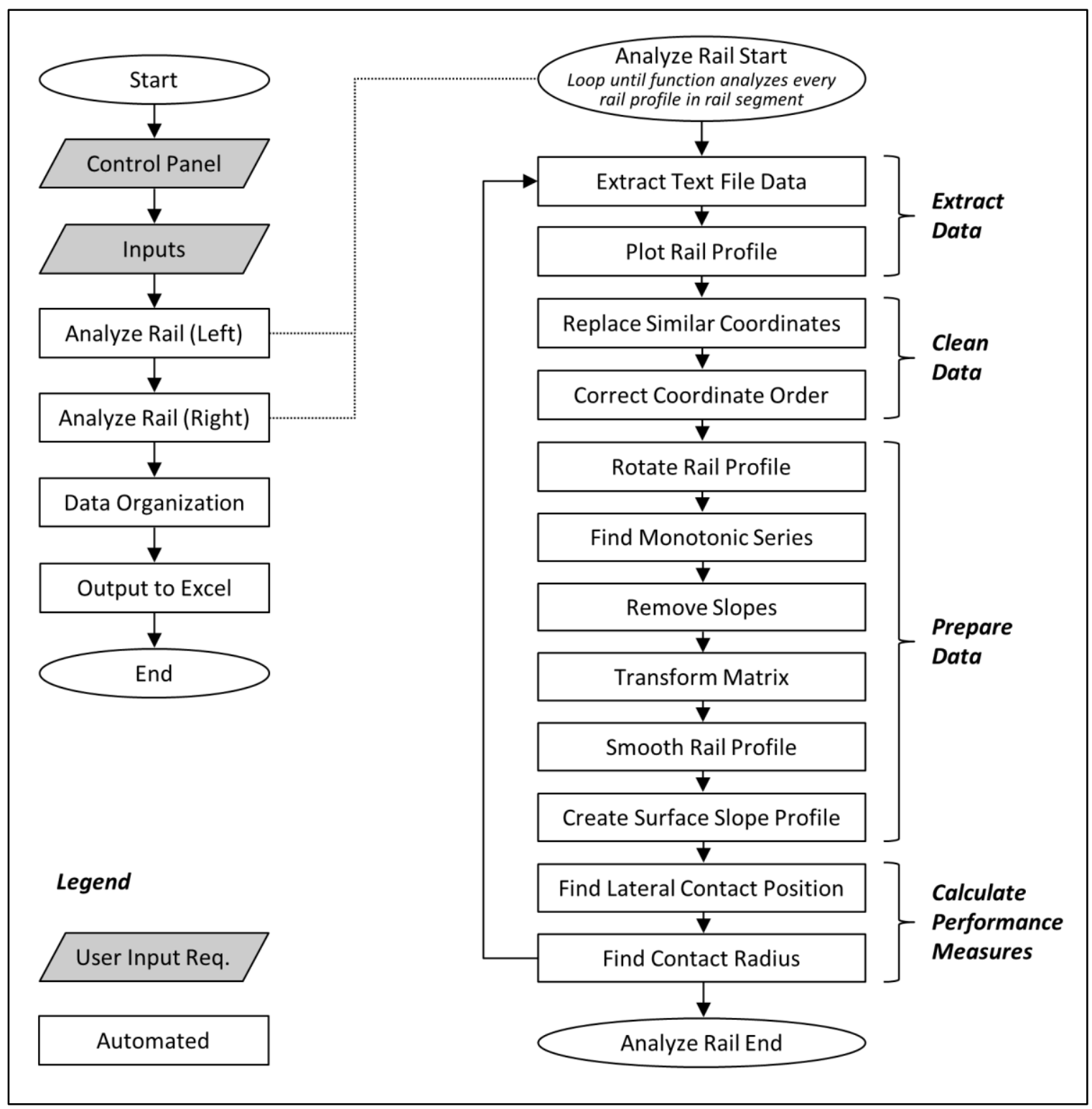

Figure 16: Flow chart diagram of MATLAB code

For each rail profile, the code

- extracts the rail profile data from the rail profile text file;

- cleans the rail profile data to ensure the $x, y$-coordinate data is in the correct order; 
- prepares the $x, y$-coordinate data for calculation of the rail profile performance measures by removing unnecessary data as well as rotating, transforming, and smoothing the data; and

- calculates the lateral contact position and contact radius performance measures.

Sections 3.2.1 to 3.2.5 provide details on the algorithm. Illustrative examples, using data from a Canadian rail transit property, help explain certain steps in the algorithm.

The structure of this section follows:

- Each section contains one function within the code. Relative to Section 3.2, the first level of sections contain the main functions (i.e., the function on the left side of the flow chart diagram in Figure 16). The second level of headings contain functions within the main functions (e.g., the analyze rail functions on the right side of the flow chart diagram in Figure 16).

- The section/functions are in the order of operation when running the algorithm.

- Each section begins with a diagram highlighting the current function in a simplified flow diagram. There are separate diagrams for the main function (Figure 17) and the analyze rail functions (Figure 18).

- After the diagram, there is a description of the functions purpose.

- After the purpose, there are two lists containing the inputs and output variables of the function, respectively, if applicable.

- The main body of the section contains detailed information on how the algorithm 
operates, which supports the transfer of this technology to rail maintenance operations looking to apply this research in the field.

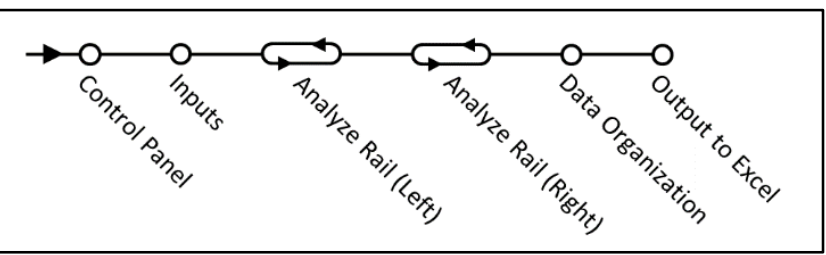

\section{Figure 17: Simplified flow diagram of main functions}

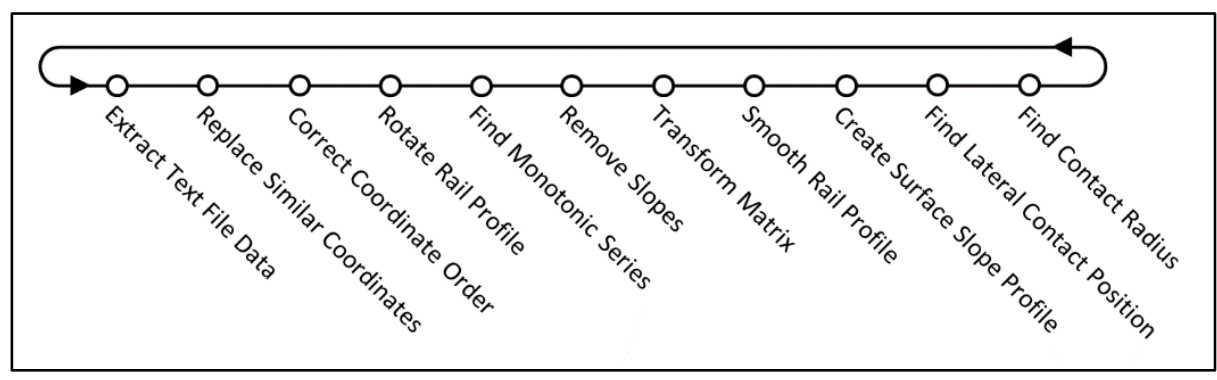

Figure 18: Simplified flow diagram of analyze rail functions

\subsubsection{Control Panel}

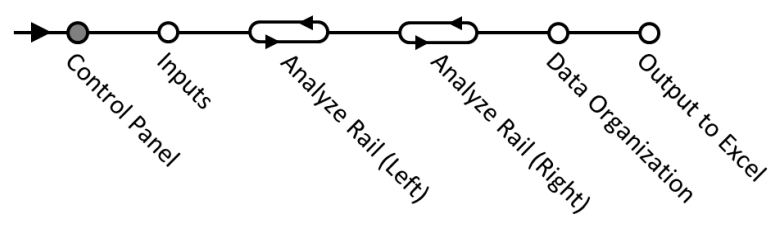

Purpose: The control panel, located in the main algorithm function in MATLAB, is where the user manually enters parameters about the track segment and the wheel profile into the algorithm. All parameter inputs are required. The default parameters are the values used in the previous analysis.

Inputs:

Outputs:

- run name

- mirror left rail

- mirror right rail 
- none

The algorithm input parameters follow:

- Run name (string input) is the first part of the Rangecam assigned name to the rail profile text file (i.e., the text file name up to the first underscore). The input string must match the first part of the text file exactly and is case-sensitive. In the case of the transit property with a rail profile text file named

TRANSIT LINE_SB_0004_1141_E_26_82,

TRANSIT LINE would be the input string.

- Mirror left rail (numeric input) is set to either 1 (yes) or 0 (no) to indicate whether the algorithm needs to mirror left rail profiles due to their representation in the rail profile text files. The orientation of the rail profile in the rail profile text file may not be correct due to a discrepancy between the direction of data collection and the direction of increasing track mileage.

The best method of correctly setting this parameter is to use Rangecam to export a single rail profile text file from the rail segment and check if the transformation matrix indicates the rail profile requires mirroring (i.e., if the number in the upper left corner of the transformation matrix is negative, the rail profile requires mirroring). The algorithm cannot use the transformation matrix in the rail profile text file to automatically determine mirroring requirements due to limitations of Rangecam's bulk export option, covered in Section 3.1.2 (Rail Profile Text Files). 
- Mirror right rail (numeric input) is set to either 1 (yes) or 0 (no) to indicate whether the algorithm needs to mirror right rail profiles due to their representation in the rail profile text files. Appropriate corrections are similar to those described for the mirror left rail parameter.

- Left wheel slope $\left(\alpha_{L}\right)$ (numeric input) is the slope that the left wheel profile makes with track level (see wheel slope in Section 1.6, Terminology). Slopes are a commonly used industry measurement for wheel profiles. The user inputs the slope input as fraction, as shown in the equation

$$
\alpha_{L, R}=1 / x
$$

where $\alpha$ is the wheel tread in degrees and $x$ is the user inputted value to analyze a wheel tread slope of 1: $x$. The input value is always positive.

- Right wheel slope $\left(\alpha_{R}\right)$ (numeric input) is the slope that the right wheel profile makes with track level (see wheel slope in Section 1.6, Terminology). The user inputs the slope input as fraction, as shown in the Equation 3.1. The input value is always positive.

The user inputs the left and right wheel slope independently to accommodate the potential cases of non-symmetric left and right wheel slopes and the wheelset not being parallel to track level.

Once the control panel in the main MATLAB function is set properly, the user can run the algorithm's MATLAB code by clicking the run button when the main algorithm function is open in MATLAB. 


\subsubsection{Inputs}

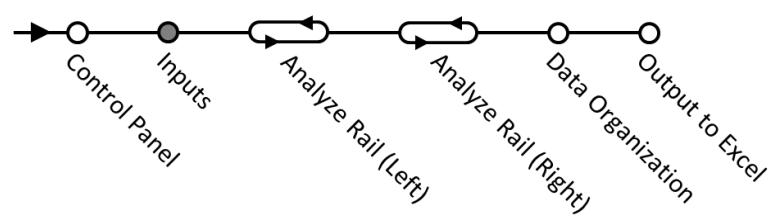

Purpose: This function obtains the track segment the user wants to analyze and returns file lists and file paths for the two rail segments. A file list contains the text file name and file path (i.e., the location on the computer drive) of every rail profile text file in a rail segment. The algorithm uses the file list to locate rail profile text files on the computer drive in order to extract rail profile information.

Inputs:

- none
Outputs:

- folder location (left rail)

- file list (left rail)

- folder location (right rail)

- file list (right rail)

- collection date

- segment name

The algorithm uses the built-in MATLAB function uigetdir to prompt the user to "Select the track segment folder" via the folder selection dialog box. Using the dialog box, the user navigates to the desired track segment folder on the computer drive, clicks on the track segment folder, and selects OK. The function uigetdi returns the folder path of the selected track segment folder.

Using the network path of the track segment folder, the algorithm builds the folder paths for the left rail segment and right rail segment using the built-in MATLAB function fullfile. The algorithm constructs the left rail segment and right rail segment folder paths in the forms 
(track segment folder path)\Left Rail

and

(track segment folder path)\Right Rail.

Using the rail segment folder paths, the algorithm constructs a file list of rail profile text files for each rail segment using the built-in MATLAB function dir. The file list contains the text file name and file path of each rail profile text file in the rail segment.

To uniquely identify the track segment analysis, the algorithm stores the collection date and the segment name. The collection date is the date the rail profile data was collected in numeric form (i.e., YYYYMMDD). The algorithm extracts the collection date from the data collection run folder and stores it as the collection date variable. The segment name is the name this research assigns to the track segment using the naming convention described in Section 3.1.4 (Folder Structure and Naming Requirements). The algorithm extracts the segment name from the track segment folder and stores it as the segment name variable.

After the input function is completed, the algorithm executes the rail segment analysis function twice (once for each rail segment of the track segment) which analyzes the rail segment on a profile-by-profile basis. The following sections detail the analysis of a single profile of a rail segment, unless otherwise noted. The algorithm completes the rail profile analysis sequentially in increasing mileage for every rail profile in the rail segment. 


\subsubsection{Analyzing Rail Profiles of a Rail Segment}

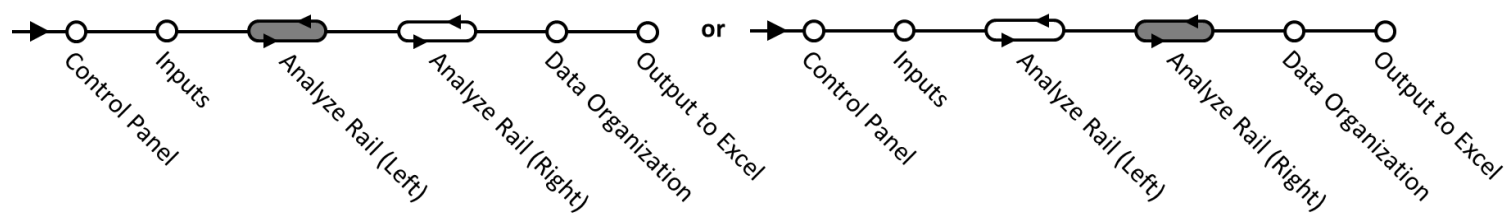

This section covers the twelve functions (Sections 3.2.3.1 to 3.2.3.12) that the algorithm runs to analyze a rail profile within a rail segment. At a high-level, the analyze rail function extracts data from the rail profile text file, cleans and prepares the data, and then calculates the lateral contact position and contact radius performance measures. This algorithm repeats this process for every rail profile in the rail segment. Figure 19 shows the flow chart diagram of the analyze rail functions. Specifically, the functions are:

1. input text file to MATLAB;

2. plot rail profile;

3. remove similar $x, y$-coordinates;

4. correct $x, y$-coordinate order;

5. rotate rail profile;

6. find largest monotonically increasing set of $x, y$-coordinates;

7. remove rail profile field and gauge sides;

8. transform head $x, y$-matrix;

9. smooth rail profile data;

10. create surface angle matrix;

11. calculate lateral contact position; and

12. calculate contact radius. 


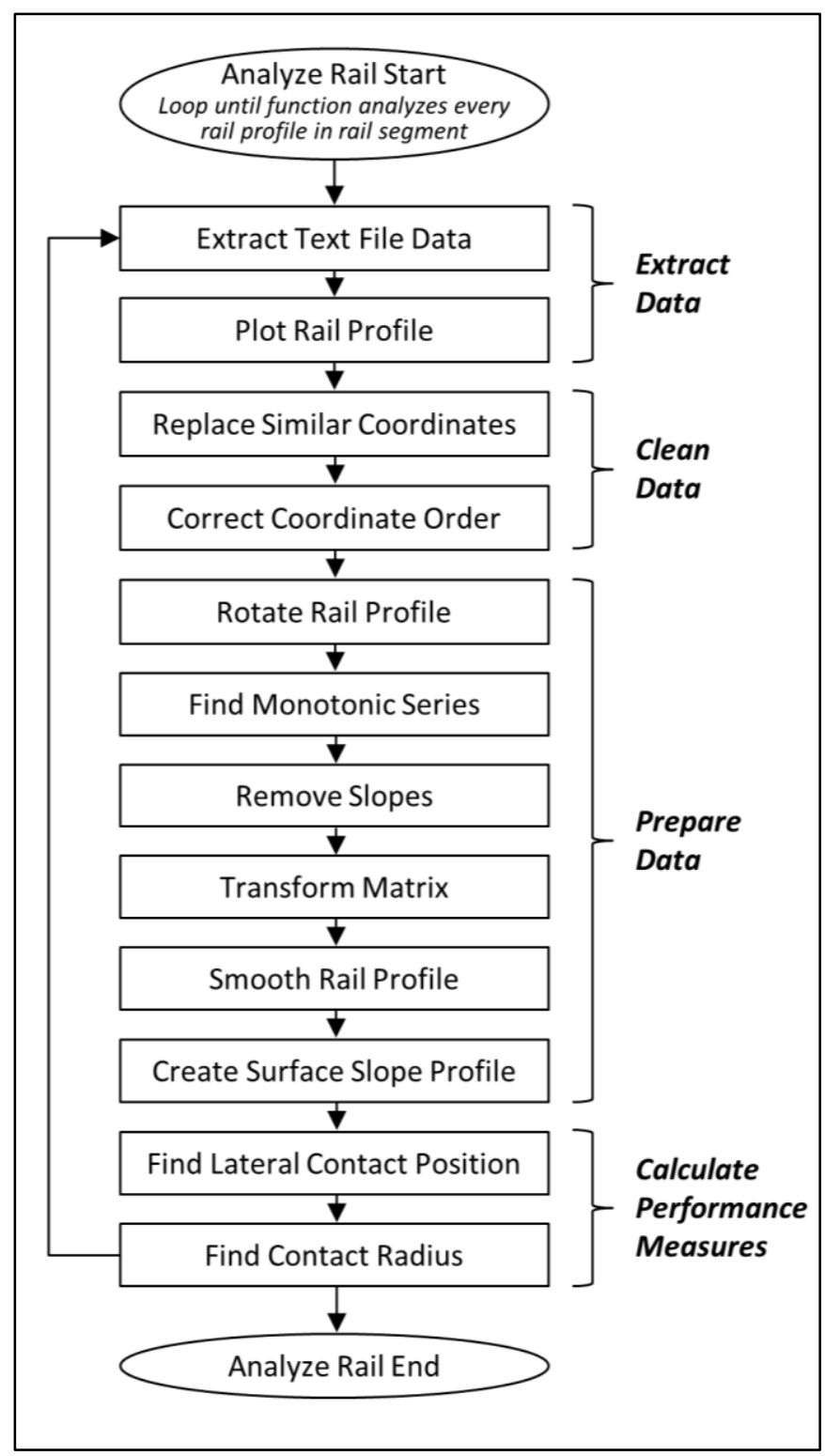

Figure 19: Flow chart diagram of analyze rail functions

To complete a track analysis, the algorithm sequentially analyzes every measured rail profile in the left rail segment in increasing mileage order and then sequentially analyzes every measured rail profile in the right rail segment in increasing mileage order. Since the algorithm orients rail profiles such that the gauge side is always in the upper right quadrant of a Cartesian coordinate system independent of which side-of-track the rail profile is from, the algorithm is able to use the same functions for both the left and right 
rail profiles.

In addition to the control panel and input variables, there are two other variables that the algorithm stores when analyzing the rail profiles of a rail segment:

- rail side (string) stores the value 'L' or 'R' denoting the left rail segment or right rail segment, respectively. The algorithm uses this variable to in a following function to mirror the rail profile for each rail correctly.

- profile count (numeric) stores the profile count of the current profile the algorithm is analyzing. The algorithm updates the value at the beginning of each rail profile analysis. The profile count is also referred to as the profile $I D$, for which a user can reference a particular profile in a rail segment across multiple data outputs. The count represents the ordering of the rail profile within the rail segment, with respect to increasing mileage (e.g., the algorithm stores a profile $I D$ of four for the fourth rail profile in the rail segment).

Prior to the algorithm running any functions, it pre-allocates two matrices for the rail segment: the rail segment analysis matrix (Table 1) and the rail segment function analysis matrix (Table 2). Pre-allocating a matrix refers to creating an empty matrix prior to storing data instead of incrementally increasing the size of a matrix; this saves computational resources and results in a faster analysis. The columns of the matrix are attributes of the rail profiles and the rows are the individual rail profiles in the rail segment. Using the file list, the algorithm identifies the $n$ number of measured rail profiles. The sizes of the pre-allocated matrices follow:

- Left rail segment analysis matrix $\left(n_{L} \times 7\right)$

- Left rail segment function performance matrix $\left(n_{L} \times 4\right)$ 
- $\quad$ Right rail segment analysis matrix $\left(n_{R} \times 7\right)$

- $\quad$ Right rail segment function performance matrix $\left(n_{R} \times 4\right)$

Table 1: Rail segment analysis matrix

\begin{tabular}{|c|c|c|c|c|c|c|}
\hline \multirow[b]{2}{*}{ column } & \multicolumn{6}{|c|}{ (Left or Right) Rail Segment Analysis } \\
\hline & 1 & 2 & 3 & 4 & 5 & 6 \\
\hline & 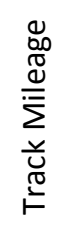 & 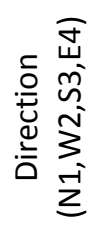 & $\begin{array}{l}\text { 으 } \\
\frac{0}{4} \\
\frac{1}{0} \\
\frac{1}{0}\end{array}$ & 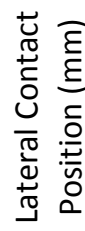 & 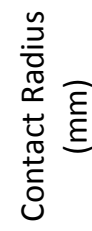 & $\begin{array}{l}0 \\
0 \\
0 \\
0 \\
0 \\
\stackrel{0}{0} \\
0 \\
10\end{array}$ \\
\hline row 1 & & & & & & \\
\hline$\ldots$ & & & & & & \\
\hline row $n$ & & & & & & \\
\hline
\end{tabular}

Table 2: Rail segment function performance matrix

\begin{tabular}{|c|c|c|c|c|}
\hline \multirow[b]{2}{*}{ column } & \multicolumn{4}{|c|}{$\begin{array}{l}\text { (Left or Right) Rail Segment } \\
\text { Function Performance }\end{array}$} \\
\hline & 1 & 2 & 3 & 4 \\
\hline & 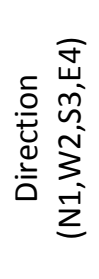 & $\begin{array}{l}\frac{0}{0} \\
\frac{0}{4} \\
\frac{0}{0}\end{array}$ & 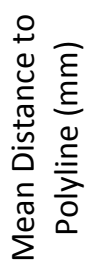 & 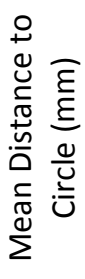 \\
\hline row 1 & & & & \\
\hline$\ldots$ & & & & \\
\hline row $n$ & & & & \\
\hline
\end{tabular}

The algorithm stores the profile ID under column 3 in the rail segment analysis matrix (Table 1) and column 2 in the rail segment function performance matrix (Table 2) for the 
row of that particular profile. The row of the profile matches the profile $I D$ for both matrices.

\subsubsection{Text File to MATLAB}

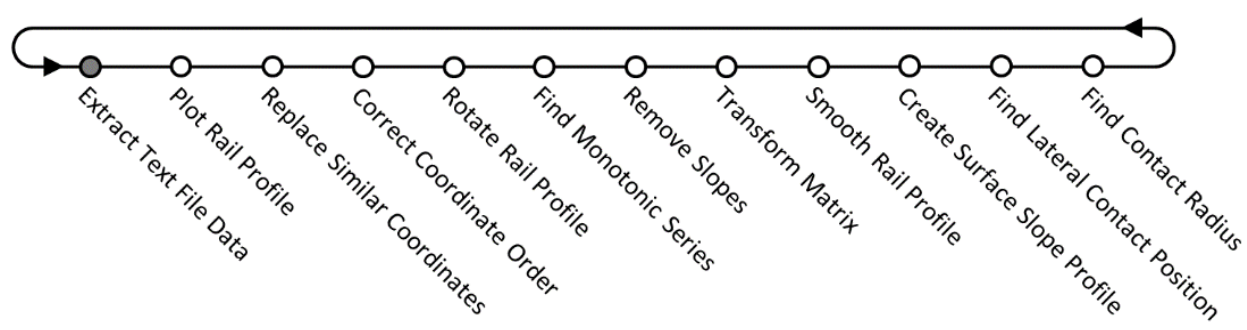

Purpose: Using a Rangecam exported text file, this function creates a matrix containing rail profile $x, y$-coordinates and extracts the corresponding rail profile metadata. The $x, y$ coordinates are the measured shape of the rail profile recorded during data collection which allows for the rail profile to be computationally stored, transformed, and analyzed in the subsequent functions. The metadata contains the information on both the identification and spatial location of the rail profile and are fundamental inputs to certain functions.

Inputs:

Outputs:

- file identification

- mirror [left/right] rail
- mileage location

- compass direction

- track gauge

- cant angle

- railhead $x, y$-matrix $\mathrm{v} 1.0$

- remove profile

The algorithm first obtains the file path of the rail profile text file from the rail segment file list and then opens the rail profile text file for read access using the built-in MATLAB function fopen. Using the file identification, the built-in MATLAB function textscan reads the data from the rail profile text file into a cell array in MATLAB; each row of the rail 
profile text file is stored as a single string in a cell within the array.

To obtain the necessary metadata, the algorithm parses rows six and seven of the cell array, obtains the necessary metadata, and applies the proper data transformations. The algorithm obtains the following metadata:

- $\quad$ compass direction (numeric; 1 = north, 2 = west, 3 = south, 4 = east)

- mileage (numeric)

- $\quad$ start row of the rail profile head segments (numeric)

- number of rail profile head segments (numeric)

- $\quad$ track gauge (numeric); and

- cant angle (numeric).

The algorithm then parses and transforms each string that contains a line segment that represents the railhead into four numeric values, $x_{1}, y_{1}$ (start of line segment) and $x_{2}, y_{2}$ (end of line segment) and stores them in a temporary matrix. The algorithm uses the location of the starting row and the number of line segments to extract only the line segments representing the railhead. Since the end $x, y$-coordinate and start $x, y$ coordinate of neighboring line segments are expected - but not guaranteed - to be identical, the algorithm finds every unique $x, y$-coordinate in the temporary matrix using the function unique and stores those values in an $i \times 2$ matrix called the railhead $x, y$ matrix. Table 3 shows an example of MATLAB's unique function using a selection of rail profile data. 
Table 3: Example of selection unique $x, y$-coordinates from the temporary matrix (a) and storing them in the railhead $x, y$-matrix (b)

(a)

\begin{tabular}{|c|c|c|c|}
\hline \multicolumn{5}{|c}{ Temporary Matrix } \\
\hline $\boldsymbol{x}_{\mathbf{1}}$ & $\boldsymbol{y}_{\mathbf{1}}$ & $\boldsymbol{x}_{\mathbf{2}}$ & $\boldsymbol{y}_{\mathbf{2}}$ \\
\hline-34.29 & 104.902 & -34.643 & 108.164 \\
-34.643 & 108.164 & -34.72 & 110.618 \\
-34.72 & 110.618 & -34.702 & 111.217 \\
-34.702 & 111.217 & -34.636 & 111.766 \\
-34.636 & 111.766 & -34.497 & 116.464 \\
-34.497 & 116.464 & -34.53 & 117.015 \\
-34.426 & 120.513 & -34.46 & 121.064 \\
-34.46 & 121.064 & -34.292 & 126.712 \\
-34.292 & 126.712 & -34.029 & 128.855 \\
-34.029 & 128.855 & -33.915 & 129.302 \\
-33.915 & 129.302 & -33.587 & 130.243 \\
-33.587 & 130.243 & -33.162 & 131.08 \\
\hline
\end{tabular}

unique value duplicate value (b)

railhead $x, y$-matrix

\begin{tabular}{|c|c|}
\hline $\boldsymbol{x}$ & $\boldsymbol{y}$ \\
\hline-34.29 & 104.902 \\
-34.643 & 108.164 \\
-34.72 & 110.618 \\
-34.702 & 111.217 \\
-34.636 & 111.766 \\
-34.497 & 116.464 \\
-34.53 & 117.015 \\
-34.426 & 120.513 \\
-34.46 & 121.064 \\
-34.292 & 126.712 \\
-34.029 & 128.855 \\
-33.915 & 129.302 \\
-33.587 & 130.243 \\
-33.162 & 131.08 \\
\hline
\end{tabular}

There are two independent conditions that require the algorithm to mirror the rail profile $x, y$-coordinates along the $y$-axis:

1. The rail profile is from the right rail segment. The algorithm will mirror the rail profile so that the gauge side $x, y$-coordinates contain positive $x$-values.

2. The rail profile text file contains a mirrored rail profile, as shown by the transformation matrix if the rail profile text file is exported as a single profile. The algorithm will mirror the rail profile to return it to the correct orientation. The rail profile may require mirroring due to the direction of data collection relative to the direction of increasing mileage of the track. The algorithm uses the variable mirror [left/right] rail to determine if the rail profile requires mirroring for this condition. 
If both conditions require the algorithm to mirror the rail profile, mirroring in not required as mirroring the rail profile twice is identical to not mirroring the rail profile.

To mirror a rail profile, the algorithm multiplies every $x$-value in the railhead $x, y$-matrix by -1 , mirroring the $x, y$-coordinates along the line $x=0$ (i.e., the vertical centreline of the non-wearing areas of the rail profile). The algorithm then reverses the order of the rows of the updated railhead $x, y$-matrix using the built-in MATLAB function flipud. Reversing the row order ensures the first row of the railhead $x, y$-matrix remains the bottom most coordinate on the field side of the rail profile and that each successive row contains the next $x, y$-coordinate moving clockwise around the rail profile.

Figure 20 shows the $x, y$-coordinates from a railhead $x, y$-matrix.

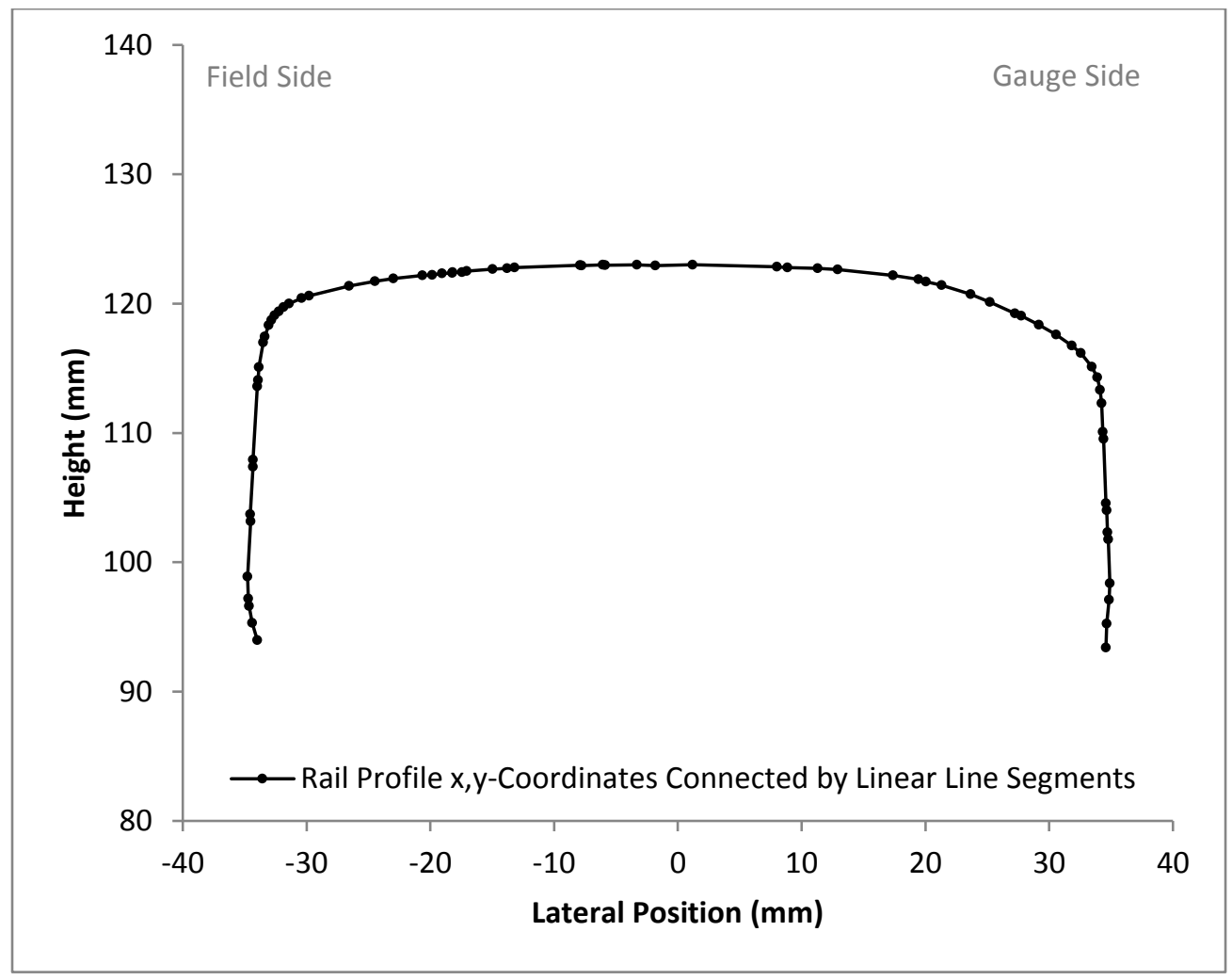

Figure 20: $x, y$-coordinates from a railhead $x, y$-matrix representing the railhead 
To ensure algorithm only analyzes reasonable railhead profile shapes, the algorithm checks if the $x, y$-coordinates are within an expected range. If any $x, y$-coordinates of a railhead profile fall outside the expect range, the algorithm plots the profile in the following function (Section 3.2.3.2, Plot Rail Profile), but does not analyze the profile (i.e., the algorithm does not run the functions in Section 3.2.3.3, Removing and Replacing Similar $\boldsymbol{x}, \boldsymbol{y}$ to Section 3.2.3.12, Determining the Rail Profile Radius). The algorithm sets the binary variable remove profile to indicate whether the profile will be analyzed. The range of expected coordinates extends approximately $10 \mathrm{~mm}$ outside the expected profile shape to accommodate small, expected variations in profile shapes. Figure 21 shows an example of a reasonable rail profile with the range of expected values highlighted. Figure 22 shows an example of a rail profile that the algorithm does not analyze. The range of expected coordinates varies depending on the properties of the track (e.g., the rail type) but is typically consistent for a rail property.

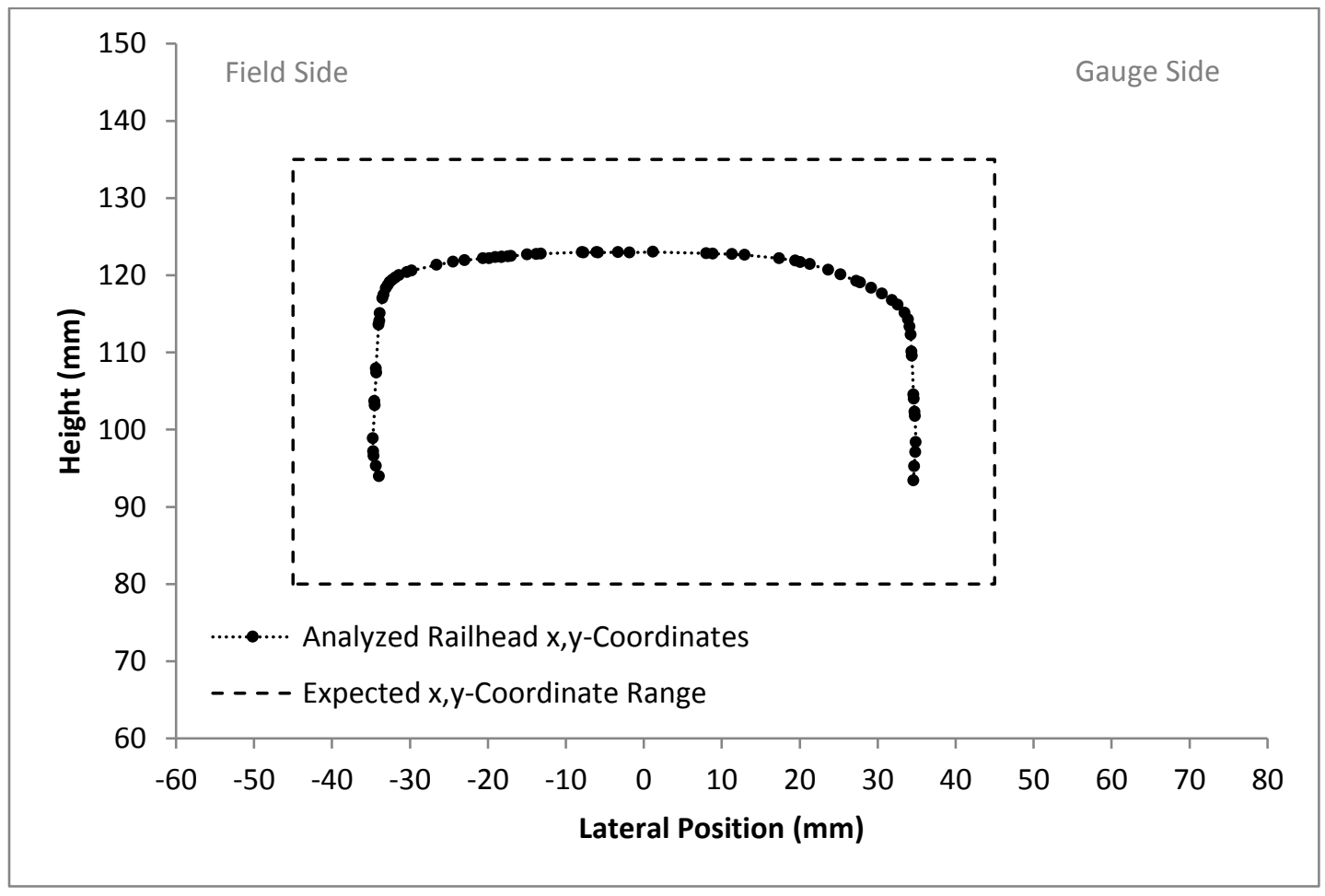

Figure 21: Example of an expected railhead profile shape 


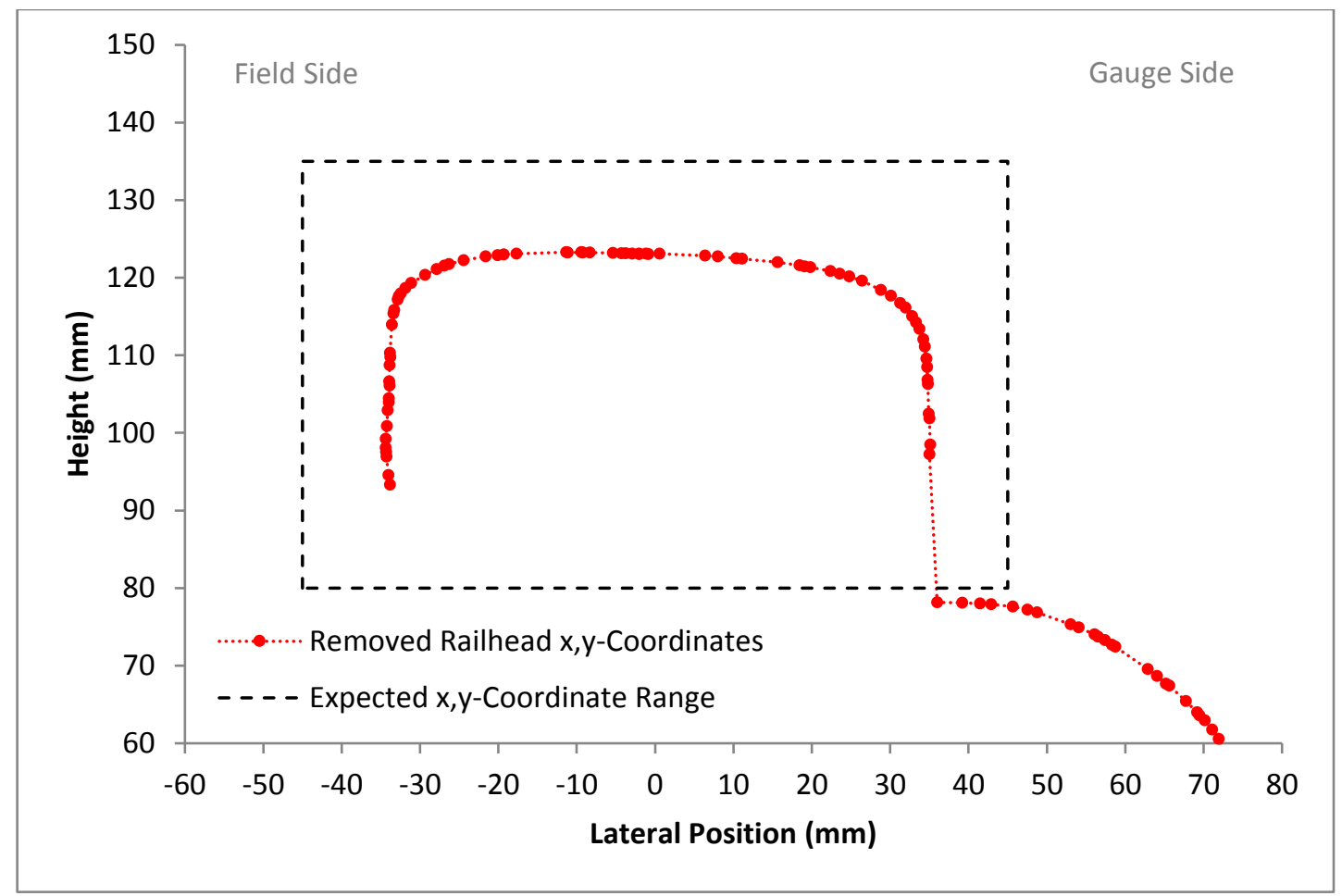

Figure 22: Example of a railhead profile removed from the analysis

The algorithm stores the mileage, compass direction, and track gauge under columns 1,2 , and 6 for the particular rail profile in the rail segment analysis matrix (Table 1), respectively. The algorithm stores the compass direction under column 1 in the rail segment function performance matrix (Table 2).

\subsubsection{Plot Rail Profile}

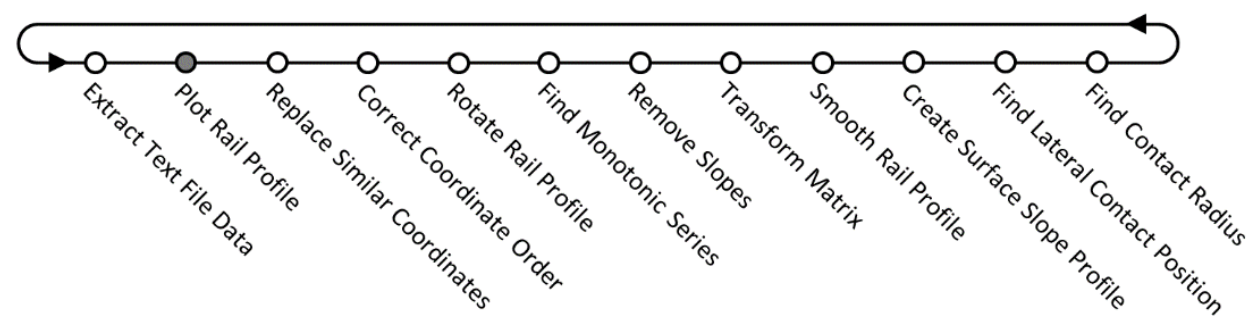

Purpose: This function plots the $x, y$-coordinates from the railhead $x, y$-matrix to the current figure in MATLAB. The function superimposes every rail profile in the rail 
segment to the same figure. The purpose of this function is to allow the user to observe the distribution of rail profile shapes of the rail segment and correct any errors that may have occurred. Examples of errors include incorrect input parameters, the rail segment folders contains the incorrect rail segment rail profile text files, or if a rail profile text file does not contain a typical rail profile shape due to either a measurement or Rangecam export error.

Inputs:

Outputs:

- $\quad$ railhead $x, y$-matrix $\mathrm{v} 1.0$

- none

- rail side

- profile count

- remove profile

Using the variables profile count and rail side, the algorithm creates a figure, which is unique for each rail side, if the profile is the first profile in the rail segment; this new figure is the current figure. The left rail segment figure differs from the right rail segment figure by the figure titles, the side labels, and the orientation of the $x$-axis. The algorithm uses the built-in MATLAB function plot to plot the $x, y$-coordinates from the railhead $x, y$ matrix to the current figure in MATLAB. If the algorithm is removing the rail profile from the analysis (based on the binary variable remove profile), the algorithm plots the removed profile using the color red instead of black. Figure 23 shows the rail profile plots after a track analysis. 
(a)

Left Rail Profiles

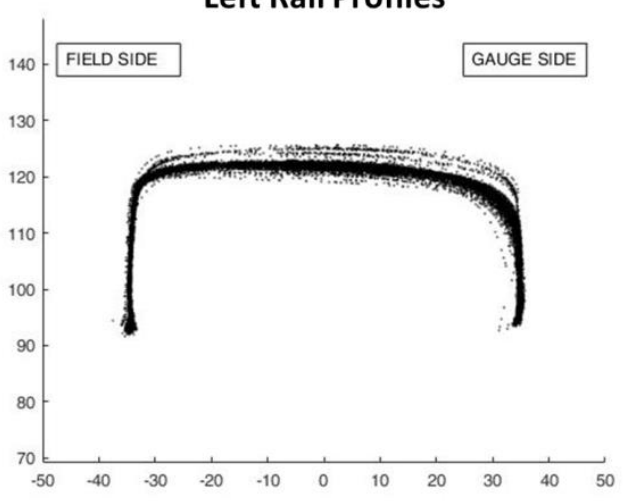

(b)

Right Rail Profiles

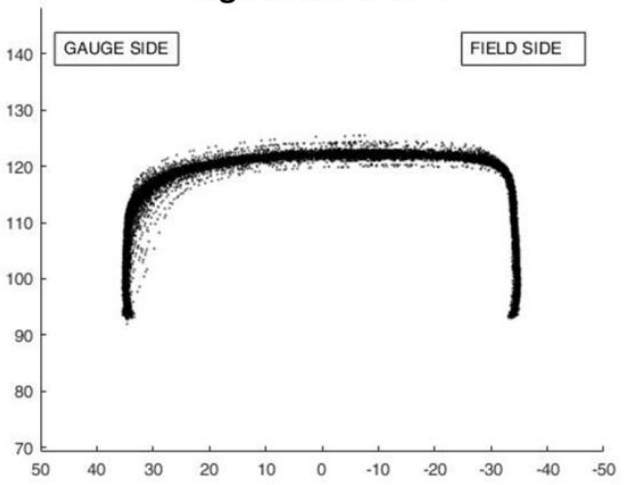

Figure 23: The post track analysis left rail profiles plot (a) and right rail profiles plot (b)

At the end of the track analysis and after inspection, the user manually closes the two figures prior to the next analysis.

\subsubsection{Removing and Replacing Similar $x, y$-Coordinates}

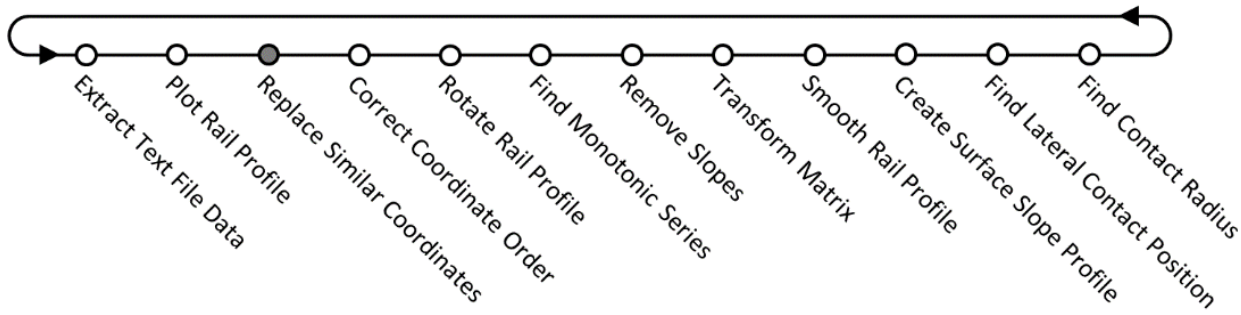

Purpose: This function scans for occurrences where two $x, y$-coordinates are too close in proximity and replaces them with a single interpolated $x, y$-coordinate. This function is necessary to avoid errors during the ordering function (Section 3.2.3.4). Due to the inherent noise in the $x, y$-coordinate data and the methodology used to correctly order the $x, y$-coordinates, two coordinates that are too close in proximity may either be: (1) originally in the incorrect order and then missed by the ordering function or (2) originally in the correct order then incorrectly ordered by the ordering function. This function nearly 
eliminates these issues and does not significantly affect the shape of the rail profile.

Inputs:

- $\quad$ railhead $x, y$-matrix $\mathrm{v} 1.0$

\section{Outputs:}

- $\quad$ railhead $x, y$-matrix v2.0

The algorithm uses a threshold of $\Delta \theta<0.001$ from polar $\theta, \rho$-coordinates to determine whether any two adjacent $x, y$-coordinates are too close in proximity. The following two steps convert the Cartesian $x, y$-coordinates to polar $\theta, \rho$-coordinates:

1. Shift the $x, y$-coordinates in the $y$-direction so that the rail profile's lowest $y$-coordinate is $0.1 \mathrm{~mm}$ above the line $y=0$. The amount the algorithm shifts the $y$-coordinates is given by the following two equations

$$
\begin{gathered}
\text { shift }=\min (\text { railhead } y \text {-matrix })-0.1, \\
y_{\text {shift }}=y-\text { shift },
\end{gathered}
$$

where $\min ($ railhead $y$-matrix $)$ is the minimum $y$-value of the railhead $x, y$ matrix, shift is the amount the algorithm shifts the $y$-coordinates, and $y_{\text {shift }}$ are the shifted $y$-coordinates. This step places the origin approximately equidistant from all $x, y$-coordinates, allowing for $\theta$ measurements that reflect the clockwise ordering of the rail profile $x, y$-coordinates.

2. Use the built-in MATLAB function cart2pol to convert the $x, y_{\text {shift }}$-coordinates to polar $\theta, \rho$-coordinates and stores them in a temporary matrix.

Using the $\theta, \rho$-coordinates, the algorithm uses the MATLAB function diff to determine the difference in $\theta$-values between adjacent coordinates, flagging any occurrences where 
two adjacent coordinates have a $\Delta \theta<0.001$. The threshold is an arbitrary value that this research determined using a trial-and-error process until the algorithm consistently produced the correct $x, y$-coordinate order in the following Removing and Replacing Similar $\boldsymbol{x}, \boldsymbol{y}$-Coordinates function (Section 3.2.3.4).

For each occurrence flagged, the algorithm takes the corresponding $x, y$-coordinates from the railhead $x, y$-matrix and replaces them with a single $x, y$-coordinate linearly interpolated at the mean $x$-value of the $x, y$-coordinate pair using the MATLAB function interp1. Figure 24 illustrates the locations of the removed $x, y$-coordinates and the replacement $x, y$-coordinate; the $x$-axis and $y$-axis are both scaled to observe these coordinates.

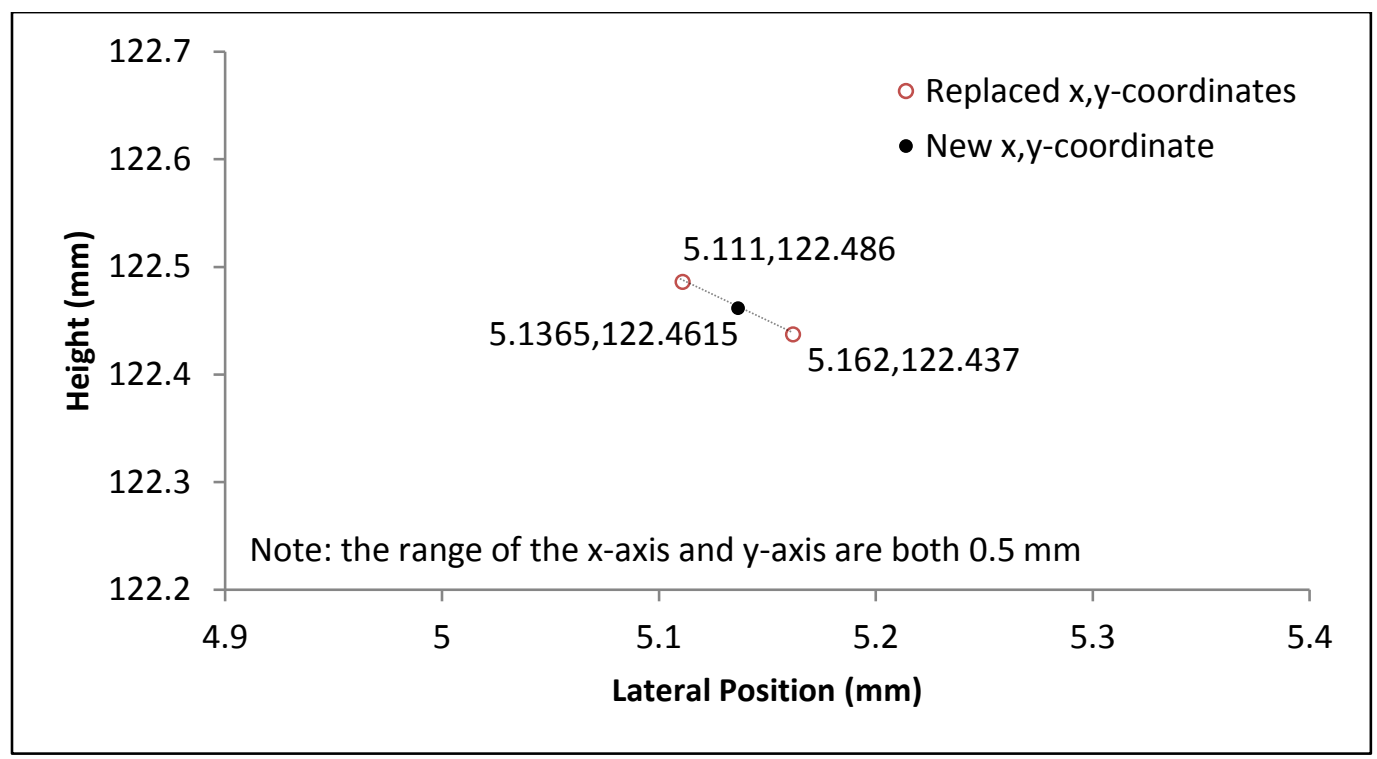

Figure 24: Example of removing and replacing similar $x, y$-coordinates 


\subsubsection{Correcting Order of $x, y$-Coordinates}

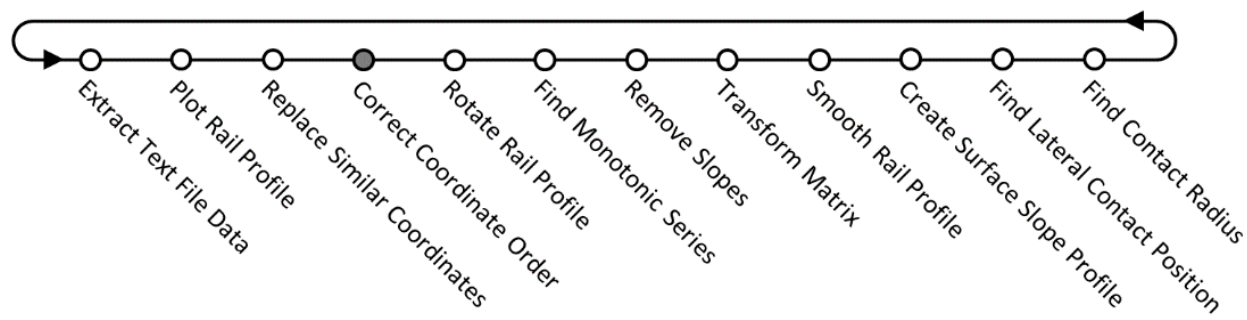

Purpose: This function corrects all $x, y$-coordinate ordering errors in the railhead $x, y$ matrix. The correct ordering of the $x, y$-coordinates is critical as many of the subsequent functions rely on the $x, y$-coordinates being in the correct order; if not, the results may not be correct or the algorithm execution could terminate due to error. When the $x, y$ coordinates are in the correct order in the railhead $x, y$-matrix, the $x_{i}, y_{i}$-coordinate in the rail profile, starting from the bottom left of the railhead (field side) and moving clockwise, is located in row $i$ of the railhead $x, y$-matrix. The algorithm encounters $x, y$-coordinate ordering errors in up to approximately $10 \%$ of rail profiles in a rail segment.

Inputs:

Outputs:

- $\quad$ railhead $x, y$-matrix $\mathrm{v} 2.0$

- $\quad$ railhead $x, y$-matrix $\mathrm{v} 3.0$

To achieve the correct $x, y$-coordinate order in the railhead $x, y$-matrix, the algorithm first finds the polar $\theta, \rho$-coordinates values using the same method found in the Removing and Replacing Similar $\boldsymbol{x}, \boldsymbol{y}$-Coordinates function (Section 3.2.3.3).

Using the $\theta, \rho$-coordinates, the algorithm sorts the rows of the temporary matrix by decreasing $\theta$-coordinates using the built-in MATLAB function sortrows. Sorting the $\theta$ coordinates by descending order ensures all railhead coordinates are in clockwise order, starting from the bottom left (field side) of the rail profile. 
After sorting, the algorithm converts the sorted $\theta, \rho$-coordinates to Cartesian coordinates and reverses the $y$-direction shift using the following steps:

1. Use the built-in MATLAB function pol2cart to convert the sorted $\theta, \rho$ coordinates to $x, y_{\text {shift }}$-coordinates.

2. Shift the $x, y_{\text {shift }}$-coordinates in the $y$-direction to reverse the original downward shift. The amount the algorithm shifts the $y$-coordinates is given by the following equations

$$
y=y_{\text {shift }}+\text { shift },
$$

Where $y$ is the new Cartesian coordinate, $y_{\text {shift }}$ the converted $y$-coordinate before the shift is reversed, and shift is the amount the algorithm shifts the coordinates when converting to polar coordinates at the beginning of the function.

The new $x, y$-coordinates over-write the current railhead $x, y$-matrix.

\subsubsection{Rotate Rail Profile}

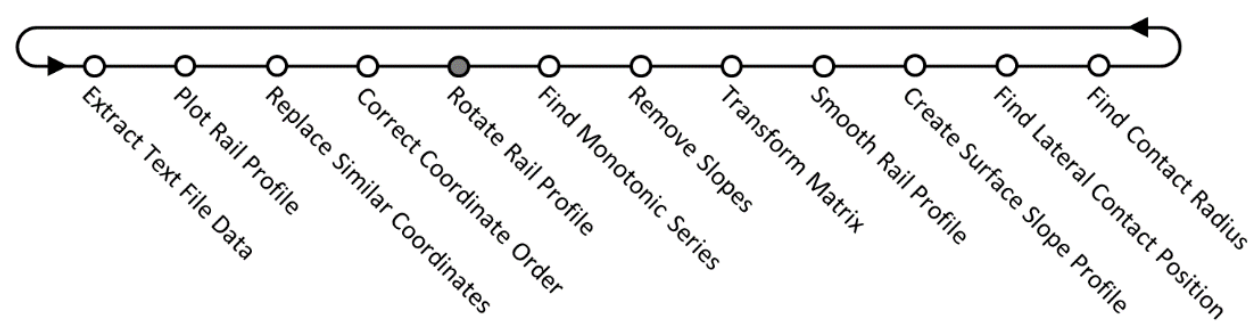

Purpose: This function rotates the rail profile $x, y$-coordinates around the origin by a combination of the cant angle (to situate the rail profile as oriented in the field when measured) and the wheel tread angle (to align the linear wheel profile parallel to the $x$ - 
axis). Based on rigid contact theory, the wheel and rail profiles will be parallel at the point of contact; therefore, in the rotated orientation, the surface angle of wheel profile and rail profile at the point of wheel-rail contact is 0 degrees (see Section 2.4.1, Rigid Contact Theory). The functions that follow utilize the known surface angle of contact to calculate to lateral contact point and the contact radius. Prior to rotation, the rail profile is in its original orientation (see Section 3.1.2, Rail Profile Text Files).

Inputs:

- $\quad$ railhead $x, y$-matrix v3.0

- cant angle

- $\quad$ left/right] wheel slope

\section{Outputs:}

- $\quad$ railhead $x, y$-matrix $\mathrm{v} 3.0$

- rotation matrix $(R)$

The algorithm calculates the total rotation of the rail profile using the equation

$$
\beta=\text { cant angle }-\tan ^{-1}(\alpha),
$$

where $\beta$ is the total amount the algorithm will rotate the rail profile in degrees, cant angle is the cant angle of the rail profile in degrees, and $\alpha$ is the wheel slope given by the variable [left/right] wheel slope.

The rotation matrix the algorithm uses rotates the rail profile to the gauge side for positive $\beta$ values and to the field side for negative $\beta$ values. The algorithm stores the rotation matrix as the variable $R$, shown by the equation

$$
R=\left[\begin{array}{cc}
\cos \beta & \sin \beta \\
-\sin \beta & \cos \beta
\end{array}\right]
$$

where $\beta$ is the total amount the algorithm will rotate the rail profile in degrees. The cant angle is positive, rotating the rail profile to the gauge side to situate the rail profile as oriented in the field, independently of wheel slope. Conceptually, Figure 25 shows the 
rail profile prior to rotation in its original orientation and Figure 26 shows the canted rail with a superimposed wheel profile; at this time, the orientation of the wheel profile is the inputted wheel profile and is not rotated. The wheel slope is negative in equation (3.5), rotating both the line representing wheel slope and the rail profile so that the wheel slope is parallel to the $x$-axis, as seen conceptually in Figure 27 . Based on rigid contact theory, the wheel and rail profiles will be parallel at the point of contact (Bosso et al., 2013); therefore, the surface angle of wheel profile and rail profile at the point of wheel-rail contact is 0 degrees in this orientation (see Section 2.4.1, Rigid Contact Theory). The functions that follow utilize the known surface angle of contact to calculate to lateral contact point and the contact radius.

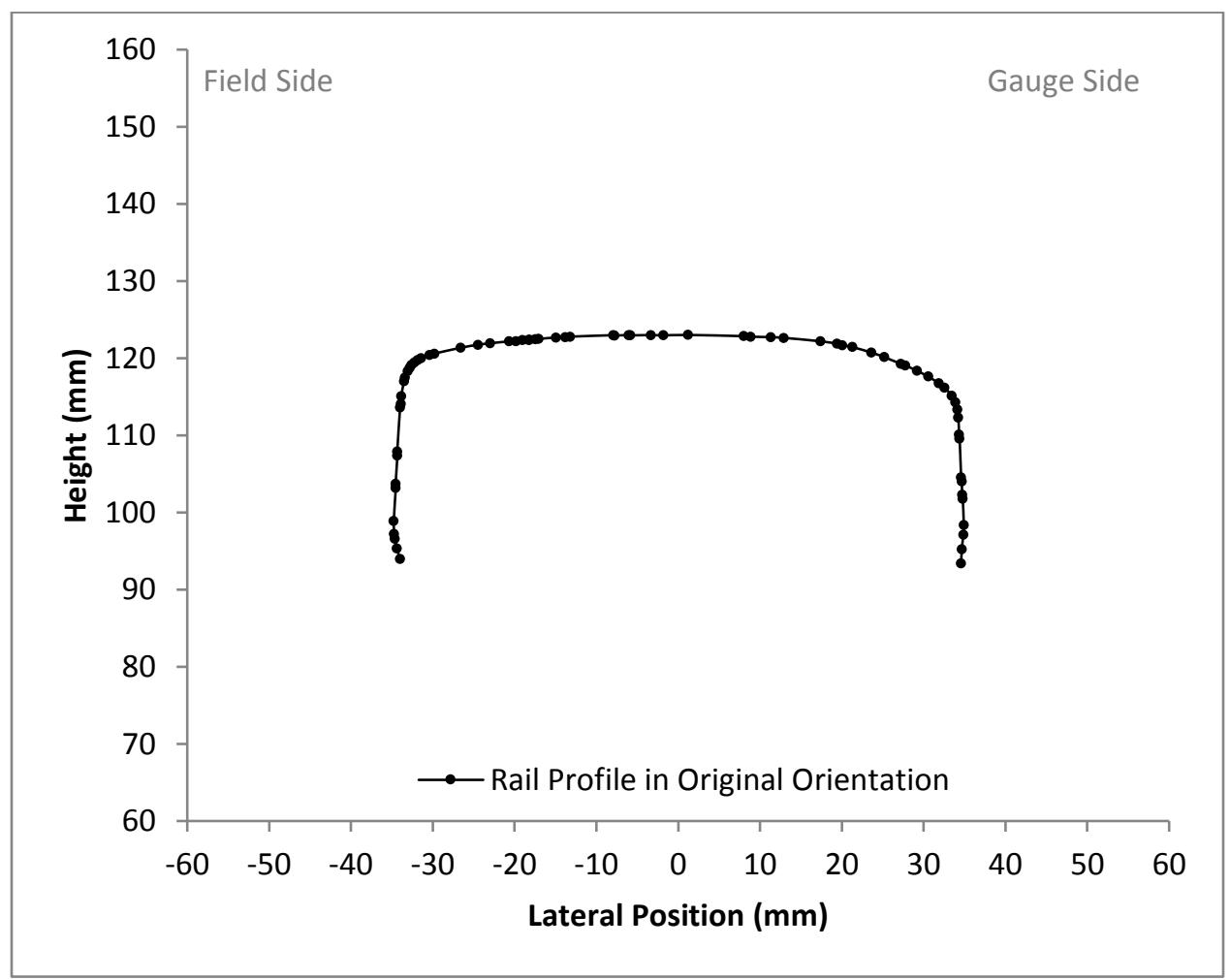

Figure 25: Rail profile in original orientation prior to rotation 


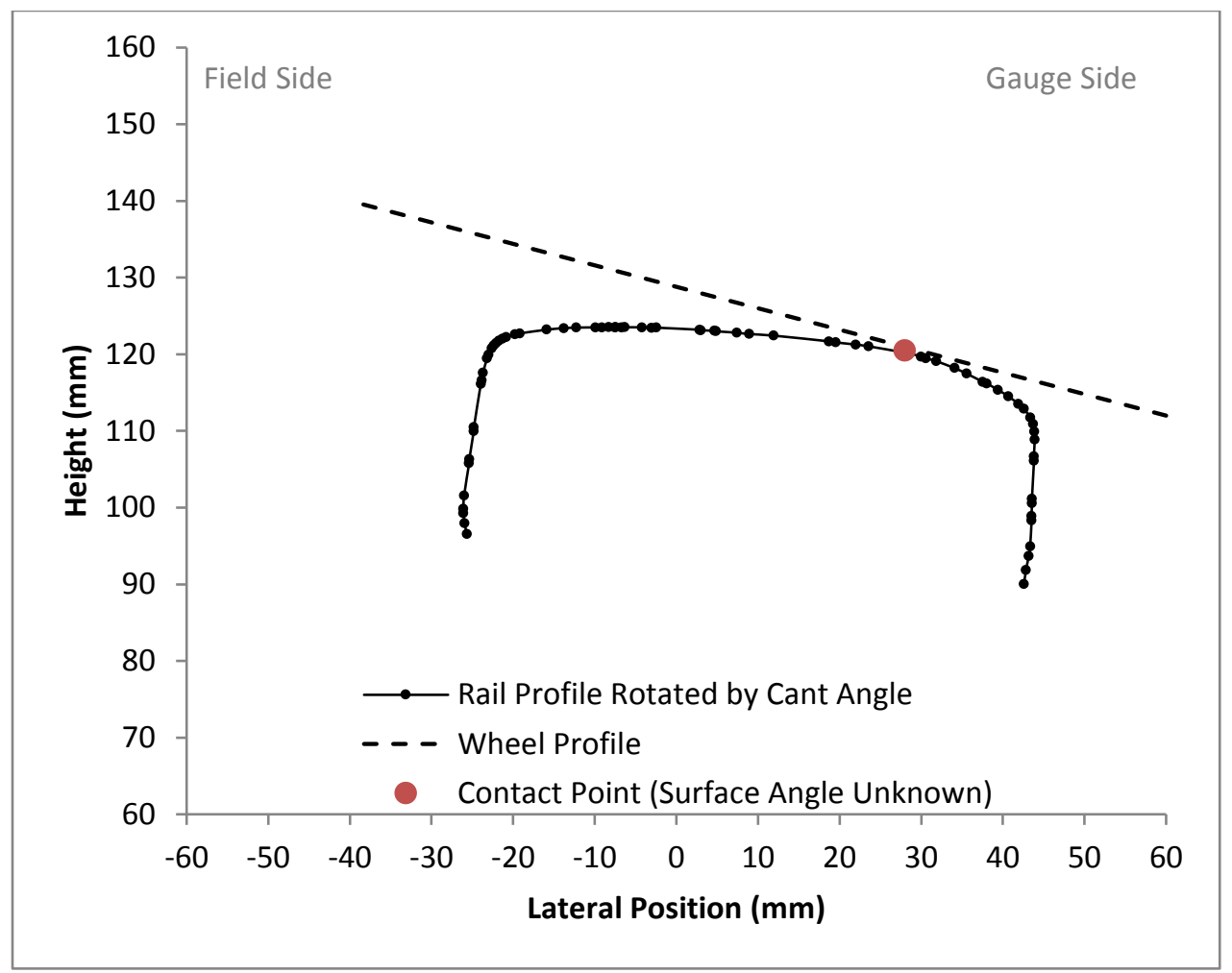

Figure 26: Rail profile rotated by cant angle with a superimposed wheel profile

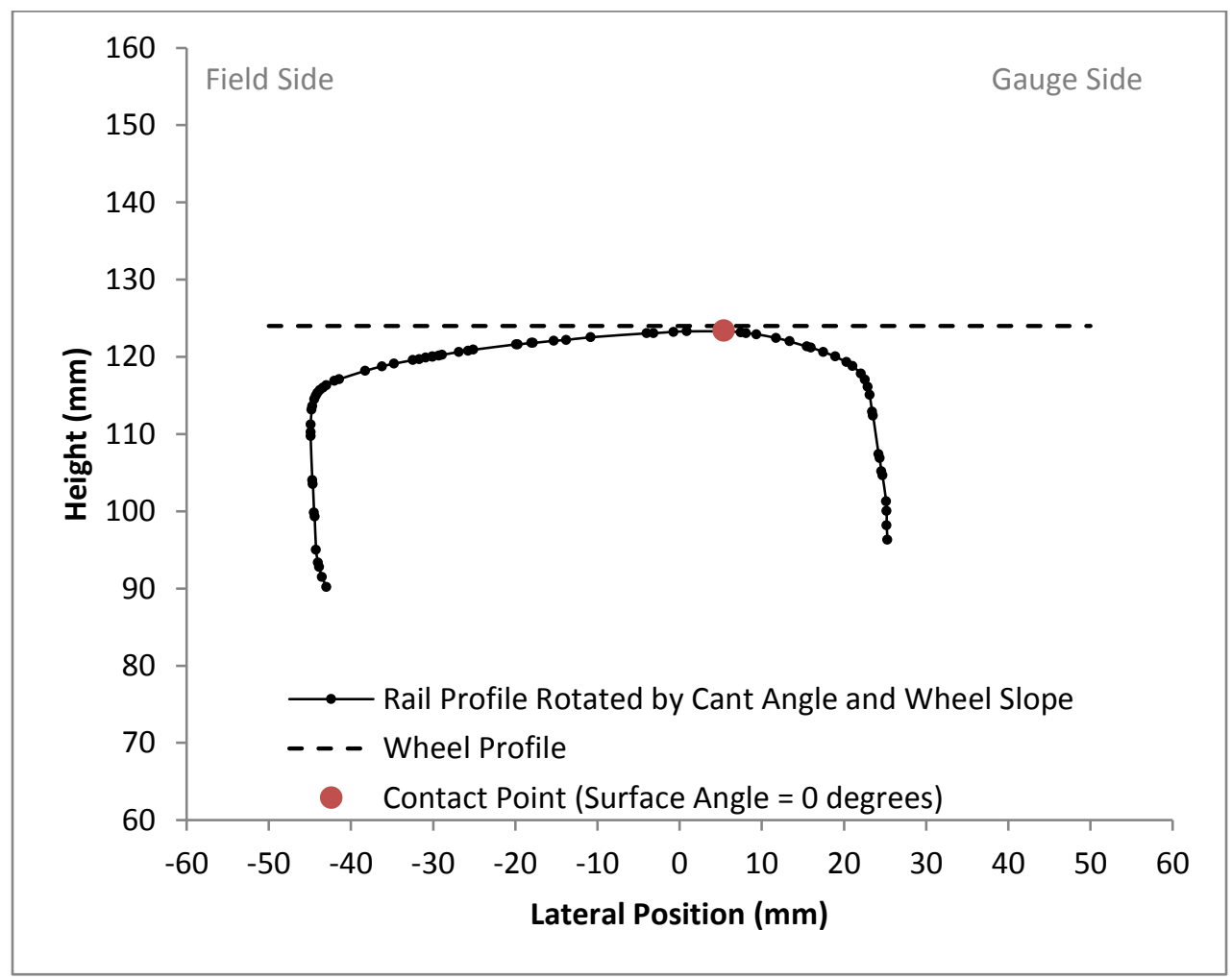

Figure 27: Rail profile rotated by cant angle and then rotated by the wheel slope 
The algorithm calculates the rotated $x^{\prime}, y^{\prime}$-coordinates using the equation

$$
\left(x_{i}{ }^{\prime}, y_{i}{ }^{\prime}\right)=\left([R]\left(\begin{array}{l}
x_{i} \\
y_{i}
\end{array}\right)\right)^{T},
$$

where the $2 \times 2$ rotation matrix is multiplied by the non-rotated $x, y$-coordinate. Figure 28 shows the original and rotated rail profile.

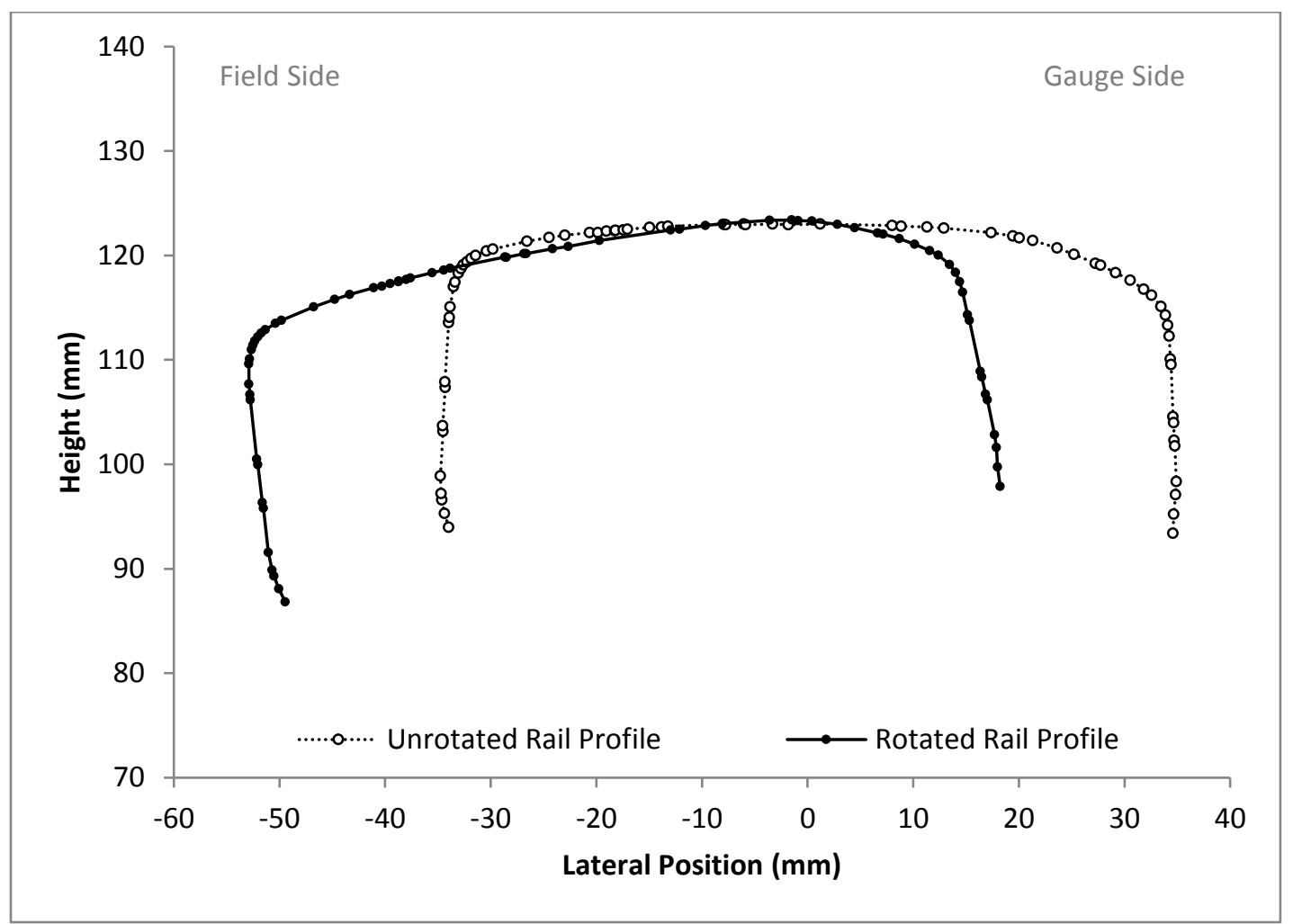

Figure 28: Comparison of rail profile before and after rotation using a 1:5 wheel slope

The algorithm overwrites the $x, y$-coordinates in the railhead $x, y$-matrix with the rotated $x^{\prime}, y^{\prime}$-coordinates. This research will hereby refer to the rotated $x^{\prime}, y^{\prime}$-coordinates as $x, y$ coordinates, as all the remaining functions use the rotated coordinates. 


\subsubsection{Find Monotonically Increasing Series of $x$, $y$-Coordinates}

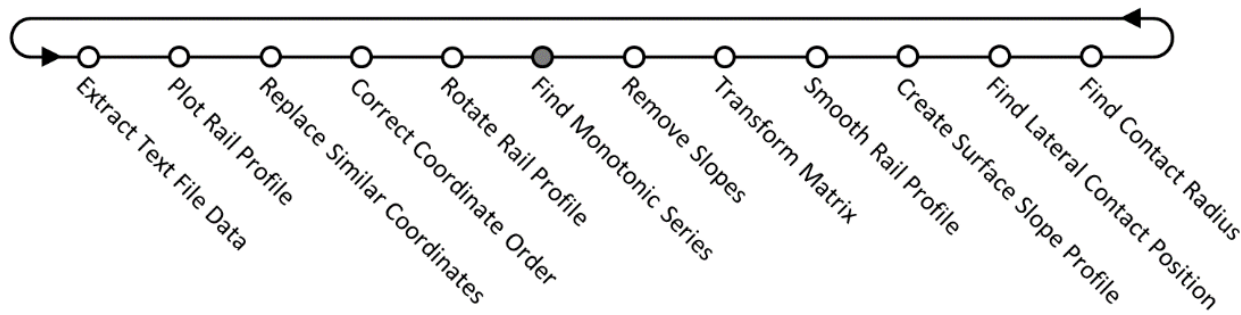

Purpose: This function removes the extreme field side and extreme gauge side of the rail profile by finding the largest series of $x, y$-coordinates that are monotonically increasing. The algorithm requires a series of monotonically increasing $x, y$-coordinates when fitting a polynomial to the rail profile data in Section 3.2.3.8 (Smooth Rail Profile Data). The extreme field and extreme gauge sides of the rail profile do not affect the type of wheelrail interaction this research focuses on.

Inputs:

- $\quad$ railhead $x, y$-matrix $\mathrm{v} 4.0$
Outputs:

- $\quad$ railhead $x, y$-matrix $\mathrm{v} 5.0$

The algorithm finds the $x, y$-coordinate that in the middle row of the railhead $x, y$-matrix. In the case where there is an even number of rows, the algorithm chooses the lower row.

The algorithm then finds the lateral distance between adjacent $x, y$-coordinates using the built-in MATLAB function diff.

Starting from the first $x, y$-coordinate in the railhead $x, y$-matrix and ending at the middle $x, y$-coordinate (i.e., starting from the field side and working clockwise), the algorithm scans the lateral distances between adjacent $x, y$-coordinates to find the last instance where the lateral distance $<0$; this is the start of a monotonically increasing set of $x, y$ - 
coordinates.

Starting from the last $x, y$-coordinate in the railhead $x, y$-matrix and ending at the middle $x, y$-coordinate (i.e., starting from the gauge side and working counter-clockwise), the algorithm scans the lateral distances between adjacent $x, y$-coordinates to find the last instances where the lateral distance $>0$; this is the end of the monotonically increasing set of $x, y$-coordinates.

The algorithm then removes all $x, y$-coordinates not contained within the start and end $x, y$-coordinates from the railhead $x, y$-matrix. Figure 29 shows the monotonically increasing set of $x, y$-coordinates from the railhead $x, y$-matrix.

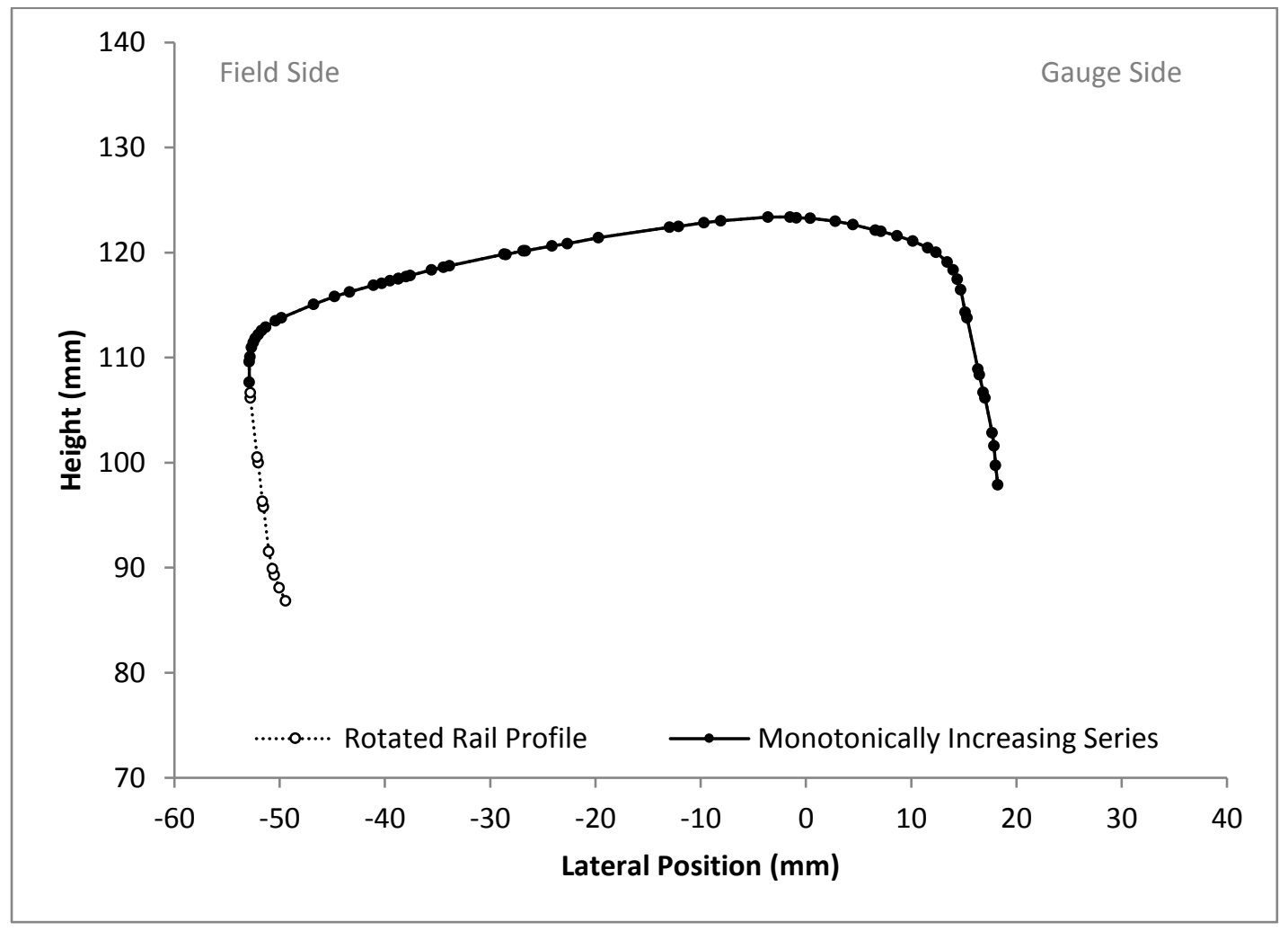

Figure 29: Largest set of $x, y$-coordinates that are monotonically increasing 


\subsubsection{Remove Slopes}

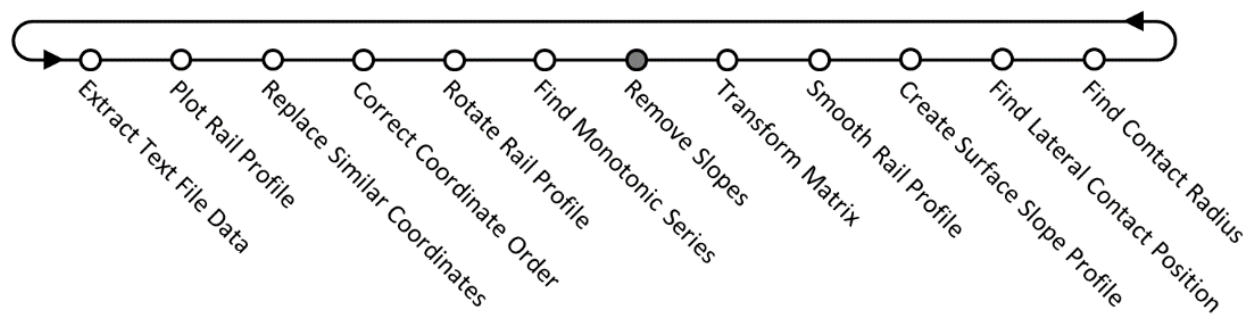

Purpose: This function removes $x, y$-coordinates that have an average slope (gradient) outside a predetermined range, typically $0 \pm 0.364$, which is based on a surface angle of $0^{\circ} \pm 20$ (due to the rotation of the rail profile, the wheel surface has a gradient slope of 0). This function is required for the proper execution of the Smooth Rail Profile Data function (Section 3.2.3.8), where a polynomial is fit to the top of the railhead to reduce noise in the data and extract a denser set of $x, y$-coordinates. Fitting a polynomial to the entire railhead would result in a poor polynomial fit that would not accurately represent the entire railhead.

Inputs:

- $\quad$ railhead $x, y$-matrix $\mathrm{v} 5.0$

- $\quad$ left/right] wheel slope

\section{Outputs:}

- $\quad$ railhead $x, y$-matrix $v 6.0$

The algorithm calculates the average slope (gradient) for each $x, y$-coordinate. This research defines the average slope of a $x, y$-coordinate to be the average of the slope(s) between it and the adjacent coordinate(s) to either side. The algorithm uses the average slope to account for the rounded shape of an actual rail profile compared to the approximated linear segmentation. To calculate the average slope $(\overline{\text { slope }})$ for the $x_{i}, y_{i^{-}}$ coordinate, the algorithm uses the equation 


$$
\overline{\text { slope }_{\iota}}=\frac{\left(\left(\frac{y_{i}-y_{i-1}}{x_{i}-x_{i-1}}\right)+\left(\frac{y_{i+1}-y_{i}}{x_{i+1}-x_{i}}\right)\right)}{2}
$$

The algorithm sets maximum rail profile surface slope (gradient) for the field side of the rail profile at 0.364 and the gauge side of the rail profile at -0.364 , with respect to a slope of 0 being a potential wheel-rail contact point. Due to the current orientation of the rail profile, the location where the wheel contacts the rail profile occurs where the surface slope is 0 . There may be multiple points on the rail profile that have a slope of 0 ; this research refers to these points as potential contact points.

This research selects a range of $0 \mp 0.364$, which was determined through a trial-anderror process. Increasing the range may negatively affect the fit of the polynomial during the Smooth Rail Profile Data function. Reducing the range may result in too few $x, y$ coordinates remaining to fit a polynomial in the Smooth Rail Profile Data function (Section 3.2.3.8)

Starting from the first $x, y$-coordinate in the railhead $x, y$-matrix and ending at the middle $x, y$-coordinate (i.e., starting from the field side and working clockwise) the algorithm finds the first $x, y$-coordinate that has an average slope (gradient) within the maximum field and gauge slopes of $0 \mp 0.364$. This $x, y$-coordinate is the first coordinate in the railhead $x, y$-matrix in the series kept for further analysis.

Starting from the last $x, y$-coordinate in the railhead $x, y$-matrix and ending at the middle $x, y$-coordinate (i.e., starting from the gauge side and working counter-clockwise), the algorithm finds the first $x, y$-coordinate that has an average slope (gradient) within the maximum field and gauge slopes of $0 \mp 0.364$. This $x, y$-coordinate is the last coordinate 
in the railhead $x, y$-matrix kept for further analysis.

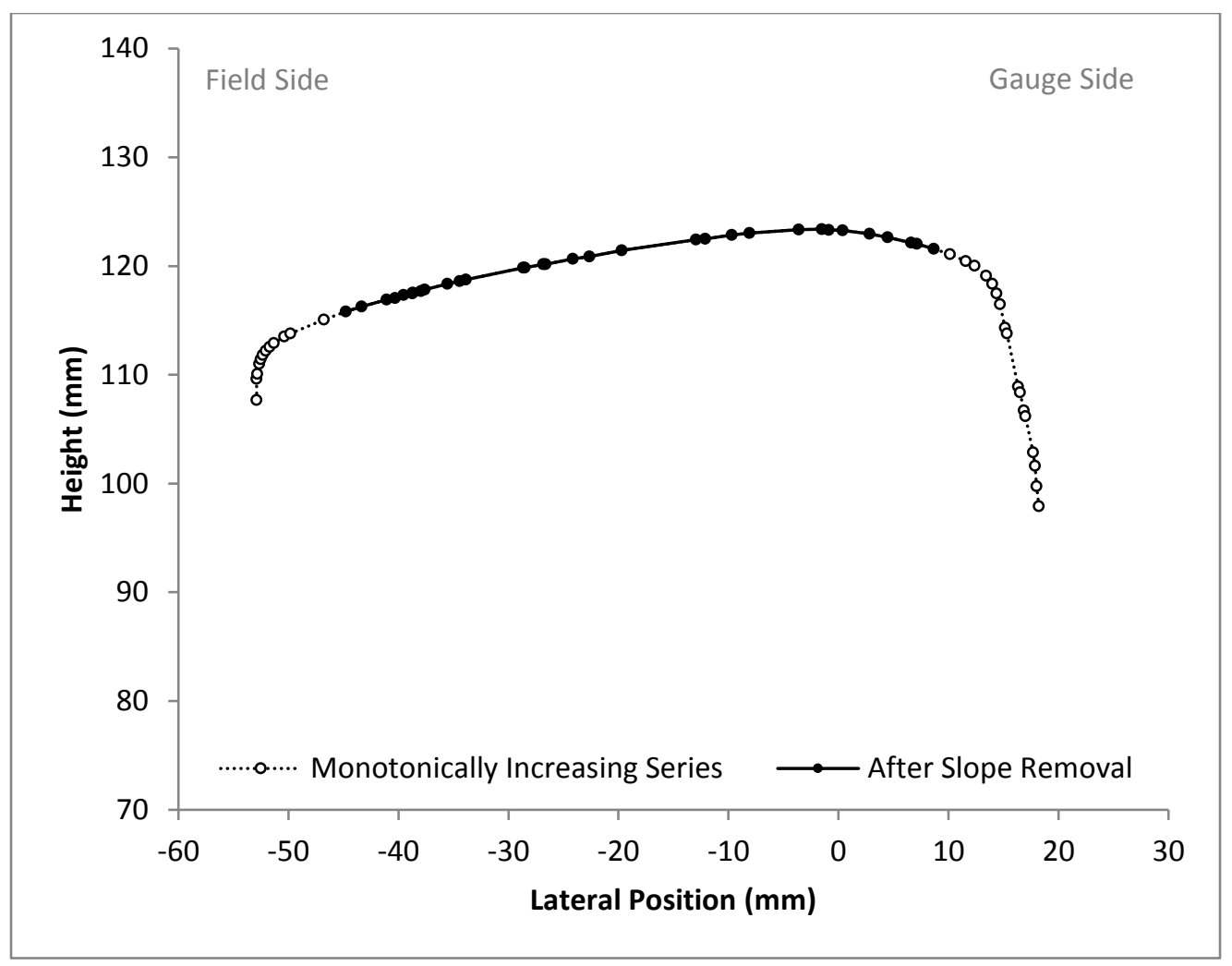

Figure 30: Removal of $x, y$-coordinates with average slopes larger than 20 degrees

The algorithm temporarily removes any $x, y$-coordinates outside the first and last coordinate found previously in this function, and evaluates if there are greater than or equal to fourteen $x, y$-coordinates remaining. If yes, the function overwrites the railhead $x, y$-matrix with the remaining $x, y$-coordinates. Otherwise, the algorithm expands the range by $\mp 0.0875$ (based on a surface angle of \pm 5 degrees) and re-runs the function. The algorithm repeats the process until at least fourteen $x, y$-coordinates remain. Having under fourteen $x, y$-coordinates remaining is undesirable. The closer the number of remaining $x, y$-coordinates is to seven, the resulting fit of the polynomial can oscillate between $x, y$-coordinates and approaches an exact fit of the data, consequently reducing the effectiveness of the polynomial to remove noise in the data. If there are less than 
seven $x, y$-coordinates remaining, there are infinite possible fits of the polynomial. The fourteen $x, y$-coordinate minimum is set through a trial and error process and is twice the minimum number of coordinates needed to avoid infinite possible fits. If the quantity of $x, y$-coordinate data measured changes (e.g., if the optical rail measurement system can extract a denser set of $x, y$-coordinate data) the minimum number of $x, y$-coordinate should be re-evaluated.

Figure 30 shows the remaining $x, y$-coordinates after the conclusion of this function.

\subsubsection{Transform Matrix}

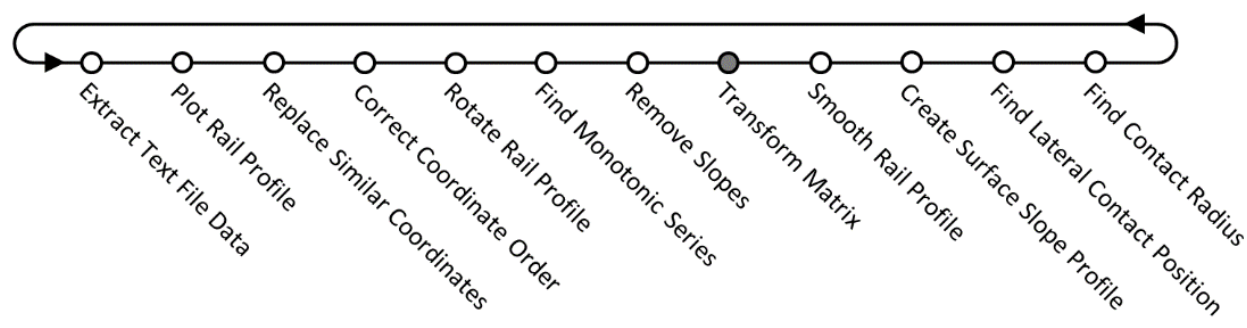

Purpose: This function centers and scales the $x, y$-coordinates in the railhead matrix to avoid poor conditioning of the fitted $6^{\text {th }}$ polynomial in the following function (Section 3.2.3.8, Smooth Rail Profile Data). An explanation of poor polynomial conditioning follows.

The amount of space allocated towards storing a variable on a computer is finite; otherwise known as finite precision. In high order, poorly conditioned polynomials, the solution of equations contains rounding errors due to the finite precision (i.e., certain values exceed the allotted computer storage space and then rounded to fit) (MathWorks, 2018). The rounding compromises the fit of the polynomial to the $x, y$-coordinates. By center and scaling down the $x, y$-coordinate values, rounding is not necessary and the fit 
of the polynomial is optimized.

Inputs:

- $\quad$ railhead $x, y$-matrix v6.0
Outputs:

- $\quad$ railhead $x, y$-matrix $\mathrm{v} 7.0$

- centre

- $\min x$

- $\max x$

- $\max y$

The algorithm calculates the mean $x$-value in the railhead $x, y$-matrix and stores the value as the variable centre.

The algorithm calculates the transformed $x^{\prime}$-coordinates using the equation

$$
x^{\prime}=(x-\text { centre }) / 10 .
$$

which centres the $x$-values by subtracting the mean $x$-value (centre) and scales the centred $x$-values by a factor of 0.1 .

The algorithm calculates the transformed $y^{\prime}$-coordinates using the equation

$$
y^{\prime}=y / 10 .
$$

which scales the $y$-coordinates by a factor of 0.1 .

Figure 31 shows the transformation matrix. 


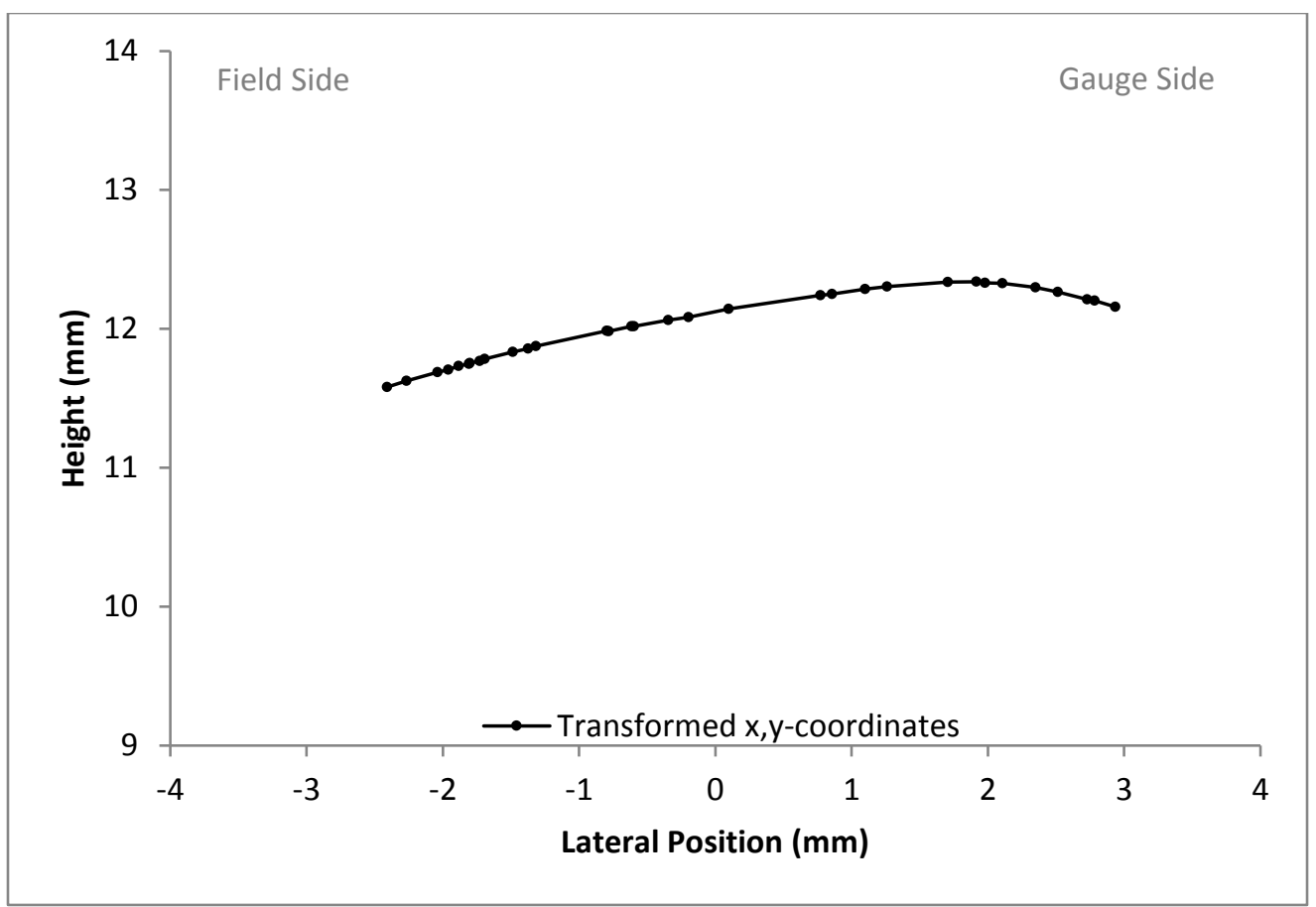

Figure 31: $x, y$-coordinates after transformation

The algorithm overwrites the $x, y$-coordinates in the railhead $x, y$-matrix with the transformed $x^{\prime}, y^{\prime}$-coordinates. This research hereby refers to the transformed $x^{\prime}, y^{\prime}$ coordinates as $x, y$-coordinates as all the remaining functions use the transformed coordinates.

The algorithm calculates the minimum $x$-value, maximum $x$-value, and maximum $y$-value from the new railhead $x, y$-matrix and stores them in the variables $\min x$, $\max x$, max $y$, respectively. 


\subsubsection{Smooth Rail Profile Data}

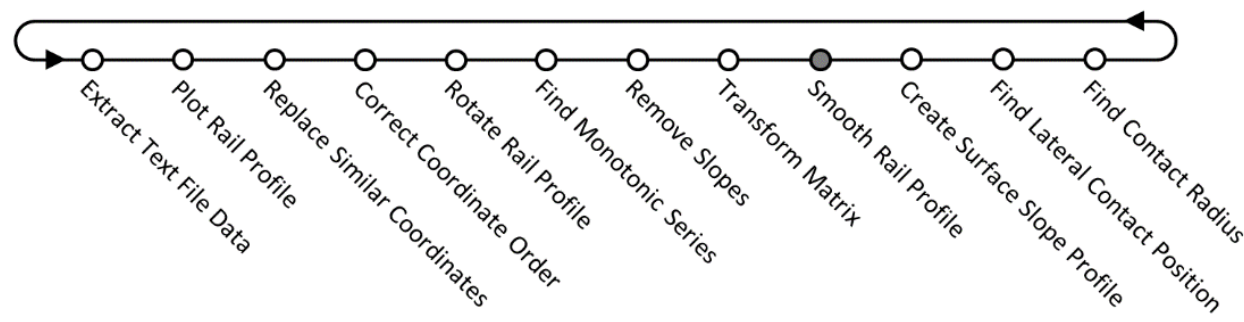

Purpose: This function takes the $x, y$-coordinates from the railhead $x, y$-matrix and fits a least-squares, $6^{\text {th }}$ order polynomial to smooth out the inherent noise in the data. The algorithm uses the railhead polynomial to determine the rail profile surface angle in Section 3.2.3.10 (Create Surface Slope), the wheel-rail contact point in Section 3.2.3.11 (Determining the Contact Point), and the rail profile radius in Section 3.2.3.12 (Determining the Rail Profile Radius).

Inputs:

Outputs:

- $\quad$ railhead $x, y$-matrix $\mathrm{v} 7.0$

- $\quad$ polynomial $R(x)$

- dist-to-poly

To remove the noise in the coordinate data, the algorithm fits a least squares, $6^{\text {th }}$ order polynomial to the $x, y$-coordinates in the railhead $x, y$-matrix using the built-in MATLAB function polyfit. This research refers to this railhead polynomial as $R(x)$. Figure 32 shows the fit of the polynomial $R(x)$ to the $x, y$-coordinates; the $y$-axis is scaled to emphasize the fit of the polynomial. 


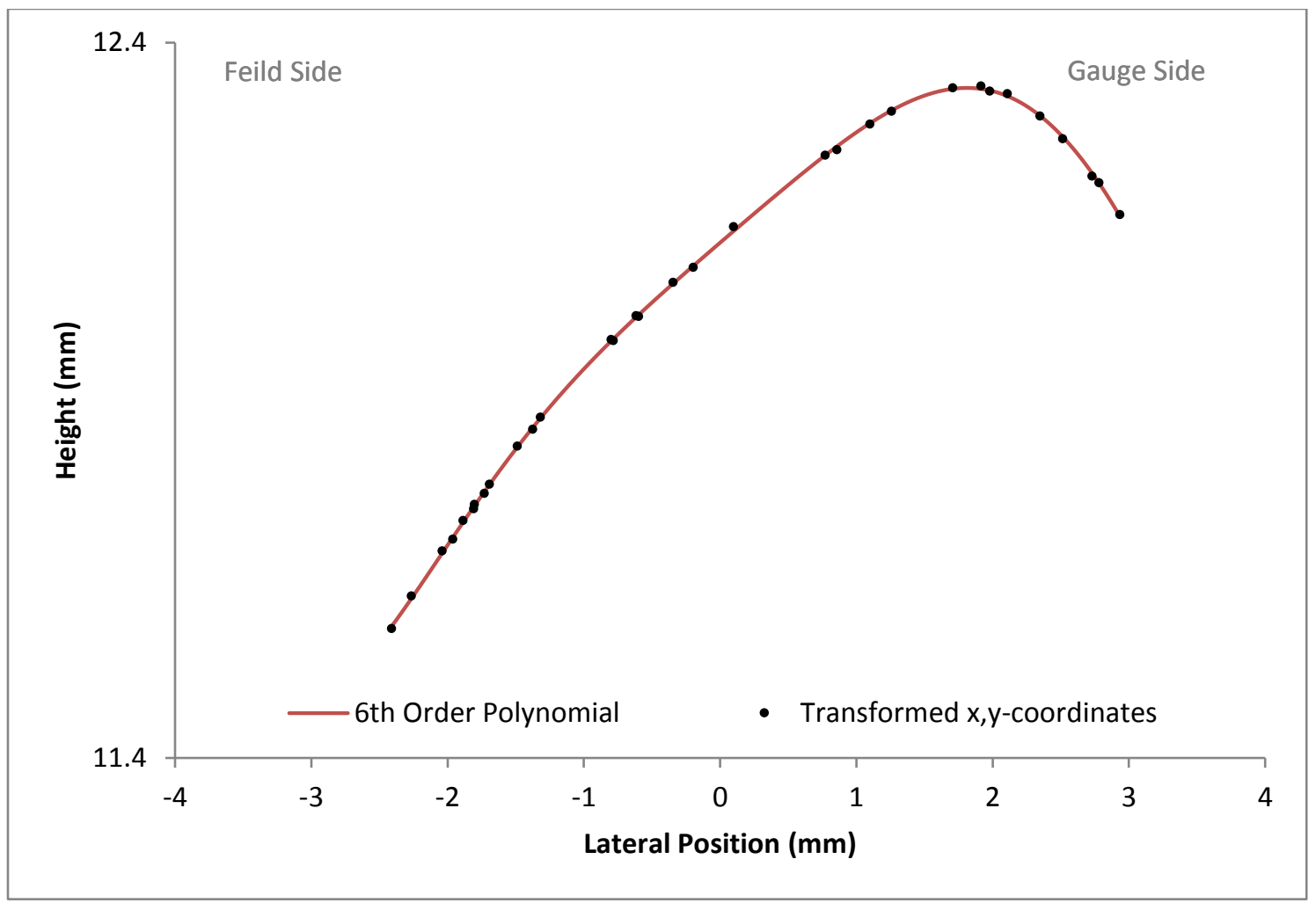

Figure 32: 6th order polynomial fit to $x, y$-coordinate data

The algorithm calculates the accuracy of polynomial fit as the average, absolute vertical distance $(\Delta y)$ between $x, y$-coordinates and the polynomial $R(x)$ using the equation

$$
\Delta y=\frac{1}{n} \sum_{i=1}^{n}\left|y_{i}-R\left(x_{i}\right)\right| .
$$

The algorithm uses the built-in MATLAB function polyval to evaluate the polynomial at each $x$-coordinate. The algorithm stores the mean, absolute vertical distance $(\Delta y)$ in the variable dist-to-poly. The algorithm stores the dist-to-poly under column 3 for the particular rail profile in the rail segment function performance matrix (Table 2).

The decision to use the average vertical distance instead of the typical sum of the squared distances is due to the varying number of $x, y$-coordinates used to represent the remaining portion of the railhead. 
This research selects a $6^{\text {th }}$ degree polynomial to fit to the $x, y$-coordinates. The order of the polynomial is set using a sensitivity analysis. The sensitivity analysis evaluated the average absolute vertical distance between the $x, y$-coordinates and the polynomial $R(x)$ versus the order of polynomial for number of rail profiles. The orders of polynomial used in the sensitivity analysis ranged from a $2^{\text {nd }}$ degree polynomial to a $9^{\text {th }}$ degree polynomial. The sensitivity analysis revealed the following:

- Lower order polynomials $\left(2^{\text {nd }}\right.$ degree polynomials to $5^{\text {th }}$ degree polynomials) resulted in a relatively high average absolute vertical distance between the $x, y$ coordinates and the polynomial, indicating the polynomial did not accurately fit the $x, y$-coordinate data. The lower the order of the polynomial, the higher the average absolute vertical distance measurement.

- An inflection point in the sensitivity analysis occurs at a $6^{\text {th }}$ order polynomial. The $6^{\text {th }}$ order polynomial provided an accurate fit of the $x, y$-coordinate data while still smoothing the $x, y$-coordinate data.

- Higher order polynomials $\left(7^{\text {th }}\right.$ degree polynomials to $9^{\text {th }}$ degree polynomials) showed minimal benefits in average absolute vertical distance between the $x, y$ coordinates and the polynomial compared to the $6^{\text {th }}$ order polynomial; however, further investigation showed that higher order polynomials started to oscillate between $x, y$-coordinates and did not achieve the desired $x, y$-coordinate smoothing. 


\subsubsection{Create Surface Slope Polynomial}

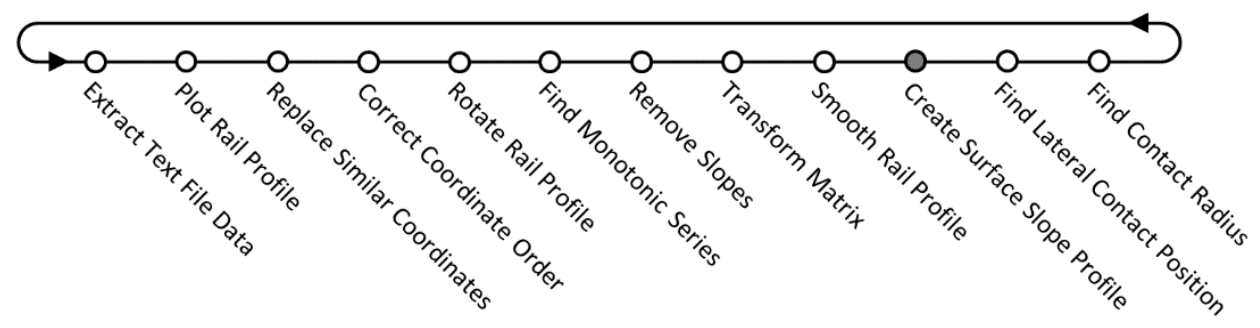

Purpose: This function calculates a railhead surface angle polynomial $S(x)$ that measures the slope (gradient) of the railhead surface across the rail profile by taking the derivative of the polynomial $R(x)$. The algorithm uses the surface slope to determine the lateral position of wheel-rail contact in Section 3.2.3.11 (Determining the Contact Point).

Inputs:

Outputs:

- $\quad$ polynomial $R(x)$

- polynomial $S(x)$

The algorithm calculates the railhead surface slope polynomial $S(x)$ by taking the derivative of the polynomial $R\left(x^{t}\right)$ with respect to $x$, using the equation

$$
S\left(x^{t}\right)=\frac{d}{d x} R\left(x^{t}\right) .
$$

Figure 33 shows the polynomial $S(x)$ in relation to the polynomial $R(x)$ across the range of the $x$-coordinates. 


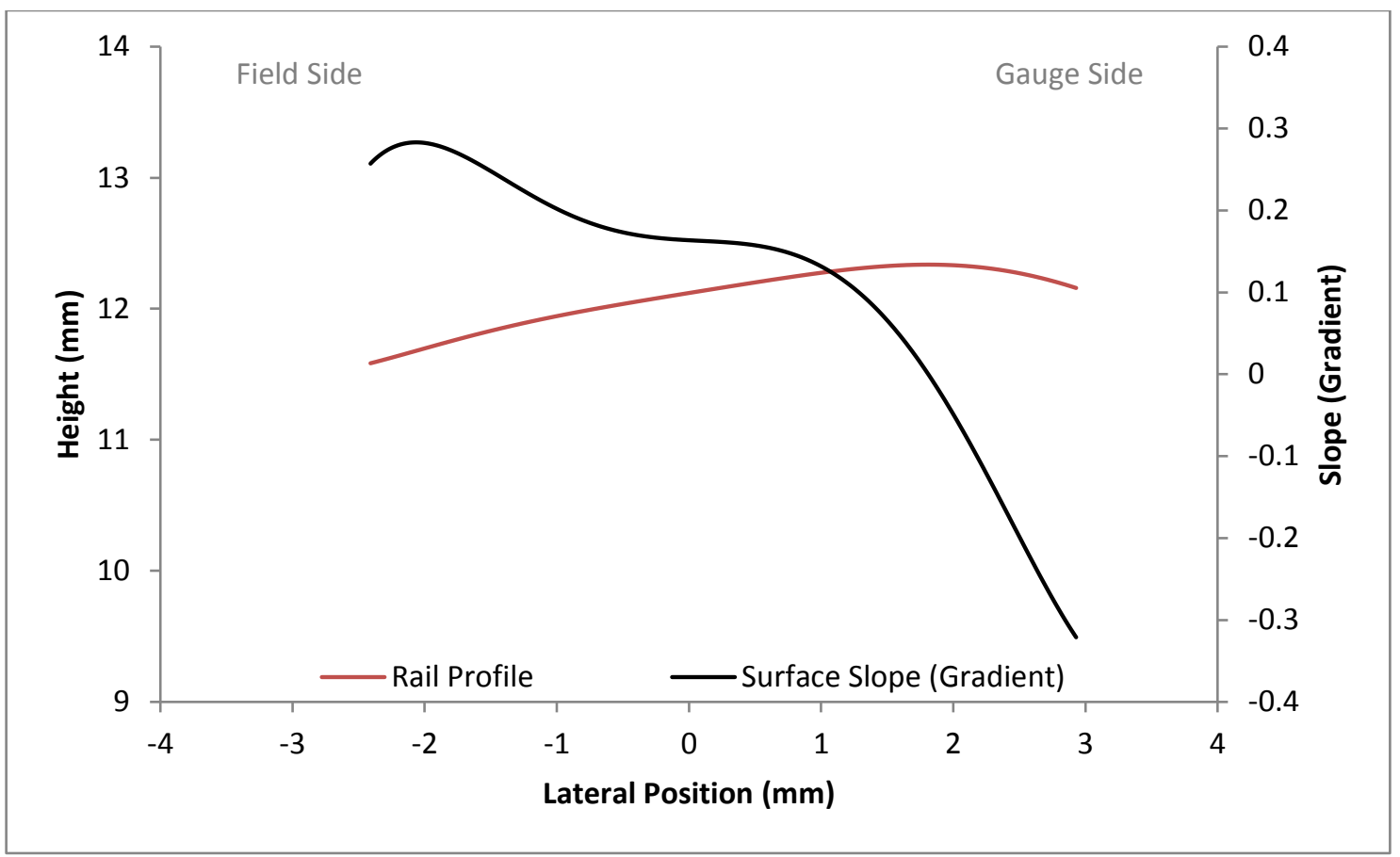

Figure 33: Surface slope (gradient) of the rail profile

\subsubsection{Determining the Contact Point}

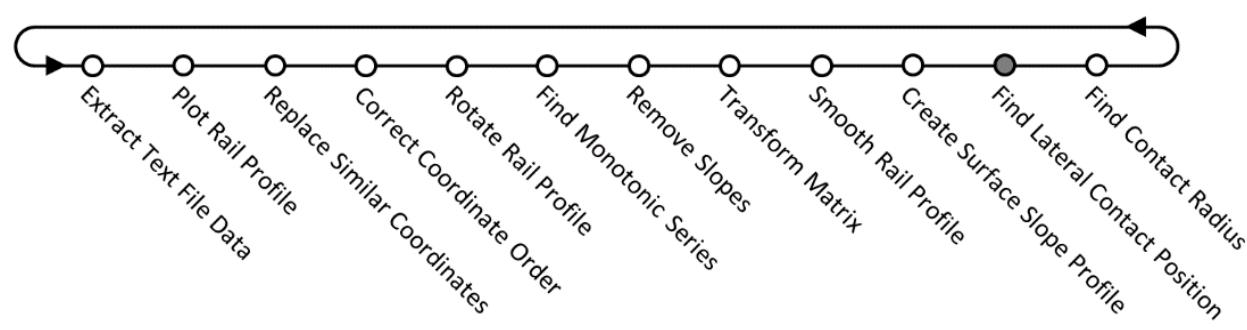

Purpose: This function estimates the lateral location of the contact point between the wheel and the rail using the surface angle polynomial $S\left(x^{t}\right)$. Due to the current orientation of the rail profile, the wheel-rail surface angle at contact is known to be 0 degrees. Of the rail profile points that have a 0 degree surface angle, the point with the largest $y$-value is selected as the contact point, as this point exhibits the least amount of interpenetration between the wheel and rail profile. The algorithm reports the lateral 
location of the contact point.

The methodology of this function uses the three principles of rigid contact:

- the profiles of the wheel and rail are parallel at the point of contact;

- the point of contact between the wheel and rail occupy the same space; and

- the wheel and rail cannot interpenetrate (Bosso et al., 2013).

Section 2.4.1 (Rigid Contact Theory) discusses rigid contact in further detail.

The algorithm records and reports the estimated location of contact when the rail profile is in the non-canted position; this allows for a consistent basis to compare between the lateral contact positions of rail profiles in a rail segment, between rail segments in a track segment, and between different track segments.

Inputs:

- polynomial $S(x)$

- polynomial $R(x)$

- centre

- $\min x$

- $\max x$
Outputs:

- contact position (rotated)

- contact position

Due to the current orientation of the rail profile, the location where the wheel contacts the rail profile occurs where the surface slope (gradient) is 0 . There may be multiple points on the rail profile that have a slope of 0 ; this research refers to these points as potential contact points.

To find locations where the rail profile surface slope is 0 , the algorithm find the roots of the surface slope polynomial $S\left(x^{t}\right)$ (i.e., locations where $y=0$ ) using the built-in MATLAB function roots. The algorithm then removes all imaginary roots and roots that fall outside the range of $x$-values in the railhead $x, y$-matrix given by the variables $\min x$ 
and $\max x$. The remaining roots are the $x$-values of the potential contact points. Figure 34 shows the $x$-value of the potential contact point. In this example, there is only one potential contact point, since the surface slope polynomial $S\left(x^{t}\right)$ only equals 0 once between $\min x$ and $\max x$.

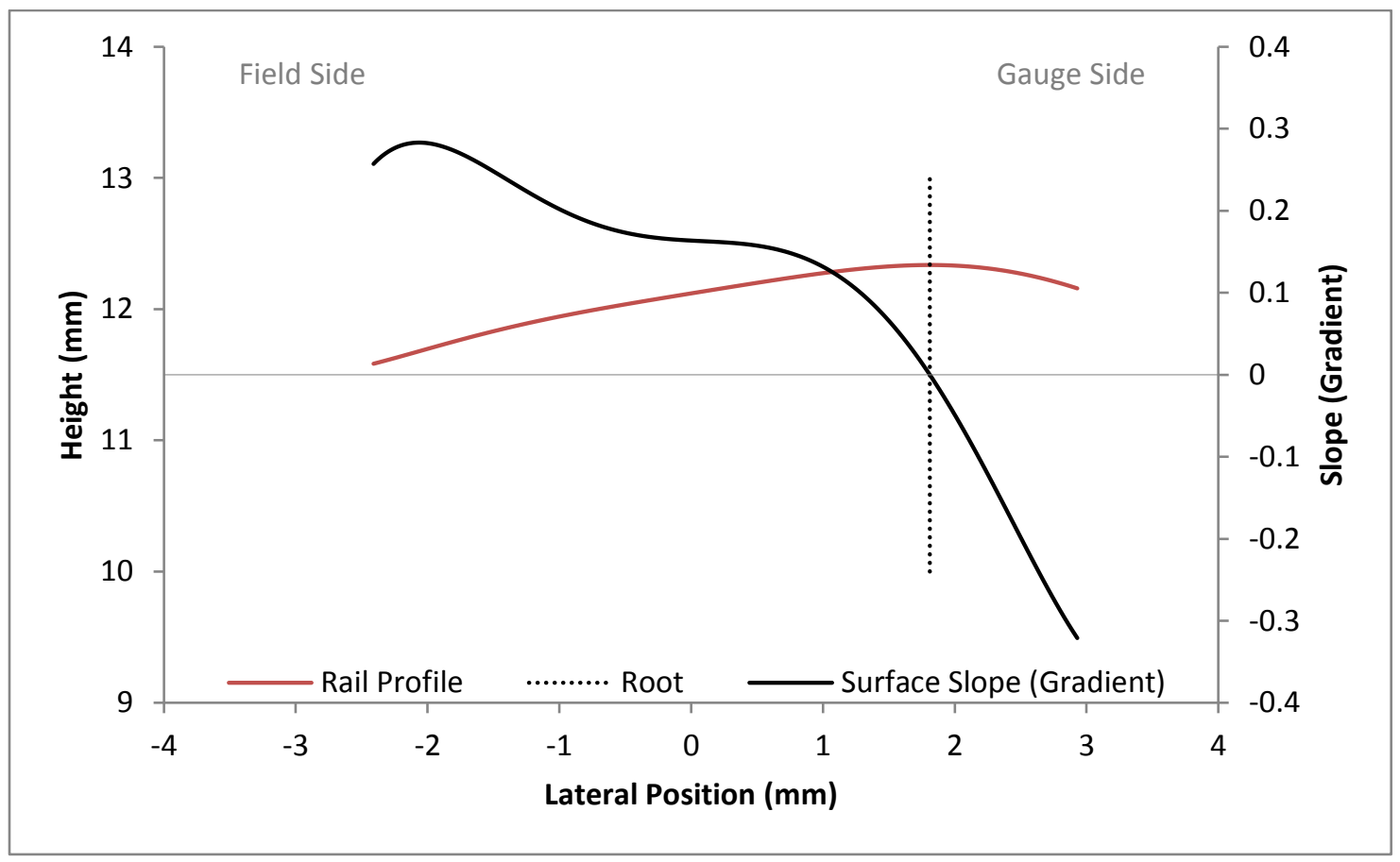

Figure 34: Root of surface slope polynomial

The algorithm evaluates the railhead polynomial $R\left(x^{t}\right)$ at the $x$-values of the potential contact points to find the corresponding $y$-values of the potential contact points.

Typically, rail profiles have only one potential contact point; however, certain rail profile shapes can exhibit more than one potential contact point. Figure 35 shows an example rail profile with two potential contact points. For these types of rail profiles, the algorithm will find more than one real root in the appropriate range of the railhead polynomial $R\left(x^{t}\right)$, with each root corresponding to a unique potential contact point. 


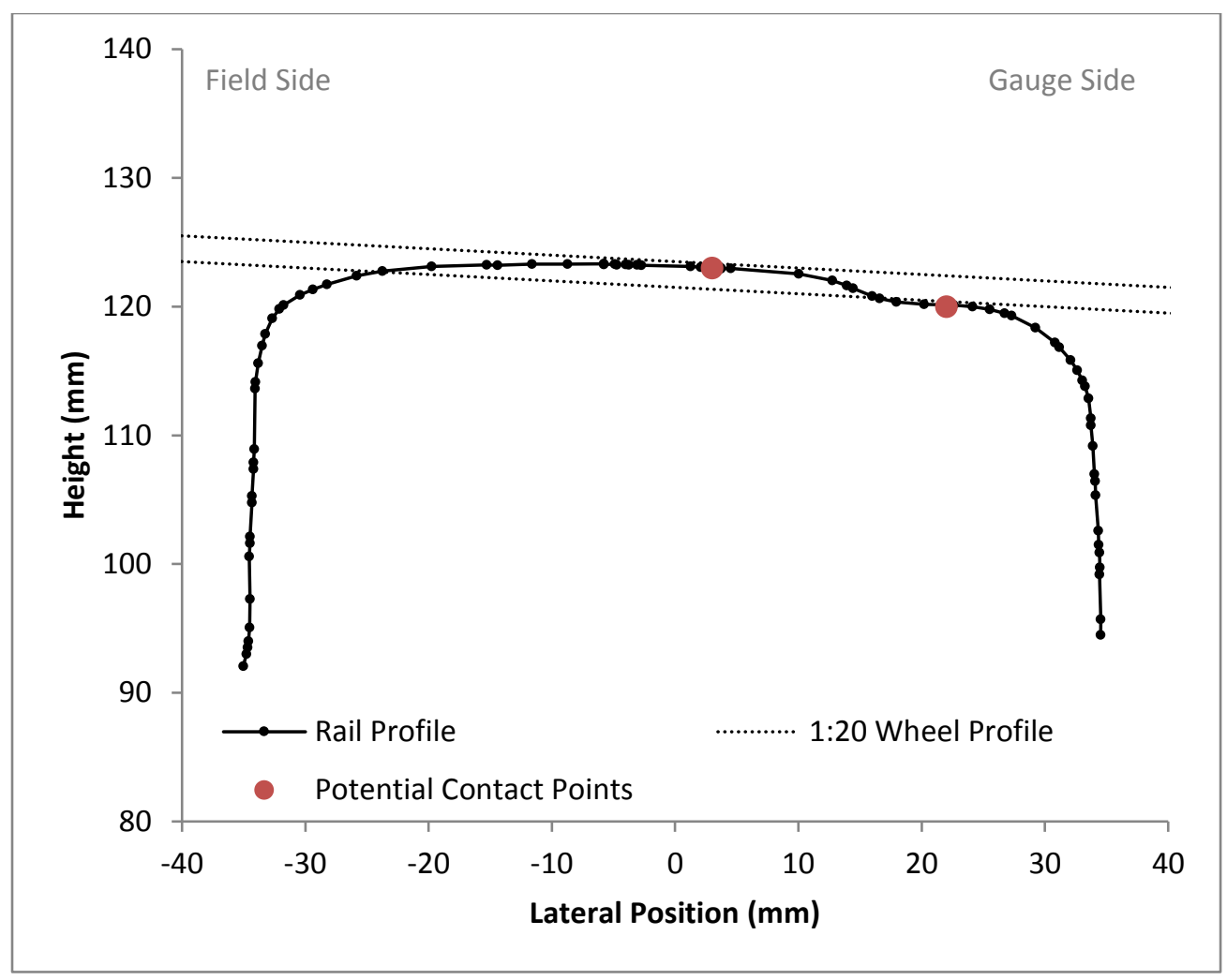

Figure 35: An example rail profile with two potential contact points

When multiple contact points exist, the algorithm selects the potential contact point that exhibits the least amount of interpenetration between the wheel and rail profile as the lateral contact point (rotated) of the rail profile. Since the wheel profile is parallel with the $x$-axis in this orientation, the potential contact point with the largest $y$-value is point with the least amount of interpenetration. Figure 36 demonstrates this concept. The algorithm stores the $x, y$-coordinate of the lateral contact point (rotated) in the contact point (rotated) variable. 


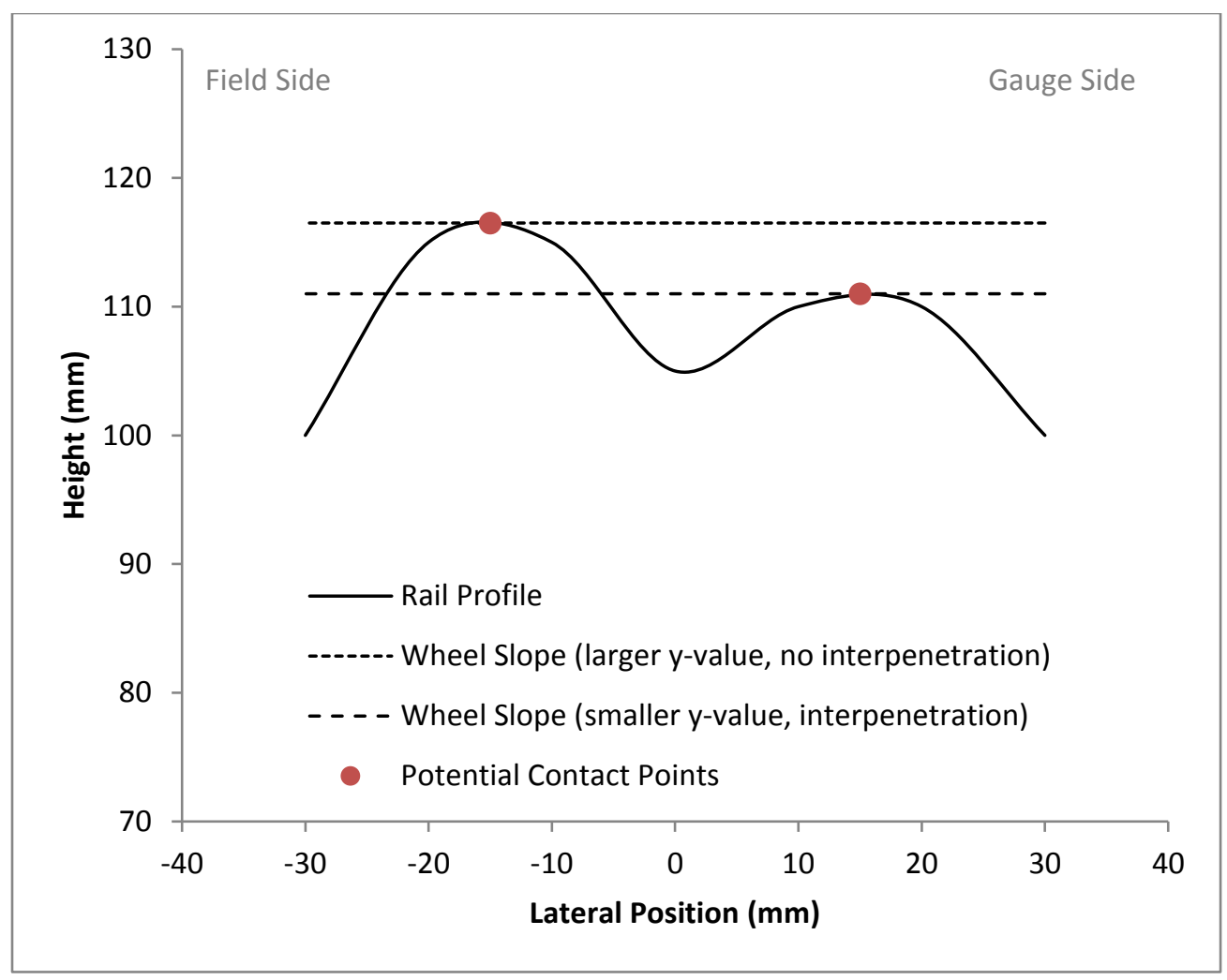

Figure 36: Interpenetration and potential contact points concept

The algorithm rotates the point back to the original orientation using the equation

$$
\left(x_{\text {temp }}, y_{\text {temp }}\right)=\left(\left[R^{T}\right]\left(\begin{array}{l}
x_{r} \\
y_{r}
\end{array}\right)\right)^{T}
$$

where the $x_{\text {temp }}, y_{\text {temp }}$-coordinate is a temporary coordinate that is in the original orientation (but is still transformed), $R^{\prime}$ is the transpose of the rotation matrix and $x_{r}, y_{r^{-}}$ coordinate is the rotated lateral contact point. The algorithm then calculates the reported lateral contact position $\left(x_{c}, y_{c}\right)$ by reversing the transformation in Section 3.2.3.8 (Transform Matrix) using the equations

$$
\begin{gathered}
x_{c}=\left(x_{\text {temp }} * 10\right)+\text { centre } \\
y_{c}=y_{\text {temp }} * 10
\end{gathered}
$$


The algorithm stores the $x_{c}, y_{c}$-coordinate in the contact point variable. Figure 37 shows the lateral contact point on a rail profile in its original orientation. The algorithm saves the contact point under column 4 for the particular rail profile in the rail segment analysis matrix (Table 1).

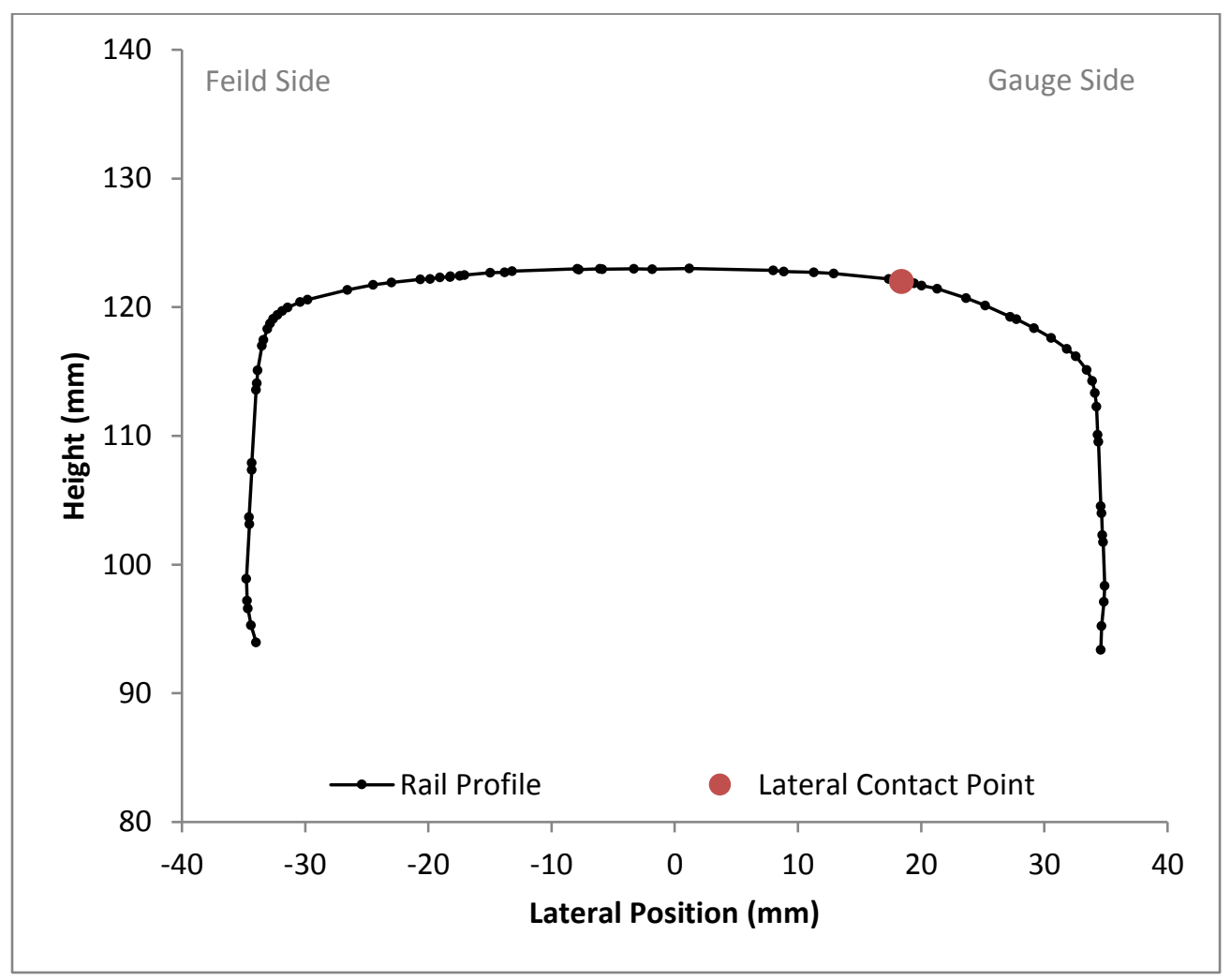

Figure 37: The lateral contact point in the original orientation (1:5 wheel slope)

\subsubsection{Determining the Rail Profile Radius}

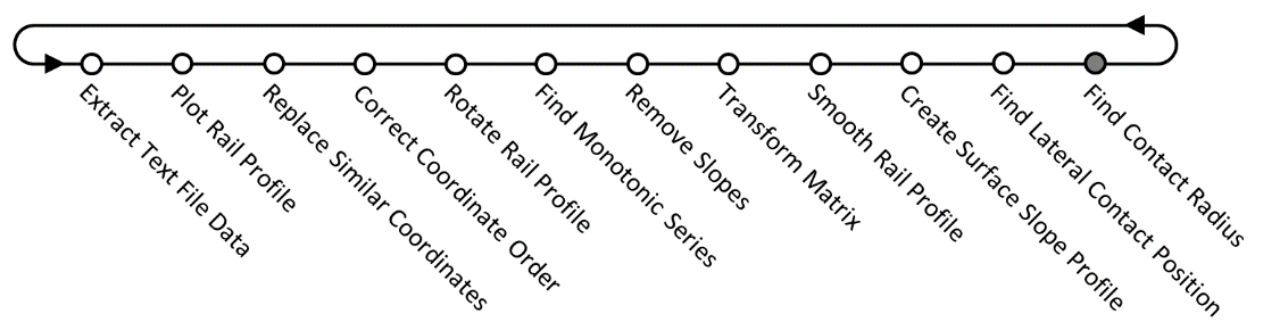

Purpose: This function calculates the radius of the rail profile around the contact point by 
fitting a least squares, constant radius circle to a selection of $x, y$-coordinates representing the width of the contact area between the wheel and rail. The $x, y$ coordinates are sampled within a predetermined $x$-value range of the contact point from the railhead polynomial $R\left(x^{t}\right)$. The predetermined $x$-value range simulates the width of the contact patch between the wheel and the rail.

Inputs:

Outputs:

- polynomial $R(x)$

- contact position (rotated)

- centre

- contact radius

- dist-to-circle

The algorithm first calculates the predetermined $x$-value range that simulates the wheelrail contact area using the equations

$$
\begin{gathered}
l=x_{r}-w / 2 \\
u=x_{r}+w / 2
\end{gathered}
$$

where $l$ is the lower bound, $u$ is the upper bound, $x_{r}$ is the $x$-value of contact point (rotated), and $w$ is the width of the contact patch. This research assumes a contact area width of $18 \mathrm{~mm}$. Prior to executing the algorithm, the width of the contact patch, $w$, can be adjusted in the code to accommodate various contact widths which may be found in different wheel-rail interaction conditions.

The algorithm then evaluates the railhead polynomial $R\left(x^{t}\right)$ at $x$-values linearly spaced at $0.1 \mathrm{~mm}$ apart between the upper and lower bounds using the built-in MATLAB function polyval. By using a $0.1 \mathrm{~mm}$ spacing, the algorithm extracts a denser and more evenly distributed set of the $x, y$-coordinates compared to the original $x, y$-coordinates. The resampling is similar in principle to the one in the crown radius "fit to all points" 
method in Rangecam. The algorithm stores the $x$-values in the variable $X$ and the corresponding $y$-values in the variable $Y$.

To calculate a contact radius, the algorithm reverses the transformation in Section 3.2.3.8 (Transform Matrix) to values in the variables $X$ and $Y$ and stores them in a radius $x, y$-matrix using the equations

$$
\begin{gathered}
\text { radius } x \text {-matrix }=(X * 10)+\text { centre } \\
\text { radius y-matrix }=Y * 10 .
\end{gathered}
$$

The algorithm then fits a least squares, constant radius circle to the $x, y$-coordinates in the radius $x, y$-matrix; the location of the centre of the circle is not restricted to origin of the coordinate system. Figure 38 shows the circle fitted to the radius $x, y$-matrix with the $y$-axis scaled to emphasize the fit of the circle. The flexibility of not pre-defining the centre of the circle, allows for a more accurate fit to the rail profile data. The algorithm stores the radius of the fitted circle to the variable contact radius; this value is the reported contact radius of the rail profile. The algorithm also stores the $x, y$-coordinates of the center of the fitted circle as $a, b$. The $a, b$ variables are later used to calculate the fit of the circle to the $x, y$-coordinate data. The algorithm saves the contact radius under column 5 for the particular rail profile in the rail segment analysis matrix (Table 1). 


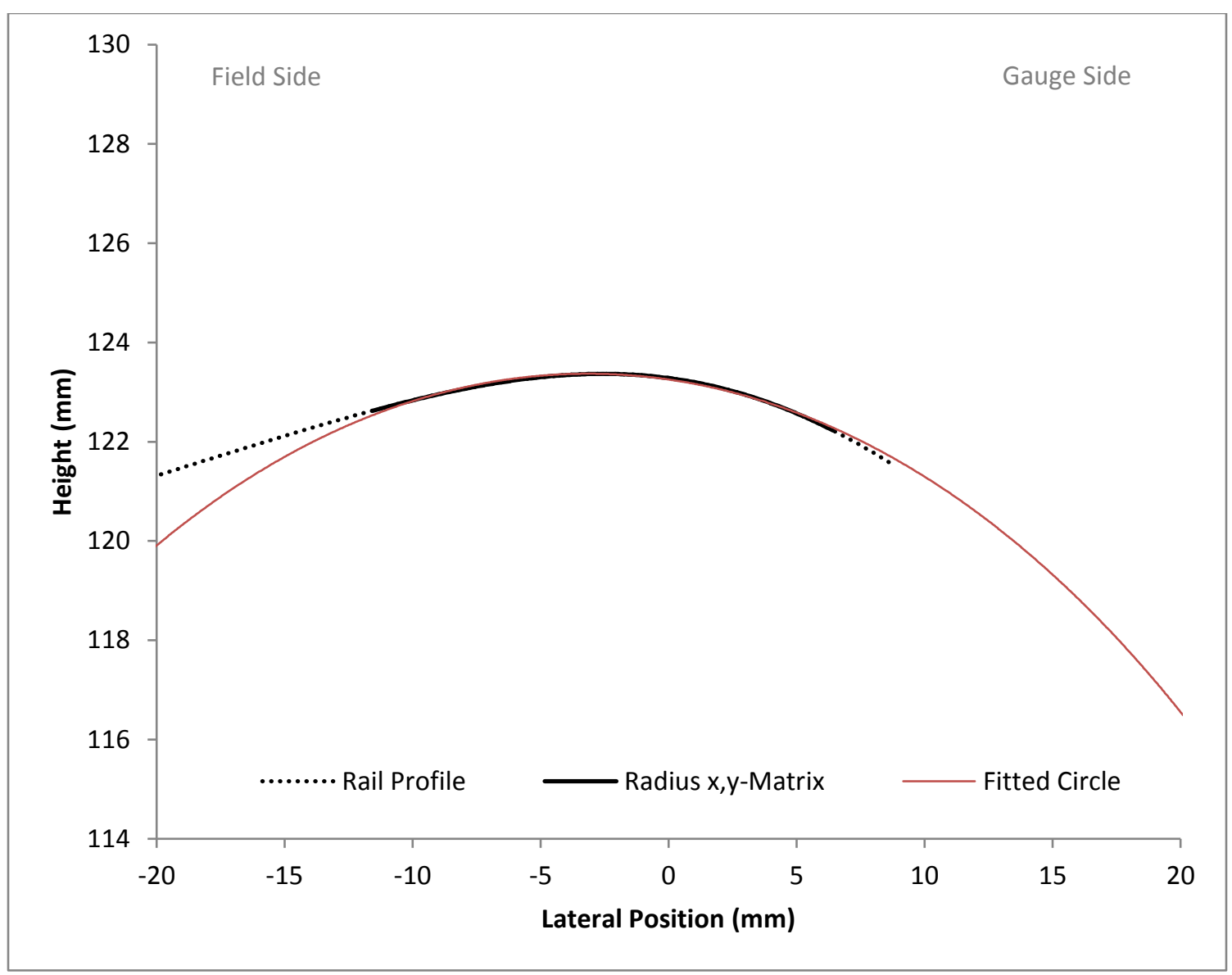

Figure 38: Fitting least squared, constant radius circle to radius $x, y$-matrix data

The measure of the fit accuracy is the average absolute Euclidean distance $(\bar{d})$ between the radius $x, y$-matrix and the fitted circle. The algorithm calculates the average absolute Euclidean distance using the equations

$$
\begin{gathered}
d_{i}=\mid \sqrt{\left(x_{i}-a\right)^{2}+\left(y_{i}-b\right)^{2}}-(\text { contact radius }) \mid \text {, and } \\
\bar{d}=\frac{\sum_{i=1}^{n} d_{i}}{n} .
\end{gathered}
$$

where $d_{i}$ is the Euclidean distance between the $x_{i}, y_{i}$-coordinate from the radius $x, y$ matrix and the fitted circle, the variables $a, b$ are the $x, y$-coordinates of the center of the fitted circle, and $n$ is the total number of coordinates in the radius $x, y$-matrix. The 
algorithm stores the value $\bar{d}$ in the variable dist-to-circle. The algorithm calculates the average distance instead of the typical sum of the squared distances due to the potential for a varying number of coordinates in the radius $x, y$-matrix, allowing for accuracy-of-fit comparisons between rail profiles. The algorithm stores the dist-to-circle under column 4 for the particular rail profile in the rail segment function performance matrix (Table 2).

This research selects the method of fitting a least squares, constant radius circle; other methods this research investigated were a closed form solution using the railhead polynomial $R\left(x^{t}\right)$ and fitting piecewise linear segments to a surface angle plot. After further investigation, this research finds that

- the closed-form solution was too sensitive to local curvatures and tends to overestimate the contact radius when comparing to the least squares, constant radius circle method; and

- fitting piecewise linear segments to a surface angle plot proves to be the most unreliable method.

This function concludes the rail profile analysis started in Section 3.2.3.1, Text File to MATLAB.

\subsubsection{Data Organization}

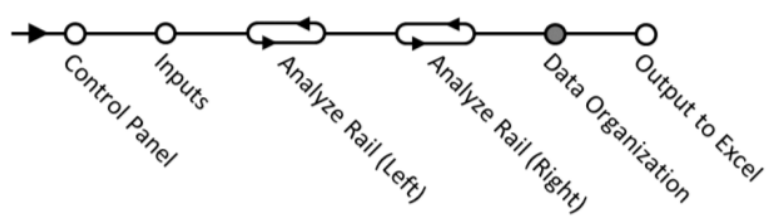

Purpose: This function collates the rail segment analysis matrices for the left rail segment and right rail segment into a track segment matrix. Collating the results 
minimizes the number of times the algorithm writes to Excel and allows for crosscomparison of left rail profile mileages and right rail profile mileages to identify missing profiles within a rail segment.

Inputs:

- left rail analysis matrix

- right rail analysis matrix
Outputs:

- track analysis matrix

The algorithm collates all the rail profile mileages from left rail segment and right rail segment. Since the optical rail measurement process attempts to collect left and right profiles simultaneously, many rail mileages in the collated list are duplicates. The algorithm uses the MALTAB function unique to remove duplicate rail mileages, resulting in $i$ unique track mileage locations remaining that contain at least one rail profile measurement.

The algorithm creates a track analysis matrix by pre-allocating an empty $i \times 10$ matrix to store the combined rail segment data, where $i$ is the number of unique track mileages. Table 4 shows the columns of the track analysis matrix.

Table 4: Track analysis matrix

\begin{tabular}{|c|c|c|c|c|c|c|c|c|c|c|}
\hline & \multicolumn{2}{|c|}{ Track } & \multicolumn{4}{|c|}{ Left Rail } & \multicolumn{4}{|c|}{ Right Rail } \\
\hline column & 1 & 2 & 3 & 4 & 5 & 6 & 7 & 8 & 9 & 10 \\
\hline & 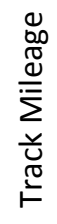 & 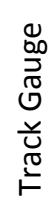 & 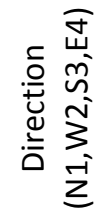 & $\begin{array}{l}\frac{0}{0} \\
\frac{0}{\underline{\underline{L}}} \\
\frac{0}{0}\end{array}$ & 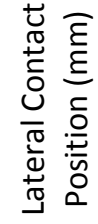 & 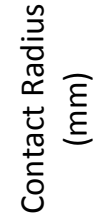 & 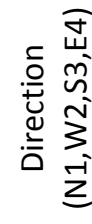 & $\begin{array}{l}\text { 으 } \\
\frac{0}{4} \\
\frac{1}{2} \\
\frac{1}{2}\end{array}$ & 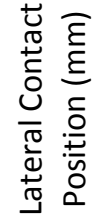 & 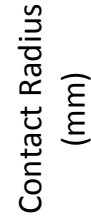 \\
\hline row 1 & & & & & & & & & & \\
\hline$\cdots$ & & & & & & & & & & \\
\hline row $i$ & & & & & & & & & & \\
\hline
\end{tabular}


The process of copying the data from the two rail analysis matrices to the track segment matrix follows:

1. Starting from row 1 and ending at row $i$, the algorithm obtains the next track mileage value from the track mileage list and copies it into the track analysis matrix $(i, 1)$.

2. The algorithm checks if the track mileage in the track analysis matrix $(i, 1)$ occurs in the left rail analysis matrix mileage (column 1) using the built-in MATLAB function find. If not, the algorithm moves to step 3. If the algorithm finds that the track mileage in the track analysis matrix $(i, 1)$ occurs in row $f$ of the left rail analysis matrix, it copies the

- $\quad$ rail side $(f, 2)$;

- $\quad$ count $(f, 3)$;

- $\quad$ contact position $(f, 4)$;

- $\quad$ contact radius $(f, 5)$; and

- $\quad$ track gauge $(f, 6)$

from the left rail analysis matrix into the respective left rail cells $(i, 3: 6)$.

3. The algorithm checks if the track mileage in the track analysis matrix $(i, 1)$ occurs in the right rail analysis matrix mileage (column 1) using the built-in MATLAB function find. If not, the algorithm moves to step 4. If the algorithm finds that the track mileage in the track analysis matrix $(i, 1)$ occurs in row $r$ of the left rail analysis matrix, it copies the

- $\quad$ rail side $(r, 2)$;

- $\quad$ count $(r, 3)$; 
- contact position $(r, 4)$; and

- contact radius $(r, 5)$

- track gauge $(r, 6)$

from the right rail analysis matrix into the respective right rail cells $(i, 7: 10)$.

4. If row $i$ in the track analysis matrix contains both a left rail profile and right rail profile, the algorithm averages the track gauge's in the left analysis matrix $(f, 6)$ and right analysis matrix $(r, 6)$ and copies the average into the track analysis matrix $(i, 2)$. Typically, the track gauges are identical. If a rail profile is missing, the track gauge in the track analysis matrix $(i, 2)$ is left blank. The reason the algorithm leaves the track gauge blank is that either the Rangecam exported rail profile text files did not contain a track gauge or the accuracy of the track gauge is compromised as one side of the rail either could not be measured or was discarded due to mismeasurement.

5. Repeat until all track mileages from the track mileage list are processed (i.e., the function completes these steps $i$ times).

To highlight the collection interval discontinuities in the rail profile data, the algorithm inserts an empty row into the track analysis matrix when the distance between two adjacent unique mileages exceeds a predetermined value. The process for identifying collection interval discontinuities and inserting the empty rows follows:

1. The algorithm finds the differences between the adjacent $k$ unique track mileage locations and stores them in the variable milediff.

2. The algorithm sets the maximum allowable collection interval $(c)$ using the 
equation

$$
c=\operatorname{mode}(\text { milediff }) * 2
$$

This research bases the maximum allowable collection interval on the most commonly observed collection interval between mileages. The multiplication by two is to account for minor variations in the collection interval that this research does not need to highlight. The user can update this equation as deemed appropriate for the particular application.

3. Using the milediff variable, the algorithm locates every consecutive pair of mileages that exceeds the maximum allowable collection interval and inserts an empty row between the mileages.

\subsubsection{Output to Microsoft Excel}

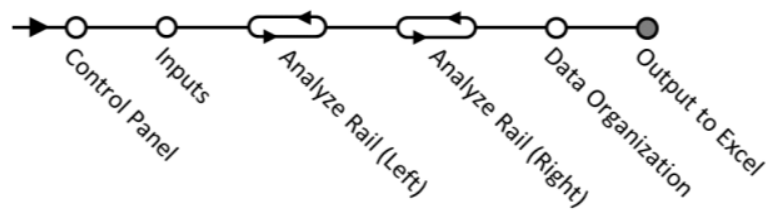

Purpose: This function exports the track analysis results and pertinent algorithm input parameters to an Excel file to facilitate data review, analysis, reporting, and sharing of the results. It is the final function of the algorithm. This section contains two subsections, which cover (1) the function in MATLAB, and (2) the layout of the Excel file and the calculations the Excel file executes. 
Inputs:

- track analysis matrix

- left rail function performance matrix

- right rail function performance matrix

- left wheel slope

- right wheel slope

- mirror left rail

- mirror right rail

- collection data

- segment name
Outputs:

- Excel file

\subsubsection{MATLAB Algorithm}

Before writing the data to the Excel file, the algorithm copies a template Microsoft Excel file located in the current folder (i.e., the folder that contains the main MATLAB function) into the Completed Excel Files folder (located in the current folder of MATLAB) and renames the file

(collection date) - (segment name).xlsm.

This research refers to this Excel file as the track segment Excel file. The template Excel file contains custom empty tables for the data, cells containing formulae for calculations, and graphics that properly reference the tables.

The algorithm uses the MATLAB functions datetime and cellstr to obtain the completion date of the MATLAB analysis in the numeric string format

YYYYMMDD.

The algorithm outputs the data from the analysis to the designated cells in the track segment Excel file using the built-in MATLAB function xlswrite. The exported data includes: 
- the track analysis matrix (Section 3.2.4, Data Organization);

- the left rail function performance matrix (Section 3.2.3, Analyzing Rail Profiles of a Rail Segment);

- the right rail function performance matrix (Section 3.2.3, Analyzing Rail Profiles of a Rail Segment);

- the left wheel tread degree (Section 3.2.1, Control Pane);

- the right wheel tread degree (Section 3.2.1, Control Panel);

- the name and date of the data collection run (Section 3.2.2, Inputs); and

- the date the algorithm ran the analysis (current section).

\subsubsection{Track Segment Excel File}

There are four Excel spreadsheet tabs in the track segment Excel file:

- the track analysis sheet;

- the smoothing function evaluation sheet;

- the least squares, circle fitting evaluation sheet; and

- an one-page summary sheet.

The track analysis sheet contains four sections:

- the track segment information tables;

- the track analysis table;

- the rail segment statistics tables; and

- the rail segment graphs.

The track segment information tables contain information about the track segment, including

- the track segment name; 
- the track segment length, which Excel calculates using the equation

$$
\text { segment length }=\text { mileage }_{\text {end }}-\text { mileage }_{\text {start }} \text {; }
$$

- the data collection date;

- the date of the analysis;

- $\quad$ the left and right wheel slopes;

- number of profiles in the left and right rail segment (separate table); and

- the left and right mirror rail settings (separate table).

Figure 39 shows the location of the track segment information tables on the track analysis sheet.

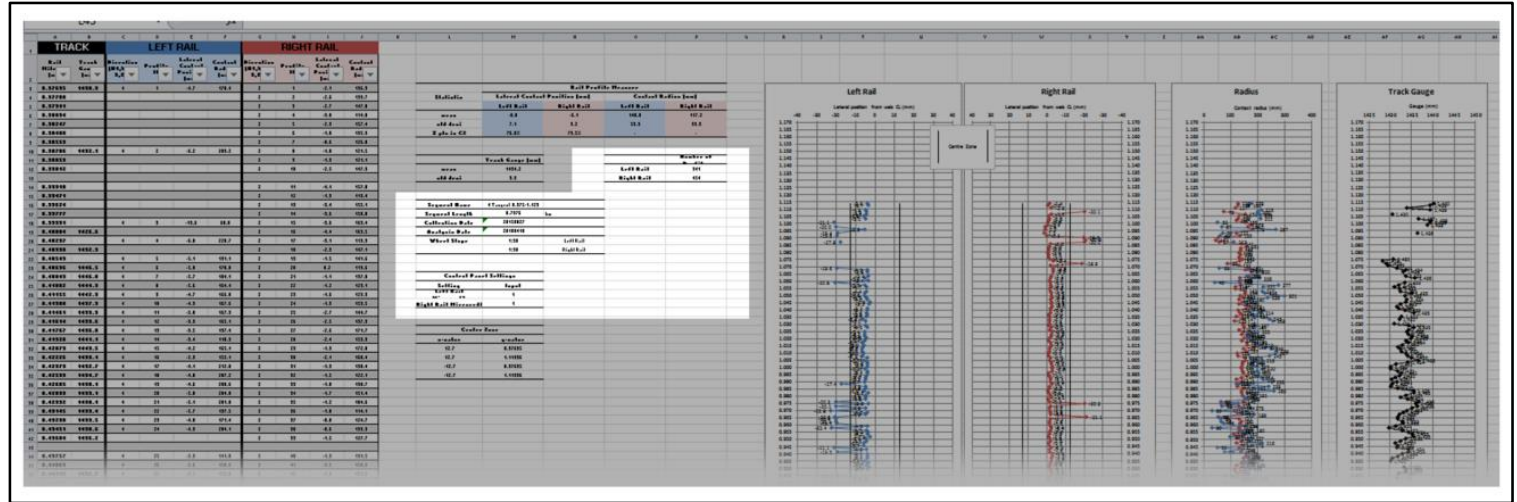

Figure 39: Location of the track segment information tables.

The track analysis table is identical to the track analysis matrix in Table 4. The table is sortable by any of the table columns. Figure 40 shows the location of the track analysis table on the track analysis sheet. 


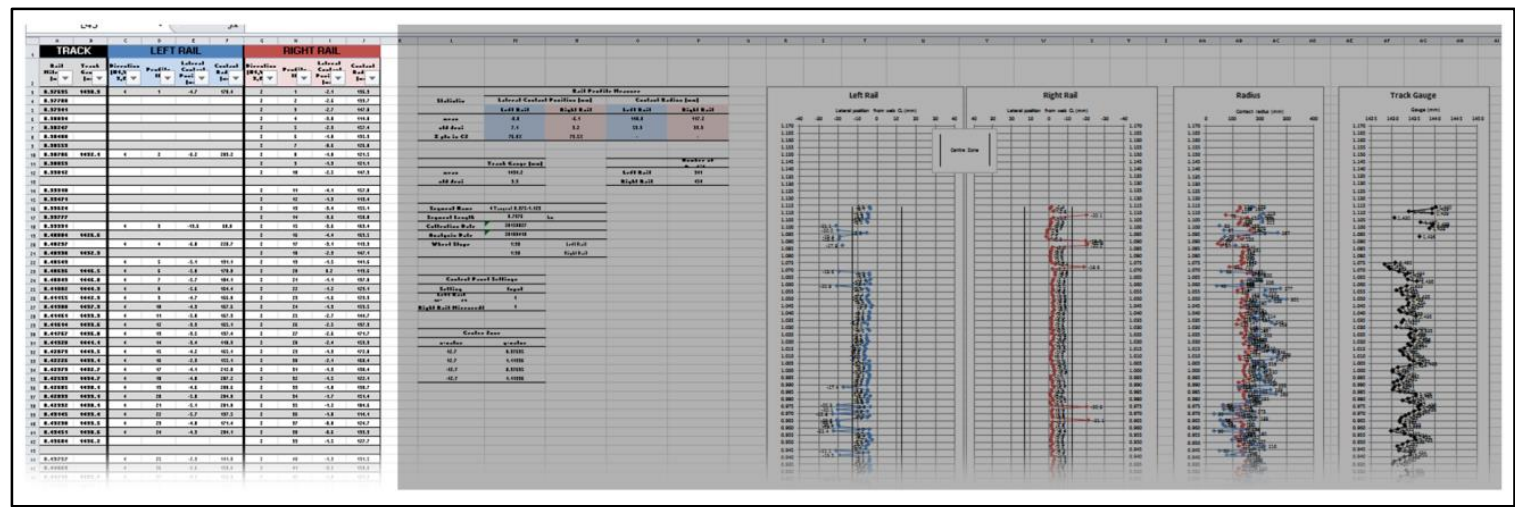

Figure 40: Location of the track analysis table.

The rail segment statistic tables provide summary statistical data for each rail segment. Table 5 shows examples of the rail segment statistics tables from Excel. The statistics Excel calculates for each rail segment include

- the mean and standard deviation of the lateral contact point;

- the mean and standard deviation of the contact radius;

- the percentage of lateral contact points within the center zone; and

- the mean and standard deviation of the track gauge (separate table).

Excel calculates the statistics using the track analysis matrix once the algorithm copies the data to Excel; this technique allows real-time updating of the statistics if the user removes a rail profile from the track analysis matrix in Excel. 
Table 5: Examples of the rail segment statistics tables containing statistics for (a) the rail segments and (b) the track segment gauge

(a)

\begin{tabular}{cccccc}
\hline \multirow{2}{*}{ Statistic } & \multicolumn{4}{c}{ Rail Profile Measure } \\
\cline { 2 - 5 } & \multicolumn{2}{c}{ Lateral Contact Point } & \multicolumn{2}{c}{ Contact Radius } \\
& Left Rail & Right Rail & Left Rail & Right Rail \\
\hline Mean $(\mathrm{mm})$ & -3.3 & -3.2 & 215.5 & 172.4 \\
Standard deviation $(\mathrm{mm})$ & 2.3 & 2.8 & 29.9 & 54.5 \\
\% within center zone $(\%)$ & $100.0 \%$ & $100.0 \%$ & - & - \\
\hline
\end{tabular}

(b)

\begin{tabular}{cc}
\hline Statistic & Track Gauge \\
\hline Mean $(\mathrm{mm})$ & 1434.2 \\
Standard deviation $(\mathrm{mm})$ & 3.3 \\
\hline
\end{tabular}

Figure 41 shows the location of the rail segment statistics tables on the track analysis sheet.

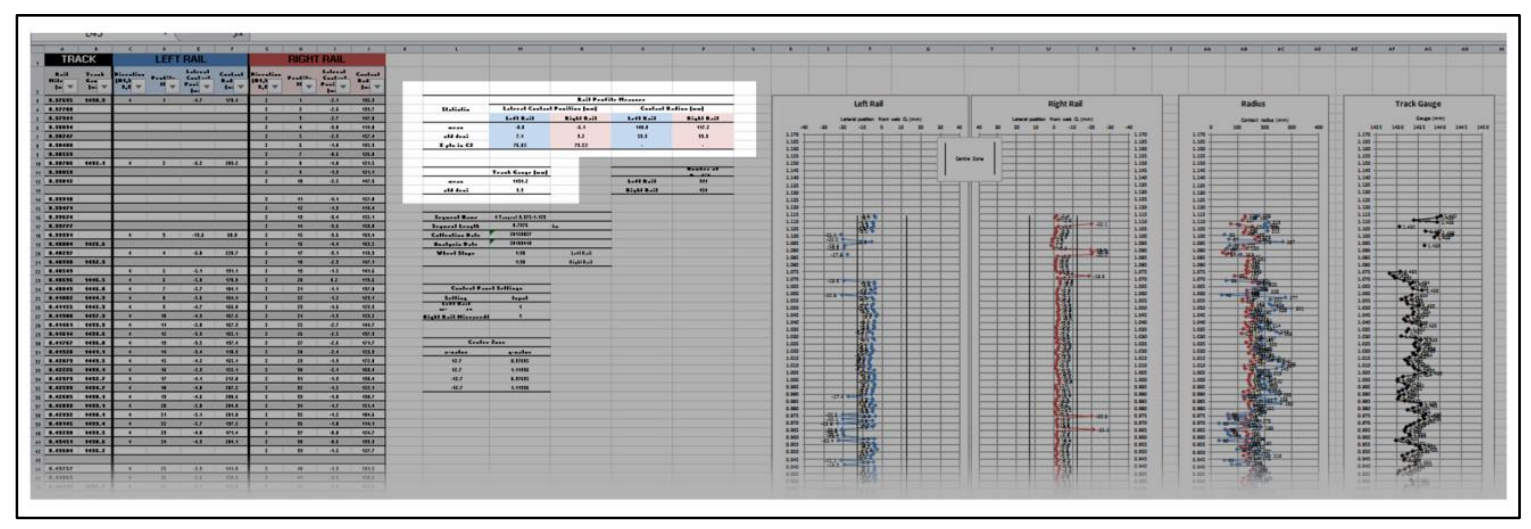

Figure 41: Location of the rail segment statistics tables

The rail segment graphs provide the user with a graphical representation of longitudinal variations in the lateral contact point (referenced with respect to the Rangecam-defined center zone) with the corresponding contact radius for the left and right rails and the track gauge. Figure 42 shows a simplified version of the rail profile graphs; the graph sizes are reformatted to fit the page. Figure 43 shows the location of the rail segments graphs on the track analysis sheets. The graphs display data from the track analysis 
matrix in Excel; therefore, user changes to the track analysis table result in corresponding changes to the rail segment graphs.

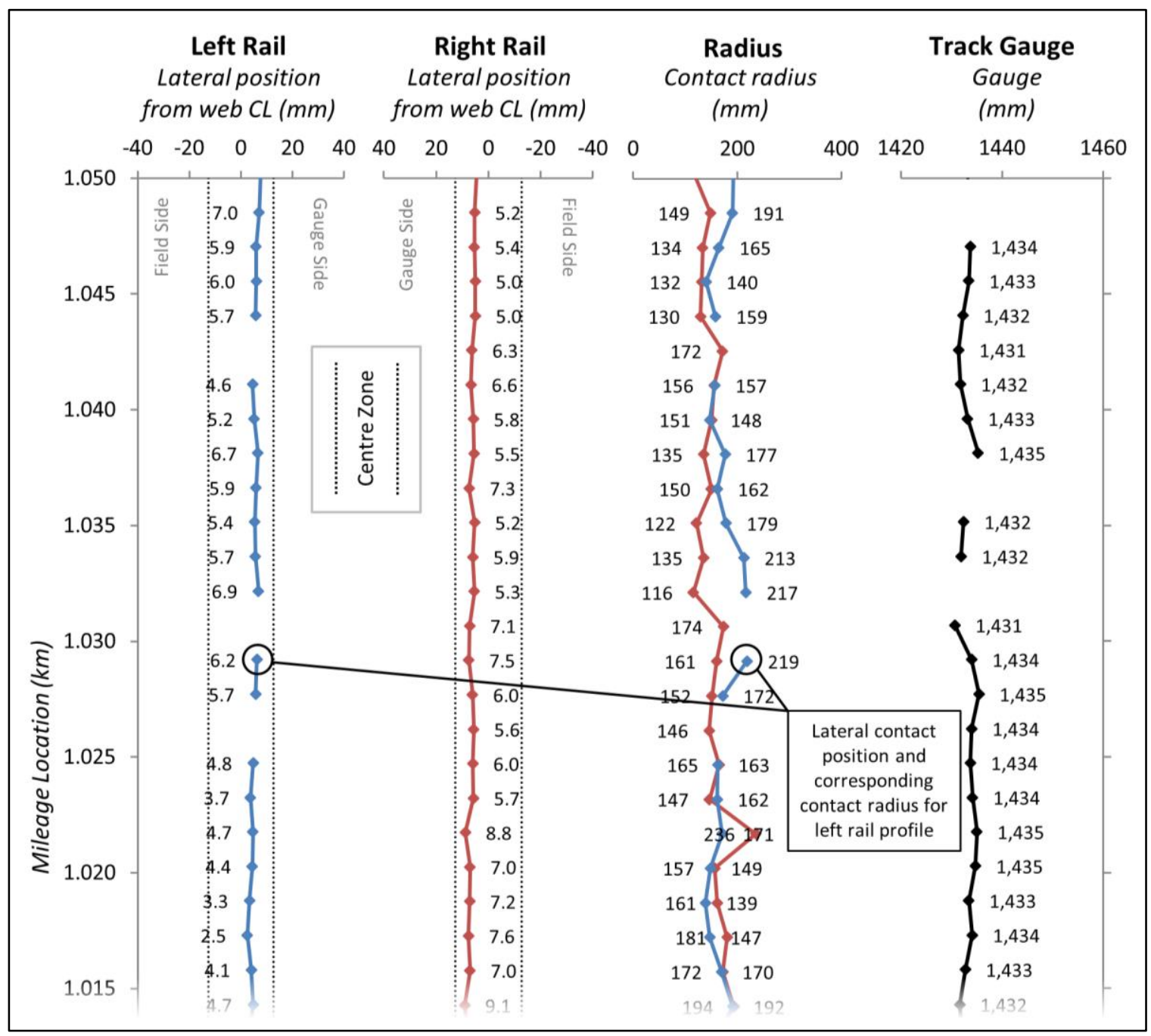

Figure 42: A reformatted version of the rail segment graphs 


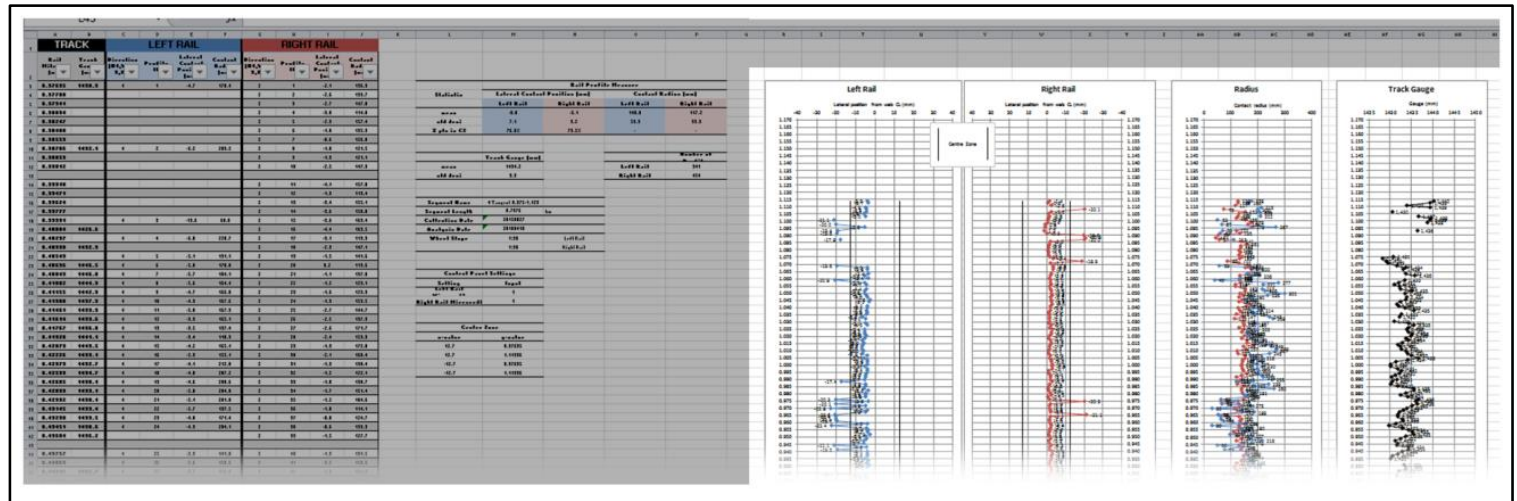

Figure 43: Location of the rail segment graphs

There are two parts to both the smoothing function evaluation sheet (the second sheet in the Excel file) and the least squares fitted circle function evaluation sheet (the third sheet in the Excel file): the function performance table and the corresponding graph. The smoothing table consist of columns 1,2, and 3 from each rail segment function performance matrix, formatted as seen in Table 6 . The least squares fitted circle table consist of columns 1,2 , and 4 from each rail segment function performance matrix, formatted as seen in Table 7. Both tables do not use a row for each unique track mileage. Instead, the tables list the rail profiles from each rail segment independent of the other rail side (i.e., the list of rail profiles for the left rail - columns 1 through 3 - may be a different length than the list of rail profiles for the right column - columns 4 through 6). The user can look-up the profile ID to locate a specific rail profile in every table in the Excel file. 
Table 6: Smoothing function performance table

\begin{tabular}{|c|c|c|c|c|c|c|}
\hline & \multicolumn{3}{|c|}{ Left Rail } & \multicolumn{3}{|c|}{ Right Rail } \\
\hline column & 1 & 2 & 3 & 4 & 5 & 6 \\
\hline & 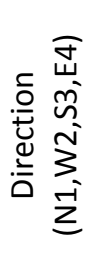 & $\begin{array}{l}\frac{}{0} \\
\frac{0}{\bar{c}} \\
\frac{0}{2}\end{array}$ & 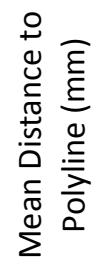 & 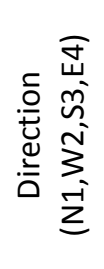 & 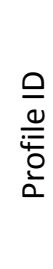 & 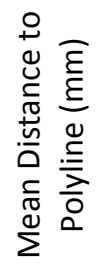 \\
\hline row 1 & & & & & & \\
\hline$\ldots$ & & & & & & \\
\hline row (end) & & & & & & \\
\hline
\end{tabular}

Table 7: Least squares, fitted circle function performance table

\begin{tabular}{|c|c|c|c|c|c|c|}
\hline \multirow[b]{2}{*}{ column } & \multicolumn{3}{|c|}{ Left Rail } & \multicolumn{3}{|c|}{ Right Rail } \\
\hline & 1 & 2 & 3 & 4 & 5 & 6 \\
\hline & 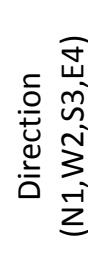 & 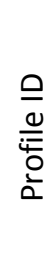 & 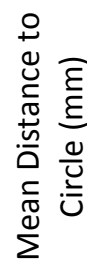 & 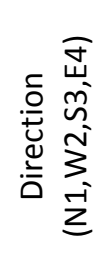 & 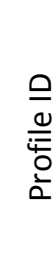 & 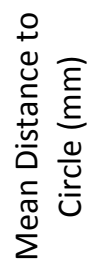 \\
\hline row 1 & & & & & & \\
\hline$\ldots$ & & & & & & \\
\hline row (end) & & & & & & \\
\hline
\end{tabular}

The smoothing function performance graph and the least squares fitted circle function performance graph provide the user with visual comparison tools to observe if the algorithm may have encountered an unexpected rail profile shape (e.g., major rail defect or optical measurement processing error) which may negatively affect the accuracy of 
the analysis results. Figure 44 and Figure 45 show examples of a smoothing function performance graph and a least squares fitted circle function performance graph, respectively; the graph sizes are reformatted to fit the page. Based on the graphs, the user may decide to investigate profiles that exhibit irregular measurements when compared to the rest of the rail profiles in the track segment, as an unexpected rail profile shape would negatively affect the accuracy-of-fit of the smoothing polynomial or the least squares, constant radius circle. After further investigation of the issue, the user can then take appropriate action to either keep or remove the profile from the track analysis table. For reference, this research would investigate profiles that had average distances $>0.3 \mathrm{~mm}$.

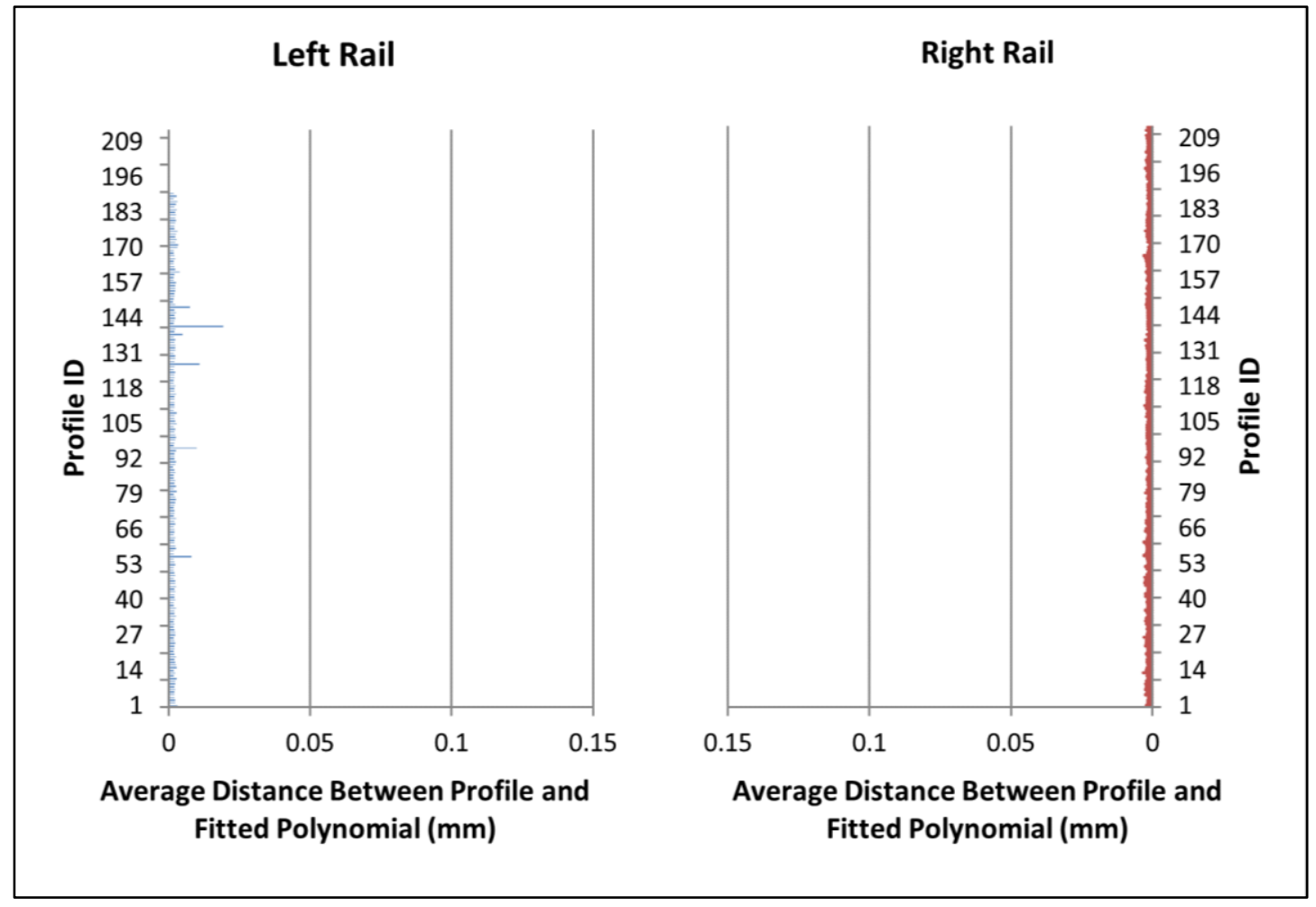

Figure 44: Reformatted analysis performance graph: average distance between profile and fitted polynomial 


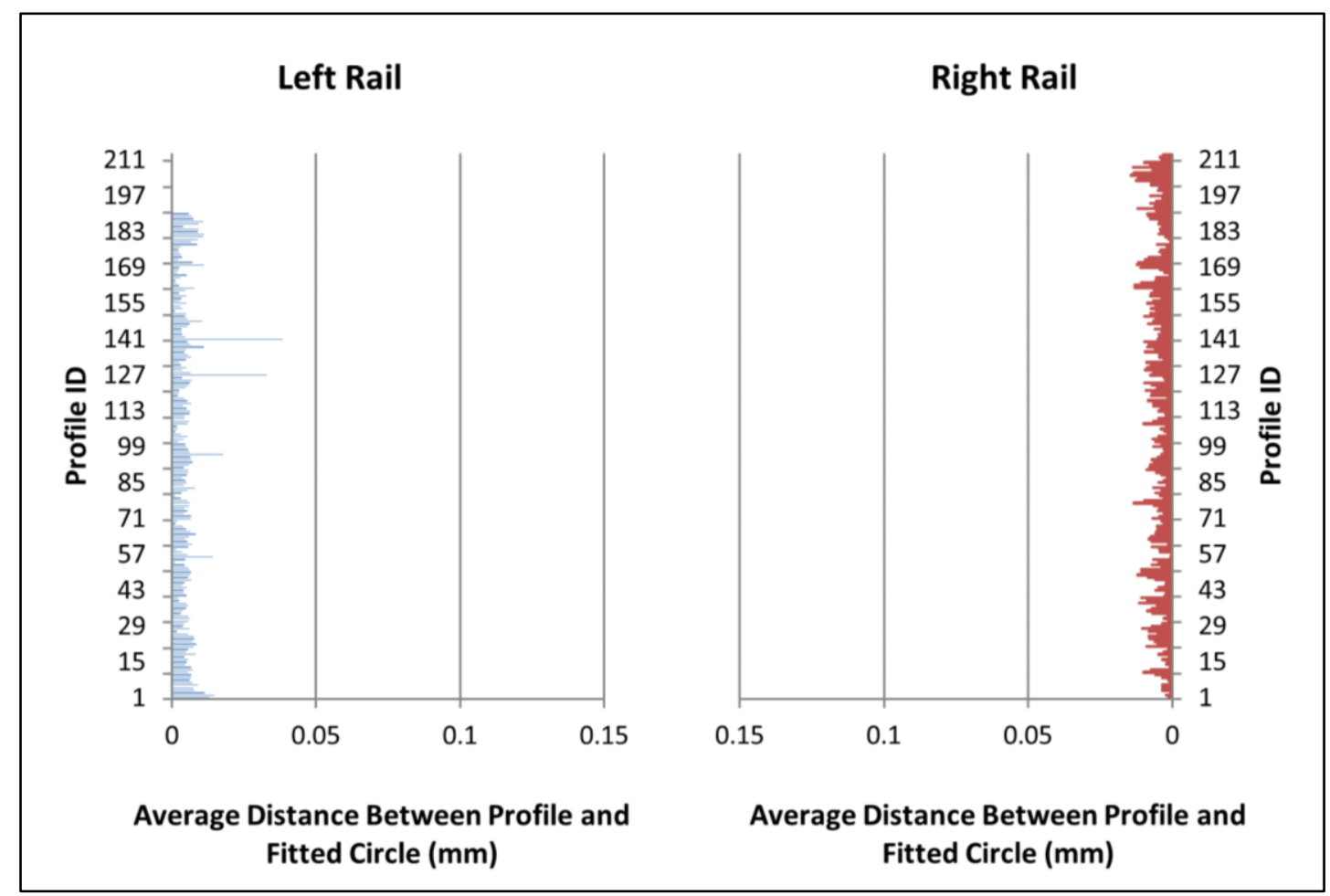

Figure 45: Reformatted analysis performance graph: average distance between profile and fitted circle

The one page summary sheet summarizes the data and reformats the graphs from the track analysis sheet onto a letter sized $(21.59 \mathrm{~cm} \times 27.94 \mathrm{~cm})$, printable page. Figure 46 shows an example of a one-page summary of a track analysis. The one-page summary contains three parts:

- The segment and analysis information section includes the track segment name, segment length, data collection date, contact analysis date, and left and right wheel slopes.

- The rail segment graphs contain a graphical representation of longitudinal variations in the lateral contact point (referenced with respect to the Rangecamdefined center zone) and the contact radius for the left and right rail segments. To increase clarity, the graphs forego lines connecting the data points. 
- The rail segment statistics table contains the means and standard deviations for the lateral contact position and contact radius for the left and right rail segments, along with the percentage of lateral contact points that are within the centre zone.

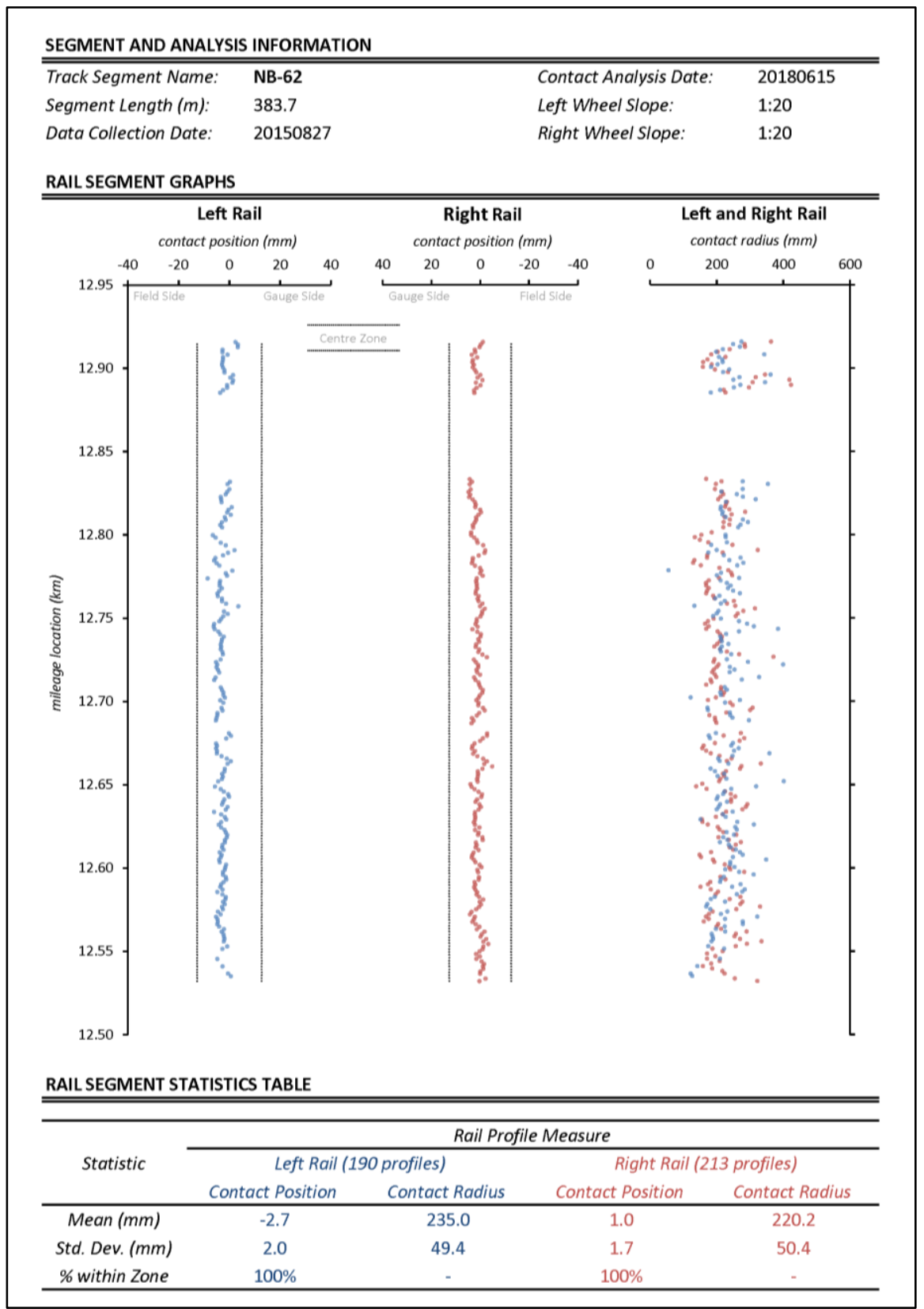

Figure 46: One-page summary of a track analysis 


\subsection{VERIFICATION OF THE PROCEDURE}

Verification of the procedure ensures proper execution of the code and the production of consistent and accurate results. The verification process involves

- comparing the algorithm's output for a rail segment comprising identical rail profiles to expected results; and

- comparing the calculated contact point and contact radius to known values.

The algorithm produces consistent and expected results when processing fictional rail segments comprising identical rail profiles. For all tests, the algorithm finds zero variation for both the lateral position of contact and the contact radius, as expected. Table 8 shows example algorithm output from this validation test.

Table 8: Rail segment statistic table from verification test using identical rail profiles

\begin{tabular}{cccccc}
\hline \multirow{2}{*}{ Statistic } & \multicolumn{4}{c}{ Rail Profile Measure } \\
\cline { 2 - 5 } & \multicolumn{2}{c}{ Lateral Contact Point } & \multicolumn{2}{c}{ Contact Radius } \\
& Left Rail & Right Rail & Left Rail & Right Rail \\
\hline Mean $(\mathrm{mm})$ & -1.2 & - & 249.7 & - \\
Standard deviation $(\mathrm{mm})$ & 0 & - & 0 & - \\
$\%$ within center zone $(\%)$ & $100.0 \%$ & - & - & - \\
\hline
\end{tabular}

The algorithm produces expected results when processing an unworn 136-lb rail with a known contact point $(x=0)$ and contact radius $(355.6 \mathrm{~mm}, 14$ ") when the wheel slope is 0 (i.e., the wheel profile is flat). Figure 47 is a drawing of the 136 -lb rail with the fitted circle for the contact radius superimposed. The algorithm output in Table 9 shows a contact point of $x=0$, which corresponds to the known contact point value, and a contact radius of $355.8 \mathrm{~mm}$, which corresponds to an error of $0.062 \%$ relative to the known value. The total error of $0.062 \%$ is acceptable for this application given the 
rounding within MATLAB along with the known uncertainty in the $x, y$-coordinate smoothing, the fit of the least squares circle, and measurement tolerances in the rail profile data for the $136-\mathrm{Ib}$ rail.

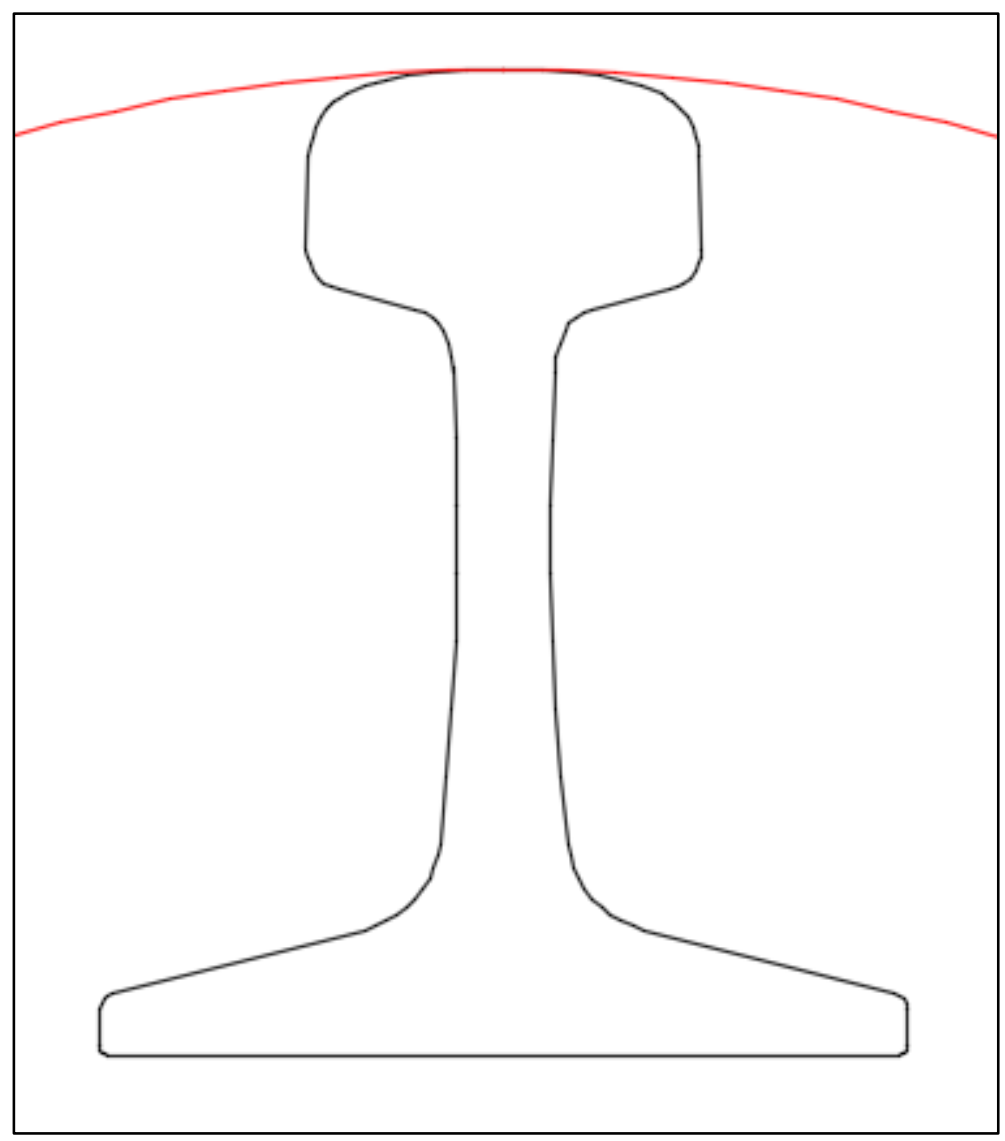

Figure 47: 136-Ib rail profile with fitted contact radius circle

Table 9: Rail segment statistics table from verification test using unworn 136-Ib rail profile

\begin{tabular}{ccccc}
\hline \multirow{2}{*}{ Statistic } & \multicolumn{4}{c}{ Rail Profile Measure } \\
\cline { 2 - 5 } & \multicolumn{2}{c}{ Lateral Contact Point } & \multicolumn{2}{c}{ Contact Radius } \\
& Left Rail & Right Rail & Left Rail & Right Rail \\
\hline Mean $(\mathrm{mm})$ & 0 & - & 355.8 & - \\
Standard deviation $(\mathrm{mm})$ & 0 & - & 0 & - \\
\% within center zone (\%) & $100.0 \%$ & - & - & - \\
\hline
\end{tabular}




\section{ALGORITHM VALIDATION THROUGH THE ANALYSIS OF RAIL PROFILE DATA}

This chapter describes the validation of the algorithm through the analysis of rail profile data from a North American rail transit property. Specifically, this chapter: (1) discusses the selection and cleaning of validation data and (2) describes the validation of the algorithm.

\subsection{SELECTION AND CLEANING OF VALIDATION DATA}

The validation process uses 36 selected track segments from a data collection run in 2015 on a closed loop, captive fleet, North American rail transit property. The property comprises a northbound line and a southbound line. The data collection run from 2015 measured 98 track segments for the northbound line -44 tangent segments and 54 curve segments - and 90 segments for the southbound line - 37 tangent segments and 53 curve segments.

Developing a set of criteria for rail segments to meet a minimal threshold for data quality is critical due to the nature of data collection methods and the needs of researchers and practitioners to have representative and reliable data. There are four criteria for selecting track segments for the algorithm validation. If a track segment meets all four criteria, it is included in the validation. The four criteria follow:

- The track segment is tangent. Only tangents segments are eligible for the validation since the increase in complexity in wheel-rail contact for non-tangent track is difficult to incorporate in an algorithm. While the algorithm is able to run non-tangent segments, the added complexity of wheel-rail contact in these 
segments reduces confidence in the accuracy of the results.

- The track segment has a minimum of 50 measured rail profiles for each rail. This criterion ensures an adequate sample size that (1) reduces the impact an unexpected profile may have on the average statistics for a rail segment and (2) enables inferences of the rail segment from the sample data (i.e., rail profile measurements). This criterion is set using a sensitivity analysis.

- The percent difference between the number of measured profiles for each rail is equal to, or less than $50 \%$. This criterion ensures that one rail is not significantly under-represented in the average statistics for a rail segment in comparison to the other rail. This criterion is set using a sensitivity analysis.

- The average collection interval (i.e., the average spacing between measured rail profiles) of the rail segment with the lowest number of measured rail profiles in the track segment is equal to, or less than $3.0 \mathrm{~m}$ per profile, calculated as

$$
(\text { ave. collection interval })=\frac{(\text { segment length })}{(\text { min.number of profiles })}
$$

This criterion ensures a relatively consistent sampling of rail profile data longitudinally along the track segment. Adequate sampling is critical for making representative inferences of the rail segment based on the sampled rail profile data. Optical rail measurement runs may discard a significant number of rail profiles due to data collection or post-processing issues (e.g., the optical rail sensor having an obstructed view of the rail profile) or where there is a significant amount of unique track infrastructure (e.g., turnouts, stations). This research 
uses a $3 \mathrm{~m}$ collection interval limit, double the typical collection interval utilized on the rail property, ensuring that the measurement process discards no more than $50 \%$ of the rail profiles of any rail segment in a track segment.

Table 10 and Table 11 display the 36 track segments that meet all four criteria for the selected northbound and southbound tracks segments, respectively. The segment name is the track direction followed by the order of the segment to increasing track mileage. For example, the first selected segment in Table 10, NB-4, is the fourth segment of the northbound line.

Table 10: Selected northbound track segments

\begin{tabular}{ccccccc}
\hline & & \multicolumn{3}{c}{ Number of Measured Rail Profiles } & Collection \\
\cline { 2 - 5 } Segment & $\begin{array}{c}\text { Length } \\
(\mathbf{m})\end{array}$ & Left Rail & Right Rail & $\begin{array}{c}\text { Percent } \\
\text { Difference }\end{array}$ & $\begin{array}{c}\text { Profiles } \\
\text { Removed } \\
\text { (Left/Right) }\end{array}$ & $\begin{array}{c}\text { Interval } \\
\text { (m/profile) }\end{array}$ \\
\hline NB-4 & 748 & 344 & 457 & $28.2 \%$ & $0 / 0$ & 2.17 \\
NB-7 & 547 & 226 & 340 & $40.3 \%$ & $0 / 1$ & 2.42 \\
NB-14 & 109 & 55 & 68 & $21.1 \%$ & $0 / 0$ & 1.98 \\
NB-17 & 650 & 361 & 409 & $12.5 \%$ & $1 / 0$ & 1.80 \\
NB-19 & 403 & 252 & 254 & $0.8 \%$ & $1 / 0$ & 1.60 \\
NB-23 & 176 & 74 & 99 & $28.9 \%$ & $0 / 0$ & 2.38 \\
NB-26 & 895 & 476 & 525 & $9.8 \%$ & $1 / 0$ & 1.88 \\
NB-34 & 1049 & 594 & 640 & $7.5 \%$ & $1 / 3$ & 1.77 \\
NB-56 & 286 & 179 & 184 & $2.8 \%$ & $0 / 0$ & 1.60 \\
NB-60 & 189 & 86 & 126 & $37.7 \%$ & $1 / 0$ & 2.20 \\
NB-62 & 385 & 190 & 213 & $11.4 \%$ & $0 / 0$ & 2.03 \\
NB-66 & 214 & 142 & 143 & $0.7 \%$ & $0 / 0$ & 1.51 \\
NB-68 & 348 & 165 & 211 & $24.5 \%$ & $0 / 0$ & 2.11 \\
NB-78 & 314 & 167 & 183 & $9.1 \%$ & $0 / 0$ & 1.88 \\
NB-80 & 878 & 400 & 448 & $11.3 \%$ & $0 / 0$ & 2.20 \\
NB-91 & 1602 & 812 & 888 & $8.9 \%$ & $2 / 0$ & 1.97 \\
NB-98 & 151 & 65 & 87 & $28.9 \%$ & $0 / 0$ & 2.32 \\
\hline
\end{tabular}


Table 11: Selected southbound track segments

\begin{tabular}{|c|c|c|c|c|c|c|}
\hline \multirow[b]{2}{*}{ Segment } & \multirow[b]{2}{*}{$\begin{array}{l}\text { Length } \\
\text { (m) }\end{array}$} & \multicolumn{4}{|c|}{ Number of Measured Rail Profiles } & \multirow{2}{*}{$\begin{array}{c}\text { Collection } \\
\text { Interval } \\
\text { (m/profile) }\end{array}$} \\
\hline & & Left Rail & Right Rail & $\begin{array}{l}\text { Percent } \\
\text { Difference }\end{array}$ & $\begin{array}{l}\text { Profiles } \\
\text { Removed } \\
\text { (Left/Right) }\end{array}$ & \\
\hline SB-11 & 626 & 406 & 260 & $43.8 \%$ & $0 / 1$ & 2.41 \\
\hline SB-13 & 847 & 521 & 418 & $21.9 \%$ & $0 / 3$ & 2.03 \\
\hline SB-16 & 897 & 527 & 475 & $10.4 \%$ & $0 / 1$ & 1.89 \\
\hline SB-22 & 1051 & 650 & 598 & $8.3 \%$ & $1 / 2$ & 1.76 \\
\hline SB-25 & 252 & 161 & 106 & $41.2 \%$ & $0 / 0$ & 2.38 \\
\hline SB-37 & 373 & 245 & 213 & $14.0 \%$ & $0 / 0$ & 1.75 \\
\hline SB-41 & 182 & 120 & 120 & $0.0 \%$ & $0 / 0$ & 1.52 \\
\hline SB-45 & 208 & 135 & 124 & $8.5 \%$ & $0 / 0$ & 1.68 \\
\hline SB-49 & 107 & 69 & 68 & $1.5 \%$ & $0 / 0$ & 1.57 \\
\hline SB-58 & 134 & 79 & 54 & $37.6 \%$ & $0 / 0$ & 2.48 \\
\hline SB-66 & 552 & 280 & 275 & $1.8 \%$ & $0 / 0$ & 2.01 \\
\hline SB-68 & 341 & 196 & 198 & $1.0 \%$ & $0 / 0$ & 1.74 \\
\hline SB-70 & 869 & 442 & 442 & $0.0 \%$ & $0 / 0$ & 1.97 \\
\hline SB-74 & 119 & 77 & 73 & $5.3 \%$ & $0 / 0$ & 1.63 \\
\hline SB-77 & 127 & 57 & 52 & $9.2 \%$ & $0 / 0$ & 2.44 \\
\hline SB-81 & 809 & 405 & 392 & $3.3 \%$ & $0 / 1$ & 2.06 \\
\hline SB-83 & 704 & 429 & 423 & $1.4 \%$ & $0 / 0$ & 1.66 \\
\hline SB-87 & 302 & 125 & 118 & $5.8 \%$ & 0/0 & 2.56 \\
\hline SB-90 & 171 & 94 & 73 & $25.1 \%$ & $1 / 0$ & 2.34 \\
\hline
\end{tabular}

After selecting track segments, the cleaning process removes atypical rail profiles with issues in either the text file or the optical rail measurement; this cleaning process prevents algorithm execution errors and erroneous analysis results. The rail profile data has previously gone through an automated cleaning process integrated within the optical measurement process and data import to the Rangecam software; however, the automated process does not catch all rail profile issues. The cleaning process applied in this research identifies and removes atypical rail profiles through manual observation of the rail segment $x, y$-coordinate plots (see Section 3.2.3.2, Plot Rail Profile). The 
cleaning process finds two main forms of errors in the rail profiles: (1) missmeasurement during the optical measurement process and (2) the rail profile text file contains an incorrect value for the number of rows that contain railhead $x, y$-coordinates, resulting in the inclusion of a portion of the rail web as part of the railhead. A rail profile showing either form of error is removed from the data set. Figure 48 provides an example of a miss-measurement of a rail profile where the gauge side of the rail profile deviates significantly from an expected rail profile. Figure 49 provides an example of a rail profile from a text file that contains an incorrect value for number of rows that contain railhead $x, y$-coordinates resulting in the inclusion of the rail web as part of the railhead.

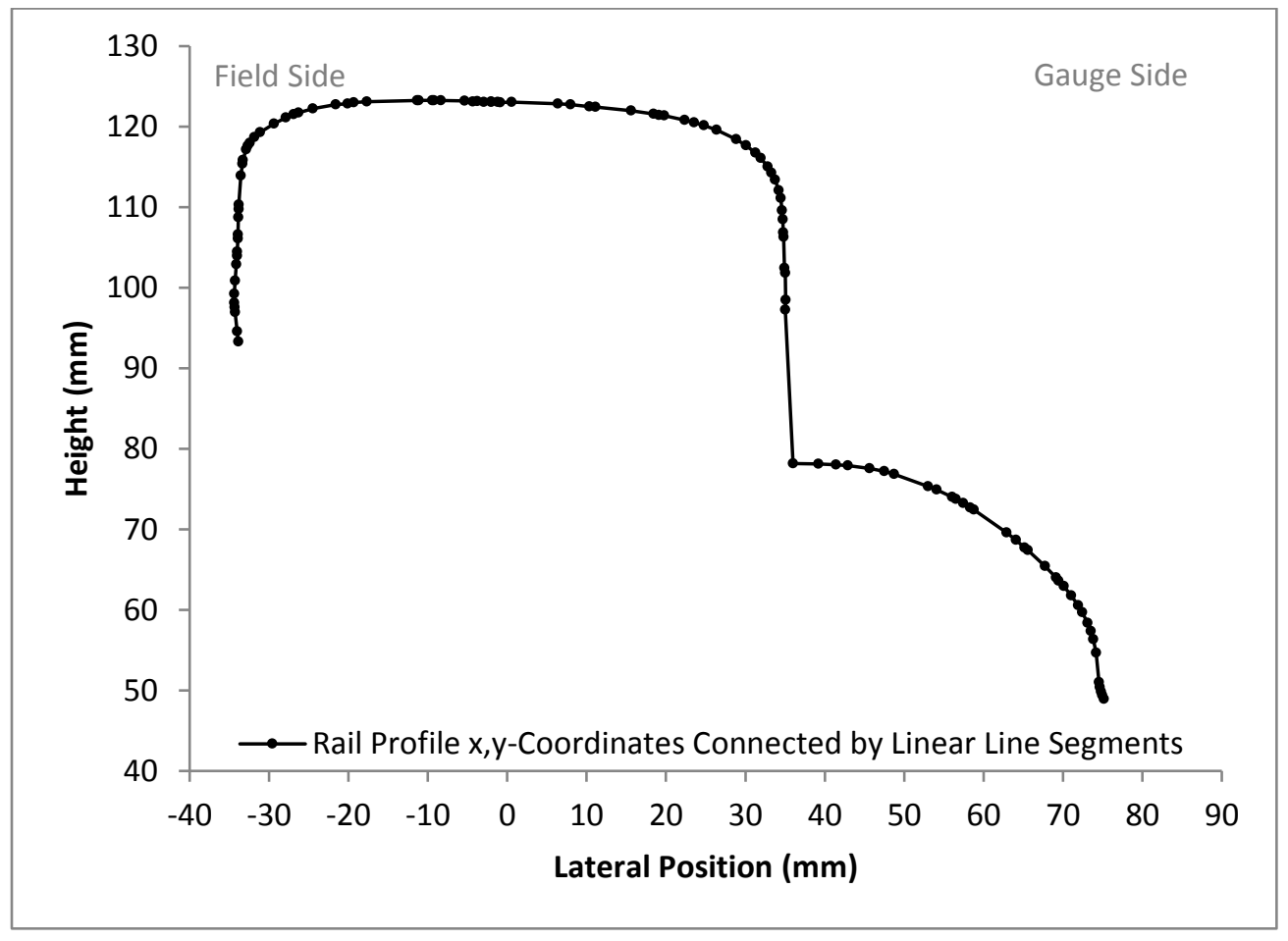

Figure 48: An example of rail profile removed due to miss-measurement 


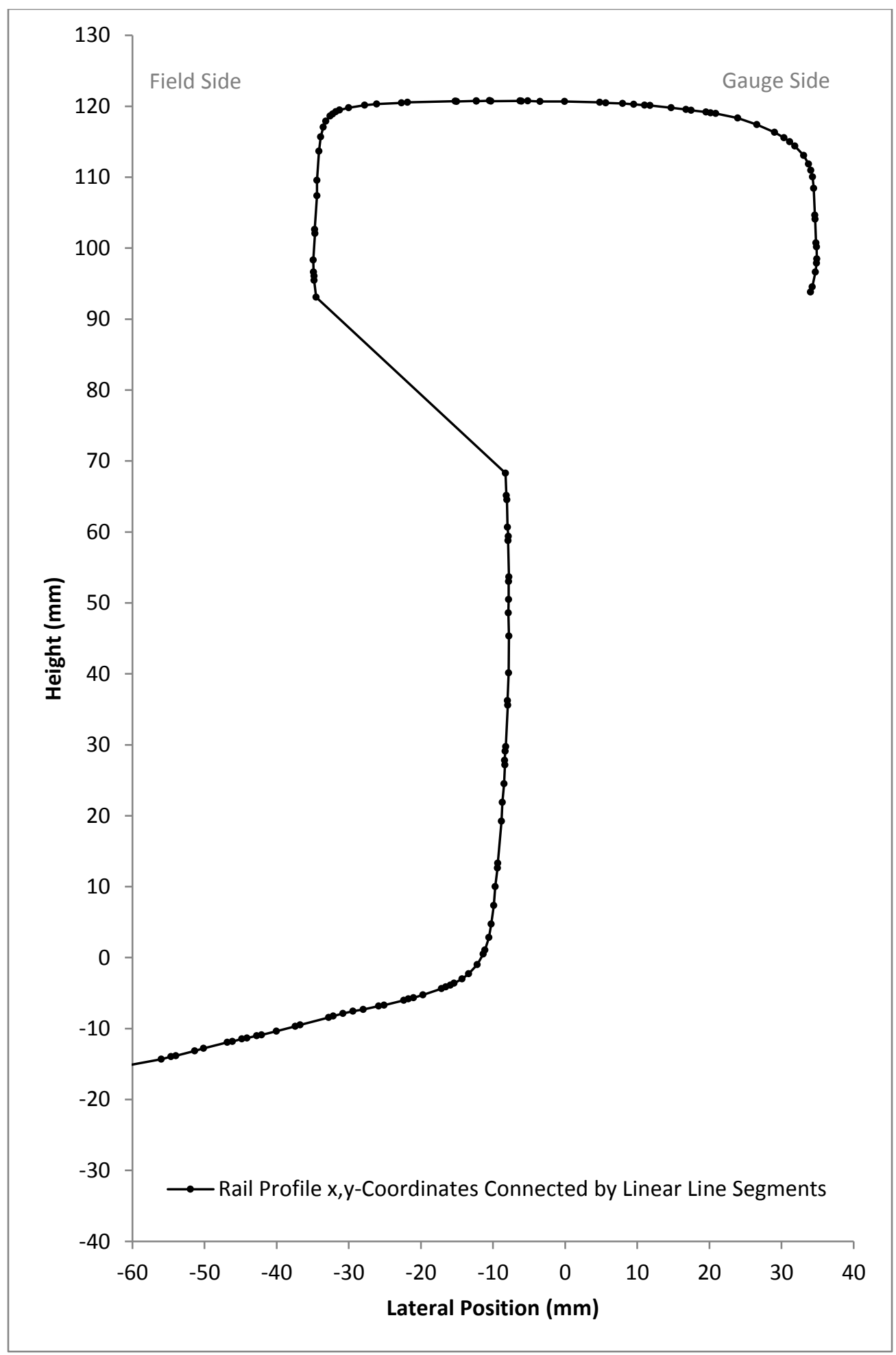

Figure 49: An example of a rail profile removed due to the inclusion of the rail web as part of the railhead. 


\subsection{VALIDATION OF THE PROCEDURE}

This research validates the algorithm by analyzing the reasonableness of algorithm output for rail profile data collected from a North American rail transit property. Typical validation compares research results with independent ground truth data; however, there is currently no accessible rail profile data sources suitable for validating this algorithm. Therefore, this research compares algorithm output to a series of expected results. The algorithm is valid for each expected result if the research finds the algorithm output reasonable compared to the expected result.

\subsubsection{Analysis of Track Segments}

The algorithm analyzed the 36 track segments using the six wheel slopes in Table 12. Figure 50 shows the approximate locations of the six wheel slopes on the specified wheel profile for the transit property.

\section{Table 12: Six wheel slope conditions used in validation}

\begin{tabular}{cc}
\hline \multicolumn{2}{c}{ Wheel Slope } \\
\hline Gradient & Degrees \\
\hline flat & $0^{\circ}$ \\
$1: 20$ & $2.86^{\circ}$ \\
$1: 10$ & $5.72^{\circ}$ \\
$1: 6.6$ & $8.59^{\circ}$ \\
$1: 4.9$ & $11.45^{\circ}$ \\
$1: 3.9$ & $14.31^{\circ}$ \\
\hline
\end{tabular}




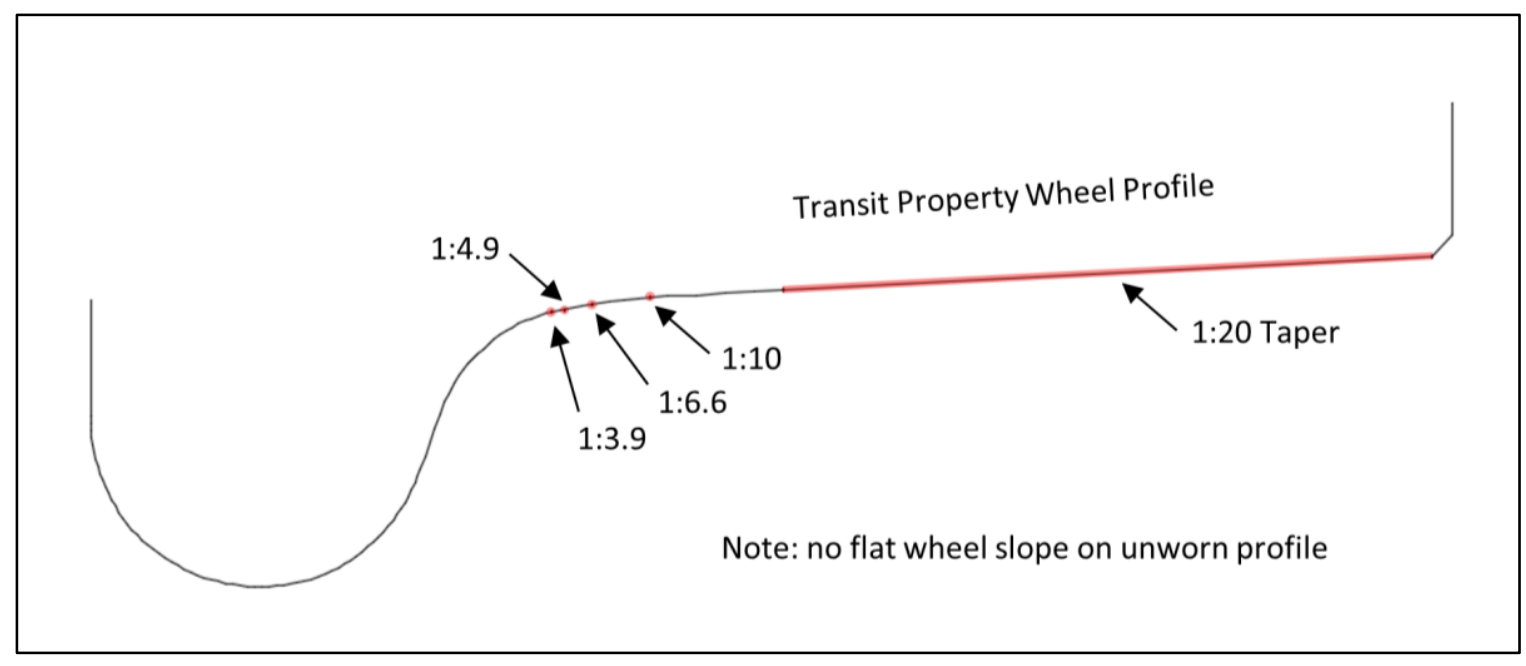

Figure 50: The approximate locations of the wheel slopes used in the validation on the transit property specified wheel profile

The flat, 1:20, and 1:10 wheel slopes are typically specified for unworn, linear wheel tread profiles; they are also of interest in worn profiles (e.g., a wheel tread profile with a 1:20 slope may eventually wear down to a flat wheel tread profile). While the 1:6.6, 1:4.9, and 1:3.9 wheel slopes are not typically specified in linear wheel treads, they do occur in the transition from the wheel flange to the wheel tread and ensure even spacing between the wheel slopes, in terms of degrees.

The shape of the transit property specified wheel profile differs from typical AAR standard wheel profiles. As Figure 51 highlights in red, the significant difference between the wheel profiles is length of the wheel tread (i.e., the linear component of the wheel profile). The linear wheel tread component of the specified wheel profile for the transit property is significantly shorty compared to an AAR standard 1:20 wheel profile shape due to the former profile's larger transition from the flange to the 1:20 taper. The specified wheel profile results in a single point of contact in any situation of lateral axle shift. In a majority of situations, the contact point occurs on the transition of the wheel profile. 


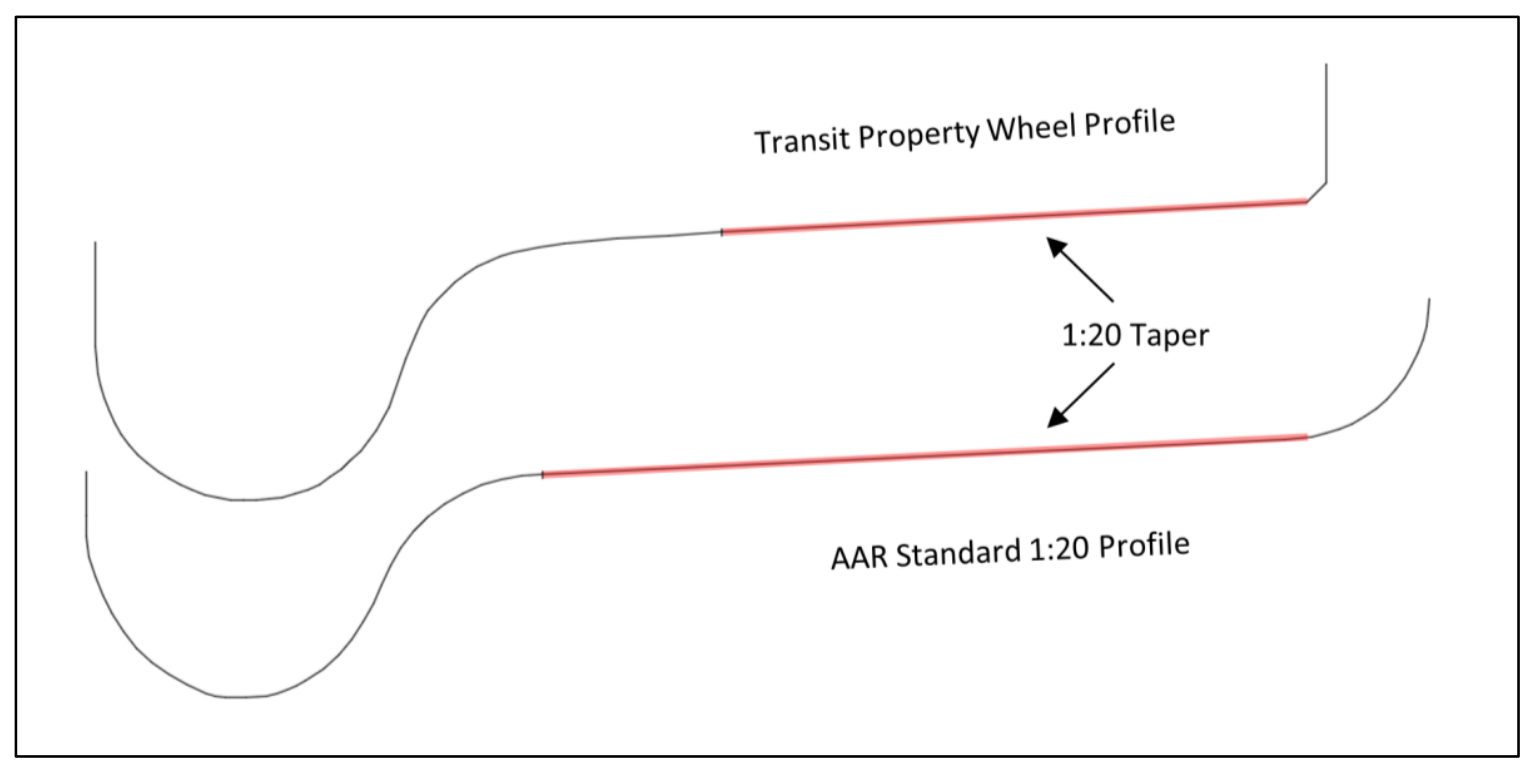

Figure 51: Comparison of transit property and AAR standard wheel profiles

Using the specified unworn wheel profile and rail profile (a 115 LB RE rail) drawings with a zero lateral shift of the wheelset, an external wheel-rail contact evaluation finds that

- the wheel-rail profile contact occurs at a profile surface angle of 8.06 degrees; and

- rail profile contact occurs $17.44 \mathrm{~mm}$ to the gauge side of the vertical centerline of the rail profile.

This research assumes that there is zero lateral shift of the wheelset when traversing tangent track. The external evaluation results are from CFIT-WRCON ${ }^{\circledR}$, a program from the multi-body dynamic simulation integration engine, NUCARS ${ }^{\circledR}$.

By combining the analysis results from the six different wheel slopes, the resulting sensitivity analysis shows the sensitivity of the lateral contact position and the contact radius to changes in wheel slope. This algorithm does not directly output the sensitivity analysis; instead, this research produces the sensitivity analysis as a tool for validating the algorithm using additional MATLAB coding. Figure 52 shows a sensitivity analysis for 
a track segment. An overview of the sensitivity analysis follows:

- The first section of the page, under the heading [Left/Right] Rail Sensitivity Analysis, contains the left and right rail sensitivity of the lateral contact position to changes in wheel slope graphs and corresponding distributions followed by the left and right rail sensitivity of the contact radius to changes in wheel slope graphs and corresponding distributions.

- The second section of the page, under the heading [Left/Right] Rail Profiles, contains all the $x, y$-coordinates of the rail segment prior to canting the rail profile.

- Both the wheel slope versus lateral contact position and wheel slope versus contact radius graphs contain a smoothed line connecting the mean contact radius for each wheel slope. The smoothed line in Excel is a type of cubic spline; however, the equation of the line is unknown.

- The black dot on the wheel contact versus lateral contact position graph represents the externally calculated location of wheel/rail contact between the unworn wheel profile and rail profile specified by the property.

- Each data point represents one rail profile. For a rail segment, each series of points for a particular wheel slope contains the same number of rail profiles.

- The bin widths for the lateral contact position and the contact radius distributions are one and ten, respectively. The sensitivity analysis for the contact radius bins all radii larger than $600 \mathrm{~mm}$ together. 

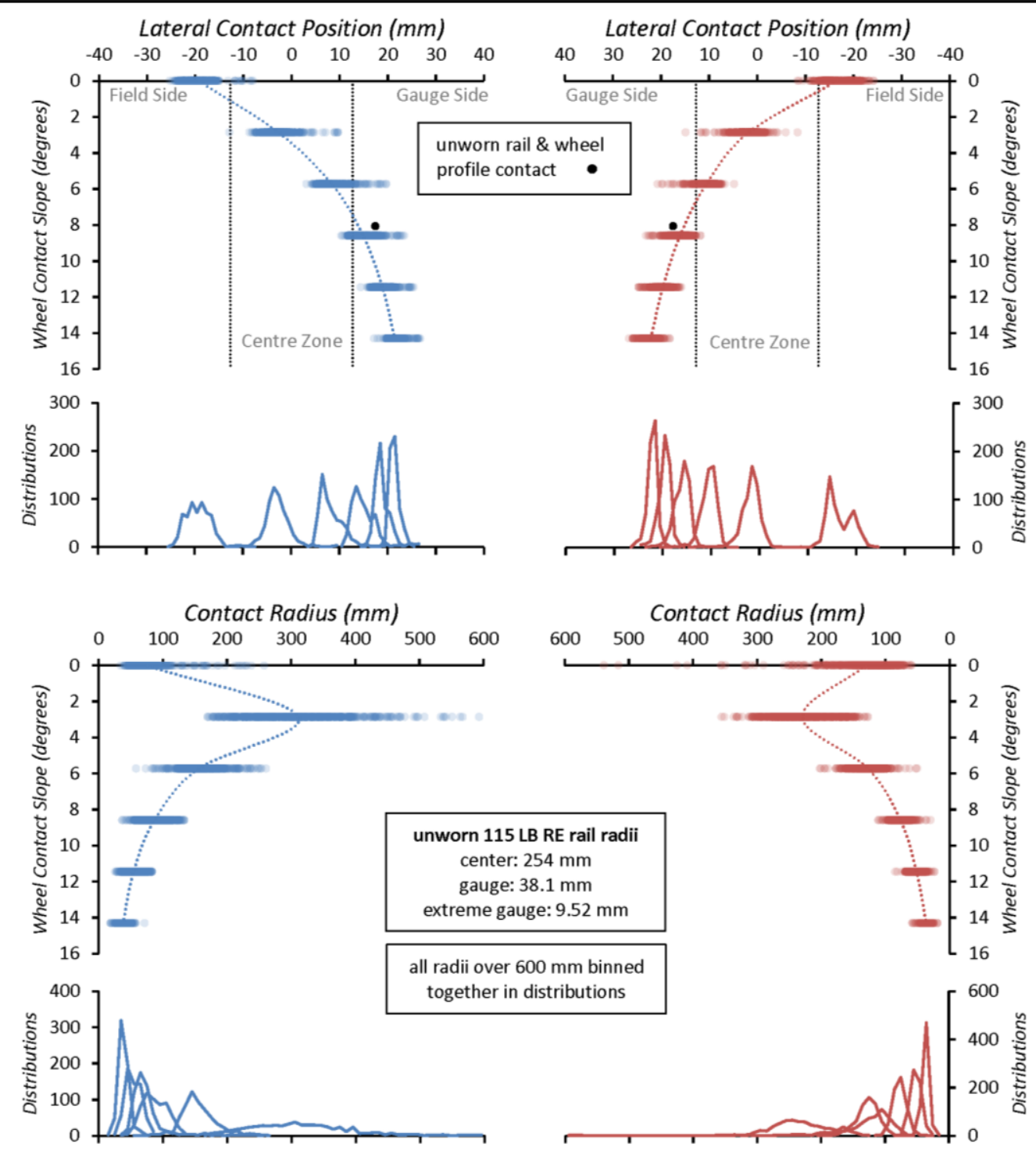

LEFT RAIL PROFILES

RIGHT RAIL PROFILES
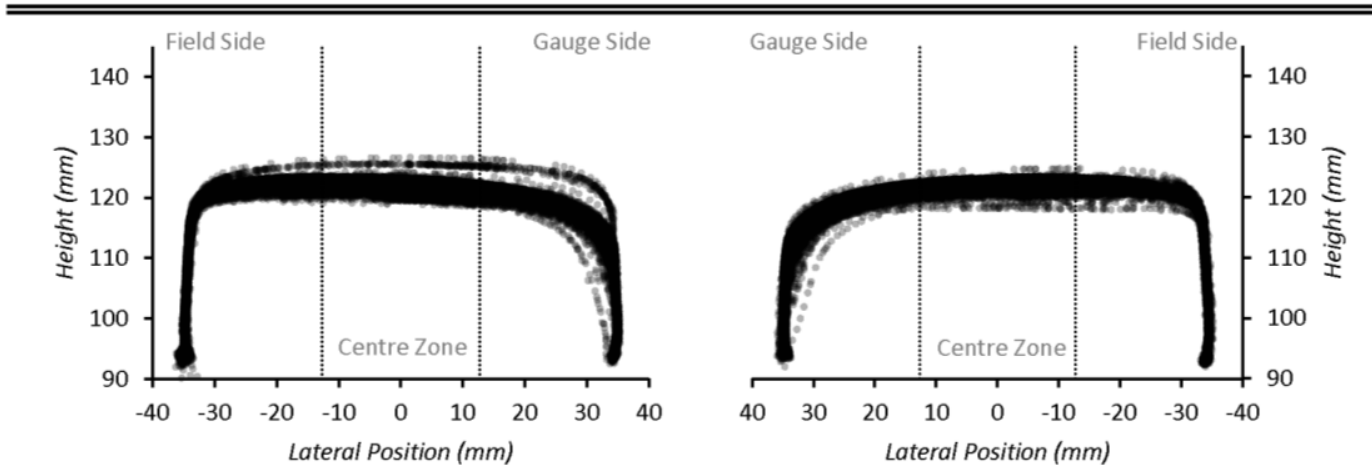

Figure 52: Example of a track segment sensitivity analysis 
The resulting files after analyzing all the selected track segments are 216 track segment analysis results files (36 track segments using 6 different wheel slopes) and 36 sensitivity analyses files (one for each track segment). Appendix D and Appendix E contain the northbound and southbound track segment analysis results one-page summaries for the 1:20 wheel slope and the corresponding sensitivity analysis, respectively. The other track segment analysis results are not included in the appendices.

\subsubsection{Algorithm Validation Criteria and Results}

To validate the algorithm, this research compiles the algorithm output for the six wheel slopes into a sensitivity analysis and then compares the sensitivity analysis to a series of expected results. The expected results for a rail segment algorithm output follow:

1. Each consecutive wheel slope, in increasing order, will result in the mean lateral contact position shifting to the gauge side of the rail profile.

2. Of the six wheel slopes, a 1:20 wheel slope will result in the largest mean contact radius.

3. For northbound segments, the line connecting the mean lateral contact position for the different wheel slopes will be to the field side of the unworn wheel profile and rail profile lateral contact position found in the external evaluation. For southbound segments, the mean lateral contact position line will be either close to, or slightly to the gauge side of the unworn wheel-rail contact position.

4. Of the 1:20 and 1:3.9 wheel slopes, the 1:20 wheel slope will result in the larger standard deviation for the lateral contact position. 
The algorithm is valid for each expected result if the research finds the algorithm output reasonable compared to the expected result. This research considers results reasonable when

- a clear majority of rail segments follow the expected result; and

- reasonable explanations exist as to why the remaining rail segments did not follow the expected result.

In this way, despite a lack of independent ground truth data, the validation process involves both a quantitative and qualitative assessment of reasonableness. The development of the expected results takes into account the shape of rail profiles, rail wear characteristics, principles of rigid contact theory, and industry feedback.

\subsubsection{Expected Result 1}

For a given rail segment, each consecutive wheel slope, in increasing order, will result in the mean lateral contact position shifting to the gauge side of the rail profile; Figure 53 illustrates this concept.

Using the principles of rigid contact, at the point of wheel-rail contact the surface angle of the wheel profile (i.e., the wheel slope when using a linear wheel profile) must match the surface angle of the rail profile (see Section 2.4.1, Rigid Contact Theory). Typically, the surface angle increases moving from the top of rail to the gauge side of the rail profile under normal wearing conditions; therefore, as the wheel slope increases, the contact point should shift further to the gauge side of the profile. 


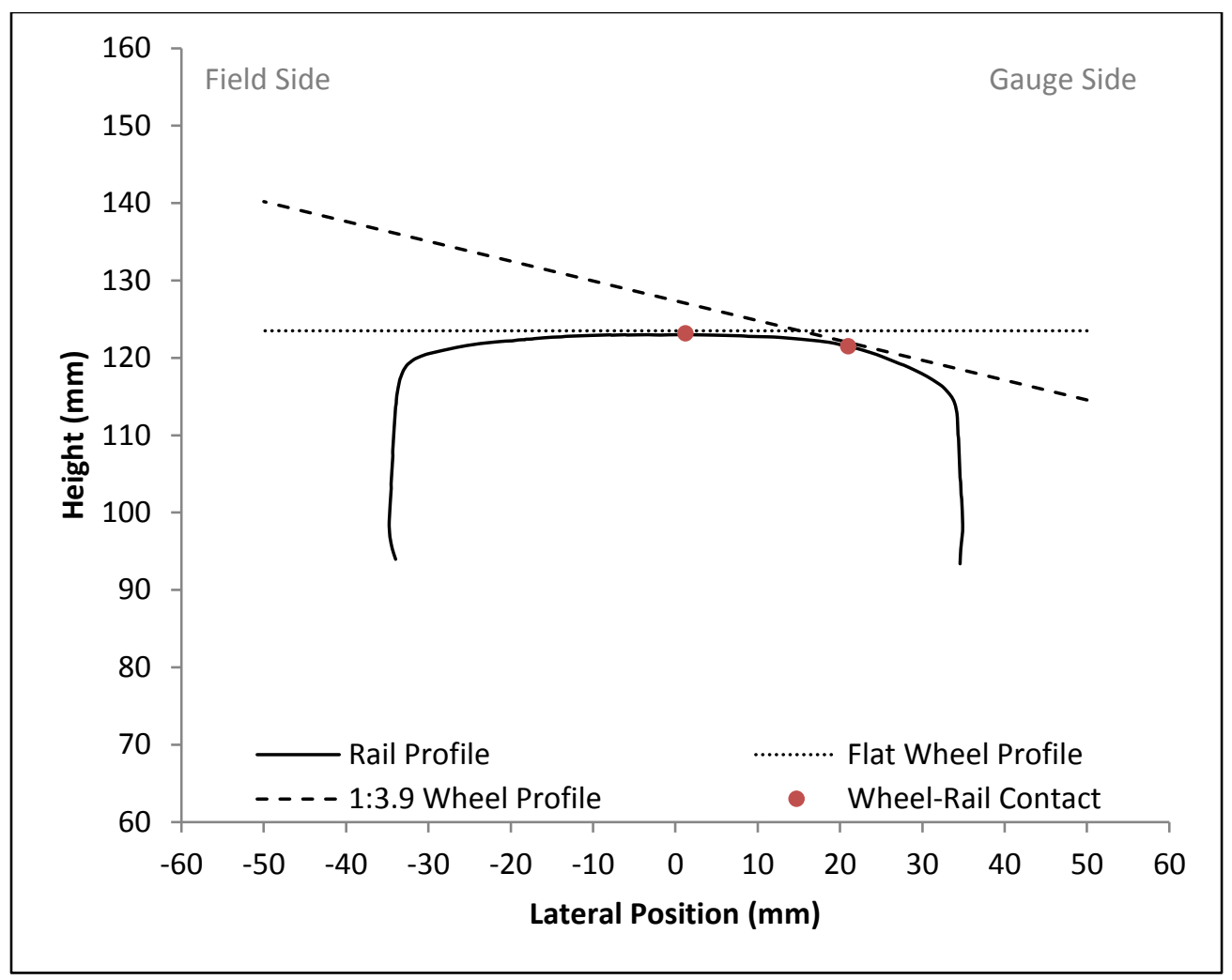

Figure 53: Location of the lateral contact position with changes in wheel slope

The validation test examines the lateral contact position sensitivity analysis qualitatively and the mean lateral contact position from average rail segment statistics quantitatively. It is expected that each consecutive, incremental increase in wheel slope from flat to 1:3.9 will result in a lateral contact position that is shifted further to the gauge side of the rail profile

The validation shows that all 72 rail segments (100\%) follow the expected result. The following is an example of the validation process for the NB-4 track segment where the algorithm output for both rail segments followed the expected result. Qualitatively, both the lateral contact position sensitivity analysis in Figure 54 show the $x$-value of the line connecting the mean lateral contact positions to increase (i.e., shift toward the gauge side) for each corresponding increase in wheel slope. Quantitatively, the mean lateral 
contact positions in Table 13 show consistent increases for each corresponding increase in wheel slope.

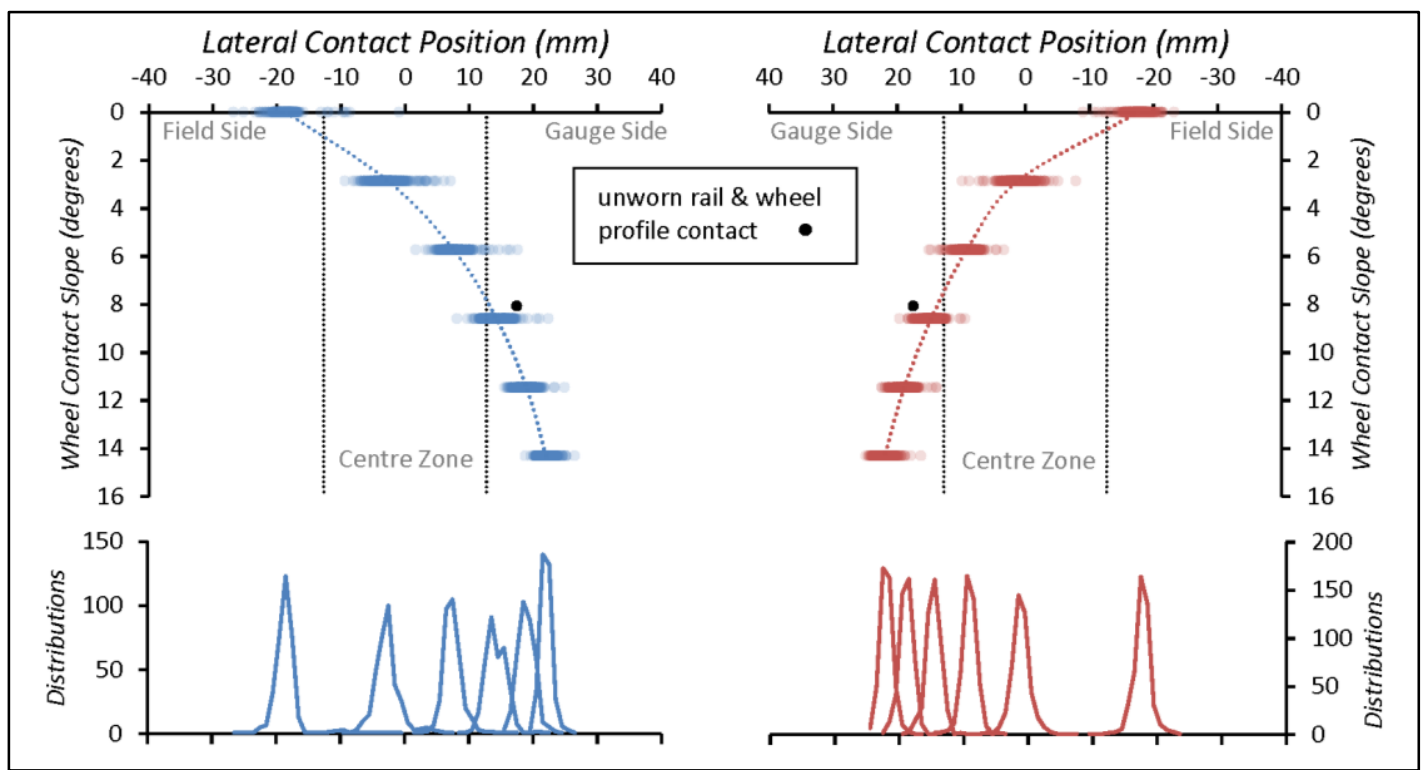

Figure 54: NB-4 left (blue) and right (red) lateral contact point sensitivity analysis

Table 13: NB-4 mean lateral contact positions

\begin{tabular}{cccccc}
\hline \multirow{2}{*}{ Segment } & \multicolumn{2}{c}{ Wheel Slope } & & \multicolumn{2}{c}{ Mean Lateral Contact Position (mm) } \\
\cline { 2 - 3 } \cline { 5 - 6 } & Gradient & Degrees & & Left Rail & Right Rail \\
\hline NB-4 & flat & $0^{\circ}$ & & -18.6 & -17.6 \\
NB-4 & $1: 20$ & $2.86^{\circ}$ & & -2.7 & 1.2 \\
NB-4 & $1: 10$ & $5.72^{\circ}$ & & 7.6 & 9.1 \\
NB-4 & $1: 6.6$ & $8.59^{\circ}$ & & 14.2 & 14.7 \\
NB-4 & $1: 4.9$ & $11.45^{\circ}$ & & 18.9 & 18.9 \\
NB-4 & $1: 3.9$ & $14.31^{\circ}$ & & 22 & 22 \\
\hline
\end{tabular}

Since the algorithm output for all 72 rail segments follow the expected result, the algorithm is considered valid for this test.

\subsubsection{Expected Result 2}

Of the six wheel slopes, a 1:20 wheel slope will result in the largest mean contact radius. 
Intermittently, a flat wheel slope may result in the largest mean contact radius under certain conditions.

The rate of change of the rail profile surface angle around the lateral contact point is directly related to the contact radius. Unless the rail profile shape is unusually or heavily worn, the top the rail profile is the location where the surface angle has the lowest rate of change; therefore, the largest contact radius is when the wheel profile contacts the top of the rail profile. Figure 55 highlights this concept. Specifically, this contact condition occurs when the cant angle of the rail profile matches the slope of the wheel profile. If the contact radius moves toward either the field side or the gauge side of the rail profile, a reduction in the contact radius is expected.

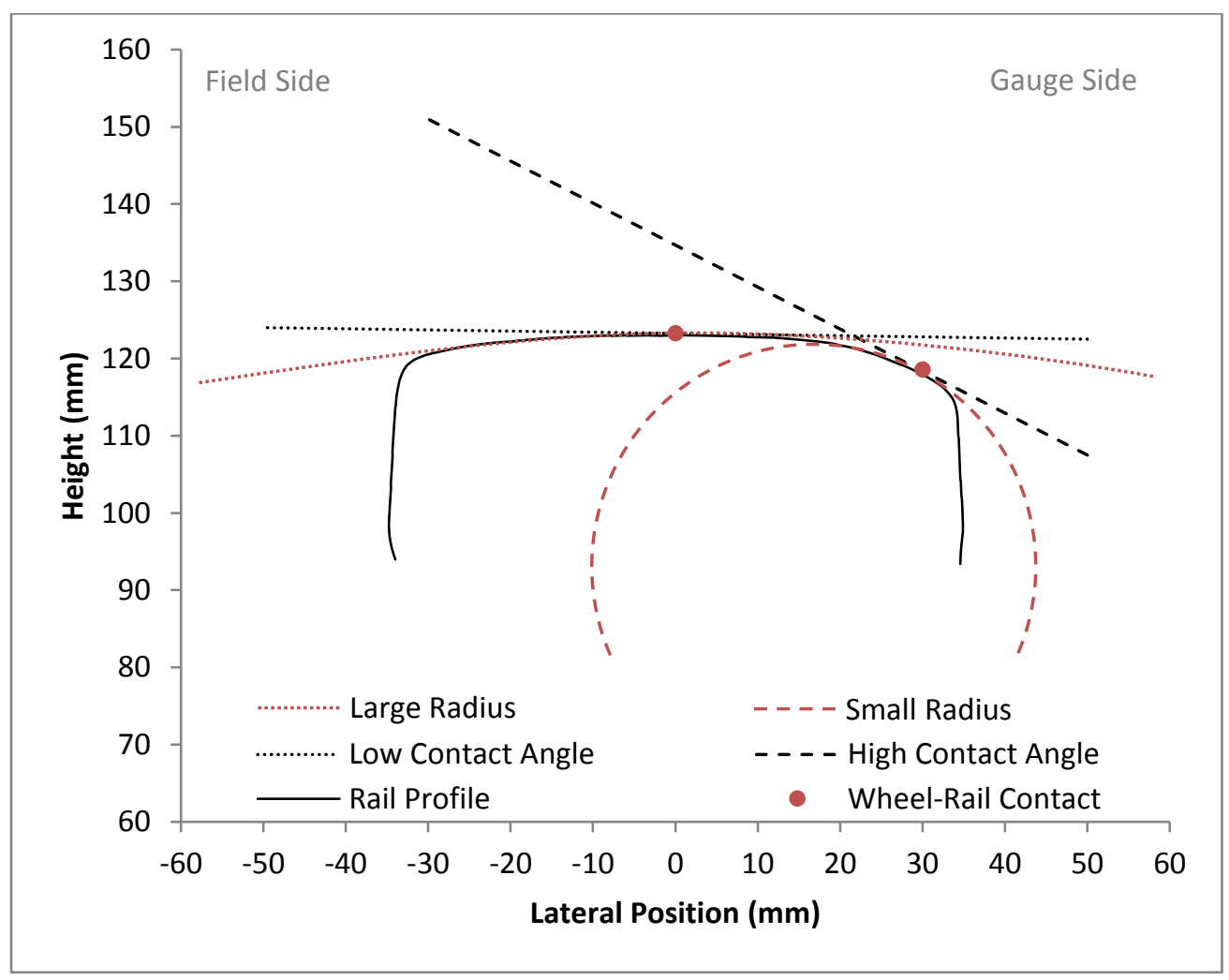

Figure 55: Contact radius relationship to the lateral contact position

The validation test examines the contact radius sensitivity analysis qualitatively and the 
contact radii from average rail segment statistics quantitatively. It is expected that the 1:20 wheel slope contains the largest mean contact radii for a given rail segment, which is the closest of the six wheel slopes to the average subdivision cant angle of 1.54 degrees. As a secondary evaluation, if the 1:20 wheel slope does not contain the largest mean contact radius, it is anticipated that the flat wheel slope will result in the largest mean contact radius since the average cant angle is between the flat and 1:20 wheel slopes.

The validation shows that 68 of the 72 rail segments (94.4\%) follow the initial expected result of a 1:20 wheel slope resulting in the largest mean contact radius. As anticipated, a flat wheel slope results in the largest mean contact radii for the other four rail segments. The following is an example of the validation process for track segment NB-4 where the algorithm output for both the left rail and right rail followed the expected result. The contact radius sensitivity analysis in Figure 56 shows that the contact radii for the 1:20 wheel slope have a greater mean than any other wheel slope. Quantitatively, the mean contact radius in Table 14 is greatest with a 1:20 wheel slope for both the left and right rail. 


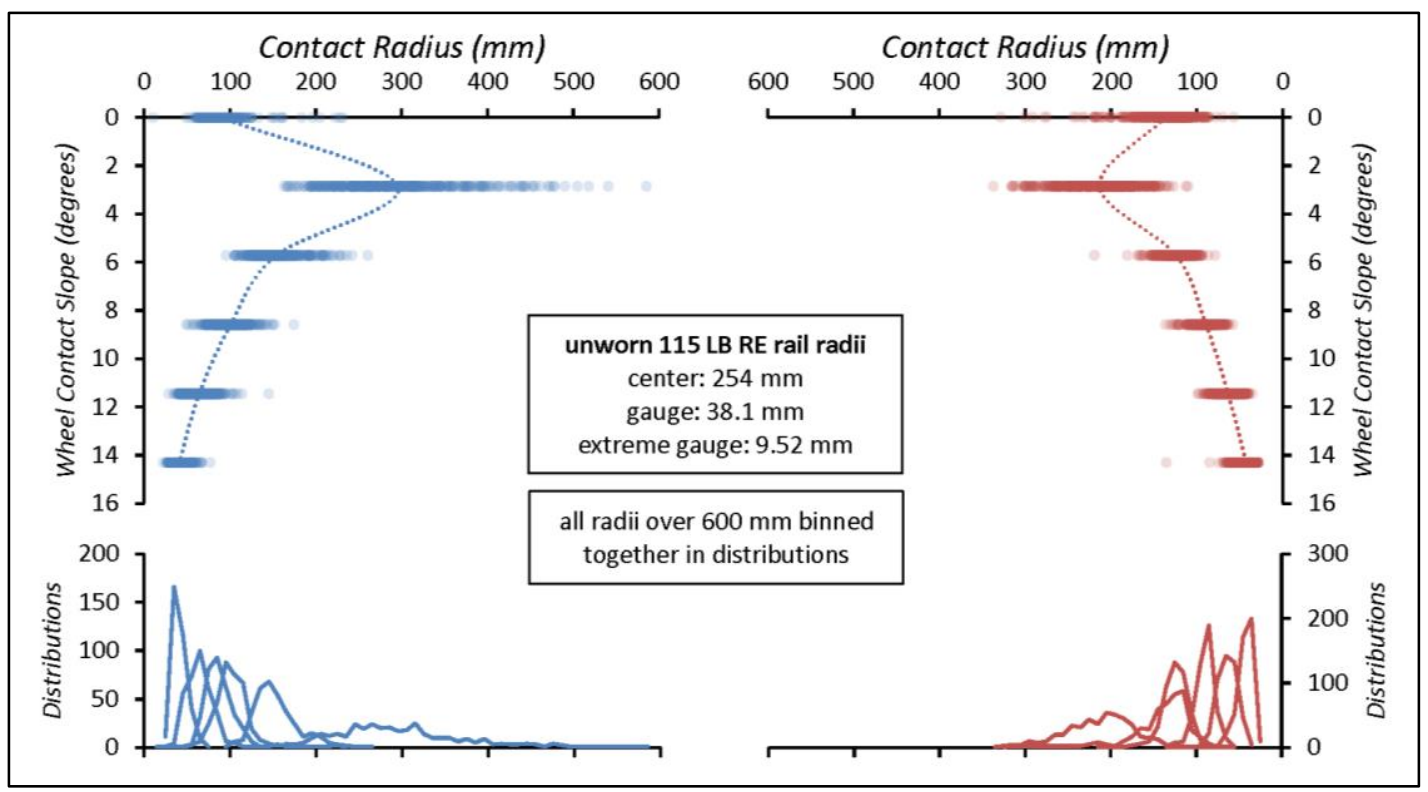

Figure 56: NB-4 left (blue) and right (red) contact radius sensitivity analysis

Table 14: NB-4 mean contact radii

\begin{tabular}{cccccc}
\hline \multirow{2}{*}{ Segment } & \multicolumn{2}{c}{ Wheel Slope } & & \multicolumn{2}{c}{ Mean Contact Radius (mm) } \\
\cline { 2 - 3 } \cline { 5 - 6 } \cline { 5 - 6 } Gradient & Degrees & & Left Rail & Right Rail \\
\hline NB-4 & flat & $0^{\circ}$ & & 90.1 & 137.3 \\
NB-4 & $1: 20$ & $2.86^{\circ}$ & & 295.7 & 213.3 \\
NB-4 & $1: 10$ & $5.72^{\circ}$ & & 153.8 & 124.0 \\
NB-4 & $1: 6.6$ & $8.59^{\circ}$ & & 100.9 & 89.4 \\
NB-4 & $1: 4.9$ & $11.45^{\circ}$ & & 64.5 & 63.5 \\
NB-4 & $1: 3.9$ & $14.31^{\circ}$ & & 41.0 & 42.9 \\
\hline
\end{tabular}

The following is an example of the validation process for the left rail segment in SB-68 where the algorithm output did not follow the expected result, the wheel slope of the exception was predicted correctly. The left rail sensitivity analysis in Figure 57 shows both the flat wheel slope and 1:20 wheel slope have a similar mean contact radius, with the 1:20 wheel slope demonstrating slightly more variability. The left rail mean contact radii in Table 15 support this observation, with the flat wheel slope having a mean contact radii only $11.2 \mathrm{~mm}$ greater than the 1:20 wheel slope. 


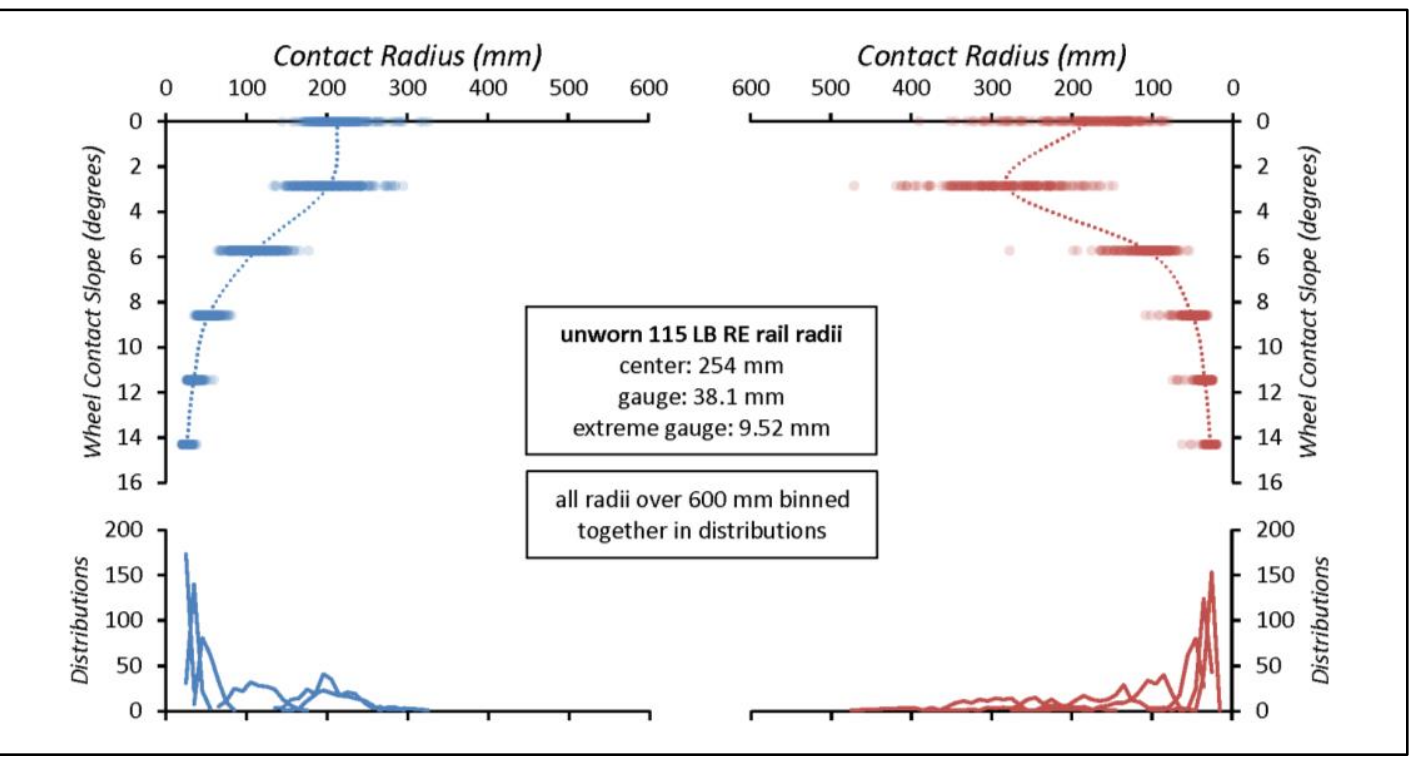

Figure 57: SB-68 left (blue) and right (red) contact radius sensitivity analysis

Table 15: SB-68 mean contact radii

\begin{tabular}{cccccc}
\hline \multirow{2}{*}{ Segment } & \multicolumn{2}{c}{ Wheel Slope } & & \multicolumn{2}{c}{ Mean Contact Radius (mm) } \\
\cline { 2 - 3 } \cline { 5 - 6 } & Gradient & Degrees & & Left Rail & Right Rail \\
\hline SB-68 & flat & $0^{\circ}$ & & 213.7 & 177.7 \\
SB-68 & $1: 20$ & $2.86^{\circ}$ & & 202.5 & 281.7 \\
SB-68 & $1: 10$ & $5.72^{\circ}$ & & 111.9 & 106.2 \\
SB-68 & $1: 6.6$ & $8.59^{\circ}$ & & 53.0 & 50.7 \\
SB-68 & $1: 4.9$ & $11.45^{\circ}$ & & 34.7 & 35.0 \\
SB-68 & $1: 3.9$ & $14.31^{\circ}$ & & 26.0 & 27.9 \\
\hline
\end{tabular}

Visual comparison of the rail profiles in Figure 58 provide insight to why the left rail segment may not have followed the expect result. The left rail profile has a slightly more rounded profile on the top part of the rail (highlighted by the red line) and the rate of surface angle change appears to be relatively consistent when compared to the right rail profile, which has a flatter profile on the top portion of the rail (highlighted by red line). Based on the comparison, it is expected that the left segment will have similar mean contact radii for the flat and 1:20 wheel slope, and the left rail profile will have a smaller mean contact radius for the flat and 1:20 wheel slopes compared to the right rail profile. 
The analysis results in Table 15 support both these expectations.

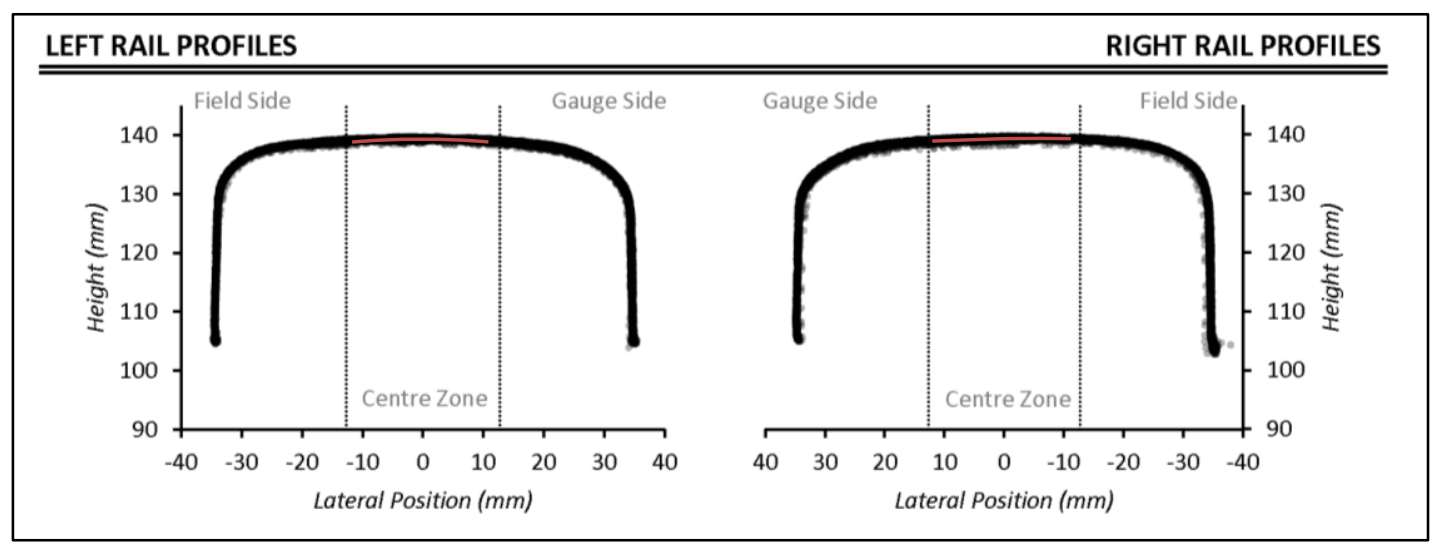

Figure 58: SB-68 rail profiles

The other rail segments that did not follow the expected result are NB-78 (Right), SB-25 (Left), and SB-45 (Left), which all follow similar reasoning as to why the expected result was not followed as SB-68 (left). Despite the not following the expected result, the wheel slope for the exceptions that resulted in the largest mean contact radius was correctly predicted as flat.

Since a clear majority of the algorithm output for the rail segments follow the initial expected result and the secondary evaluation accurately predicted the wheel slope that resulted in the largest mean contact radii for remaining rail segments, the algorithm is considered valid for this test.

\subsubsection{Expected Result 3}

For rail segments that show significant wear characteristics, the mean lateral contact position is expected to be on the field side of the unworn wheel/rail contact point from the external evaluation. Figure 59 illustrates this concept. The rail wearing process reshapes the gauge side of the rail profile, spreading the high rate of surface angle 
change at the gauge corner over a greater width of the profile.

For newer rail segments that are unworn or closely resemble unworn rail, the unworn wheel-rail contact point will be near the mean lateral contact position, though not necessary identical due to rail wearing and variations in the rail measurement process as well as track structure.

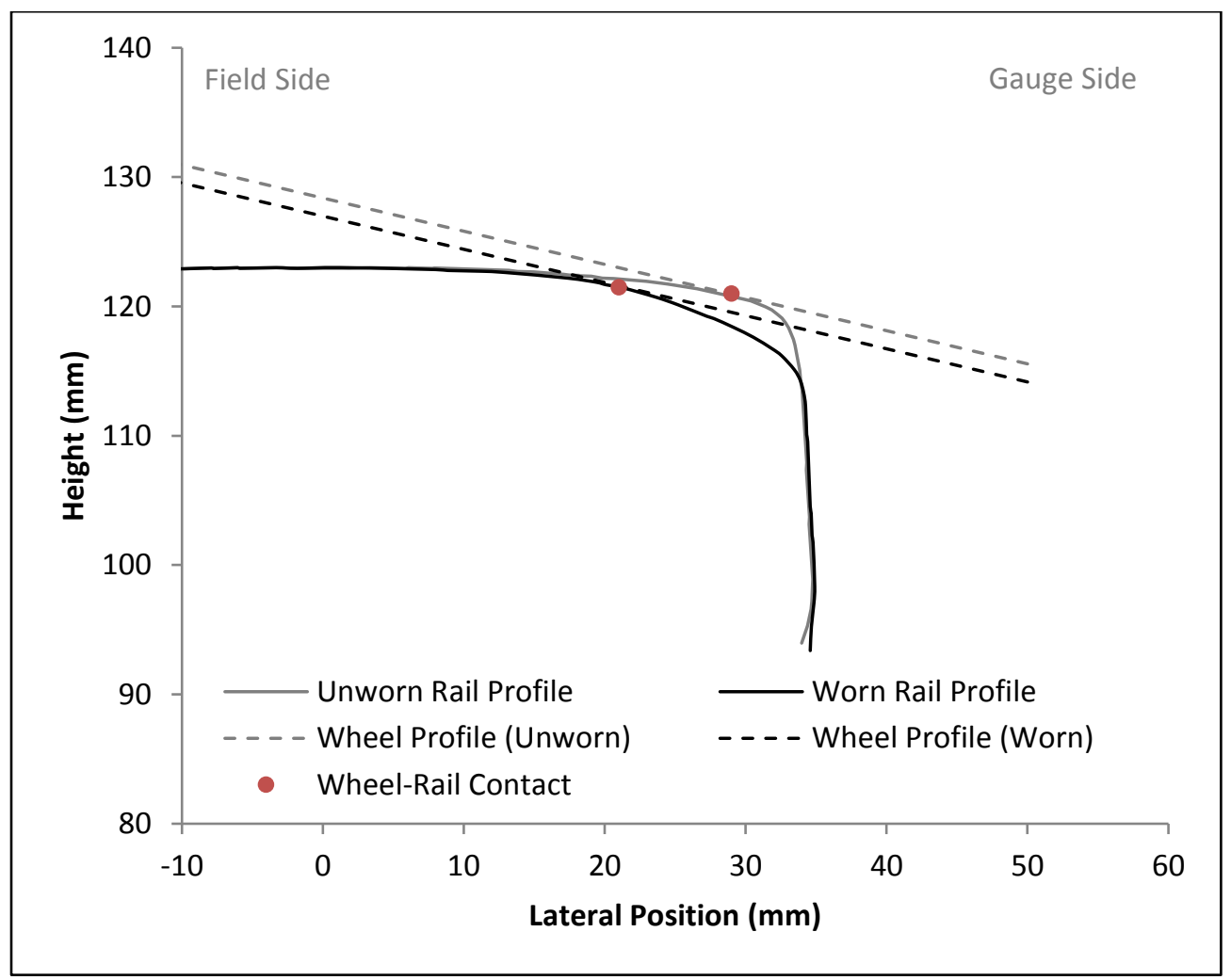

Figure 59: Worn vs. unworn lateral contact position with identical wheel slopes

The validation test examines the contact radius sensitivity analysis qualitatively, looking at the position of the mean lateral contact position line compared to the unworn wheelrail contact point. It is expected that the mean lateral contact position line connecting each of the mean lateral contact points

- will be to the field side of the unworn wheel-rail contact point, as the northbound track rail profiles show relatively high wear characteristics compared to the 
specified rail profile; and

- will be near, or will intersect, the unworn wheel-rail contact point as the southbound track rail profiles show relatively low wear characteristics compared to the specified rail profile.

Information on the subdivision wear characteristics is obtained through manual observation of the rail profile plots and is confirmed by personnel associated with the transit property. The wear differences between the northbound and southbound tracks are likely due to artificial wear (i.e., grinding) (G. Bachinsky, personal communication, 2018).

The validation shows that 34 of the 34 northbound rail segments (100\%) and 36 of 38 southbound rail segments $(94.7 \%)$ follow the expected results. The following is an example of the validation processes for track segments NB-19 and SB-13 where the algorithm output for all rail segments followed the expected result. The lateral contact position sensitivity analysis for the northbound track segment in Figure 60 shows that the line connecting the mean lateral contact positions is to the gauge side of the unworn rail and wheel profile contact point, following the expected result for the northbound segments. The lateral contact position sensitivity analysis for the northbound track segment in Figure 61 shows that the mean lateral contact line passes through the unworn wheel-rail contact point, following the expected result for the southbound segments. 


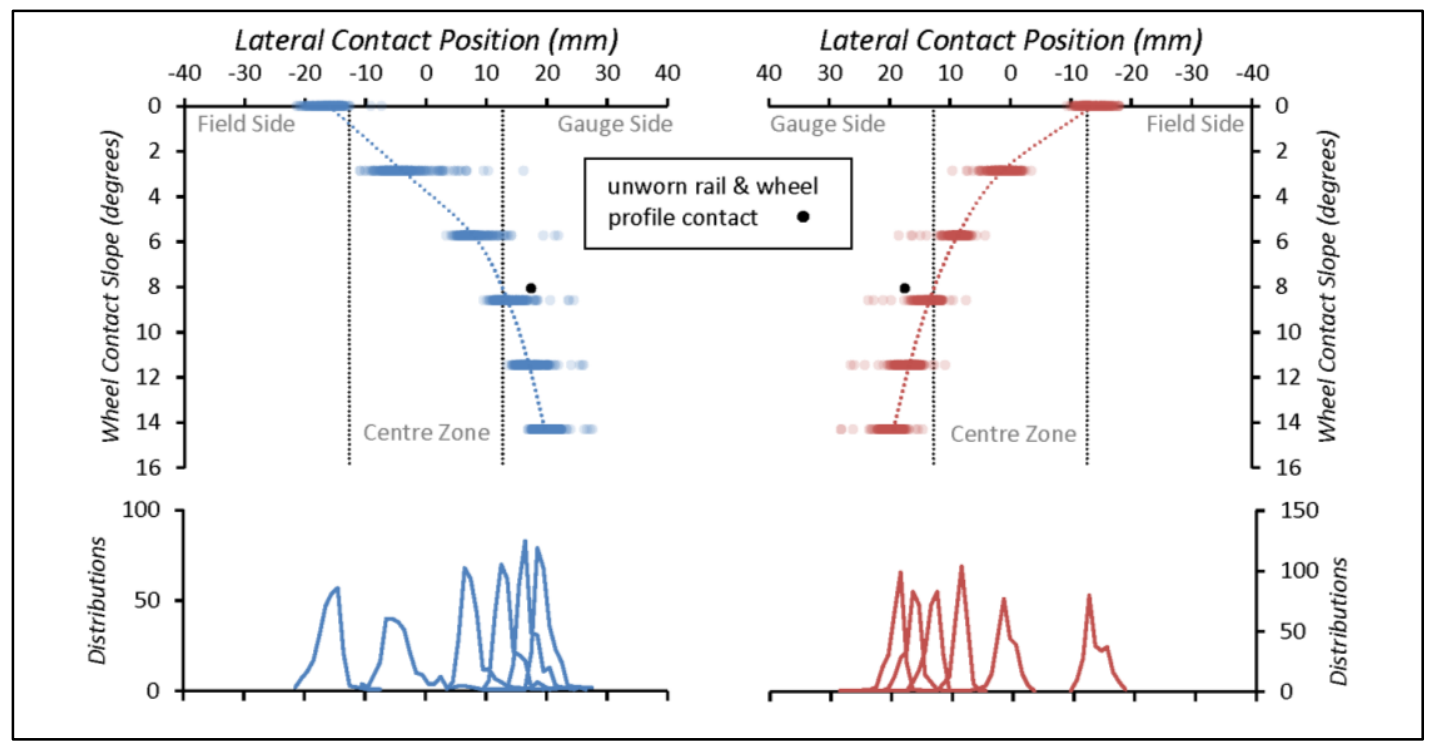

Figure 60: NB-19 left (blue) and right (red) lateral contact position sensitivity analysis

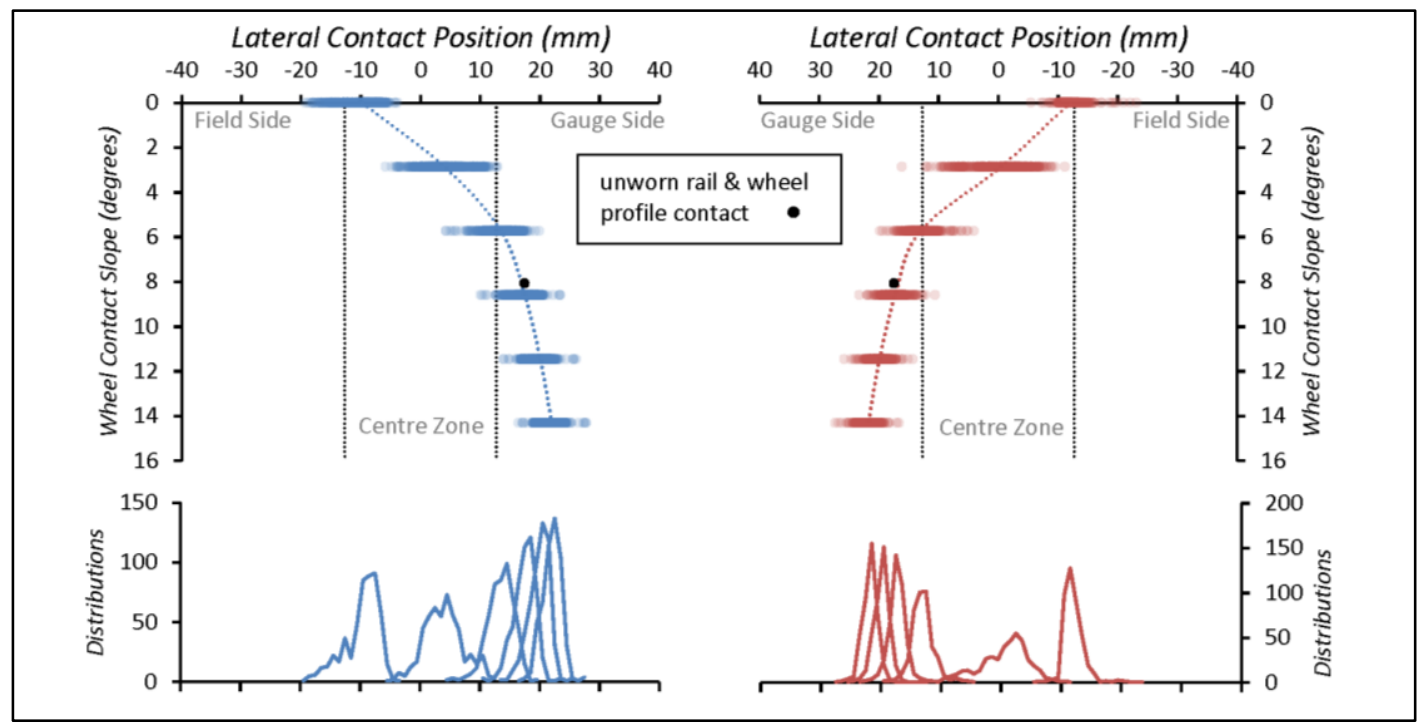

Figure 61: SB-13 left (blue) and right (red) lateral contact position sensitivity analysis

The following is an example of the validation process for only two rail segments, both from track segment SB-45, where the algorithm output did not follow the expected result. The lateral contact position sensitivity analysis in Figure 62 shows the mean lateral contact position line to the field side of the unworn wheel-rail contact point. Further 
investigation using the rail profile plots in Figure 63 show that the rail profiles do exhibit gauge corner wear similar to the northbound tracks which likely explains the analysis results. Two potential reasons for the worn rail segments follow:

- The transit property may not have replaced or maintained the SB-45 rail on the same schedule as the rest of the southbound segment; however, the transit property maintenance schedule shows that the SB-45 rails received grinding at the same time as the rest of the southbound rail segments.

- The SB-45 track segment experiences more wear than other southbound track segments.

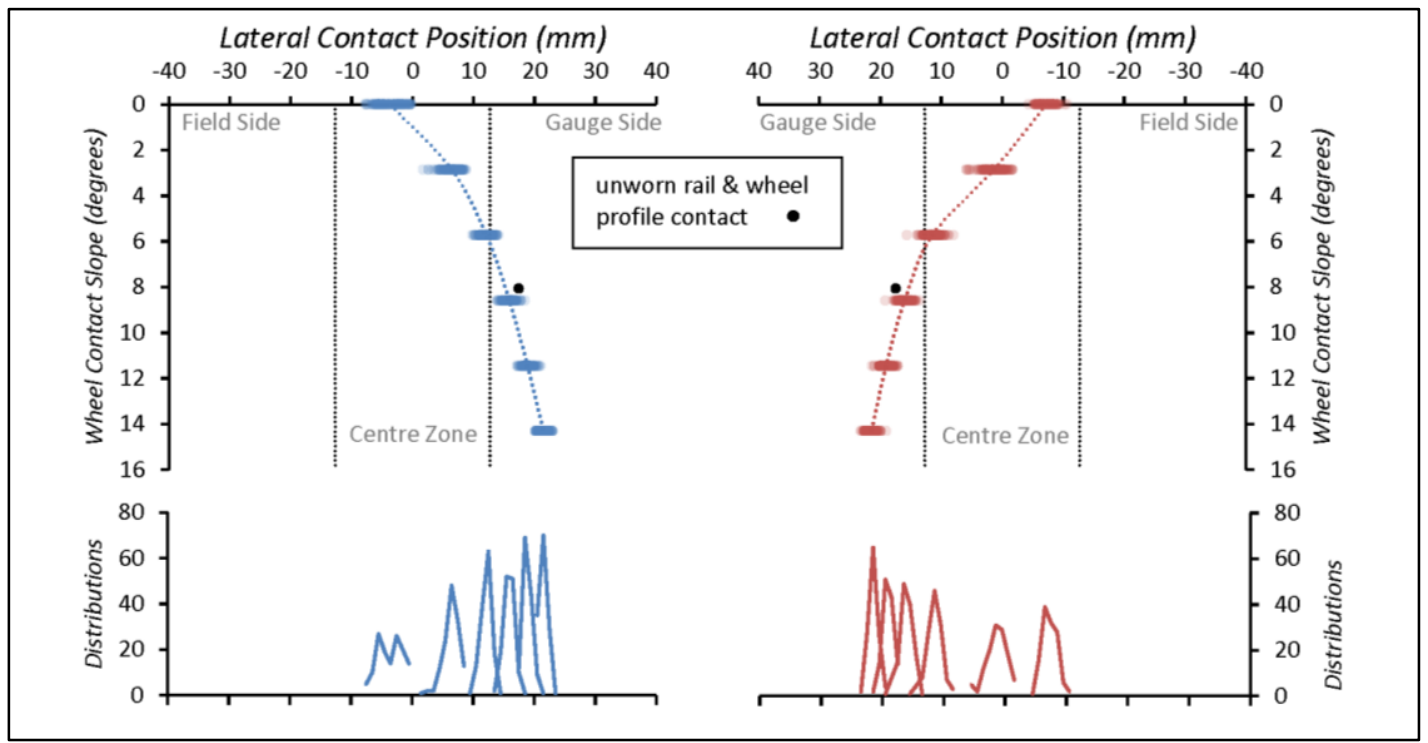

Figure 62: SB-45 left (blue) and right (red) lateral contact position sensitivity analysis 


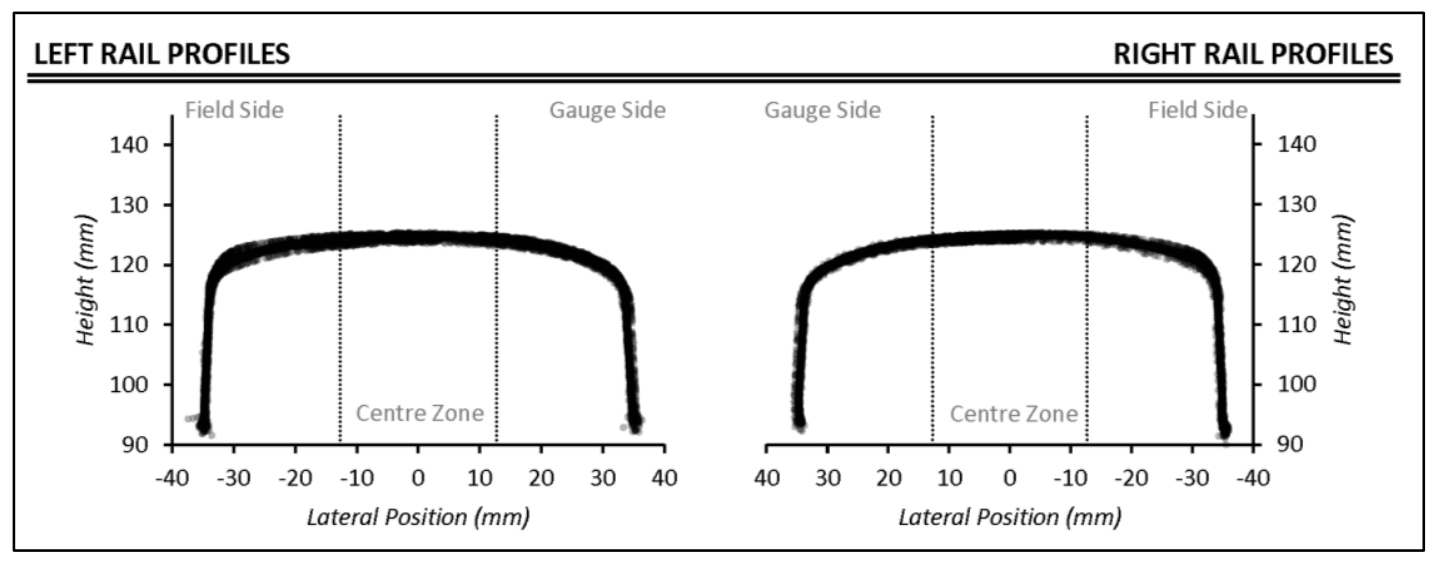

Figure 63: SB-45 rail profiles

All the northbound rail segments and a clear majority of the southbound segments follow the expect result. Southbound rail segments that do not follow the expected result show higher wear characteristics and algorithm output that corresponds to northbound segments. Based on these findings, the algorithm is considered valid for this test.

\subsubsection{Expected Result 4}

Of the 1:20 and 1:3.9 wheel slopes, the 1:20 wheel slope will result in the larger standard deviation for the lateral contact position.

For a given rail segment, the variability of the lateral contact position is larger when the wheel contacts the top portion of the railhead (i.e., the wheel slope is approximately equal to the cant angle of the rail profile) compared to the wheel contacts near the gauge corners. Figure 64 illustrates this concept. Using the principles of rigid contact, at the point of wheel-rail contact the surface angle of the wheel profile (i.e., the wheel slope when using a linear wheel profile) must match the surface angle of the rail profile (see Section 2.4.1, Rigid Contact Theory). Typically, there is an increasing rate of change of the surface angle moving from the top of rail to the gauge side of the rail profile; therefore, small changes in the cant angle or shape of the rail profile will result in larger 
variations on the top of the railhead, relative to the gauge corner of the rail.

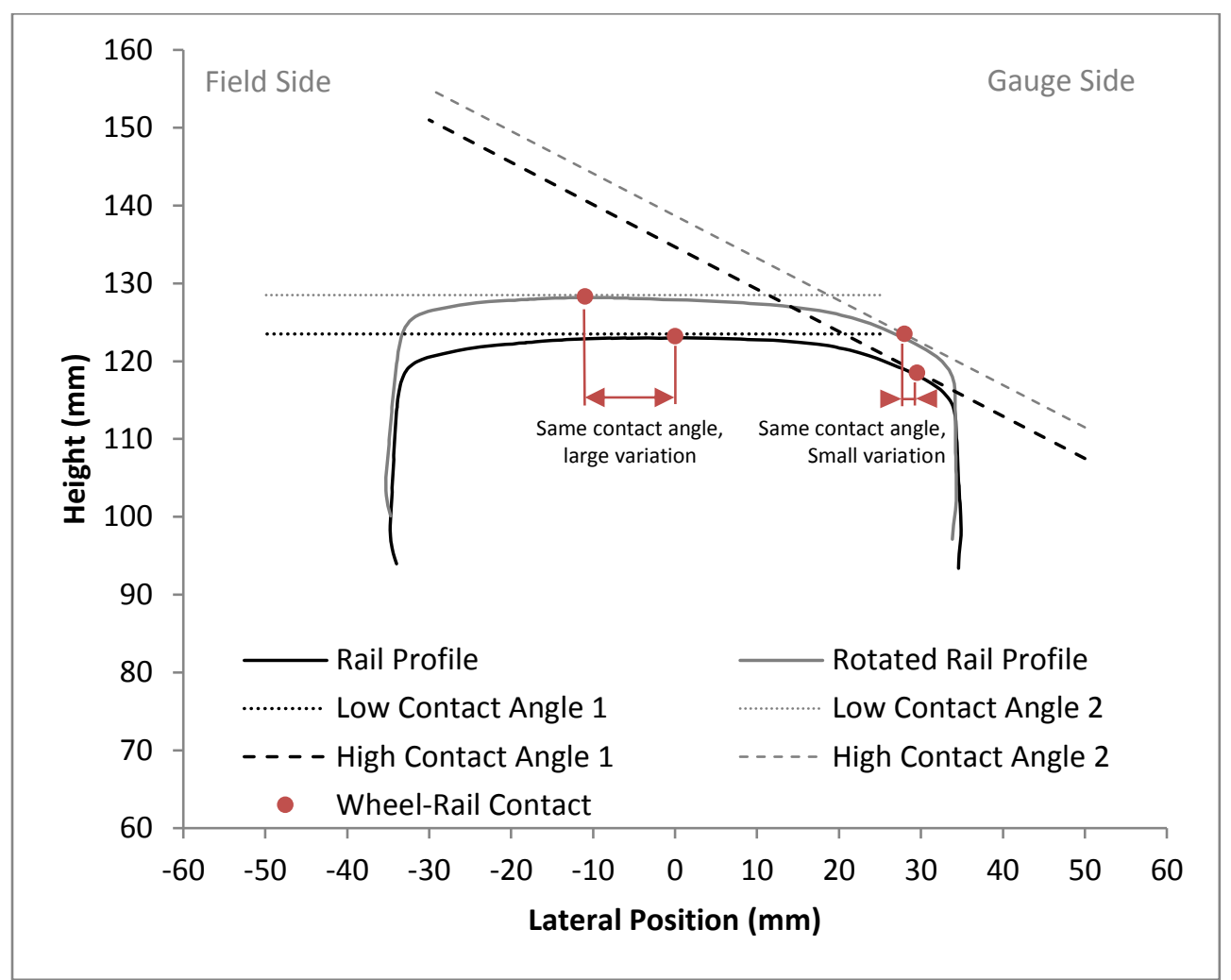

Figure 64: Sensitivity of the lateral contact position on the top of the railhead

The validation test examines the lateral contact position sensitivity analysis qualitatively and the mean lateral contact position from average rail segment statistics quantitatively. It is expected that the lateral contact position for the 1:20 wheel slope will have a larger standard deviation than the 1:3.9 wheel slope.

The validation shows that 71 of the 72 rail segments $(98.6 \%)$ follow the expected results. The following is an example of the validation process for the track segment NB-14 where the algorithm output for both rail segments follow the expected result. The lateral contact point sensitivity analysis in Figure 54 shows a significantly wider distribution of lateral contact position points for the 1:20 wheel slope compared to the 1:3.9 wheel slope for both the left and right rails. The standard deviations of the lateral contact point in Table 
13 show that the 1:20 wheel slope has a larger standard deviation for both the left and right rail segments than the 1:3.9 wheel slope.

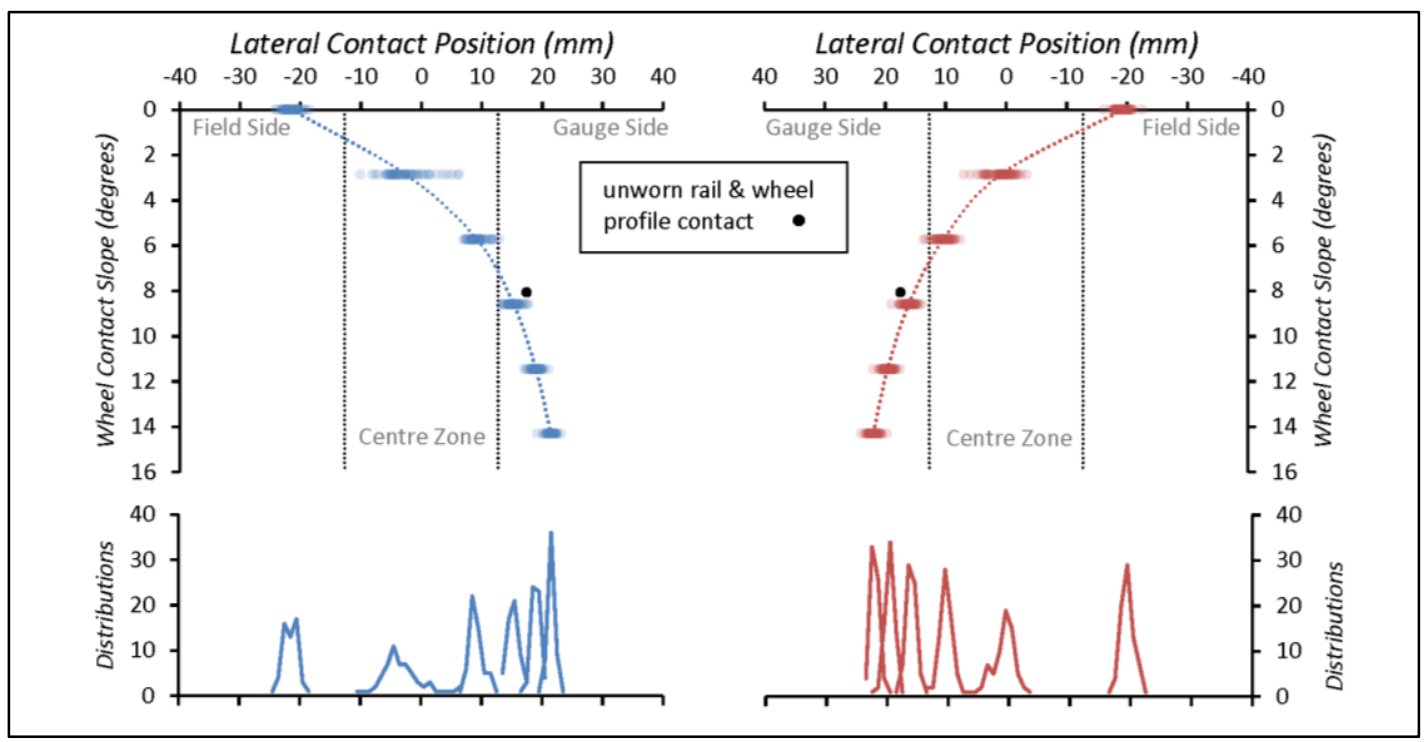

Figure 65: NB-14 left (blue) and right (red) lateral contact point sensitivity analysis

Table 16: NB-14 standard deviation of the lateral contact position

\begin{tabular}{cccccc}
\hline \multirow{2}{*}{ Segment } & \multicolumn{2}{c}{ Wheel Slope } & & \multicolumn{2}{c}{$\begin{array}{c}\text { Standard Deviation of } \\
\text { Contact Position }(\mathrm{mm})\end{array}$} \\
\cline { 2 - 3 } \cline { 5 - 6 } & Gradient & Degrees & & Left Rail & Right Rail \\
\hline NB-14 & flat & $0^{\circ}$ & & 3.5 & 2.0 \\
NB-14 & $1: 3.9$ & $14.31^{\circ}$ & & 0.7 & 0.7 \\
\hline
\end{tabular}

The following is the validation process for the only rail segment, the right rail segment of NB-60, where the algorithm output did not follow the expected result. The lateral contact position sensitivity analysis in Figure 66 shows the right rail segment having approximately identical standard deviations. The rail profiles in Figure 67 show that the rate of change across the railhead for the rail profiles does not significantly increase, while the gauge corner remains relatively unworn, supporting the similar standard deviations observed in the sensitivity analysis. The lateral contact point standard deviations for the right rail in Table 17 show that the 1:3.9 wheel slope produces a larger 
variation, but not significantly larger than the 1:20 wheel slope. The lateral contact point standard deviations for the left rail segment has similar results as the right rail segment; however, the 1:20 wheel slope is slightly larger than the 1:3.9 wheel slope. Based on these results, this type of profile shape results in comparable standard deviations for the different wheel slopes, with the chance of the larger standard deviation of any particular wheel slope likely due to minute variations in the rail profile shape.

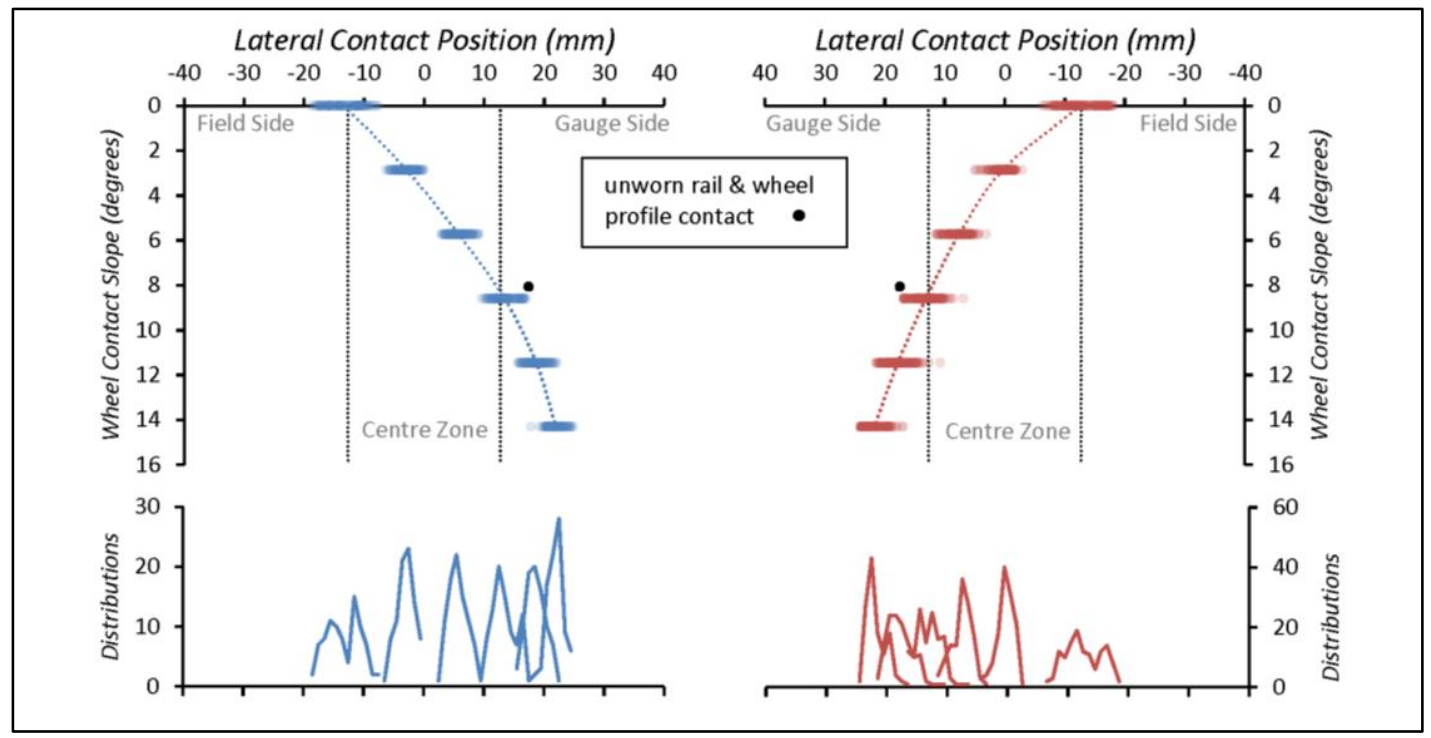

Figure 66: NB-60 left (blue) and right (red) lateral contact position sensitivity analysis

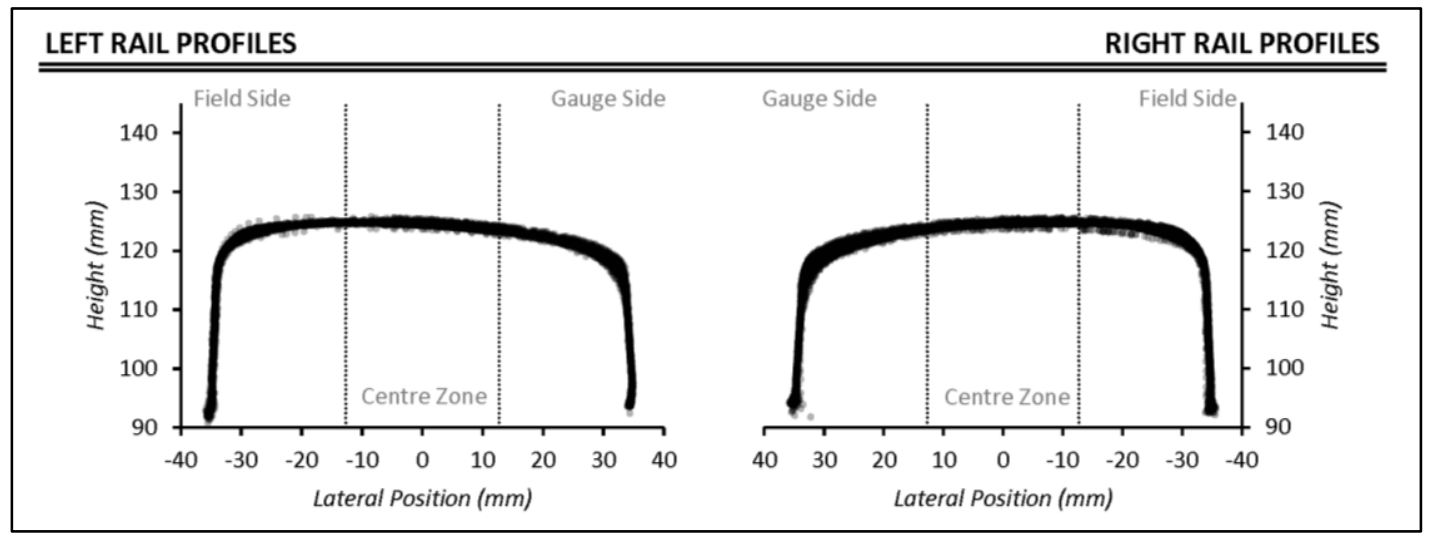

Figure 67: NB-60 rail profiles 
Table 17: NB-60 standard deviation of the lateral contact position

\begin{tabular}{cccccc}
\hline \multirow{2}{*}{ Segment } & \multicolumn{2}{c}{ Wheel Slope } & & \multicolumn{2}{c}{$\begin{array}{c}\text { Standard Deviation of } \\
\text { Contact Position }(\mathrm{mm})\end{array}$} \\
\cline { 2 - 3 } \cline { 5 - 6 } & Gradient & Degrees & & Left Rail & Right Rail \\
\hline NB-60 & flat & $0^{\circ}$ & & 1.5 & 1.4 \\
NB-60 & $1: 3.9$ & $14.31^{\circ}$ & & 1.3 & 1.6 \\
\hline
\end{tabular}

A clear majority of the rail segments follow the expect result. The one rail segment that was an exception was due to the unique wear pattern on top of the rail profile. Based on these findings, the algorithm is considered valid for this test.

\subsubsection{Summary of Validation}

The algorithm is validated by analyzing the reasonableness of the algorithm outputs to expected results using 36 track segments from a North American transit property. Table 18 summarizes the results from the four validation tests. 
Table 18: Summary of algorithm validation

\begin{tabular}{|c|c|c|c|c|c|}
\hline $\begin{array}{c}\text { Validation } \\
\text { Test }\end{array}$ & Expected Result & $\begin{array}{l}\text { Algorithm } \\
\text { Result }\end{array}$ & $\begin{array}{c}\text { Validation } \\
\text { Type }\end{array}$ & $\begin{array}{l}\text { Exceptions } \\
\text { Explanation }\end{array}$ & $\begin{array}{c}\text { Model } \\
\text { Validated? }\end{array}$ \\
\hline \multirow[t]{2}{*}{1} & $\begin{array}{l}\text { Increasing the wheel } \\
\text { slope results in the mean } \\
\text { lateral contact position } \\
\text { shifting towards the } \\
\text { gauge side of the rail } \\
\text { profile. }\end{array}$ & $\begin{array}{l}72 / 72 \text { rail } \\
\text { segments } \\
(100 \%) \text { follow } \\
\text { the expect } \\
\text { result }\end{array}$ & $\begin{array}{c}\text { Qualitative } \\
\text { and } \\
\text { Quantitative }\end{array}$ & - & \multirow[t]{2}{*}{ Yes } \\
\hline & Exceptions: None & & & & \\
\hline \multirow[t]{2}{*}{2} & $\begin{array}{l}\text { A } 1: 20 \text { wheel slope } \\
\text { results in the largest } \\
\text { mean contact radius }\end{array}$ & $\begin{array}{c}68 / 72 \text { rail } \\
\text { segments } \\
(94.4 \%) \text { follow } \\
\text { the expected } \\
\text { result }\end{array}$ & $\begin{array}{l}\text { Qualitative } \\
\text { and } \\
\text { Quantitative }\end{array}$ & $\begin{array}{l}\text { The flat wheel } \\
\text { slope results in } \\
\text { the largest } \\
\text { mean contact } \\
\text { radius, as } \\
\text { predicted }\end{array}$ & \multirow[t]{2}{*}{ Yes } \\
\hline & Exceptions: NB-78 (right $r$ & i), SB-25 (left rail & SB-45 (left rai & ), SB-68 (left rail) & \\
\hline \multirow[t]{4}{*}{3} & $\begin{array}{l}\text { The mean lateral contact } \\
\text { position line will be to } \\
\text { the field side of the } \\
\text { unworn wheel-rail } \\
\text { contact point }\end{array}$ & $\begin{array}{l}\text { 34/34 NB rail } \\
\text { segments } \\
(100 \%) \text { follow } \\
\text { the expected } \\
\text { result }\end{array}$ & Qualitative & - & \multirow[t]{2}{*}{ Yes } \\
\hline & \multicolumn{4}{|l|}{ Exceptions: None } & \\
\hline & $\begin{array}{l}\text { The mean lateral contact } \\
\text { position line will be to } \\
\text { the near or will pass } \\
\text { through the unworn } \\
\text { wheel-rail contact point }\end{array}$ & $\begin{array}{l}36 / 38 \text { SB rail } \\
\text { segments } \\
(94.7 \%) \text { follow } \\
\text { the expected } \\
\text { result }\end{array}$ & Qualitative & $\begin{array}{l}\text { Rail profiles } \\
\text { show more } \\
\text { wear than } \\
\text { typical SB } \\
\text { segment }\end{array}$ & \multirow[t]{2}{*}{ Yes } \\
\hline & \multicolumn{4}{|c|}{ Exceptions: SB-45 (left and right rails) } & \\
\hline \multirow[t]{2}{*}{4} & $\begin{array}{l}\text { A } 1: 20 \text { wheel slope } \\
\text { results in a higher lateral } \\
\text { contact position } \\
\text { variability compared to a } \\
\text { 1:3.9 wheel slope. }\end{array}$ & $\begin{array}{l}71 / 72 \text { rail } \\
\text { segments } \\
(98.6 \%) \text { follow } \\
\text { the expected } \\
\text { results }\end{array}$ & $\begin{array}{c}\text { Qualitative } \\
\text { and } \\
\text { Quantitative }\end{array}$ & $\begin{array}{l}\text { Rail profile } \\
\text { shape shows } \\
\text { consistent rate- } \\
\text { of-change } \\
\text { across railhead }\end{array}$ & \multirow[t]{2}{*}{ Yes } \\
\hline & Exceptions: NB-60 (right $r$ & & & & \\
\hline
\end{tabular}




\section{CONCLUSION AND RECOMMENDATIONS}

This chapter summarizes the key findings from this research and provides recommendations for future research.

\subsection{SUMMARY OF KEY FINDINGS AND LIMITATIONS}

This research develops, verifies, and validates an algorithm to estimate the lateral position of wheel-rail contact and corresponding rail profile radius. The algorithm uses rail profile data measured from an in-service rail segment to estimate the lateral position of wheel-rail contact and corresponding radius of rail profiles along that rail segment.

The following sections discussion key findings, contributions to both rail research and the rail maintenance industry, and research limitations.

\subsubsection{Key Contributions and Findings}

There are three main contributions to rail research and the rail maintenance industry: (1) the development of the lateral contact point and the contact radius performance measures, (2) the methodology to clean and analyze rail profile data, and (3) the systematic approach to analyzing wheel-rail contact with only rail profile information.

The first contribution is the development of the lateral contact point and contact radius rail profile performance measures. Rail maintenance personnel can use these performance measures to (1) numerically quantify and graphically depict wheel-rail contact in short-term application, (2) predict rail conditions using trend analysis in longterm application, and (3) quantify and demonstrate the effectiveness of rail grinding efforts using pre-grind and post-grind rail profile measurements or to predict rail 
conditions using a long-term trend analysis. The development of the lateral contact point and contact radius performance measures contributes to the current effort within the rail maintenance industry to develop tools for the monitoring and management of the wheelrail interface.

The second contribution is the methodologies for the cleaning and analysis of Rangecam rail profile data to calculate the lateral contact point and contact radius, allowing for efficient analysis of bulk rail profile data. Rail maintenance personnel can reasonably apply this methodology to large historical rail profile databases and current rail profile data measurement efforts in an efficient manner bring increased value to rail profile data collection.

The third contribution is the development of a systematic approach to wheel-rail contact using only rail profile measurements and approximating the wheel profile as a straight line. Rail profile data and wheel profile data collection is typically asynchronous; therefore, datasets for wheel profiles might not directly relate temporally to the rail profile data set, or may not exist. Being able to systematically analyze rail profile data without measured wheel profile data increases the value of rail profile measurements.

More specifically, the algorithm passes the two verification tests, ensuring proper execution of the code and the production of consistent and accurate results. The verification process involved comparing the algorithm output to expected results or known values:

- The algorithm output shows zero variation for both the lateral contact position and the contact radius when processing fictional rail segments composed of identical rail profiles, as expected. 
- The algorithm calculates a contact radius of $355.8 \mathrm{~mm}$ at $x=0$ when processing an unworn 136-lb rail with a contact radius of $355.6 \mathrm{~mm}$ (14") at $x=0$; an error of $0.062 \%$ relative to the known value. The error is likely a function of the resampling of $x, y$-coordinates, rounding within MATLAB, and measurement tolerances in the rail profile data for the 136 -lb rail. Taking all the possible sources of error into consideration (e.g., such as rail measurement tolerances) the total error of $0.062 \%$ is acceptable for this application.

Finally, the research validates the algorithm by analyzing the reasonableness of the algorithm output when compared to a series of expected results; the algorithm passes all validation tests. Specifically, the algorithm analyzed 36 track segments from a North American rail transit property under six wheel slope contact conditions ranging from flat $\left(0^{\circ}\right)$ to $1: 3.9\left(14.31^{\circ}\right)$. The algorithm output is combined into a sensitivity analysis that shows the sensitivity of the lateral contact position and contact radius to changes in wheel slope. The algorithm output and the resulting sensitivity analysis is then evaluated against the following four expected results:

1. Increasing the wheel slope results in the mean lateral contact position shifting towards the gauge side of the rail profile.

2. A 1:20 wheel slope results in the largest mean contact radius.

3. The mean lateral contact position line will be to the field side of the unworn wheel-rail contact point for the northbound segments and the mean lateral contact position line will be near or will pass through the unworn wheel-rail contact point for the southbound segments. 
4. A 1:20 wheel slope results in a higher lateral contact position variability compared to a 1:3.9 wheel slope.

The algorithm output closely follows the expect results, with $100 \%, 94.4 \%, 94.4 \%$, and $98.6 \%$ of rail segments passing each of the four validation tests, respectively. This research finds the algorithm valid based on the reasonableness of the results when compared to the four expected results.

\subsubsection{Limitations}

There are five research limitations identified throughout the development, verification, and validation of the algorithm:

- the inclusion of only tangent segments in the validation;

- the simplification of the wheel profile to a straight line;

- $\quad$ validation with a lack of independent ground truth data;

- limited rail transit property maintenance information; and

- the applicability of the algorithm using other rail profile data sources and future implementation of the algorithm.

\subsubsection{Inclusion of only Tangent Segments in the Validation}

The validation of the algorithm includes only tangent segments of track, as the algorithm functions are not optimized to handle the complexities of the wheel-rail contact during curving. The validation of the algorithm applied contact angles from flat $\left(0^{\circ}\right)$ to $1: 3.9$ $\left(14.31^{\circ}\right)$. The algorithm has not been validated for contact angles outside this range, such as those often observed when curving (see Figure 68). 


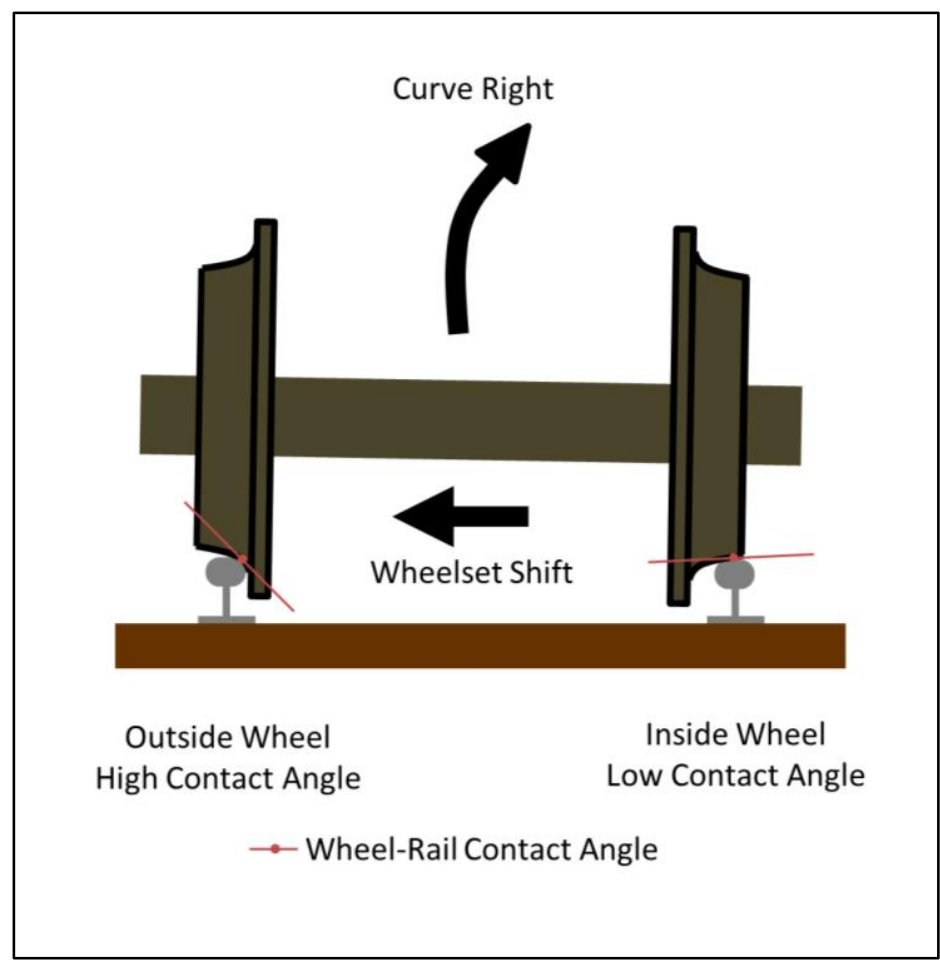

Figure 68: Wheelset shift and contact angles during curve segments

One of the factors that contributes to the complexity of wheel-rail contact in curve and spiral track segments is the dynamic shift of the wheelset laterally while traversing the track. This research assumes the wheelset remains static relative to track level in tangent track; however, even on tangent track the wheelset will still shift lateral and oscillate on the track to compensate for lateral forces. The research assumes a static lateral positioning of the wheelset along the length of a segment for tangent segments but not curves since the wheel-rail contact dynamics on tangent track is significantly less than when curving.

Currently, the accuracy of the algorithm output for curve track segments is uncertain. The algorithm requires further validation and possibly further development to ensure accurate output. 


\subsubsection{The Simplification of the Wheel Profile to a Straight Line}

This research models the wheel profile as a straight line to simplify the theoretical and computational complexities of wheel-rail contact. By modeling the wheel profile as a straight line, the algorithm can run without measured wheel profiles while still providing a wheel-rail system based approach in the analysis. However, the simplification of the wheel profile to a straight line is a limitation since most wheel profiles are not linear, due to wearing processes or specified design. This limitation is compensated for by assuming six wheel slopes in the validation. The six wheel slopes represent different locations of the transit specified wheel profile of the same wheel slope where the rail may contact. Using a cubic spline to connect the data point allows for inferences of the mean lateral contact position or contact radius for a particular wheel slope between the six wheel slopes. Figure 50 in Section 4.2.1, Analysis of Track Segments, illustrates the approximate locations of the six wheel slope on the specified transit wheel profile.

\subsubsection{Lack of Independent Ground Truth Data}

This research cannot establish a comparison between algorithm output and ground truth measurements due to a lack of independent ground truth data, restricting the validation to a comparison of the algorithm output to expect results. The verification of the algorithm compares algorithm output to unworn rail profiles with a known contact location and corresponding contact radius; however, this verification does not validate the algorithm for analyzing worn profiles of unknown and varying lateral contact location and contact radii.

The lack of ground truth data also affects function development; namely, the rail profile smoothing and contact radius calculation functions. Both functions apply the best methodology based on a comparison of alternatives; however, not knowing the true rail 
profile shape makes it difficult to judge the effectiveness of an alternative to accurately represent the rail profile shape.

As the industry continues to automate data collection and analysis, the current lack of independent ground truth rail profile data is to be expected when considering the equipment needed and time required obtaining ground truth data to match the optical measured data.

\subsubsection{Limited Rail Transit Property Maintenance Information}

The lack of availability of maintenance information for the rail transit property limits the scope of the validation and the types of conclusions the validation can make. Information on the rail maintenance activities is from a rail maintenance consulting company; there are no readily available records from the rail transit property or the companies who performed the maintenance. With additional maintenance information, there is potential for increased confidence in the validation results and expansion of the validation.

The following highlight two areas of the research affected by the limited rail maintenance information:

- The research validation does not include trend analysis as the collection of maintenance information (e.g., rail profile measurements, grind summaries, rail replacement schedules) for multiple years are incomplete.

- Findings about the general rail profile wear characteristics for the northbound and southbound lines are based on observations of the rail profile graphs in the sensitivity analysis and discussion with rail maintenance consultants related to the project and are not record in any transit property maintenance documents. 


\subsubsection{The Applicability of the Algorithm Using Other Rail Profile Data Sources and Future Implementation}

This research designs the algorithm around the input of rail profile data output from the proprietary Rangecam software suite. Consequently, the use of rail profile data exported from a different software may diminish the applicability of the algorithm. Nevertheless, the rail industry can use the main concepts this research develops to assist in rail maintenance practices.

The types of software used as well as the concerns of input data quality and type largely affect the future implementation of the algorithm. Currently, a user exports the rail profile data out of Rangecam and the algorithm imports it into a MATLAB environment. Further work is required to develop a more streamlined application with additional capabilities for industry use.

\subsection{RECOMMENDATIONS FOR FUTURE RESEARCH AND WORK}

This research identifies the need for future research to

- investigate the relationships of the lateral contact position and the contact radius with other rail performance measures, such as average rail profile, GQI, gauge wear, vertical wear, to provide a more comprehensive, quantifiable understanding of rail wear conditions;

- further validate the algorithm and test the rail profile smoothing and contact radius calculation methodologies if independent ground truth data becomes available to increase the confidence in the algorithm output; 
- further validate the algorithm using multiple data collection runs to form a trend analysis if additional maintenance records for the transit property become available;

- adapt the algorithm to be able to input and analyze segments using non-linear wheel profiles;

- investigate wheelset shift in tangent track segments and if necessary, revise and re-validate the algorithm as this research only validates the algorithm for tangent track segments with an assumed static wheelset;

- test, revise (if necessary), and validate the algorithm for curved track segments, with a focus on understanding and incorporating the lateral shift of the wheelset (i.e., wheelset shift) relative to the track; and

- develop a more streamlined application of the algorithm that can be integrated into current rail maintenance monitoring programs and commercially available software.

\subsection{CONCLUSION}

In conclusion, this research develops, verifies, and validates an algorithm to estimate the lateral position of wheel-rail contact and corresponding rail profile radius using measured rail profile data from a transit property. This work directly builds on the past work by Hornaday $(2006,2010)$ and Abadpour \& Alfa (2007), both of which focused on the concept of the top-of-rail measurement. The completion of this research contributes to the current effort within the rail maintenance industry to develop tools for the monitoring and management of the wheel-rail interface. Incorporating the algorithm as part of a 
comprehensive management strategy for the wheel-rail interface can help improve railroad safety and operational performance proactively in both short-term and long-term applications. 


\section{WORKS CITED}

Abadpour, A., \& Alfa, A. S. (2007). Railroad Profile. University of Manitoba.

Bosso, N., Spiryagin, M., Gugliotta, A., \& Somà, A. (2013). Review of Wheel-Rail Contact Models. In Mechatronic Modeling of Real-Time Wheel-Rail Contact (pp. 5-19). Springer Berlin Heidelberg. https://doi.org/10.1007/978-3-642-36246-0_2

Cambridge Systematics. (2006). Performance Measures and Targets for Transportation Asset Management: (NCHRP Report 551) (No. 551). Washington D.C.: $\begin{array}{llll}\text { Transportation } & \text { Research } & \text { Board. } & \text { Retrieved from }\end{array}$ http://onlinepubs.trb.org/onlinepubs/nchrp/nchrp_rpt_551.pdf

Cannon, D. F., Edel, K.-O., Grassie, S. L., \& Sawley, K. (2003). Rail defects: an overview. Fatigue \& Fracture of Engineering Materials \& Structures, 26(10), 865886. https://doi.org/10.1046/j.1460-2695.2003.00693.x

Chiacchiari, L., \& Loprencipe, G. (2015). Measurement methods and analysis tools for rail irregularities: a case study for urban tram track. Journal of Modern Transportation, 23(2), 137-147. https://doi.org/10.1007/s40534-015-0070-6

de Pater, A. D. (1988). The Geometrical Contact between Track and Wheelset. Vehicle System Dynamics, 17(3), 127-140. https://doi.org/10.1080/00423118808968898 
Grassie, S. L. (2009). Maintenance of the wheel-rail interface. In R. Lewis \& U. Olofsson (Eds.), Wheel-Rail Interface Handbook (pp. 576-607). Woodhead Publishing. Retrieved from https://www.elsevier.com/books/wheel-rail-interfacehandbook/lewis/978-1-84569-412-8

Hornaday, J. (2006, October 18). Practical rail maintenance gains that can be achieved by calculation of the peak lateral position of a rail head's section profile, followed by serial comparisons of derived rail head peak positions in any segment of track. Memorandum.

Hornaday, J. (2010, September 20). A Preliminary Correlation of Rails' Gauge and Vertical Wear Variation in Top-of-Rail Peak Position in a Short Caltrain Tangent Track Section. Memorandum.

Industrial Metrics. (2006, December). Grind Analyst Rangecam 10.1. Industrial Metrics.

Industrial Metrics / Holland LP. (2012, December). Rangecam Office System 12.3. Industrial Metrics / Holland LP.

Iwnicki, S., Björklund, S., \& Enblom, R. (2009). Wheel-rail contact mechanics. In R. Lewis \& U. Olofsson (Eds.), Wheel-Rail Interface Handbook (pp. 58-92). Woodhead Publishing. Retrieved from https://www.elsevier.com/books/wheelrail-interface-handbook/lewis/978-1-84569-412-8 
Kalay, S. (2001). Vehicle/Track Inspection and Condition Monitoring. In J. Leeper \& R. Allen (Eds.), Guidelines to Best Practices for Heavy Haul Railway Operations: Management of the Wheel and Rail Interface (pp. 6.1-6.53). Simmons-Boardman Books.

Lewis, R., \& Olofsson, U. (2009). Basic tribology of the wheel-rail contact. In R. Lewis \& U. Olofsson (Eds.), Wheel-Rail Interface Handbook (pp. 34-57). Woodhead Publishing. Retrieved from https://www.elsevier.com/books/wheel-rail-interfacehandbook/lewis/978-1-84569-412-8

Lunden, R., \& Paulsson, B. (2009). Introduction to wheel-rail interface research. In R. Lewis \& U. Olofsson (Eds.), Wheel-Rail Interface Handbook (pp. 3-33). Woodhead Publishing. Retrieved from https://www.elsevier.com/books/wheelrail-interface-handbook/lewis/978-1-84569-412-8

Magel, E. (2018a, January). Designing Rail Profiles to Reduce Wheel Wear and Promote Steering in New York City Transit Curve. Slideshow Presentation presented at the Transportation Research Board, Washington D.C.

Magel, E. (2018b, January). Techniques for Monitoring Rail Surface Condition. Slideshow Presentation presented at the Transportation Research Board, Washington D.C.

Magel, E., Kalousek, J., \& Caldwell, R. (2005). A numerical simulation of wheel wear. Wear, 258(7), 1245-1254. https://doi.org/10.1016/j.wear.2004.03.033 
MathWorks. (2018, July 10). Polynomial curve fitting - MATLAB polyfit. Retrieved July 10, 2018, from https://www.mathworks.com/help/matlab/ref/polyfit.html

Parida, A., Kumar, U., Galar, D., \& Stenström, C. (2015). Performance measurement and management for maintenance: a literature review. Journal of Quality in Maintenance Engineering, 21(1), 2-33. https://doi.org/10.1108/JQME-10-20130067

Parsons Brinckerhoff, Incorporated. (2012). Track Design Handbook for Light Rail Transit (2nd Edition): (TCRP Report 155). Transportation Research Board. Retrieved from https://app.knovel.com/hotlink/toc/id:kpTDHLRTEL/track-designhandbook/track-design-handbook

Regehr, S. (2016). A repeatable Procedure to Determine a Representative Average Rail Profile (Thesis). University of Manitoba, Winnipeg, MB.

Regehr, S., Grande, G., Regehr, J., \& Bachinsky, G. (2017). Repeatable Procedure for Determining a Representative Average Rail Profile. Transportation Research Record: Journal of the Transportation Research Board, 2607, 1-6. https://doi.org/10.3141/2607-01

Roney, M. (2001). Wheel/Rail Maintenance Processes. In J. Leeper \& R. Allen (Eds.), Guidelines to Best Practices for Heavy Haul Railway Operations: Management of the Wheel and Rail Interface (pp. 7.1-7.58). Simmons-Boardman Books. 
Schupp, G., Weidemann, C., \& Mauer, L. (2004). Modelling the Contact Between Wheel and Rail Within Multibody System Simulation. Vehicle System Dynamics, 41(5), 349-364. https://doi.org/10.1080/00423110412331300326

Shabana, A. A., Zaazaa, K. E., \& Sugiyama, H. (2007). Railroad Vehicle Dynamics: A Computational Approach. CRC Press.

Solomon, B. (2001). Railway Maintenance Equipment. Voyageur Press.

Tournay, H. (2001). The Fundamentals of Vehicle/Track Interaction. In J. Leeper \& R. Allen (Eds.), Guidelines to Best Practices for Heavy Haul Railway Operations: Management of the Wheel and Rail Interface (pp. 2.1-2.71). Simmons-Boardman Books.

Tunna, J. (2009). Managing the wheel-rail interface: the US experience. In R. Lewis \& U. Olofsson (Eds.), Wheel-Rail Interface Handbook (pp. 686-700). Woodhead Publishing. Retrieved from https://www.elsevier.com/books/wheel-rail-interfacehandbook/lewis/978-1-84569-412-8

Vollebregt, E. A. H. (2008). Survey of programs on contact mechanics developed by J.J. Kalker. Vehicle System Dynamics, 46(1-2), 85-92. https://doi.org/10.1080/00423110701586451

VORtech b.v. (n.d.-a). Home. Retrieved August 28, 2018, from https://www.kalkersoftware.org/index.php?mid=home

VORtech b.v. (n.d.-b). Products: contact tables. Retrieved August 28, 2018, from https://www.kalkersoftware.org/index.php?mid=products\&cid=tables 
VORtech b.v. (n.d.-c). Products: fastsim. Retrieved August 28, 2018, from https://www.kalkersoftware.org/index.php?mid=products\&cid=fastsim

VORtech b.v. (n.d.-d). Products: free version. Retrieved August 28, 2018, from https://www.kalkersoftware.org/index.php?mid=products\&cid=free_ver

VORtech b.v. (n.d.-e). Products: overview. Retrieved August 28, 2018, from https://www.kalkersoftware.org/index.php?mid=products\&cid=products

Wu, H. (2006). Effects of wheel and rail profiles on vehicle performance. Vehicle System Dynamics, 44(sup1), 541-550. https://doi.org/10.1080/00423110600875393

Zarembski, A. M., Palese, J. W., \& Euston, T. (2005). Monitoring grinding effectiveness. Railway Track \& Structures; New York, 101(6), 45-48. 


\section{APPENDIX A}

PROCEDURE TO EXPORT RAIL PROFILE DATA FROM

RANGECAM 
Rangecam's bulk export process creates ASCII ttxt files for each rail profile in the subdivision mileage range from the proprietary $\mathrm{HSH}$ file Rangecam uses to store the collected track measurement data. Common computer script applications (e.g., MATLAB) cannot read or have difficulty reading. $\mathrm{HSH}$ files; therefore, exporting to a commonly used .txt file type is a necessary action.

The lateral contact position and contact radius algorithm requires a certain form of rail profile text file. The Rangecam export process allows users to change rail profile export settings that can affect the form of the rail profile text file. The process to export rail profile text files from Rangecam in the correct form for the contact algorithm follows:

1. Open the desired data collection run in Rangecam.

2. From the profile view window, click on the Profile menu. Select Bulk Export. The Bulk Profile Export dialog box that will appear.

3. Select the desired mileage range for the analysis in the Bulk Profile Export dialog box. There are options to export all rail profiles in the run or for the user to enter a start and end mileage.

4. Select Both for the side option in the Bulk Profile Export dialog box; this will export both sides of the track.

5. Select Ascii for the format option in the Bulk Profile Export dialog box; this will export .txt files.

6. Click the O.K. button in the Bulk Profile Export dialog box. A new dialog box will appear. 
7. Select a folder to store the newly created rail profile text files using the current dialog box. Do not specify a file name. Click the OK button. Rangecam will now create the new text files in the selected folder. 


\section{APPENDIX B}

\section{PROCEDURE TO EXPORT THE TRACK SEGMENT INFORMATION FROM RANGECAM}


Prior to running the rail profile text file sorting algorithm, the user must export the track segment information from Rangecam. The track segment information provides the necessary information to create the rail segment folders and sort the rail profiles into those folders.

The process to export the track segment information from Rangecam follows:

1. Open the desired data collection run from Rangecam. The data collection run should be identical to the data collection run selected in Appendix A.

If the data collection run does not include geometry data, the user may select a data collection run that includes geometry data from a different date but on the same subdivision line. The user must ensure the geometry data is still applicable to the rail profile data being analyzed (i.e., no significant changes in track infrastructure or track measurement occurred during that time).

2. From the profile view window, click on the Reports menu. Select Track Segment Report. A dialog box that will appear.

3. Select $O K$ in the current dialog box. The correct options should be already be selected. Export all track segment types (i.e., curves and tangents) even if one type is not needed for the analysis.

4. An error message appears. Note the file path under the Embedded command heading. Despite the error message, Rangecam still exports a track segment report.

5. Open the computer file explorer and navigate to the noted file path from the 
previous step.

6. Open Microsoft Excel.

7. Drag the .xml file named TRACKSEGMENTRPT into the excel window.

8. In the current Excel Open XML dialog box, select the option to open the file as an XML table.

9. Select $O K$ for any of the following dialog boxes.

10. Save the Excel file using the default Excel .xlsx format. The user can choose any file name. 


\section{APPENDIX C}

RAIL PROFILE TEXT FILE SORTING SUBROUTINE 
This appendix provides a general guide for the rail profile text file sorting subroutine. Implementation of the rail profile text file sorting subroutine occurs within the MATLAB environment and alleviates the need to manually review, sort, and revise the rail profile text files to meet a predetermined set of criteria.

The subroutine works by implementing the following steps:

1. The user inputs the following information about the subdivision rail lines:

- Run name (string) is the first part of the Rangecam assigned name to the rail profile text file (i.e., the text file name up to the first underscore). The input string must match the first part of the text file exactly and is casesensitive. In the case of the transit property with a rail profile text file named

TRANSIT LINE_SB_0004_1141_E_26_82,

TRANSIT LINE would be the input string.

- Left rail (string) is the compass direction of the rail that appears on the left side of the screen when the data collection run that corresponds to the track segments for analysis is open in Rangecam. The four options are 'N', 'W', 'S', and 'E'.

- Right rail (string) is the compass direction of the rail that appears on the right side of the screen when the data collection run that corresponds to the track segment is open in Rangecam. The four options are 'N', 'W', 'S', and 'E'. 
- Units (numeric) is the conversion from either miles to feet (input would in 5280 ) or kilometers to metres (input would be 1000).

2. The user sets the include spirals binary variable from which the algorithm will either exclude or include the entry and exit spirals of curves.

3. The user sets the use criteria binary variable. If set to 0 (no), the results from the subroutine will include all track segments. If set to 1 (yes), the results of the subroutine contain only segments that meet all criteria.

Developing a set of criteria for rail segments to meet a minimal threshold for data quality is critical due to the nature of data collection methods and the needs of researchers and practitioners to have representative and reliable data. The rail profile text file sorting subroutine applies a set of criteria to each rail profile text file to sort it into the appropriate rail segment folder or remove the rail profile.

The criteria follow:

- Minimum number of profiles (numeric) is the minimum allowable number of profiles in a rail segment.

- Maximum percent difference of profiles (percentage) is the maximum allowable percent difference between the number of rail profiles in the left and right rail segment.

- Maximum collection interval (numeric) is the maximum allowable average rail profile collection interval for the segment measured in meters per profile or feet per profile. 
- Couple rail segments (binary) criteria affects weather the subroutine results can include single rail segments. If set to 1 (yes), the subroutine will discard a track segment if one or both rail segments fail to meet the all other criteria. If set to $0(\mathrm{no})$, the subroutine will keep a rail segment that passes the profiles in segment criterion even if the other rail segment fails and is rejected.

Expanding the criteria of the subroutine is possible through additional coding. Examples of additional criteria include severity and direction of curve, super elevation, or acquisition rate of rail profiles. The track segment information Excel file or calculations done in MATLAB during execution would provide the data the subroutine uses to apply the criteria.

4. The subroutine prompts the user to locate the track segment information Excel file on the local network. Appendix A describes how to export the track segment information Excel file from Rangecam.

5. Using the track segment information Excel file, the subroutine extracts the list of track segments with the segment type (i.e. tangent or curve number), start and end mileage, degree and direction of curve, and the length of the entry and exit spiral.

6. If the segment type is a curve and include spirals criterion is set to 0 (no), the subroutine recalculates the start mileage $(S M)$ and end mileage (EM) of the track segment using the length of the entry and exit spirals. The following two equations find the updated start mileage $(S M)$ and end mileage $(E M)$. 


$$
\begin{gathered}
S M_{\text {updated }}=S M+(\text { entry spiral length }) \\
E M_{\text {updated }}=E M-(\text { exit spiral length })
\end{gathered}
$$

7. The subroutine creates the names for track segments using the following naming convention. For tangents:

(segment sequence number) Tangent (start mileage)-(end mileage)

$$
\text { e.g., } 4 \text { Tangent } 0.375-1.123
$$

For curves:

(segment sequence number) (curve number) (degree and direction of curve)

(start mileage)-(end mileage)

e.g., $18 \mathrm{C \# 381} 1.2 \mathrm{~L} 3.804-3.984$

The segment sequence number of a track segment starts at one for the segment with the lowest mileage and increases by one for every consecutive track segment. The sequence number properly orders the track segment folders in increasing mileage order within the data collection folder.

8. The subroutine prompts the user to select the folder that contains all the rail profile text files from the subdivision rail line. Appendix B describes the process of exporting the rail profile text files from Rangecam.

9. The subroutine finds the character location of the compass direction (i.e., either $\mathrm{N}, \mathrm{W}, \mathrm{S}, \mathrm{E})$ in the rail profile text file name. The character location of the compass direction is consistent for all rail profile text files in the subdivision. 
10. To determine which segments to remove, the subroutine applies the different criteria to the segments. The process of applying the criteria follow:

a. The subroutine first counts the number of rail profile in every rail segment. This sub-step extracts the mileage location and the rail of the track segment (given by a compass direction) from the file name of each rail profile text file from the export folder (step 8), and matches those values to the mileage range and compass direction of a rail segment. If the include spirals criterion is set to 'no', a rail profile measured in a spiral will not fall within the updated mileage of any rail segment set in step 3 , and the subroutine will skip that rail profile.

b. If use criteria is set to 1 (yes), move to the next step c. If set to 0 , move to step 11.

c. Using the profiles in segment criterion, the subroutine checks if the rail segment meets or exceeds the minimum number of rail profiles criteria. If not, the subroutine flags the rail segment.

d. Using the maximum collection interval criterion, the subroutine checks if the segment meets or is under the maximum collection interval of the rail segment. The subroutine calculates the average collection interval as

$$
\text { average collection interval }=\frac{(E M-S M) *(\text { units })}{(\text { number of profiles in segment })}
$$

Where $E M$ is the end mileage, $S M$ is the start mileage, and units is the conversion from $\mathrm{km}$ to $\mathrm{m}$ or miles to feet. The subroutine flags the rail 
segment if it does not meet the criterion.

e. If the couple rail segments criterion is set to 1 (yes), the subroutine calculates the percent different in rail profile between corresponding rail segments of a track segment, using the equation

$$
\% \text { difference }=\frac{\mid(\text { diffence in number of profiles }) \mid}{\overline{(\text { number of prof lles })}}
$$

where the difference in number of profiles is the difference between the number of profiles in the left and right rail segments and $\overline{\text { number of prof lles }}$ is the mean number of profiles in the left and right rail segments. Using the maximum percent difference criterion, the subroutine flags both rail segments if they do not meet the criterion.

f. If the couple rail segments criterion is set to 1 (yes), the subroutine removes any track segment (i.e., both rail segments) that contains a rail segment that the subroutine has flagged. If set to 0 (no), the subroutine removes any rail segment that is flagged.

11. Based on the remaining segments, the subroutine creates the track segment folders in the Segments folder (located in the current MATLAB folder) and then creates the rail segment folders within each track segment folder. The subroutine names the track segment folders using the track segment names created in step 7 and the rail segment folders using the names "Left Rail" and "Right Rail".

12. To sort the rail profile text files, the subroutine first extracts the mileage location and the rail of the track segment (given by a compass direction) from the file 
name of each rail profile text file from the export folder (step 8). Next, the subroutine attempts to match those values to the mileage range of the remaining track segments folder and the corresponding rail segment folders. If the rail profile text file fits within a rail segment, the subroutine copies the rail profile text file into the rail segment folder using MATLAB's copyfile function. If the rail profile text file does not match, the subroutine ignores the rail profile.

13. To verify if the execution was successful, the subroutine checks if the sum of the number of rail profile text files moved and the number of rail profile text files rejected equals the total number of rail profile text files exported by Rangecam. If the numbers are not equal, the algorithm warns the user an error has occurred during execution.

14. Prior to rerunning the rail profile text file sorting subroutine executing again, the resulting file structure in the Segments folder (located in the current MATLAB folder) must be moved to a different location on the local network (i.e., the segments folder must be empty).

15. If the analysis requires only track segment of a certain track geometry (i.e., only tangent segment or only curve segments), the user manually deletes the other track segment folders in the computer's file explorer.

The resulting file structure containing the rail profile text files is now suitable for lateral contact position and contact radius analysis using the algorithm. 


\section{APPENDIX D}

NORTHBOUND TRACK SEGMENT ANALYSIS AND SENSITIVITY

ANALYSIS RESULTS 
SEGMENT AND ANALYSIS INFORMATION

\begin{tabular}{llll}
\hline \hline Track Segment Name: & NB-4 & Contact Analysis Date: & 20180615 \\
Segment Length $(m):$ & 746.5 & Left Wheel Slope: & $1: 20$ \\
Data Collection Date: & 20150827 & Right Wheel Slope: & $1: 20$
\end{tabular}

RAIL SEGMENT GRAPHS

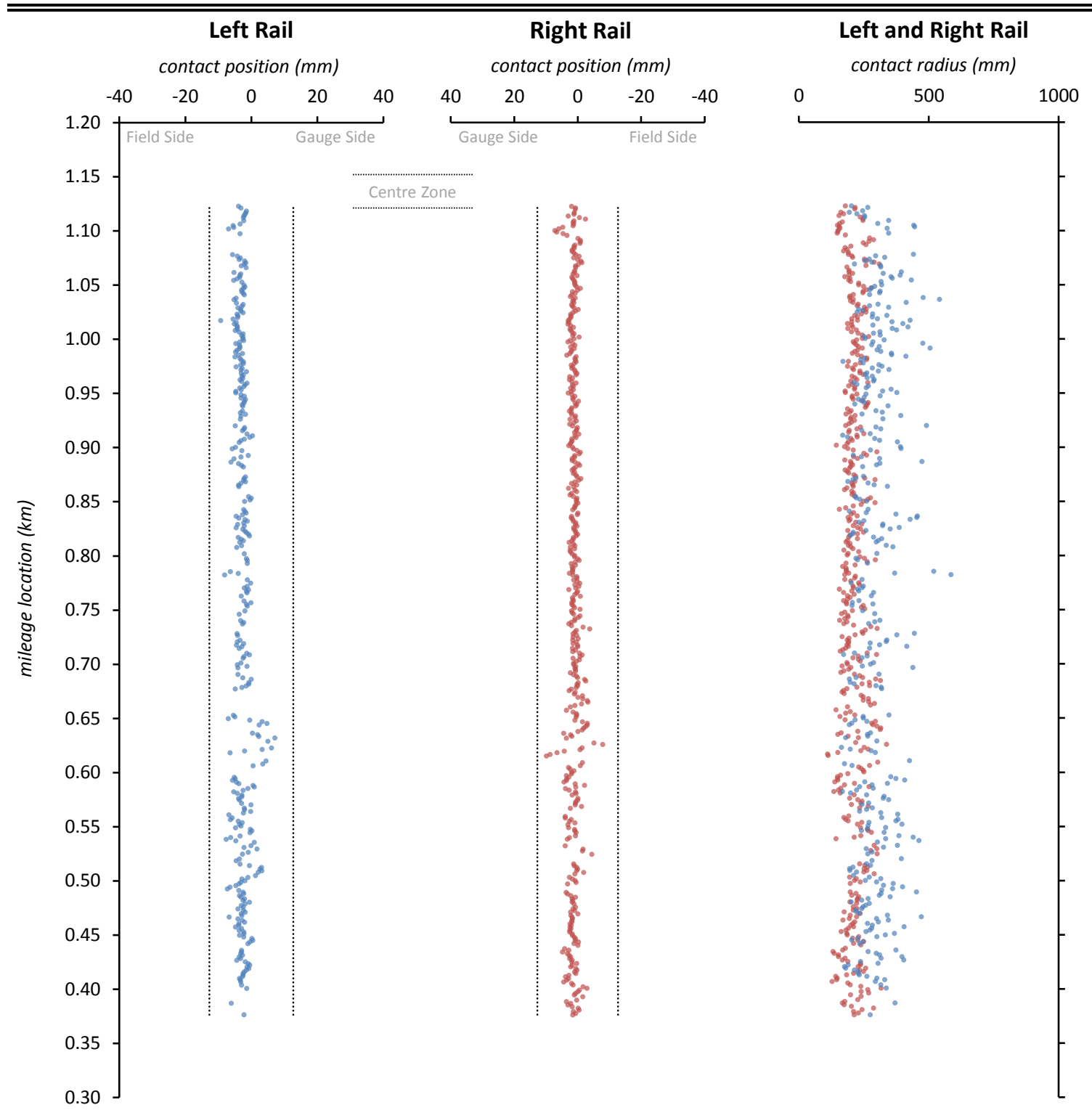

RAIL SEGMENT STATISTICS TABLE

\begin{tabular}{|c|c|c|c|c|}
\hline \multirow{3}{*}{ Statistic } & \multicolumn{4}{|c|}{ Rail Profile Measure } \\
\hline & \multicolumn{2}{|c|}{ Left Rail (344 profiles) } & \multicolumn{2}{|c|}{ Right Rail (457 profiles) } \\
\hline & Contact Position & Contact Radius & Contact Position & Contact Radius \\
\hline Mean $(\mathrm{mm})$ & -2.7 & 295.7 & 1.2 & 213.3 \\
\hline Std. Dev. $(\mathrm{mm})$ & 2.1 & 72.8 & 1.6 & 38.9 \\
\hline$\%$ within Zone & $100 \%$ & - & $100 \%$ & - \\
\hline
\end{tabular}

D-1 
Lateral Contact Position ( $\mathrm{mm}$ )
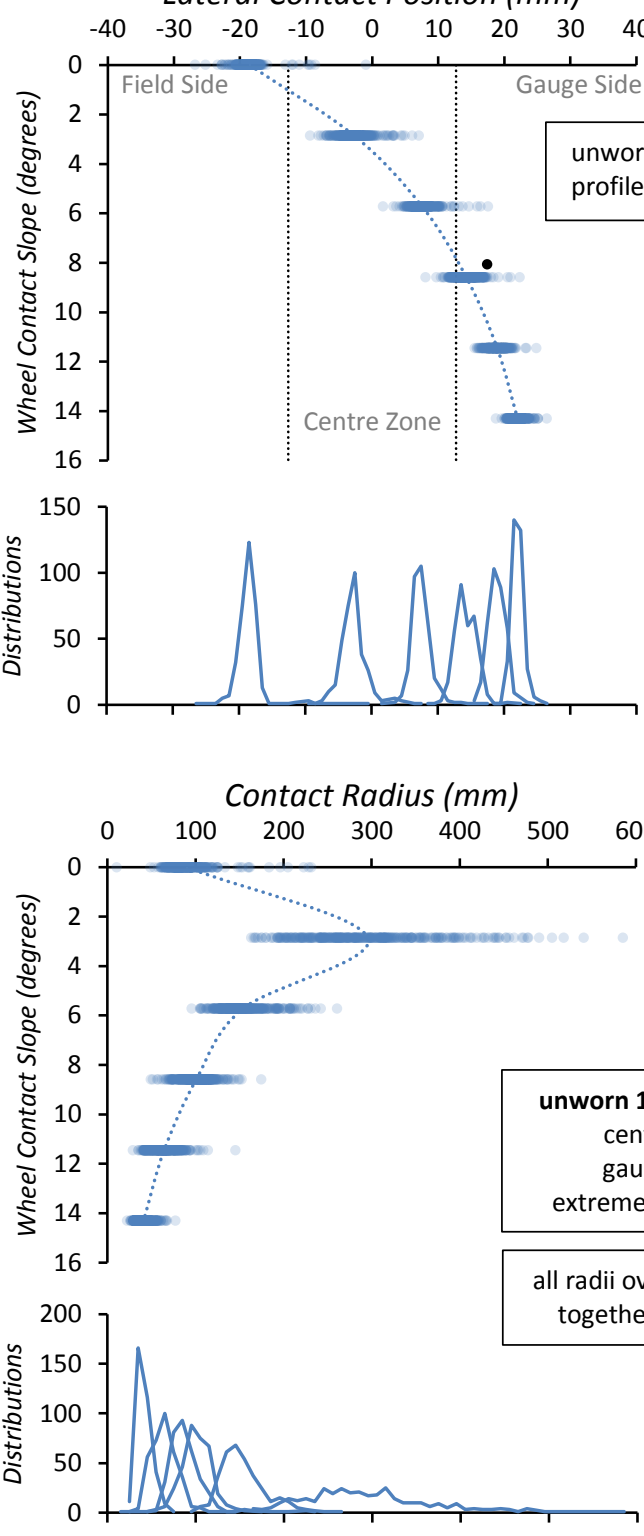

LEFT RAIL PROFILES

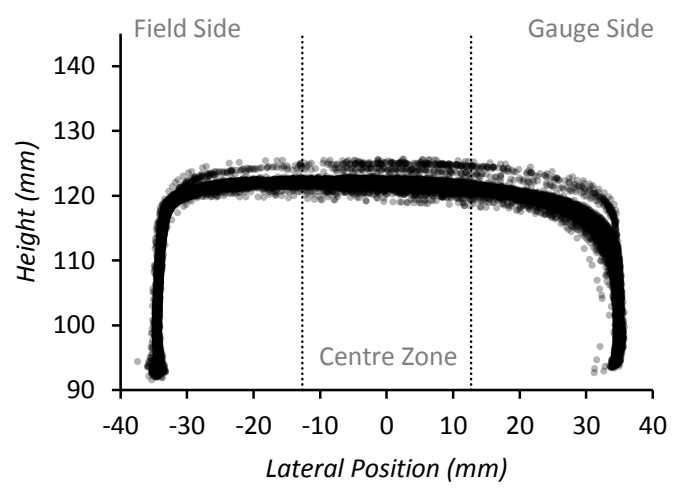

Lateral Contact Position ( $\mathrm{mm}$ )
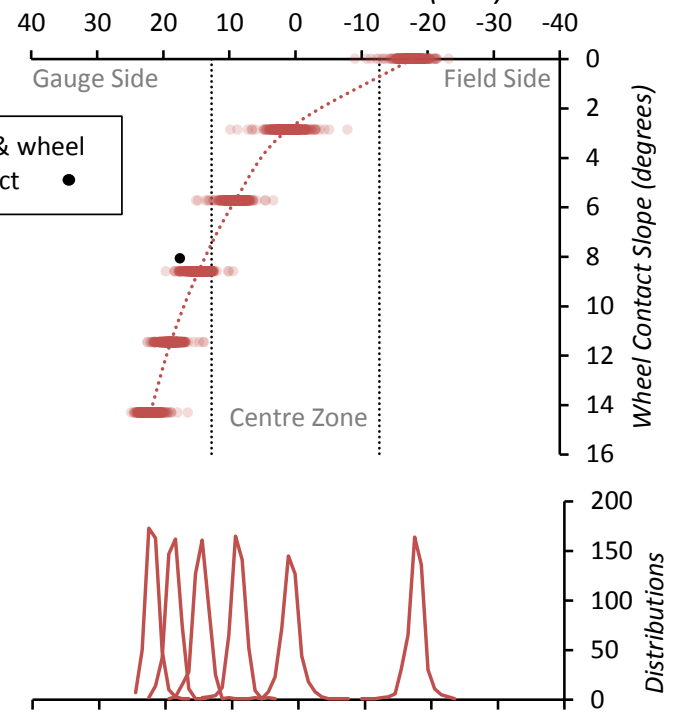

\section{Contact Radius ( $\mathrm{mm}$ )}
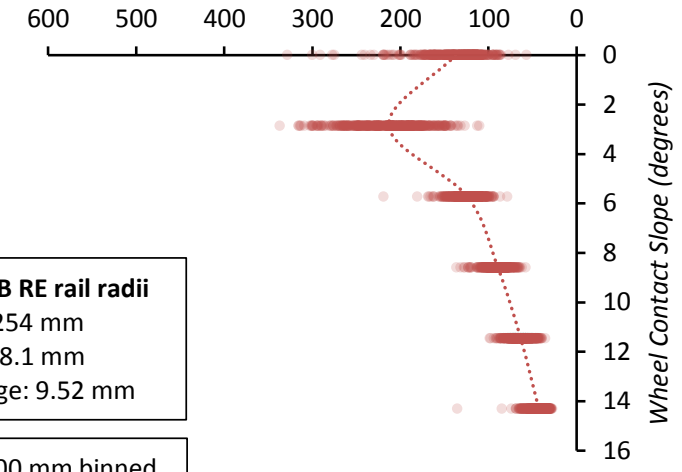

enter: $254 \mathrm{~mm}$

uge: $38.1 \mathrm{~mm}$

reme gauge: $9.52 \mathrm{~mm}$

$600 \mathrm{~mm}$ binne

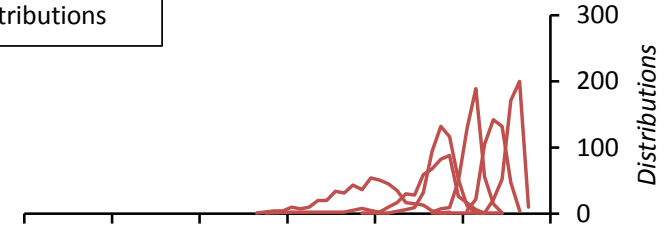

RIGHT RAIL PROFILES

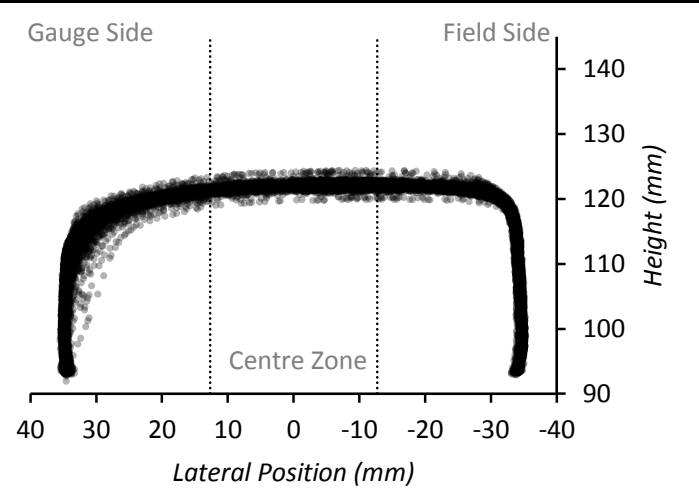

D-2 
SEGMENT AND ANALYSIS INFORMATION

\begin{tabular}{llll}
\hline \hline Track Segment Name: & NB-7 & Contact Analysis Date: & 20180615 \\
Segment Length $(\mathrm{m}):$ & 544.9 & Left Wheel Slope: & $1: 20$ \\
Data Collection Date: & 20150827 & Right Wheel Slope: & $1: 20$
\end{tabular}

RAIL SEGMENT GRAPHS

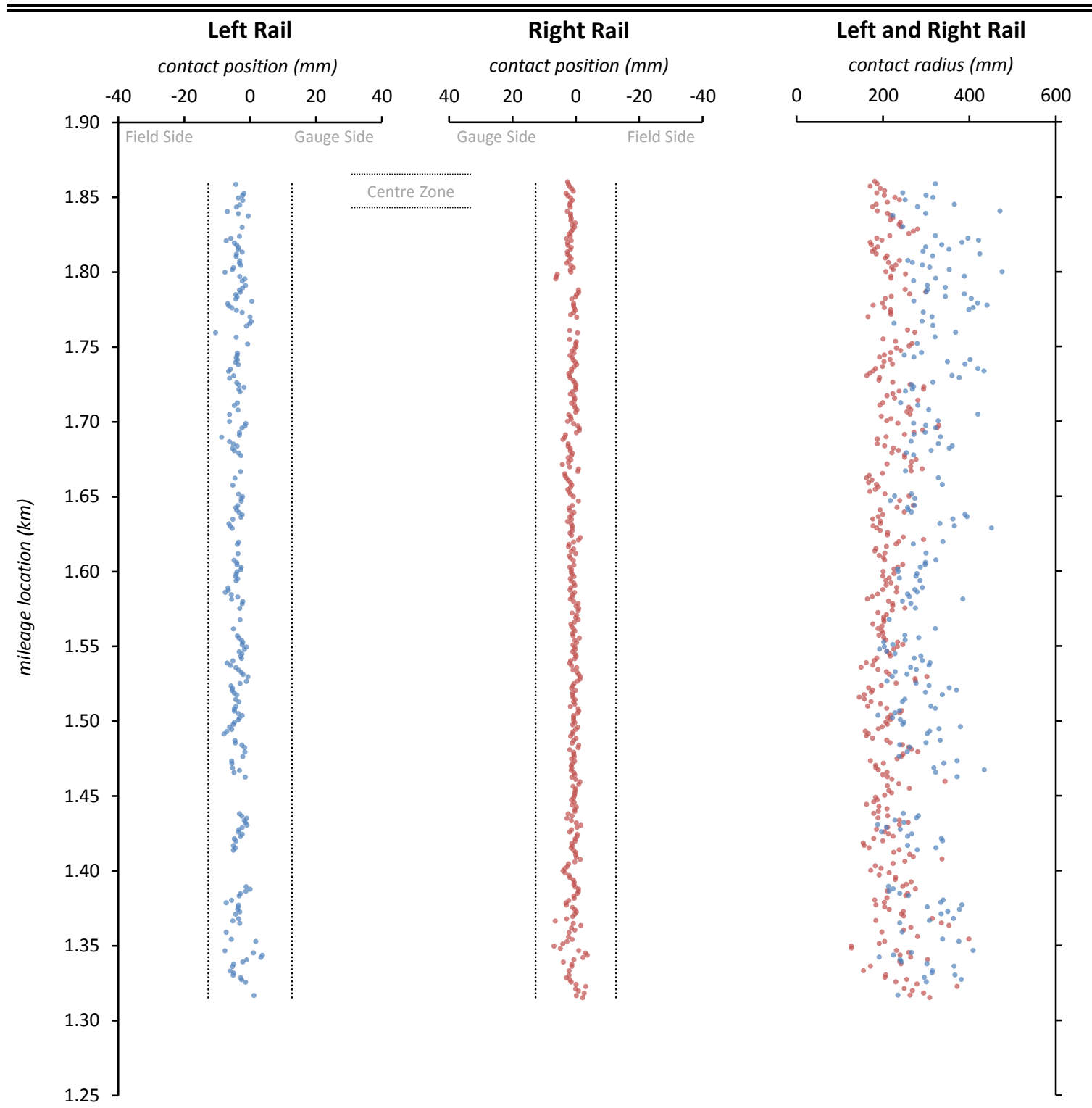

RAIL SEGMENT STATISTICS TABLE

\begin{tabular}{|c|c|c|c|c|}
\hline \multirow{3}{*}{ Statistic } & \multicolumn{4}{|c|}{ Rail Profile Measure } \\
\hline & \multicolumn{2}{|c|}{ Left Rail (226 profiles) } & \multicolumn{2}{|c|}{ Right Rail (340 profiles) } \\
\hline & Contact Position & Contact Radius & Contact Position & Contact Radius \\
\hline Mean $(\mathrm{mm})$ & -3.8 & 299.4 & 1.0 & 218.3 \\
\hline Std. Dev. $(\mathrm{mm})$ & 2.0 & 60.1 & 1.4 & 40.1 \\
\hline$\%$ within Zone & $100 \%$ & - & $100 \%$ & - \\
\hline
\end{tabular}


Lateral Contact Position ( $\mathrm{mm}$ )
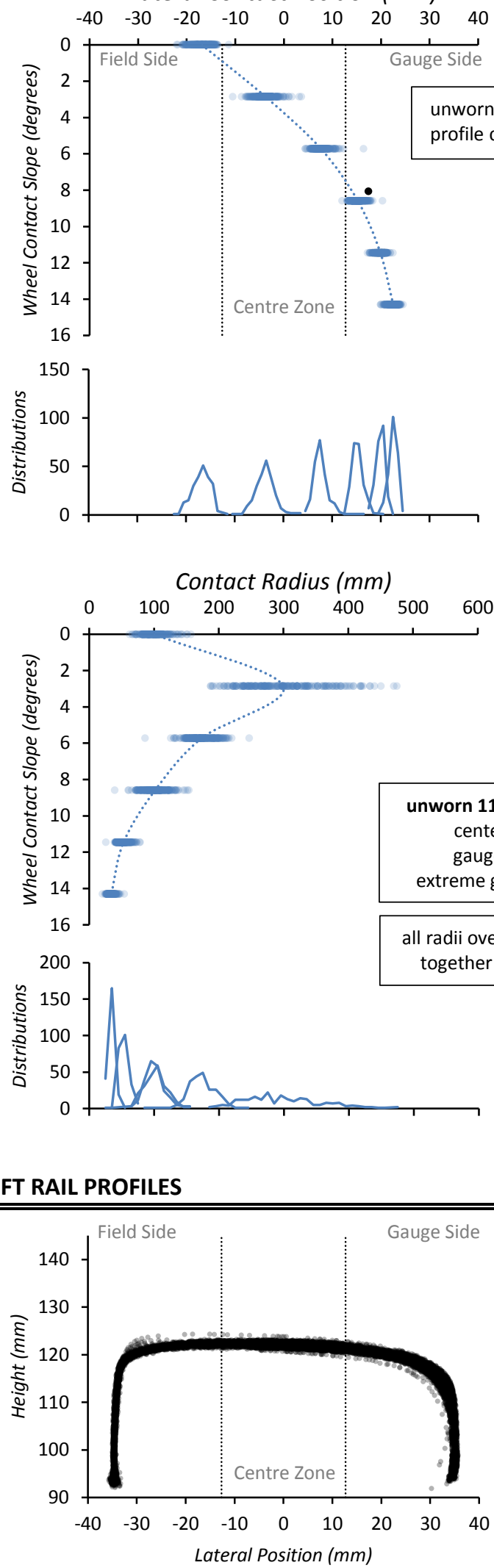

\section{LEFT RAIL PROFILES}

Lateral Contact Position ( $\mathrm{mm}$ )
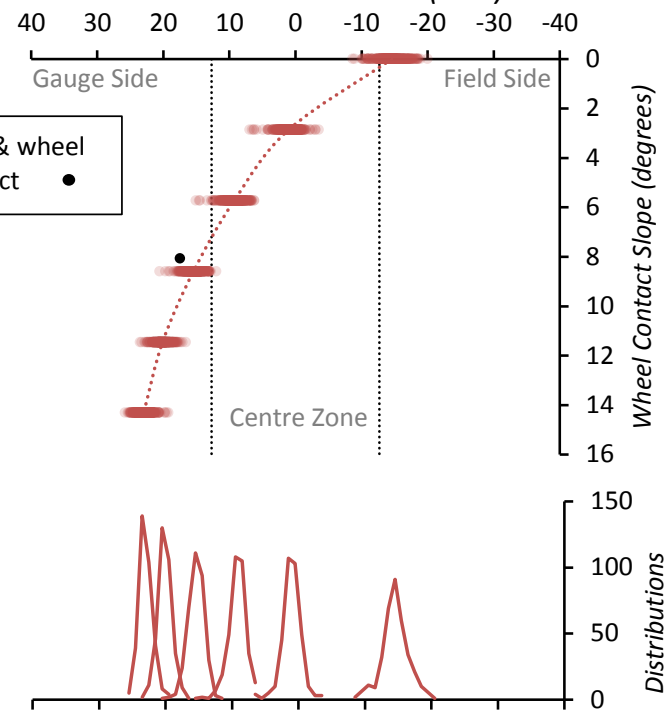

Contact Radius ( $\mathrm{mm}$ )

nter: $254 \mathrm{~mm}$

uge: $38.1 \mathrm{~mm}$

$\begin{array}{llllll}500 & 400 & 300 & 200 & 100 & 0\end{array}$
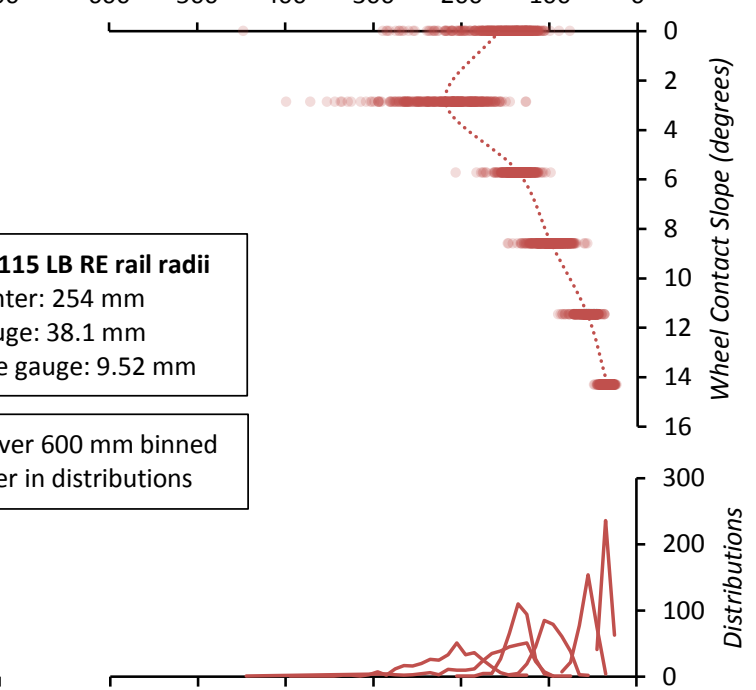

RIGHT RAIL PROFILES

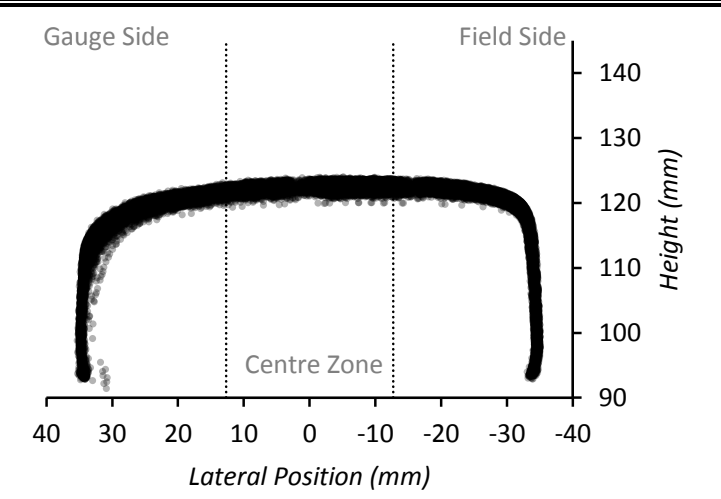

D-4 
SEGMENT AND ANALYSIS INFORMATION

\begin{tabular}{llll}
\hline \hline Track Segment Name: & NB-14 & Contact Analysis Date: & 20180615 \\
Segment Length $(m):$ & 107.0 & Left Wheel Slope: & $1: 20$ \\
Data Collection Date: & 20150827 & Right Wheel Slope: & $1: 20$
\end{tabular}

RAIL SEGMENT GRAPHS

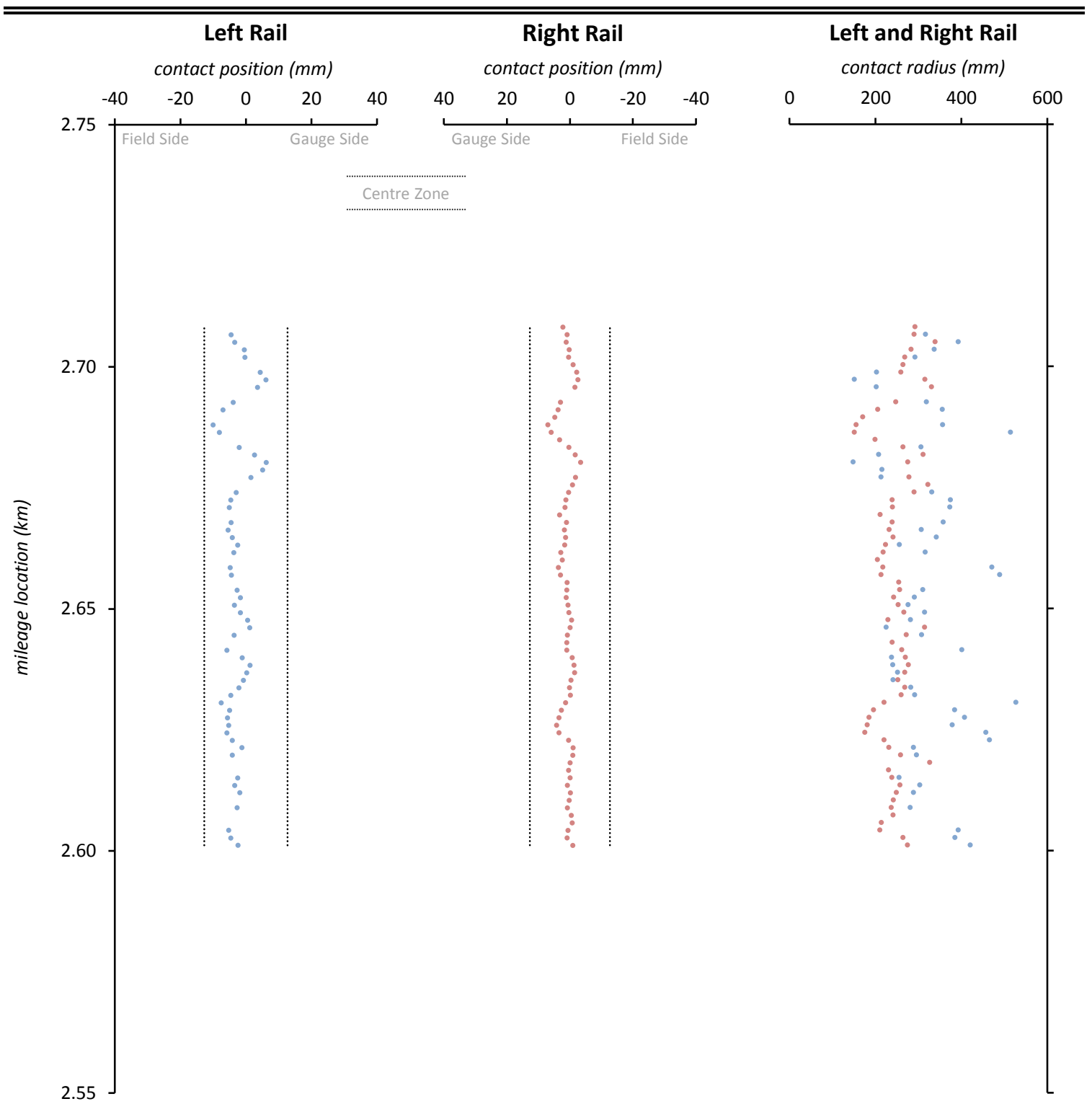

RAIL SEGMENT STATISTICS TABLE

\begin{tabular}{|c|c|c|c|c|}
\hline \multirow{3}{*}{ Statistic } & \multicolumn{4}{|c|}{ Rail Profile Measure } \\
\hline & \multicolumn{2}{|c|}{ Left Rail (55 profiles) } & \multicolumn{2}{|c|}{ Right Rail (68 profiles) } \\
\hline & Contact Position & Contact Radius & Contact Position & Contact Radius \\
\hline Mean $(\mathrm{mm})$ & -2.6 & 320.0 & 0.9 & 247.0 \\
\hline Std. Dev. $(\mathrm{mm})$ & 3.5 & 86.5 & 2.0 & 40.7 \\
\hline$\%$ within Zone & $100 \%$ & - & $100 \%$ & - \\
\hline
\end{tabular}


Lateral Contact Position ( $\mathrm{mm}$ )
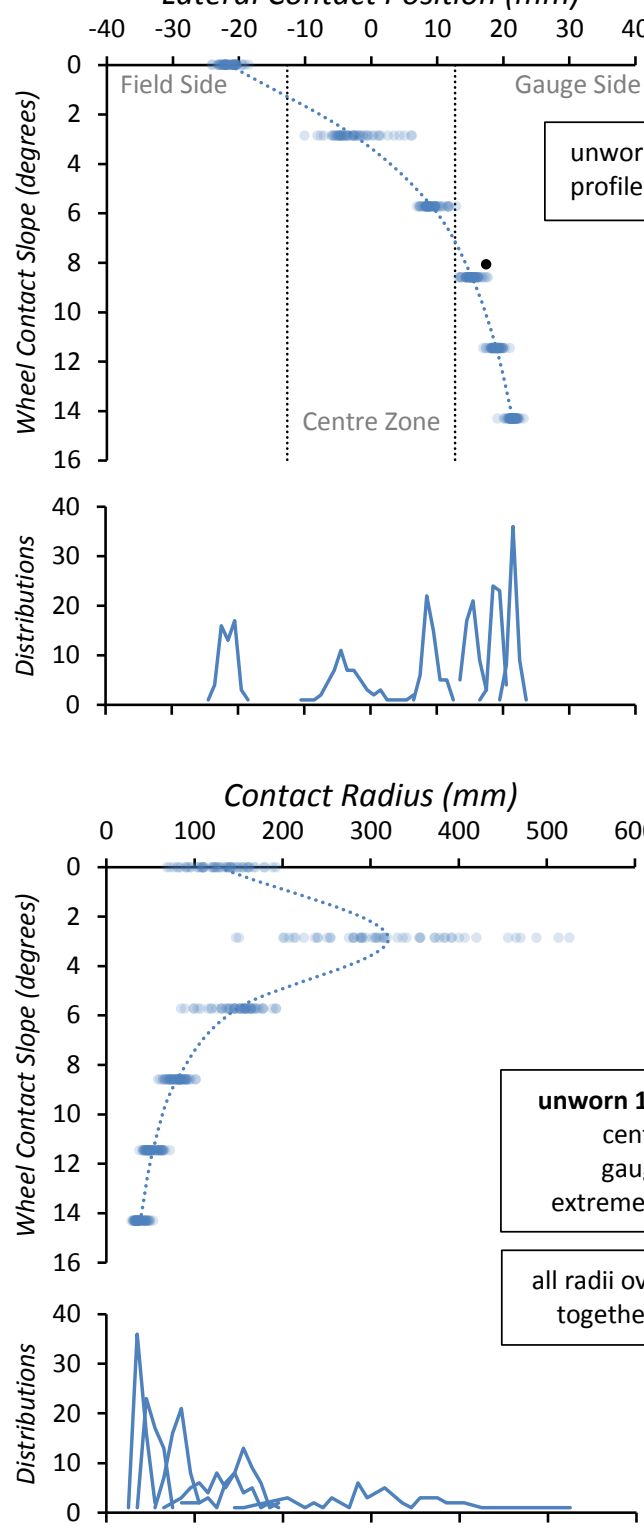

LEFT RAIL PROFILES

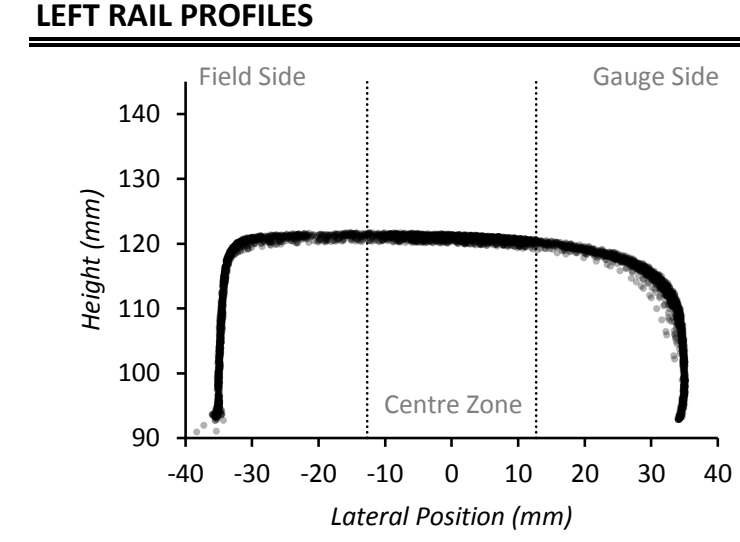

Lateral Contact Position ( $\mathrm{mm}$ )
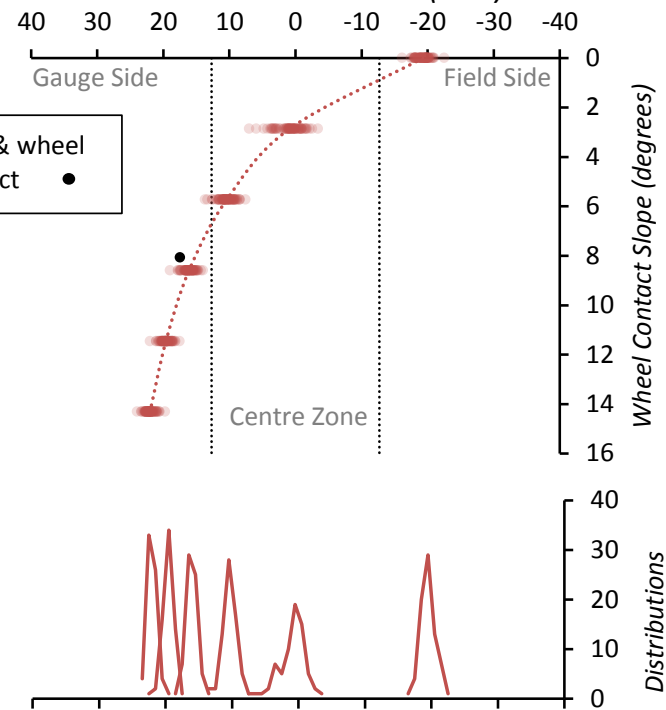

Contact Radius ( $\mathrm{mm}$ )

nter: $254 \mathrm{~mm}$

uge: $38.1 \mathrm{~mm}$

$\begin{array}{llllll}500 & 400 & 300 & 200 & 100 & 0\end{array}$
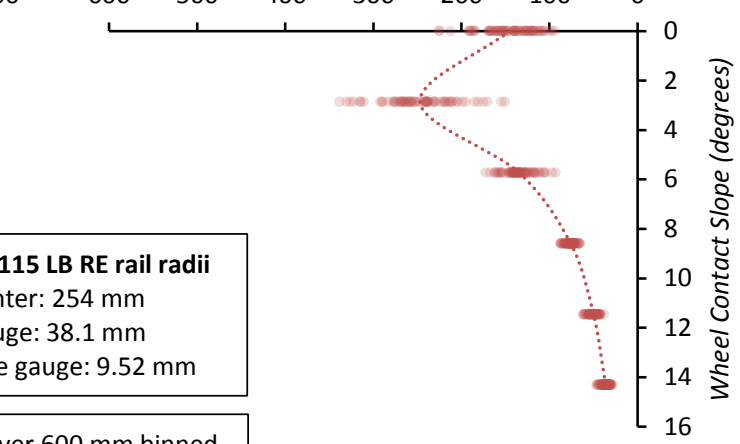

tributions

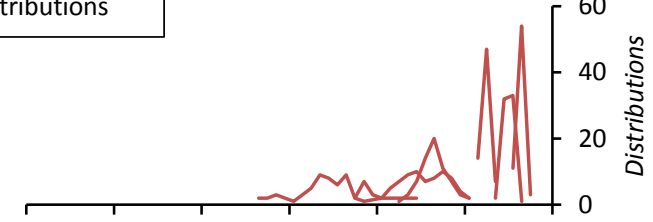

RIGHT RAIL PROFILES

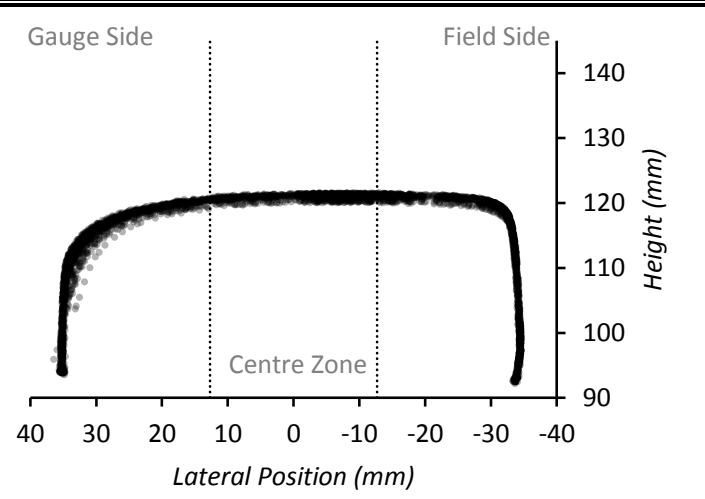

D-6 
SEGMENT AND ANALYSIS INFORMATION

\begin{tabular}{llll}
\hline \hline Track Segment Name: & NB-17 & Contact Analysis Date: & 20180615 \\
Segment Length $(\mathrm{m}):$ & 648.3 & Left Wheel Slope: & $1: 20$ \\
Data Collection Date: & 20150827 & Right Wheel Slope: & $1: 20$
\end{tabular}

RAIL SEGMENT GRAPHS

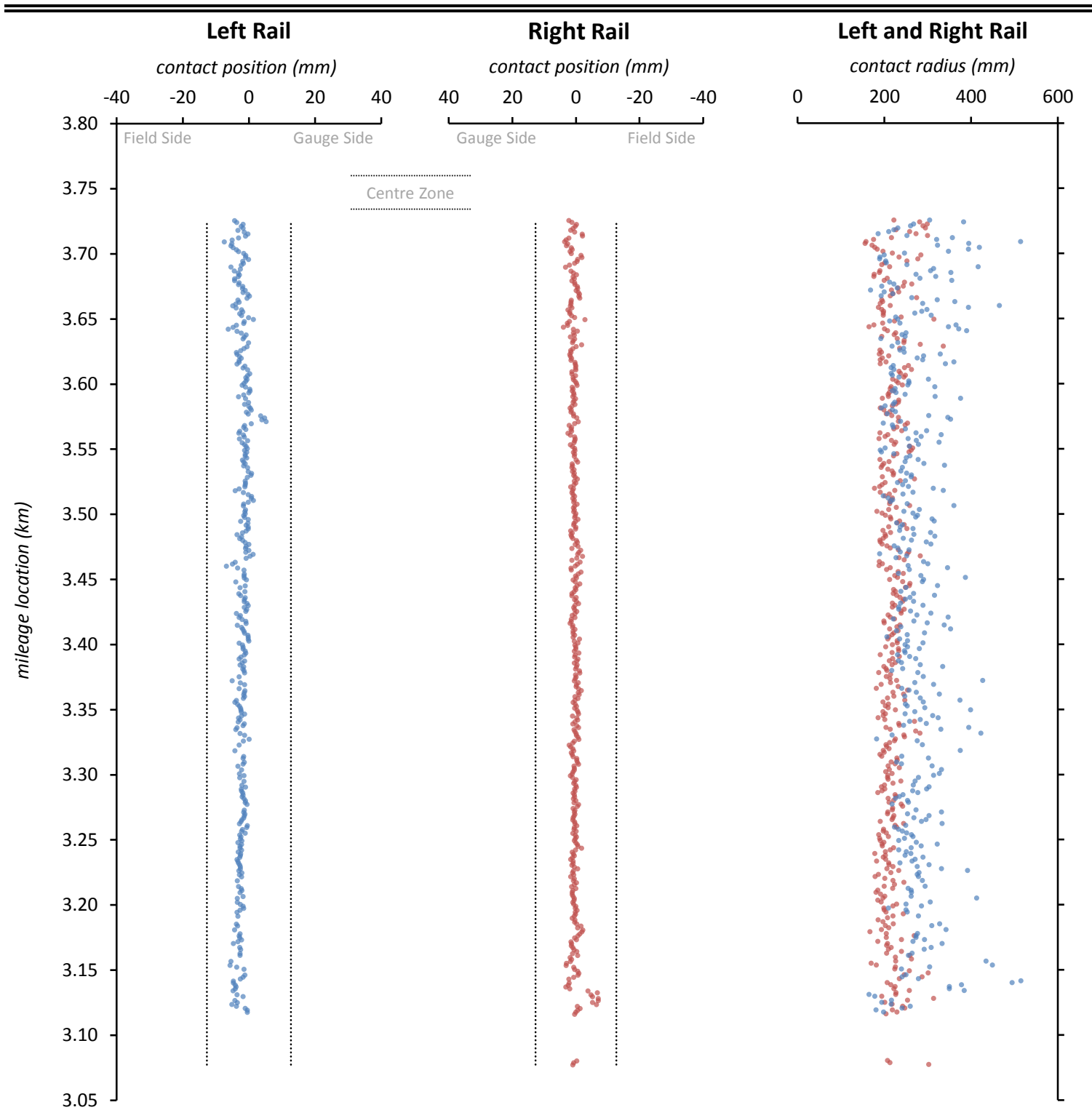

RAIL SEGMENT STATISTICS TABLE

\begin{tabular}{|c|c|c|c|c|}
\hline \multirow{3}{*}{ Statistic } & \multicolumn{4}{|c|}{ Rail Profile Measure } \\
\hline & \multicolumn{2}{|c|}{ Left Rail (361 profiles) } & \multicolumn{2}{|c|}{ Right Rail (409 profiles) } \\
\hline & Contact Position & Contact Radius & Contact Position & Contact Radius \\
\hline Mean $(\mathrm{mm})$ & -2.0 & 274.7 & 0.4 & 218.4 \\
\hline Std. Dev. $(\mathrm{mm})$ & 1.6 & 57.1 & 1.3 & 26.7 \\
\hline$\%$ within Zone & $100 \%$ & - & $100 \%$ & - \\
\hline
\end{tabular}

D-7 
Lateral Contact Position ( $\mathrm{mm}$ )
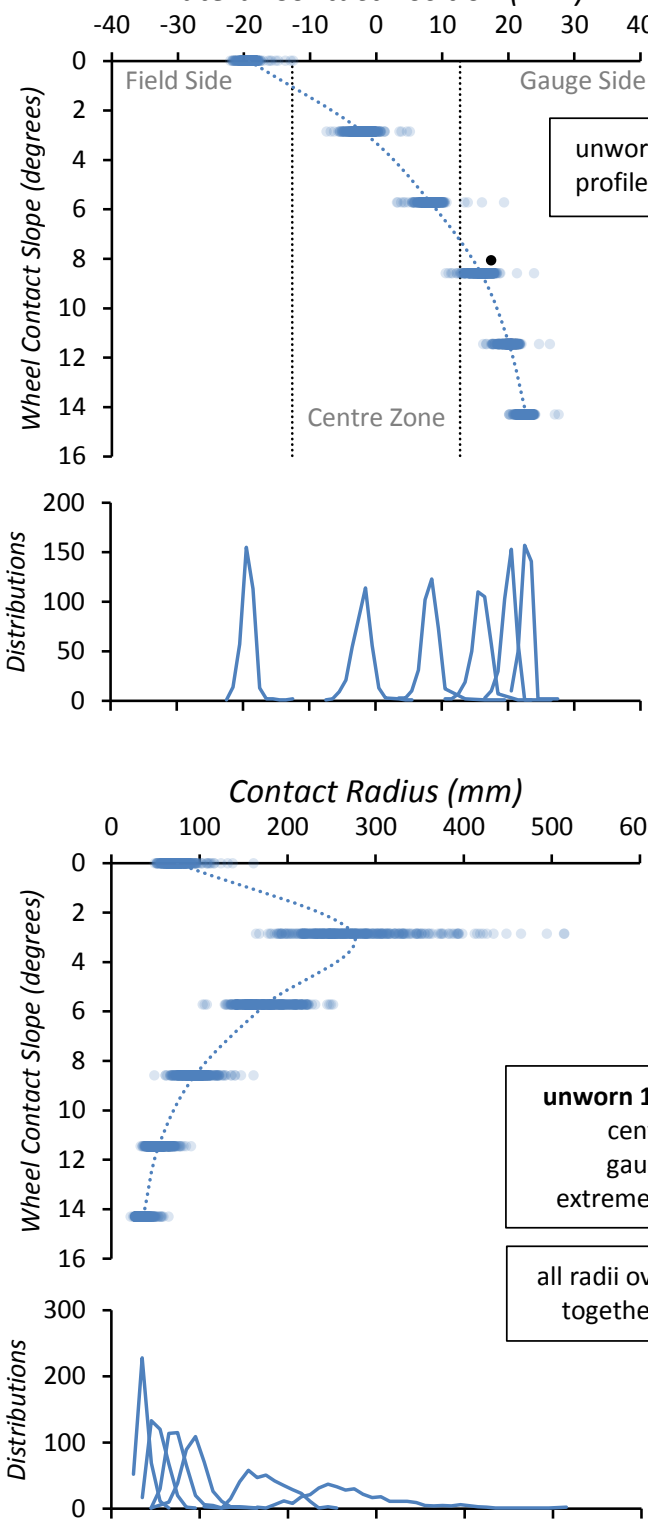

LEFT RAIL PROFILES

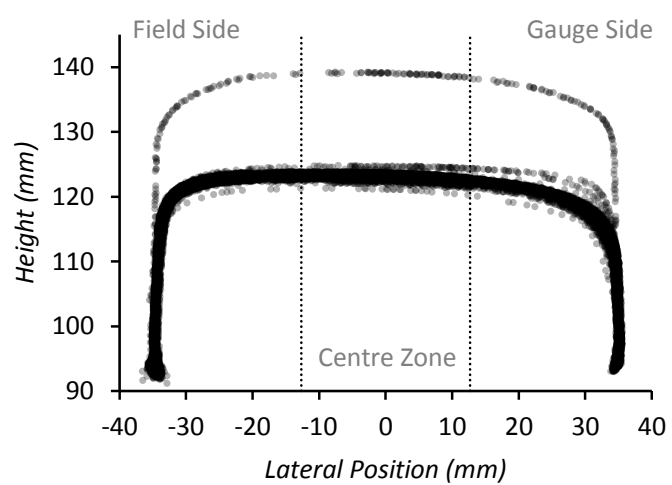

Lateral Contact Position ( $\mathrm{mm}$ )
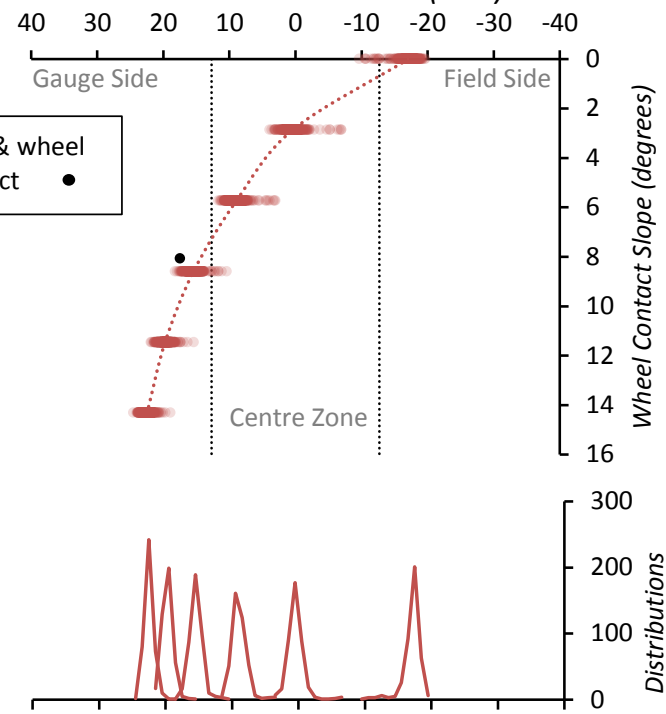

Contact Radius ( $\mathrm{mm}$ )
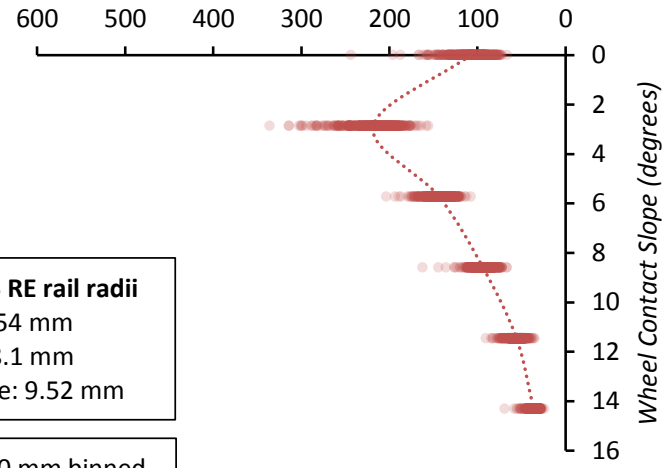

nter: $254 \mathrm{~mm}$

auge: $38.1 \mathrm{~mm}$

eme gauge: $9.52 \mathrm{~mm}$

$\mathrm{mm}$ binned

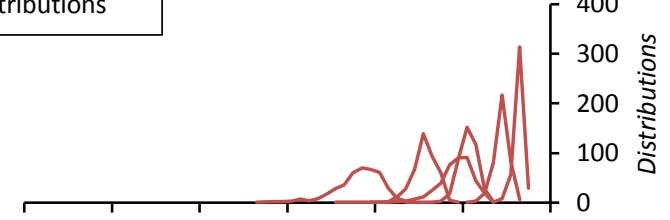

RIGHT RAIL PROFILES

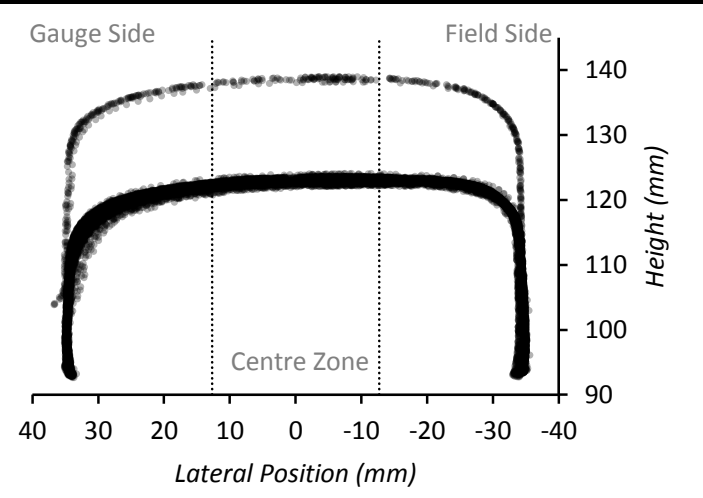

D-8 
SEGMENT AND ANALYSIS INFORMATION

\begin{tabular}{llll}
\hline \hline Track Segment Name: & NB-19 & Contact Analysis Date: & 20180615 \\
Segment Length $(m):$ & 402.6 & Left Wheel Slope: & $1: 20$ \\
Data Collection Date: & 20150827 & Right Wheel Slope: & $1: 20$
\end{tabular}

RAIL SEGMENT GRAPHS

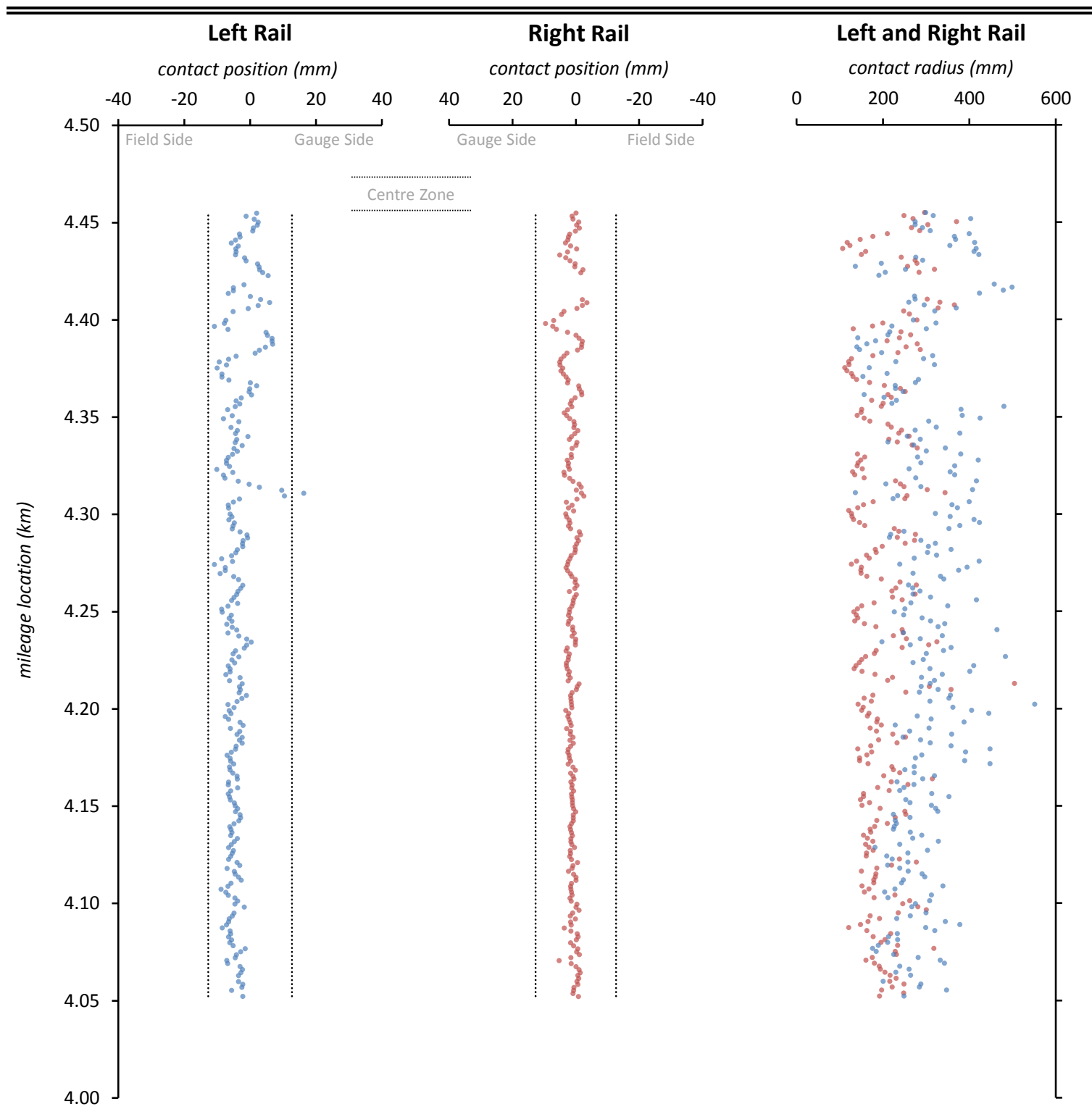

RAIL SEGMENT STATISTICS TABLE

\begin{tabular}{|c|c|c|c|c|}
\hline \multirow{3}{*}{ Statistic } & \multicolumn{4}{|c|}{ Rail Profile Measure } \\
\hline & \multicolumn{2}{|c|}{ Left Rail (252 profiles) } & \multicolumn{2}{|c|}{ Right Rail (254 profiles) } \\
\hline & Contact Position & Contact Radius & Contact Position & Contact Radius \\
\hline Mean $(\mathrm{mm})$ & -3.9 & 295.8 & 1.3 & 202.4 \\
\hline Std. Dev. (mm) & 3.7 & 74.7 & 1.7 & 58.4 \\
\hline$\%$ within Zone & $100 \%$ & - & $100 \%$ & - \\
\hline
\end{tabular}


Lateral Contact Position ( $\mathrm{mm}$ )

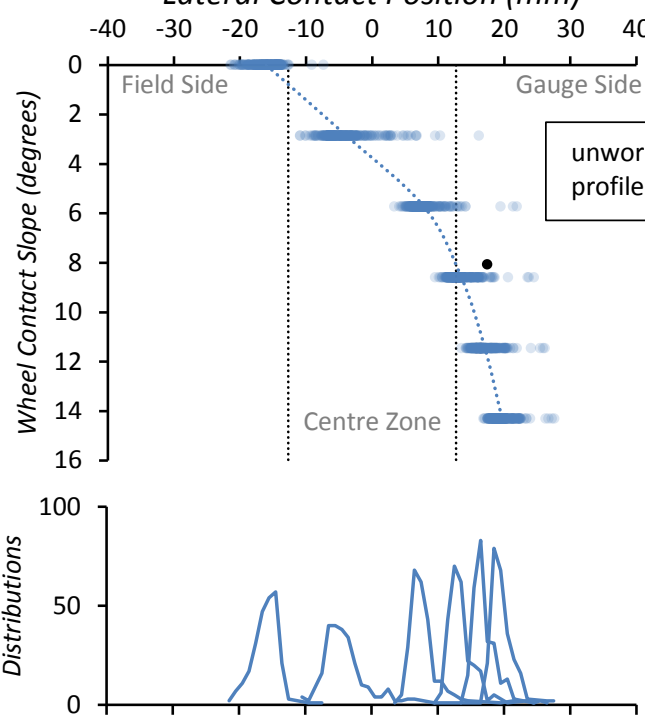

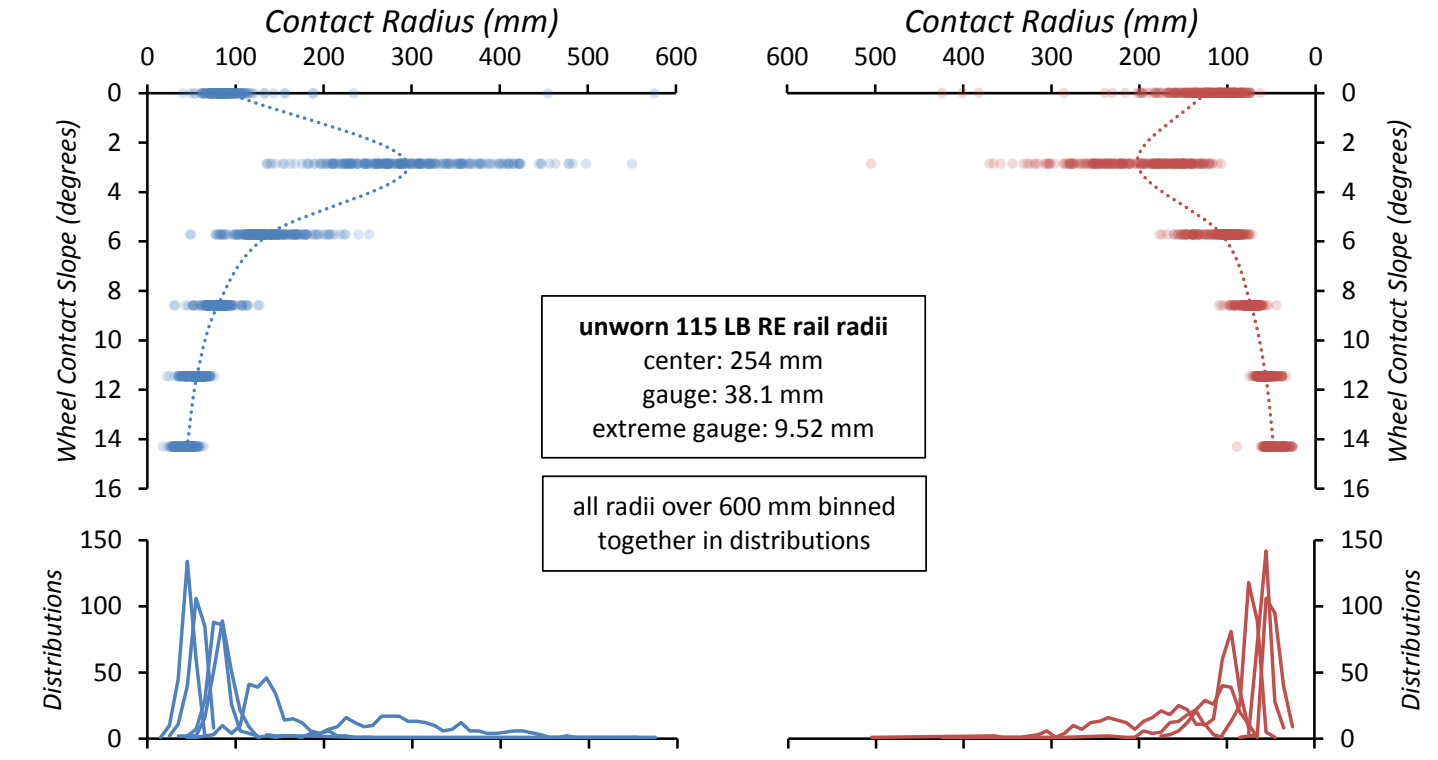

Lateral Contact Position ( $\mathrm{mm}$ )
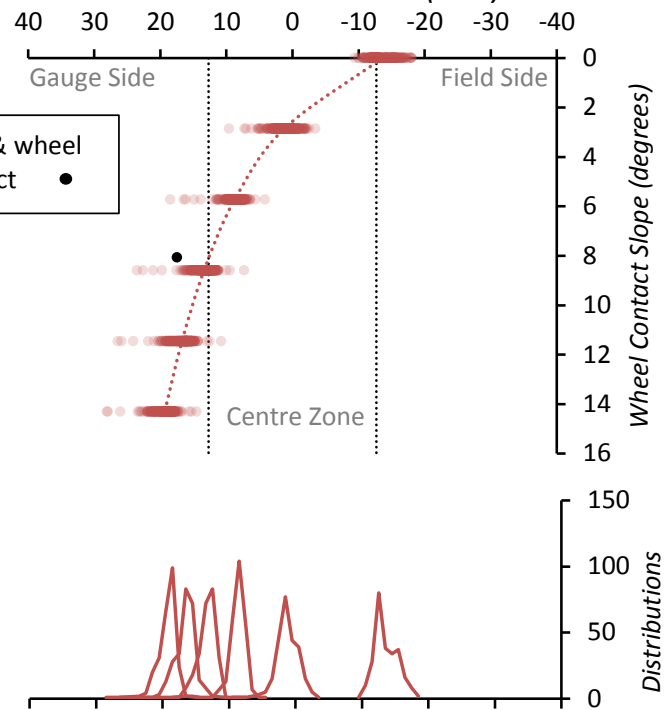

Contact Radius ( $\mathrm{mm}$ )

LEFT RAIL PROFILES

RIGHT RAIL PROFILES
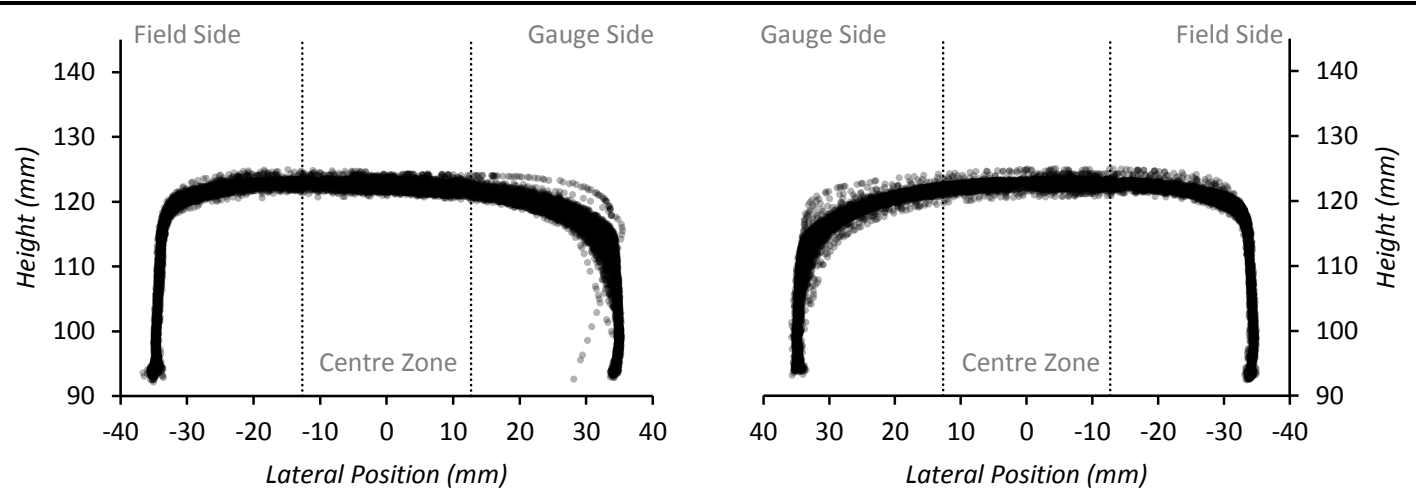

D-10 
SEGMENT AND ANALYSIS INFORMATION

\begin{tabular}{llll}
\hline \hline Track Segment Name: & NB-23 & Contact Analysis Date: & 20180615 \\
Segment Length $(m):$ & 173.6 & Left Wheel Slope: & $1: 20$ \\
Data Collection Date: & 20150827 & Right Wheel Slope: & $1: 20$
\end{tabular}

RAIL SEGMENT GRAPHS

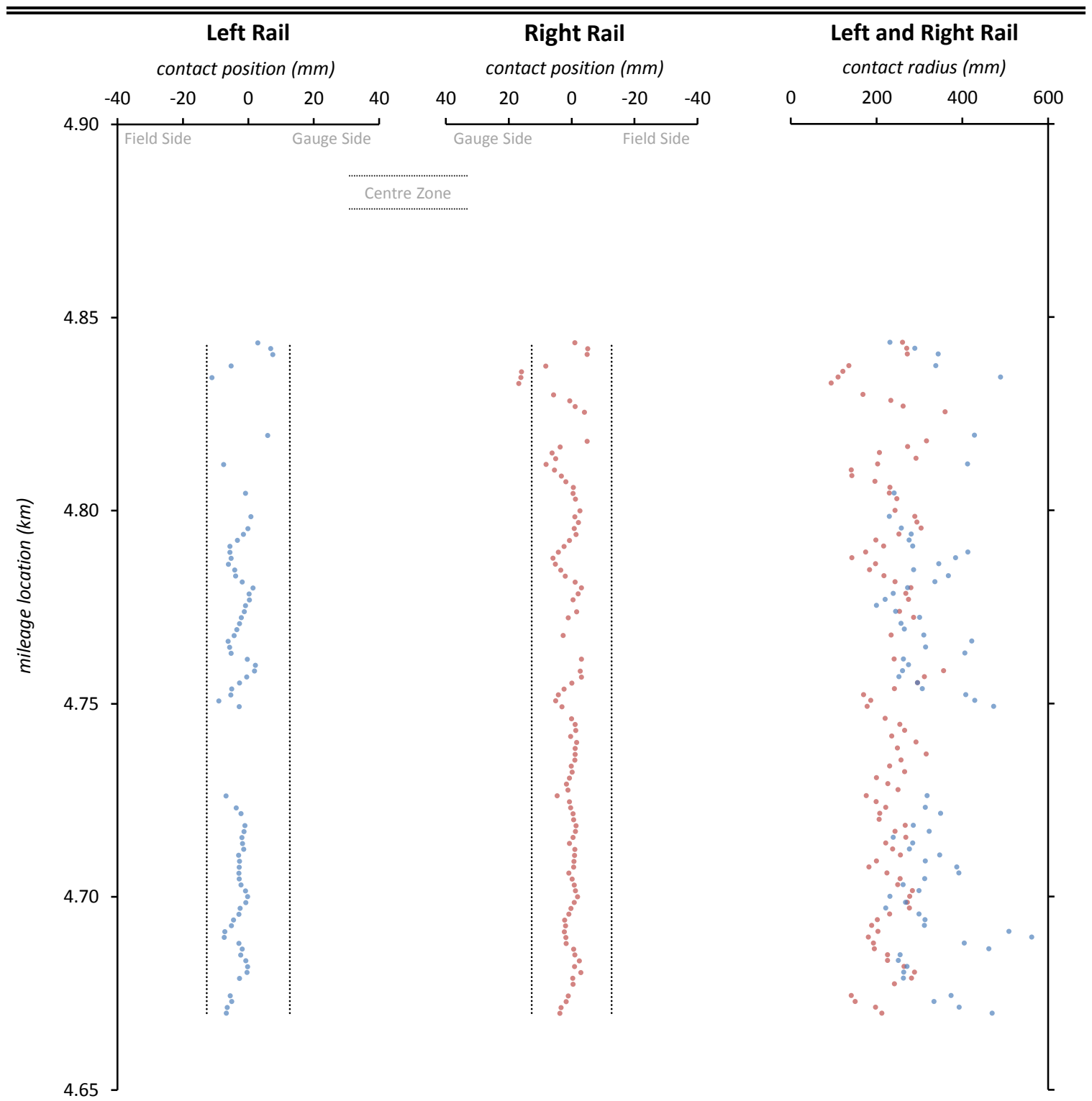

RAIL SEGMENT STATISTICS TABLE

\begin{tabular}{|c|c|c|c|c|}
\hline \multirow{3}{*}{ Statistic } & \multicolumn{4}{|c|}{ Rail Profile Measure } \\
\hline & \multicolumn{2}{|c|}{ Left Rail (74 profiles) } & \multicolumn{2}{|c|}{ Right Rail (99 profiles) } \\
\hline & Contact Position & Contact Radius & Contact Position & Contact Radius \\
\hline Mean $(\mathrm{mm})$ & -2.6 & 321.3 & 1.0 & 231.0 \\
\hline Std. Dev. $(\mathrm{mm})$ & 3.3 & 77.3 & 3.8 & 51.0 \\
\hline$\%$ within Zone & $100 \%$ & - & $97 \%$ & - \\
\hline
\end{tabular}


Lateral Contact Position ( $\mathrm{mm}$ )
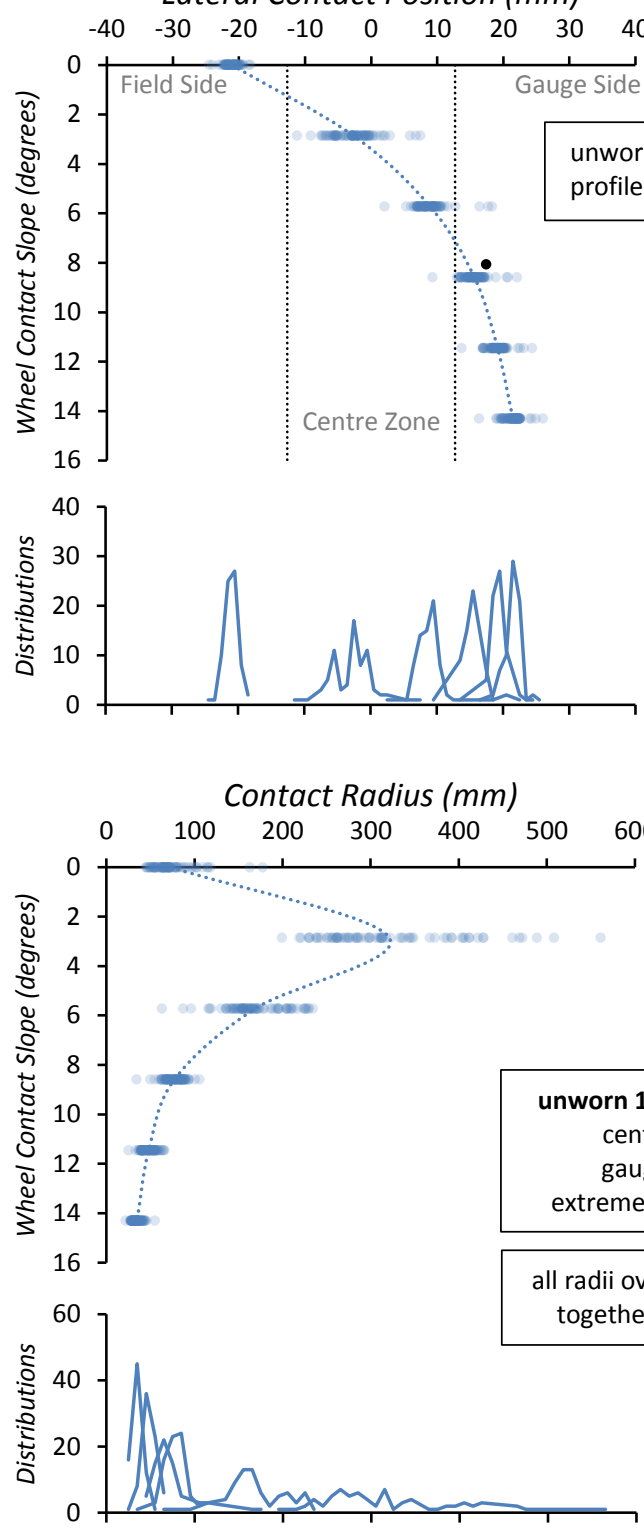

LEFT RAIL PROFILES

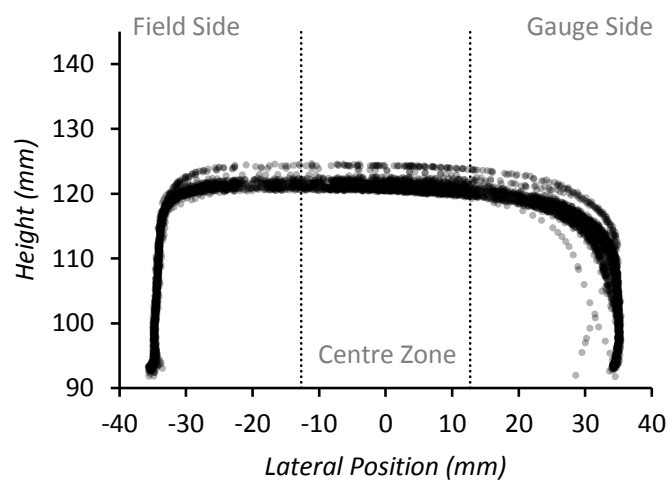

Lateral Contact Position ( $\mathrm{mm}$ )
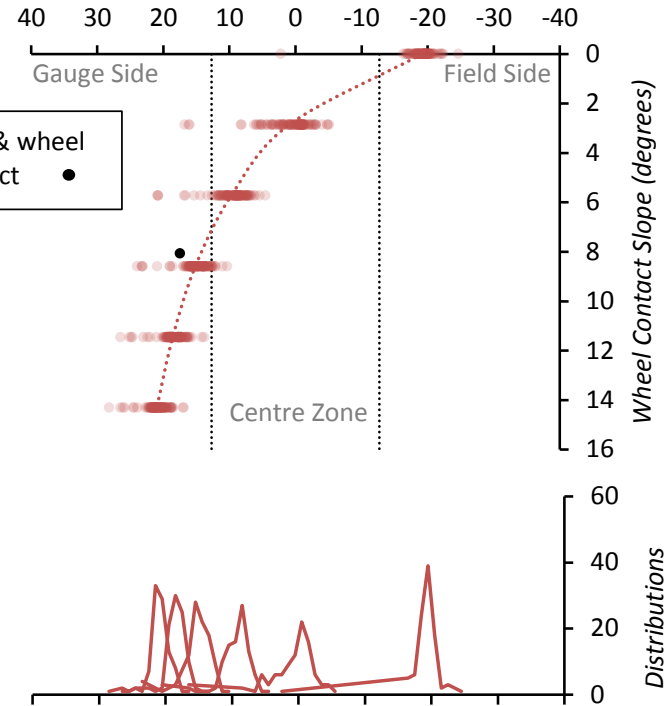

Contact Radius ( $\mathrm{mm}$ )
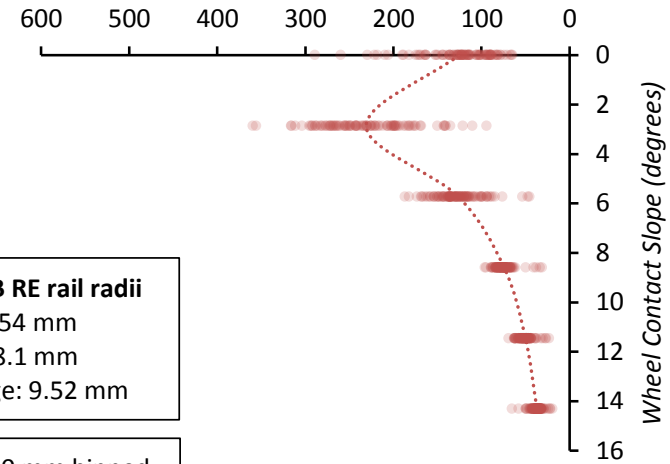

er: $254 \mathrm{~mm}$

uge: $38.1 \mathrm{~mm}$

gauge: $9.52 \mathrm{~mm}$

tributions

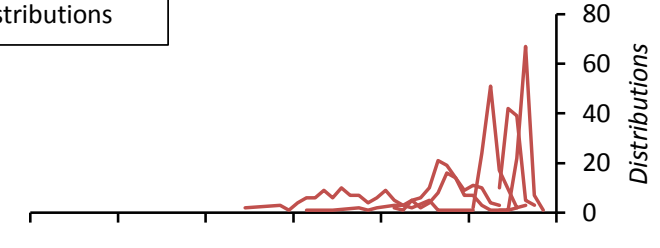

RIGHT RAIL PROFILES

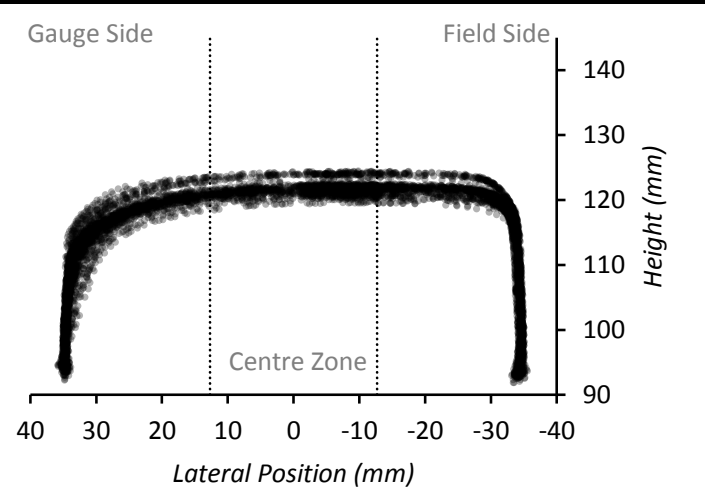

D-12 
SEGMENT AND ANALYSIS INFORMATION

\begin{tabular}{llll}
\hline \hline Track Segment Name: & NB-26 & Contact Analysis Date: & 20180615 \\
Segment Length $(m):$ & 884.2 & Left Wheel Slope: & $1: 20$ \\
Data Collection Date: & 20150827 & Right Wheel Slope: & $1: 20$
\end{tabular}

RAIL SEGMENT GRAPHS

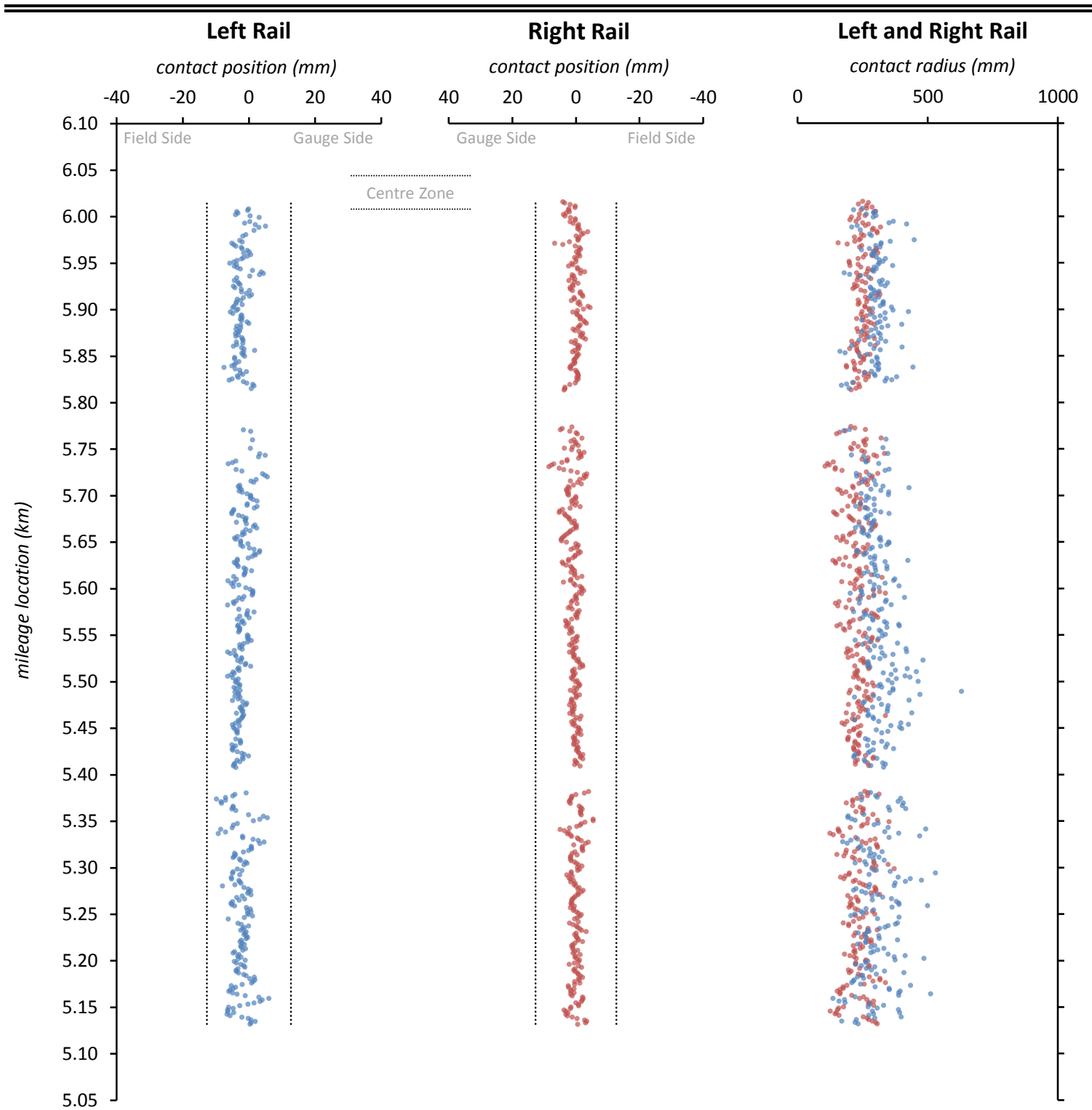

RAIL SEGMENT STATISTICS TABLE

\begin{tabular}{|c|c|c|c|c|}
\hline \multirow{3}{*}{ Statistic } & \multicolumn{4}{|c|}{ Rail Profile Measure } \\
\hline & \multicolumn{2}{|c|}{ Left Rail (476 profiles) } & \multicolumn{2}{|c|}{ Right Rail (525 profiles) } \\
\hline & Contact Position & Contact Radius & Contact Position & Contact Radius \\
\hline Mean (mm) & -2.2 & 304.7 & 0.4 & 236.3 \\
\hline Std. Dev. $(\mathrm{mm})$ & 2.6 & 65.0 & 1.8 & 44.2 \\
\hline$\%$ within Zone & $100 \%$ & - & $100 \%$ & - \\
\hline
\end{tabular}


Lateral Contact Position ( $\mathrm{mm}$ )
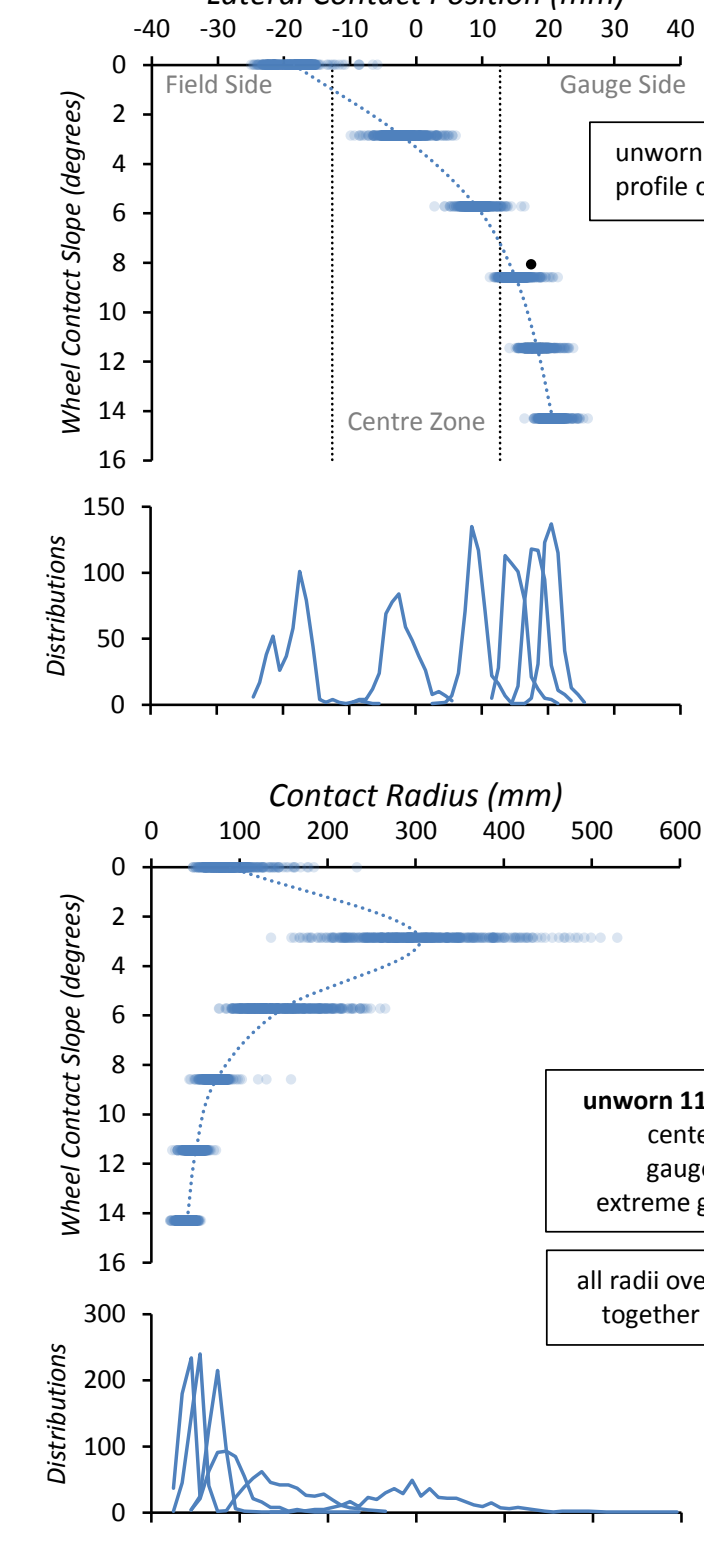

Lateral Contact Position ( $\mathrm{mm}$ )
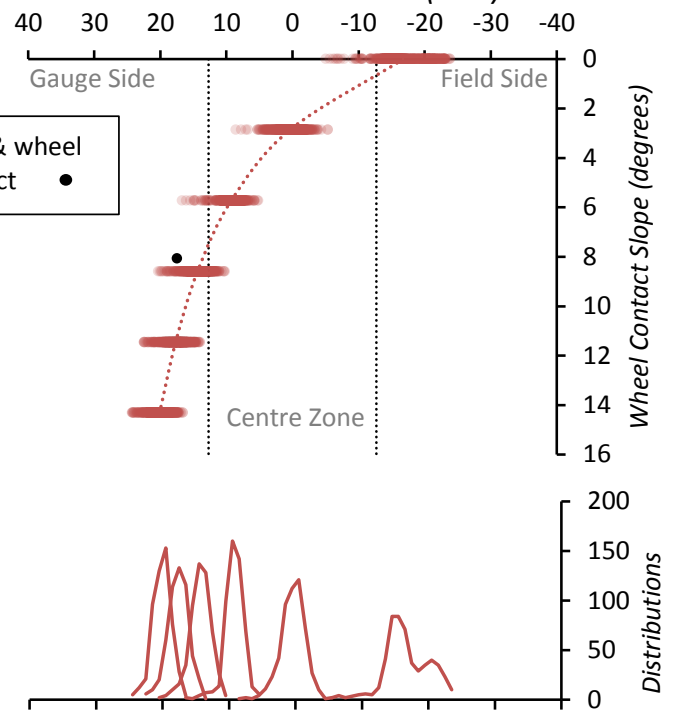

Contact Radius ( $\mathrm{mm}$ )

$\begin{array}{lllllll}600 & 500 & 400 & 300 & 200 & 100 & 0\end{array}$

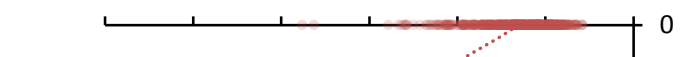

115 LB $\mathrm{RE}$ rail radii nter: $254 \mathrm{~mm}$ uge: $38.1 \mathrm{~mm}$ gauge: $9.52 \mathrm{~mm}$

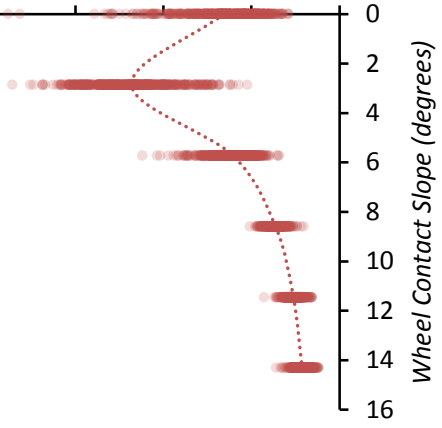

$00 \mathrm{~mm}$ binned
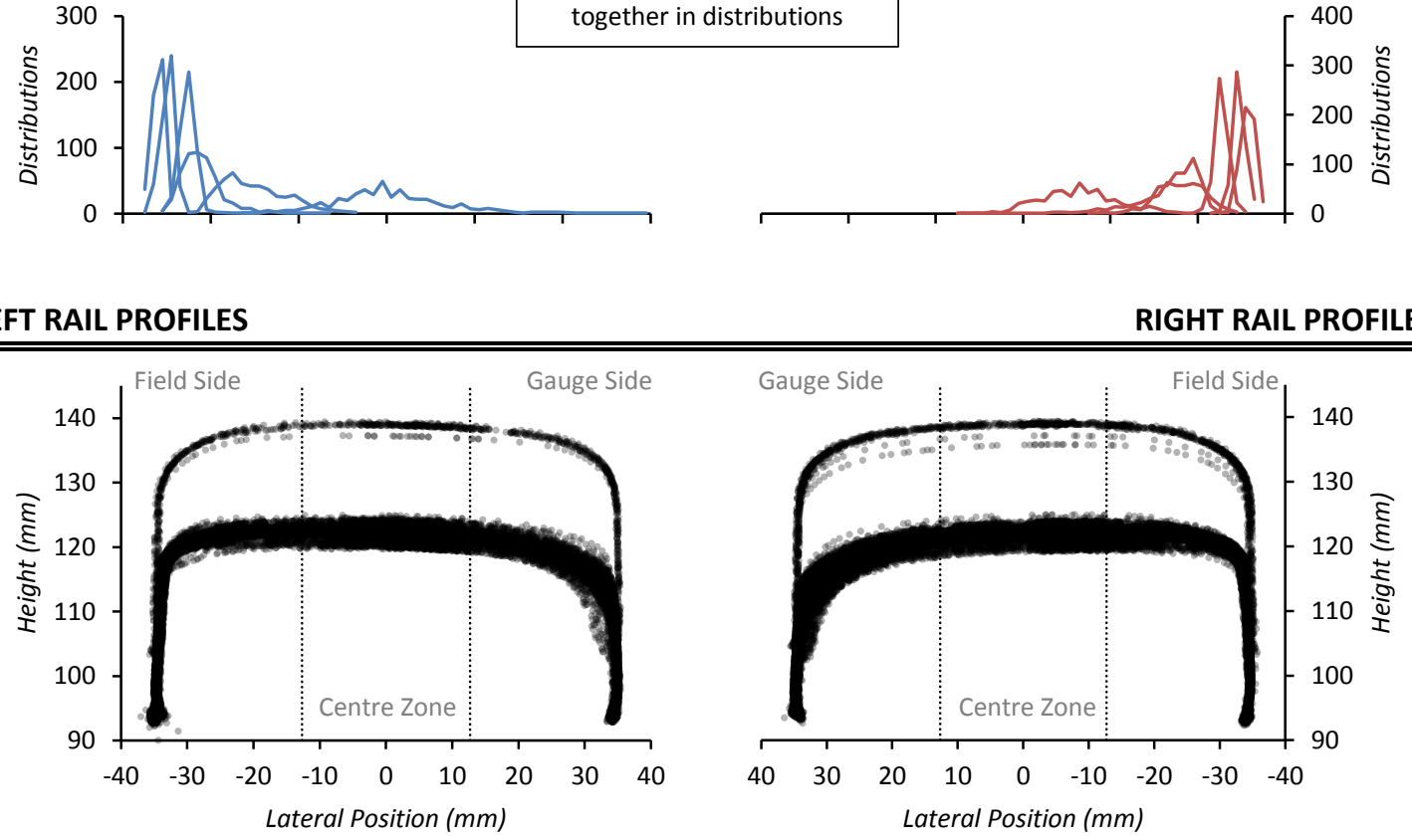
SEGMENT AND ANALYSIS INFORMATION

\begin{tabular}{llll}
\hline \hline Track Segment Name: & NB-34 & Contact Analysis Date: & 20180615 \\
Segment Length $(\mathrm{m}):$ & 1047.7 & Left Wheel Slope: & $1: 20$ \\
Data Collection Date: & 20150827 & Right Wheel Slope: & $1: 20$
\end{tabular}

RAIL SEGMENT GRAPHS

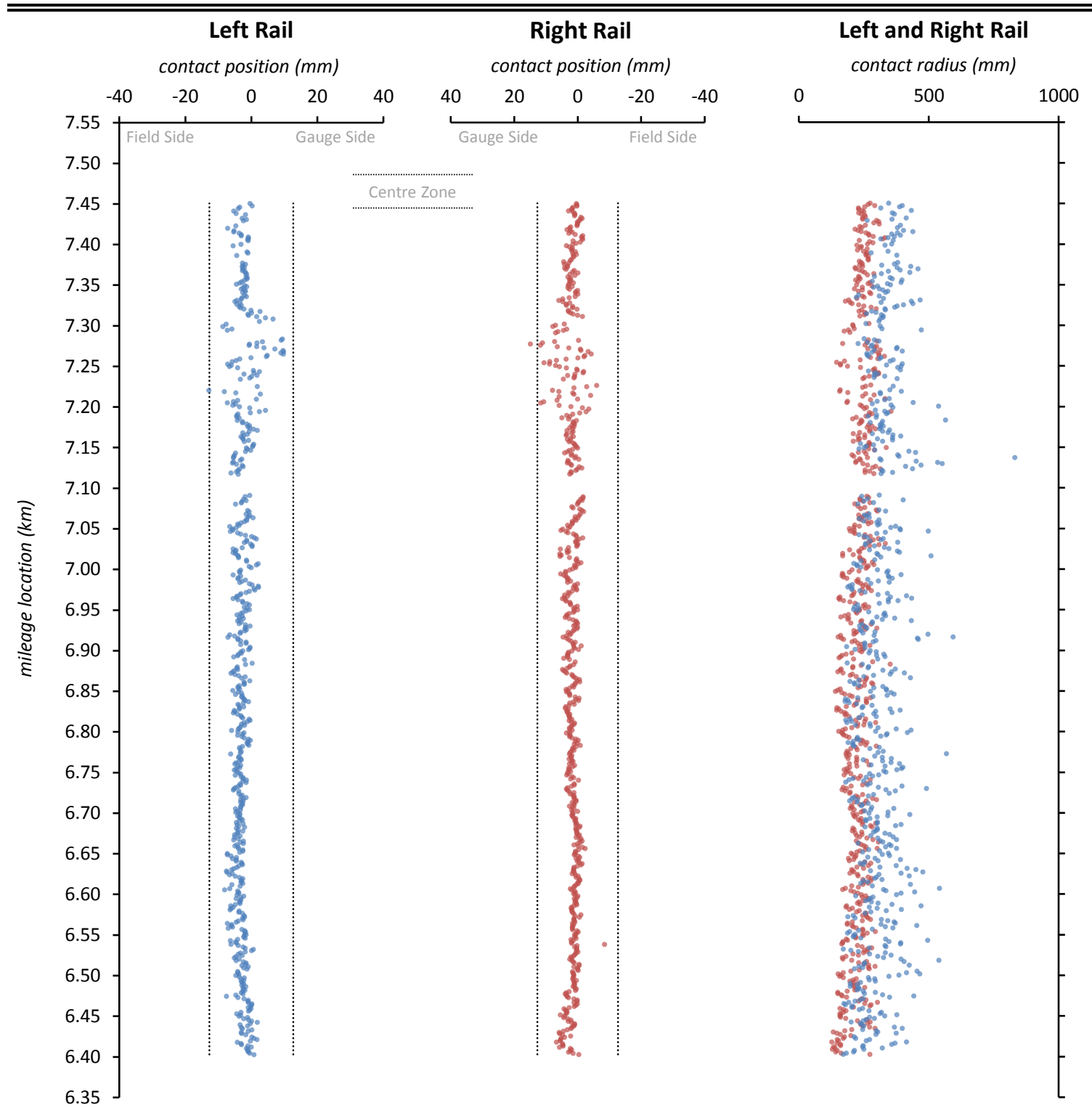

RAIL SEGMENT STATISTICS TABLE

\begin{tabular}{|c|c|c|c|c|}
\hline \multirow{3}{*}{ Statistic } & \multicolumn{4}{|c|}{ Rail Profile Measure } \\
\hline & \multicolumn{2}{|c|}{ Left Rail (594 profiles) } & \multicolumn{2}{|c|}{ Right Rail (640 profiles) } \\
\hline & Contact Position & Contact Radius & Contact Position & Contact Radius \\
\hline Mean $(\mathrm{mm})$ & -2.7 & 311.5 & 1.8 & 229.9 \\
\hline Std. Dev. $(\mathrm{mm})$ & 2.5 & 76.9 & 2.1 & 42.0 \\
\hline$\%$ within Zone & $100 \%$ & - & $100 \%$ & - \\
\hline
\end{tabular}


Lateral Contact Position ( $\mathrm{mm}$ )
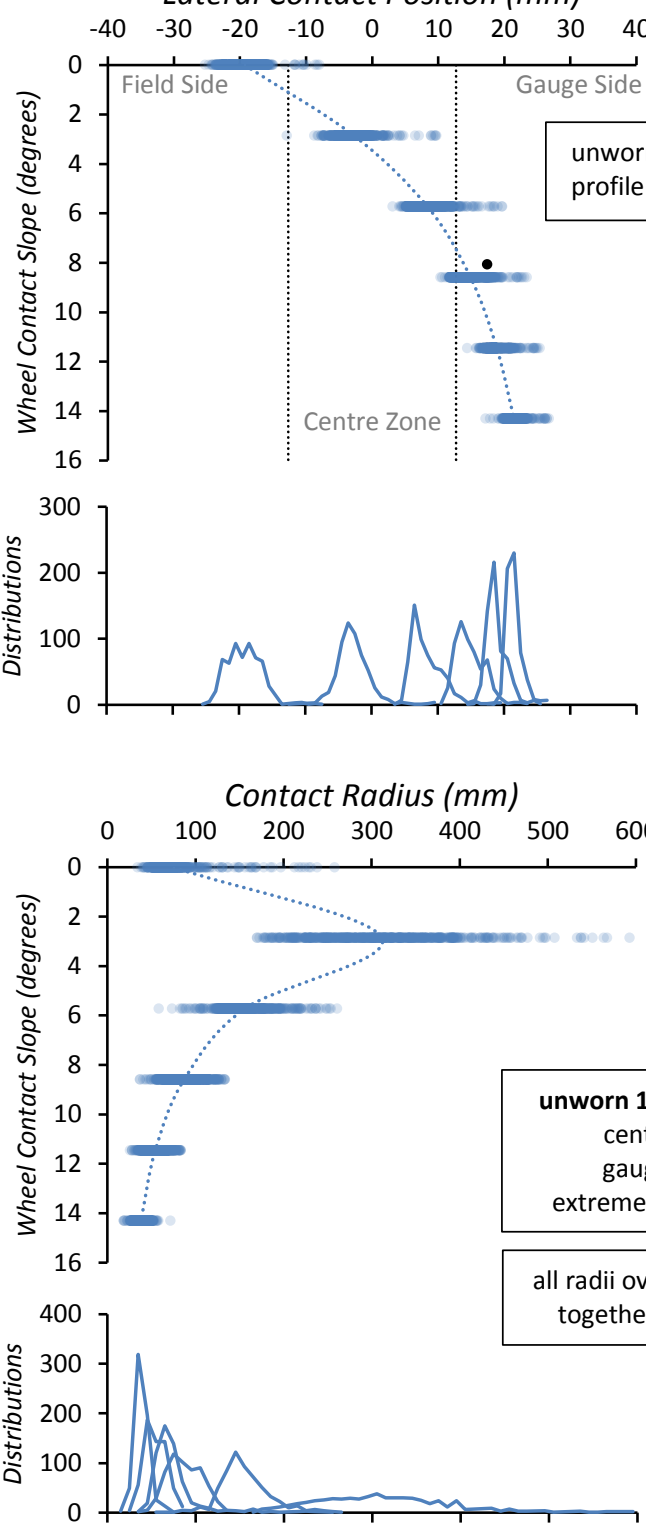

LEFT RAIL PROFILES

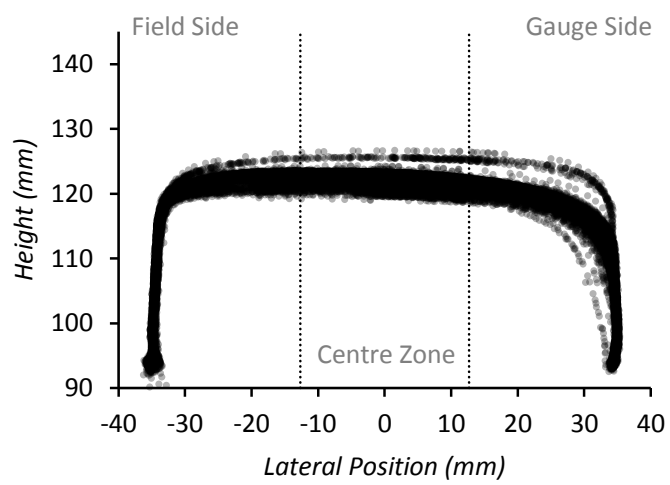

Lateral Contact Position ( $\mathrm{mm}$ )
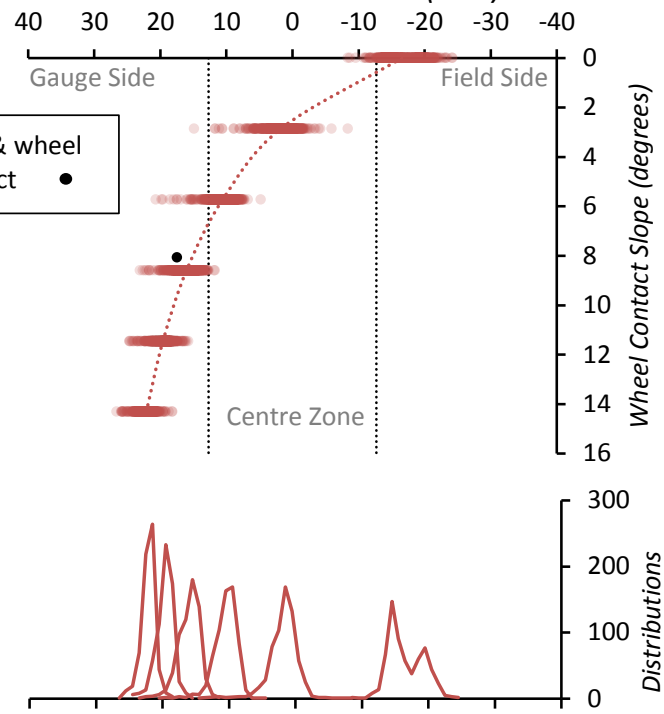

Contact Radius ( $\mathrm{mm}$ )
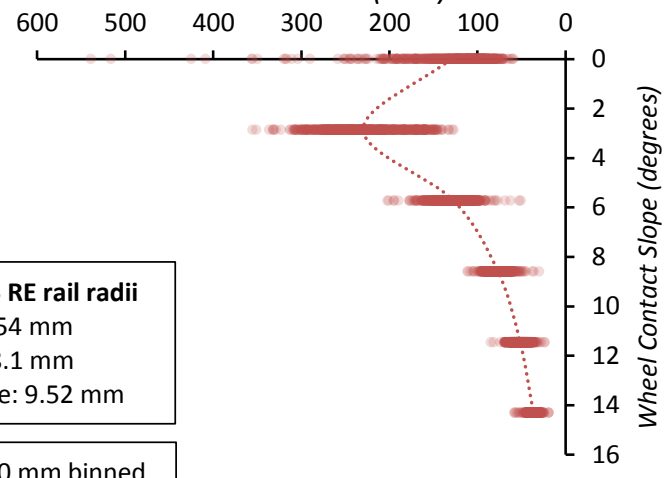

nter: $254 \mathrm{~mm}$

gauge: $38.1 \mathrm{~mm}$

eme gauge: $9.52 \mathrm{~mm}$

$600 \mathrm{~mm}$ binned

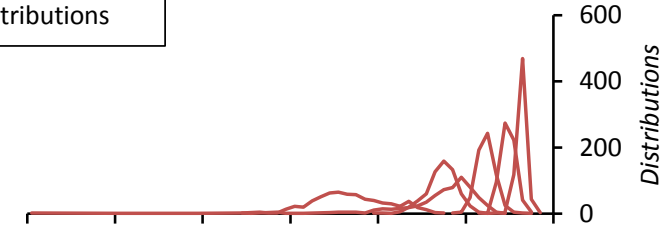

RIGHT RAIL PROFILES

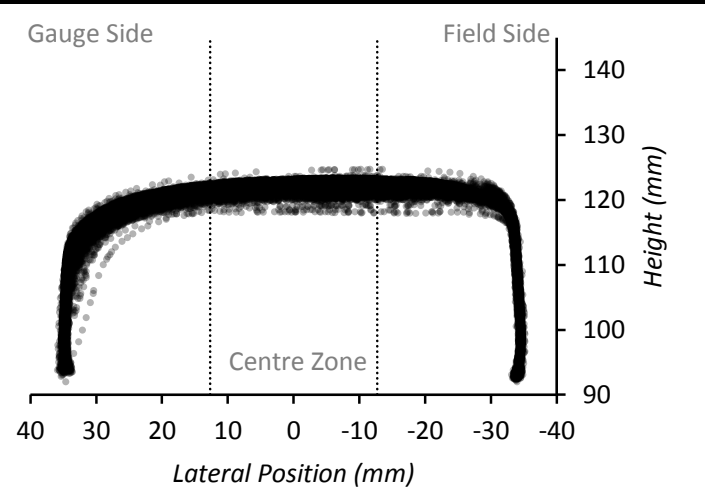

D-16 
SEGMENT AND ANALYSIS INFORMATION

\begin{tabular}{llll}
\hline \hline Track Segment Name: & NB-56 & Contact Analysis Date: & 20180615 \\
Segment Length $(m):$ & 283.8 & Left Wheel Slope: & $1: 20$ \\
Data Collection Date: & 20150827 & Right Wheel Slope: & $1: 20$
\end{tabular}

RAIL SEGMENT GRAPHS

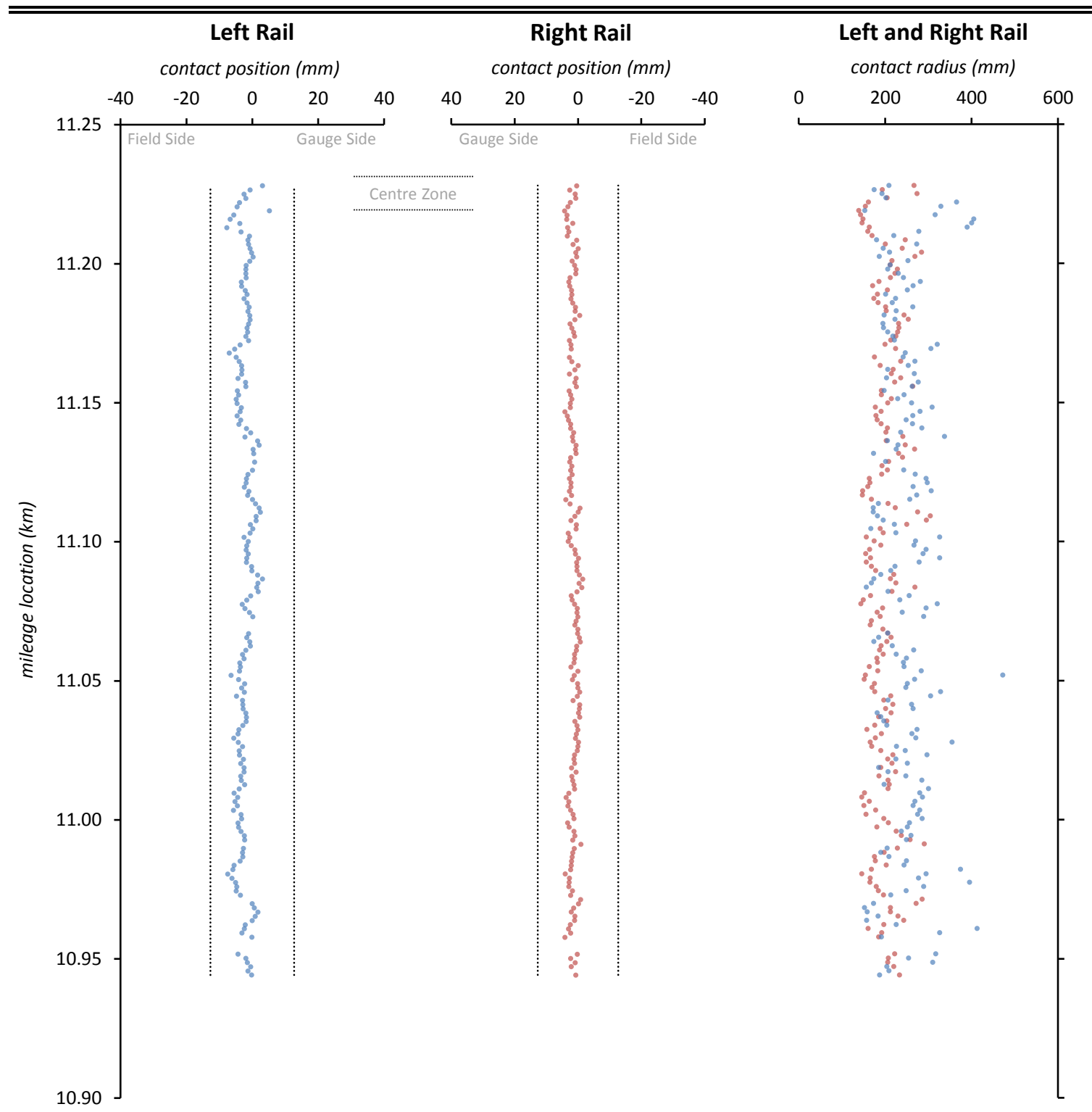

RAIL SEGMENT STATISTICS TABLE

\begin{tabular}{|c|c|c|c|c|}
\hline \multirow{3}{*}{ Statistic } & \multicolumn{4}{|c|}{ Rail Profile Measure } \\
\hline & \multicolumn{2}{|c|}{ Left Rail (179 profiles) } & \multicolumn{2}{|c|}{ Right Rail (184 profiles) } \\
\hline & Contact Position & Contact Radius & Contact Position & Contact Radius \\
\hline Mean $(m m)$ & -2.3 & 247.4 & 1.5 & 199.0 \\
\hline Std. Dev. $(\mathrm{mm})$ & 2.2 & 55.6 & 1.2 & 34.4 \\
\hline$\%$ within Zone & $100 \%$ & - & $100 \%$ & - \\
\hline
\end{tabular}


Lateral Contact Position ( $\mathrm{mm}$ )
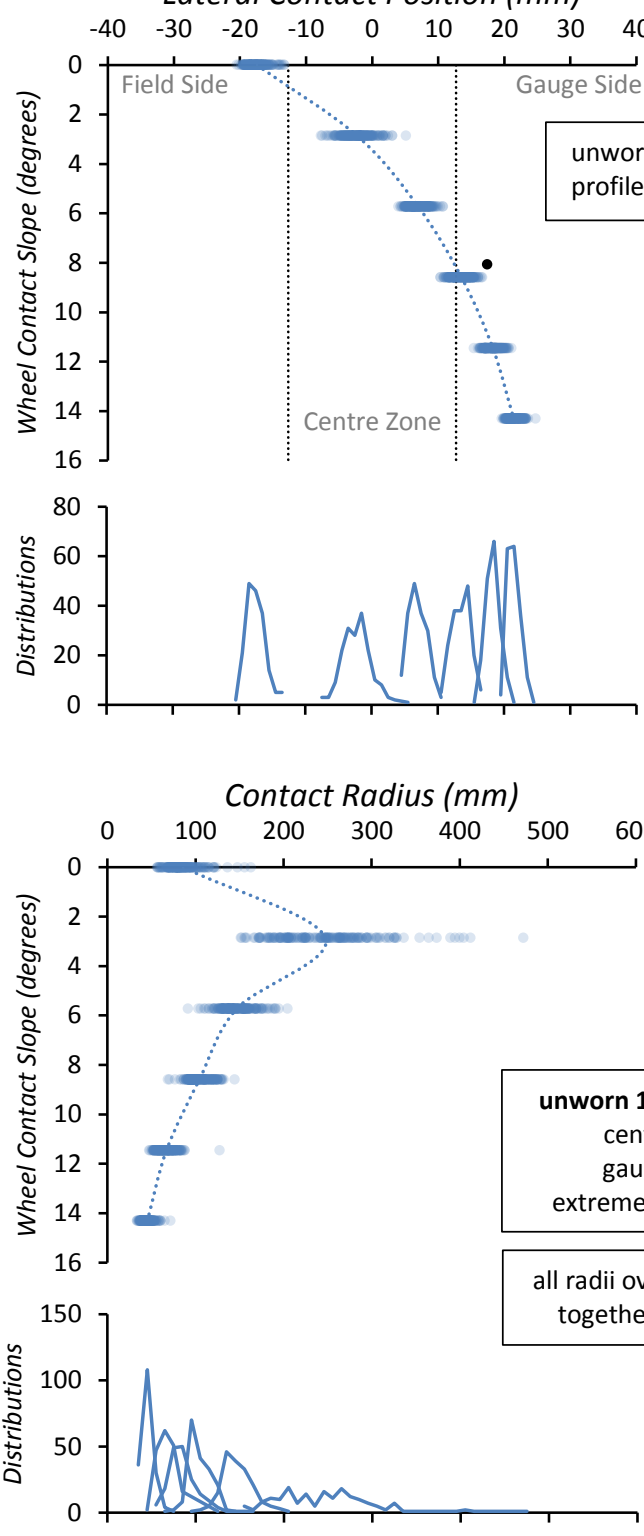

LEFT RAIL PROFILES

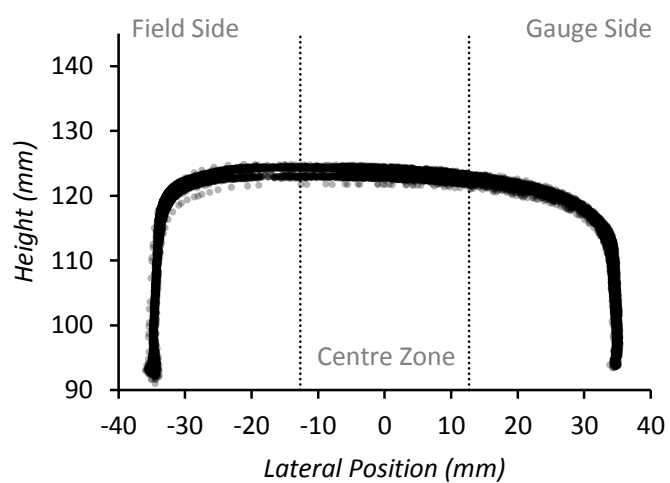

Lateral Contact Position ( $\mathrm{mm}$ )
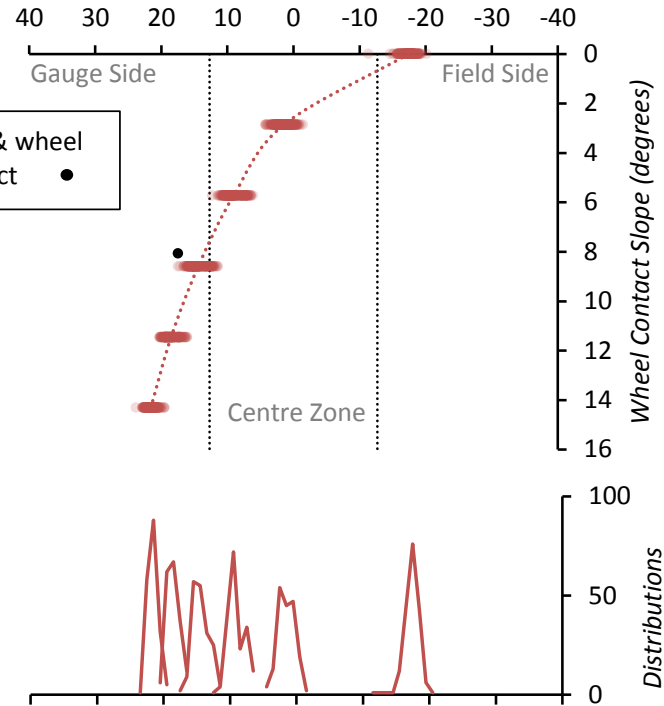

Contact Radius ( $\mathrm{mm}$ )
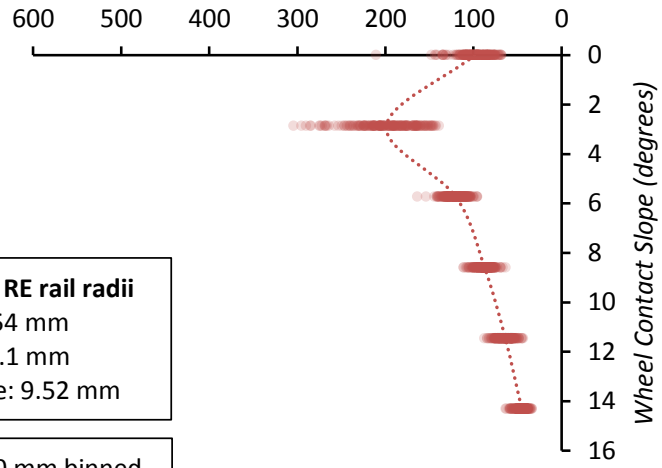

nter: $254 \mathrm{~mm}$

auge: $38.1 \mathrm{~mm}$

eme gauge: $9.52 \mathrm{~mm}$

$600 \mathrm{~mm}$ binned

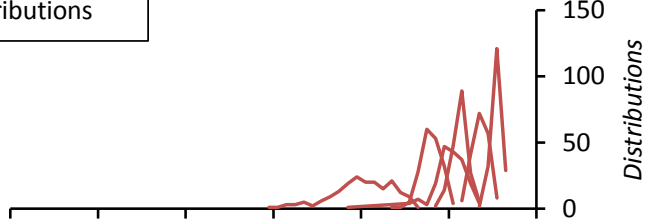

RIGHT RAIL PROFILES

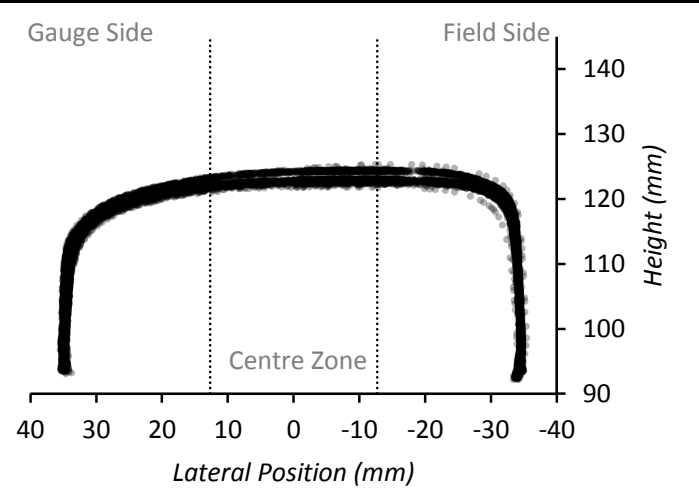

D-18 
SEGMENT AND ANALYSIS INFORMATION

\begin{tabular}{llll}
\hline \hline Track Segment Name: & NB-60 & Contact Analysis Date: & 20180615 \\
Segment Length $(m):$ & 187.4 & Left Wheel Slope: & $1: 20$ \\
Data Collection Date: & 20150827 & Right Wheel Slope: & $1: 20$ \\
& & & \\
RAIL SEGMENT GRAPHS & & \\
\hline \hline
\end{tabular}

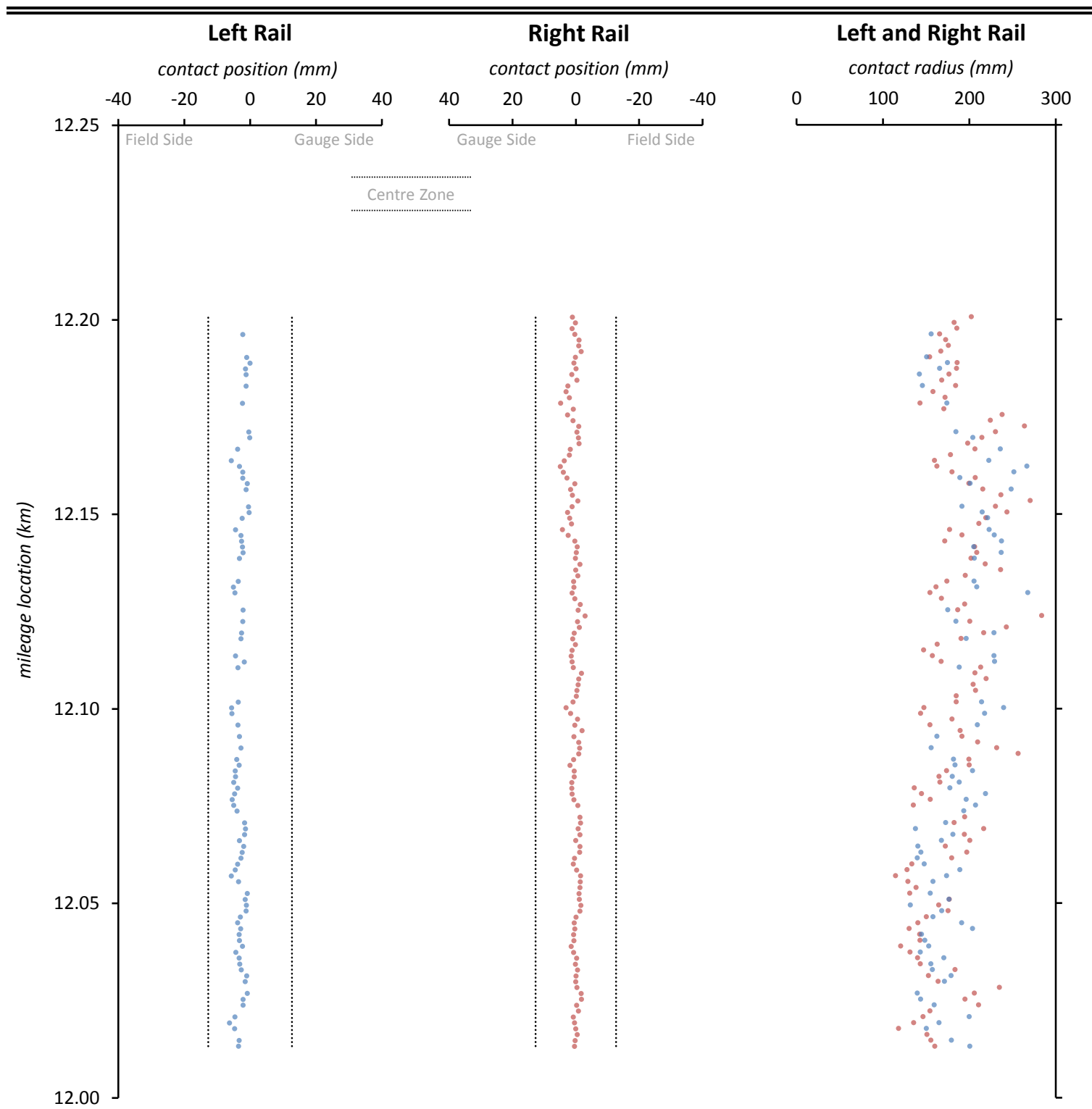

RAIL SEGMENT STATISTICS TABLE

\begin{tabular}{|c|c|c|c|c|}
\hline \multirow{3}{*}{ Statistic } & \multicolumn{4}{|c|}{ Rail Profile Measure } \\
\hline & \multicolumn{2}{|c|}{ Left Rail (86 profiles) } & \multicolumn{2}{|c|}{ Right Rail (126 profiles) } \\
\hline & Contact Position & Contact Radius & Contact Position & Contact Radius \\
\hline Mean $(\mathrm{mm})$ & -3.0 & 186.0 & 0.3 & 181.4 \\
\hline Std. Dev. $(\mathrm{mm})$ & 1.5 & 32.6 & 1.4 & 34.2 \\
\hline$\%$ within Zone & $100 \%$ & - & $100 \%$ & - \\
\hline
\end{tabular}

D-19 

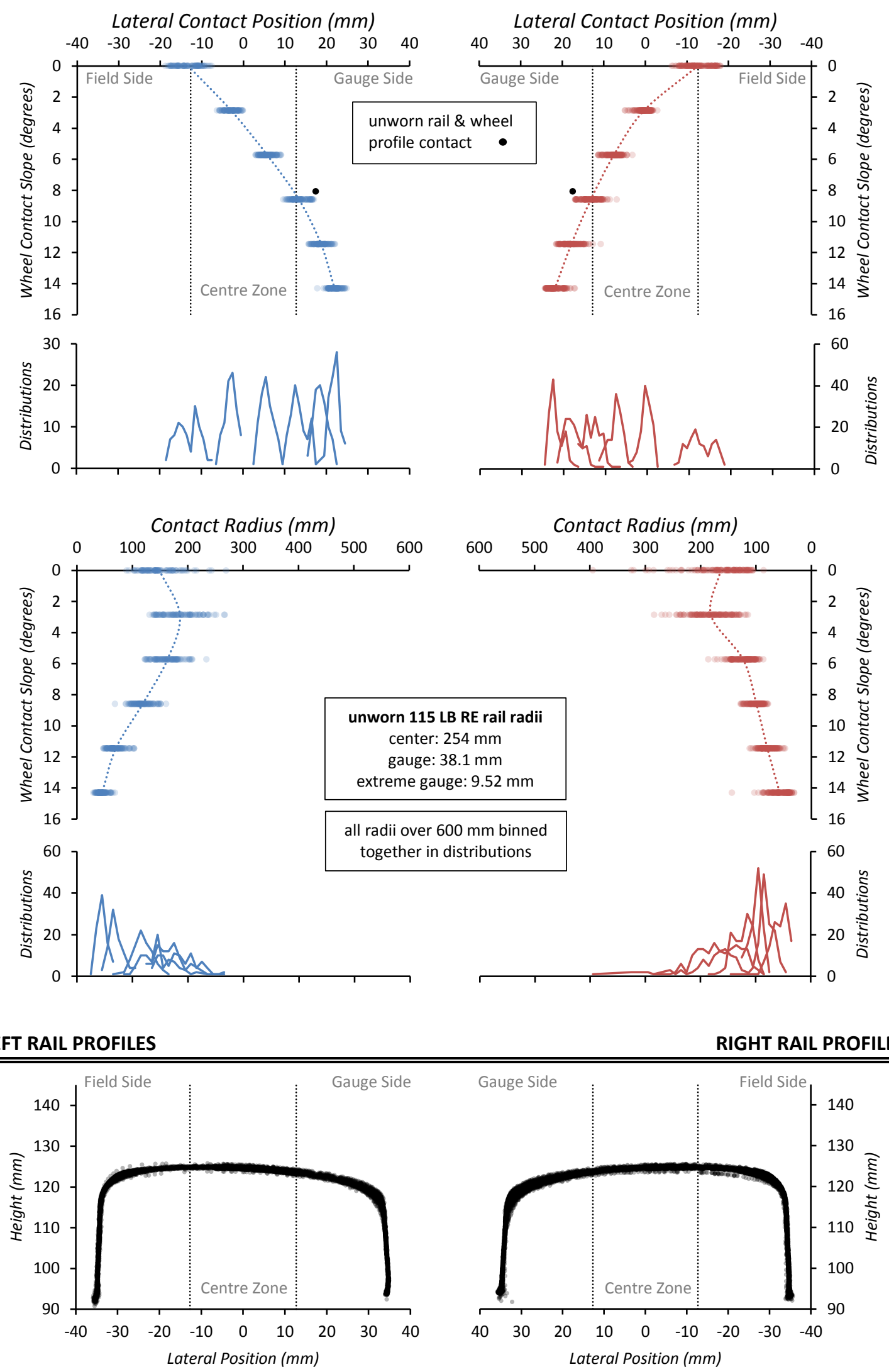
SEGMENT AND ANALYSIS INFORMATION

\begin{tabular}{llll}
\hline \hline Track Segment Name: & NB-62 & Contact Analysis Date: & 20180615 \\
Segment Length $(\mathrm{m}):$ & 383.7 & Left Wheel Slope: & $1: 20$ \\
Data Collection Date: & 20150827 & Right Wheel Slope: & $1: 20$
\end{tabular}

RAIL SEGMENT GRAPHS

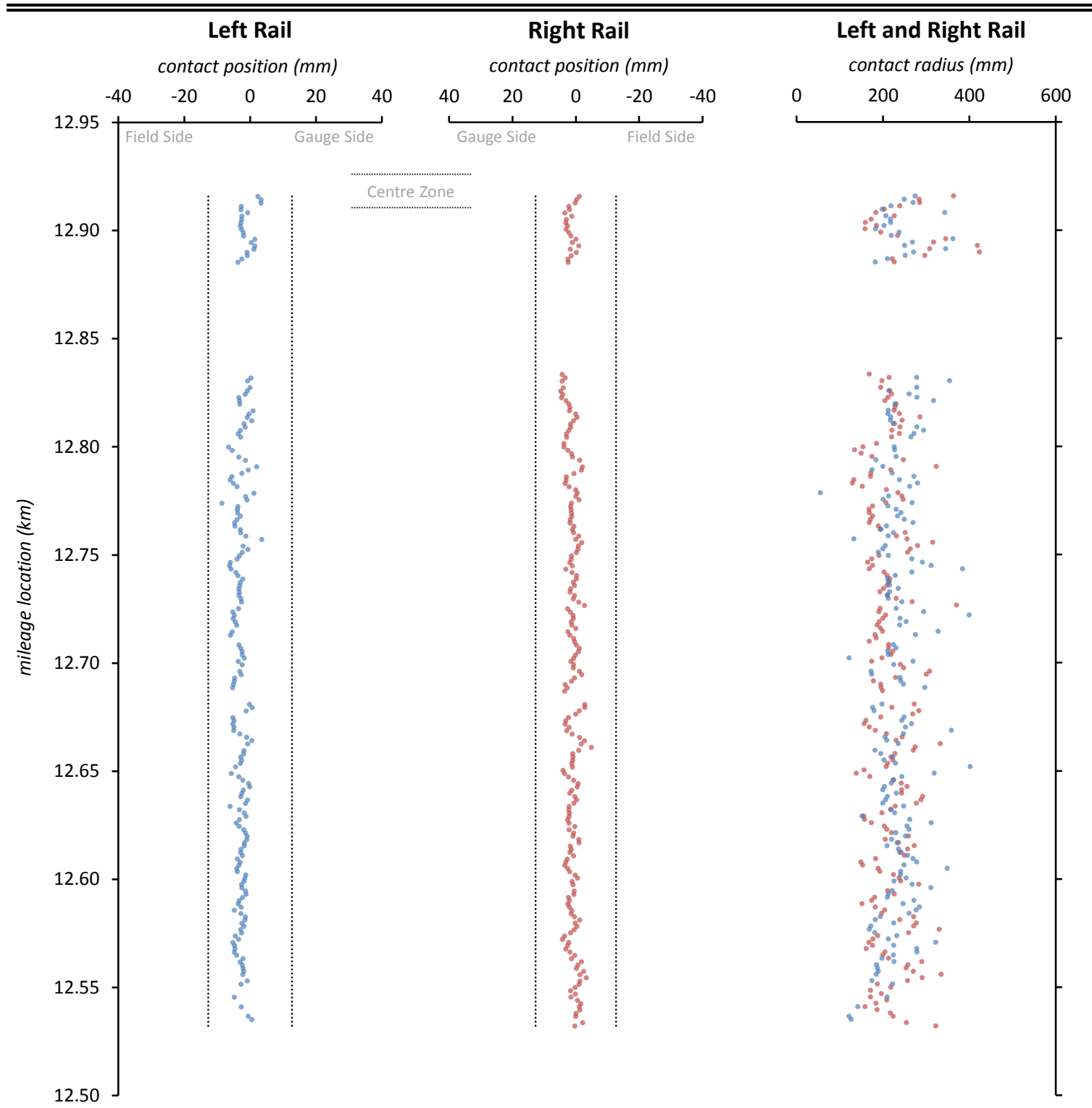

RAIL SEGMENT STATISTICS TABLE

\begin{tabular}{|c|c|c|c|c|}
\hline \multirow{3}{*}{ Statistic } & \multicolumn{4}{|c|}{ Rail Profile Measure } \\
\hline & \multicolumn{2}{|c|}{ Left Rail (190 profiles) } & \multicolumn{2}{|c|}{ Right Rail (213 profiles) } \\
\hline & Contact Position & Contact Radius & Contact Position & Contact Radius \\
\hline Mean $(\mathrm{mm})$ & -2.7 & 235.0 & 1.0 & 220.2 \\
\hline Std. Dev. $(\mathrm{mm})$ & 2.0 & 49.4 & 1.7 & 50.4 \\
\hline$\%$ within Zone & $100 \%$ & - & $100 \%$ & - \\
\hline
\end{tabular}


Lateral Contact Position ( $\mathrm{mm}$ )

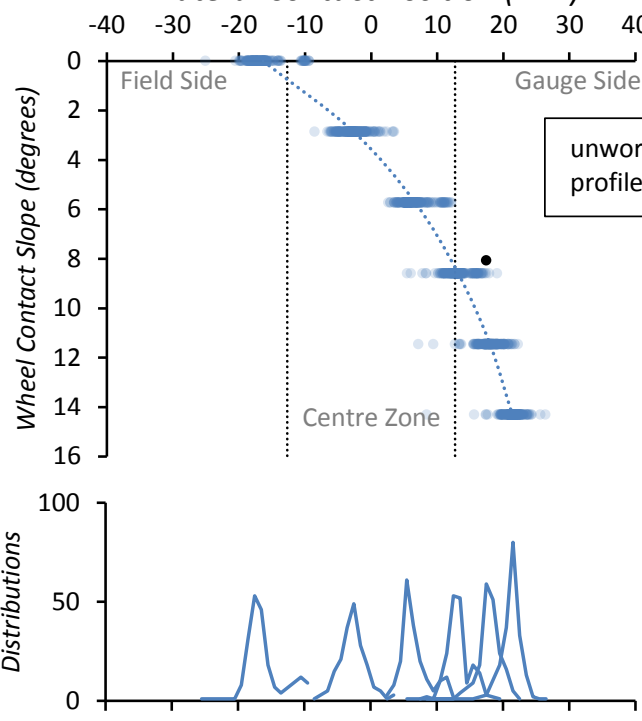

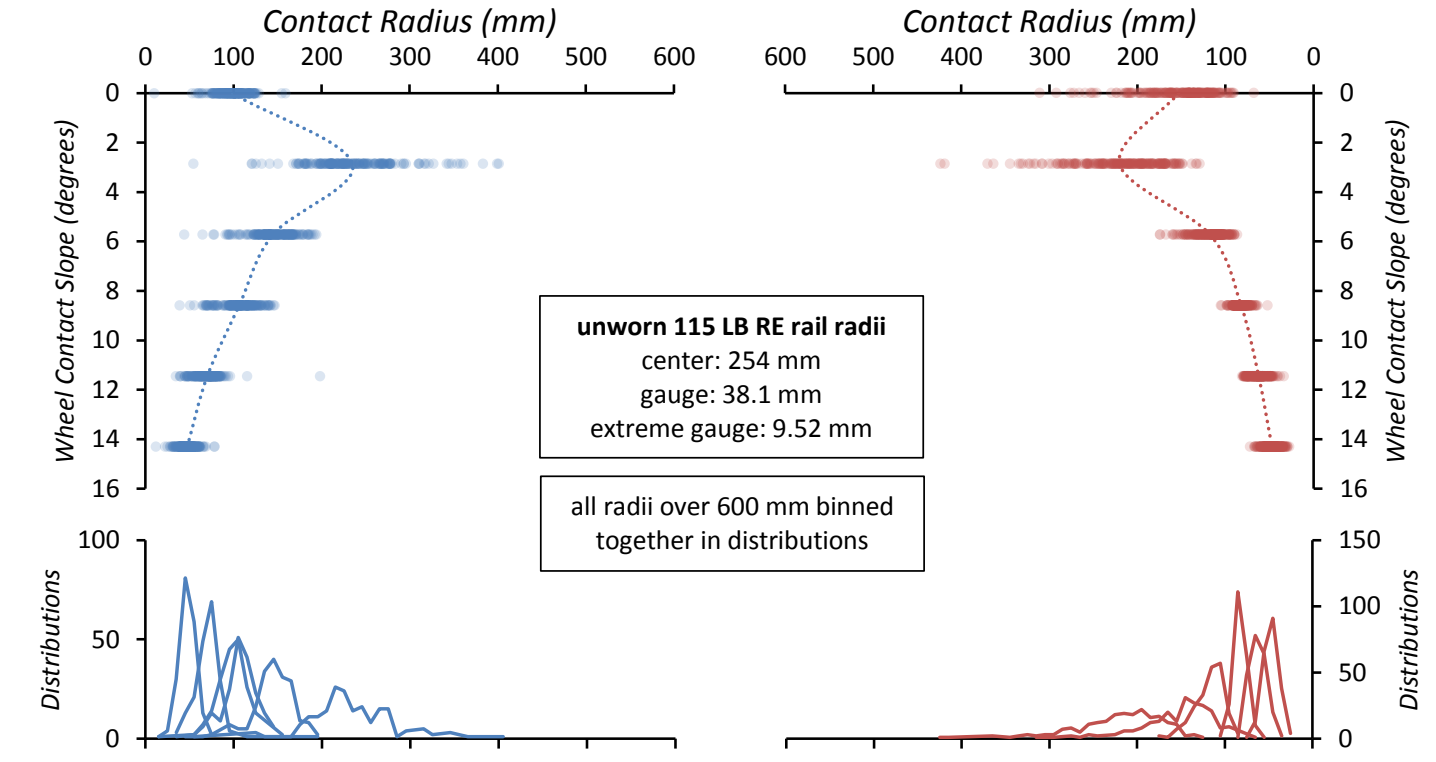

Lateral Contact Position ( $\mathrm{mm}$ )
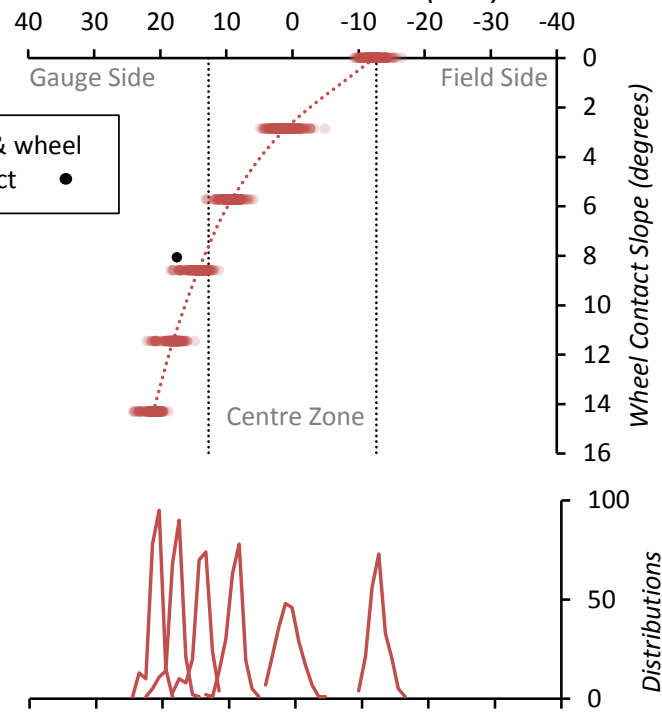

Contact Radius ( $\mathrm{mm}$ )

LEFT RAIL PROFILES

RIGHT RAIL PROFILES
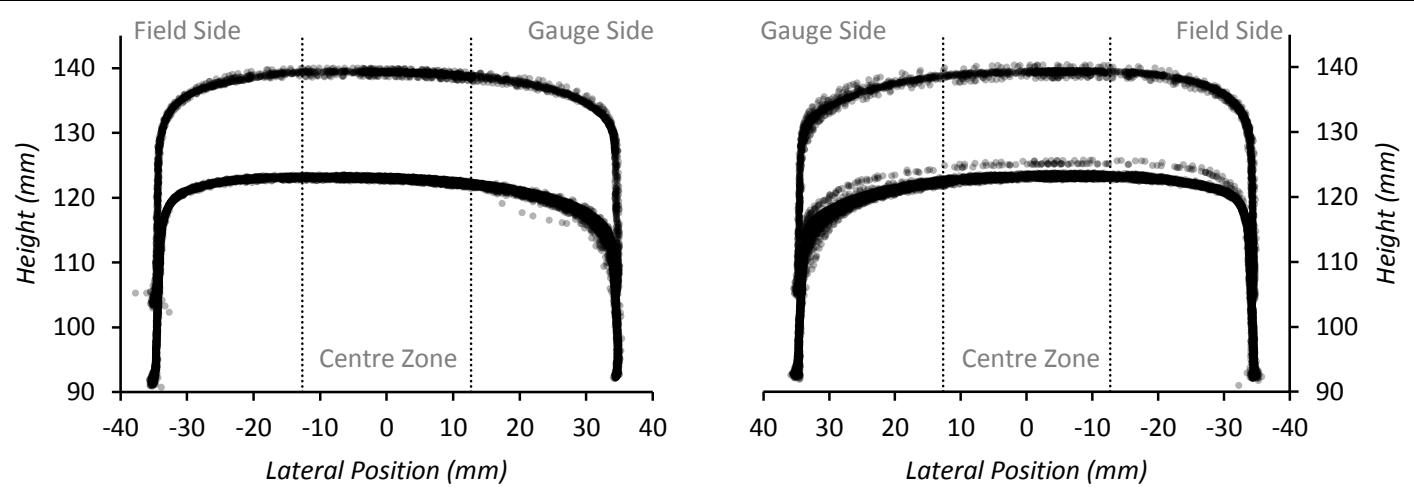
SEGMENT AND ANALYSIS INFORMATION

\begin{tabular}{llll}
\hline \hline Track Segment Name: & NB-66 & Contact Analysis Date: & 20180615 \\
Segment Length $(m):$ & 212.5 & Left Wheel Slope: & $1: 20$ \\
Data Collection Date: & 20150827 & Right Wheel Slope: & $1: 20$ \\
& & & \\
RAIL SEGMENT GRAPHS & & \\
\hline \hline
\end{tabular}

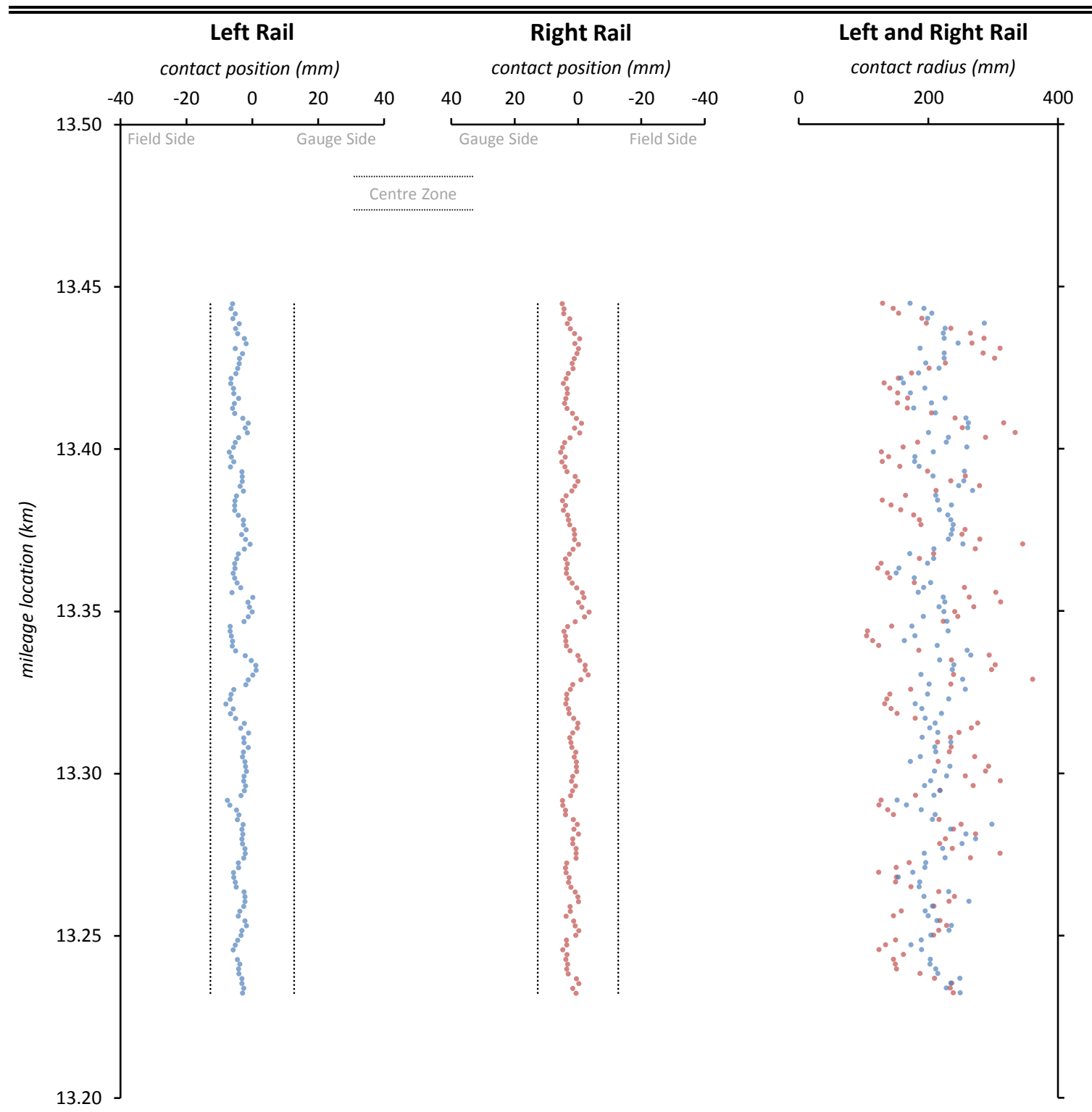

RAIL SEGMENT STATISTICS TABLE

\begin{tabular}{|c|c|c|c|c|}
\hline \multirow{3}{*}{ Statistic } & \multicolumn{4}{|c|}{ Rail Profile Measure } \\
\hline & \multicolumn{2}{|c|}{ Left Rail (142 profiles) } & \multicolumn{2}{|c|}{ Right Rail (143 profiles) } \\
\hline & Contact Position & Contact Radius & Contact Position & Contact Radius \\
\hline Mean $(\mathrm{mm})$ & -3.8 & 212.5 & 2.0 & 206.8 \\
\hline Std. Dev. $(\mathrm{mm})$ & 1.9 & 29.4 & 1.9 & 60.5 \\
\hline$\%$ within Zone & $100 \%$ & - & $100 \%$ & - \\
\hline
\end{tabular}


Lateral Contact Position ( $\mathrm{mm}$ )

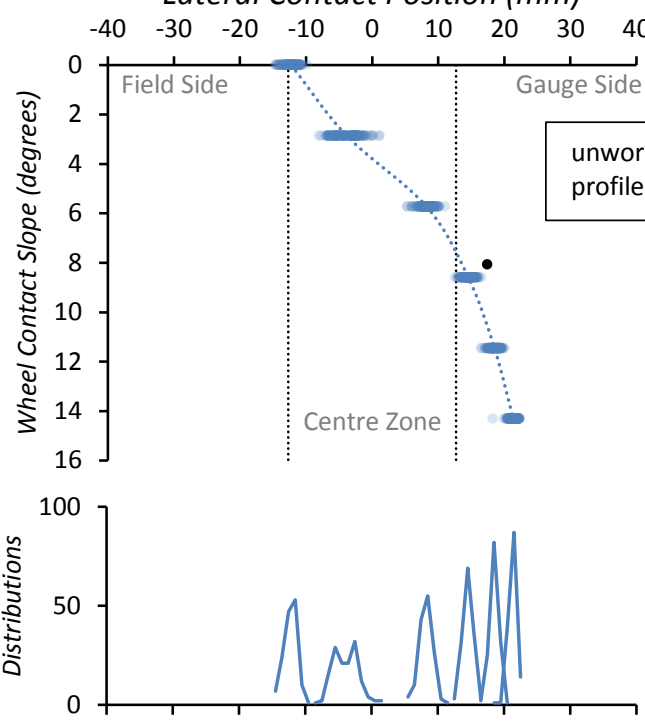

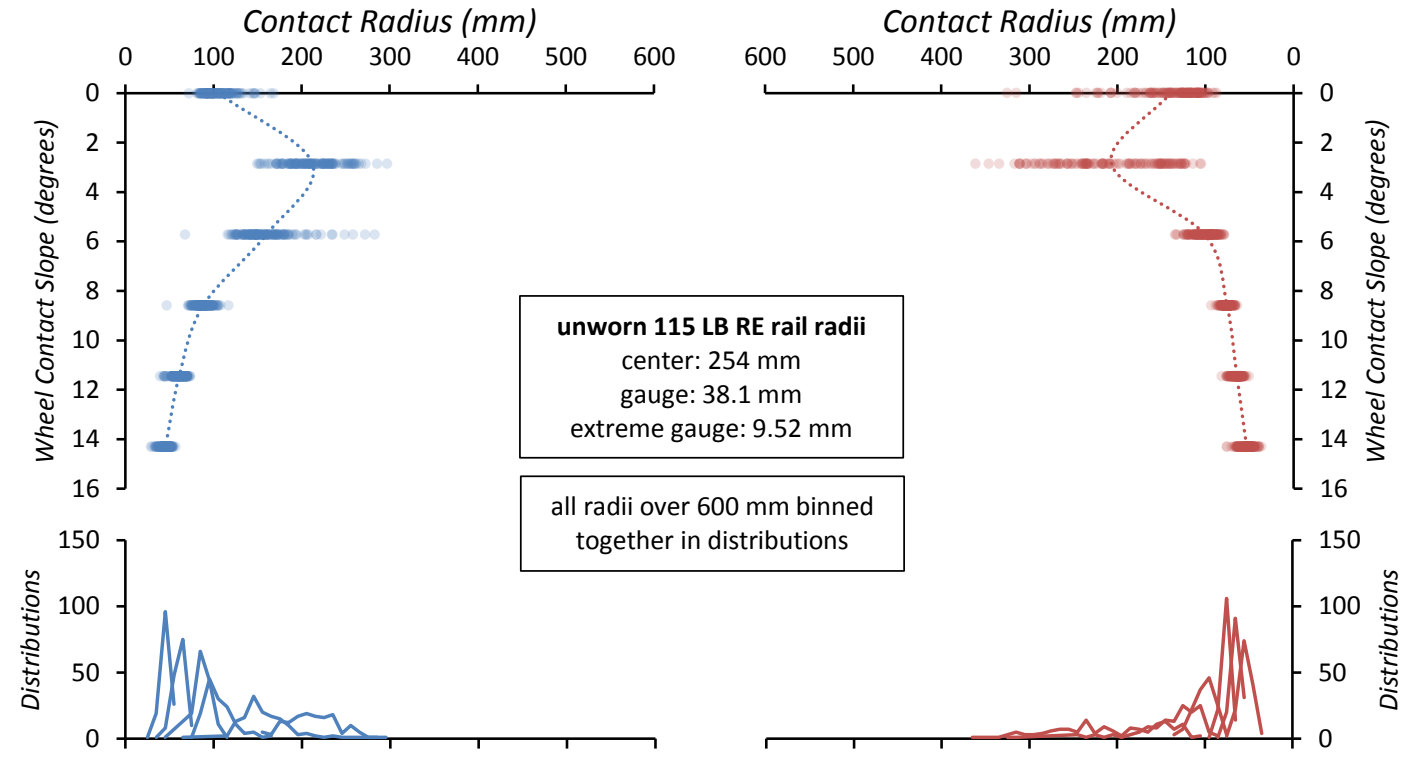

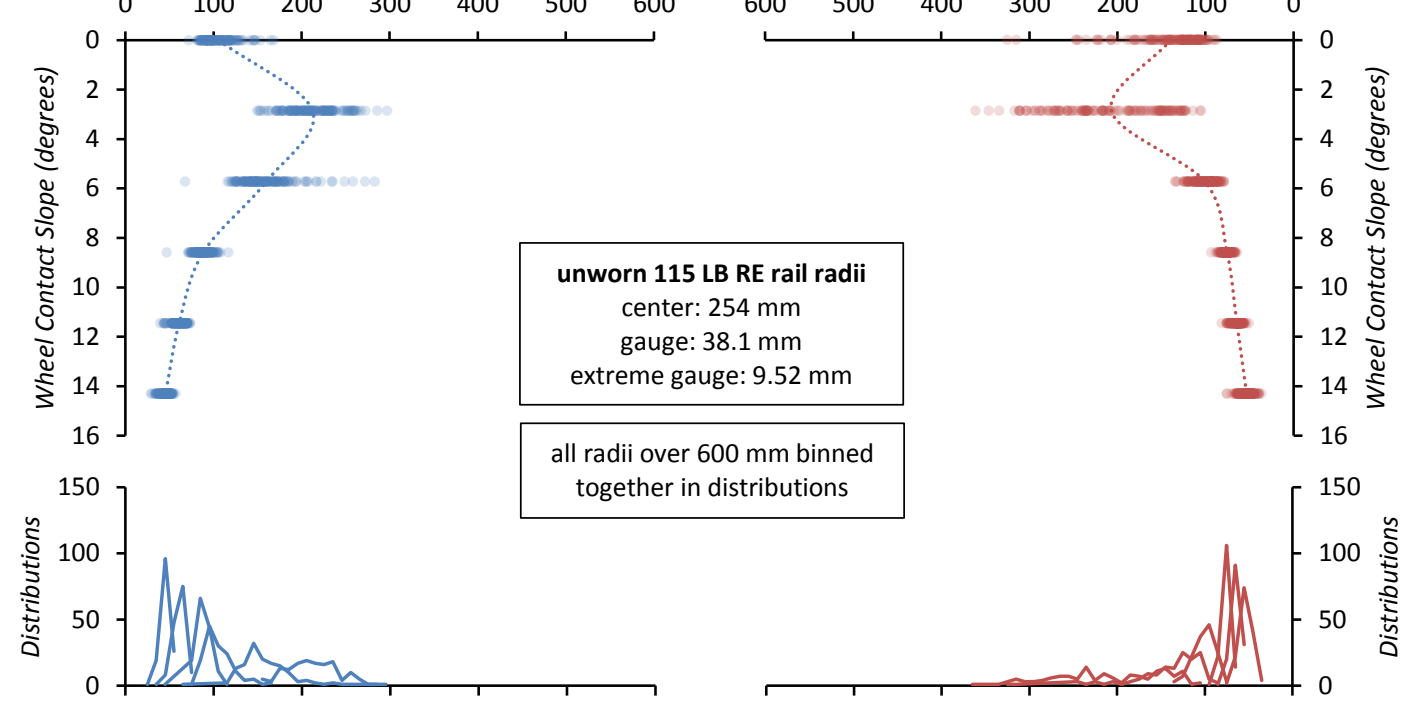

Lateral Contact Position ( $\mathrm{mm}$ )
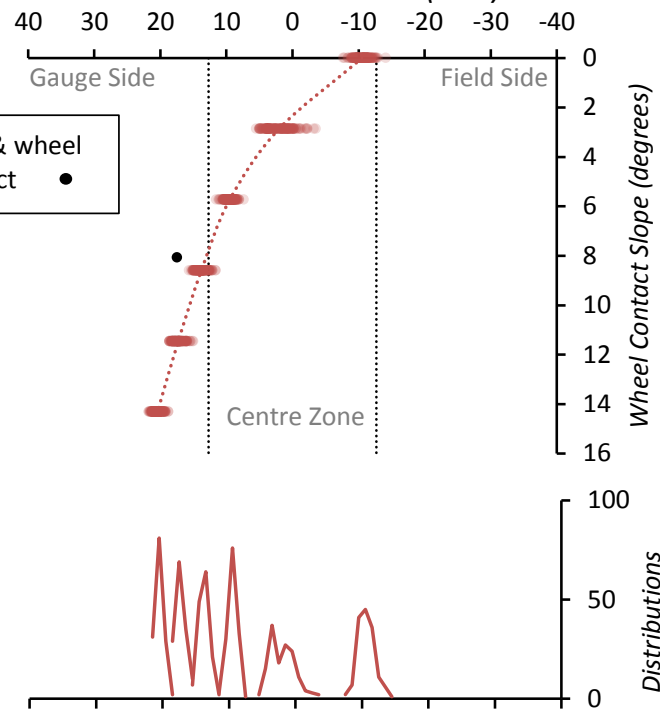
center: $254 \mathrm{~mm}$ gauge: $38.1 \mathrm{~mm}$ extreme gauge: $9.52 \mathrm{~mm}$

Il radii over $600 \mathrm{~mm}$ binned Contact Radius (mm)

LEFT RAIL PROFILES

RIGHT RAIL PROFILES
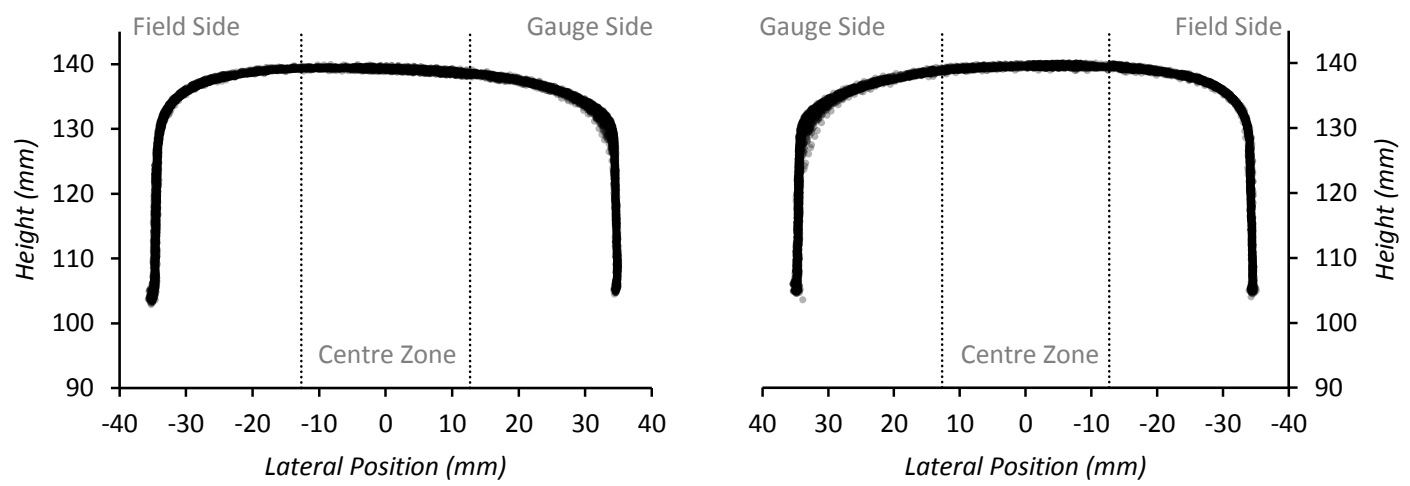
SEGMENT AND ANALYSIS INFORMATION

\begin{tabular}{llll}
\hline \hline Track Segment Name: & NB-68 & Contact Analysis Date: & 20180615 \\
Segment Length $(\mathrm{m}):$ & 346.3 & Left Wheel Slope: & $1: 20$ \\
Data Collection Date: & 20150827 & Right Wheel Slope: & $1: 20$
\end{tabular}

RAIL SEGMENT GRAPHS

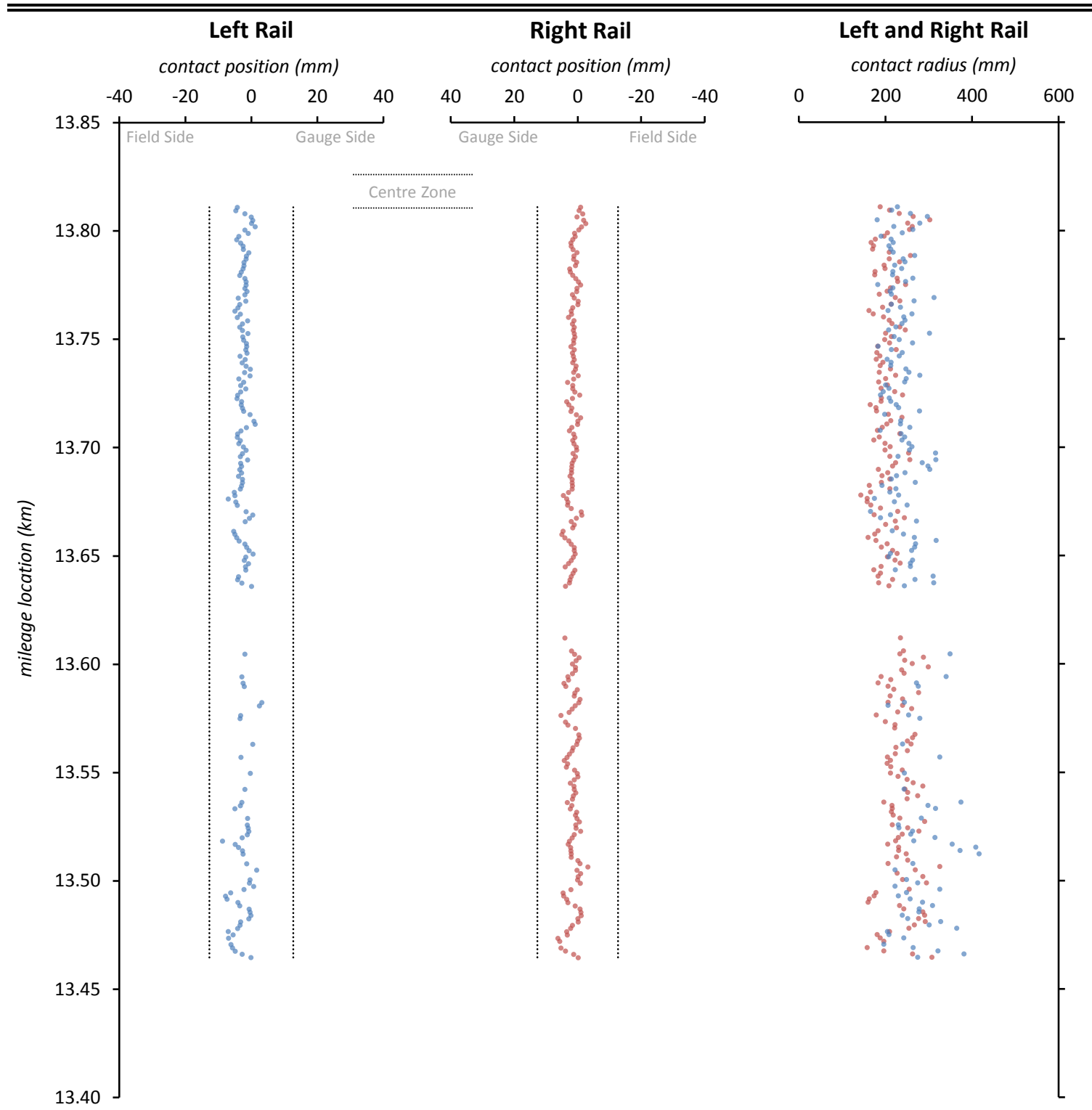

RAIL SEGMENT STATISTICS TABLE

\begin{tabular}{|c|c|c|c|c|}
\hline \multirow{3}{*}{ Statistic } & \multicolumn{4}{|c|}{ Rail Profile Measure } \\
\hline & \multicolumn{2}{|c|}{ Left Rail (165 profiles) } & \multicolumn{2}{|c|}{ Right Rail (211 profiles) } \\
\hline & Contact Position & Contact Radius & Contact Position & Contact Radius \\
\hline Mean $(\mathrm{mm})$ & -2.6 & 251.2 & 1.5 & 217.1 \\
\hline Std. Dev. $(\mathrm{mm})$ & 1.9 & 46.0 & 1.5 & 34.7 \\
\hline$\%$ within Zone & $100 \%$ & - & $100 \%$ & - \\
\hline
\end{tabular}


Lateral Contact Position ( $\mathrm{mm}$ )
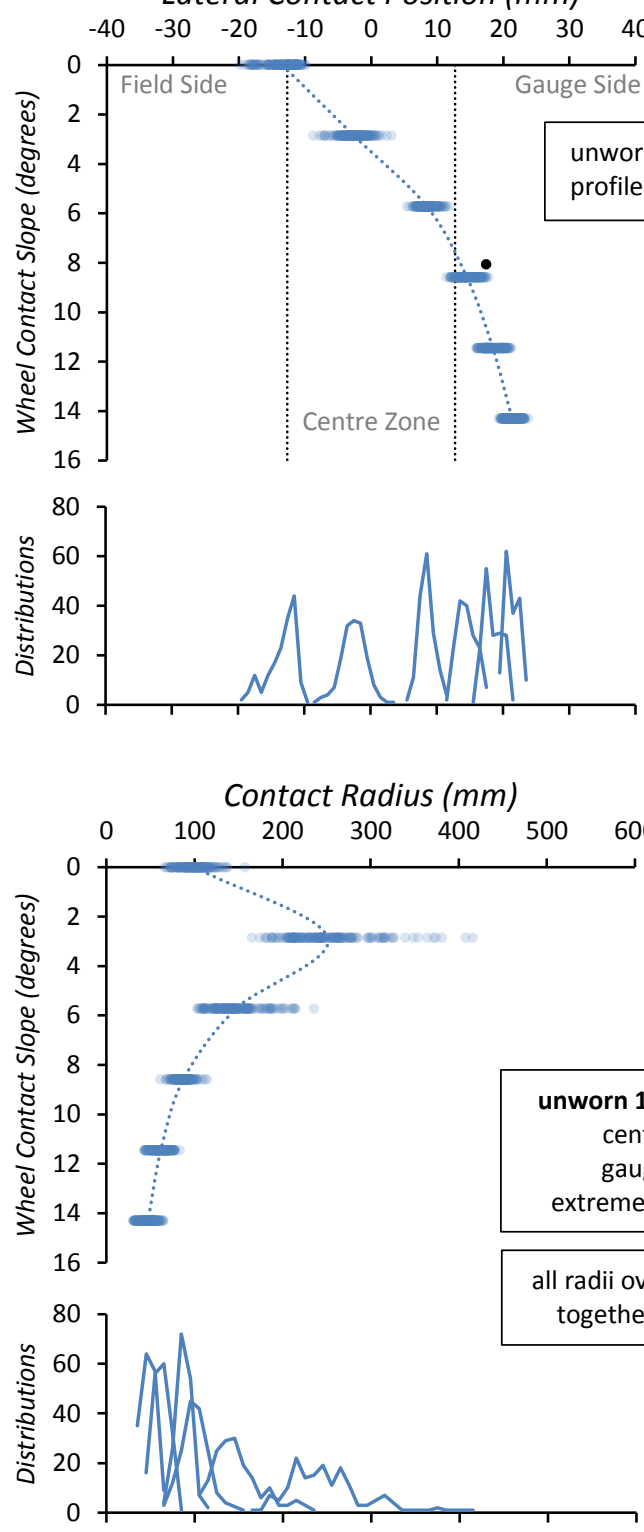

LEFT RAIL PROFILES

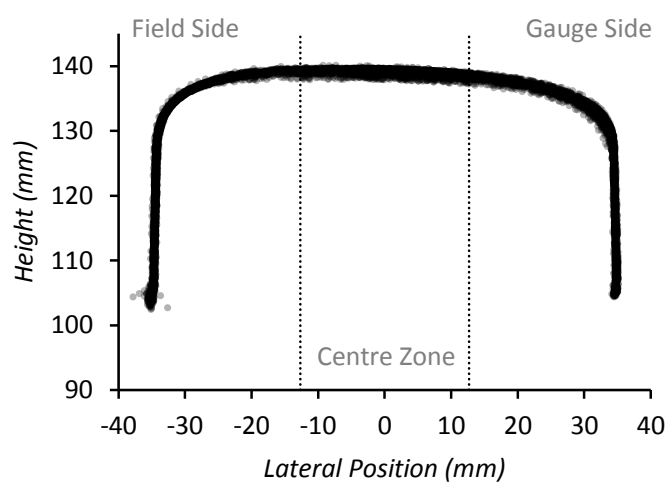

Lateral Contact Position ( $\mathrm{mm}$ )
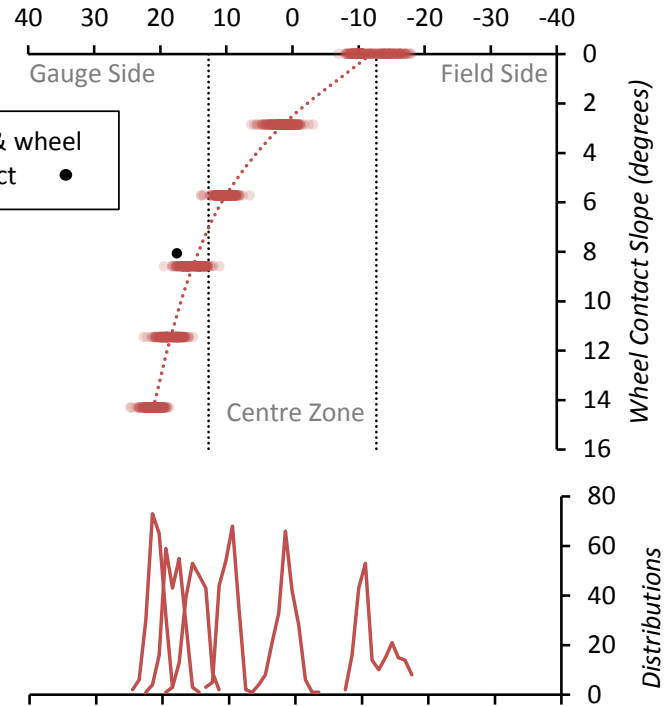

Contact Radius ( $\mathrm{mm}$ )
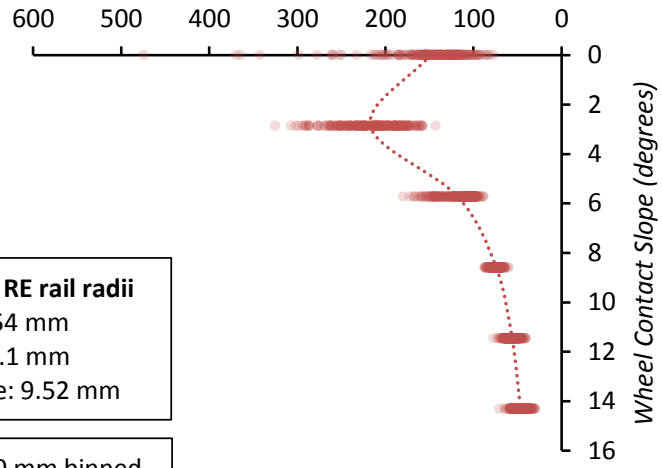

nter: $254 \mathrm{~mm}$

auge: $38.1 \mathrm{~mm}$

eme gauge: $9.52 \mathrm{~mm}$

$600 \mathrm{~mm}$ binned

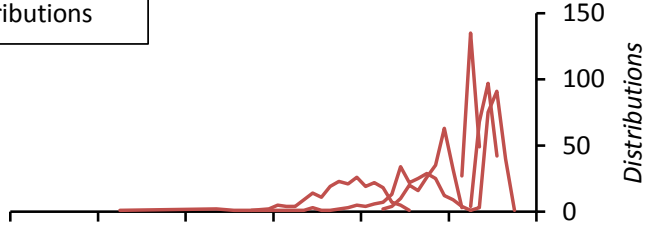


SEGMENT AND ANALYSIS INFORMATION

\begin{tabular}{llll}
\hline \hline Track Segment Name: & NB-78 & Contact Analysis Date: & 20180615 \\
Segment Length $(\mathrm{m}):$ & 312.7 & Left Wheel Slope: & $1: 20$ \\
Data Collection Date: & 20150827 & Right Wheel Slope: & $1: 20$
\end{tabular}

RAIL SEGMENT GRAPHS

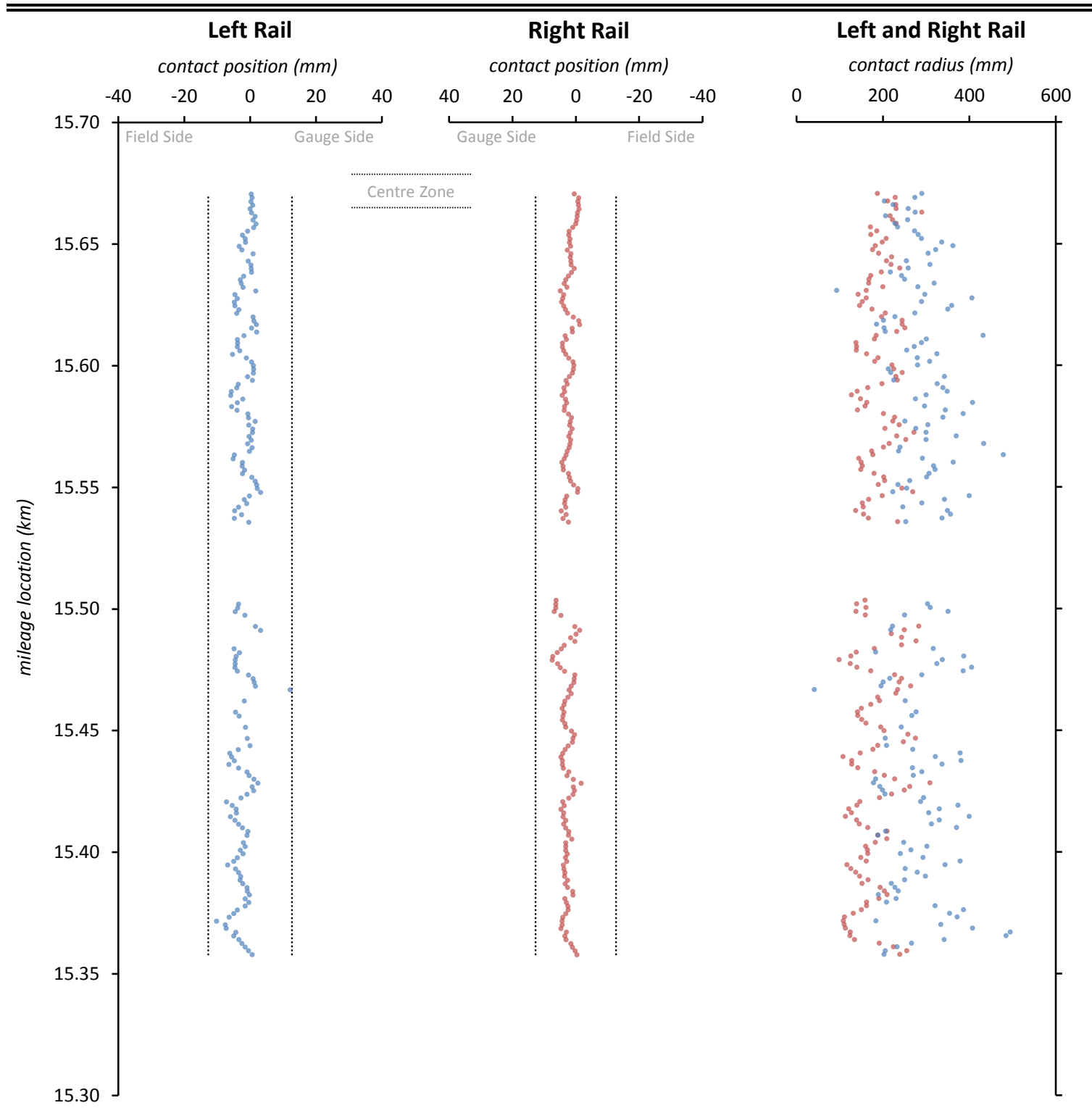

RAIL SEGMENT STATISTICS TABLE

\begin{tabular}{|c|c|c|c|c|}
\hline \multirow{3}{*}{ Statistic } & \multicolumn{4}{|c|}{ Rail Profile Measure } \\
\hline & \multicolumn{2}{|c|}{ Left Rail (167 profiles) } & \multicolumn{2}{|c|}{ Right Rail (183 profiles) } \\
\hline & Contact Position & Contact Radius & Contact Position & Contact Radius \\
\hline Mean $(\mathrm{mm})$ & -1.9 & 286.3 & 2.6 & 184.8 \\
\hline Std. Dev. $(\mathrm{mm})$ & 2.8 & 70.3 & 1.7 & 44.1 \\
\hline$\%$ within Zone & $100 \%$ & - & $100 \%$ & - \\
\hline
\end{tabular}


Lateral Contact Position ( $\mathrm{mm}$ )

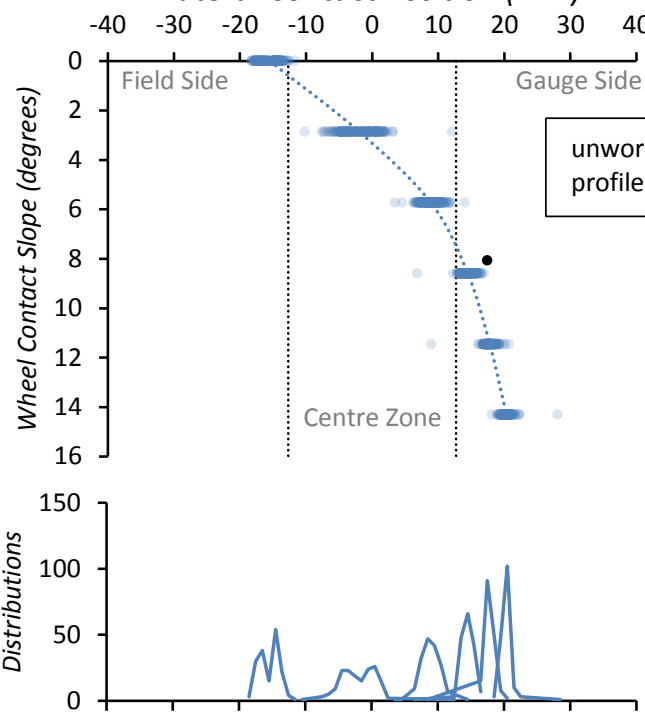

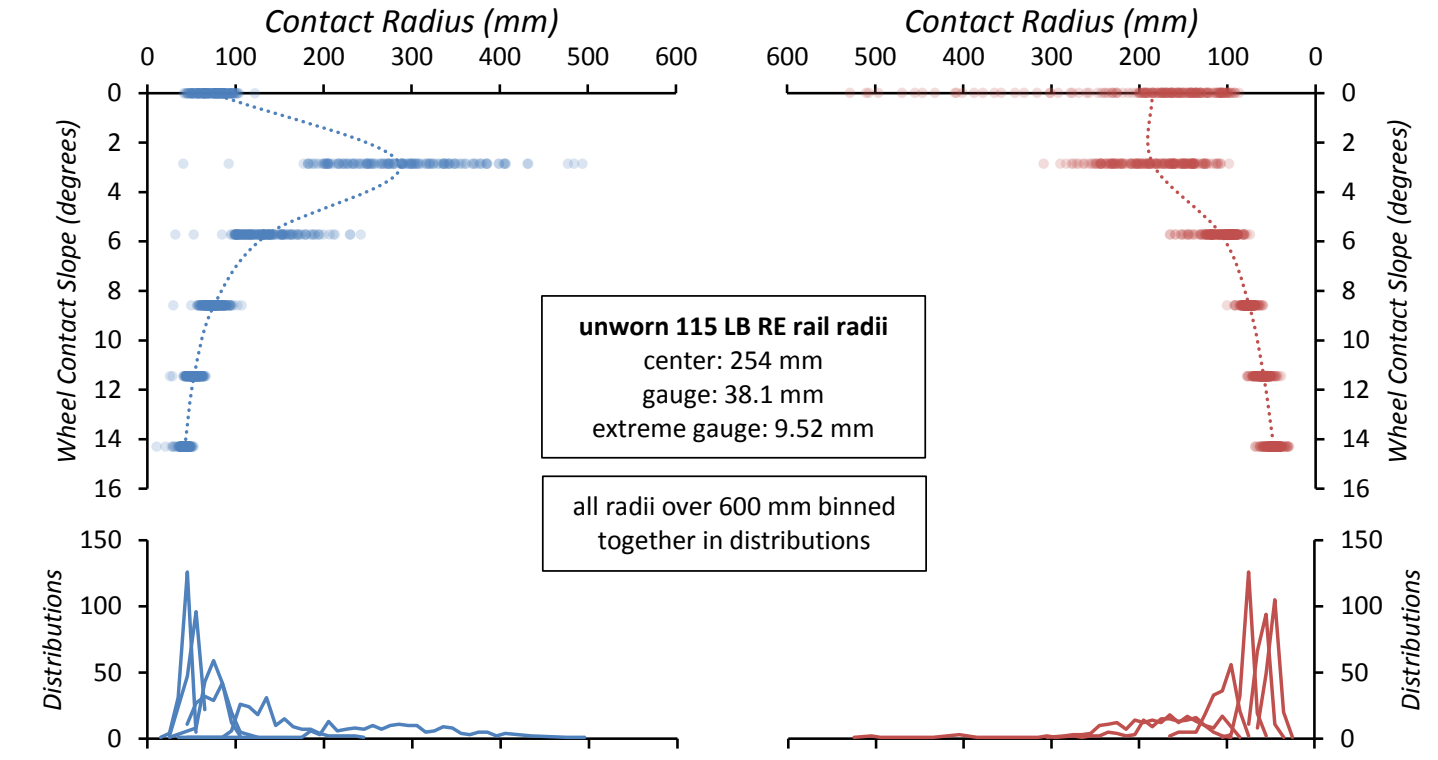

Lateral Contact Position ( $\mathrm{mm}$ )
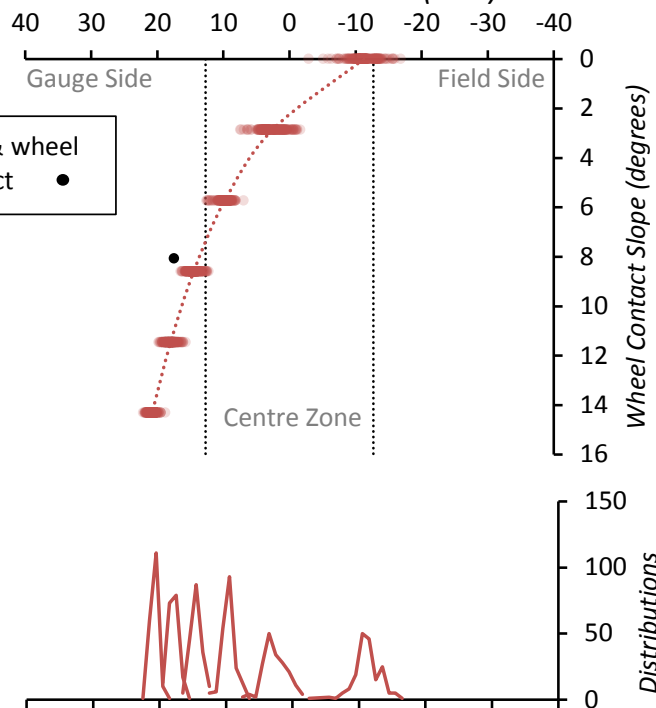

Contact Radius ( $\mathrm{mm}$ )

LEFT RAIL PROFILES

RIGHT RAIL PROFILES
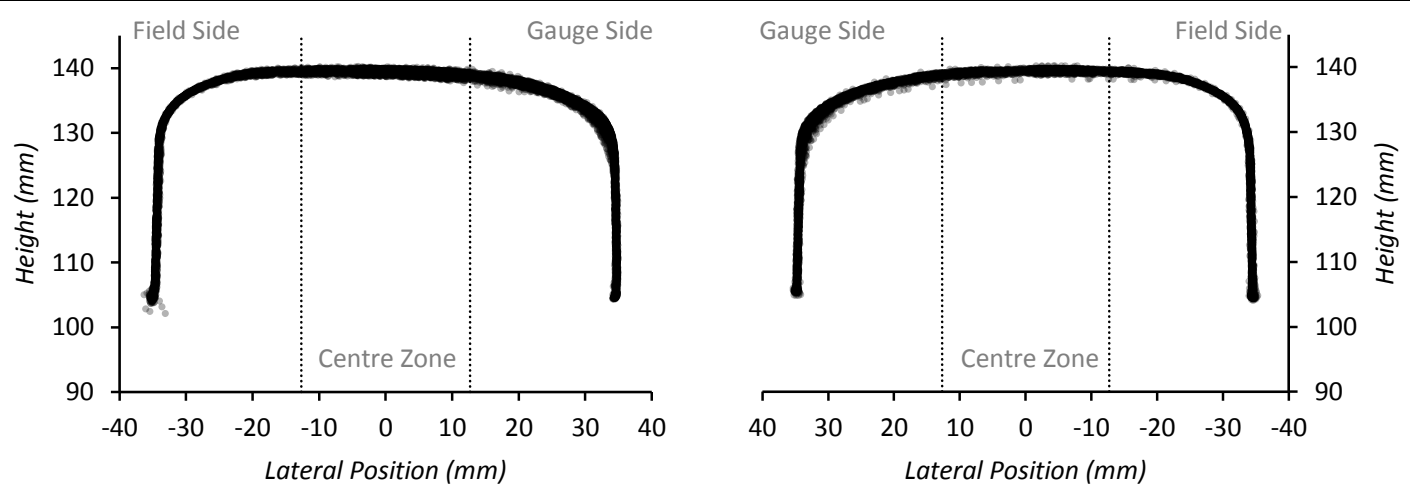
SEGMENT AND ANALYSIS INFORMATION

\begin{tabular}{llll}
\hline \hline Track Segment Name: & NB-80 & Contact Analysis Date: & 20180615 \\
Segment Length $(\mathrm{m}):$ & 876.6 & Left Wheel Slope: & $1: 20$ \\
Data Collection Date: & 20150827 & Right Wheel Slope: & $1: 20$
\end{tabular}

RAIL SEGMENT GRAPHS

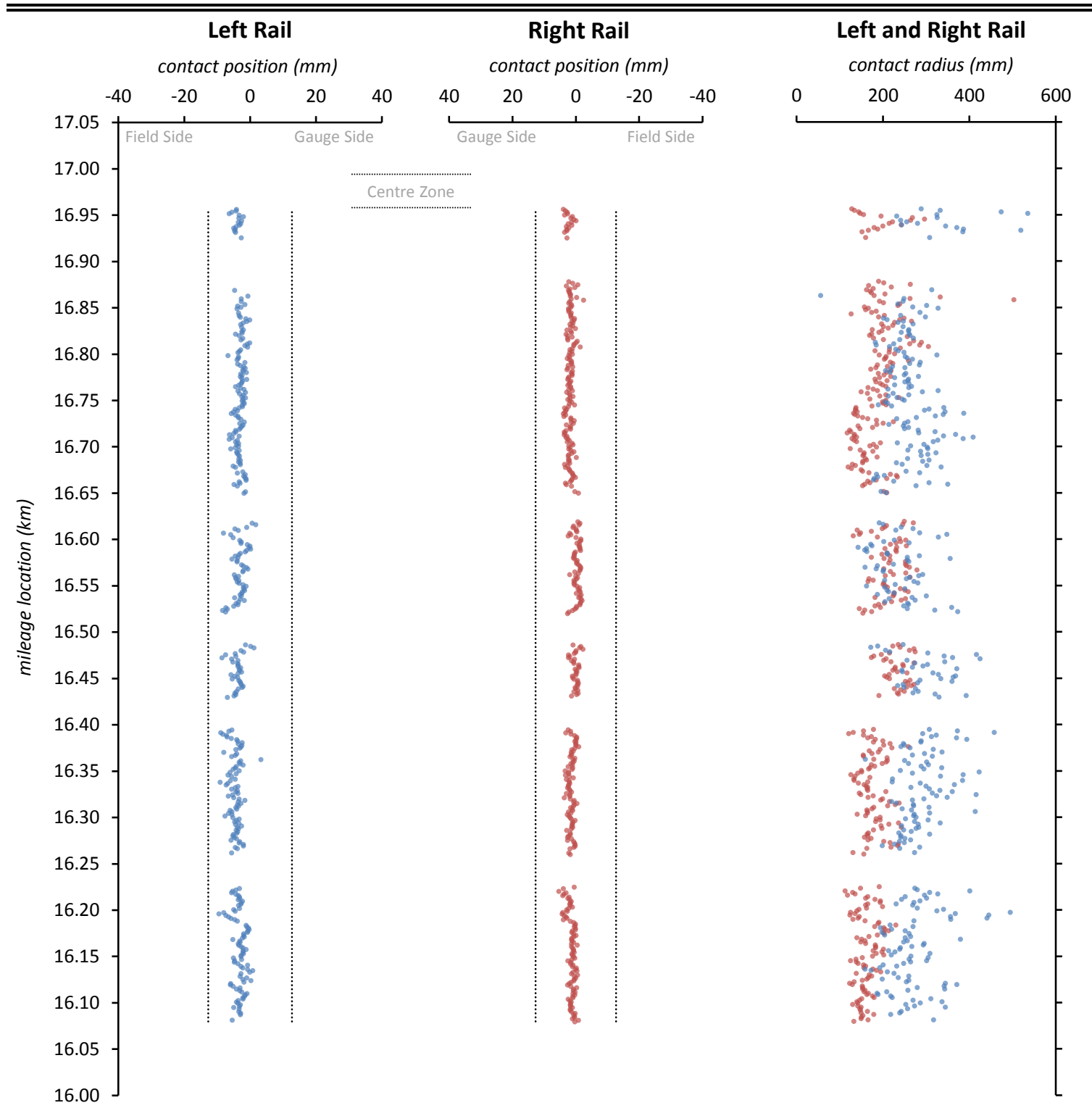

RAIL SEGMENT STATISTICS TABLE

\begin{tabular}{|c|c|c|c|c|}
\hline \multirow{3}{*}{ Statistic } & \multicolumn{4}{|c|}{ Rail Profile Measure } \\
\hline & \multicolumn{2}{|c|}{ Left Rail (400 profiles) } & \multicolumn{2}{|c|}{ Right Rail (448 profiles) } \\
\hline & Contact Position & Contact Radius & Contact Position & Contact Radius \\
\hline Mean (mm) & -3.5 & 273.3 & 1.2 & 188.0 \\
\hline Std. Dev. $(\mathrm{mm})$ & 1.8 & 62.0 & 1.3 & 42.3 \\
\hline$\%$ within Zone & $100 \%$ & - & $100 \%$ & - \\
\hline
\end{tabular}


Lateral Contact Position ( $\mathrm{mm}$ )

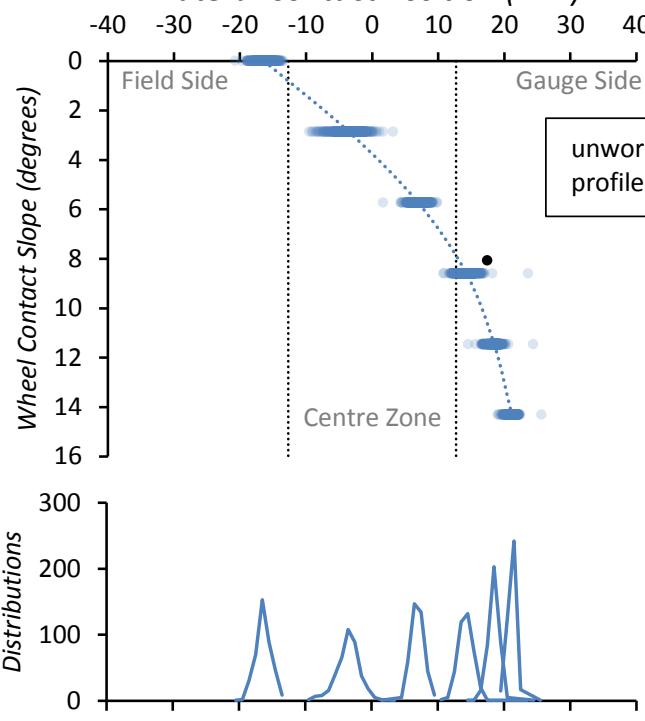

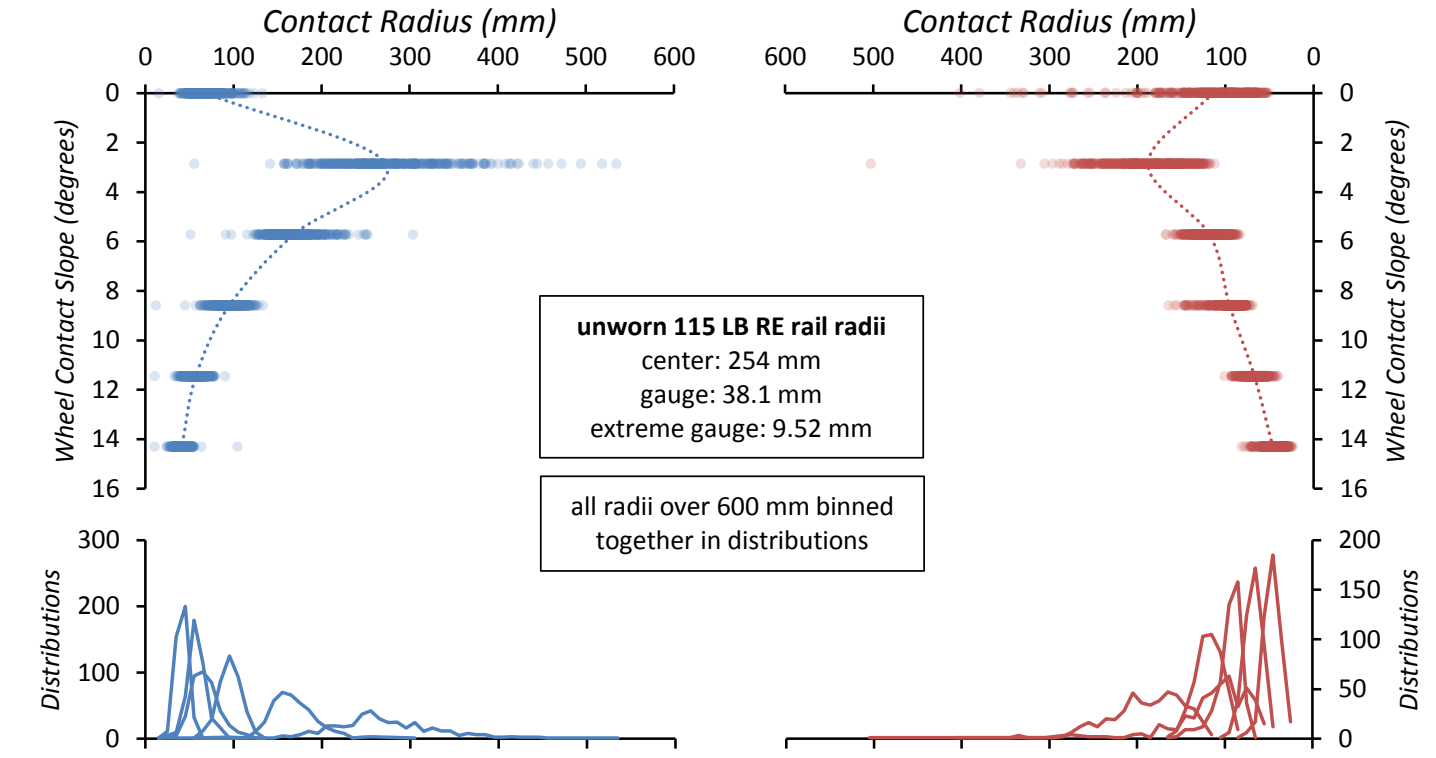

Lateral Contact Position ( $\mathrm{mm}$ )
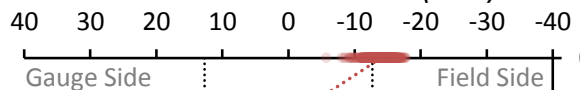

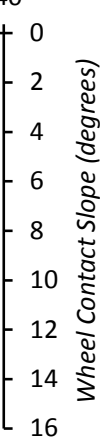

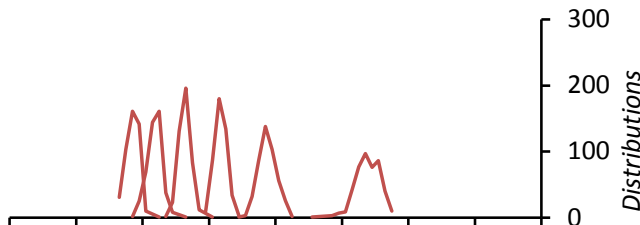

Contact Radius ( $\mathrm{mm}$ )

LEFT RAIL PROFILES

RIGHT RAIL PROFILES
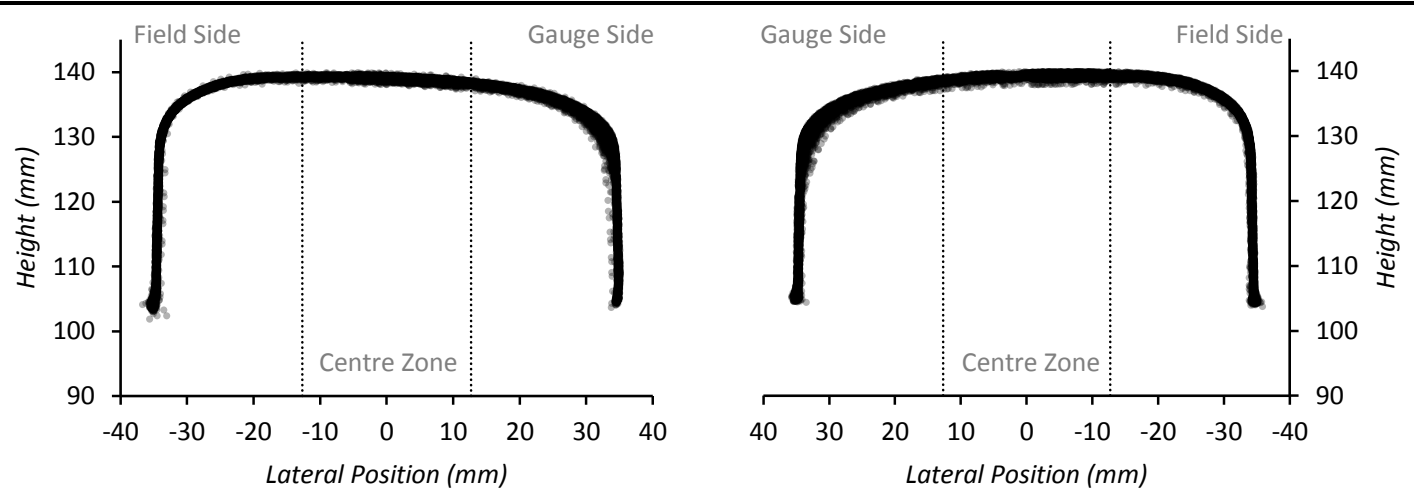
SEGMENT AND ANALYSIS INFORMATION

\begin{tabular}{llll}
\hline \hline Track Segment Name: & NB-91 & Contact Analysis Date: & 20180615 \\
Segment Length $(\mathrm{m}):$ & 1600.2 & Left Wheel Slope: & $1: 20$ \\
Data Collection Date: & 20150827 & Right Wheel Slope: & $1: 20$
\end{tabular}

RAIL SEGMENT GRAPHS

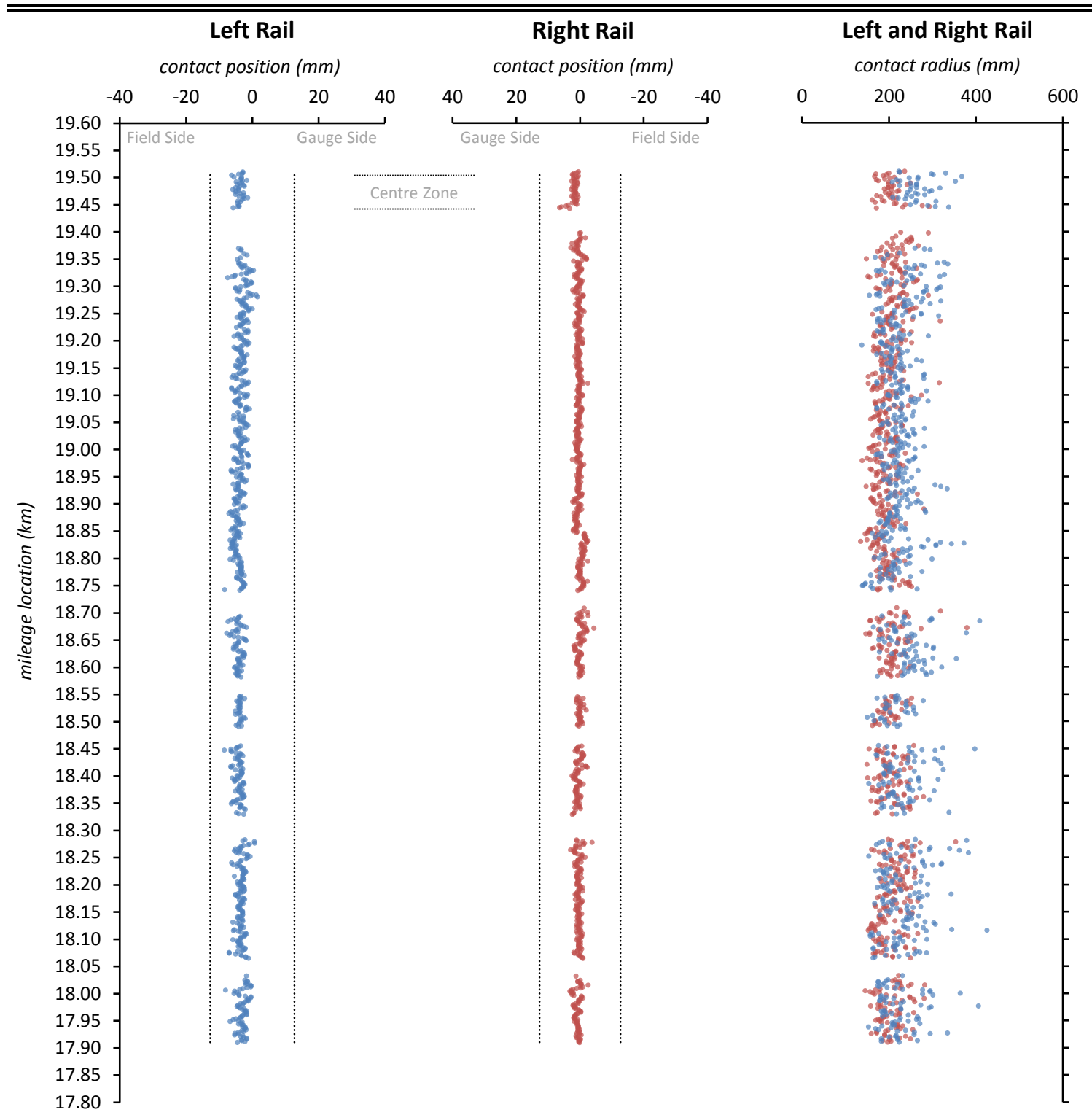

RAIL SEGMENT STATISTICS TABLE

\begin{tabular}{|c|c|c|c|c|}
\hline \multirow{3}{*}{ Statistic } & \multicolumn{4}{|c|}{ Rail Profile Measure } \\
\hline & \multicolumn{2}{|c|}{ Left Rail (812 profiles) } & \multicolumn{2}{|c|}{ Right Rail (888 profiles) } \\
\hline & Contact Position & Contact Radius & Contact Position & Contact Radius \\
\hline Mean $(\mathrm{mm})$ & -3.7 & 229.6 & 0.4 & 202.3 \\
\hline Std. Dev. $(\mathrm{mm})$ & 1.4 & 44.6 & 1.0 & 29.9 \\
\hline$\%$ within Zone & $100 \%$ & - & $100 \%$ & - \\
\hline
\end{tabular}


Lateral Contact Position ( $\mathrm{mm}$ )
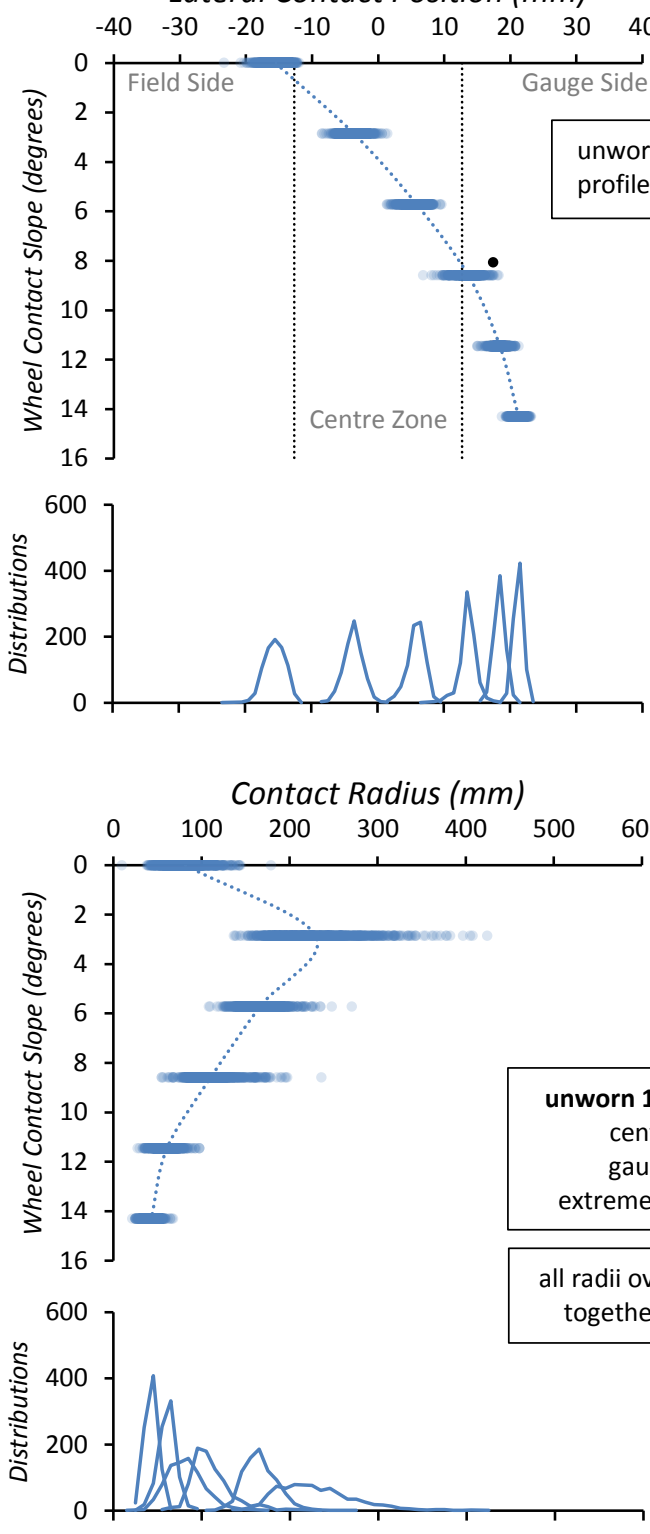

LEFT RAIL PROFILES

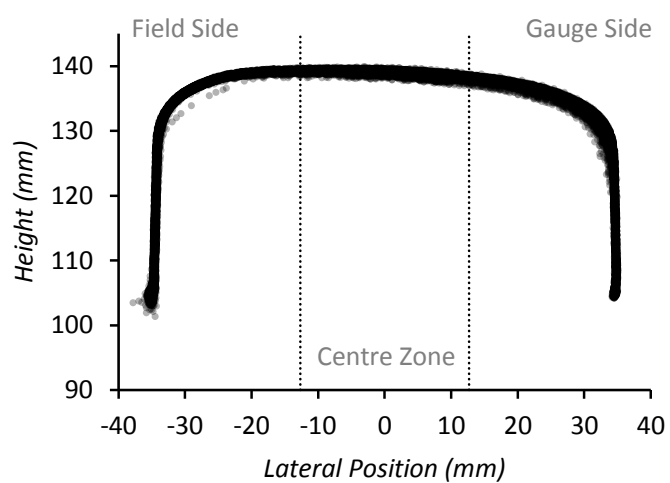

Lateral Contact Position ( $\mathrm{mm}$ )
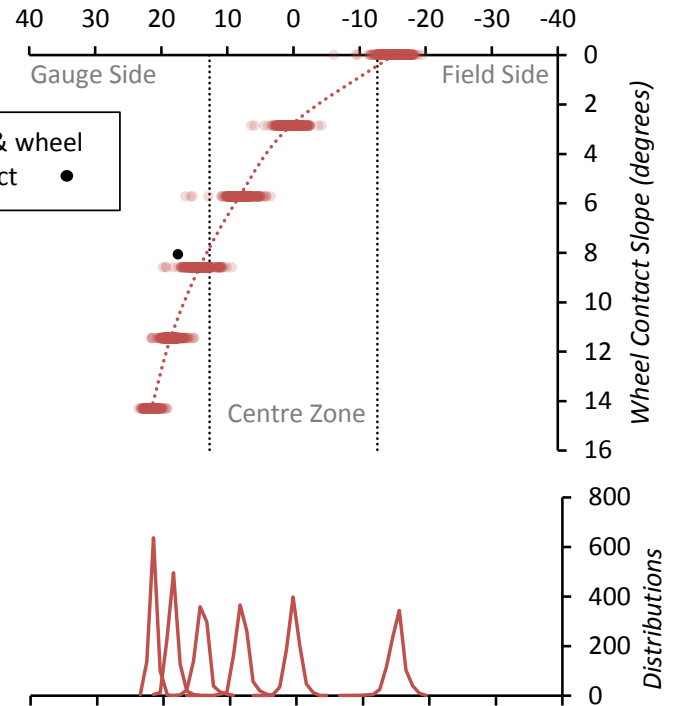

Contact Radius ( $\mathrm{mm}$ )
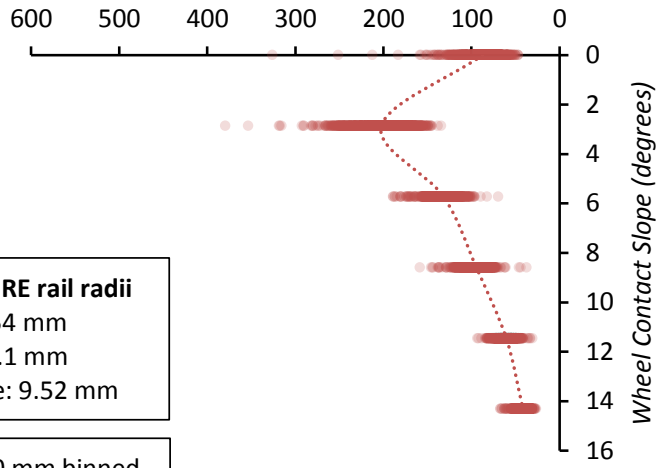

enter: $254 \mathrm{~mm}$

gauge: $38.1 \mathrm{~mm}$

eme gauge: $9.52 \mathrm{~mm}$

$600 \mathrm{~mm}$ binned

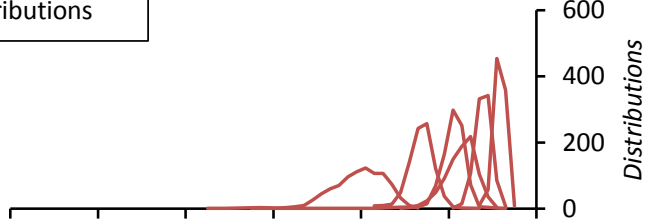


SEGMENT AND ANALYSIS INFORMATION

\begin{tabular}{llll}
\hline \hline Track Segment Name: & NB-98 & Contact Analysis Date: & 20180615 \\
Segment Length $(m):$ & 143.3 & Left Wheel Slope: & $1: 20$ \\
Data Collection Date: & 20150827 & Right Wheel Slope: & $1: 20$
\end{tabular}

RAIL SEGMENT GRAPHS

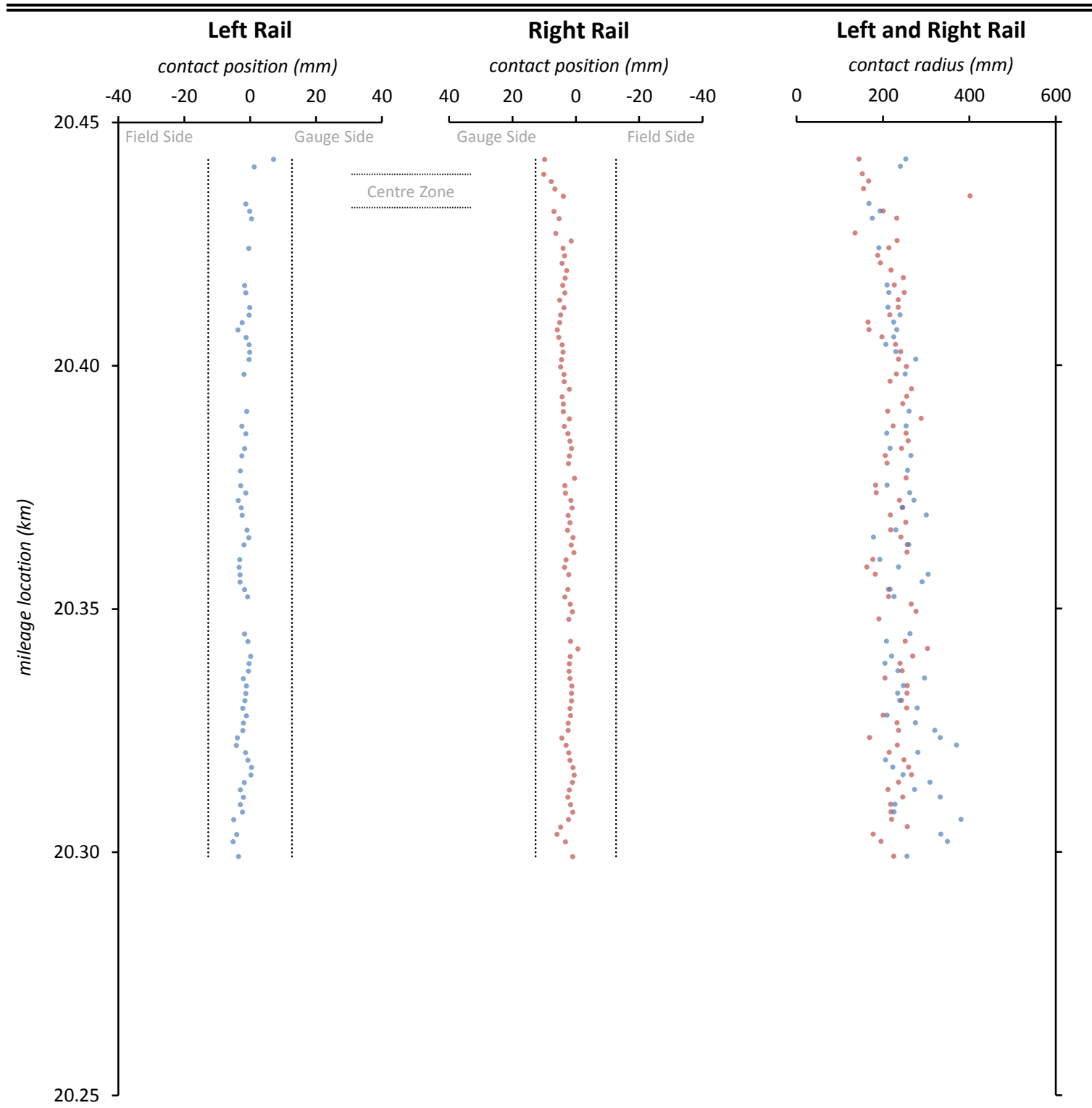

RAIL SEGMENT STATISTICS TABLE

\begin{tabular}{|c|c|c|c|c|}
\hline \multirow{3}{*}{ Statistic } & \multicolumn{4}{|c|}{ Rail Profile Measure } \\
\hline & \multicolumn{2}{|c|}{ Left Rail (65 profiles) } & \multicolumn{2}{|c|}{ Right Rail (87 profiles) } \\
\hline & Contact Position & Contact Radius & Contact Position & Contact Radius \\
\hline Mean $(\mathrm{mm})$ & -1.7 & 248.9 & 3.1 & 225.6 \\
\hline Std. Dev. $(\mathrm{mm})$ & 1.8 & 46.4 & 1.9 & 38.9 \\
\hline$\%$ within Zone & $100 \%$ & - & $100 \%$ & - \\
\hline
\end{tabular}


Lateral Contact Position ( $\mathrm{mm}$ )
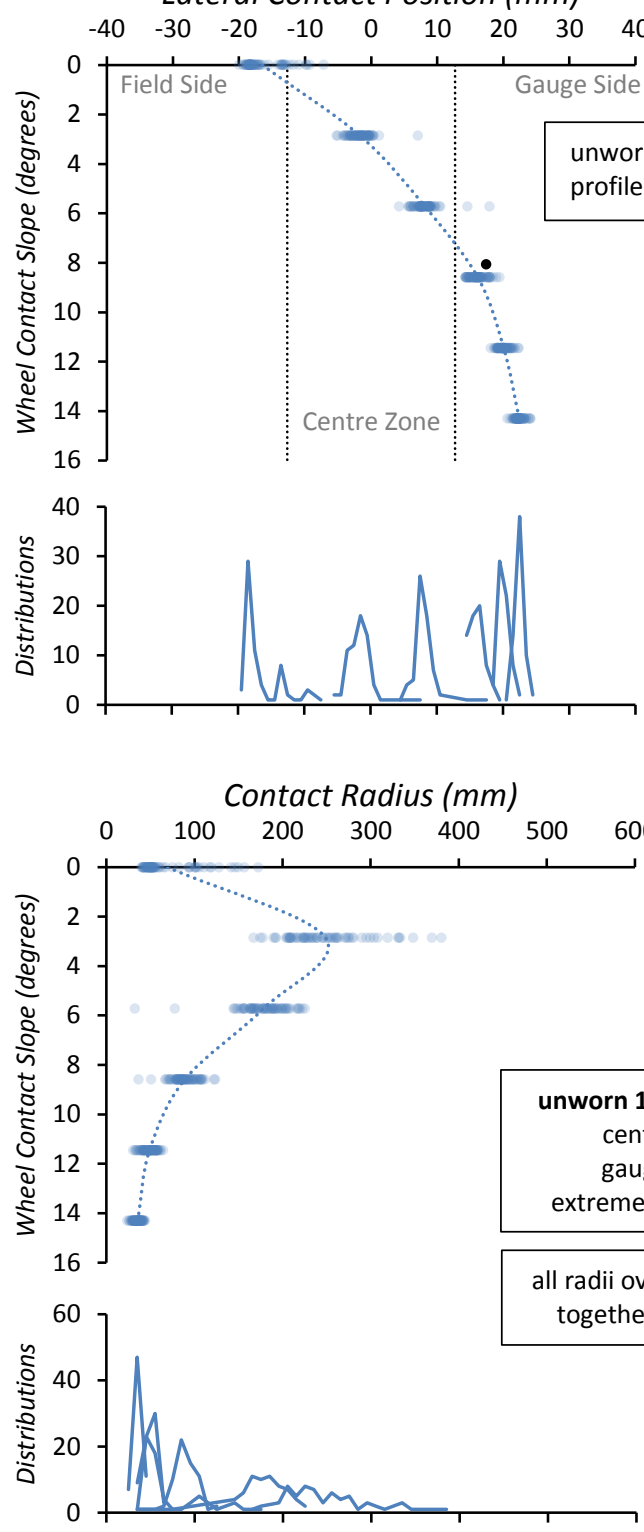

Lateral Contact Position ( $\mathrm{mm}$ )
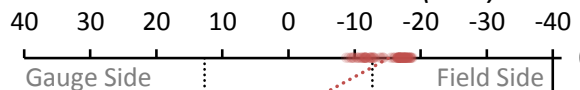

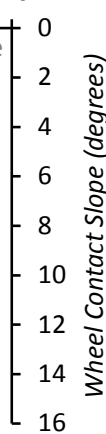

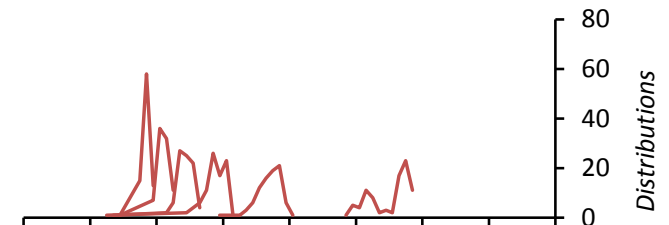

Contact Radius ( $\mathrm{mm}$ ) worn 115 LB $R E$ rail radi

center: $254 \mathrm{~mm}$

gauge: $38.1 \mathrm{~mm}$

treme gauge: $9.52 \mathrm{~mm}$

radii over $600 \mathrm{~mm}$ binned

$$
\text { ibutions }
$$

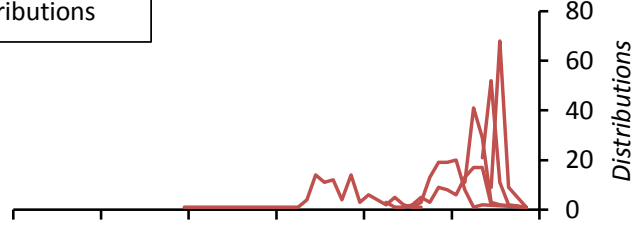

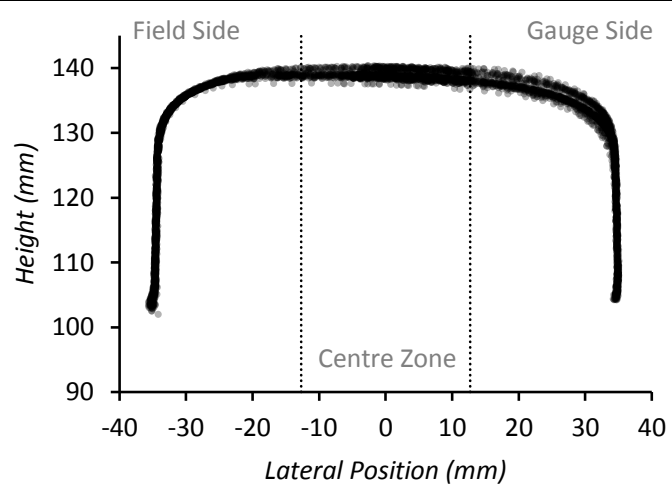

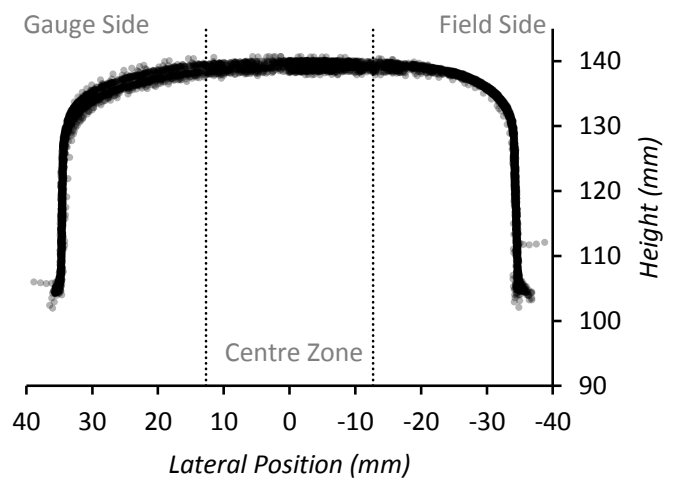


APPENDIX E

SOUTHBOUND TRACK SEGMENT ANALYSIS AND SENSITIVITY

ANALYSIS RESULTS 
SEGMENT AND ANALYSIS INFORMATION

\begin{tabular}{llll}
\hline \hline Track Segment Name: & SB-11 & Contact Analysis Date: & 20180615 \\
Segment Length $(\mathrm{m}):$ & 624.8 & Left Wheel Slope: & $1: 20$ \\
Data Collection Date: & 20150827 & Right Wheel Slope: & $1: 20$
\end{tabular}

RAIL SEGMENT GRAPHS

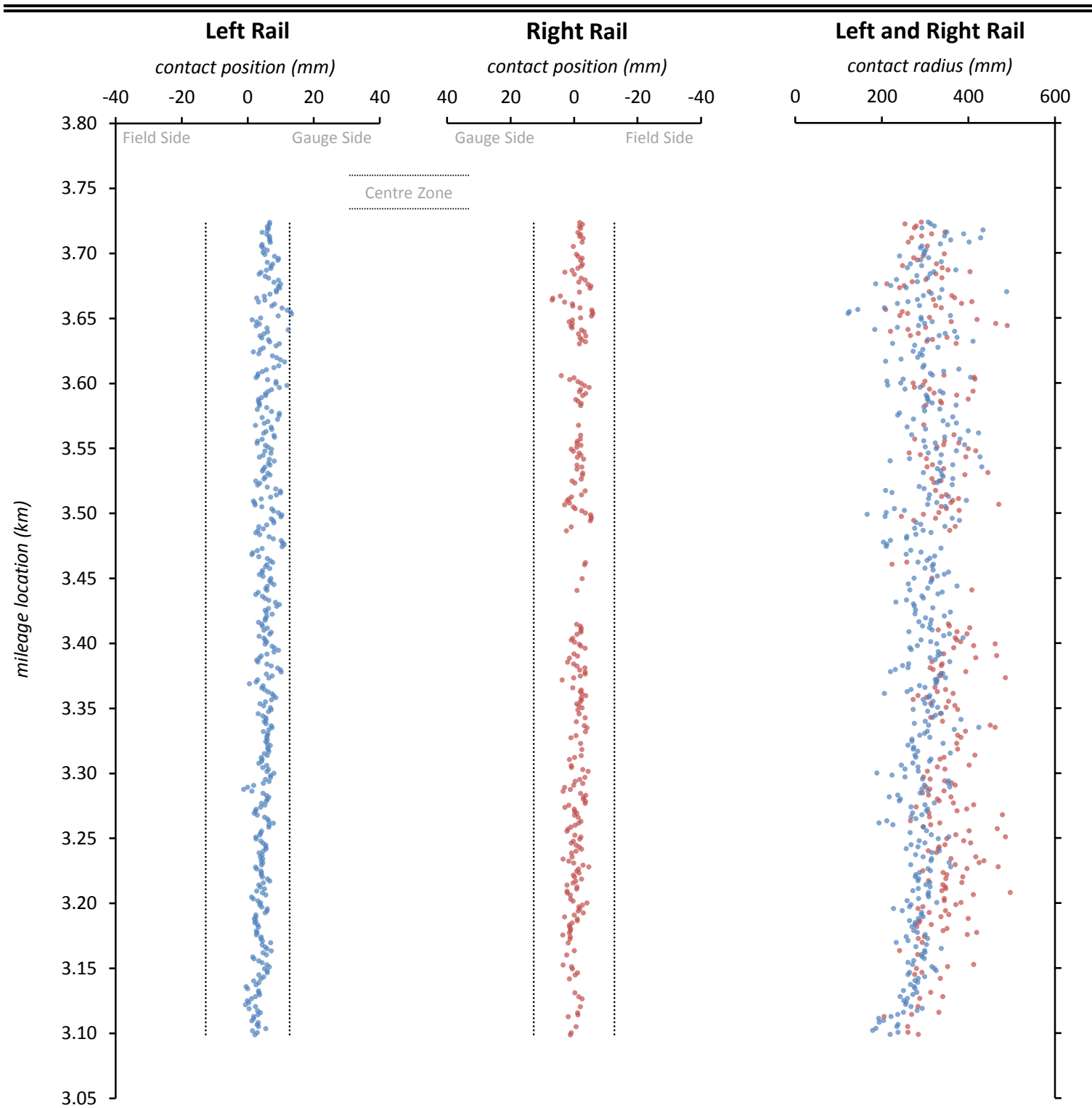

RAIL SEGMENT STATISTICS TABLE

\begin{tabular}{|c|c|c|c|c|}
\hline \multirow{3}{*}{ Statistic } & \multicolumn{4}{|c|}{ Rail Profile Measure } \\
\hline & \multicolumn{2}{|c|}{ Left Rail (406 profiles) } & \multicolumn{2}{|c|}{ Right Rail (260 profiles) } \\
\hline & Contact Position & Contact Radius & Contact Position & Contact Radius \\
\hline Mean $(\mathrm{mm})$ & 5.3 & 294.3 & -0.9 & 338.8 \\
\hline Std. Dev. $(\mathrm{mm})$ & 2.4 & 49.7 & 2.2 & 56.0 \\
\hline$\%$ within Zone & $100 \%$ & - & $100 \%$ & - \\
\hline
\end{tabular}

$\mathrm{E}-1$ 
Lateral Contact Position ( $\mathrm{mm}$ )
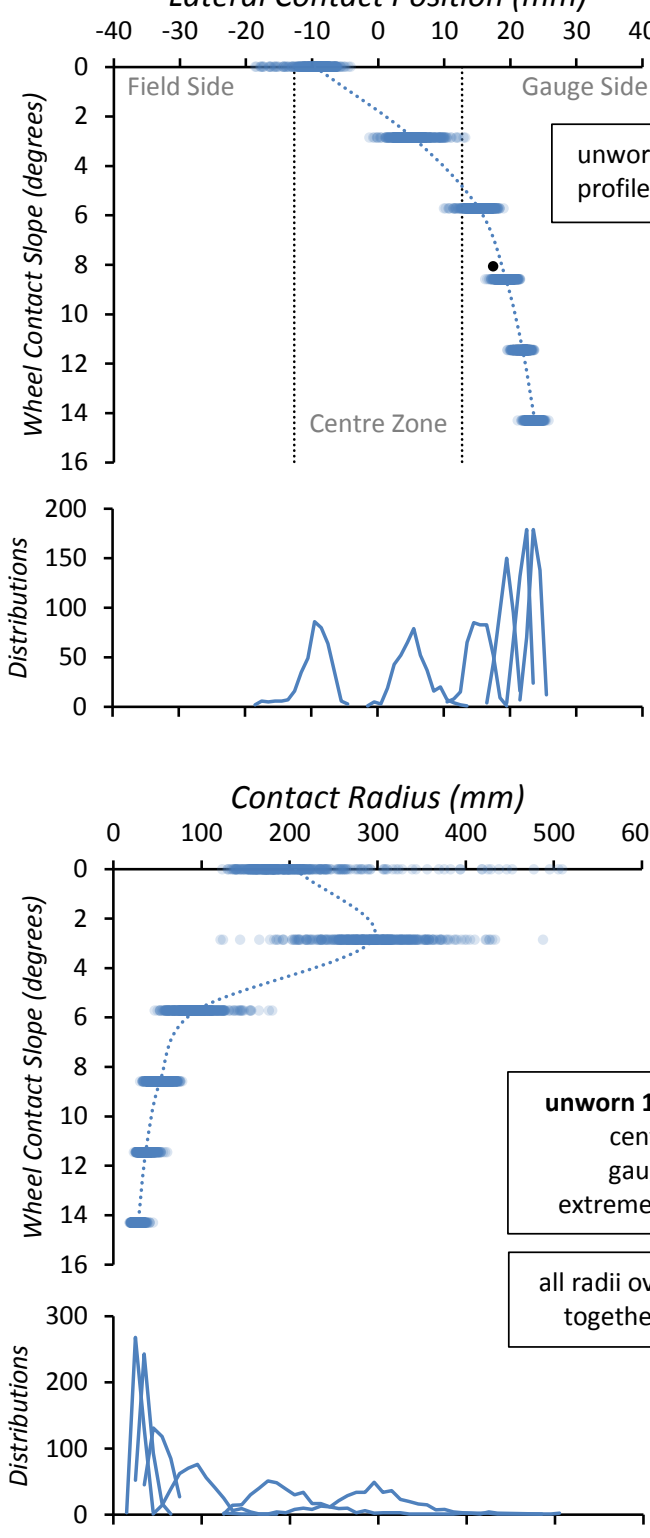

LEFT RAIL PROFILES

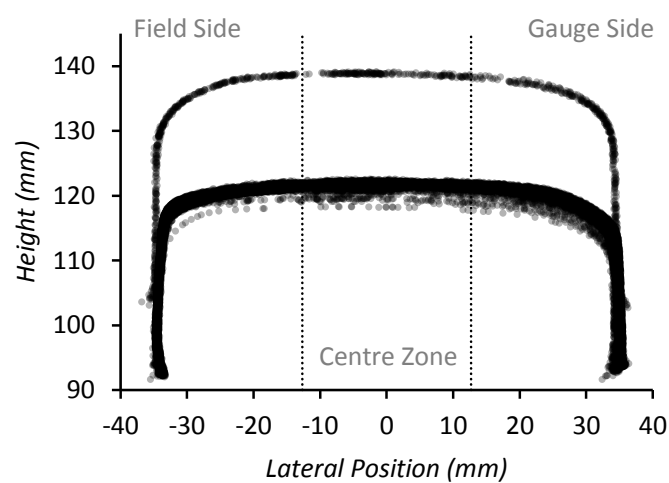

Lateral Contact Position ( $\mathrm{mm}$ )

$\begin{array}{lllllllll}40 & 30 & 20 & 10 & 0 & -10 & -20 & -30 & -40\end{array}$
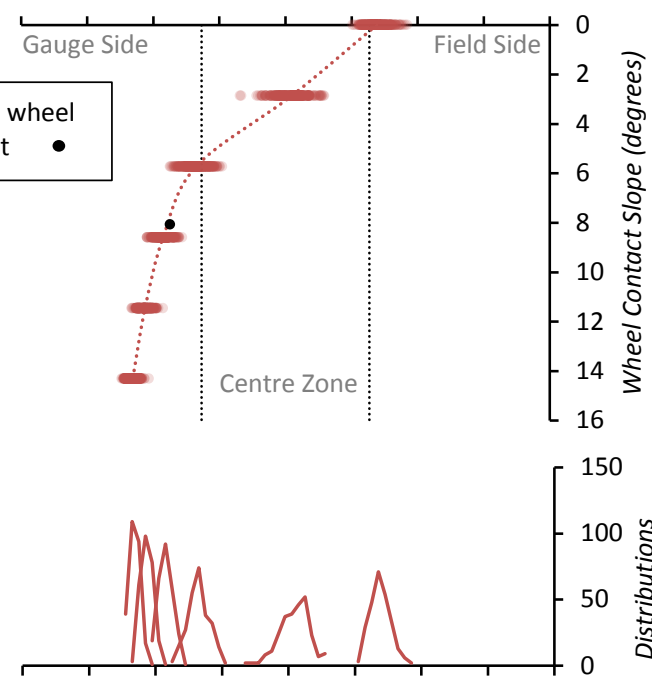

\section{Contact Radius ( $\mathrm{mm}$ )}

$\begin{array}{lllllll}600 & 500 & 400 & 300 & 200 & 100 & 0\end{array}$

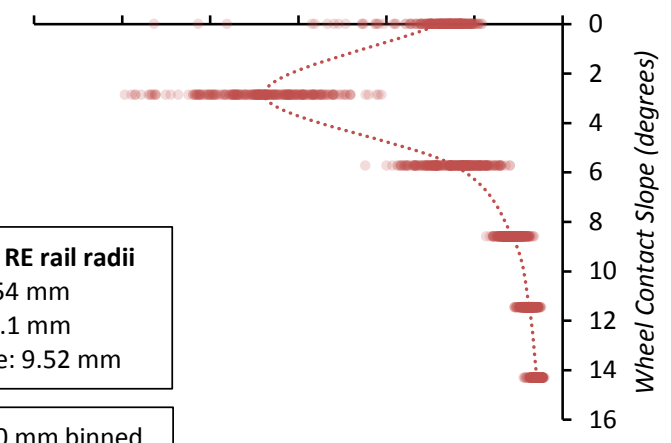

nter: $254 \mathrm{~mm}$

auge: $38.1 \mathrm{~mm}$

eme gauge: $9.52 \mathrm{~mm}$

$600 \mathrm{~mm}$ binned

ibutions

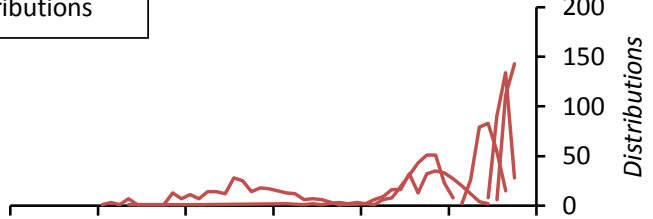

RIGHT RAIL PROFILES

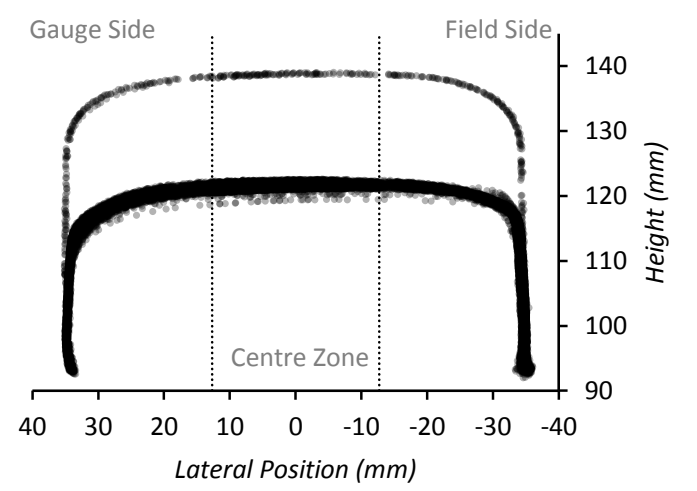

E-2 
SEGMENT AND ANALYSIS INFORMATION

\begin{tabular}{llll}
\hline \hline Track Segment Name: & SB-13 & Contact Analysis Date: & 20180615 \\
Segment Length $(\mathrm{m}):$ & 837.6 & Left Wheel Slope: & $1: 20$ \\
Data Collection Date: & 20150827 & Right Wheel Slope: & $1: 20$
\end{tabular}

RAIL SEGMENT GRAPHS

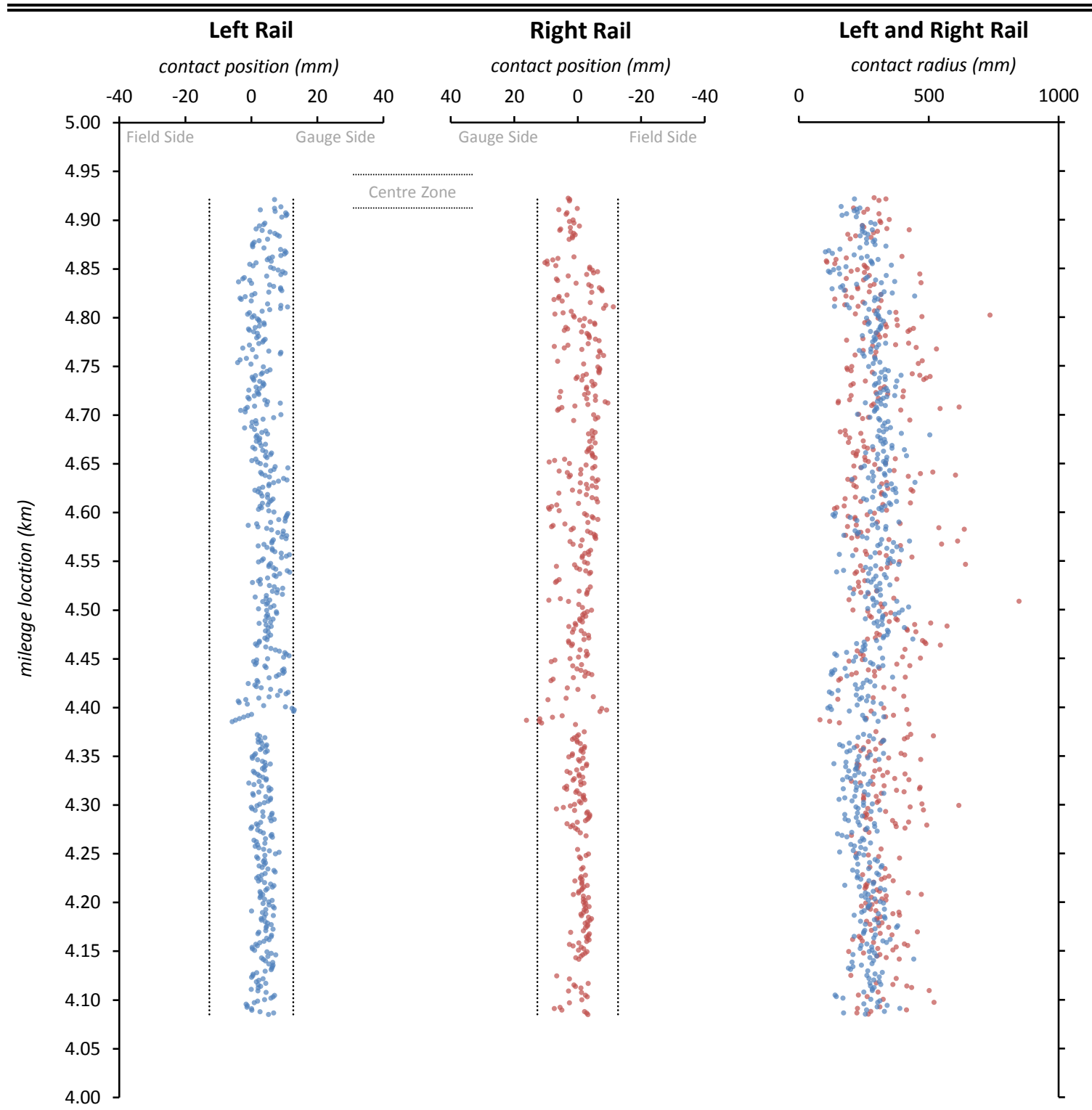

RAIL SEGMENT STATISTICS TABLE

\begin{tabular}{|c|c|c|c|c|}
\hline \multirow{3}{*}{ Statistic } & \multicolumn{4}{|c|}{ Rail Profile Measure } \\
\hline & \multicolumn{2}{|c|}{ Left Rail (521 profiles) } & \multicolumn{2}{|c|}{ Right Rail (418 profiles) } \\
\hline & Contact Position & Contact Radius & Contact Position & Contact Radius \\
\hline Mean $(\mathrm{mm})$ & 4.0 & 271.5 & -0.4 & 311.3 \\
\hline Std. Dev. $(\mathrm{mm})$ & 3.3 & 65.3 & 4.2 & 104.0 \\
\hline$\%$ within Zone & $100 \%$ & - & $100 \%$ & - \\
\hline
\end{tabular}


Lateral Contact Position ( $\mathrm{mm}$ )

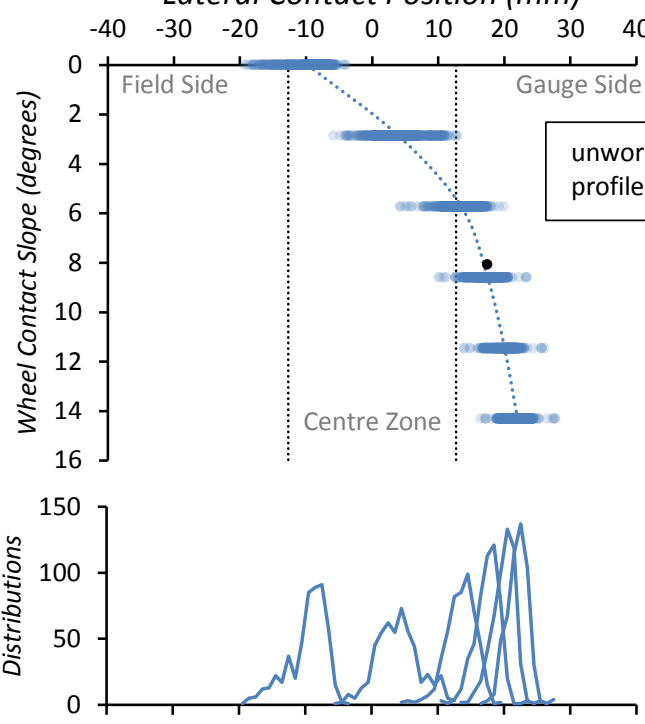

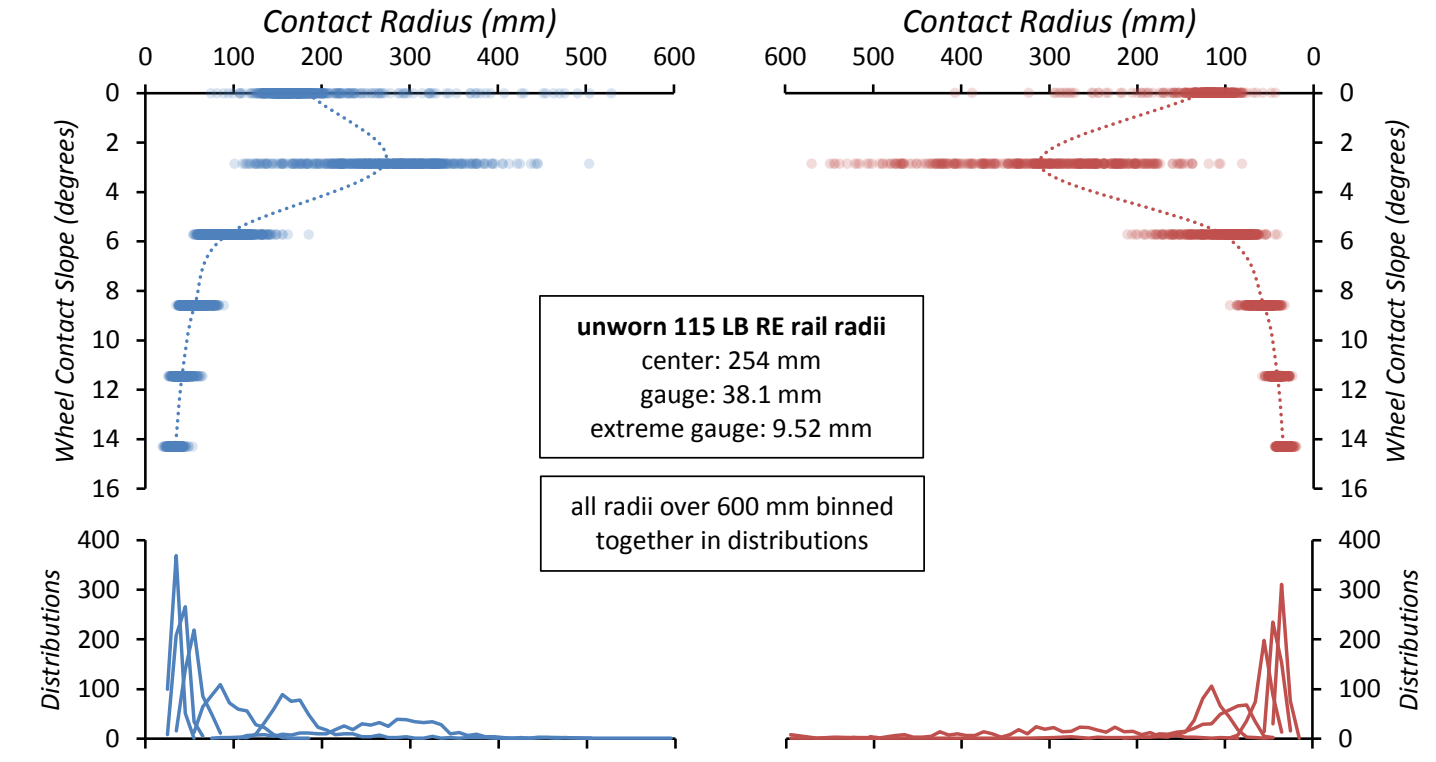

Lateral Contact Position ( $\mathrm{mm}$ )
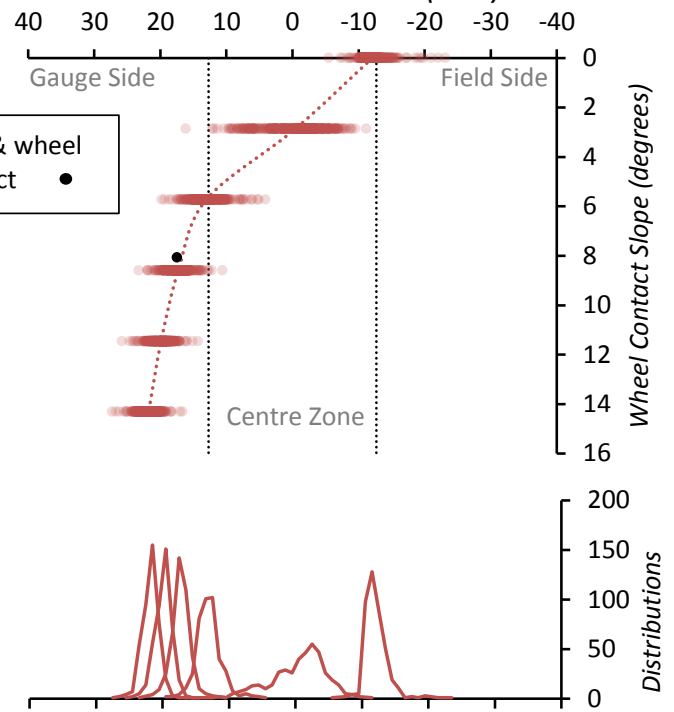

Contact Radius ( $\mathrm{mm}$ )

LEFT RAIL PROFILES

RIGHT RAIL PROFILES
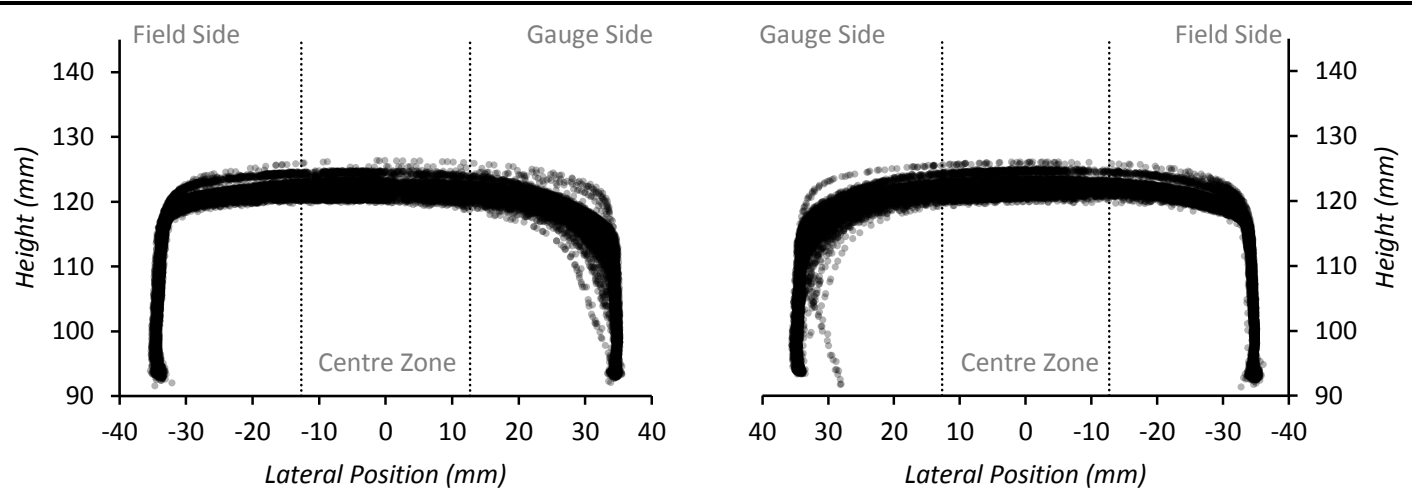
SEGMENT AND ANALYSIS INFORMATION

\begin{tabular}{llll}
\hline \hline Track Segment Name: & SB-16 & Contact Analysis Date: & 20180615 \\
Segment Length $(\mathrm{m}):$ & 882.9 & Left Wheel Slope: & $1: 20$ \\
Data Collection Date: & 20150827 & Right Wheel Slope: & $1: 20$
\end{tabular}

RAIL SEGMENT GRAPHS

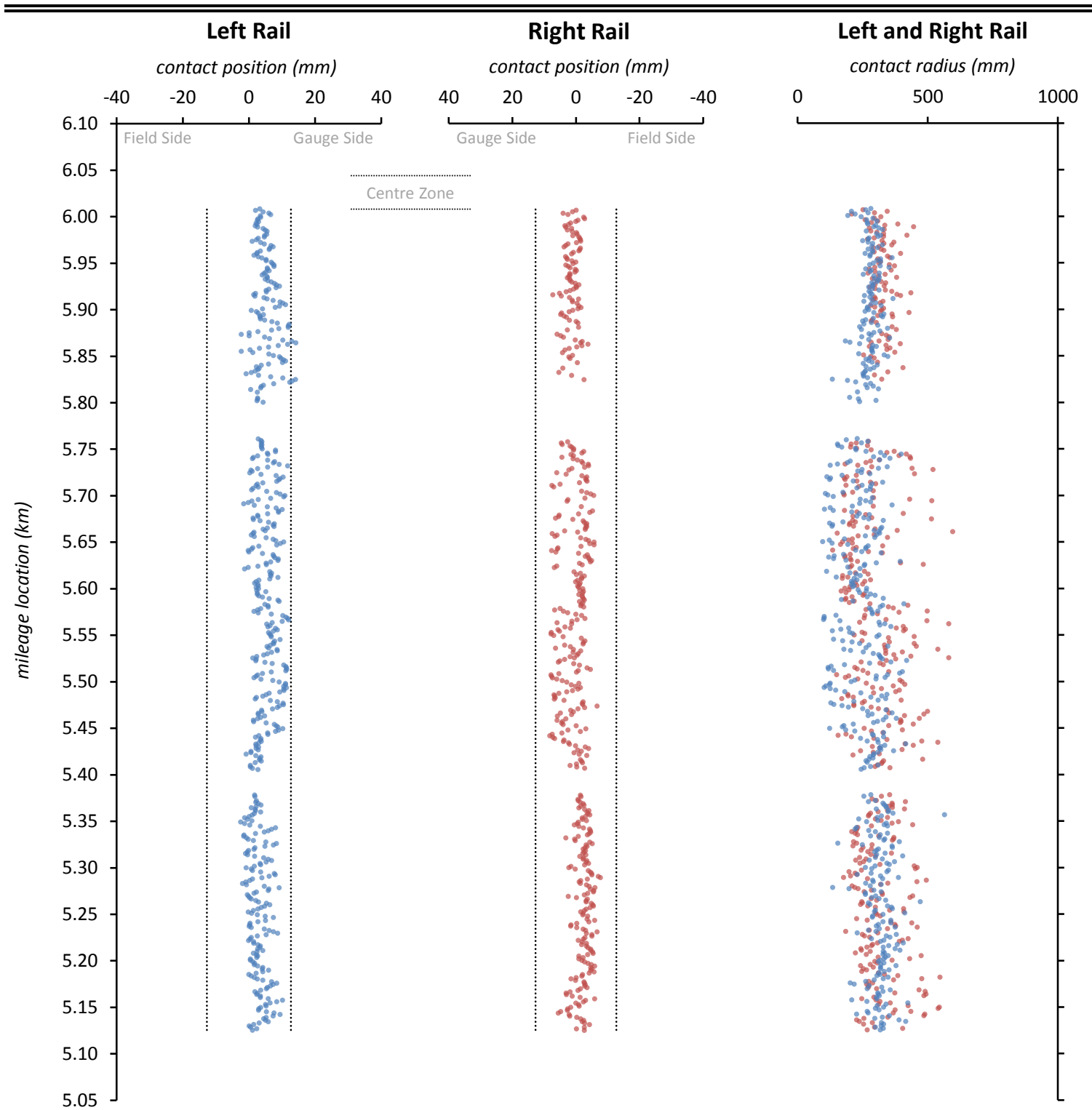

RAIL SEGMENT STATISTICS TABLE

\begin{tabular}{|c|c|c|c|c|}
\hline \multirow{3}{*}{ Statistic } & \multicolumn{4}{|c|}{ Rail Profile Measure } \\
\hline & \multicolumn{2}{|c|}{ Left Rail (527 profiles) } & \multicolumn{2}{|c|}{ Right Rail (475 profiles) } \\
\hline & Contact Position & Contact Radius & Contact Position & Contact Radius \\
\hline Mean $(\mathrm{mm})$ & 4.5 & 274.7 & -0.2 & 311.8 \\
\hline Std. Dev. $(\mathrm{mm})$ & 3.3 & 68.8 & 3.4 & 86.1 \\
\hline$\%$ within Zone & $99 \%$ & - & $100 \%$ & - \\
\hline
\end{tabular}


Lateral Contact Position ( $\mathrm{mm}$ )

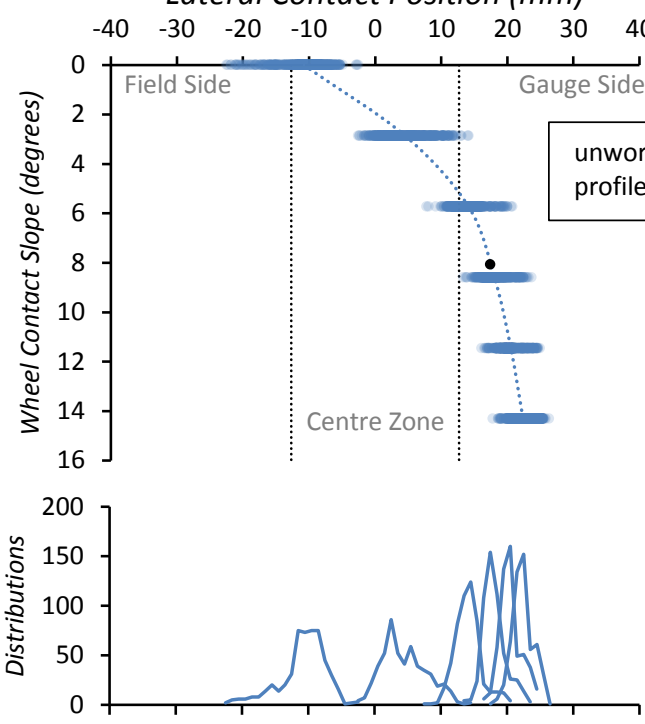

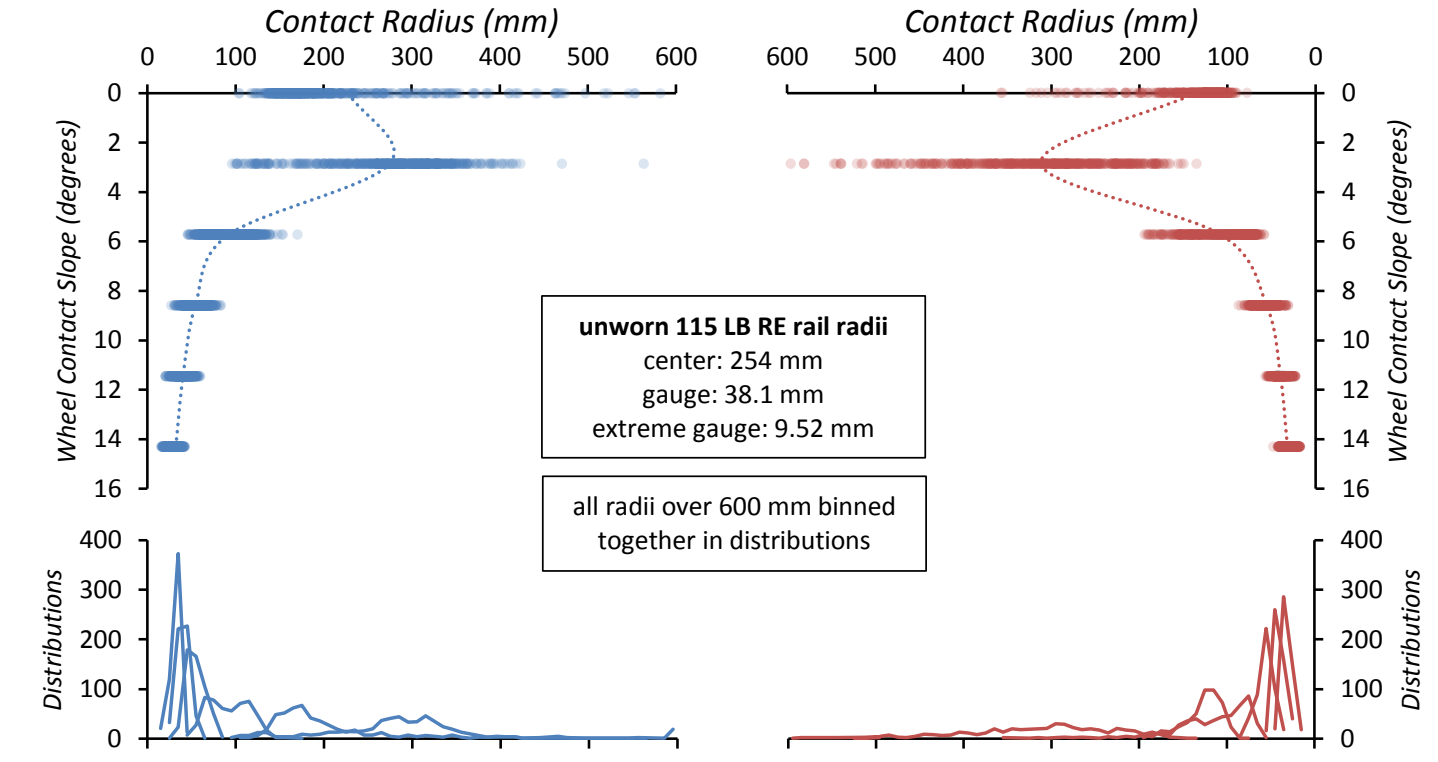

Lateral Contact Position ( $\mathrm{mm}$ )
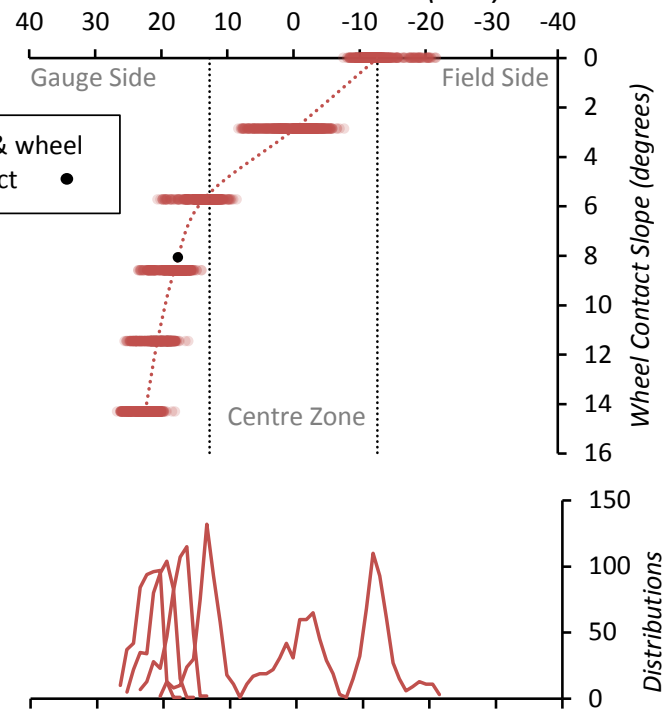

Contact Radius ( $\mathrm{mm}$ )

LEFT RAIL PROFILES

RIGHT RAIL PROFILES
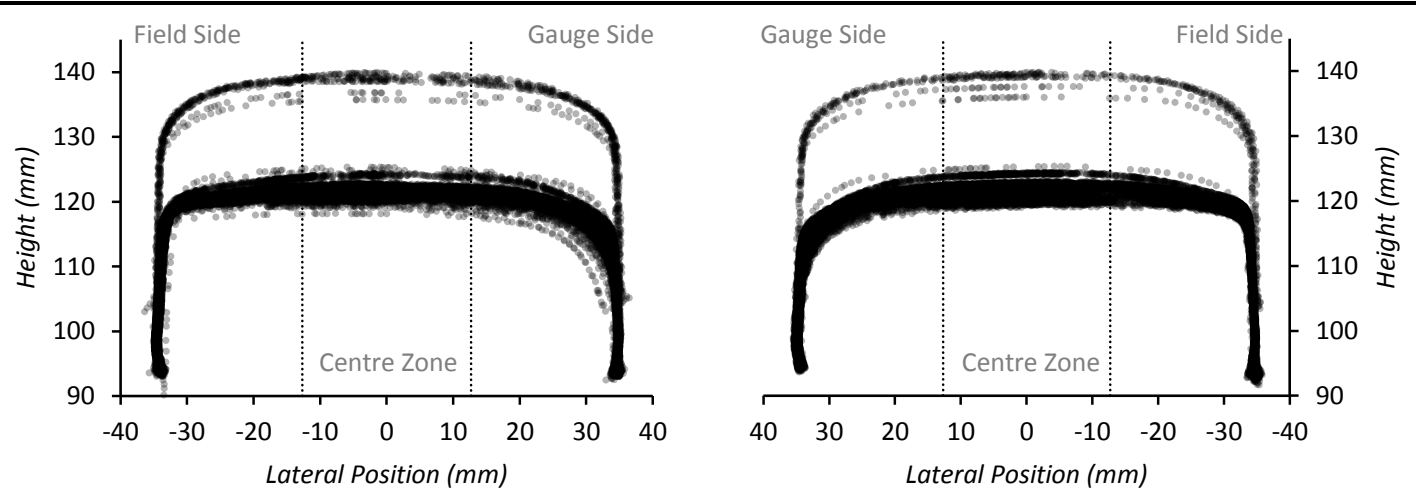

E-6 
SEGMENT AND ANALYSIS INFORMATION

\begin{tabular}{llll}
\hline \hline Track Segment Name: & SB-22 & Contact Analysis Date: & 20180615 \\
Segment Length $(\mathrm{m}):$ & 1050.0 & Left Wheel Slope: & $1: 20$ \\
Data Collection Date: & 20150827 & Right Wheel Slope: & $1: 20$
\end{tabular}

RAIL SEGMENT GRAPHS

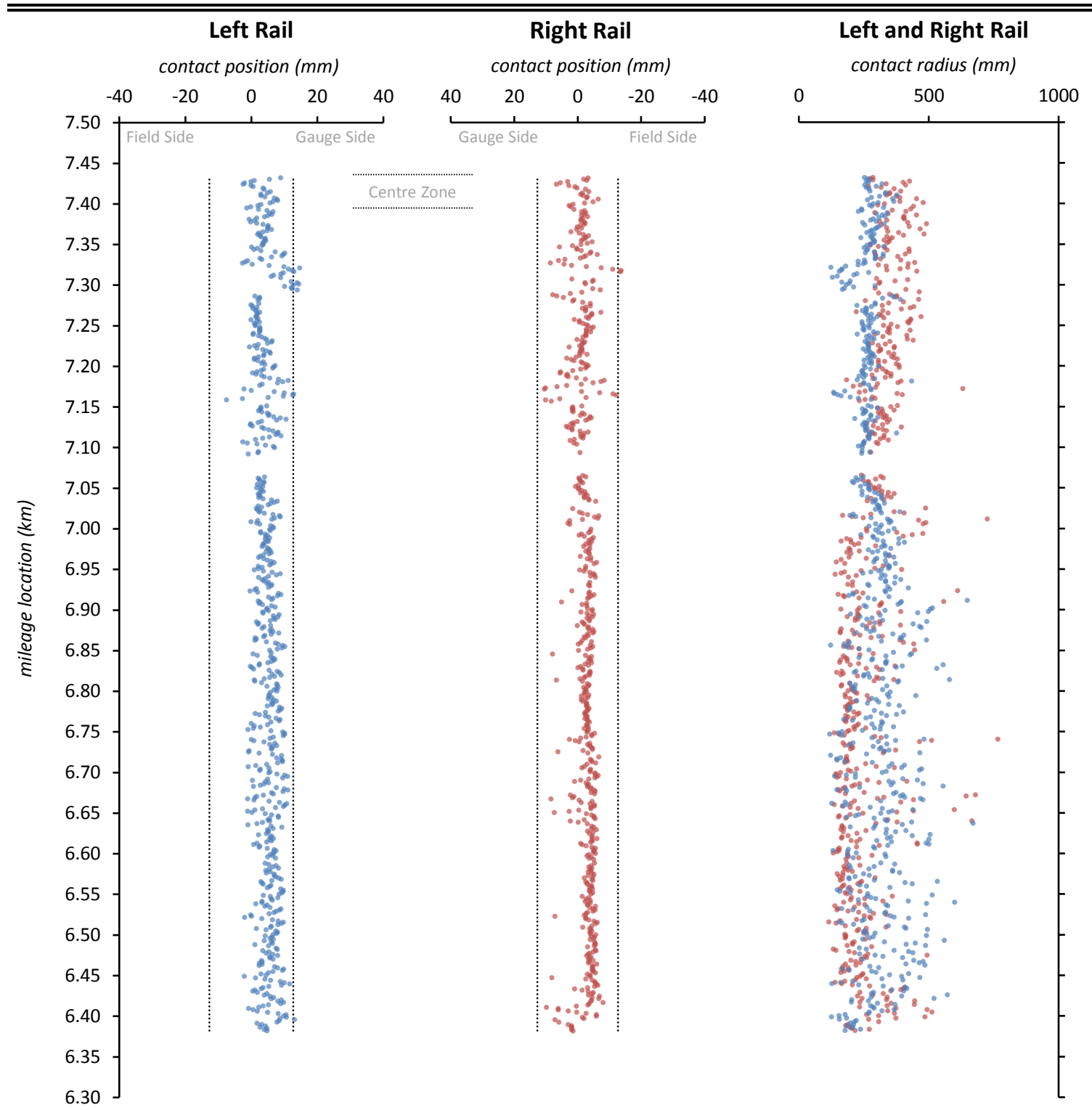

RAIL SEGMENT STATISTICS TABLE

\begin{tabular}{|c|c|c|c|c|}
\hline \multirow{3}{*}{ Statistic } & \multicolumn{4}{|c|}{ Rail Profile Measure } \\
\hline & \multicolumn{2}{|c|}{ Left Rail (650 profiles) } & \multicolumn{2}{|c|}{ Right Rail (598 profiles) } \\
\hline & Contact Position & Contact Radius & Contact Position & Contact Radius \\
\hline Mean $(\mathrm{mm})$ & 4.8 & 295.8 & -2.2 & 282.6 \\
\hline Std. Dev. $(\mathrm{mm})$ & 3.2 & 86.9 & 3.2 & 101.1 \\
\hline$\%$ within Zone & $99 \%$ & - & $100 \%$ & - \\
\hline
\end{tabular}


Lateral Contact Position ( $\mathrm{mm}$ )
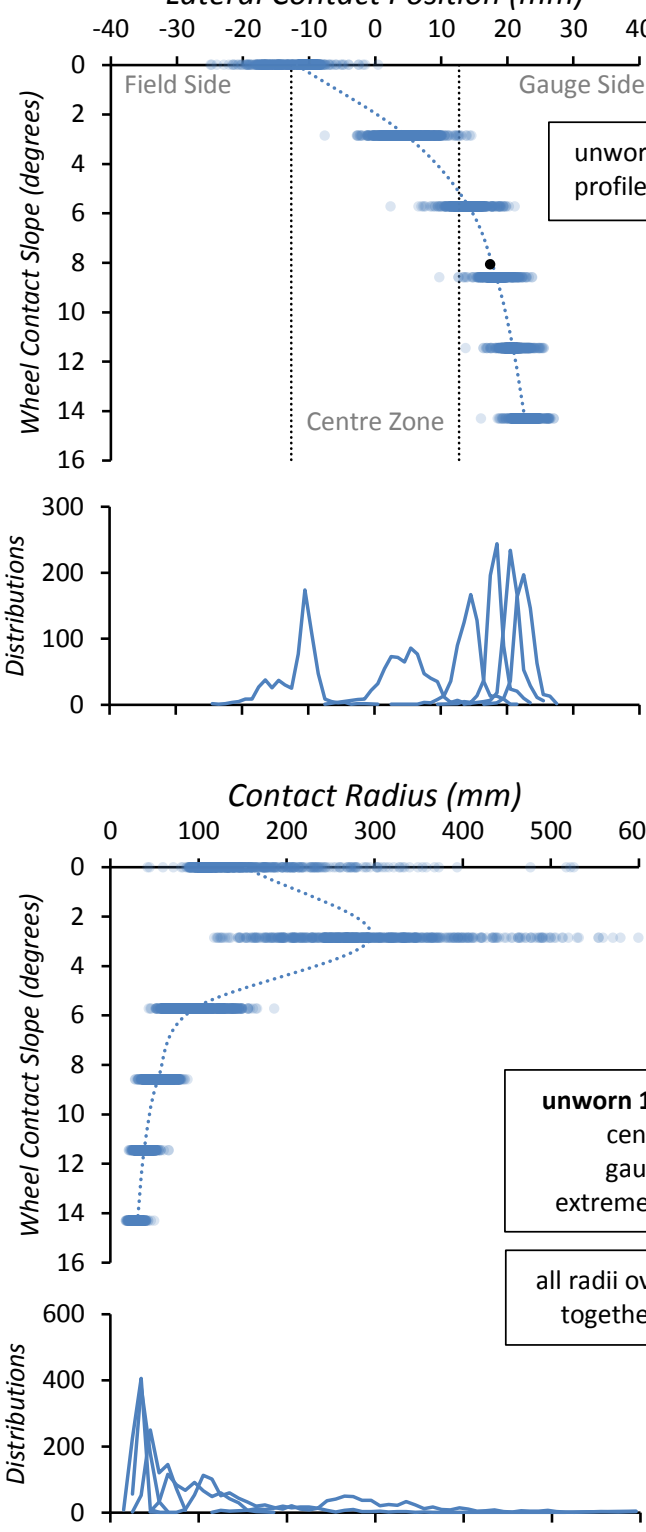

Lateral Contact Position ( $\mathrm{mm}$ )
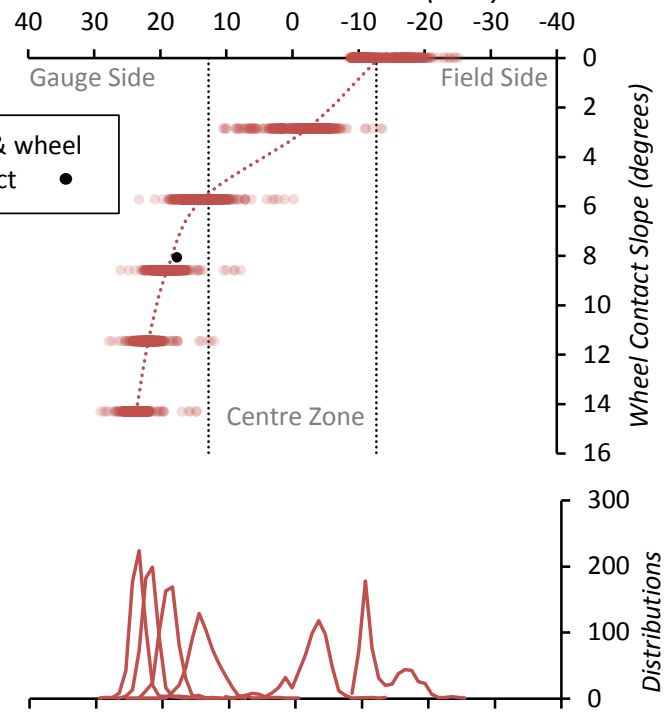

\section{Contact Radius ( $\mathrm{mm}$ )}
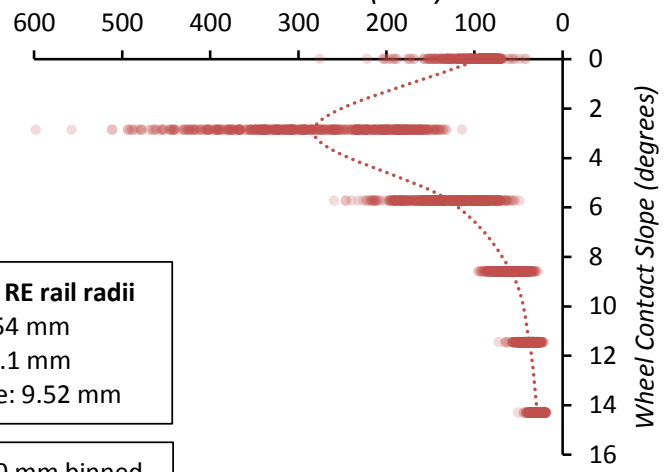

ver $600 \mathrm{~mm}$ binned

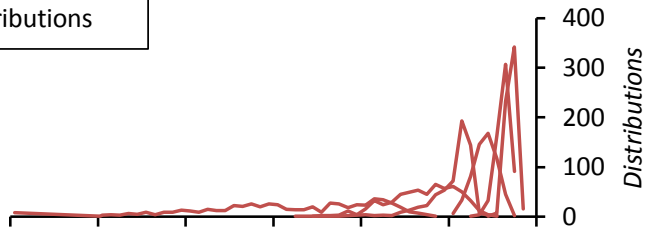

LEFT RAIL PROFILES

RIGHT RAIL PROFILES
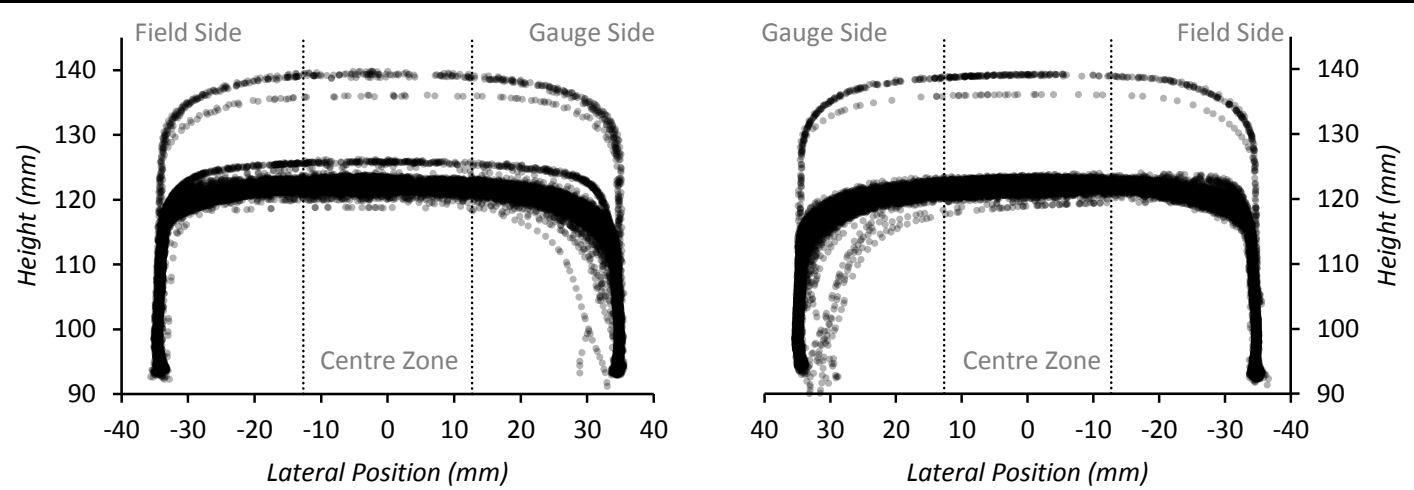

E-8 
SEGMENT AND ANALYSIS INFORMATION

\begin{tabular}{llll}
\hline \hline Track Segment Name: & SB-25 & Contact Analysis Date: & 20180615 \\
Segment Length $(\mathrm{m}):$ & 250.2 & Left Wheel Slope: & $1: 20$ \\
Data Collection Date: & 20150827 & Right Wheel Slope: & $1: 20$
\end{tabular}

RAIL SEGMENT GRAPHS

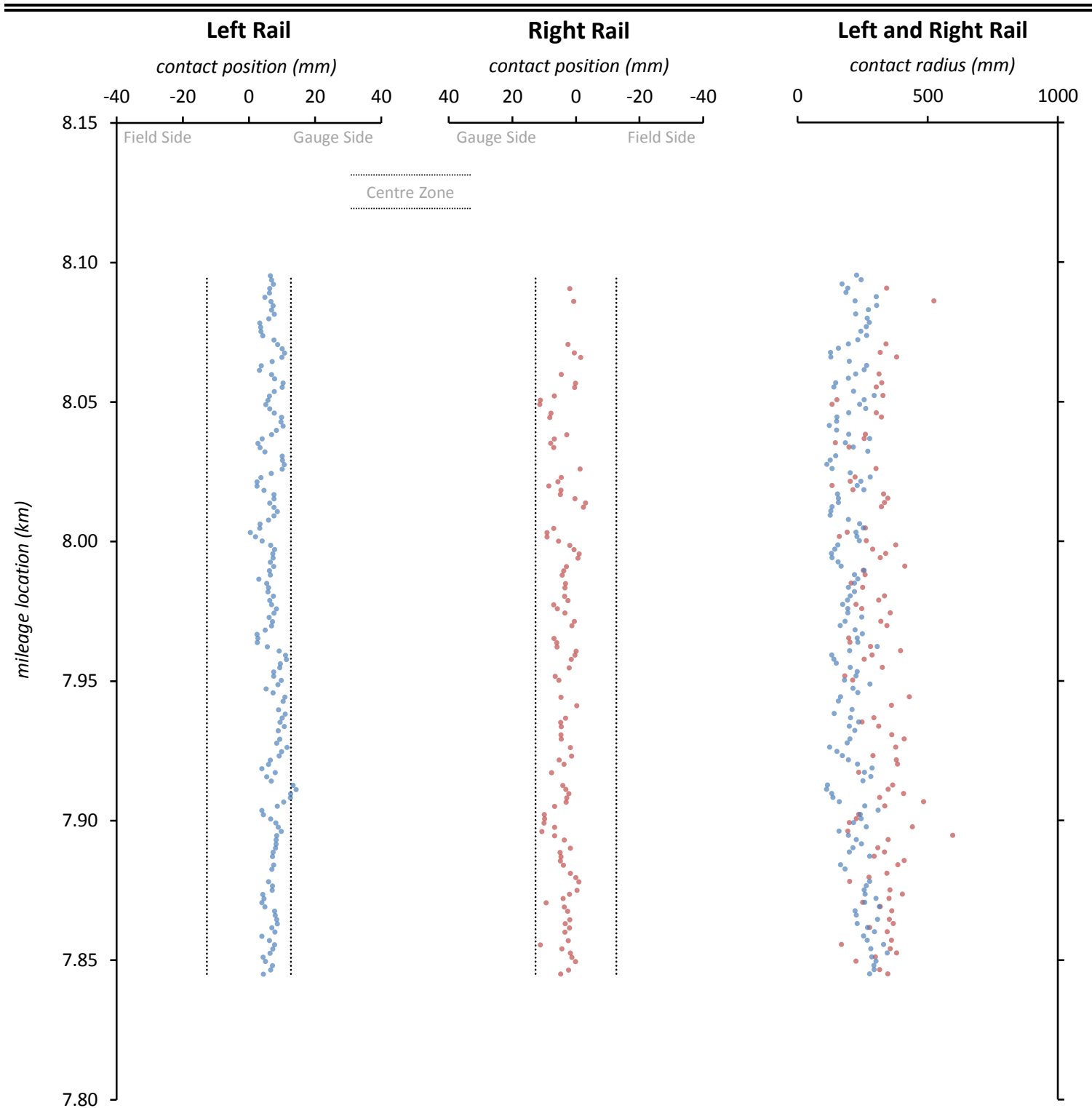

RAIL SEGMENT STATISTICS TABLE

\begin{tabular}{|c|c|c|c|c|}
\hline \multirow{3}{*}{ Statistic } & \multicolumn{4}{|c|}{ Rail Profile Measure } \\
\hline & \multicolumn{2}{|c|}{ Left Rail (161 profiles) } & \multicolumn{2}{|c|}{ Right Rail (106 profiles) } \\
\hline & Contact Position & Contact Radius & Contact Position & Contact Radius \\
\hline Mean $(\mathrm{mm})$ & 7.0 & 214.2 & 4.0 & 304.7 \\
\hline Std. Dev. $(\mathrm{mm})$ & 2.5 & 54.5 & 3.2 & 82.0 \\
\hline$\%$ within Zone & $99 \%$ & - & $100 \%$ & - \\
\hline
\end{tabular}


Lateral Contact Position ( $\mathrm{mm}$ )

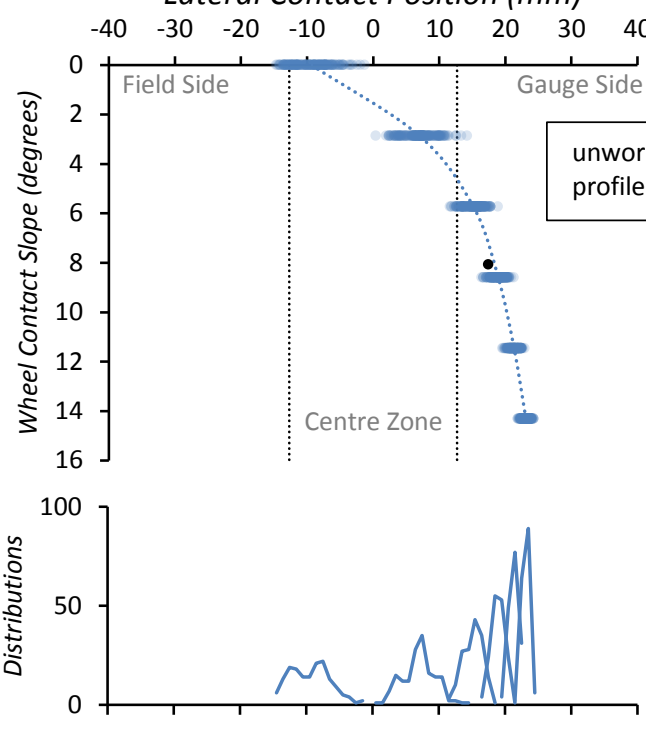

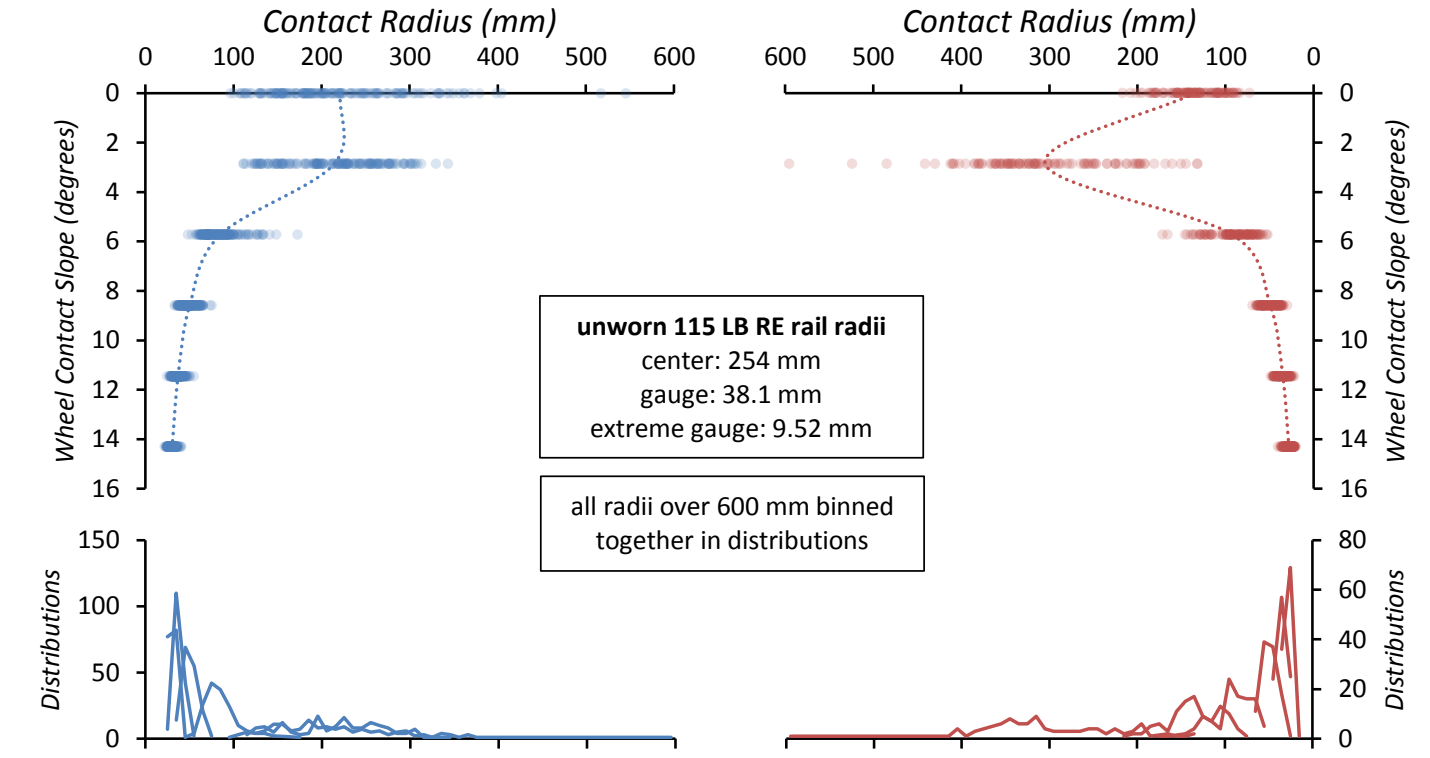

Lateral Contact Position ( $\mathrm{mm}$ )

$\begin{array}{lllllllll}40 & 30 & 20 & 10 & 0 & -10 & -20 & -30 & -40\end{array}$
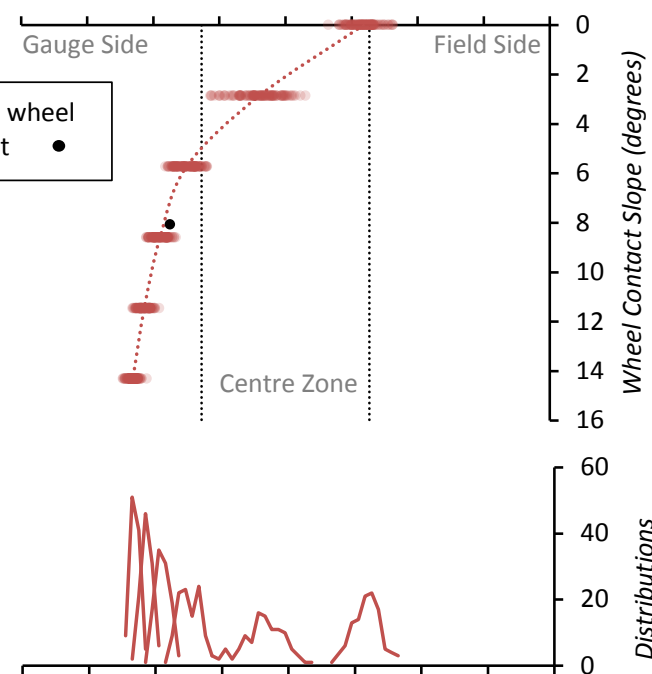

Contact Radius ( $\mathrm{mm}$ )

LEFT RAIL PROFILES

RIGHT RAIL PROFILES
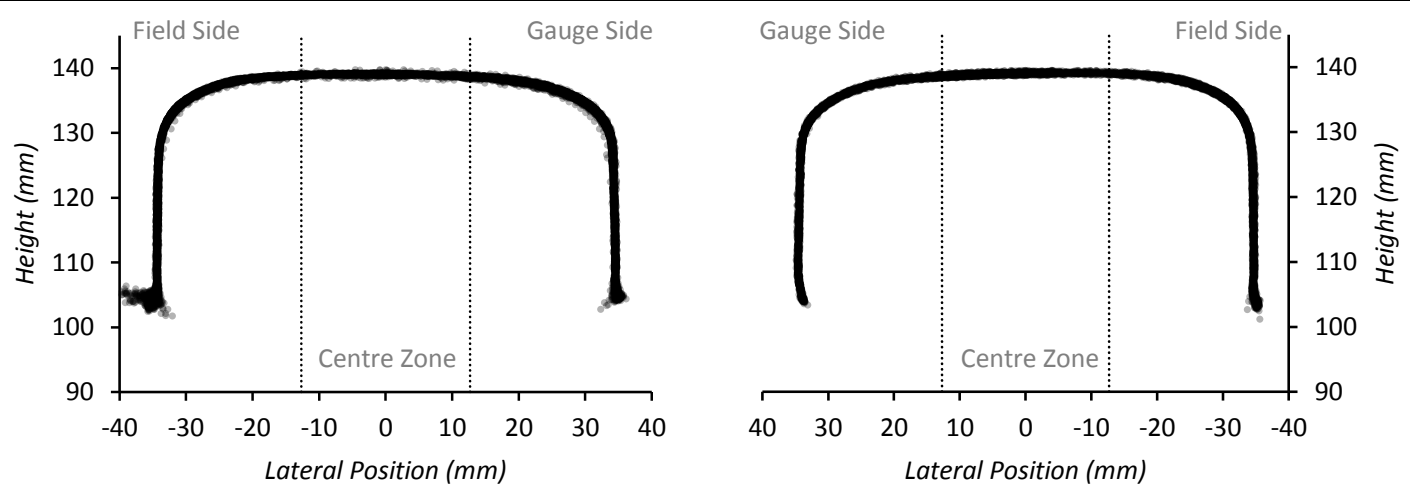

E-10 
SEGMENT AND ANALYSIS INFORMATION

\begin{tabular}{llll}
\hline \hline Track Segment Name: & SB-37 & Contact Analysis Date: & 20180615 \\
Segment Length $(\mathrm{m}):$ & 370.6 & Left Wheel Slope: & $1: 20$ \\
Data Collection Date: & 20150827 & Right Wheel Slope: & $1: 20$
\end{tabular}

RAIL SEGMENT GRAPHS

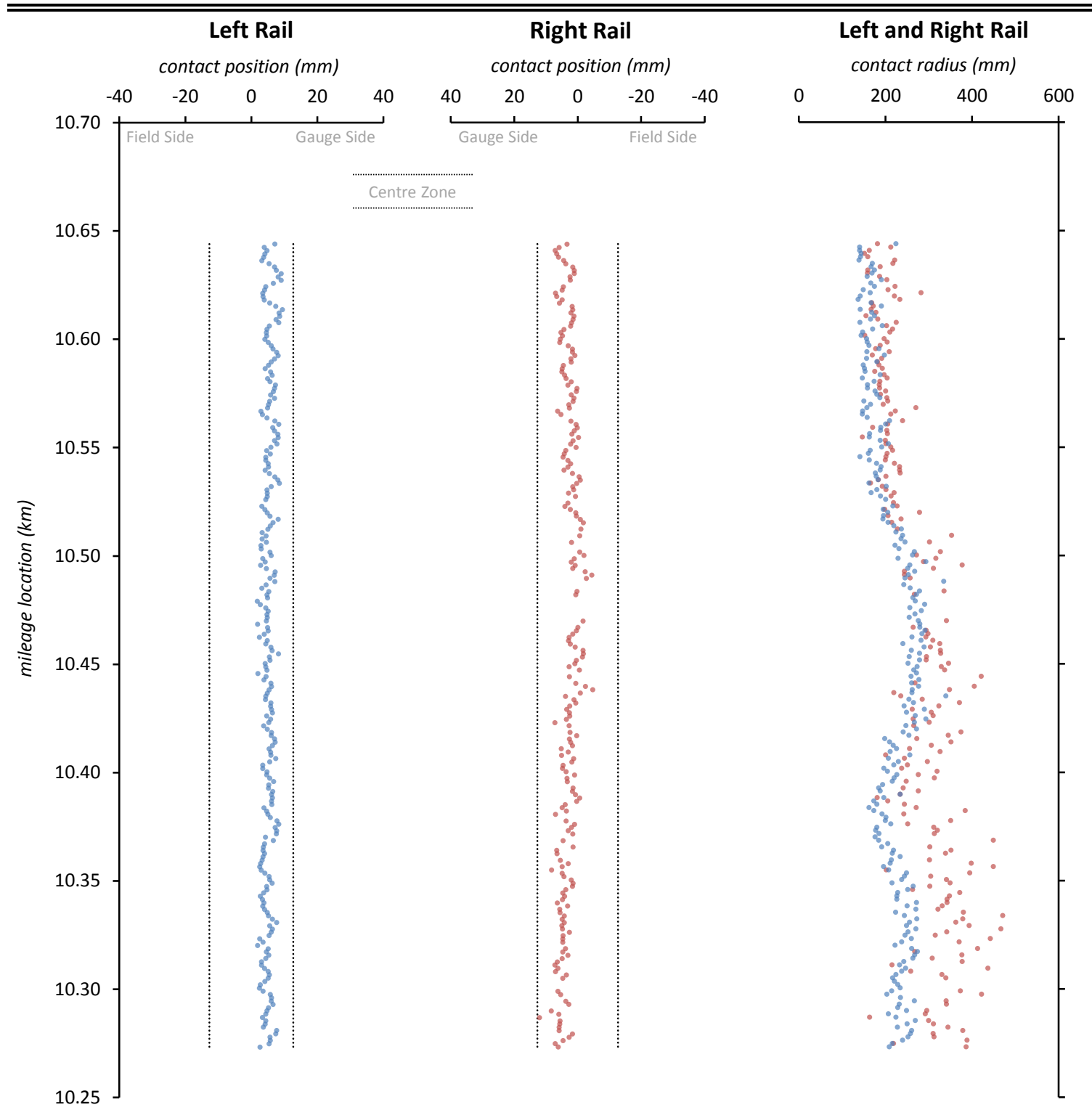

RAIL SEGMENT STATISTICS TABLE

\begin{tabular}{|c|c|c|c|c|}
\hline \multirow{3}{*}{ Statistic } & \multicolumn{4}{|c|}{ Rail Profile Measure } \\
\hline & \multicolumn{2}{|c|}{ Left Rail (245 profiles) } & \multicolumn{2}{|c|}{ Right Rail (213 profiles) } \\
\hline & Contact Position & Contact Radius & Contact Position & Contact Radius \\
\hline Mean $(\mathrm{mm})$ & 5.2 & 216.2 & 2.9 & 270.3 \\
\hline Std. Dev. $(\mathrm{mm})$ & 1.6 & 43.8 & 2.5 & 75.6 \\
\hline$\%$ within Zone & $100 \%$ & - & $100 \%$ & - \\
\hline
\end{tabular}


Lateral Contact Position ( $\mathrm{mm}$ )
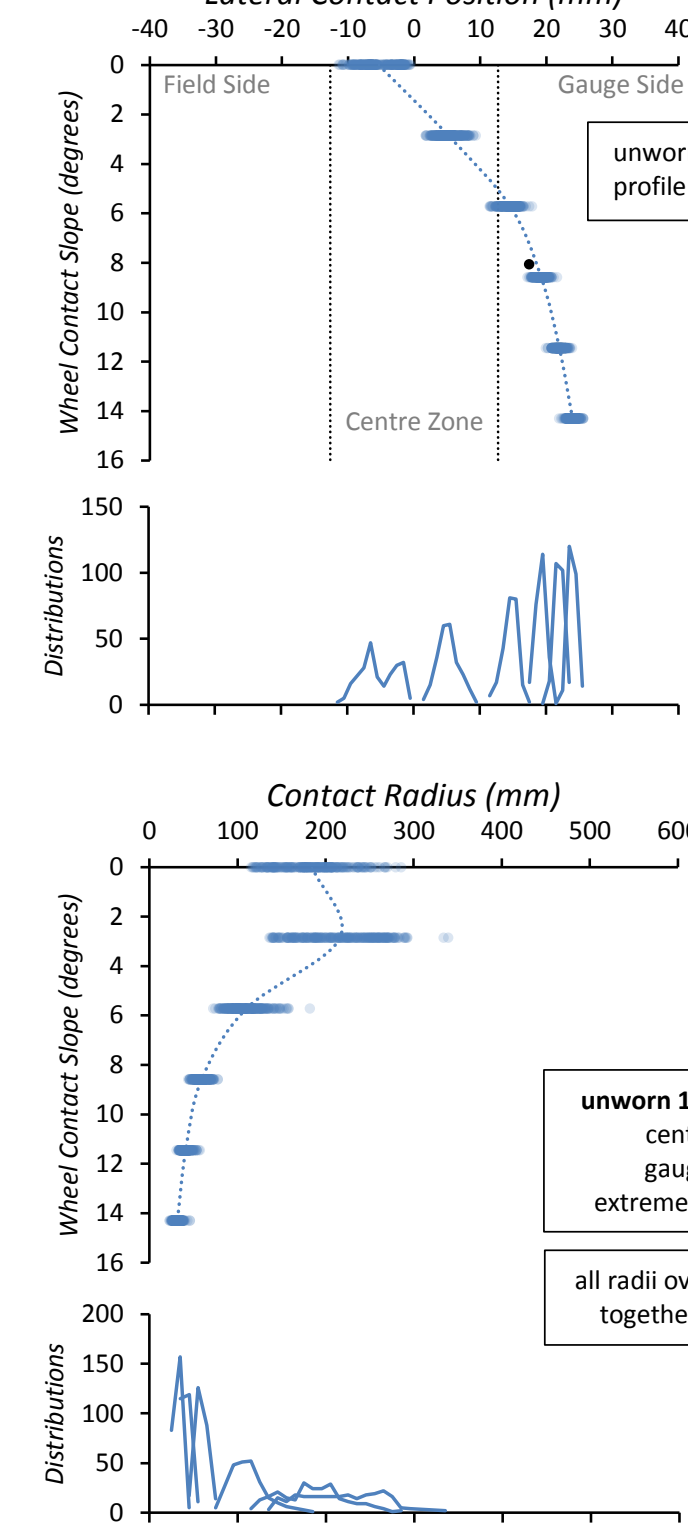

Lateral Contact Position ( $\mathrm{mm}$ )
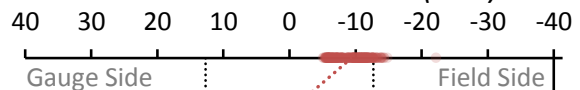

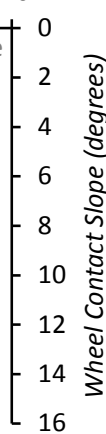

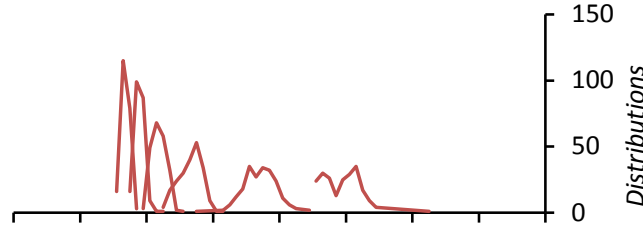

Contact Radius ( $\mathrm{mm}$ )

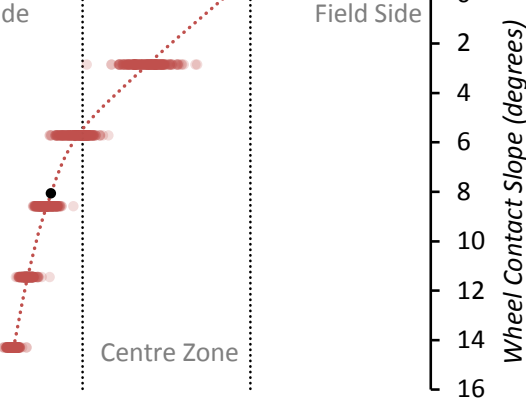

$\begin{array}{lllllll}600 & 500 & 400 & 300 & 200 & 100 & 0\end{array}$

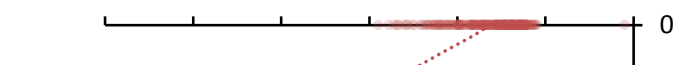

nter: $254 \mathrm{~mm}$

uge: $38.1 \mathrm{~mm}$ 
SEGMENT AND ANALYSIS INFORMATION

\begin{tabular}{llll}
\hline \hline Track Segment Name: & SB-41 & Contact Analysis Date: & 20180615 \\
Segment Length $(m):$ & 181.1 & Left Wheel Slope: & $1: 20$ \\
Data Collection Date: & 20150827 & Right Wheel Slope: & $1: 20$
\end{tabular}

RAIL SEGMENT GRAPHS

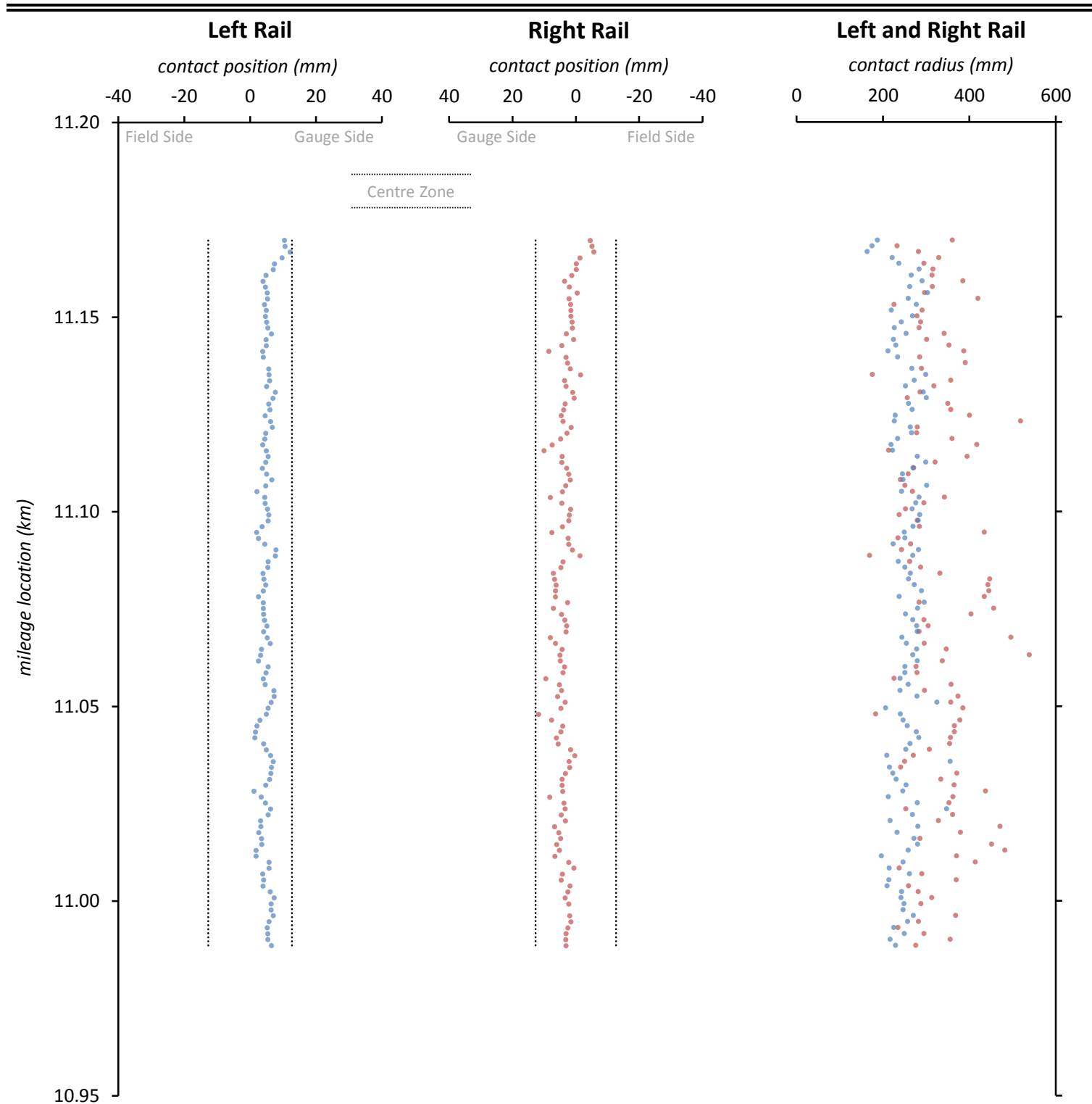

RAIL SEGMENT STATISTICS TABLE

\begin{tabular}{ccccc}
\hline \hline \multirow{2}{*}{ Statistic } & \multicolumn{4}{c}{ Rail Profile Measure } \\
\cline { 2 - 5 } & \multicolumn{4}{c}{ Left Rail } \\
\cline { 2 - 5 } & Contact Position & Contact Radius & Contact Position & Contact Radius \\
\hline Mean $(\mathrm{mm})$ & 5.0 & 254.1 & 3.5 & 325.0 \\
Std. Dev. $(\mathrm{mm})$ & 1.8 & 30.9 & 2.7 & 72.5 \\
$\%$ within Zone & $100 \%$ & - & $100 \%$ & - \\
\hline
\end{tabular}


Lateral Contact Position ( $\mathrm{mm}$ )
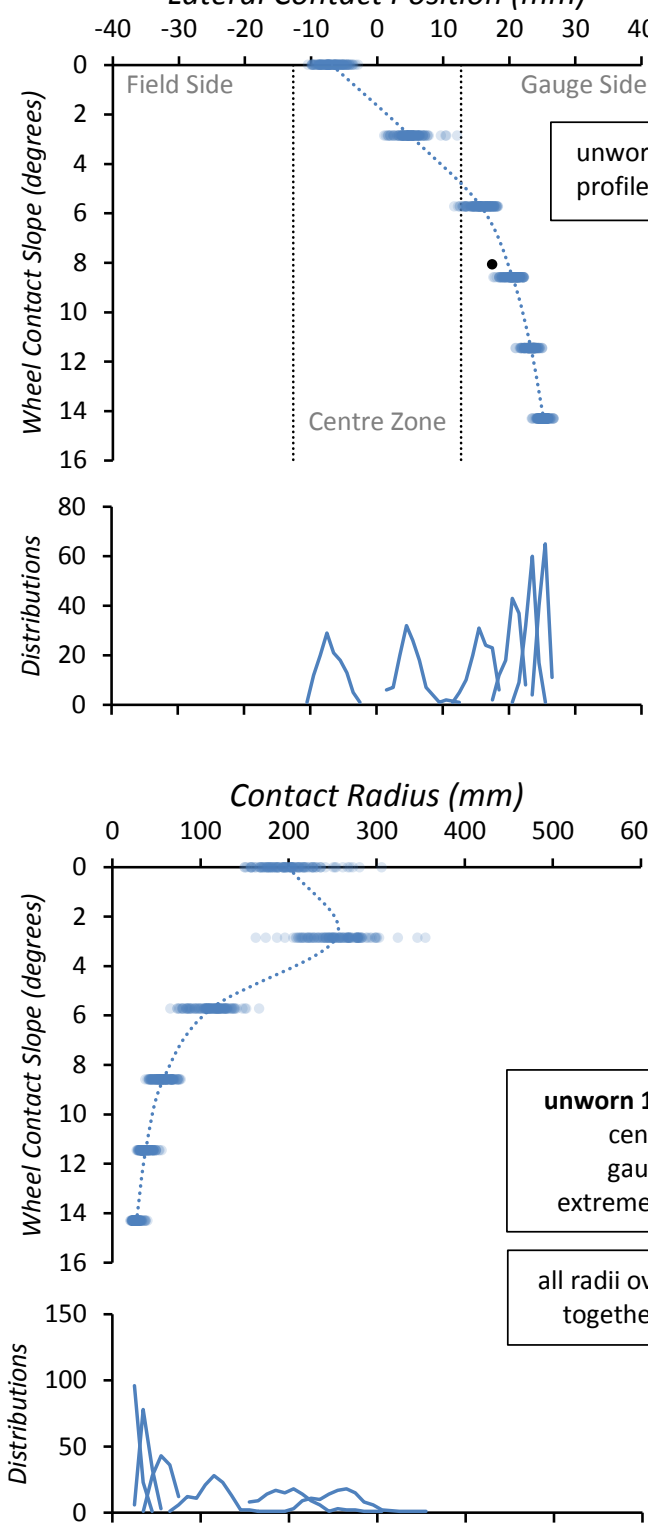

Lateral Contact Position ( $\mathrm{mm}$ )
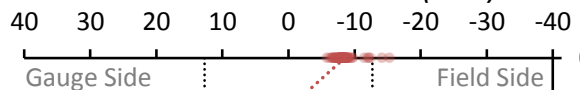

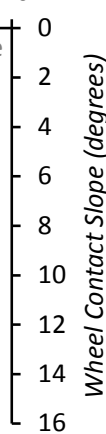

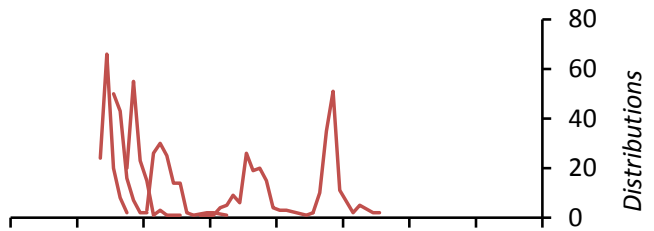

Contact Radius ( $\mathrm{mm}$ )

$\begin{array}{lllllll}600 & 500 & 400 & 300 & 200 & 100 & 0\end{array}$
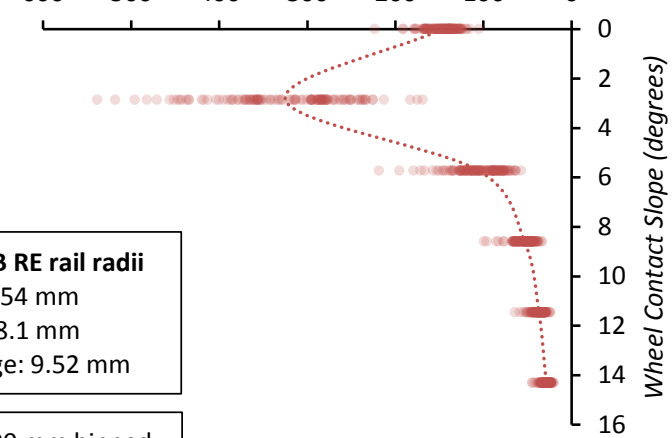

enter: $254 \mathrm{~mm}$

gauge: $38.1 \mathrm{~mm}$

treme gauge: $9.52 \mathrm{~mm}$

radii over $600 \mathrm{~mm}$ binned

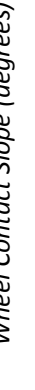

LEFT RAIL PROFILES

RIGHT RAIL PROFILES
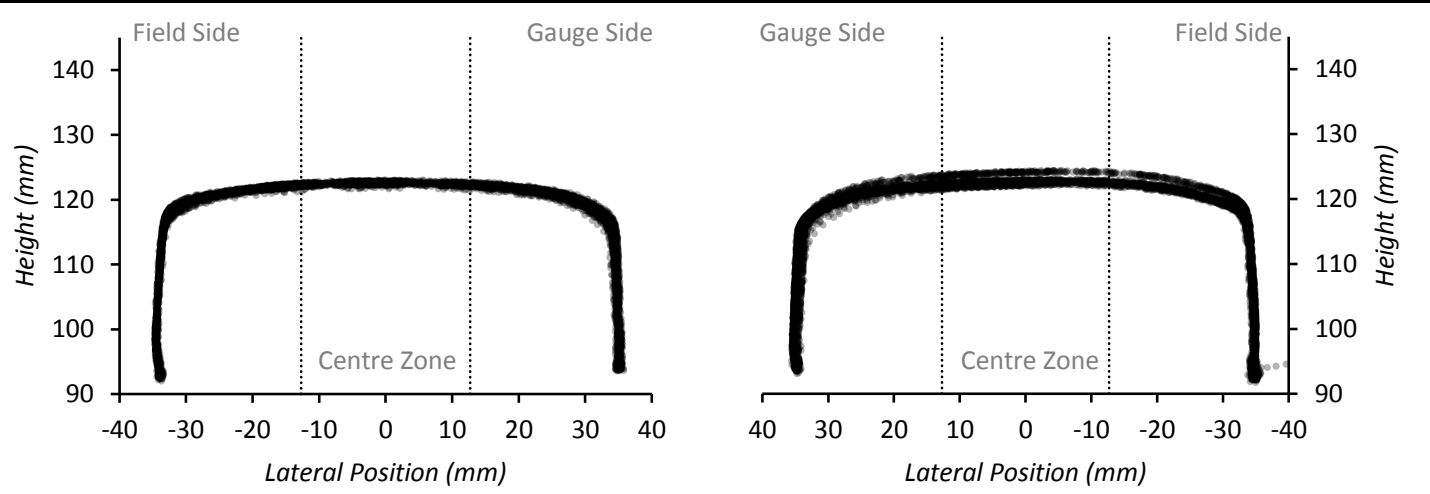

E-14 
SEGMENT AND ANALYSIS INFORMATION

\begin{tabular}{llll}
\hline \hline Track Segment Name: & SB-45 & Contact Analysis Date: & 20180615 \\
Segment Length $(\mathrm{m}):$ & 206.4 & Left Wheel Slope: & $1: 20$ \\
Data Collection Date: & 20150827 & Right Wheel Slope: & $1: 20$
\end{tabular}

RAIL SEGMENT GRAPHS

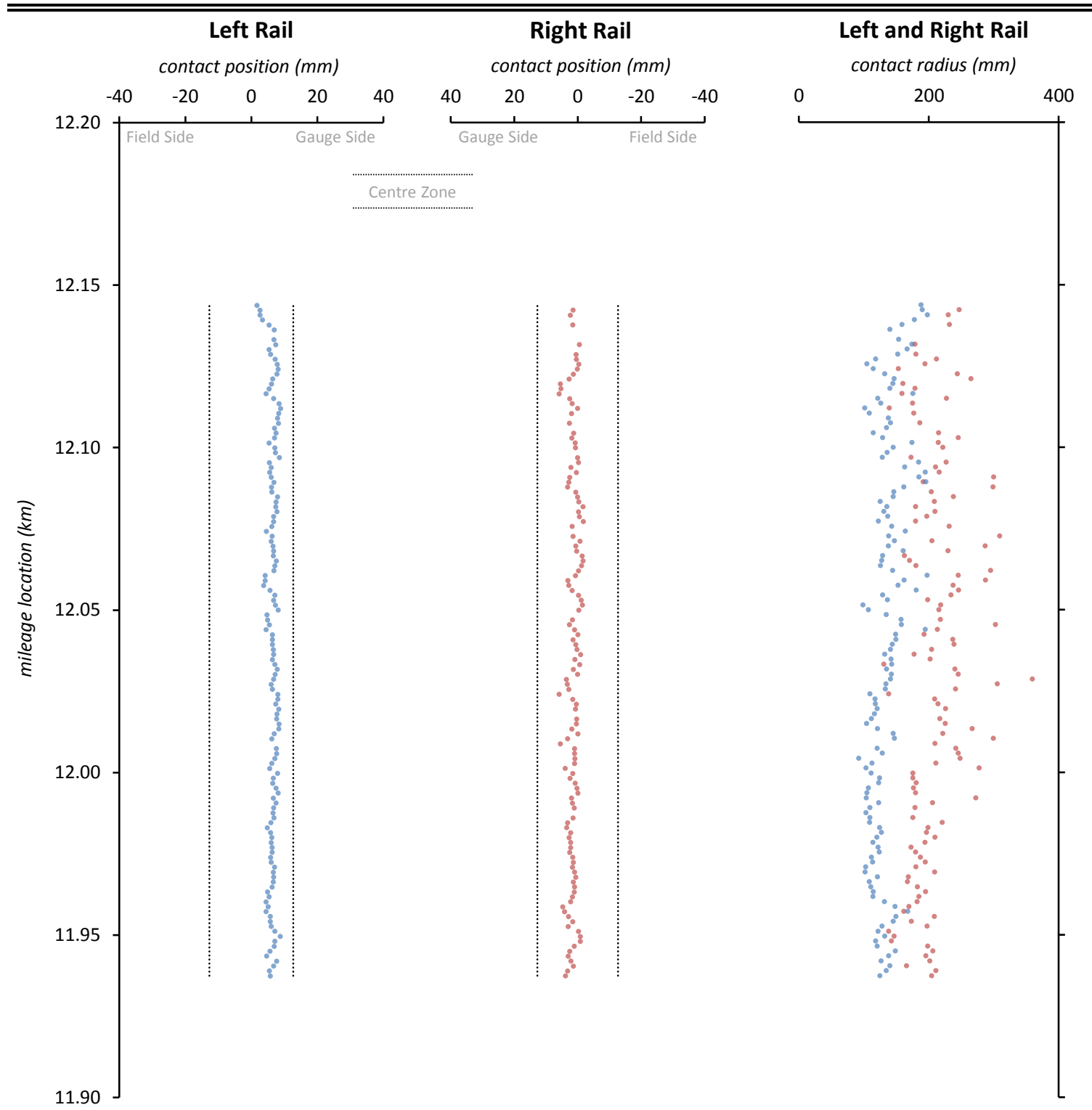

RAIL SEGMENT STATISTICS TABLE

\begin{tabular}{|c|c|c|c|c|}
\hline \multirow{3}{*}{ Statistic } & \multicolumn{4}{|c|}{ Rail Profile Measure } \\
\hline & \multicolumn{2}{|c|}{ Left Rail (135 profiles) } & \multicolumn{2}{|c|}{ Right Rail (124 profiles) } \\
\hline & Contact Position & Contact Radius & Contact Position & Contact Radius \\
\hline Mean $(\mathrm{mm})$ & 6.4 & 135.5 & 1.4 & 209.8 \\
\hline Std. Dev. $(\mathrm{mm})$ & 1.3 & 24.0 & 1.6 & 41.2 \\
\hline$\%$ within Zone & $100 \%$ & - & $100 \%$ & - \\
\hline
\end{tabular}


Lateral Contact Position ( $\mathrm{mm}$ )
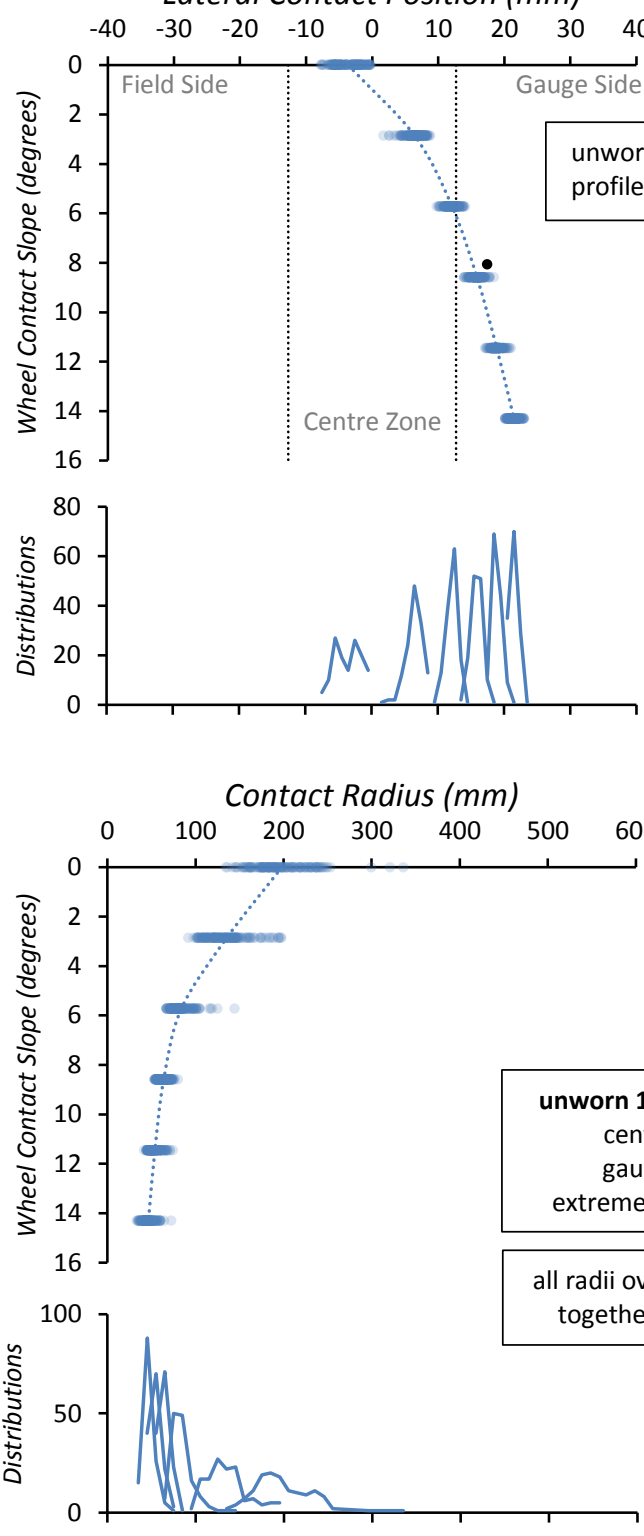

LEFT RAIL PROFILES

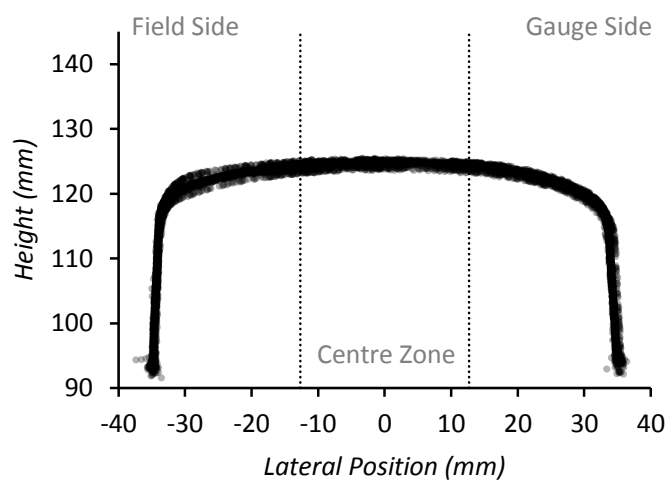

Lateral Contact Position ( $\mathrm{mm}$ )
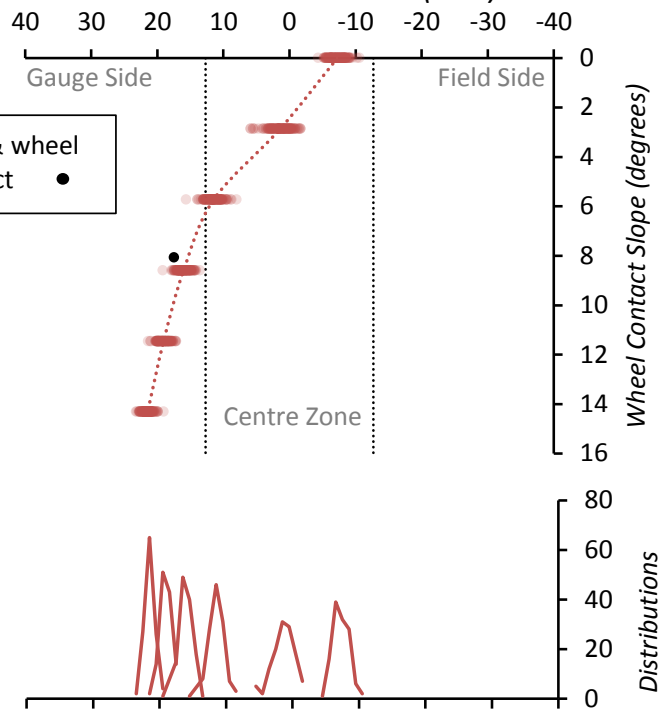

Contact Radius ( $\mathrm{mm}$ )
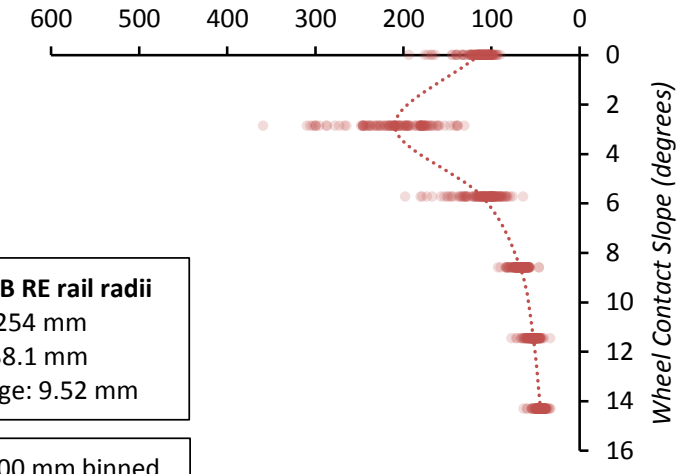

enter: $254 \mathrm{~mm}$

uge: $38.1 \mathrm{~mm}$

reme gauge: $9.52 \mathrm{~mm}$

$00 \mathrm{~mm}$ binned

$$
\text { tributions }
$$

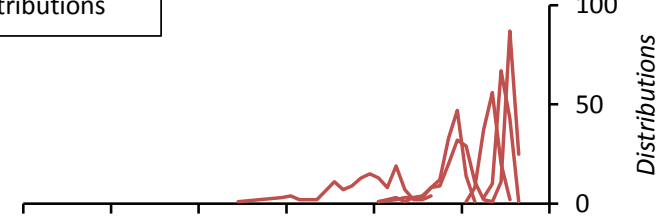


SEGMENT AND ANALYSIS INFORMATION

\begin{tabular}{llll}
\hline \hline Track Segment Name: & SB-49 & Contact Analysis Date: & 20180615 \\
Segment Length $(m):$ & 106.0 & Left Wheel Slope: & $1: 20$ \\
Data Collection Date: & 20150827 & Right Wheel Slope: & $1: 20$
\end{tabular}

RAIL SEGMENT GRAPHS

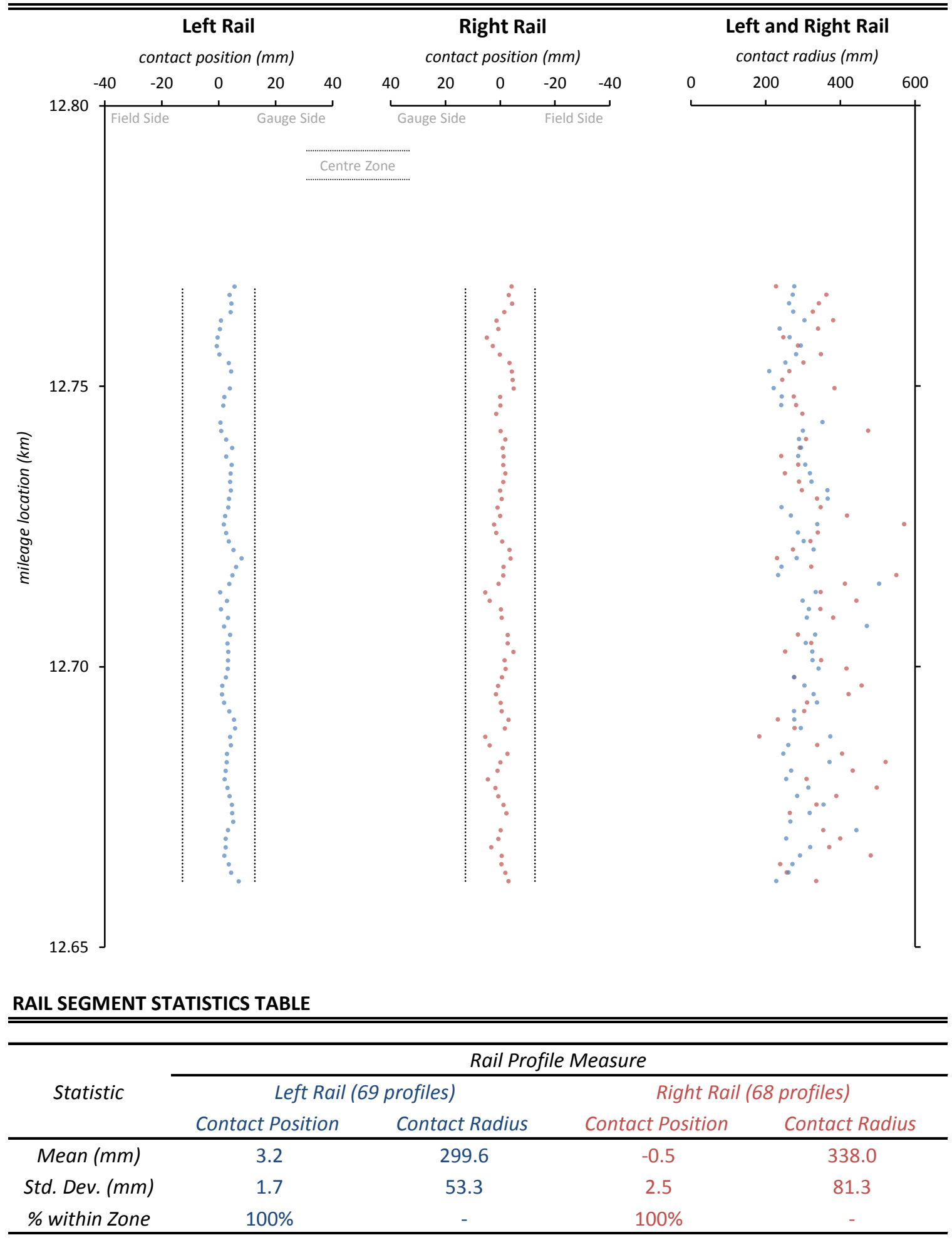


Lateral Contact Position ( $\mathrm{mm}$ )
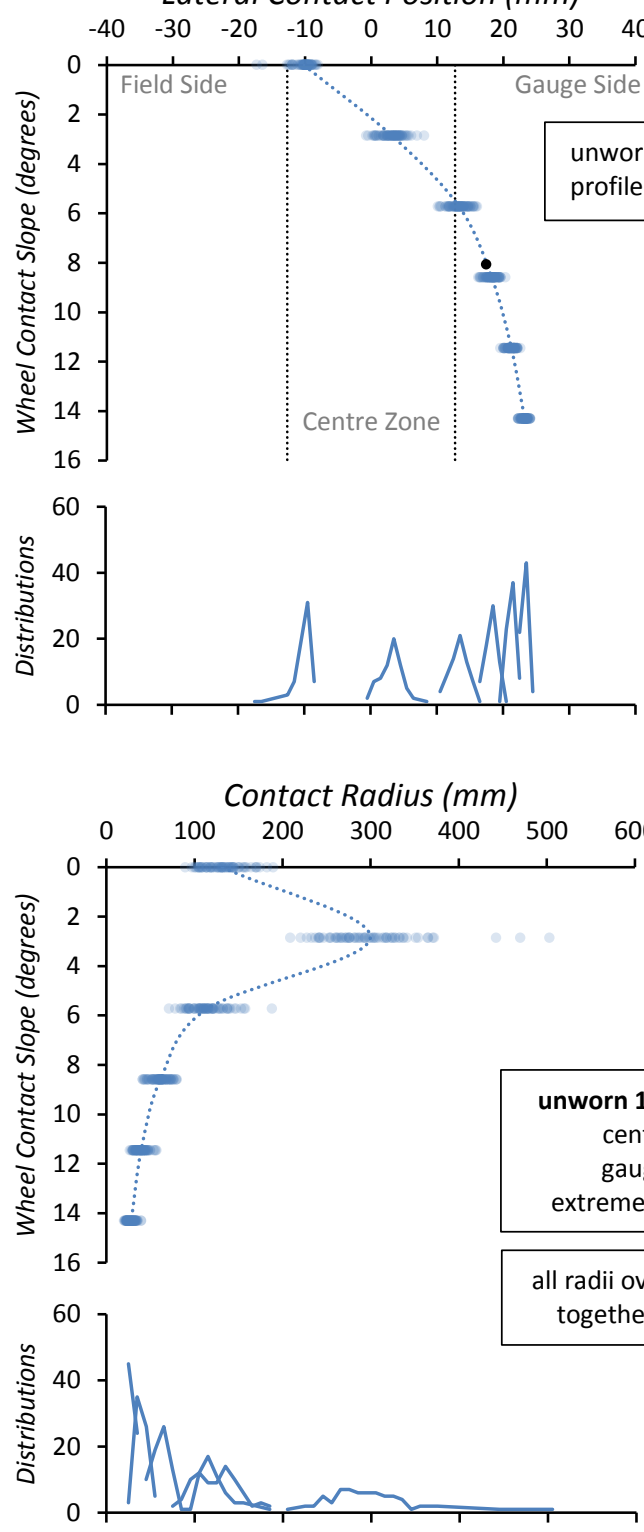

Lateral Contact Position ( $\mathrm{mm}$ )
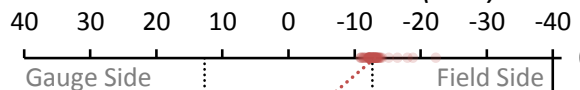

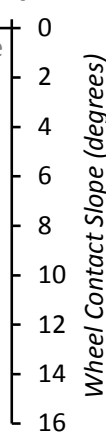

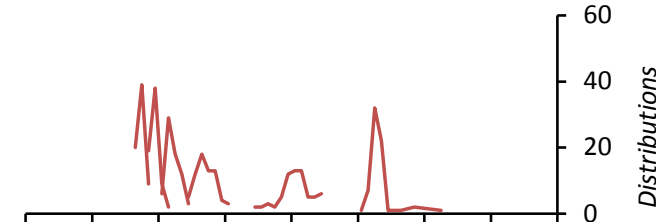

Contact Radius ( $\mathrm{mm}$ )

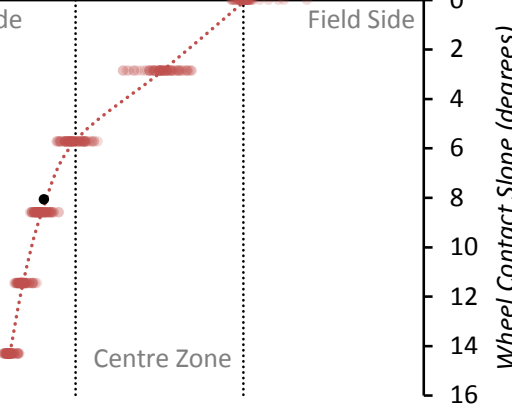

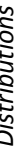

115 LB RE rail radi

center: $254 \mathrm{~mm}$

gauge: $38.1 \mathrm{~mm}$

treme gauge: $9.52 \mathrm{~mm}$

radii over $600 \mathrm{~mm}$ binned ogether in distributions
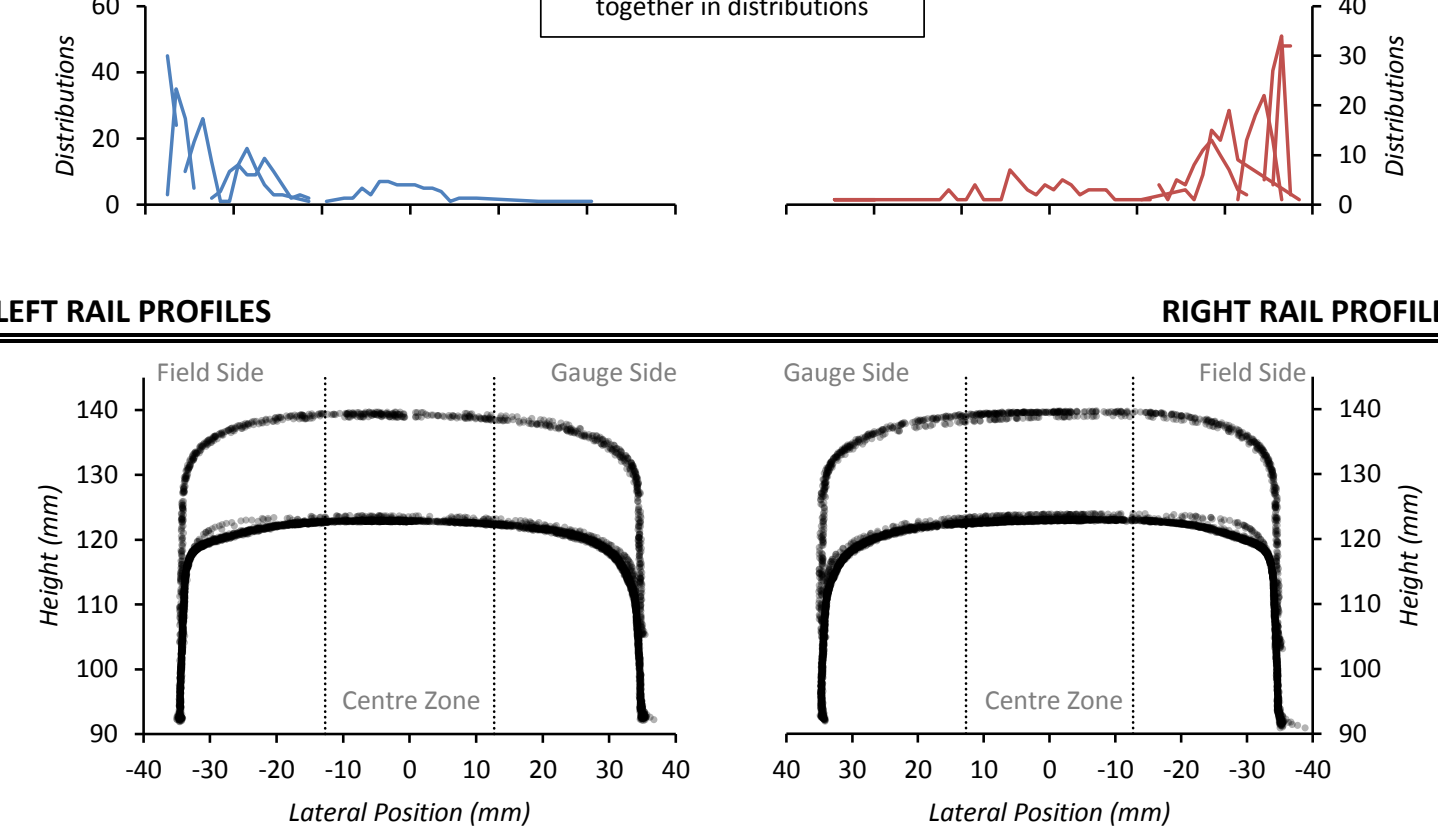

E-18 
SEGMENT AND ANALYSIS INFORMATION

\begin{tabular}{llll}
\hline \hline Track Segment Name: & SB-58 & Contact Analysis Date: & 20180615 \\
Segment Length $(m):$ & 119.8 & Left Wheel Slope: & $1: 20$ \\
Data Collection Date: & 20150827 & Right Wheel Slope: & $1: 20$
\end{tabular}

RAIL SEGMENT GRAPHS

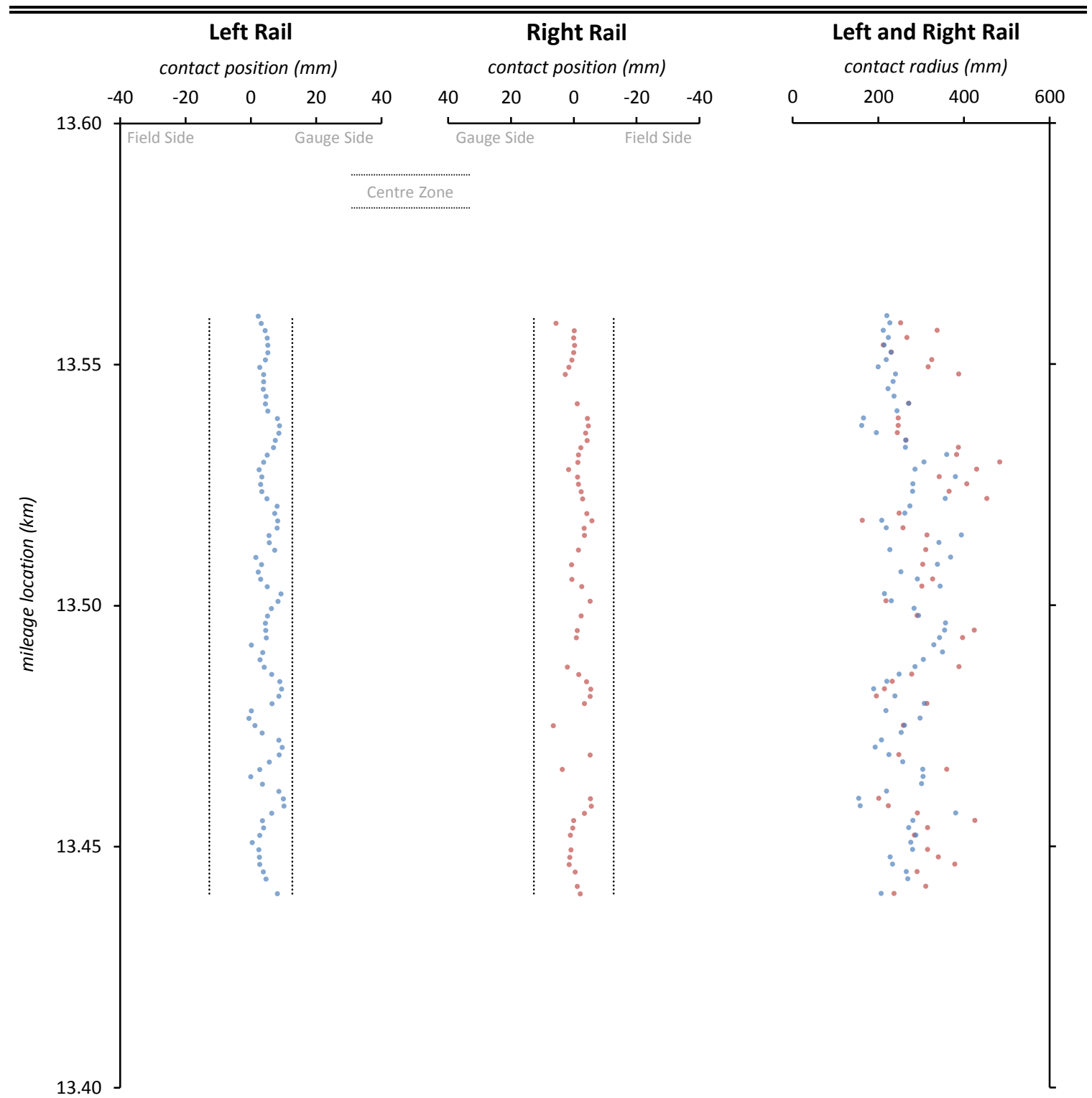

RAIL SEGMENT STATISTICS TABLE

\begin{tabular}{|c|c|c|c|c|}
\hline \multirow{3}{*}{ Statistic } & \multicolumn{4}{|c|}{ Rail Profile Measure } \\
\hline & \multicolumn{2}{|c|}{ Left Rail (79 profiles) } & \multicolumn{2}{|c|}{ Right Rail (54 profiles) } \\
\hline & Contact Position & Contact Radius & Contact Position & Contact Radius \\
\hline Mean $(\mathrm{mm})$ & 5.0 & 264.2 & -1.3 & 305.4 \\
\hline Std. Dev. (mm) & 2.6 & 56.6 & 2.8 & 72.8 \\
\hline$\%$ within Zone & $100 \%$ & - & $100 \%$ & - \\
\hline
\end{tabular}


Lateral Contact Position ( $\mathrm{mm}$ )
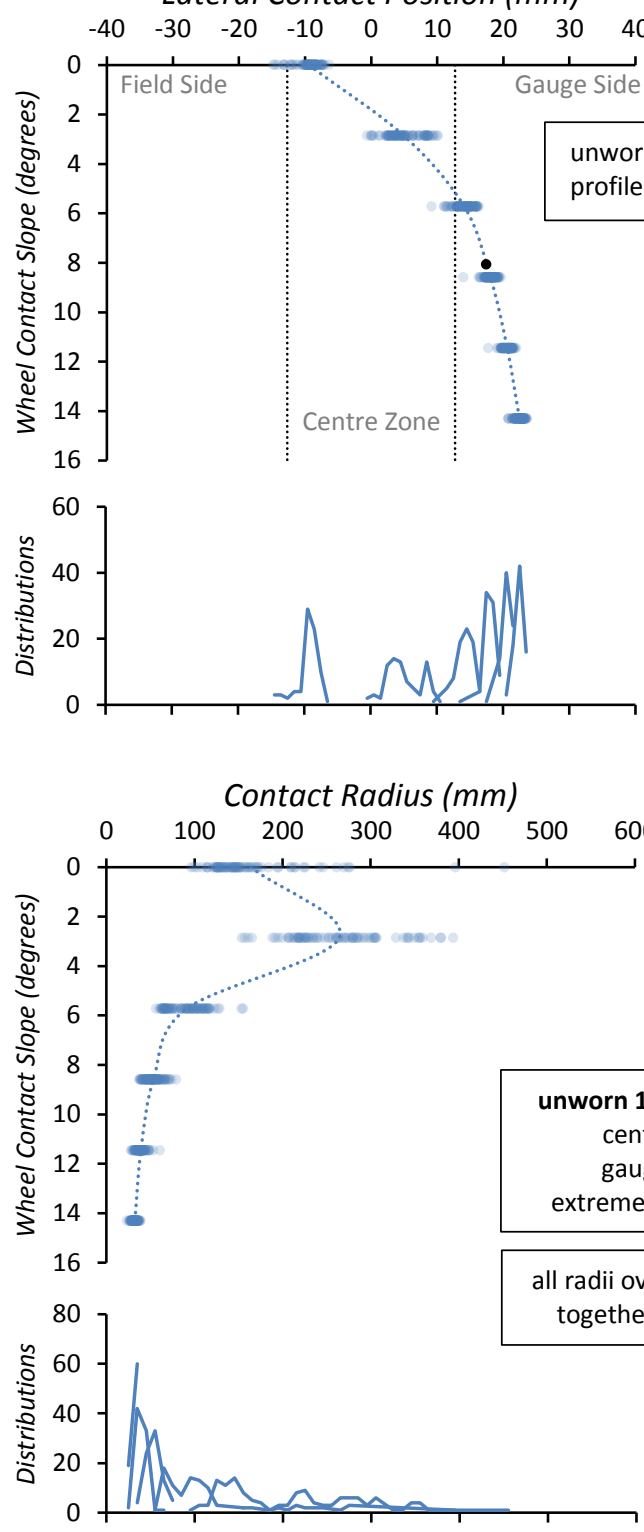

LEFT RAIL PROFILES

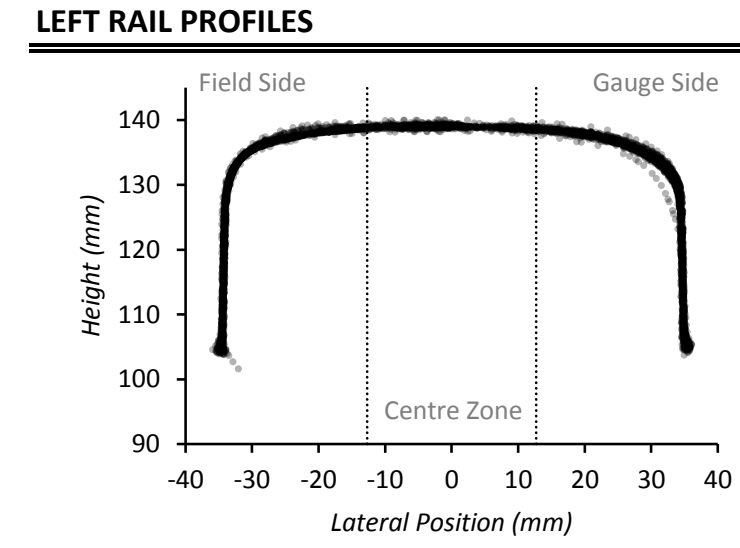

Lateral Contact Position ( $\mathrm{mm}$ )
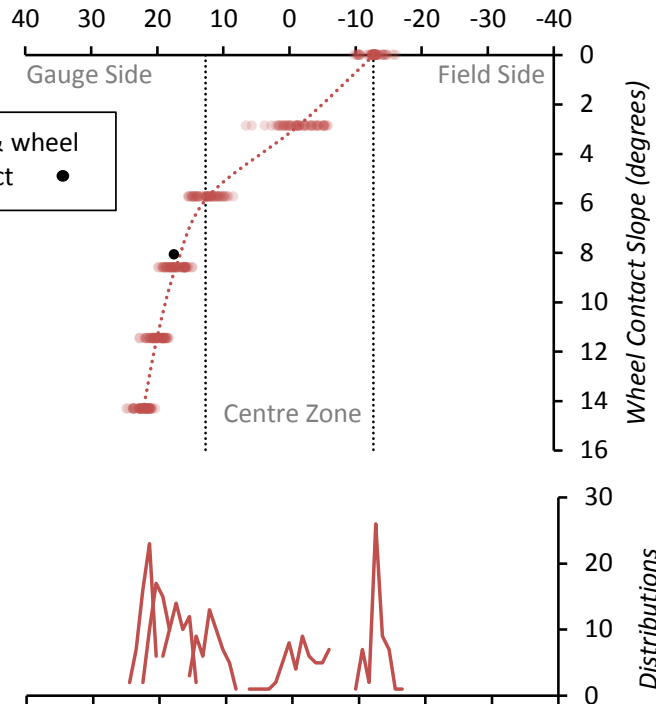

Contact Radius ( $\mathrm{mm}$ )
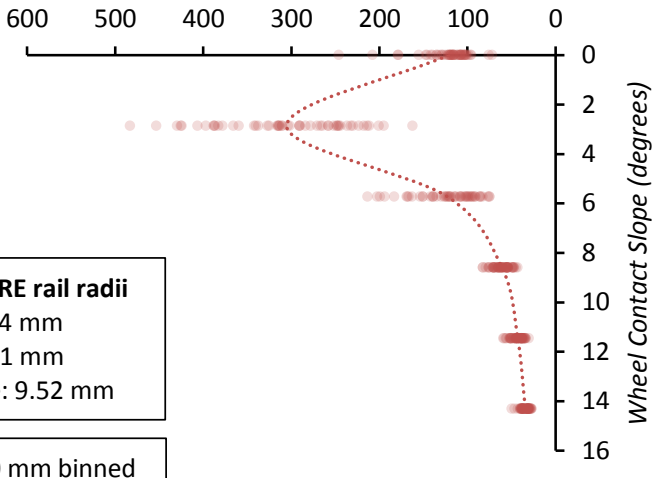

ter: $254 \mathrm{~mm}$

uge: $38.1 \mathrm{~mm}$

binned

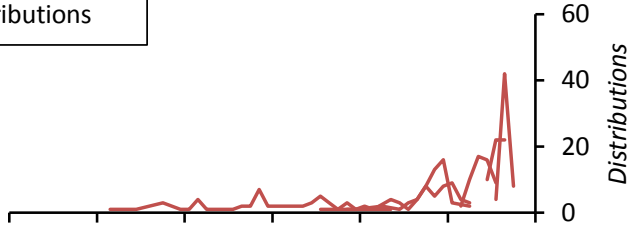

RIGHT RAIL PROFILES

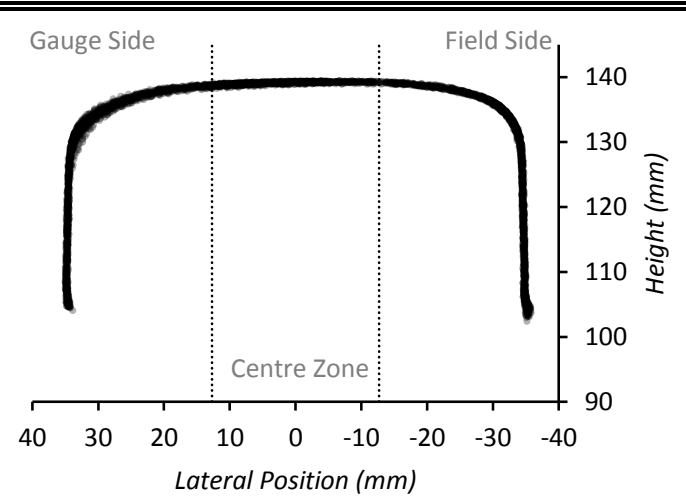

E-20 
SEGMENT AND ANALYSIS INFORMATION

\begin{tabular}{llll}
\hline \hline Track Segment Name: & SB-66 & Contact Analysis Date: & 20180615 \\
Segment Length $(\mathrm{m}):$ & 513.3 & Left Wheel Slope: & $1: 20$ \\
Data Collection Date: & 20150827 & Right Wheel Slope: & $1: 20$
\end{tabular}

RAIL SEGMENT GRAPHS

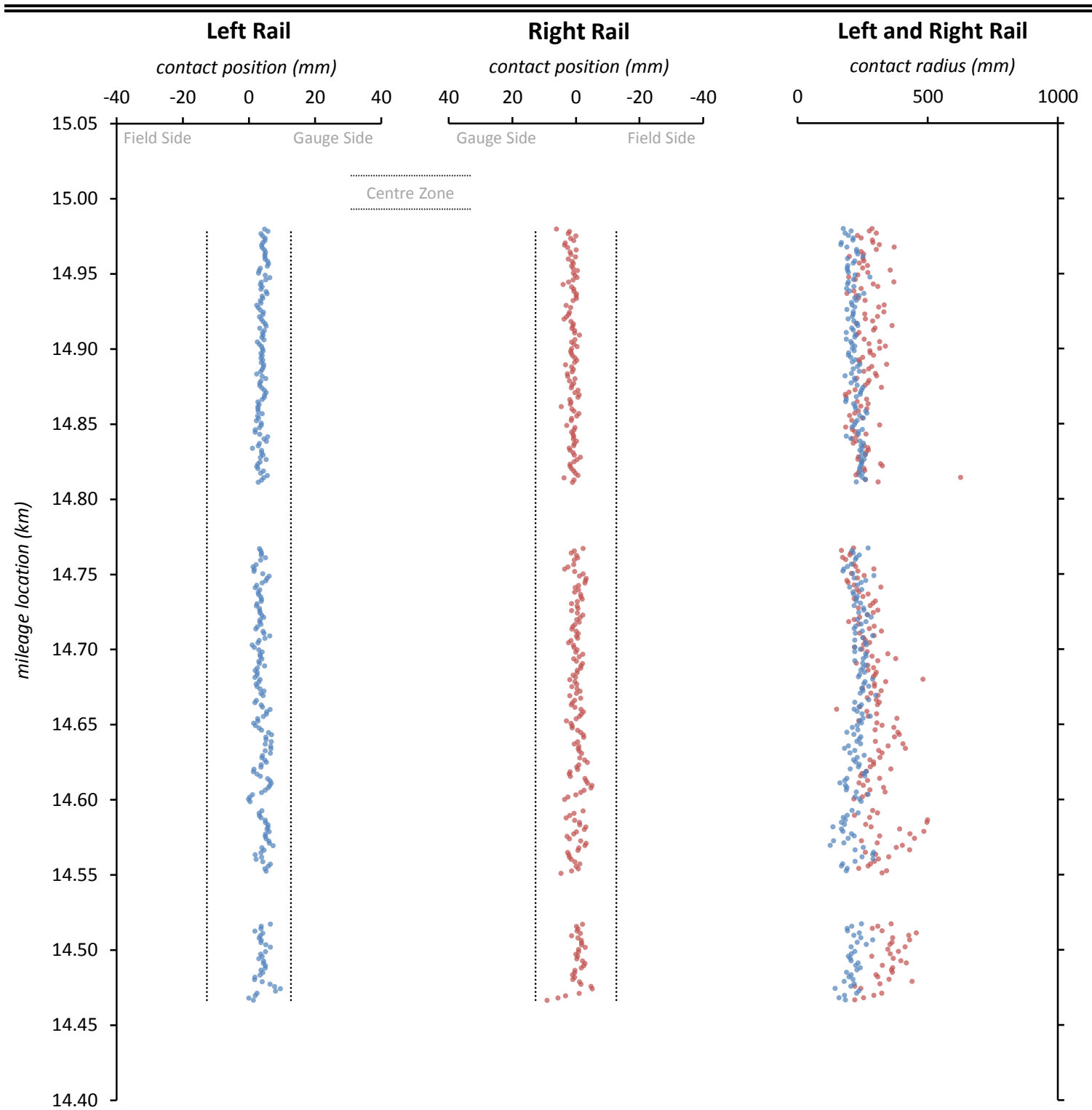

RAIL SEGMENT STATISTICS TABLE

\begin{tabular}{|c|c|c|c|c|}
\hline \multirow{3}{*}{ Statistic } & \multicolumn{4}{|c|}{ Rail Profile Measure } \\
\hline & \multicolumn{2}{|c|}{ Left Rail (280 profiles) } & \multicolumn{2}{|c|}{ Right Rail (275 profiles) } \\
\hline & Contact Position & Contact Radius & Contact Position & Contact Radius \\
\hline Mean $(\mathrm{mm})$ & 3.9 & 222.4 & 0.3 & 286.8 \\
\hline Std. Dev. $(\mathrm{mm})$ & 1.4 & 30.0 & 1.9 & 65.9 \\
\hline$\%$ within Zone & $100 \%$ & - & $100 \%$ & - \\
\hline
\end{tabular}


Lateral Contact Position ( $\mathrm{mm}$ )
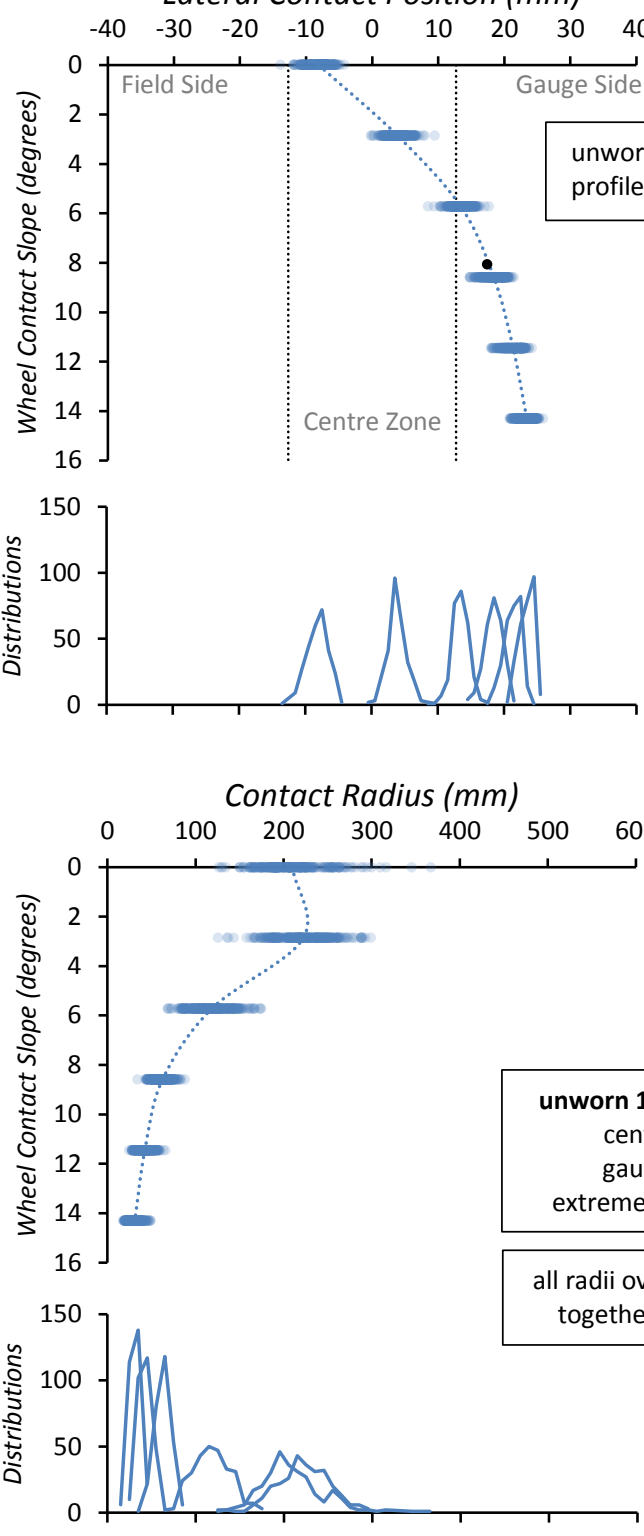

LEFT RAIL PROFILES

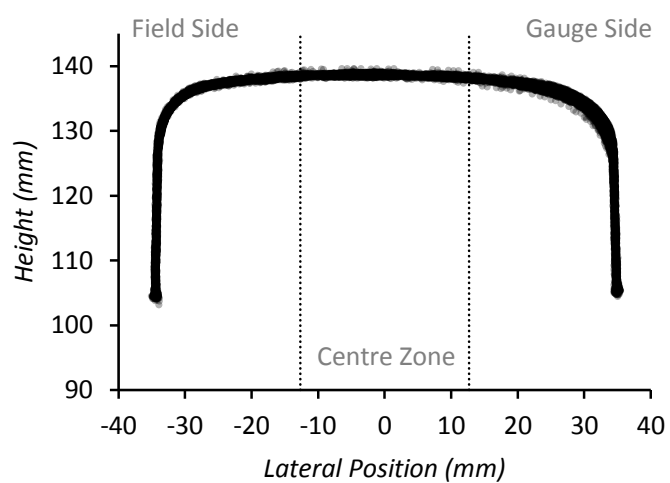

Lateral Contact Position ( $\mathrm{mm}$ )

$\begin{array}{lllllllll}40 & 30 & 20 & 10 & 0 & -10 & -20 & -30 & -40\end{array}$
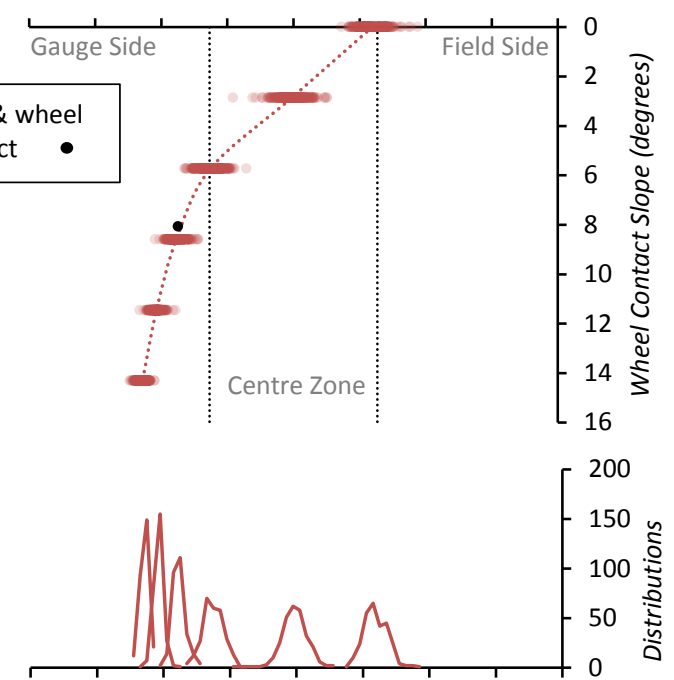

\section{Contact Radius ( $\mathrm{mm}$ )}
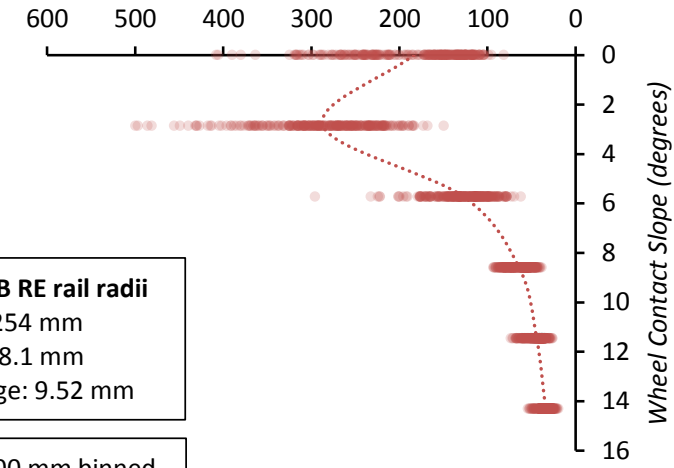

enter: $254 \mathrm{~mm}$

uge: $38.1 \mathrm{~mm}$

reme gauge: $9.52 \mathrm{~mm}$

$600 \mathrm{~mm}$ binned

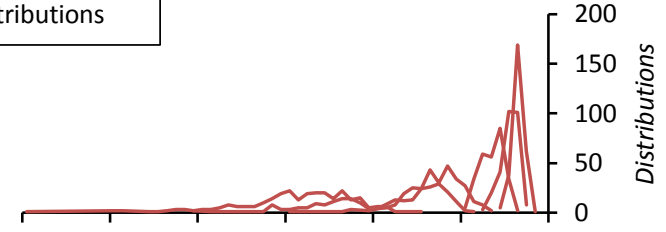

RIGHT RAIL PROFILES

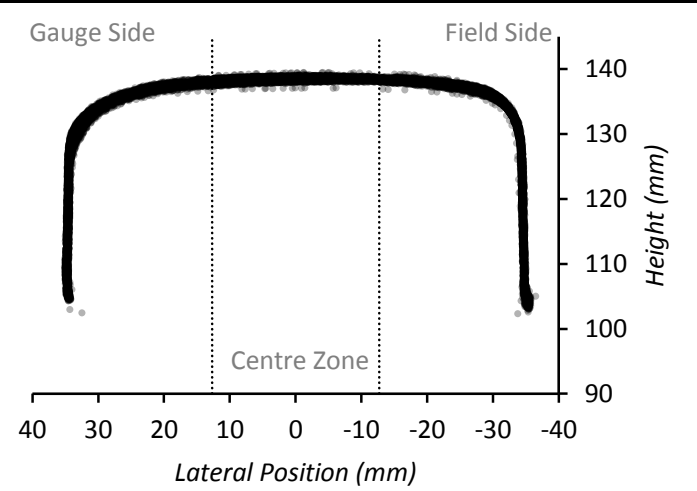

E-22 
SEGMENT AND ANALYSIS INFORMATION

\begin{tabular}{llll}
\hline \hline Track Segment Name: & SB-68 & Contact Analysis Date: & 20180615 \\
Segment Length $(\mathrm{m}):$ & 340.5 & Left Wheel Slope: & $1: 20$ \\
Data Collection Date: & 20150827 & Right Wheel Slope: & $1: 20$
\end{tabular}

RAIL SEGMENT GRAPHS

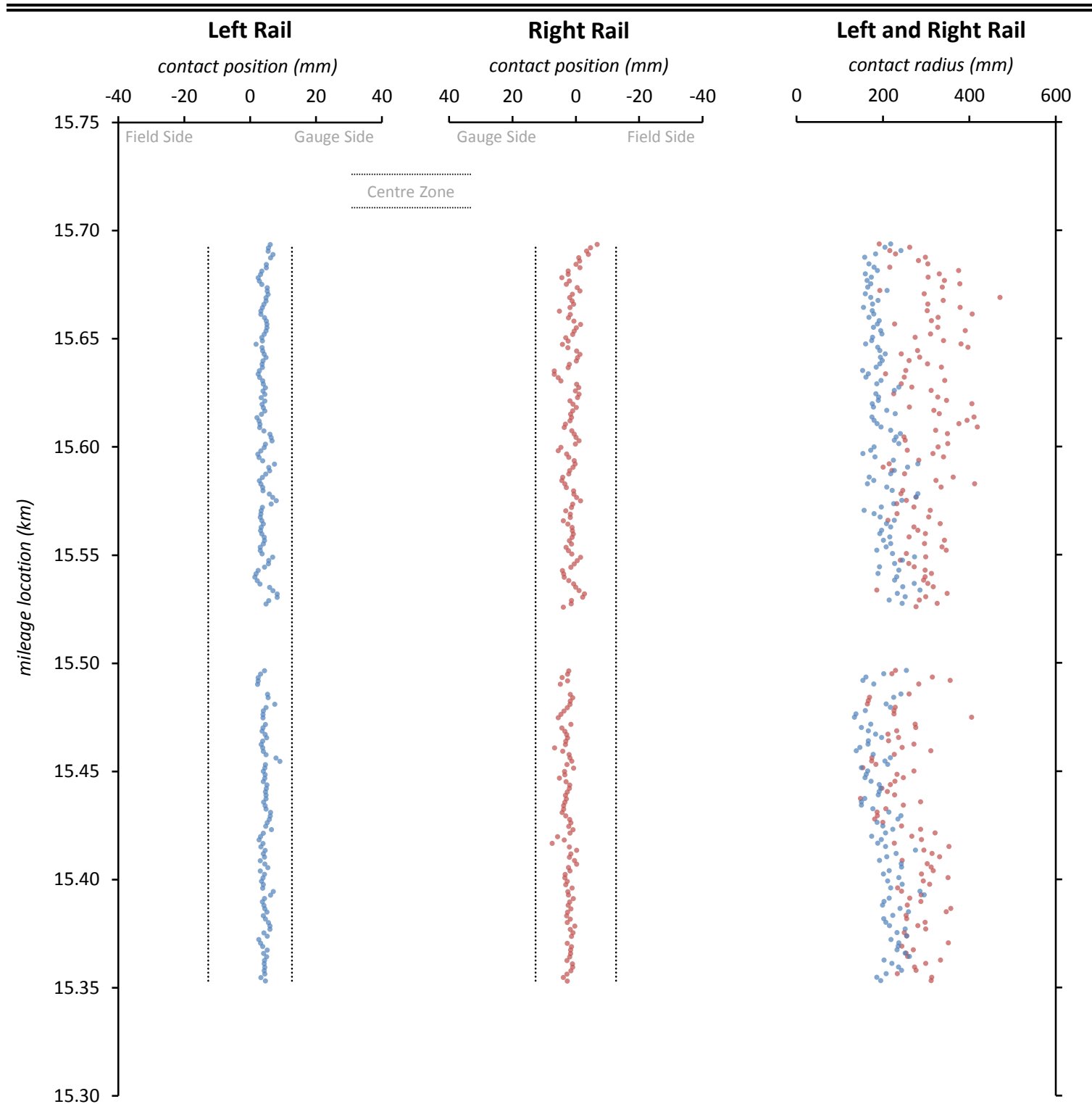

RAIL SEGMENT STATISTICS TABLE

\begin{tabular}{|c|c|c|c|c|}
\hline \multirow{3}{*}{ Statistic } & \multicolumn{4}{|c|}{ Rail Profile Measure } \\
\hline & \multicolumn{2}{|c|}{ Left Rail (196 profiles) } & \multicolumn{2}{|c|}{ Right Rail (198 profiles) } \\
\hline & Contact Position & Contact Radius & Contact Position & Contact Radius \\
\hline Mean $(\mathrm{mm})$ & 4.3 & 202.5 & 1.9 & 281.7 \\
\hline Std. Dev. $(\mathrm{mm})$ & 1.3 & 33.6 & 2.0 & 58.8 \\
\hline$\%$ within Zone & $100 \%$ & - & $100 \%$ & - \\
\hline
\end{tabular}


Lateral Contact Position ( $\mathrm{mm}$ )

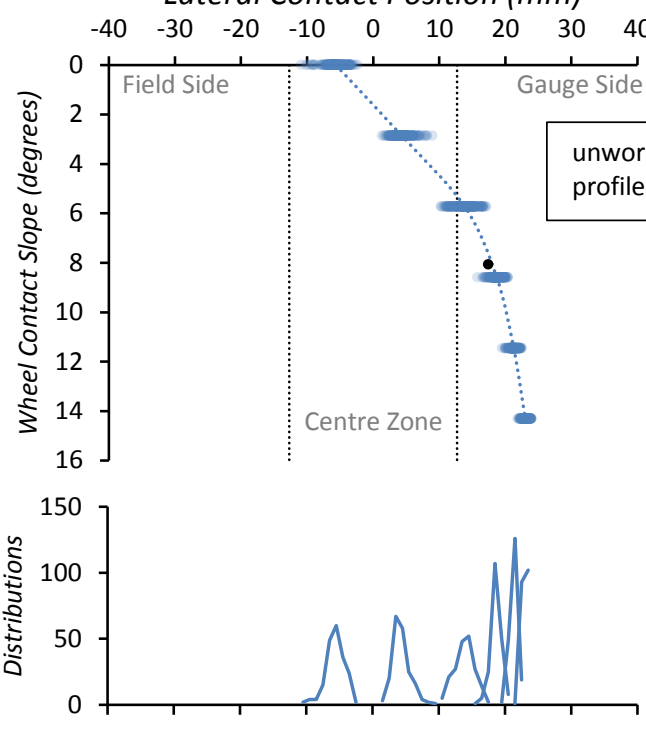

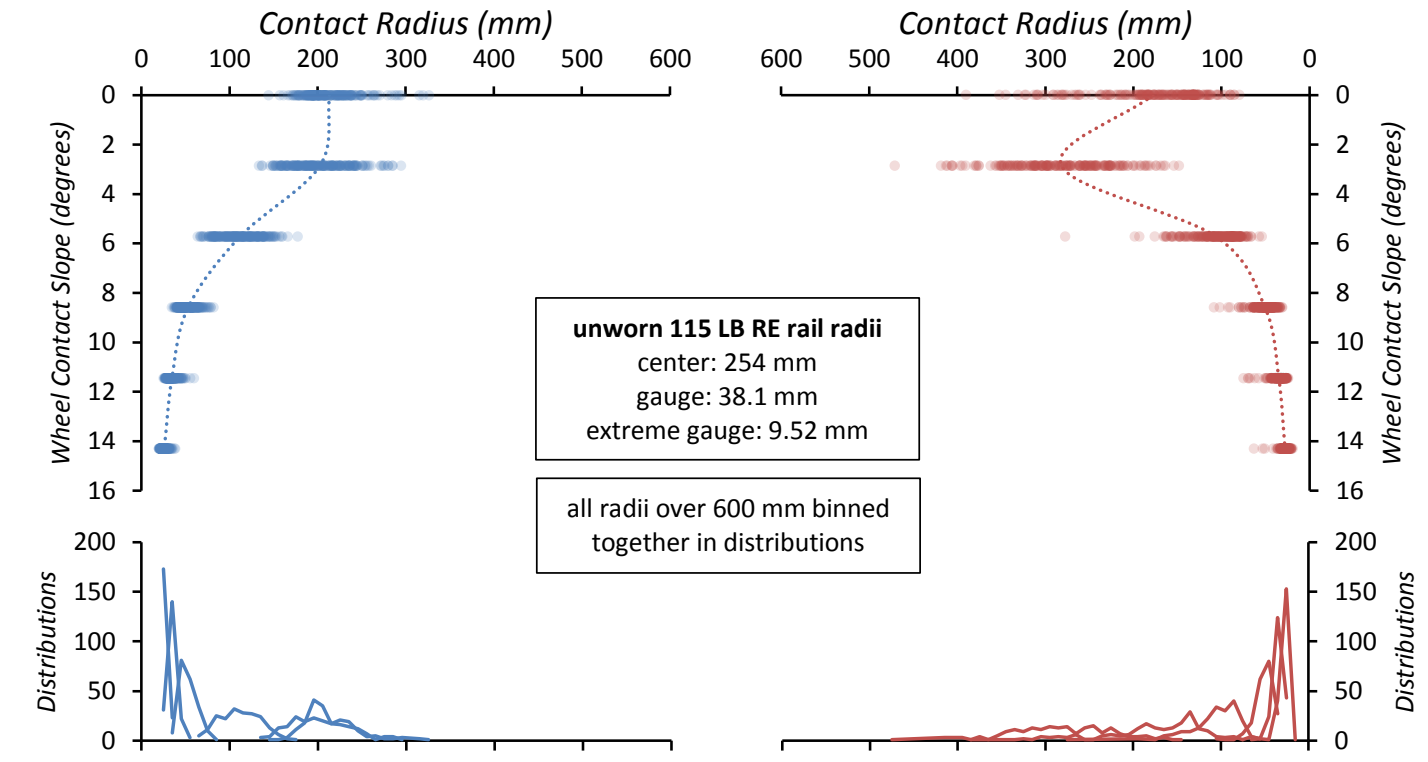

Lateral Contact Position ( $\mathrm{mm}$ )

$\begin{array}{lllllllll}40 & 30 & 20 & 10 & 0 & -10 & -20 & -30 & -40\end{array}$
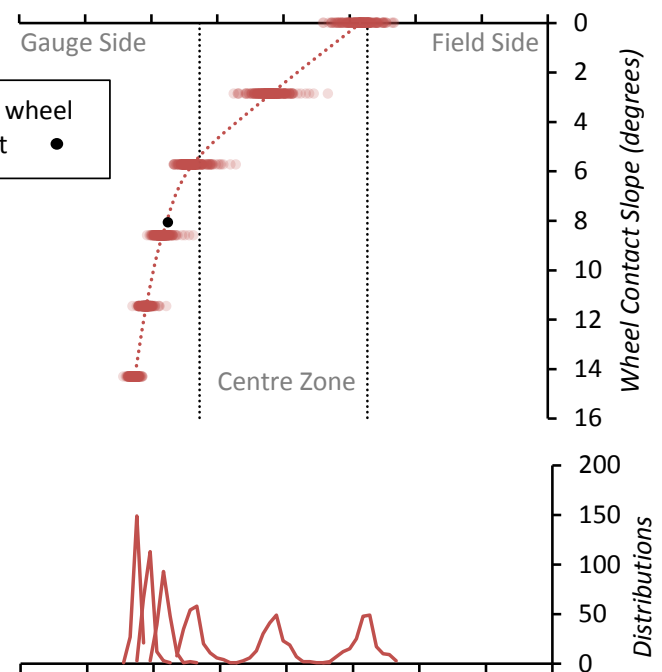

Contact Radius ( $\mathrm{mm}$ )

LEFT RAIL PROFILES

RIGHT RAIL PROFILES
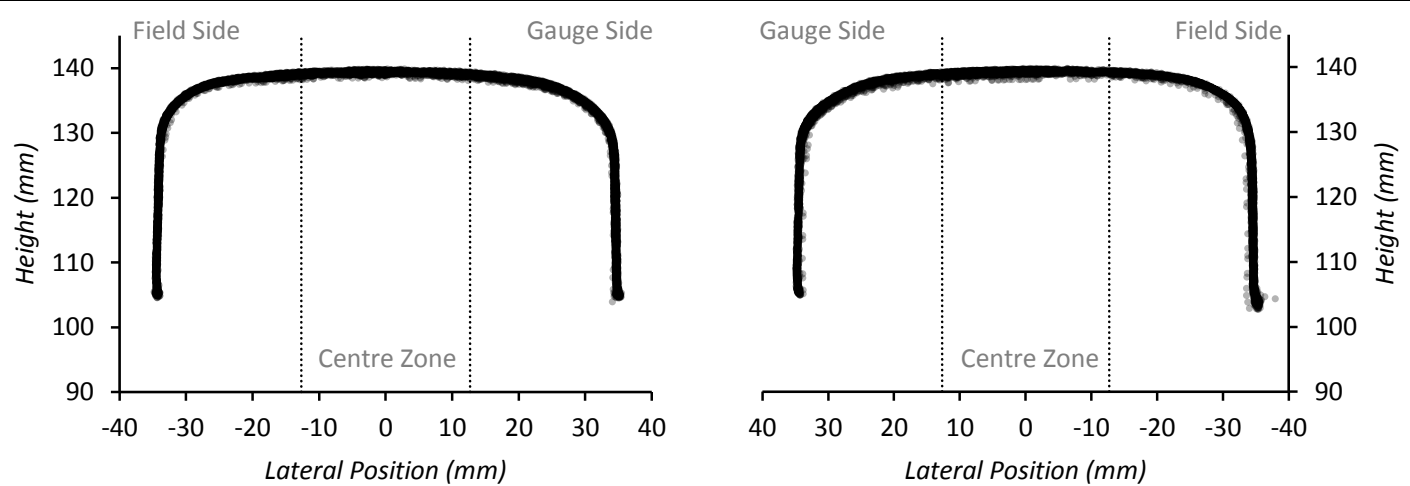
SEGMENT AND ANALYSIS INFORMATION

\begin{tabular}{llll}
\hline \hline Track Segment Name: & SB-70 & Contact Analysis Date: & 20180615 \\
Segment Length $(\mathrm{m}):$ & 868.4 & Left Wheel Slope: & $1: 20$ \\
Data Collection Date: & 20150827 & Right Wheel Slope: & $1: 20$
\end{tabular}

RAIL SEGMENT GRAPHS

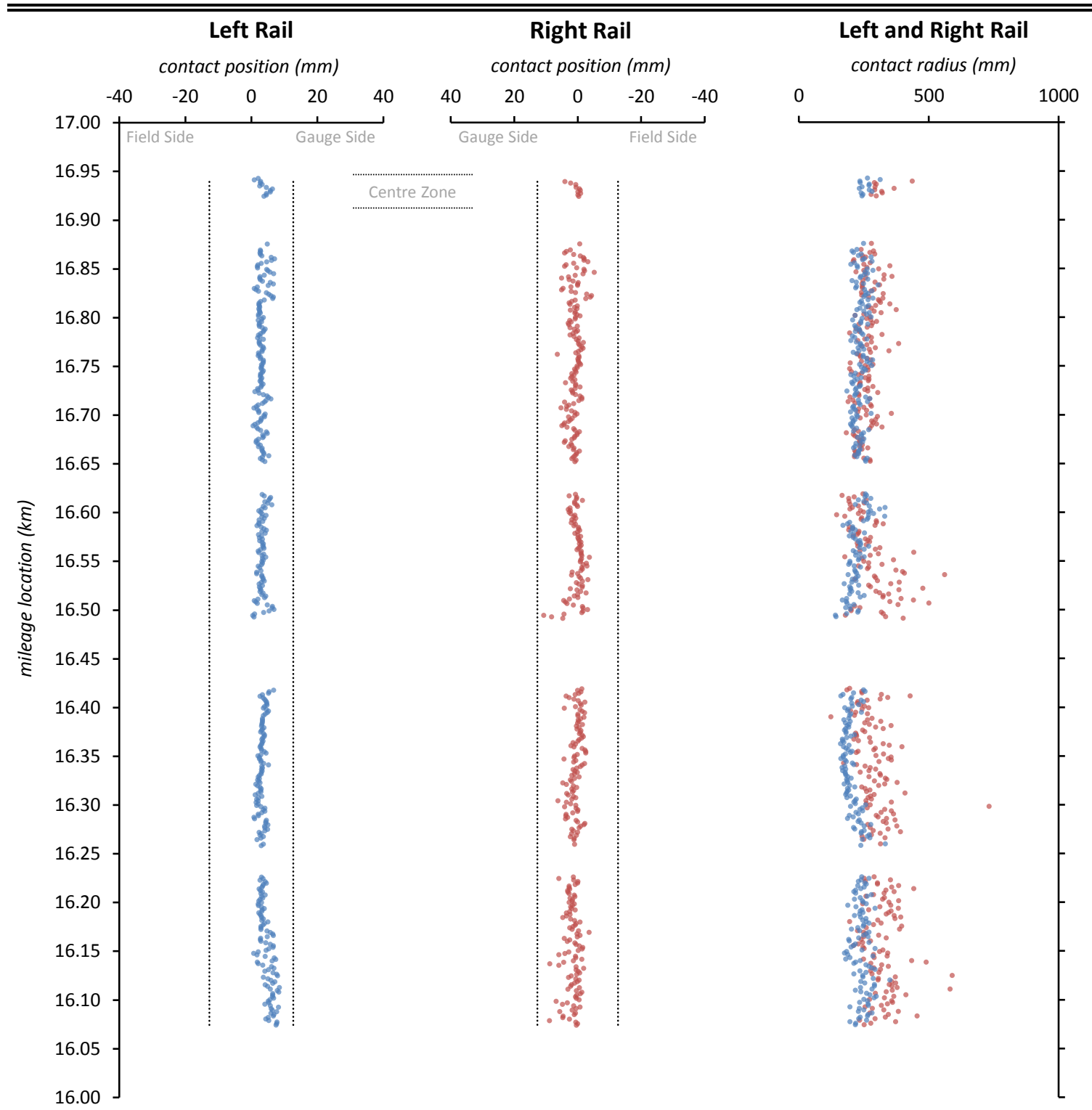

RAIL SEGMENT STATISTICS TABLE

\begin{tabular}{|c|c|c|c|c|}
\hline \multirow{3}{*}{ Statistic } & \multicolumn{4}{|c|}{ Rail Profile Measure } \\
\hline & \multicolumn{2}{|c|}{ Left Rail (442 profiles) } & \multicolumn{2}{|c|}{ Right Rail (442 profiles) } \\
\hline & Contact Position & Contact Radius & Contact Position & Contact Radius \\
\hline Mean (mm) & 3.5 & 228.5 & 0.9 & 287.1 \\
\hline Std. Dev. $(\mathrm{mm})$ & 1.5 & 33.4 & 2.1 & 66.8 \\
\hline$\%$ within Zone & $100 \%$ & - & $100 \%$ & - \\
\hline
\end{tabular}


Lateral Contact Position ( $\mathrm{mm}$ )
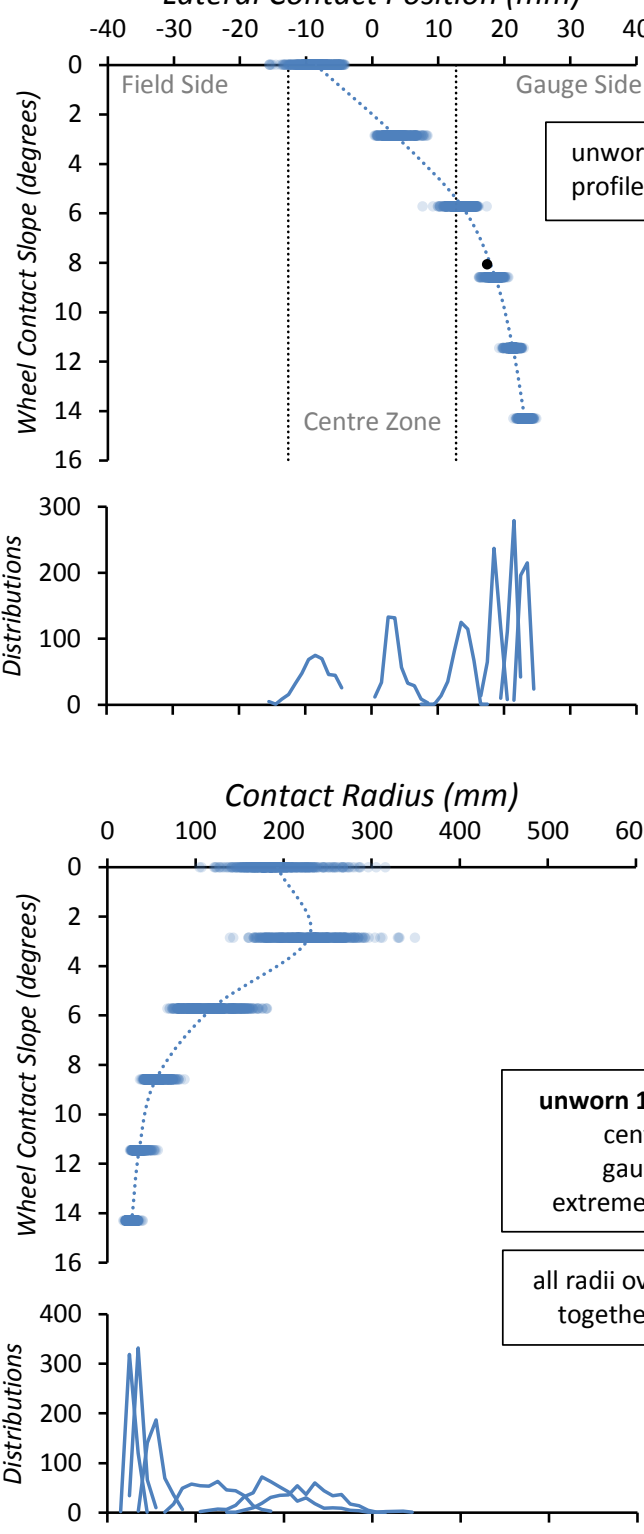

LEFT RAIL PROFILES

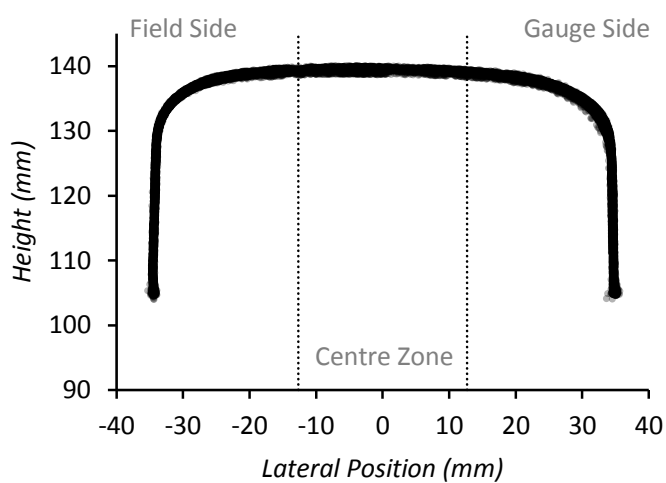

Lateral Contact Position ( $\mathrm{mm}$ )

$\begin{array}{lllllllll}40 & 30 & 20 & 10 & 0 & -10 & -20 & -30 & -40\end{array}$
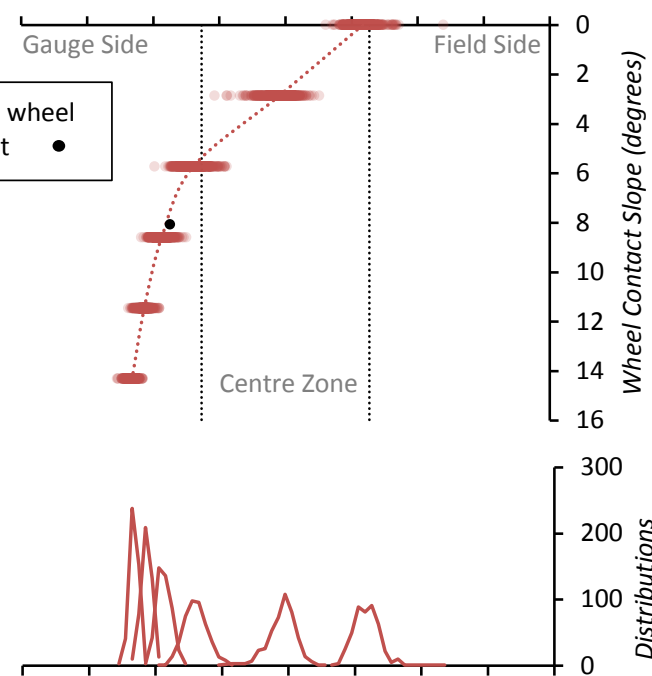

\section{Contact Radius ( $\mathrm{mm}$ )}
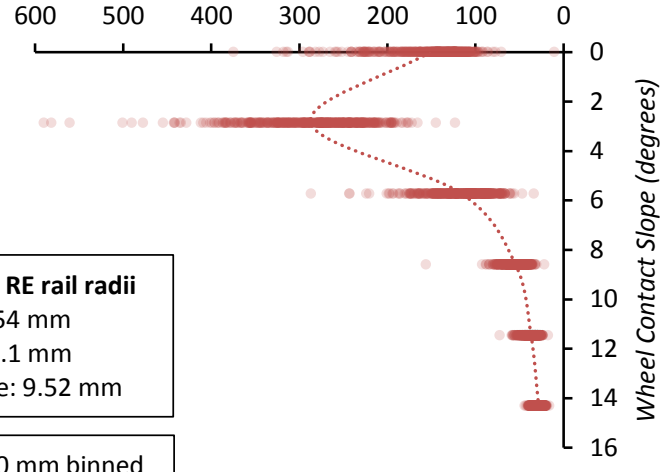

nter: $254 \mathrm{~mm}$

auge: $38.1 \mathrm{~mm}$

eme gauge: $9.52 \mathrm{~mm}$

$\mathrm{mm}$ binne

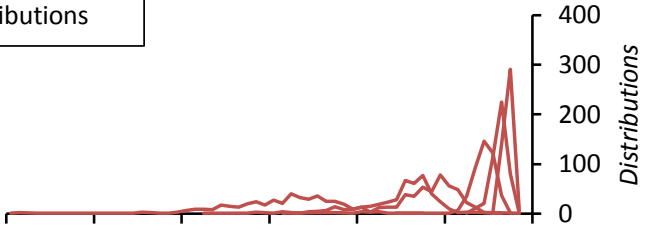


SEGMENT AND ANALYSIS INFORMATION

\begin{tabular}{llll}
\hline \hline Track Segment Name: & SB-74 & Contact Analysis Date: & 20180615 \\
Segment Length $(\mathrm{m}):$ & 117.2 & Left Wheel Slope: & $1: 20$ \\
Data Collection Date: & 20150827 & Right Wheel Slope: & $1: 20$
\end{tabular}

RAIL SEGMENT GRAPHS

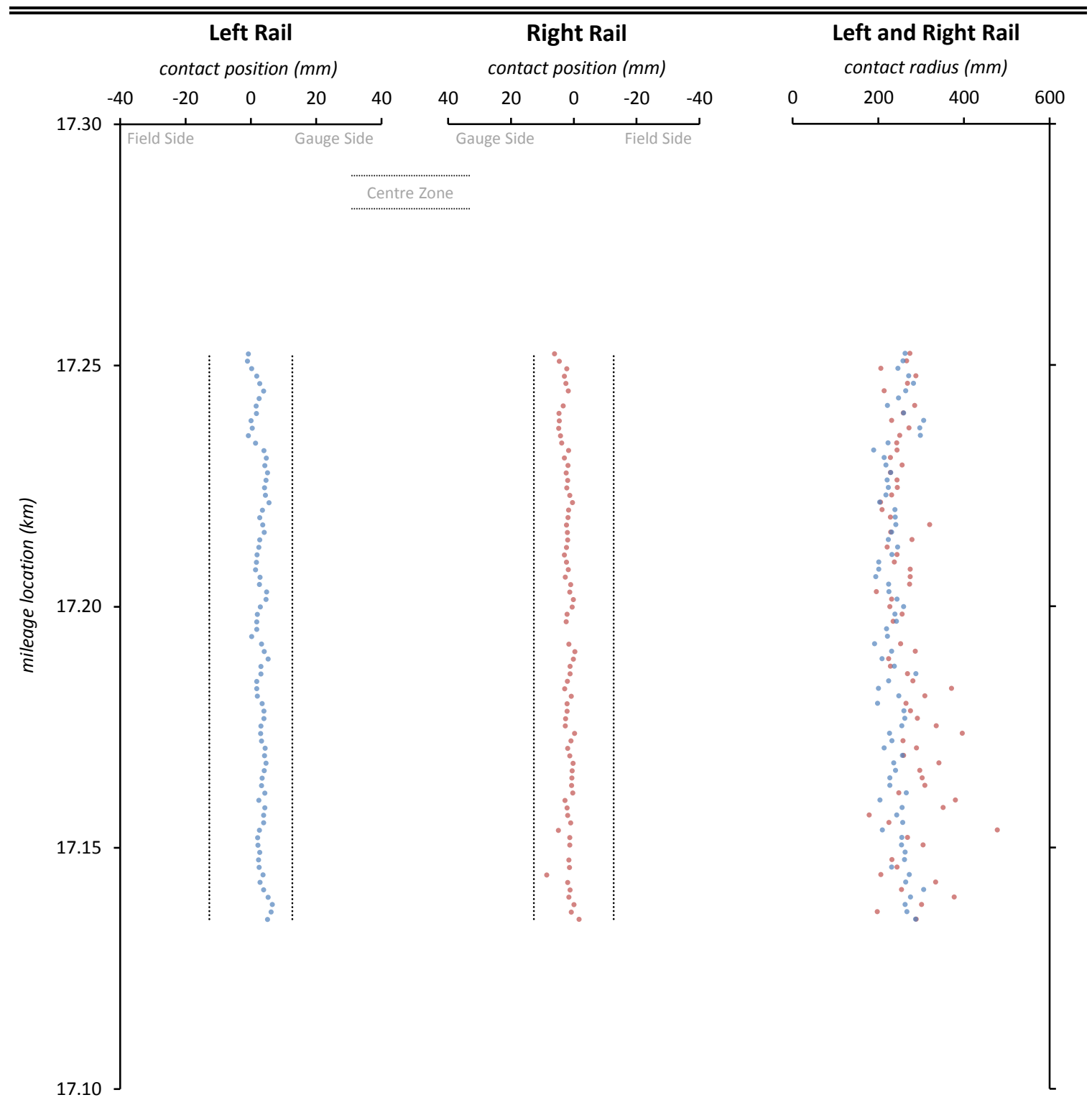

RAIL SEGMENT STATISTICS TABLE

\begin{tabular}{|c|c|c|c|c|}
\hline \multirow{3}{*}{ Statistic } & \multicolumn{4}{|c|}{ Rail Profile Measure } \\
\hline & \multicolumn{2}{|c|}{ Left Rail (77 profiles) } & \multicolumn{2}{|c|}{ Right Rail (73 profiles) } \\
\hline & Contact Position & Contact Radius & Contact Position & Contact Radius \\
\hline Mean $(\mathrm{mm})$ & 3.0 & 240.8 & 2.0 & 267.8 \\
\hline Std. Dev. (mm) & 1.6 & 27.5 & 1.6 & 52.0 \\
\hline$\%$ within Zone & $100 \%$ & - & $100 \%$ & - \\
\hline
\end{tabular}


Lateral Contact Position ( $\mathrm{mm}$ )
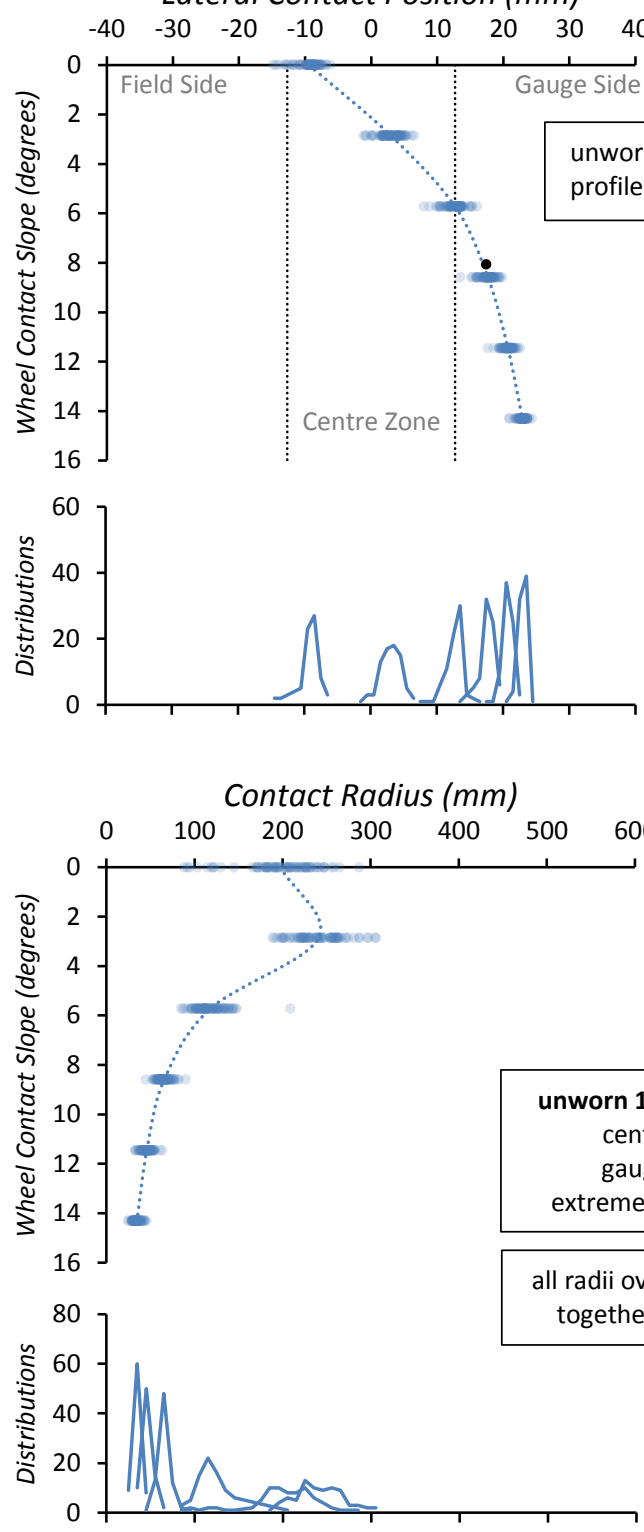

Lateral Contact Position ( $\mathrm{mm}$ )
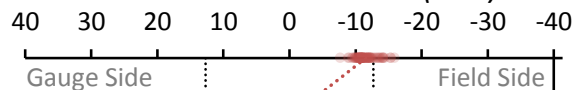

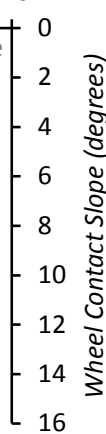

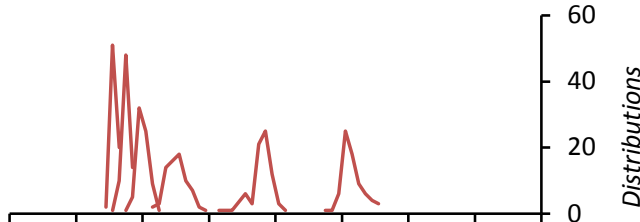

Contact Radius ( $\mathrm{mm}$ )
115 LB RE rail radi

center: $254 \mathrm{~mm}$

gauge: $38.1 \mathrm{~mm}$

treme gauge: $9.52 \mathrm{~mm}$

radii over $600 \mathrm{~mm}$ binned

ibutions

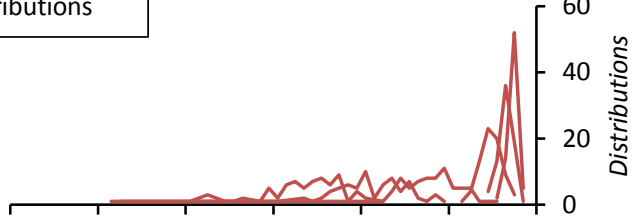

LEFT RAIL PROFILES

RIGHT RAIL PROFILES
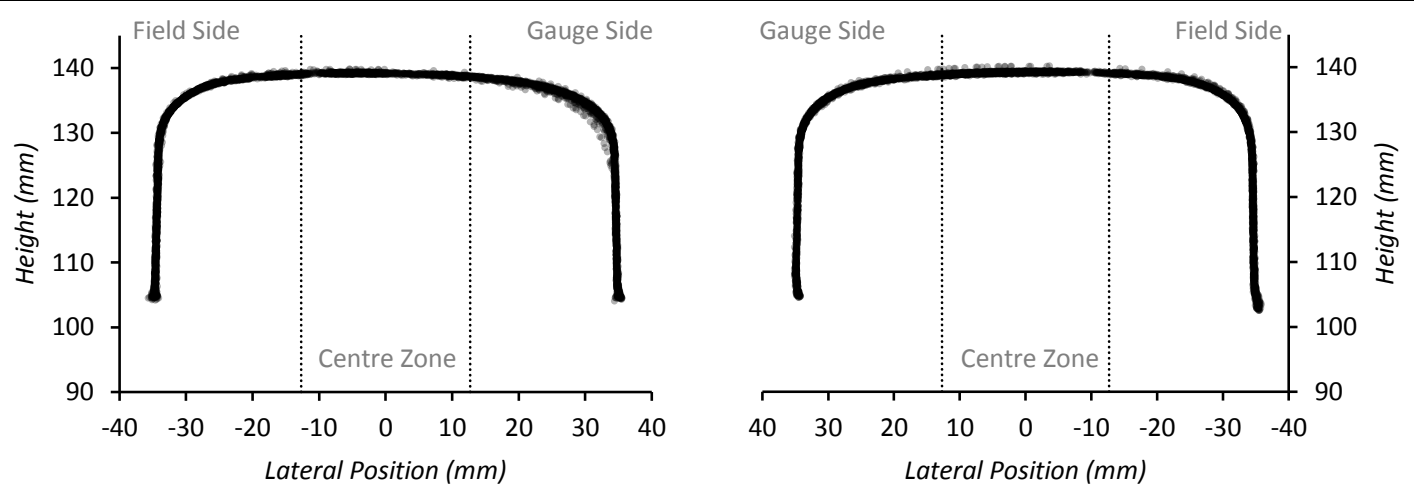

E-28 
SEGMENT AND ANALYSIS INFORMATION

\begin{tabular}{llll}
\hline \hline Track Segment Name: & SB-77 & Contact Analysis Date: & 20180615 \\
Segment Length $(\mathrm{m}):$ & 125.3 & Left Wheel Slope: & $1: 20$ \\
Data Collection Date: & 20150827 & Right Wheel Slope: & $1: 20$
\end{tabular}

RAIL SEGMENT GRAPHS

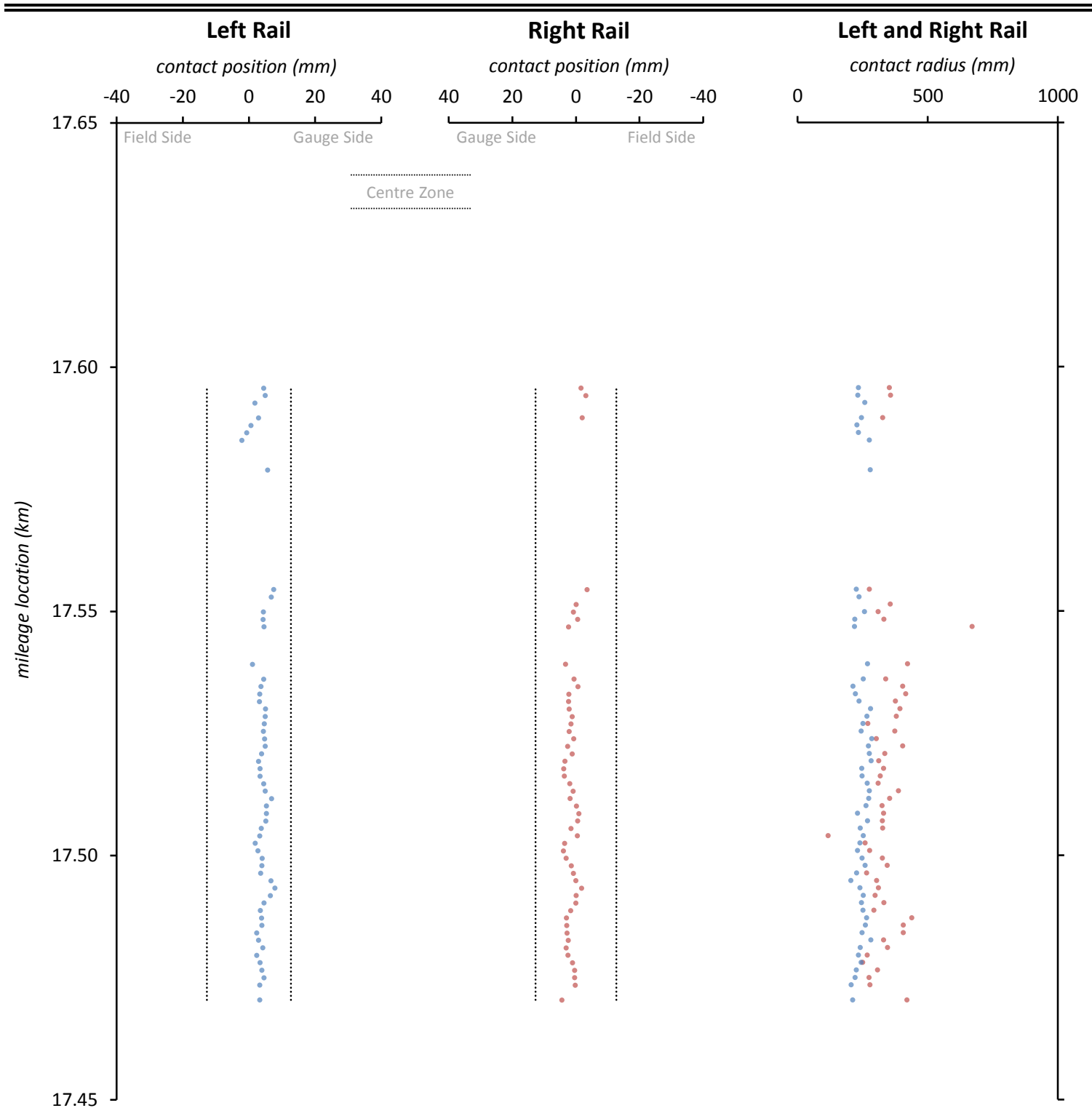

RAIL SEGMENT STATISTICS TABLE

\begin{tabular}{|c|c|c|c|c|}
\hline \multirow{3}{*}{ Statistic } & \multicolumn{4}{|c|}{ Rail Profile Measure } \\
\hline & \multicolumn{2}{|c|}{ Left Rail (57 profiles) } & \multicolumn{2}{|c|}{ Right Rail (52 profiles) } \\
\hline & Contact Position & Contact Radius & Contact Position & Contact Radius \\
\hline Mean $(\mathrm{mm})$ & 3.9 & 246.8 & 1.2 & 337.2 \\
\hline Std. Dev. $(\mathrm{mm})$ & 1.8 & 21.1 & 1.8 & 73.3 \\
\hline$\%$ within Zone & $100 \%$ & - & $100 \%$ & - \\
\hline
\end{tabular}


Lateral Contact Position ( $\mathrm{mm}$ )
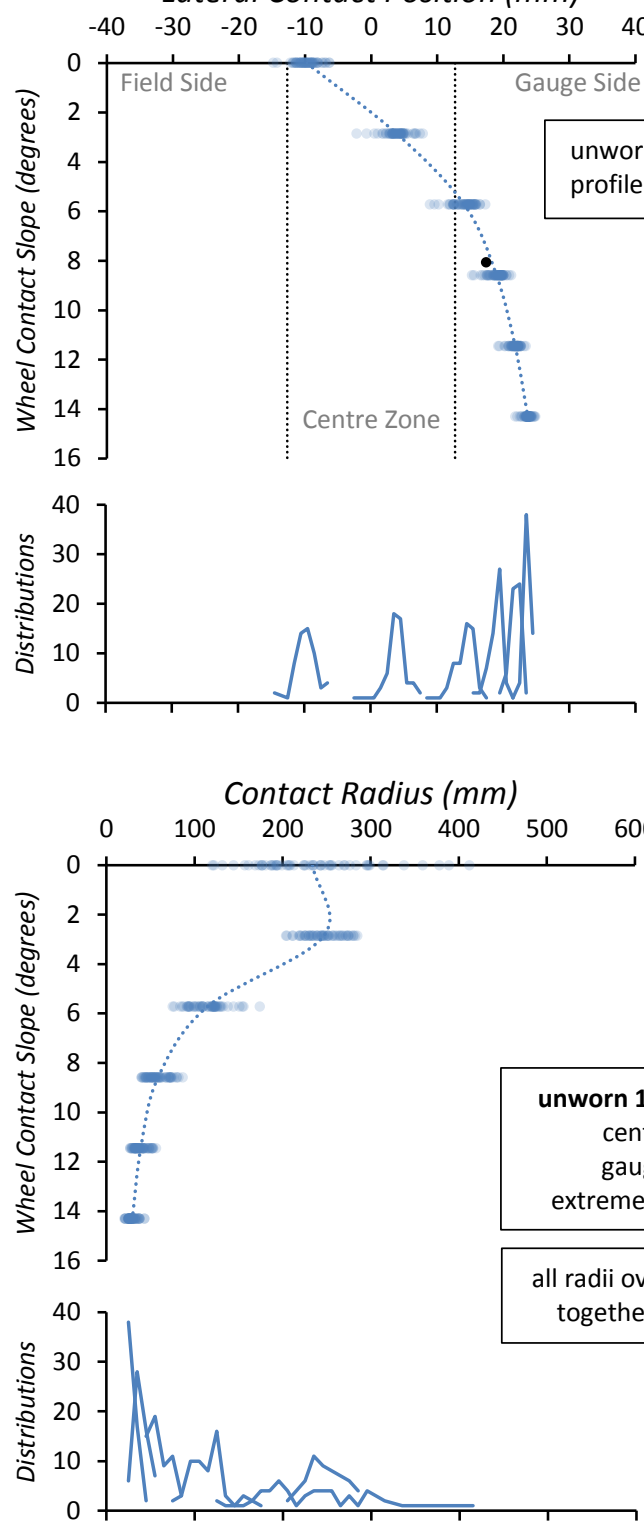

LEFT RAIL PROFILES

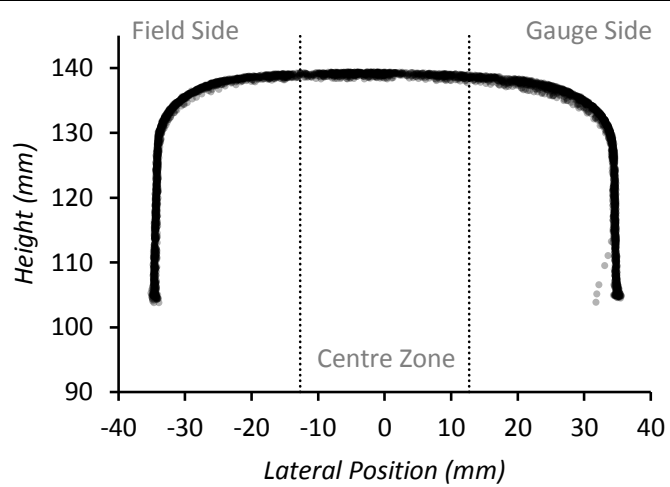

Lateral Contact Position ( $\mathrm{mm}$ )

$\begin{array}{lllllllll}40 & 30 & 20 & 10 & 0 & -10 & -20 & -30 & -40\end{array}$
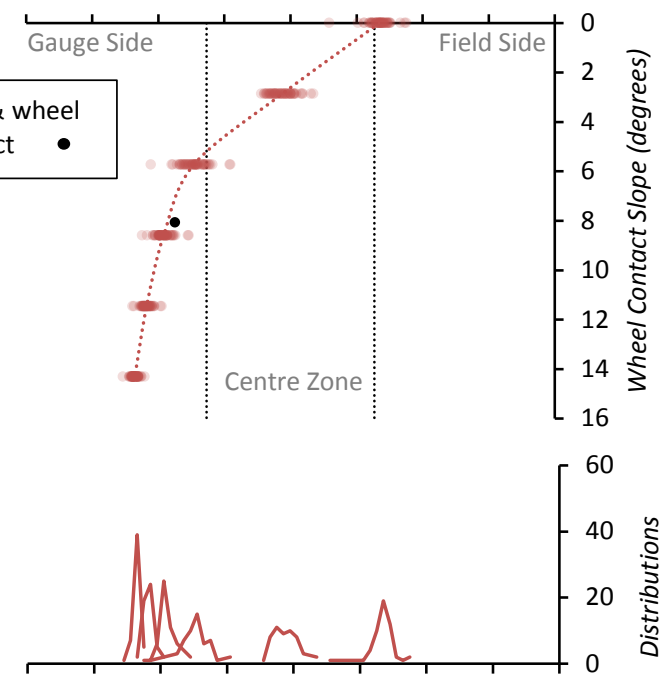

Contact Radius ( $\mathrm{mm}$ )
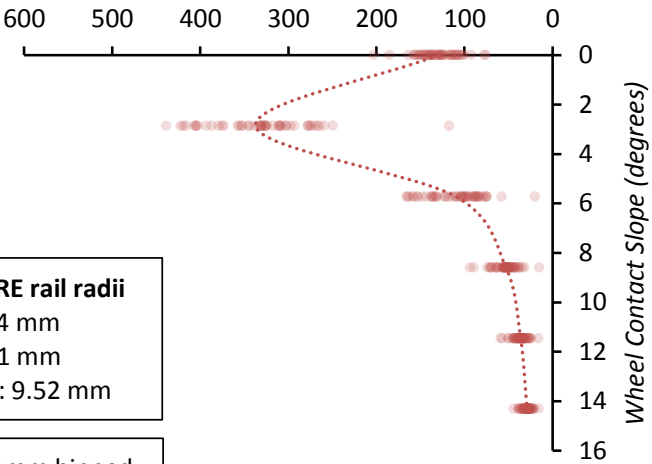

er: $254 \mathrm{~mm}$

uge: $38.1 \mathrm{~mm}$

gauge: $9.52 \mathrm{~mm}$

m binned

(ributions

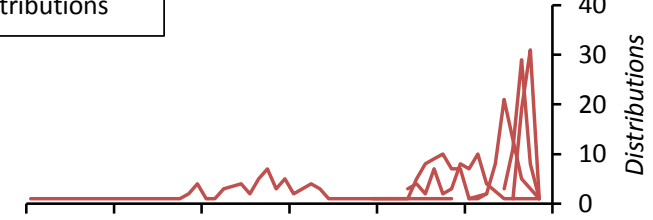


SEGMENT AND ANALYSIS INFORMATION

\begin{tabular}{llll}
\hline \hline Track Segment Name: & SB-81 & Contact Analysis Date: & 20180615 \\
Segment Length $(\mathrm{m}):$ & 807.4 & Left Wheel Slope: & $1: 20$ \\
Data Collection Date: & 20150827 & Right Wheel Slope: & $1: 20$
\end{tabular}

RAIL SEGMENT GRAPHS

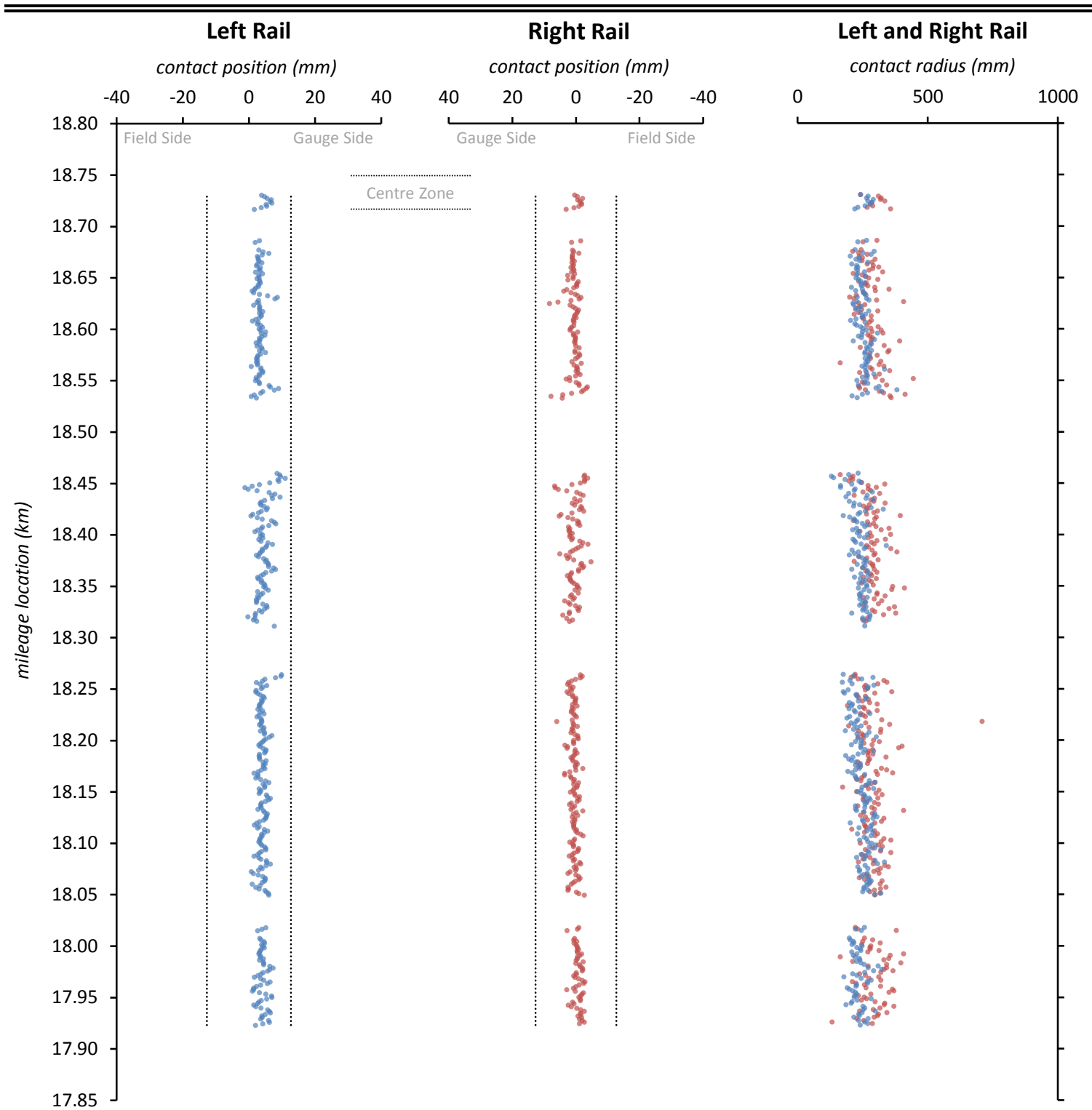

RAIL SEGMENT STATISTICS TABLE

\begin{tabular}{|c|c|c|c|c|}
\hline \multirow{3}{*}{ Statistic } & \multicolumn{4}{|c|}{ Rail Profile Measure } \\
\hline & \multicolumn{2}{|c|}{ Left Rail (405 profiles) } & \multicolumn{2}{|c|}{ Right Rail (392 profiles) } \\
\hline & Contact Position & Contact Radius & Contact Position & Contact Radius \\
\hline Mean $(\mathrm{mm})$ & 4.0 & 245.5 & 0.4 & 286.4 \\
\hline Std. Dev. $(\mathrm{mm})$ & 1.7 & 32.4 & 1.8 & 50.9 \\
\hline$\%$ within Zone & $100 \%$ & - & $100 \%$ & - \\
\hline
\end{tabular}


Lateral Contact Position ( $\mathrm{mm}$ )
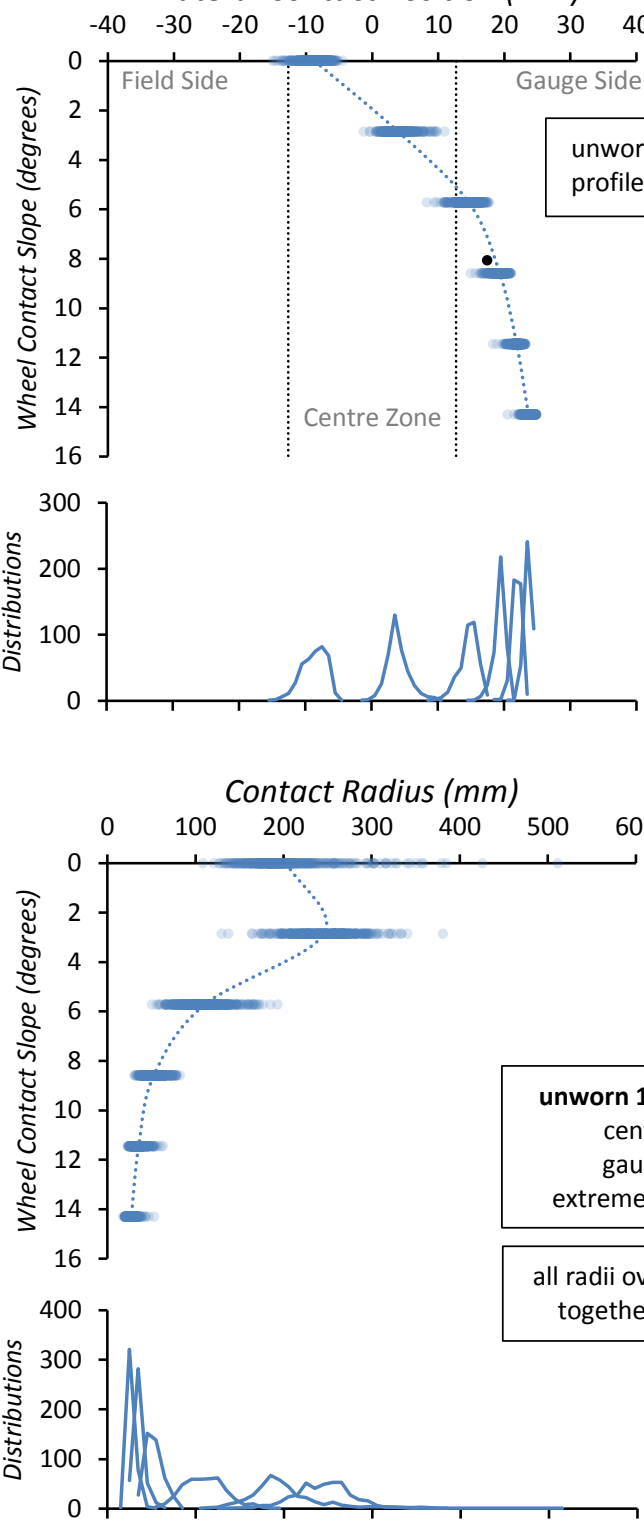

LEFT RAIL PROFILES

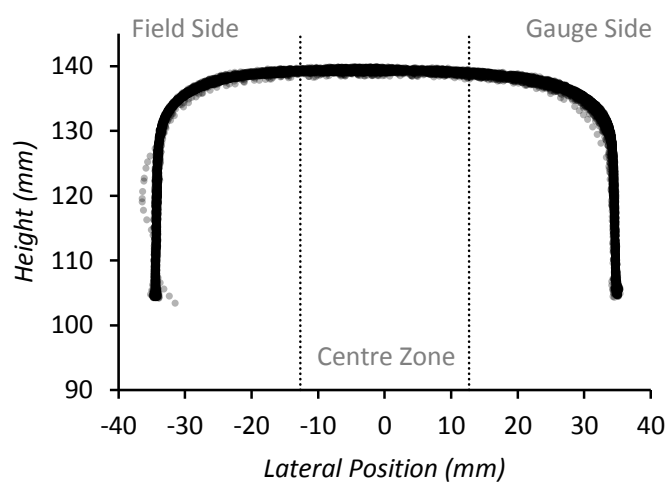

Lateral Contact Position ( $\mathrm{mm}$ )

$\begin{array}{lllllllll}40 & 30 & 20 & 10 & 0 & -10 & -20 & -30 & -40\end{array}$
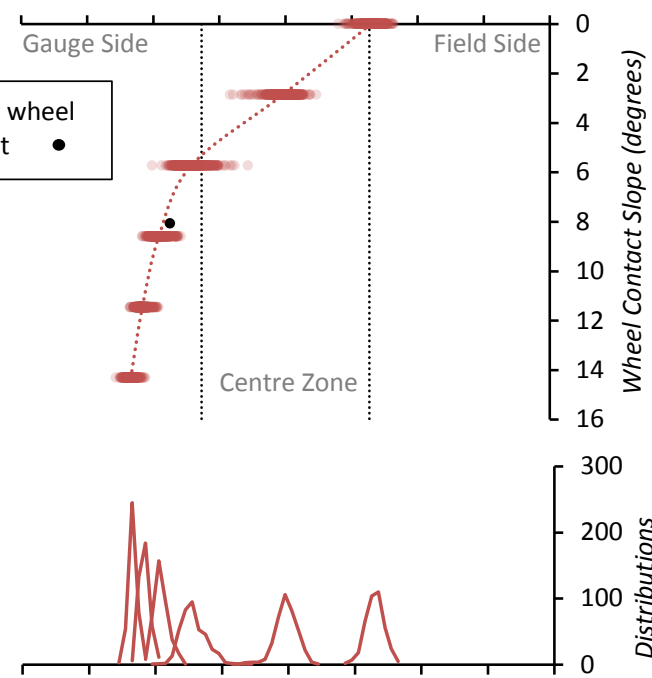

Contact Radius ( $\mathrm{mm}$ )
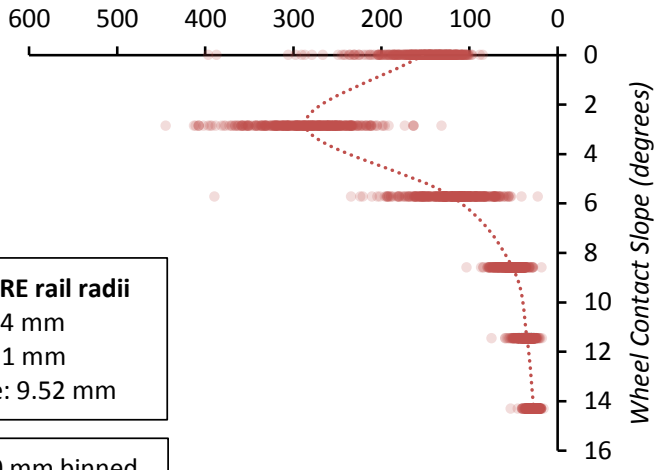

ter: $254 \mathrm{~mm}$

uge: $38.1 \mathrm{~mm}$

eme gauge: $9.52 \mathrm{~mm}$

$$
\text { ributions }
$$

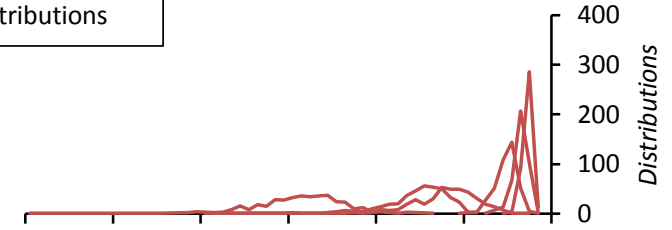


SEGMENT AND ANALYSIS INFORMATION

\begin{tabular}{llll}
\hline \hline Track Segment Name: & SB-83 & Contact Analysis Date: & 20180615 \\
Segment Length $(\mathrm{m}):$ & 701.8 & Left Wheel Slope: & $1: 20$ \\
Data Collection Date: & 20150827 & Right Wheel Slope: & $1: 20$
\end{tabular}

RAIL SEGMENT GRAPHS

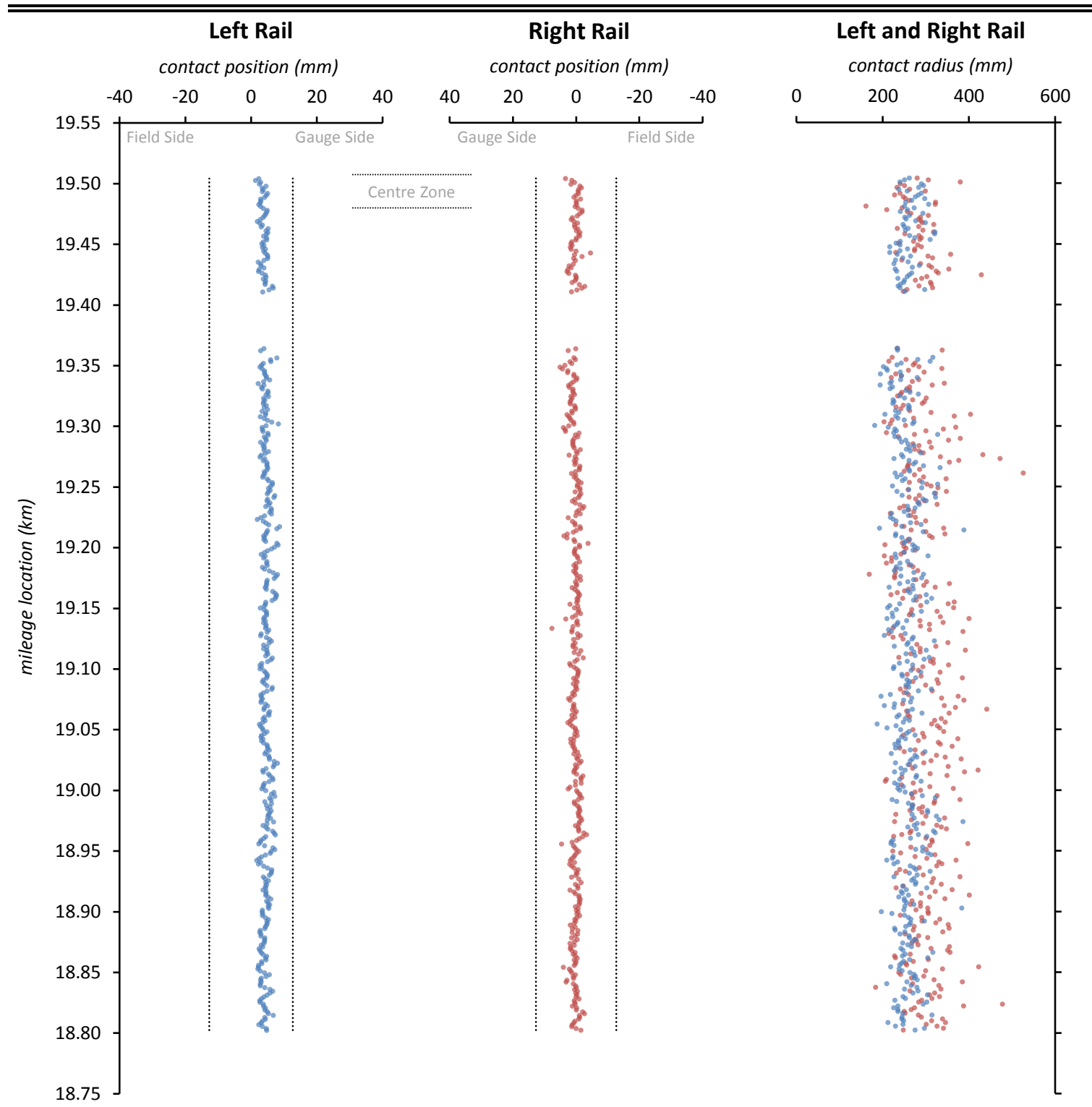

RAIL SEGMENT STATISTICS TABLE

\begin{tabular}{|c|c|c|c|c|}
\hline \multirow{3}{*}{ Statistic } & \multicolumn{4}{|c|}{ Rail Profile Measure } \\
\hline & \multicolumn{2}{|c|}{ Left Rail (429 profiles) } & \multicolumn{2}{|c|}{ Right Rail (423 profiles) } \\
\hline & Contact Position & Contact Radius & Contact Position & Contact Radius \\
\hline Mean $(\mathrm{mm})$ & 4.4 & 257.2 & 0.3 & 292.0 \\
\hline Std. Dev. $(\mathrm{mm})$ & 1.3 & 30.2 & 1.3 & 49.8 \\
\hline$\%$ within Zone & $100 \%$ & - & $100 \%$ & - \\
\hline
\end{tabular}


Lateral Contact Position ( $\mathrm{mm}$ )
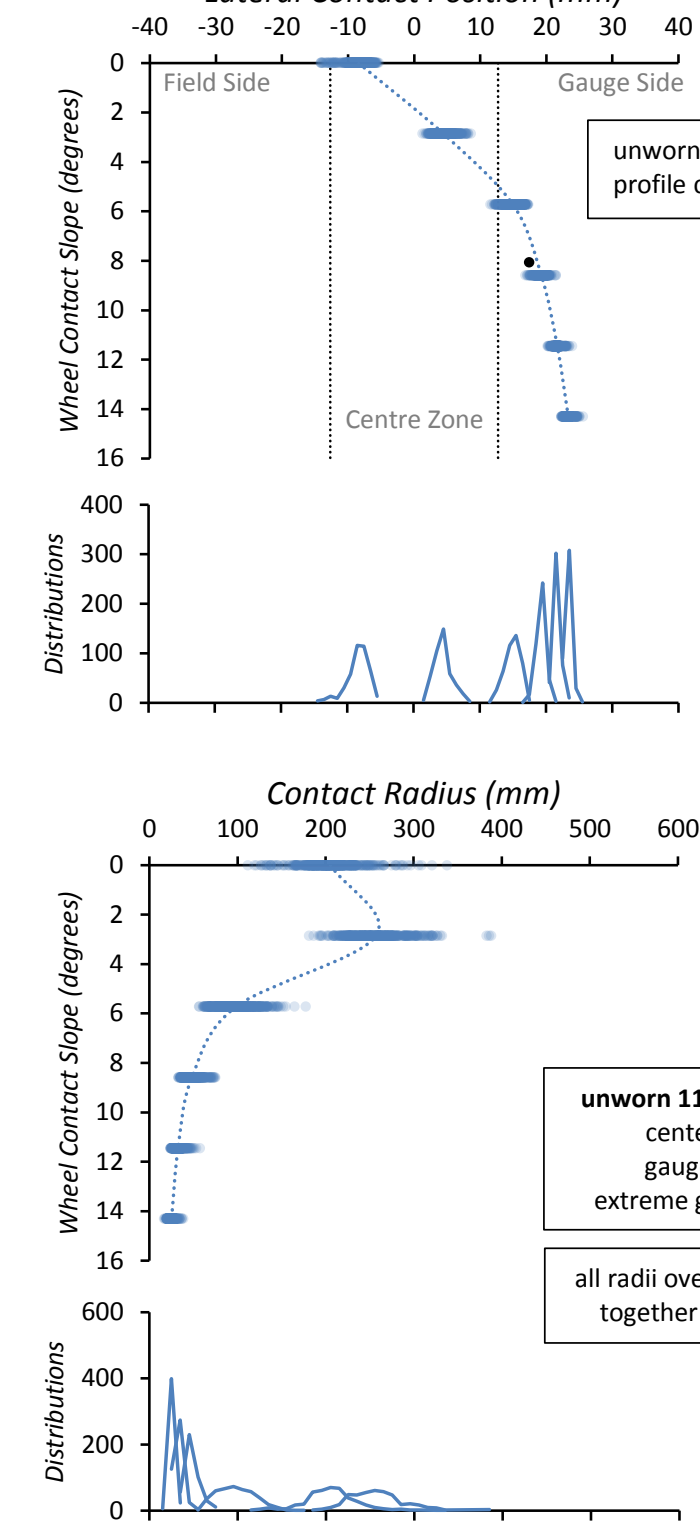

Lateral Contact Position ( $\mathrm{mm}$ )

$\begin{array}{lllllllll}40 & 30 & 20 & 10 & 0 & -10 & -20 & -30 & -40\end{array}$
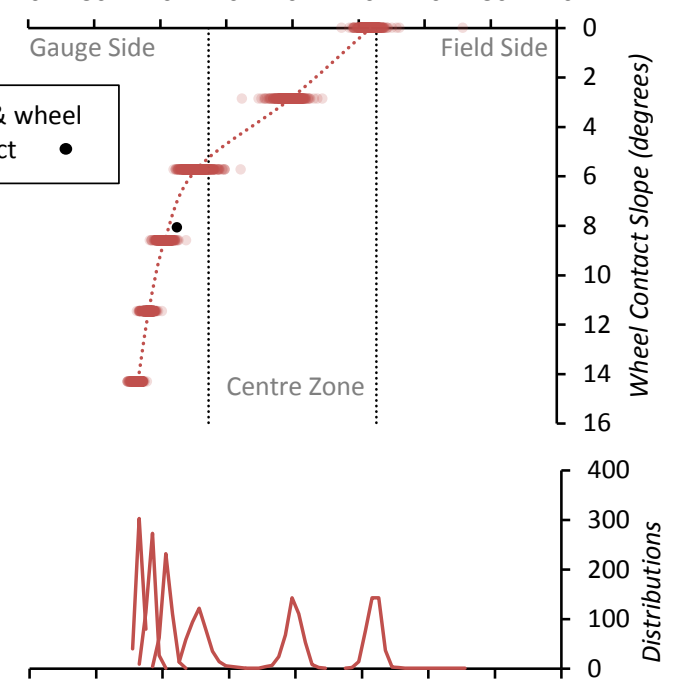

Contact Radius ( $\mathrm{mm}$ )

$\begin{array}{lllllll}500 & 500 & 400 & 300 & 200 & 100 & 0\end{array}$

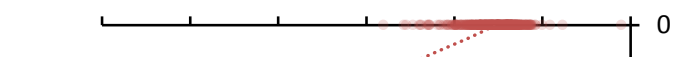

115 LB $\mathrm{RE}$ rail radii nter: $254 \mathrm{~mm}$ uge: $38.1 \mathrm{~mm}$ gauge: $9.52 \mathrm{~mm}$
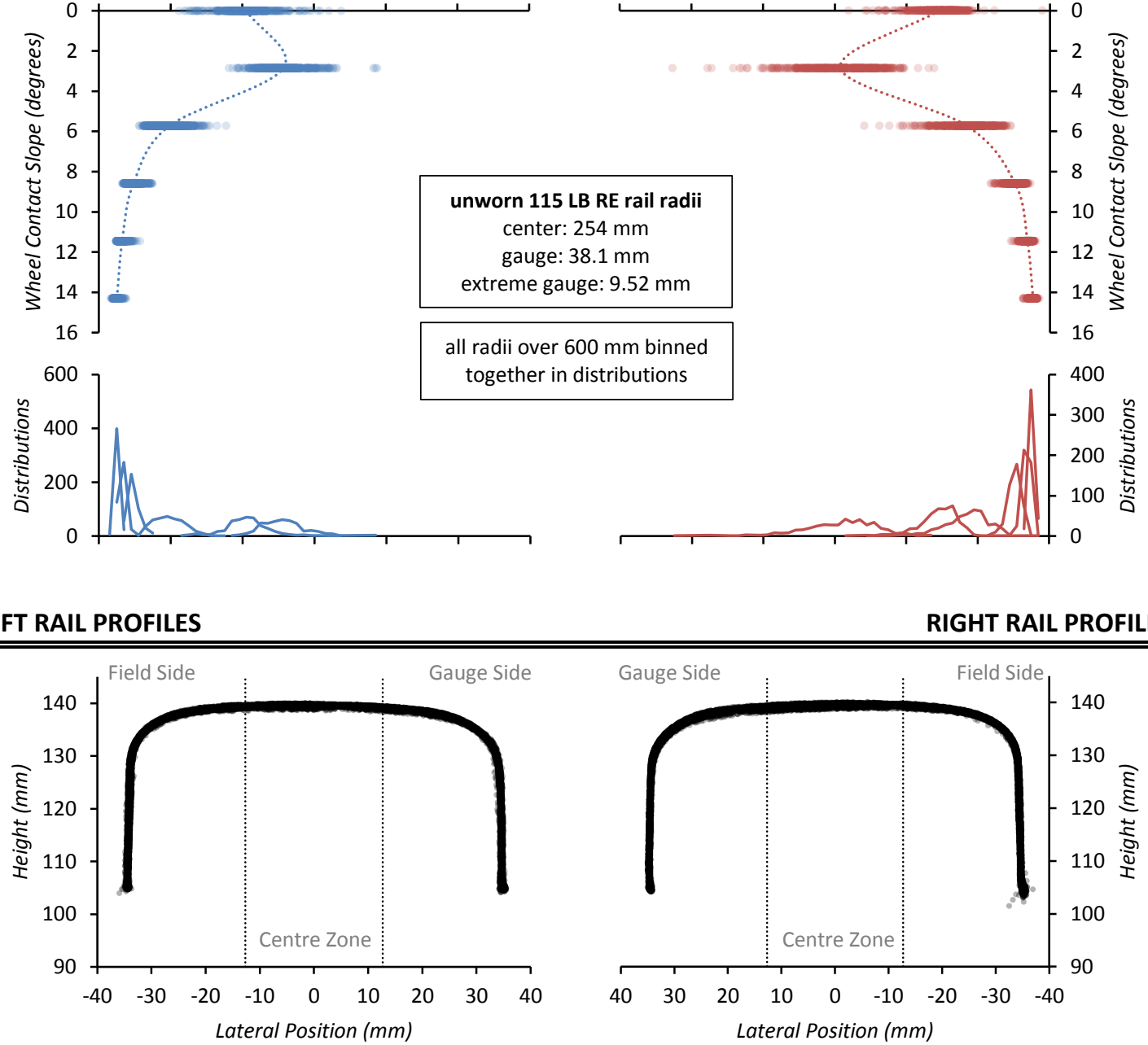
SEGMENT AND ANALYSIS INFORMATION

\begin{tabular}{llll}
\hline \hline Track Segment Name: & SB-87 & Contact Analysis Date: & 20180615 \\
Segment Length $(\mathrm{m}):$ & 301.0 & Left Wheel Slope: & $1: 20$ \\
Data Collection Date: & 20150827 & Right Wheel Slope: & $1: 20$
\end{tabular}

RAIL SEGMENT GRAPHS

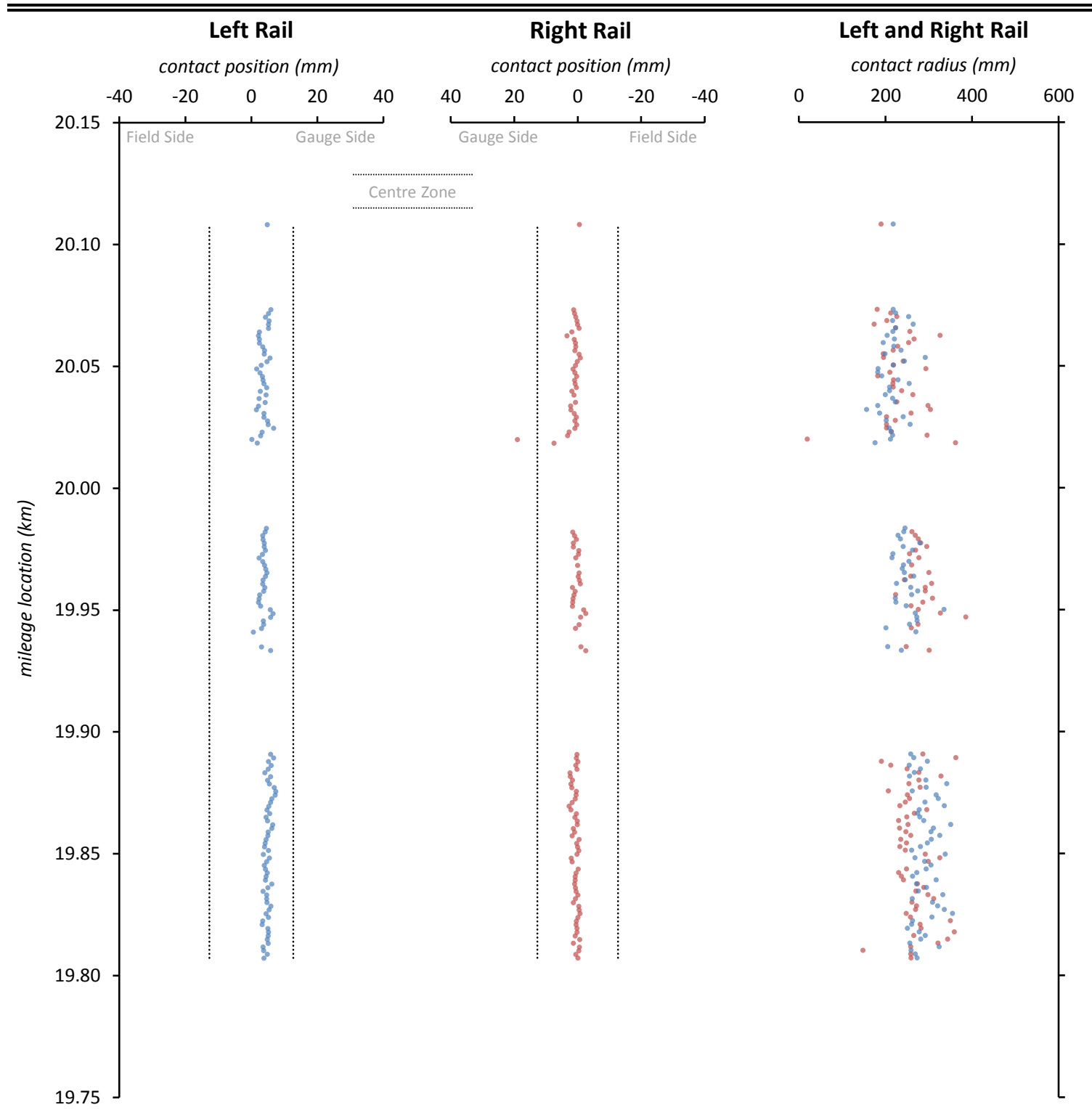

RAIL SEGMENT STATISTICS TABLE

\begin{tabular}{ccccc}
\hline \hline \multirow{2}{*}{ Statistic } & \multicolumn{4}{c}{ Rail Profile Measure } \\
\cline { 2 - 5 } & \multicolumn{4}{c}{ Left Rail } \\
\cline { 2 - 5 } & Contact Position & Contact Radius & Contact Position & Contact Radius \\
\hline Mean $(\mathrm{mm})$ & 4.3 & 257.4 & 0.9 & 257.3 \\
Std. Dev. $(\mathrm{mm})$ & 1.3 & 42.3 & 2.1 & 48.2 \\
$\%$ within Zone & $100 \%$ & - & $99 \%$ & - \\
\hline
\end{tabular}


Lateral Contact Position ( $\mathrm{mm}$ )
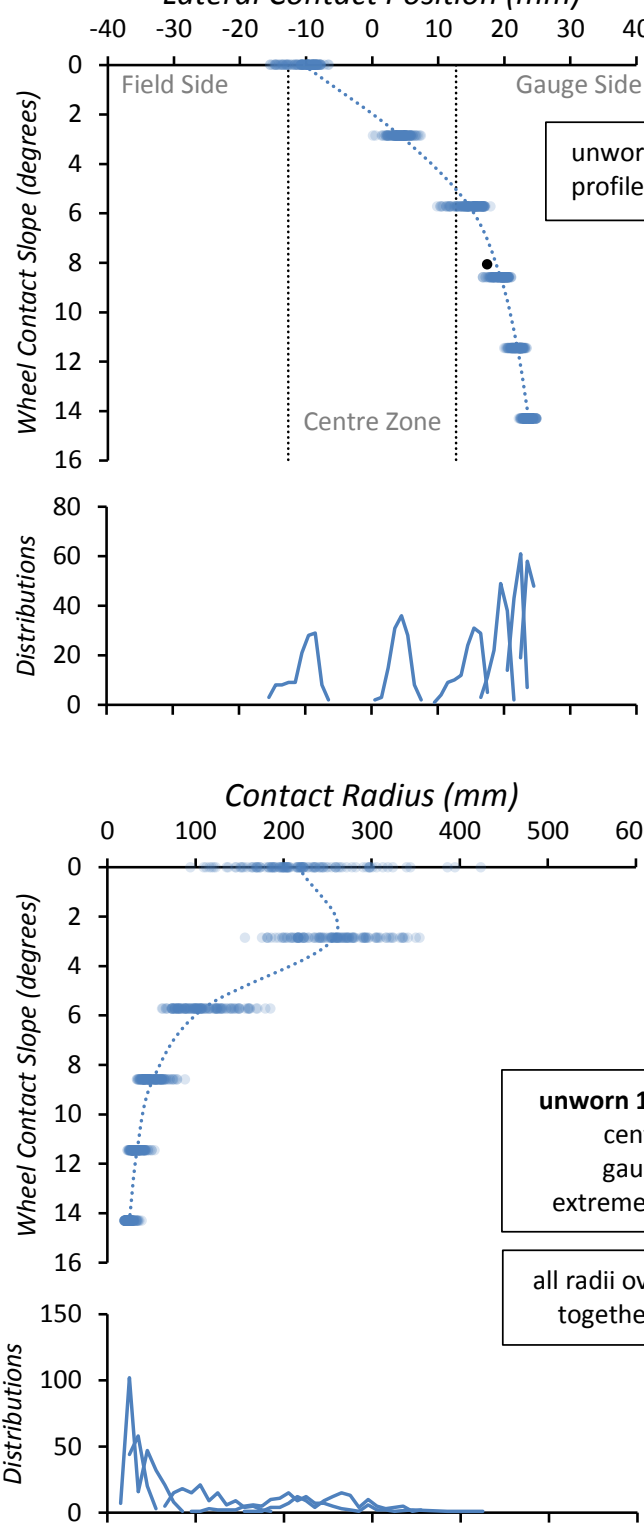

LEFT RAIL PROFILES

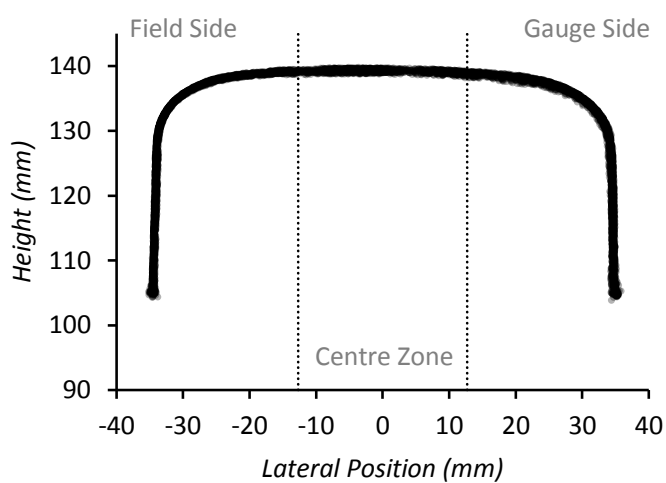

Lateral Contact Position ( $\mathrm{mm}$ )

$\begin{array}{lllllllll}40 & 30 & 20 & 10 & 0 & -10 & -20 & -30 & -40\end{array}$
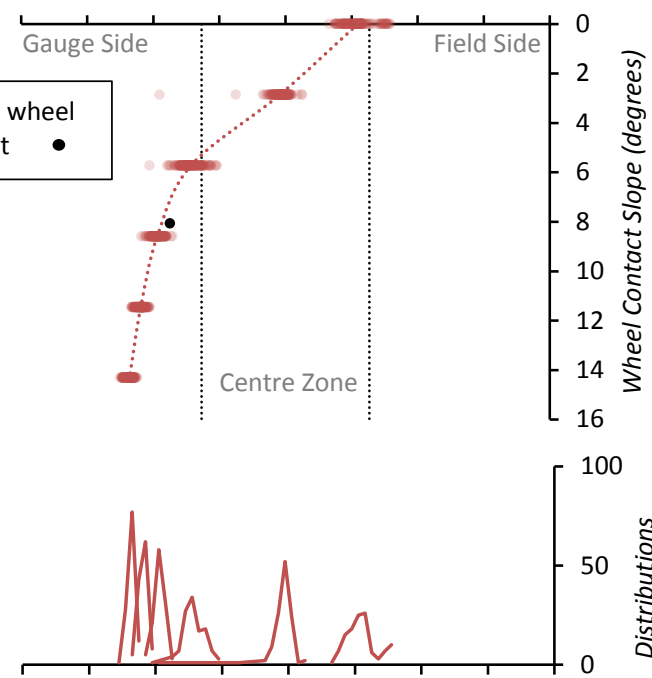

Contact Radius ( $\mathrm{mm}$ )
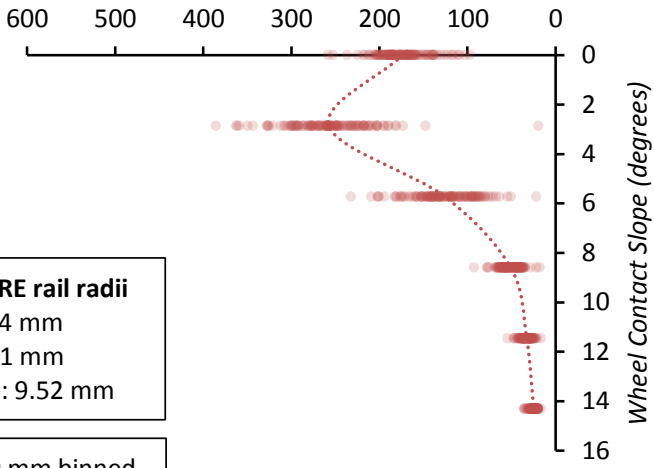

er: $254 \mathrm{~mm}$

uge: $38.1 \mathrm{~mm}$

eme gauge: $9.52 \mathrm{~mm}$

ributions

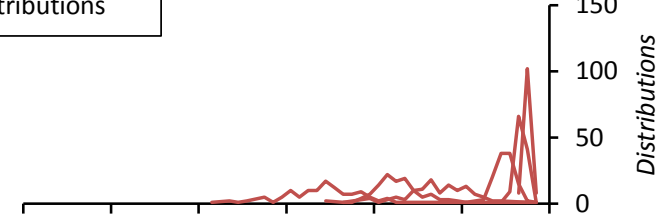


SEGMENT AND ANALYSIS INFORMATION

\begin{tabular}{llll}
\hline \hline Track Segment Name: & SB-90 & Contact Analysis Date: & 20180618 \\
Segment Length $(\mathrm{m}):$ & 144.6 & Left Wheel Slope: & $1: 20$ \\
Data Collection Date: & 20150827 & Right Wheel Slope: & $1: 20$
\end{tabular}

RAIL SEGMENT GRAPHS

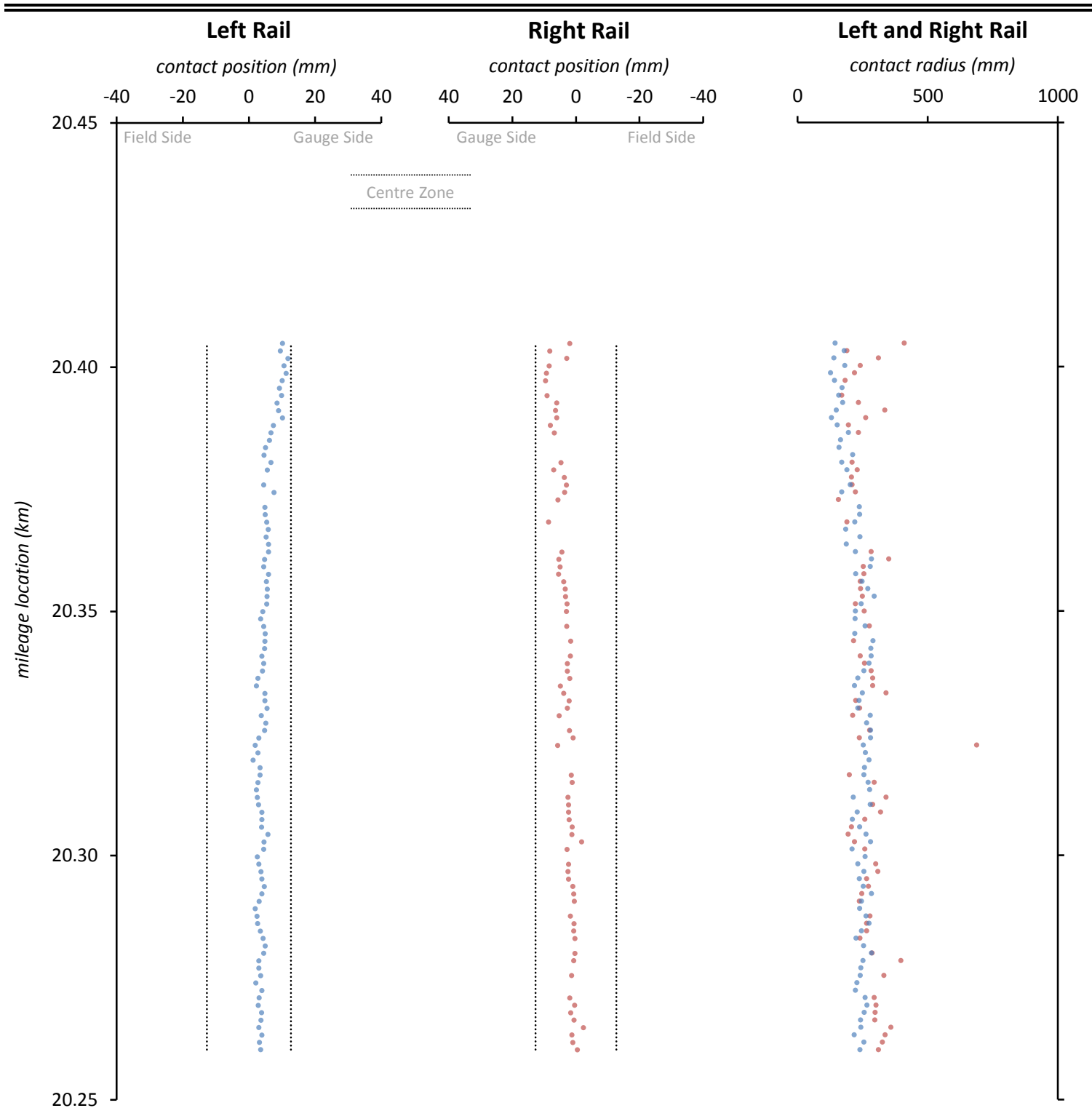

RAIL SEGMENT STATISTICS TABLE

\begin{tabular}{|c|c|c|c|c|}
\hline \multirow{3}{*}{ Statistic } & \multicolumn{4}{|c|}{ Rail Profile Measure } \\
\hline & \multicolumn{2}{|c|}{ Left Rail (94 profiles) } & \multicolumn{2}{|c|}{ Right Rail (73 profiles) } \\
\hline & Contact Position & Contact Radius & Contact Position & Contact Radius \\
\hline Mean $(\mathrm{mm})$ & 4.8 & 230.8 & 3.2 & 268.2 \\
\hline Std. Dev. $(\mathrm{mm})$ & 2.3 & 42.0 & 2.6 & 71.8 \\
\hline$\%$ within Zone & $100 \%$ & - & $100 \%$ & - \\
\hline
\end{tabular}


Lateral Contact Position ( $\mathrm{mm}$ )
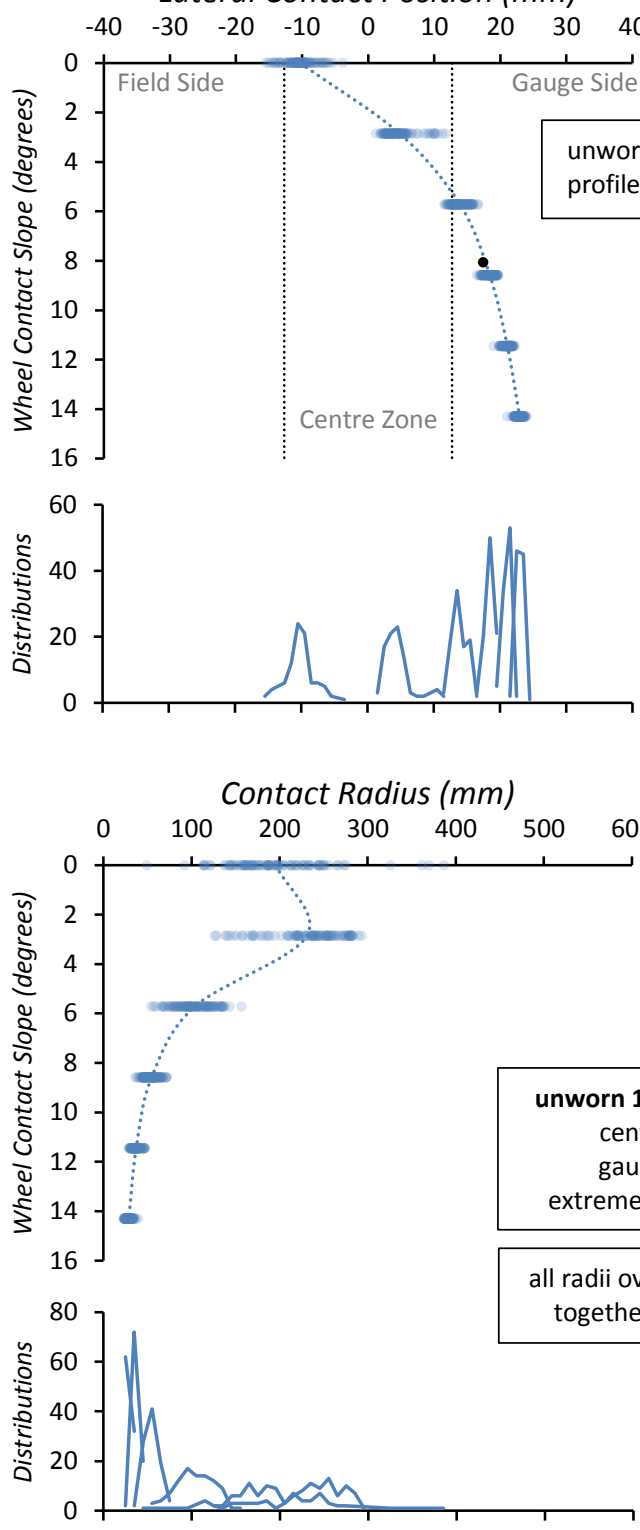

LEFT RAIL PROFILES

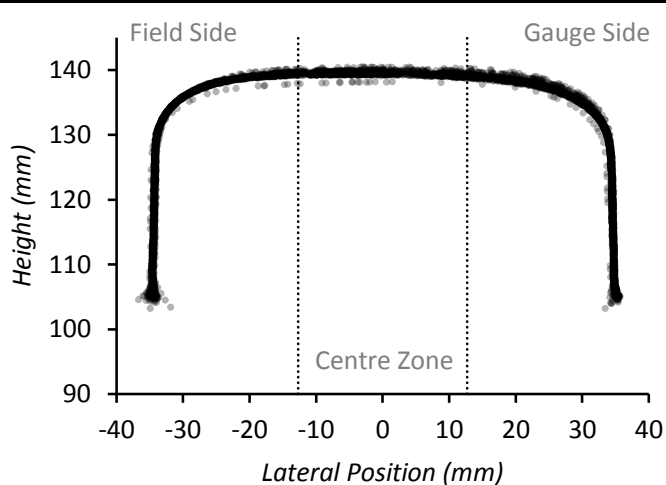

Lateral Contact Position ( $\mathrm{mm}$ )

$\begin{array}{lllllllll}40 & 30 & 20 & 10 & 0 & -10 & -20 & -30 & -40\end{array}$
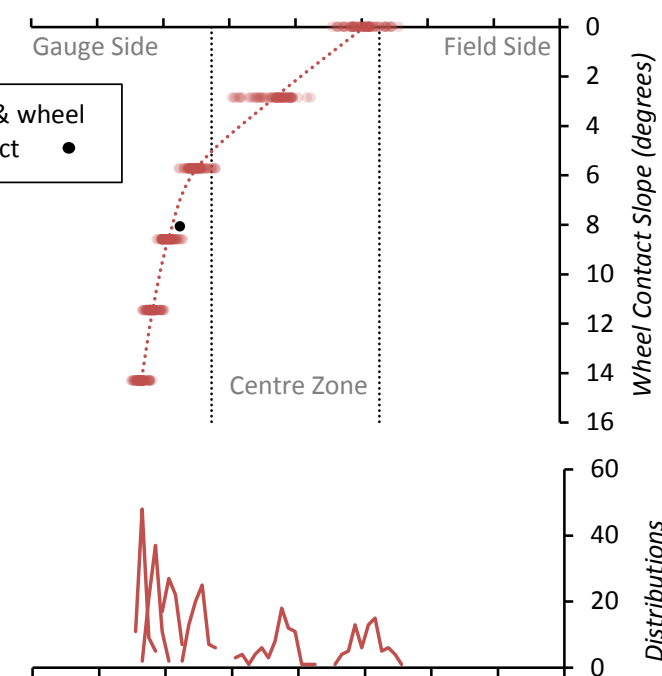

Contact Radius ( $\mathrm{mm}$ )
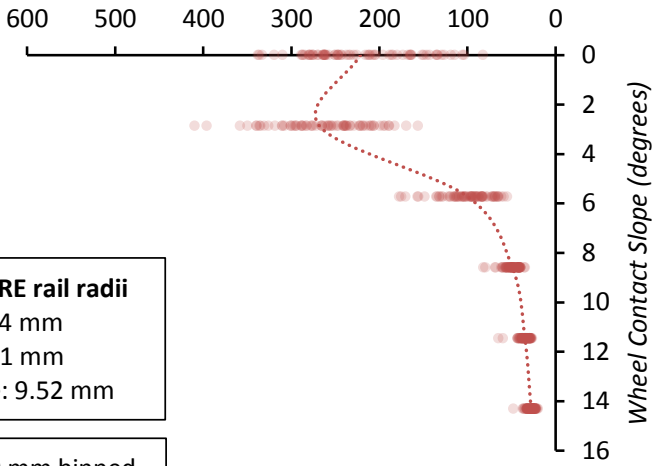

ter: $254 \mathrm{~mm}$

uge: $38.1 \mathrm{~mm}$

$600 \mathrm{~mm}$ binned

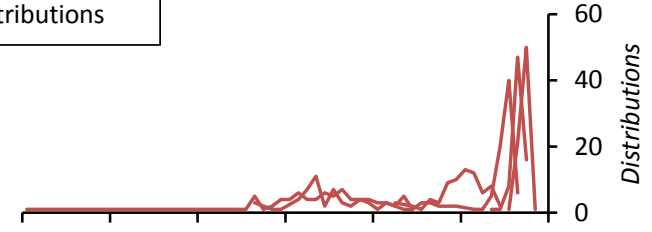

RIGHT RAIL PROFILES

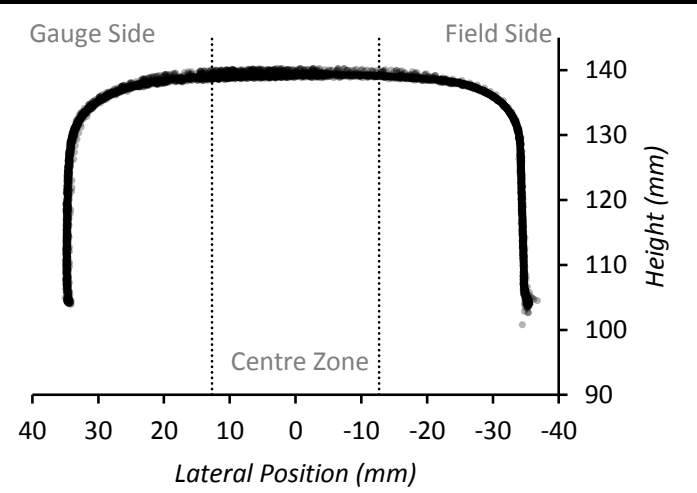

